OAK RIDGE NATIONAL LABORATORY

MARTUN MAGRETRA

\section{AMPX-77: A Modular Code System for Generating Coupled Multigroup Neutron-Gamma Cross-Section Libraries from ENDF/B-IV and/or ENDF/B-V}

\author{
N. M. Greene \\ W. E. Ford III \\ L. M. Petrie \\ J. W. Arwood
}


ORNL/CSD/TM--283

DE93 005750

\title{
AMPX-77: A MODULAR CODE SYSTEM FOR GENERATING COUPLED MULTIGROUP NEUTRON- GAMMA CROSS-SECTION LIBRARIES FROM ENDF/B-IV AND/OR ENDF/B-V
}

\author{
N. M. Greene, W. E. Ford III, L. M. Petrie, and J. W. Arwood \\ Computing and Telecommunications Division \\ at Oak Ridge National Laboratory \\ P.O. Box 2008 \\ Oak Ridge, Tennessee 37831-6370
}

Date Published: October 1992

\author{
Prepared for the \\ Office of Nuclear Energy \\ U.S. Department of Energy \\ Activity No. KK05022060
}
Prepared by
Martin Marietta Energy Systems, Inc.
managing the
Oak Ridge National Laboratory Oak Ridge Y-12 Plant
Oak Ridge K-25 Site Paducah Gaseous Diffusion Plant
for the
U.S. Department of Energy
under contract DE-AC05-84OR21400


This report has been reproduced directly from the best available copy.

Available to DOE and DOE contractors from the Office of Scientific and Technical Information, P.O. Box 62, Oak Ridge, TN 37831; prices available from (615) 576-8401, FTS 626-8401.

Available to the public from the National Technical Information Service, U.S. Depiartment of Commerce, 5285 Port Royal Rd., Springfield, VA 22161.

This report was prepared as an account of work sponsored by an agency of the United States Government. Neither the United States Government nor any agency thereof, nor any of their employees, makes any warranty, express or implied, or assumes any legal liability or responsibility for the accuracy, completeness, or usefulness of any information, apparatus, product, or process disclosed, or represents that its use would not infringe privately owned rights. Reference herein to any specific comrnercial product, process, or service by trade name, trademark, manufacturer, or otherwise, does not necessarily constitute or imply its endorsement, recommendation, or favoring by the United States Government or any agency thereof. The views and opinions of authors expressed herein do not necessarily state or reflect those of the United States Government or any agency thereof. 


\section{DEDICATION}

Much of the work and many of the ideas that are contained in the codes described in this report are due to the efforts of a dear colleague, James L. Lucius, who died in October 1987. Although he does not show as the primary author on very many of the modules (his primary work assignments were in areas external to AMPX), he worked on a large fraction of the AMPX modules at one time or another.

Certainly Jim's largest contributions came in the form of the several codes he developed in the 1960s, which served to prove the ideas that were later incorporated, either virtually intact, or in spirit, in the final AMPX codes. We sincerely miss his input in providing advice on ways to solve problems, but, more than this, we miss his daily camaraderie.

It is for these reasons that we dedicate this document in memory of our very dear friend. 


\section{PREFACE}

There is perhaps a plethora of documents to be located that lay claim to the intention of serving as a "living document," inherently implying an association with an effort that is expected to exist over a long period of time or even in perpetuity.

AMPX is an example of an effort of this type, though neither of the previous two documents that described the codes in the system were anything like living, and were constructed in such a manner as to make even simple updates and corrections very awkward. It is probably because of the lack of preplanning on the structure of the document that led to this situation, where only the "master copies" of the principal authors are anything like up-to-date, and these are certainly not maintained in a formal structure, but are loose-leaf collections of notes, both typed and handwritten.

There have been at least two or three aborted and abandoned efforts to produce a new user's guide for AMPX that would update the previous issue and contain current information, but in each of these cases the "living effort" ran away from the documentation.

The same situation still exists. At least two or three quite useful modules have been written since this document was frozen, and there are at least 10 to 15 older codes that exist in varying levels of completion, but that should (and probably will) be included in a later version of this user's guide.

In order to allow for an expandable document that will accommodate the aforementioned needs, we have "invented" a new page-numbering scheme that is certainly unconventional, but that we hope our users will find acceptable. We have adopted the practice of starting the information for a major section on an odd (right-hand) page, with the numbers in a section ranging from 1 to whatever number of pages is required for the section. In addition, each right-hand page will contain a one- or two-word title at the lower outside margin of the page, which we hope will alleviate the need for tabs or other artifices to make locating a particular section easier.

Since the primary information is users' information for almost 50 separate modules arranged in alphabetical order by code name, this should make locating information on a particular code quite easy. This arrangement will make adding a new code trivial, because it can be issued and merely inserted in its proper alphabetical location. Renumbering of any other section will not be required. Other changes will be limited to the Table of Contents and whatever part of the Introduction that might be affected. Furthermore, if a new sample problem is required to demonstrate the new module, it can be added at the end of that section without requiring much, if any change to other parts of the section.

In the event pages from a section, or a whole section, require modification, a reissue will list the date of the current issue under the module name at the bottom of the page. 


\section{CONTENTS}

ACKNOWLEDGMENTS . . . . . . . . . . . . . . . . . . . . . xix

ABSTRACT . . . . . . . . . . . . . . . . . . . . . . . . . . . .

1. INTRODUCTION . . . . . . . . . . . . . . . . . . . . . . . . . . . . 1

2. OVERVIEW OF AMPX . . . . . . . . . . . . . . . . . . . . . . . . . . . . . 1

2.OVERVIEW.1 AMPX CAPABILITIES . . . . . . . . . . . . . . . . . . . . . 2

2.OVERVIEW.1.1 Basic Data Processing . . . . . . . . . . . . . 3

2.0VERVIEW.1.2 Resonance Self-Shielding . . . . . . . . . . . . 3

2.OVERVIEW.1.3 Spectral Collapsing . . . . . . . . . . . . . . 3

2.OVERVIEW.1.4 Format Conversion . . . . . . . . . . . . . . 3

2.OVERVIEW.1.5 Service Functions . . . . . . . . . . . . . . . 4

3. INPUT INSTRUCTIONS FOR AMPX-77 MODULES . . . . . . . . . . . . . . . . . . 1

3.AIM-MODULE TO CONVERT MASTER CROSS-SECTION

LIBRARIES FROM BCD TO BINARY FORMAT

(OR VICE VERSA) . . . . . . . . . . . . . . . . . . . . . . . . 1

3.AlM.1.1 AlM Input Data . . . . . . . . . . . . . . . . . . . . . . 1

3.AIM.1.2 Scattering Matrix Structure . . . . . . . . . . . . . . . . . . . . . 8

3.AIM.1.3 AIM Input/Output Specifications . . . . . . . . . . . . . . . 11

3.AJAX-MODULE TO MERGE, COLLECT, ASSEMBLE, REORDER, JOIN, AND/OR COPY SELECTED DATA SETS

FROM AMPX MASTER INTERFACES . . . . . . . . . . . . . . . . 1

3.AJAX.1 AJAX Input Data . . . . . . . . . . . . . . . . . . . . . 1

3.AJAX.2 AJAX Input/Output Specifications . . . . . . . . . . . . . 2

3.ALE-MODULE TO LIST INFORMATION FROM MASTER

OR WORKING LIBRARIES . . . . . . . . . . . . . . . . . . . . . . 1

3.ALE.1 ALE Input Data . . . . . . . . . . . . . . . . . . . . . . . 1

3.ALE.2 ALE Input/Output Specifications . . . . . . . . . . . . . . . . 3

3.ALPO-MODULE FOR PRODUCING ANISN LIBRARIES FROM

AMPX WORKING LIBRARIES . . . . . . . . . . . . . . . . . . . . . 1

3.ALPO.1 ALPO Input Data . . . . . . . . . . . . . . . . . . . . . 1

3.ALPO.2 ALPO Input/Output Specifications . . . . . . . . . . . . . . 2

3.BONAMI-MODULE TO PERFORM BONDARENKO

RESONANCE SELF-SHIELDING . . . . . . . . . . . . . . . . . . . 1

3.BONAMI.1 BONAMI Input Data . . . . . . . . . . . . . . . . . 3

3.BONAMI.1.1 Notes on input . . . . . . . . . . . . . . 5

3.BONAMI.2 BONAMI Input/Output Specifications . . . . . . . . . . . 7

vii 
3.CASTROL-MODULE TO WRITE "TAB1" RECORDS WITH

CROSS SECTIONS INPUT FROM CARDS . . . . . . . . . . . . . 1

3.CASTROL.1 CASTROL Input Data . . . . . . . . . . . . . . . 1

3.CASTROL.2 CASTROL Input/Output Specifications . . . . . . . . 2

3.CLAROL-A MODULE TO REPLACE CROSS SECTIONS ON

AN AMPX MASTER INTERFACE . . . . . . . . . . . . . . . . . . 1

3.CLAROL.1 CLAROL Input Data . . . . . . . . . . . . . . 1

3.CLAROL.2 CLAROL Input/Output Specifications . . . . . . . . . 3

3.CLAROL.3 Special CLAROL Cases . . . . . . . . . . . . . . . . 3

3.CLAROL.3.1 Title card replacement . . . . . . . . . 3

3.CLAROL.3.2 Resonance parameter replacernent . . . . 4

3.CLAROL.3.3 Creating new cross-section arrays . . . . . 4

3.CLAROL.3.4 Special vector commands . . . . . . . . 4

3.CLAROL.3.5 Replacement of entries in

the master library directories . . . . . . 5

3.CLAROL.3.6 Replacement of values in

the Bondarenko reference

cross sections . . . . . . . . . . . . . 5

3.CLAROL.3.7 Replacement of Bondarenko

factor values . . . . . . . . . . . 5

3.COLLINS-MODULE TO COLLECT INELASTIC SCATTERING

INTO A SINGLE MATRIX . . . . . . . . . . . . . . . . . . . . . 1

3.COLLINS.1 COLLINS Input Data . . . . . . . . . . . . . . . . . 1

3.COLLINS.2 COLLINS Input/Output Specifications . . . . . . . . . 2

3.COMAND-MODULE TO COLLAPSE ANISN CROSS-SECTION

LIBRARIES . . . . . . . . . . . . . . . . . . . . . . . . . . . . 1

3.COMAND.1 COMAND Input Data . . . . . . . . . . . . . . . 1

3.COMAND.2 COMAND Input/Output Specifications . . . . . . . 2

3.COMAND.3 COMAND Special Notes . . . . . . . . . . . . . 3

3.COMET-MODULE TO FORCE CONSISTENCY BETWEEN

AVERAGED VALUES AND SCATTERING MATRICES

ON AMPX MASTER LIBRARIES . . . . . . . . . . . . . . . . . . 1

3.COMET.1 COMET Input Data . . . . . . . . . . . . . . . . . . . 1

3.COMET.2 COMET Input/Output Specifications . . . . . . . . . . . 2

3.CONTAC-MODULE TO PRODUCE ANISN OR CCCC

ISOTXS LIBRARIES FROM AMPX WORKING LIBRARIES . . . . . . 1

3.CONTAC.1 CONTAC Input Data . . . . . . . . . . . . . . . . . 1

3.CONTAC.2 CONTAC Input/Output Specifications . . . . . . . . . 3

3.CORECTOL-MODULE TO CONVERT PRE-AMPX-77

MASTER LIBRARIES TO THE AMPX-77 FORM . . . . . . . . . 1 
3.CORECTOL.1 CORECTOL Input Data . . . . . . . . . . . . 2

3.CORECTOL.2 CORECTOL Input/Output Specifications . . . . . 2

3.CREST-A MODULE TO COLLECT INFORMATION

FROM ENDF/B, MASTER, AND WORKING LIBRARIES . . . . . . . . . 1

3.CREST.1 CREST Input Data . . . . . . . . . . . . . . . . . . 1

3.CREST.2 CREST Input/Output Specifications . . . . . . . . . . . . . 2

3.FRESH-AMPX MODULE TO RENORMALIZE THERMAL

SCATTERING MATRICES .................... 1

3.FRESH.1 FRESH Input Data . . . . . . . . . . . . . . . . . . . . . 1

3.FRESH.2 FRESH Input/Output Specifications . . . . . . . . . . . . . 3

3.GERITOL-AMPX MODULE TO PLACE GRUUP

CONSTANTS INTO A "STRING" LIBRARY . . . . . . . . . . . . . 1

3.GERITOL.1 GERITOL Input Data . . . . . . . . . . . . . . . . 2

3.GERITOL.2 GERITOL Input/Output Specifications . . . . . . . . 3

3.ICE-MODULE TO MIX DATA ON A WORKING

LIBRARY TO PRODUCE MACROSCOPIC LIBRARIES . . . . . . . . . . 1

3.ICE.1 ICE Input Data . . . . . . . . . . . . . . . . . . . . . . . . . 1

3.ICE.2 ICE Input/Output Specifications . . . . . . . . . . . . . . . . . 5

3.JERGENS-MODULE TO GENERATE WEIGHT FUNCTIONS

AND TO COMBINE ENDF/B TAB1 RECORDS . . . . . . . . . . . 1

3.JERGENS.1 JERGENS Input Data . . . . . . . . . . . . . . . . . 1

3.JERGENS.2 JERGENS Input/Output Specifications . . . . . . . . 5

3.LAPHNGAS-MODULE FOR GENERATING MULTIGROUP SECONDARY

GAMMA-RAY PRODUCTION CROSS SECTIONS . . . . . . . . . 1

3.LAPHNGAS.1 LAPHNGAS Input Data . . . . . . . . . . . . . 1

3.LAPHNGAS.2 LAPHNGAS Input/Output Specifications . . . . . . 5

3.LAPHNGAS.3 LAPHNGAS Error Messages . . . . . . . . . . . 6

3.LAVA-AMPX MODULE TO MAKE AN AMPX WORKING

LIBRARY FROM AN ANISN LIBRARY . . . . . . . . . . . . . . . . 1

3.LAVA.1 LAVA Input Data . . . . . . . . . . . . . . . . . . . . . . 1

3.LAVA.2 LAVA Input/Output Specifications . . . . . . . . . . . . . . 3

3.MAD-MODULE TO MAKE CHANGES IN THE NUCLIDE

DIRECTORY ON A MASTER INTERFACE . . . . . . . . . . . . . . 1

3.MAD.1 MAD Input Data . . . . . . . . . . . . . . . . . . . . . . . 2

3.MAD.2 MAD Input/Output Specifications . . . . . . . . . . . . . . . 3

3.MAD.3 MAD Examples . . . . . . . . . . . . . . . . . . . . . . . 3

3.MALOCS-MODULE TO COLLAPSE AMPX MASTER

CROSS-SECTION LIBRARIES . . . . . . . . . . . . . . . . . . .

3.MALOCS.1 MALOCS Input Data . . . . . . . . . . . . . . . . . 1 
3.NITAWL-II-MODULE TO CONVERT MASTER LIBRARIES

TO WORKING LIBRARIES AND TO PERFORM

RESONANCE SELF-SHIELDING CALCULATIONS . . . . . . . . . 1

3.NITAWL-II.1 NITAWL-II Input Data . . . . . . . . . . . . . . . 2

3.NITAWL-II.2 NITAWL-II Input/Output Specifications . . . . . . . 5

3.NPTXS-MODULE TO PRODUCE POINT DATA FOR

RESONANCE NUCLIDES . . . . . . . . . . . . . . . . . . . . . . 1

3.NPTXS.1 NPTXS Input Data . . . . . . . . . . . . . . . . . . . . 1

3.NPTXS.2 NPTXS Input/Output Specifications . . . . . . . . . . . . 3

3.NPTXS.3 NPTXS Output Format . . . . . . . . . . . . . . . . . . . 3

3.PAL-MODULE TO PUNCH 1-D CROSS SECTIONS FROM

AMPX LIBRARIES . . . . . . . . . . . . . . . . . . . . . . . . . . . 1

3.PAL.1 PAL Input Data . . . . . . . . . . . . . . . . . . . . . . . . . 1

3.PAL.2 PAL Input/Output Specifications . . . . . . . . . . . . . . . . 1

3.PERFUME-MODULE TO CORRECT LEGENDRE

POLYNOMIAL FITS TO SCATTERING MATRICES . . . . . . . . . 1

3.PERFUME.1 PERFUME Input Data . . . . . . . . . . . . . . . . . 1

3.PERFUME.2 PERFUME Input/Output Specifications . . . . . . . 1

3.POLIDENT-MODULE TO PRODUCE POINT DATA FROM

RESONANCE DATA . . . . . . . . . . . . . . . . . . 1

3.POLIDENT.1 POLIDENT Input Data . . . . . . . . . . . . . . . 1

3.POLIDENT.2 POLIDENT Input/Output Specifications . . . . . . . 3

3.PRELL--MODULE TO PRODUCE AND MANIPULATE AN

ENERGY LIMITS LIBRARY . . . . . . . . . . . . . . . . . . . . . . 1

3.PRELl.1 PRELL Input Data . . . . . . . . . . . . . . . . . . . . . . 1

3.PRELL.2 PRELL Logical Assignments . . . . . . . . . . . . . . . . . 2

3.PRUDE-AMPX MODULE TO CREATE CROSS SECTIONS FOR

THE UNRESOLVED RESONANCE ENERGY REGION . . . . . . . . . . 1

3.PRUDE.1 PRUDE Input Data . . . . . . . . . . . . . . . . . . . . 2

3.PRUDE.2 PRUDE Input/Output Specifications . . . . . . . . . . . . . 3

3.RADE-MODULE TO CHECK AMPX MASTER CROSS-

SECTION LIBRARIES . . . . . . . . . . . . . . . . . . . . . . . . . 1

3.RADE.1 RADE Input Data . . . . . . . . . . . . . . . . . 1

3.RADE.2 RADE Input/Output Specifications . . . . . . . . . . . . 3 
3.ROLAIDS-MODULE FOR TREATING RESONANCE

SHIELDING IN MULTIREGION GEOMETRIES . . . . . . . . . . . . 1

3.ROLAIDS.1 ROLAIDS Input Data . . . . . . . . . . . . . . . . . 3

3.ROLAIDS.2 ROLAIDS Input/Output Specifications . . . . . . . . . . 6

3.ROLAIDS.3 ROLAIDS Error Messages . . . . . . . . . . . . . . . . 7

3.RUFFLES-MODULE TO CHANGE THE ENERGY GKOUP STRUCTURE

FOR AN ANISN OR XSDRNPM SCALAR FLUX TAPE . . . . . . . . . 1

3.RUFFLES.1 RUFFLES Input Data . . . . . . . . . . . . . . . . . 1

3.RUFFLES.2 RUFFLES Input/Output Specifications . . . . . . . . . . 2

3.SALVAGE-AMPX MODULE TO RECOVER USABLE PORTIONS

OF MASTER LIBRARIES WHICH ARE INCOMPLETE . . . . . . . . 1

3.SALVAGE.1 SALVAGE Input Data . . . . . . . . . . . . . . . . . 1

3.SALVAGE.2 SALVAGE Input/Output Specifications . . . . . . . . . . 1

3.SMILER-AMPX MODULE TO CONVERT NJOY GENDF FILES

TO AMPX MASTER LIBRARIES . . . . . . . . . . . . . . . . . . . 1

3.SMILER.1 SMILER Input Data . . . . . . . . . . . . . . . . . . . 1

3.SMILER.2 SMILER Logical Assignments . . . . . . . . . . . . . . . 2

3.SMILER.3 SMILER Procedures . . . . . . . . . . . . . . . . . . . 3

3.SMUG-MODLIE FOR GENERATING MULTIGROUP

FHOTON CROSS SECTIONS ................. . 1

3.SMUC. I SMUG Input Data . . . . . . . . . . . . . . . . . . . . . . 1

3.SMUG.2 SMUG Input/Output Specifications . . . . . . . . . . . . . . 3

3.SMUG.3 SMUG Error Messages . . . . . . . . . . . . . . . . . . . . 3

3.SMUG.4 SMUG Warning Messages . . . . . . . . . . . . . . . . . . . 4

3.TABU-AMPX MODULE TO CREATE BONDARENKO FACTORS FOR THE

UNRESOLVED RESONANCE ENERGY REGION IN ENDF/B . . . . . . . 1

3.TABU.1 TABU Input Data . . . . . . . . . . . . . . . . . . . . . 1

3.TABU.2 TABU Input/Output Specifications . . . . . . . . . . . . . . . 2

3.TIDE-MODULE TO CORRECT AN AMPX MASTER

LIBRARY DIRECTORY . . . . . . . . . . . . . . . . . . . . . 1

3.TIDE.1 TIDE Input Data . . . . . . . . . . . . . . . . . . . . . . 1

3.TIDE.2 TIDE Input/Output Specifications . . . . . . . . . . . . . . . . 1

3.UNITAB-AMPX MODULE TO MERGE SELECTED PORTIONS

OF MASTER LIBRARIES INTO HYBRID MASTER SETS . . . . . . . . 1

3.UNITAB.1 UNITAB Input Data . . . . . . . . . . . . . . . . . . . 1

3.UNITAB.2 UNITAB Input/Output Specifications . . . . . . . . . . . . 3

3.VASELINE-AMPX MODULE TO PLOT ENDF POINT AND

AMPX GROUP-AVERAGED CROSS SECTIONS . . . . . . . . . . . 1

3.VASELINE.1 VASELINE Input Data . . . . . . . . . . . . . . . . 1

3.VASELINE.2 VASELINE Input/Output Requirements . . . . . . . . 3

$\mathrm{xi}$ 
3.VEL-A MODULE FOR GROUP-AVERAGING POINT DATA . . . . . . . . . . . 1

3.VEL.1 VEL Input Data . . . . . . . . . . . . . . . . . . . . . . . . . 1

3.VEL.2 VEL Input/Output Specifications . . . . . . . . . . . . . . . . 2

3.WAX-MODULE TO MERGE, COLLECT, ASSEMBLE, REORDER, JOIN, COPY SELECTED NUCLIDESFROM AMPX WORKING LIBRARIES . . . . 1

3.WAX.1 WAX Input Data . . . . . . . . . . . . . . . . . . . . . . . . 1

3.WAX.2 WAX Input/Output Specifications . . . . . . . . . . . . . . 2

3.WINE-AMPX MODULE TO FORCE NORMALIZATION OF

SELECTED POR'TIONS OF AMPX WORKING LIBRARIES . . . . . . . . 1

3.WINE.1 WINE Input Data . . . . . . . . . . . . . . . . . . . . . 1

3.WINE.2 WINE Input/Output Specifications . . . . . . . . . . . . . . . 2

3.WISK-A MODULE TO CREATE A SENSITIVITY LIBRARY

FROM AN AMPX MASTER INTERFACE . . . . . . . . . . . . . . . . . 1

3.WISK.1 WISK Input Data . . . . . . . . . . . . . . . . . . . . . . . 1

3.WISK.2 WISK Input/Output Specifications . . . . . . . . . . . . . . . 2

3.WORKER-A MODULE TO CONVERT FROM AMPX MASTER

FORMAT TO AMPX WORKING FORMAT . . . . . . . . . . . . . . 1

3.WORKER.1 WORKER Input Data . . . . . . . . . . . . . . . . . 1

3.WORKER.2 WORKER Input/Output Specifications . . . . . . . . . 2

3.WORM-AMPX MODULE TO CONVERT AN AMPX WORKING LIBRARY TO AN AMPX MASTER LIBRARY . . . . . . . . . . . . . . 1

3.WORM.1 WORM Input Data . . . . . . . . . . . . . . . . . . . . . 1

3.WORM.2 WORM Input/Output Specifications . . . . . . . . . . . . . 1

3.XLACS-MODULE TO PRODUCE GROUP-AVERAGED NEUTRON CROSSSECTION LIBRARIES ON AN AMPX MASTER LIBRARY . . . . . . . . 1

3.XLACS.1 XLACS-77 Input Data . . . . . . . . . . . . . . . . . 1

3.XLACS.2 XLACS-77 Input/Output Specifications . . . . . . . . . . . . 6

3.XLACS.3 XLACS-77 Error Message . . . . . . . . . . . . . . . . . . 7

3.XLACS.4 XLACS-77 Procedures . . . . . . . . . . . . . . . . . . 10

3.XLACS.4.1 File 1 processing . . . . . . . . . . . . . . 10

3.XLACS.4.2 File 2 processing . . . . . . . . . . . . . . 10

3.XLACS.4.3 File 3 processing . . . . . . . . . . . . . . 12

3.XLACS.4.4 File 4 processing . . . . . . . . . . . . . . . . 12

3.XLACS.4.5 File 5 processing . . . . . . . . . . . . . . . 15

3.XLACS.4.6 File 6 processing . . . . . . . . . . . . . . . . 17

3.XLACS.4.7 File 7 processing . . . . . . . . . . . . . . . . 18

3.XLACS.4.8 Collecting data for the

AMPX master interface . . . . . . . . . . . . 19

3.XSDRNPM-MODULE WITH ONE-DIMENSIONAL $S_{n}$

CAPABILITY FOR SPATIAL WEIGHTING . . . . . . . . . . . . . . 1

xii 
3.XSDRNPM.1 Cross-Section Weighting Options . . . . . . . . . . . 1

3.XSDRNPM.1.1 "Cell" weighting . . . . . . . . . . 2

3.XSDRNPM.1.2 "Zone" weighting . . . . . . . . . . 2

3.XSDRNPM.1.3 "Region" weighting . . . . . . . . . 3

3.XSDRNPM.1.4 "Inner-cell" weighting . . . . . . . . 3

3.XSDRNPM.1.5 Multigroup weighting equations . . . . . . . . . . . . . 3

3.XSDRNPM.1.6 Transfer matrices . . . . . . . . . . 4

3.XSDRNPM.1.7 Weighting of $\bar{\nu}$. . . . . . . . . . . 5

3.XSDRNPM.1.8 Transport cross sections . . . . . . 183

3.XSDRNPM.2 XSDRNPM Input Data . . . . . . . . . . . . . . . 186

3.XSDRNPM.2.1 Abbreviated XSDRNPM input

description . . . . . . . . . . . 196

3.XSDRNPM.2.2 Special XSDRNPM files . . . . . . 21

3.XSDRNPM.2.2.1 Activity file . . 21

3.XSDRNPM.2.2.2 Balance table file 22

3.XSDRNPM.3 XSDPN:M input/Output Specifications . . . . . . . 22

3.XSDRNPM.4 Error virss. ges . . . . . . . . . . . . . . . . . . 23

3.ZEST-A MODULE TO MANAGE STRING LIBRARIES . . . . . . . . . . . . . . 1

3.ZEST.1 ZEST Input . . . . . . . . . . . . . . . . . . . . . . . . . . 1

3.ZEST.2 ZEST Input/Output Specifications . . . . . . . . . . . . . . . . 2

4. AMPX-77 MISCELLANEOUS USEFUL INFORMATION . . . . . . . . . . . . . . . . 1

4.INFORMATION.1 AMPX MASTER LIBRARY FORMAT . . . . . . . . . . . . . . 1

4.INFORMATION.2 AMPX WORKING LIBRARY FORMAT . . . . . . . . . . . . 14

4.INFORMATION.3 ANISN LIBRARY FORMAT . . . . . . . . . . . . . . . . . . . . . 17

4.INFORMATION.4 STANDARD ENERGY GROUP STRUCTURES . . . . . . . . 19

4.INFORMATION.4.1 "Standard" Neutron Boundaries . . . . . 20

4.INFORMATION.4.2 "Standard" Gamma Boundaries . . . . . 33

4.INFORMATION.5 AMPX WEIGH'TING FUNCTION LIBRARY . . . . . . . . . . 35

4.INFORMATION.6 REACTION TYPE IDENTIFIERS . . . . . . . . . . . . . . . . . . 35

4.INFORMATION.7 GLOSSARY OF TERMS . . . . . . . . . . . . . . . . . . . . . . . 42

4.INFORMATION.7.1 General Terms Used to Describe

Cross-Section Libraries . . . . . . . . . 42

4.INFORMATION.7.2 Discrete-Ordinate Terms . . . . . . . . 43

4.INFORMATION.7.3 ENDF Terms . . . . . . . . . . . . . 45

4.INFORMATION.7.4 Multigroup Cross-Section Terms . . . . . 47

4.INFORMATION.7.5 AMPX Format Terms . . . . . . . . . 48

4.INFORMATION.7.6 CCCC Format Terms . . . . . . . . . . 49

4.INFORMATION.7.7 Resonance Region and Temperature

Effects . . . . . . . . . . . . . . . 50

4.INFORMATION.7.8 Thermal Region . . . . . . . . . . . . 51

5. AMPX-77 INSTALlatiON GUIDE . . . . . . . . . . . . . . . . . . . . . . . . . 1

xiii 
5.ILLUSTRATION.1 CONSTRUCTION OF SUBROUTINE LIBRARY . . . . . . . . 3

5.ILLUSTRATION ' 1 IBM MVS Systems . . . . . . . . . . . 3

5.ILLUSTRATION.1.2 Cray UNICOS Systems . . . . . . . . . 3

5.ILLUSTRATION.2 AMPX DRIVER PROGRAM CONSTRUCTION . . . . . . . . . 3

5.ILLUSTRATION.2.1 IBM MV System . . . . . . . . . . . . 4

5.ILLUSTRATION.2.2 Cray UNICOS System . . . . . . . . . . 4

5.ILLUSTRATION.3 AMPX-77 MODULE CONSTRUCTION . . . . . . . . . . . . 4

5.ILLUSTRATION.3.1 IBM MVS System . . . . . . . . . . . 4

5.ILLUSTRATION.3.2 Cray UNICOS System . . . . . . . . . . 5

5.ILLUSTRATION.4 EXECUTING A MODULE . . . . . . . . . . . . . . . . . . 5

5.ILLUSTRATION.4.1 IBM MVS Systems . . . . . . . . . . . 5

5.ILLUSTRATION.4.1.1 Adding or modifying

a module using

the IBM procedure 7

5.ILLUSTRATION.4.2 Cray UNICOS System . . . . . . . . . 8

6. SAMPLE PROBLEMS . . . . . . . . . . . . . . . . . . . . . . . . . . . 1

6.SAMPLE PROBLEMS.1 CREATE A MULTIGROUP NEUTRON LIBRARY . . . . . 1

6.SAMPLE PROBLEMS.2 CREATE A MUITIGROUP COUPLED NEUTRON-

GAMMA-RAY LIBRARY . . . . . . . . . . . . . . . 58

6.SAMPLE PROBLEMS.3 PERFORM A RESONANCE SELF-SHIELDING

CALCUlation . . . . . . . . . . . . . . . . . . 81

6.SAMPLE PROBLEMS.3.1 Sample Resonance Calculation by ROLAIDS . . . . . . . 82

6.SAMPLE PROBLEMS.3.2 Sample Resonance Calculation by NITAWL . . . . . . . . 82

6.SAMPLE PROBLEMS.3.3 Sample Resonance Calculation by BONAMI . . . . . . . . 82

6.SAMPLE PROBLEMS.4 HOMOGENIZE AND COLLAPSE CROSS SECTIONS . . 108

6.SAMPLE PROBLEMS.4.1 Homogenization of Cross

Sections . . . . . . . 108

6.SAMPLE PROBLEMS.4.2 Collapsing the 238-Group

Library to 25 Groups . . . . 110

6.SAMPLE PROBLEMS.5 MOVE MULTIGROUP LIBRARIES TO ANOTHER.

COMPUTING SYSTEM . . . . . . . . . . . . . . . 135

6.SAMPLE PROBLEMS.6 AVERAGE POINT CROSS SECTIONS . . . . . . . . . 145

6.SAMPLE PROBLEMS.7 CONVERT THE CROSS SECTIONS ON AN AMPX

MASTER LIBRARY FOR USE IN THE

ANISN PROGRAM . . . . . . . . . . . . . . . . . . . . . 158

7. FIDO (ANISN) INPUT SYSTEM . . . . . . . . . . . . . . . . . . . . . . . . 1

7.FIDO.1 FIXED-FIELD INPUT . . . . . . . . . . . . . . . . . . . 1

7.FIDO.2 FREE-FIELD INPUT . . . . . . . . . . . . . . . . . . . . . . . . . . 5

$7 . F I D O .3$ USER-FIELD INPUT . . . . . . . . . . . . . . . . . . . . . . . . . . 6 


\section{LIST OF FIGURES}

Figure

Page

5.ILLUSTRATION.1 IBM procedure to execute AMPX-77 . . . . . . . . . . . . 6

6.SAMPLE PROBLEMS.1 Primary neutron library production procedure . . . . . . . 4

6.SAMPLE PROBLEMS.2 Procedure to add Bondarenko factors to an AMPX master . 5

6.SAMPLE PROBLEMS.3 Procedure to perform $1 / \mathrm{E} \Sigma_{t}$ weighting . . . . . . . . . . 6

6.SAMPLE PROBLEMS.4 Procedure to combine AMPX masters . . . . . . . . . . . 7

6.SAMPLE PROBLEMS.5 Procedure to produce a coupled neutron-gamma library . . 59

6.SAMPLE PROBLEMS.6 Hypothetical reactor lattice . . . . . . . . . . . . . . . 81

6.SAMPLE PROBLEMS.7 Wigner-Seitz cell approximation . . . . . . . . . . . . . 81

6.SAMPIE PROBLEMS.8 Procedure to use ROLAIDS for resonance self-shielding . . . 83

6.SAMPLE PROBLEMS.9 Procedure to use NITAWL for resonance self-shielding $\quad$. . $\quad 84$

6.SAMPLE PROBLEMS.10 Procedure to use BONAMI for resonance self-shielding . . 84

6.SAMPLE PROBLEMS.11 Hypothetical system for demonstrating Task 4 . . . . . . 108

6.SAMPLE PROBLEMS.12 Procedure for homogenizing cross sections . . . . . . . . 109

6.SAMPLE PROBLEMS.13 Procedure to collapse cross sections . . . . . . . . . . . 110

6.SAMPLE PROBLEMS.14 Procedure for moving an AMPX master to a new computer 136

6.SAMPLE PROBLEMS.15 Procedure to weight a point cross-section array . . . . . . 146

6.SAMPLE PROBLEMS.16 Procedure to make ANISN cross sections . . . . . . . . 159 


\section{LIST OF TABLES}

Table

Page

1.INTRODUCTION.1 AMPX modules . . . . . . . . . . . . . . . . . . . . . 3

6.SAMPLE PROBLEMS.1 ENDF/B "standard" library . . . . . . . . . . . . . . . . 3 


\section{ACKNOWLEDGMENTS}

Many individuals have contributed to the AMPX system. Authors of the individual modules are identified at the front of Sect. 3.INSTRUCTIONS of this document.

This, the third, complete release of the system has been especially frustrating and difficult because of the lack of adequate funding and personnel support to prepare the requisite programs, distribution files, and documentation in a timely fashion. Perhaps the most frustrating part was the wait for a satisfactory copy of this user's guide, which was drafted in 1988. In this regard, the authors are sincerely appreciative of Lindy Norris, who took the collection of very rough output from attempts to generate a useable draft and produced this document. Her stamina, fortitude, accuracy, and attitude are all highly commendable.

And, finally, the authors are grateful for the several projects that provided the support necessary to complete this task. 


\section{ABSTRACT}

AMPX-77 is a modular system of computer programs that pertain to nuclear analyses, with a primary emphasis on tasks associated with the production and use of multigroup cross sections. All basic cross-section data are to be input in the formats used by the Evaluated Nuclear Data Files (ENDF/B), and output can be obtained in a variety of formats, including its own internal and very general formats, along with a variety of other useful formats used by major transport, diffusion theory, and Monte Carlo codes. Processing is provided for both neutron and gamma-ray data.

The present release contains codes all written in the FORTRAN-77 dialect of FORTRAN and will process ENDF/B-V and earlier evaluations, though major modules are being upgraded in order to process ENDF/B-VI and will be released when a complete collection of usable routines is available. 


\section{INTRODUCTION}

The AMPX system has been in continuous development at the Oak Ridge National Laboratory (ORNL) since the early 1970s. It is a collection of computer programs in a modular arrangement for generating multigroup or pointwise cross-section libraries. Starting with ENDF/B-IV- or ENDF/B-V-formatted nuclear data files, ${ }^{1-8 *}$ or with previously generated, pseudo-problem-independent fine-group, problem-dependent fine- or broad-group, or pointwise cross-section libraries, ${ }^{\dagger}$ the system includes a full range of features needed to: (1) produce multigroup neutron, gamrna-ray production, and/or gamma-ray interaction cross-section data; (2) resonance self-shield; (3) spectrally collapse; (4) convert cross-section libraries from one format to another format; (5) execute a one-dimensional (1-D) discrete-ordinates calculation; and (6) perform miscellaneous cross-section operations (list, punch, plot, check, etc.). AMPXproduced cross sections can be used with radiation transport codes and systems, such as ANISN,${ }^{10}$ DOT, ${ }^{11}$ FORSS, ${ }^{12}$ KENO IV,${ }^{13}$ MORSE, ${ }^{14}$ SCALE, ${ }^{15}$ SWANLAKE, ${ }^{16}$ XSDRN,${ }^{17}$ and VENTURE. ${ }^{18-19}$

The AMPX system was initially issued by the Radiation Shielding Information Center (RSIC) in October 1973 as the PSR-63/AMPX package. ${ }^{20}$ The package included sample problems and an AMPX user's guide. ${ }^{21}$ To incorporate modifications to the original AMPX modules and to add new modules, a second generation of the AMPX system (the "IBM standard version") was packaged and issued by RSIC in late 1978 as PSR-63/AMPX-II. ${ }^{22}$

Again, to incorporate literally hundreds of modifications to AMPX modules (including modifications to process ENDF/B-V data, to delete obsolete modules, and to add new modules) the third generation of the IBM version of the AMPX system was packaged and issued by RSIC as PSR-63/AMPX-77. This "User's Guide" is the documentation for AMPX-77. The designation "77" was chosen because all modules in this release are in FORTRAN-77, thereby making it easier to use AMPX on different computers.

A list of the modules included in the AMPX system and a brief statement of the purpose of each module are given in Table 1. In addition to the modules, AMPX-77 consists of subroutine libraries, sample problems, data libraries, etc. An overview of the AMPX system is given in Ser.. 2.OVERVIEW. Input instructions for the AMPX modules are given in Sect. 3.INSTRUCTIONS. A catch-all sec-

*ENDF/B-IV and ENDF/B-V data are distributed by the National Nuclear Data Center, Brookhaven National Laboratory, Upton, N.Y. ENDF/B-formatted LENDL, ${ }^{5,6}$ DNA $^{7}$ and HPICE ${ }^{8}$ data are distributed by the Radiation Shielding Information Center (RSIC), Oak Ridge National Laboratory, Oak Ridge, Tennessee.

${ }^{\dagger}$ Many previously generated cross-section libraries that can be used with the AMPX-III system are distributed by RSIC. A capsule review of the RSIC data library collection is given in ref. 9 .

INTRODUCTION 
tion that contains detailed descriptions of various AMPX-77 features is given in Sect. 4.MISCELLANEOUS.

Selected AMPX-77 modules were used in a series of sample problems that were designed to illustrate how to execute the modules. AMPX becomes a system when the DRIVER program, the modules, the subroutine libraries, the data libraries, and the $\mathrm{JCL}$ for scratch and interface devices are incorporated into a procedure. An installation guide for AMPX-77 is given in Sect. 5.INSTALLATION. Several sample problems are described in Sect. 6.SAMPLE PROBLEMS. Finally, the FIDO (ANISN) data input system, which is used throughout the AMPX system, is described in Sect. 7.FIDO. 
Table 1. AMPX modules

Module Purpose

AIM

AJAX

Converts master cross-section libraries from binary coded decimal (BCD) to binary format (or vice-versa). The BCD format is a card-image format intended primarily for passing AMPX master libraries between different computing systems.

Merges, collects, reorders, joins, and/or copies selected data sets from AMPX master interfaces. This module is used to collect data sets from multiple AMPX master libraries to a single AMPX master library.

ALE Produces listings of data from AMPX master and working interfaces. This module is an upgrade to the DIAL module.

ALPO Produces ANISN libraries from AMPX master libraries.

ARID

Obsolete. Use the COMAND module.

AVAL

Obsolete. Use the LAVA module.

BONAMI Performs Bondarenko resonance self-shielding calculation.

BRUT

Obsolete. Use the PRUDE and TABU modules.

CASTROL Creates a library of point cross sections in ENDF/B "TAB1" format. The cross sections are specified in a card-image format.

CHOX Obsolete. Use the UNITAB module.

CHOXM Obsolete.

CLAROL Allows for replacing and correcting individual terms on an AMPX master library. For example, it will allow overriding group-averaged values, nuclide titles, resonance parameters, etc.

COLLINS Sums the cross sections for multiple inelastic levels into a single process $(\mathrm{MT})=4$ matrix and discards the level cross sections, thereby making the library more compact.

COMAND Collapses ANISN cross-section libraries.

COMET Allows one to force consistency between averaged cross sections and transfer matrices on AMPX master libraries.

CONTAC Produces ANISN or CCCC ISOTXS libraries from AMPX working libraries.

CORECTOL Converts from structure used for resonance background arrays associated with the Nordheim treatment to the structure used by NITAWL-2.

CREST Collects "TAB1" records from an arbitrary number of sources.

DIAL Obsolete. Use the ALE module.

DOSE Obsolete. Use the JERGENS, CASTROL, and VEL modules.

FRESH Normalizes thermal matrices to account for several situations. 
Table 1. (continued)

\begin{tabular}{ll}
\hline Module & \multicolumn{1}{c}{ Purpose } \\
\hline GERITOL & $\begin{array}{l}\text { Processes group-integrated fluxes into energy flux pairs using least- } \\
\text { squares fitting techniques, and writes the function in a "TAB1" record } \\
\text { format. }\end{array}$
\end{tabular}

ICE Mixes cross sections on an AMPX working library and produces a macroscopic AMPX working library, ANISN libraries, or group-independent ANISN cross-section libraries.

JERGENS Performs arithmetic operations with "TAB1" records.

LAPHNGAS Generates multigroup secondary gamma-ray production cross sections and/or multiplicities from ENDF/B point data. The output is an AMPX master library.

LAVA Produces an AMPX working library from an ANISN cross-section library.

MAD Modifies entries in the directory records which describe the cross sections for nuclides on an AMPX master library.

MALOCS Collapses fine-group AMPX master libraries into broad-group AMPX master libraries.

NITAWL-II Converts from the AMPX master library format to an AMPX working library. Optionally, it will perform a Nordheim integral treatment calculation for nuclides with resonance parameters.

NPTXS Prepares point cross sections for the MT $=1,2,18$, and 102 reactions for resonance materials on an ENDF/B library. The cross sections are written in "TAB1" format for use in other AMPX modules, such as JERGENS, VEL, VASELINE, etc.

OCTAGN Obsolete.

PAL Punches group-averaged values from either an AMPX master or an AMPX working library.

PERFUME Adjusts the Legendre coefficients used to present group-to-group scattering terms on an AMPX master library so that they are physically reasonable values.

POLIDENT Prepares a point library in "TAB1" format for nuclides on an ENDF/B library. Resonarice nuclides are processed into energy cross-section pairs. All processes with MT values less than 150 are passed.

PRELL Creates, copies, modifies, punches, or lists from a library of energy group structures.

PRUDE Uses ENDF/B unresolved resonance data to produce a file used by the TABU module to create Bondarenko factors.

RADE Used to perform checks on AMPX master, AMPX working, and ANISN libraries. Checks are made to ensure values are reasonable and sum properly, etc. 
Table 1. (continued)

Module Purpose

ROLAIDS Performs an integral transport calculation for the energy-pointwise, slowing-down flux distribution in each zone of a 1-D, multiregion geometry to produce multigroup resonance-shielded constants to be folded into an existing AMPX master data set.

RUFFLES Takes the scalar fluxes output by ANISN or XSDRN on a binary file and allows for summing and/or splitting them into another group structure to produce a second binary scalar flux file. This program is used primarily to quickly produce starting flux guesses for a transport calculation.

SALVAGE Salvages readable portions of an AMPX master library tape that has gone bad.

SMILER Produce AMPX master libraries from NJOY GENDF files.

SMUG Generates a photon-interaction master library from ENDF/B data.

TABU Produces an AMPX master cross-section file which contains Bondarenko factor data for the unresolved energy region. TABU requires an input file from the PRUDE module.

TIDE Scans an AMPX master library and keeps track of attributes, such as maximum array length, etc., in order to rewrite the library with proper directory entries for each nuclide.

UNITAB Combines data for a nuclide from more than one AMPX master library to create a new AMPX master library. For example, it serves (as CHOX did) to create a coupled neutron-gamma library containing the union of data taken from AMPX masters written by XLACS-77, LAPHNGAS, and SMUG.

VASELINE Produces plots of cross sections from ENDF/B libraries or from AMPX master or working libraries.

VEL Uses data from libraries written in ENDF/B "TAB1" format (both cross sections and weighting functions) to form group-averaged values that are written onto an AMPX master library.

WAX Collects data from one or more AMPX working libraries to create a new working library. (It is equivalent to the AJAX module used for master libraries.)

WINE Forces scattering matrices and 1-D cross sections to normalize properly.

WISK Produces an AMPX working library that contains a separate set of data for each nuclear process for all nuclides on the library structured as is needed by a cross-section sensitivity analysis.

WORKER Produce a working library from a master library. Provides a capability to interpolate between scattering matrices at different temperatures. 
Table 1. (continued)

Module Purpose

WORM Reads an AMPX working library and reformats it to write an AMPX master library, thereby making it possible to use various AMPX service modules (for example, CLAROL) with data from a working library.

XLACS-77 Uses ENDF/B data to produce multigroup neutron cross sections on an AMPX master library.

XSDRNPM Performs a 1-D discrete-ordinates or diffusion theory calculation using cross sections on an AMPX working library. It can, on option, collapse its cross sections to produce a weighted library.

ZEST Merges, collects, assembles, reorders, joins, and/or copies libraries written in the ENDF/B "TAB1" format. (As such, it performs the same service for the libraries as does AJAX for master libraries and WAX for working libraries.) 


\section{REFERENCES}

1. D. Garber et al., Data Formats and Procedures for the Evaluated Nuclear Data File, ENDF, BNL/NCS-50496 (ENDF 102), Brookhaven Natl. Lab., October 1975. Formats and procedures for ENDF/B-IV.

2. D. Garber, ENDF/B Summary Documentation, BNL-17541 (ENDF 201), Brookhaven Natl. Lab., October 1975. Summary documentation for ENDF/B-IV.

3. R. Kinsey, Ed., ENDF-102, Data Formats and Procedures for the Evaluated Nuclear Data File, ENDF, BNL/NCS-50496 (ENDF 102), 2nd Ed. (ENDF/B-V), Brookhaven Natl. Lab., October 1979.

4. R. Kinsey, Ed., ENDF-201, ENDF/B Summary Documentation, BNL/NCS-17541 (ENDF 201), 3rd Ed. (ENDF/B-V), Brookhaven Natl. Lab., July 1979.

5. R. J. Howerton, The Lawrence Livermore Evaluated Nuclear Data Library (ENDL) Translated Into the ENDF/B Format, UCID-16727, University of California, March 1975.

6. R. J. Howerton, The LLL Evaluated Nuclear Data Library (ENDL): Translation of ENDL Neutron-Induced Interaction Data Into the ENDF/B Format, UCRL-50400, Vol. 15, Part C, University of California, 1976.

7. R. W. Roussin and J. B. Wright, Defense Nuclear Agency Working Cross-Section Library: Description and Contents, ORNL/RSIC-34, Vol. I, Union Carbide Corp., Nucl. Div., Oak Ridge Natl. Lab., June 1974.

8. HPICE, Evaluated Photon Interaction Library ENDF/B File 23 Format, RSIC Data Library Collection DLC-7, March 9, 1978.

9. RSIC Computer Code and Data Collections, A Capsule Review of the Computer Code Collection (CCC), Peripheral Shielding Routines (PSR), and Data Library Collection ( $D L C$ ), RSIC document, Union Carbide Corp., Nucl. Div., Oak Ridge Natl. Lab. (Revised January 1982).

10. W. W. Engle, Jr., A User's Manual for ANISN, A One-Dimensional Discrete Ordinates Transport Code with Anisotropic Scattering, K-1693, Union Carbide Corp., Nucl. Div., Oak Ridge Gaseous Diffusion Plant, March 1967.

11. W. A. Rhoades and R. L. Childs, An Updated Version of the DOT IV One- and Two-Dimensional Neutron/Photon Transport Code, ORNL-5851, Union Carbide Corp., Nucl. Div., Oak Ridge Natl. Lab., July 1982.

12. J. L. Lucius, C. R. Weisbin, J. H. Marable, J. D. Drischler, R. Q. Wright, and J. E. White, $A$ User's Manual for the FORSS Sensitivity and Uncertainty Analysis Code System, ORNL-5316 (ENDF-291), Union Carbide Corp., Nucl. Div., Oak Ridge Natl. Lab., January 1981.

13. L. M. Petrie and N. F. Cross, KENO IV: An Improved Monte Carlo Criticality Program, ORNL-4938, Union Carbide Corp., Nucl. Div., Oak Ridge Natl. Lab., November 1975. 
14. M. B. Emmett, The MORSE Monte Carlo Radiation Transport Code System, ORNL-4972, Union Carbide Corp., Nucl. Div., Oak Ridge Natl. Lab., February 1975.

15. SCALE: A Modular Code System for Performing Standardizcd Computer Analyses for Licensing Evaluation, Sect. C4 of NUREG/CR-0200, Rev. 4, Vol. 1 (Draft, February 1990). Available from Radiation Shielding Information Center as CCC545.

16. D. E. Bartine, F. R. Mynatt, and E. M. Oblow, SWANLAKE, A Computer Code Utilizing ANISN Radiation Transport Calculations for Cross-Section Sensitivity Analysis," ORNL-TM-3809, Union Carbide Corp., Nucl. Div., Oak Ridge Natl. Lab., May 1973.

17. N. M. Greene and C. W. Craven, Jr., XSDRN: A Discrete Ordinates Spectral Averaging Code, OKNL/TM-2500, Union Carbide Corp., Nucl. Div., Oak Ridge Natl. Lab., July 1969.

18. D. R. Vondy, T. B. Fowler, and G. W. Cunningham, VENTURE: A Code Block for Solving Multigroup Neutronics Problems Applying the Finite-Difference DiffusionTheory Approximation to Neutron Transport, Version II, ORNL-5062/R1, Union Carbide Corp., Nucl. Div., Oak Ridge Natl. Lab., November 1977.

19. D. R. Vondy. T. B. Fowler, and G. W. Cunningham, III, The Bold Venture Computation System for Nuclear Reacior Code Analysis, Version III, ORNL-5711, Union Carbide Corp., Nucl. Div., Oak Ridge Natl. Lab., June 1981.

20. AMPX-I, Modular Code System for Generating Coupled Multigroup NeutronGamma Libraries from $E N D F / B$, RSIC documentation for PSR-63, circa 1973.

21. N. M. Greene, J. L. Lucius, L. M. Petrie, W. E. Ford, III, J. E. White, and R. Q. Wright, AMPX: A Modular Code System for Generating Coupled Multigroup Neutron-Gamma Libraries from ENDF/B, ORNL/TM-3706, Union Carbide Corp., Nucl. Div., Oak Ridge Natl. Lab., March 1976.

22. AMPX.II, Modular Code System for Generating Coupled Multigroup Neutron Gamma-Ray Cross-Section Libraries From Data in ENDF Format, RSIC documentation for PSR-63, November 30, 1978.

\section{INTRODUCTION}




\section{OVERVIEW OF AMPX}

In early 1971, an effort was initiated to build a system of computer programs capable of producing multigroup cross sections. This work was funded by the Defense Nuclear Agency (DNA), which had a particular interest in producing coupled neutrongamma cross sections for use in weapons shielding and effects studies. The system was named AMPX, which is an acronym for Automation of MUG, ${ }^{1}$ POPOP $4,{ }^{2}$ and $\underline{X} L A C S,{ }^{3}$ three codes which were then in use at ORNL to generate multigroup gamma-ray interaction, gamma-ray production, and neutron interaction cross sections, respectively.

The production of multigroup cross sections has always been a time-consuming and complicated process. It uses its own jargon (e.g., ENDF/B, self-shielding, $S_{\alpha, \beta}, P_{\ell}$ matrices, transport corrections, etc.). (A glossary of terms used to describe multigroup cross-section processing is given in Sect. 4.MISCELLANEOUS.7.) The selection of the most appropriate treatment from a wide variety of algorithms is a challenge even for the experienced cross-section producer.

Producing multigroup coupled neutron-gamma cross sections involves several timeconsuming and expensive steps, many of which are not independent of each other and which must be performed in a certain order. An error in any step can mean a repetition of the entire sequence. It is clearly an area where stand-alone programs with all their awkward and labor-intensive data preparation procedures create an undesirable situation.

A better way of attacking the problem involves the use of a modular system that encompasses the complete collection of codes. In this system, one can set up the entire sequence, if necessary, thereby making it much easier to keep track of calculations or to repeat them.

In a well-defined modular system, there is little redundancy. Important functions are isolated into modules that are executed when needed. It is easier to maintain the system because programming for a function is only needed in one place. New modules are easier to write because they only have programming for new functions.

A key consideration for the modular system is that of communicating between the modules. It is absolutely imperative that major collections of data which must be transferred between modules be done in a manner that does not burden the user with large entries of data.

Considerations such as those cited above influenced the design of our AMPX system. Even though our present system does not totally satisfy all the goals, it is certainly much easier to use than was the previous situation.

Other considerations that went into the original system design are listed below. Even though some of these will seem either obvious or unnecessary in today's computing environment, they were made to be used with computing systems available in the early 1970s.

- All processing codes that need basic data use ENDF/B data.

- All codes use variable dimensioning, which means that the program assigns only the necessary space from a large container array for the problem being run. This was done both to conserve computer core and to keep from having to make "dimension" 
changes in the programs. Although the amounts of storage easily obtained on today's virtual systems make it possible to allocate generous array dimensions and to let the system take care of shuttling data into core, the variable dimensioning technicues a e still being used to good advantage, especially in situations where the computer cost. algorithms take account of the amount of core requested.

- Codes write their multigroup cross-section data using a well-defined format capable of handling any and all kinds of cross sections used in the system. This format is constructed in a "self-defining" manner, whereby it can be read and understood without requiring external descriptive information to be supplied. This particular feature is extremely important to AMPX because these cross-section files form the bulk of the comminication interfaces that one needs in a multigroup processing system. AMPX uses two types of these interfaces. The first is the more complicated and is called a master interface or library. It contains data at its lowest level. For example, it may contain resonance parameters or Bondarenko factors. Il may contain averaged cross sections for an arbitrary number of neutron and gamma processes, 3s well as scattering matrices independently specified by process. It is the format that all of the basic processing codes produce. In this format the cross sections are not directly usable. Resonance calculations may be needed. Certainly data for many processes will have to be summed to make "total" values prior to using the cross sections in a calculation. The programs that deal with these "summed" values use a format called the AMPX working interface. As one would expect, it does not allow the detail of the master library, but is tailored for direct use in a transport calculation.

- Codes can be executed in any order (as long as all necessary input files to a module pre-exist or are produced in the execution sequence).

- Codes use a free-form input scheme for user data. The free-form scheme chosen for AMPX (described in Sect. 7.FIDO of this document) was developed for use in several large stand-alone programs and provides a wide variety of features, making it easier to instruct the codes on what to do.

- The system is planned to be comprehensive, thereby making provisions for most, or all, functions necessary to generate and process multigroup cross sections. To a large extent, this goal has been met. To the extent that it has not is one of the major strengths of the modular system. Whenever a new function (modile) is required, it is written and combined with existing codes without requiring tive reprogramming of existing modules.

\section{OVERVIEW.1 AMPX CAPABILITIES}

AMPX modules can be loosely categorized into the following areas:

- basic data processing

- resonance self-shielding

- spectral collapsing

- format conversion

- service functions

\section{OVERVIEW}




\section{OVERVIEW.1.1 Basic Data Processing}

AMPX has several modules that use ENDF/B data to produce multigroup libraries or to produce point data. XLACS-77 is a module that produces multigroup neutron cross sections. LAPHNGAS is a module that produces multigroup gamma-ray yield information (i.e., the data that allow one to determine the gamma sources produced by neutron interactions). SMUG is analogous to XLACS-77, except that it produces multigroup gamma-ray cross sections. NPTXS, POLIDENT, and PRUDE all process ENDF/B neutron resonance parameter data to produce various kinds of point neutron cross-section libraries.

\section{OVERVIEW.1.2 Resonance Self-Shielding}

The NITAWL-II module uses an improved version of the Nordheim Integral Treatment ${ }^{4}$ for resonance self-shielding. Among improvements in the new version are (1) an ability to self-shield elastic scattering, (2) the ability to treat "multiisotope" ENDF/B evaluations, and (3) the ability to treat $\ell>0$ resonances. The BONAMI-S module provides for a Bondarenko (or shielding factor method) selfshielding treatment. ${ }^{5}$ Indicative of this module's generality is the ability to perform the calculation over energy ranges that can vary for each nuclide. The ROLAIDS module provides a 1-D integral transport solution to a system with an arbitrary number of spatial zones, each of which contains an arbitrary mixture. The fluxes from this solution are used to produce self-shielded cross sections that include self-shielded elastic transfer matrices.

\section{OVERVIEW.1.3 Spectral Collapsing}

A large number of situations require starting with an existing multigroup library and reducing the number of energy groups by spectrally collapsing the library. If the weighting spectrum is known, the COMAND or MALOCS modules can be used for collapsing. COMAND is used to collapse ANISN libraries. MALOCS is a very important AMPX module that is used to collapse AMPX master cross-section interfaces (described later) while retaining all of the generality of these libraries. For the case where the weighting spectrum is not known (most prevalent situation) but where a 1-D calculation can be made to determine an appropriate one, the XSDRNPM module is provided. It can use cross sections in a discrete ordinates, diffusion theory, or $B_{n}$ calculation whose fluxes are then used to collapse cross sections.

\section{OYERVIEW.1.4 Format Conversion}

Because of differences in their applications and heritages, the modules in AMPX and in other programs that use AMPX cross sections employ a variety of cross-section library formats. Several AMPX modules are provided to allow converting between these formats. In some cases, a simple rearrangement of the data is all that is required, while in other cases, considerable processing is necessary. The LAVA module converts ANISN $^{6}$-formatted libraries to AMPX working libraries. The ALPO and CONTAC 
modules convert AMPX working libraries to ANISN libraries. The CONTAC module can also make a CCCC ISOTXS ${ }^{7}$ library. A major function of the NITAWL module is to convert from an AMPX master library to an AMPX working library, while WORM converts from an AMPX working library to an AMPX master library. Several older AMPX modules (CONVERT, REVERT, and OCTAGN) which were for "format conversion" have been made obsolete because the codes they service are no longer in local use.

\section{OVERVIEW.1.5 Service Functions}

A myriad of operations is needed to maintain multigroup libraries. These operations include functions such as listing, plotting, checking, and modifying cross sections, combining sets of data, etc. As AMPX has evolved, most of these functions are provided in independent modules, in keeping with the modular approach. This feature is very desirable in that it makes new modules more compact and easier to maintain.

A very important function that could be needed at any stage of cross-section production is that of producing listings of group-averaged values. ALE serves this function for AMPX master and working libraries. It is an improved successor to the DIAL module.

For reasons of convenience and utility, it is often necessary to combine sets of cross sections from different libraries. The AJAX module provides this capability for AMPX. master libraries. WAX serves the same purpose for working libraries, and ZEST does this for ENDF "TAB1" record-formatted libraries. These modules can merge, delete, update, reorder, etc., sets of data.

In several cases, AMPX modules only produce part of the data needed in a crosssection set. For example, XLACS-77 makes neutron cross sections, SMUG makes gamma cross sections, and LAPHNGAS makes the gamma-production cross sections. These modules all write their output in AMPX master library format. The UNITAB program is provided to allow collecting sets of information from multiple sources, such as just described, to create a final coupled set of data. In the case of neutrons, XLACS-77 makes all necessary data except the Bondarenko factors, which are made by TABU. UNITAB provides the necessary facility for combining data from all of these libraries. UNITAB also provides the capability for splitting a library back into its original multigroup constituent components, such that one could, for example, extract a neutron-only library from a coupled library.

It is often desirable to plot point cross sections, or group-averaged values, or both on the same graph. VASELINE provides a very powerful and flexible tool for making these plots.

If it is necessary to produce punched card output, the PAL module is provided.

One of the most important modules is named RADE. It performs numerous tests of the multigroup data on a master, working, or ANISN library in order to determine if the values are reasonable and consistent. When problems are noted, the COMET module can be used to force consistent values, or, if bad values are noted in some groups, the CLAROL module provides an easy method of introducing corrected values into the complicated formats. If a portion of a cross-section library becomes unreadable, the

\section{OVERVIEW}


SALVAGE module can be used to extract the usable data. If, because of numerical problems or whatever, the Legendre coefficients used to fit some of the group-to-group transfer matrices are found to be nonphysical, the PERFUME module is employed to both find and assign plausible values.

TIDE and MAD are modules that make corrections to the directories in AMPX master libraries which describe the data for each nuclide.

If it is necessary to pass cross-section libraries between sites using different computing hardware that cannot read the same binary-formatted tapes, the AIM module will convert an AMPX master library to a special BCD form which, when delivered to the second site, can be read by AIM to create the required binary library.

JERGENS is a very powerful module for dealing with ENDF/B "TAB1" records. If, for example, it is desired to generate a $1 / \mathrm{E} \Sigma_{t}$ weighting function where $\Sigma_{t}$ is the macroscopic total cross section of a mixture containing an arbitrary number of nuclides, each with an arbitrary representation of its total cross section, JERGENS will generate the required energy, value pairs creating a new function to the accuracy specified by the user. It also contains many other useful options for generating and combining ENDF/B "TAB1" data records.

Many times a user will need to produce group-averaged values for a function that is not part of an ENDF/B library (e.g., a dose factor). With CASTROL, a function can be properly formatted for combination with a weighting function produced by JERGENS and weighted by the VEL module to produce a master library.

For reasons of convenience, the ICE module can mix multigroup cross sections to make a multigroup library of macroscopic mixture cross sections on an AMPX working library.

The COLLINS module might be used with a master library to combine the transfer matrices for inelastic levels to create a library that discards the level values in favor of a total inelastic transfer matrix to reduce the library size and make using the library more efficient to use input/output-wise.

In summary, the modular approach taken in this system has proven itself to be a very efficient and powerful means for developing a system of computer codes for producing multigroup cross sections.

\section{REFERENCES}

1. J. R. Knight and F. R. Mynatt, MUG: A Program for Generating Multigroup Photon Cross Sections, CTC-17, Union Carbide Corp., Nucl. Div., Oak Ridge Natl. Lab., January 1970.

2. W. E. Ford, III, and D. H. Wallace, POPOP $4-A$ Code for Converting GammaRay Spectra to Secondary Gamma-Ray Production Cross Sections, CTC-12, Union Carbide Corp., Nucl. Div., Oak Ridge Natl. Lab., May 1969.

3. N. M. Greene and C. W. Craven, Jr., XSDRN: A Discrete Ordinates Spectral Averaging Code, ORNL-TM-2500, Union Carbide Corp., Nucl. Div., Oak Ridge Natl. Lab., July 1969. 
4. G. D. Joanou and J. S. Dudek, GAM-II: $A B_{3}$ Code for the Calculation of FastNeutron Spectra and Associated Multigroup Constants, GA-4265, General Atomic, September 1963.

5. W. W. Engle, Jr., A User's Manual for ANISN, K-1693, Union Carbide Corp., Nucl. Div., Oak Ridge Gaseous Diffusion Plant, March 1967.

6. H. C. Honeck, THERMOS: A Thermalization Transport Theory Code for Reactor Lattice Calculations, BNL-5826, Brookhaven Natl. Lab., September 1961.

7. L. W. Nordheim, "The Theory of Resonance Absorption," Proceedings of Symposia in Applied Mathematics, American Mathematical Soc., Vol. XI, p. 58, Garrett Birkhoff and Eugene P. Wigner, Eds., 1961.

8. C. N. Kelber, G. Jensen, L. Just, and B. J. Toppel, "The Argonne Reactor Computation System, ARC," Proceedings of the International Conference on the Utilization of Research Reactors and Reactor Mathematics and Computation, Mexico City, pp. 1428-1445, 1967. See also: B. J. Toppel, The Argonne Reactor Computation System, ARC, ANL-7332, Argonne National Laboratory, 1968. See also: B. J. Toppel, "The Argonne Reactor Computation (ARC) System," Reactor Physics Division Annual Report, July 1, 1966, to June 90, 1967, ANL7310, pp. 433-436. See also: L. Just and S. G. Sparck, The ARC System, Applied Mathematics Division Internal Technical Memorandum No. 157, 1968 (unpublished).

9. E. D. Reilly and W. H. Turner, "The Automation of Reactor Design Calculations at the Knolls Atomic Power Laboratory," Proc. Conf. Application of Computing Methods to Reactor Problems, Argonne, Illinois, May 27-29, 1965; ANL-7050, Argonne National Laboratory, pp. 251-63, 1965.

10. H. C. Honeck, The JOSHUA System, DPSTM-500, Savannah River Laboratory, November 1969.

11. B. M. Carmichael, Standard Interface Files and Procedures for Reactor Physics Codes, Version III, LA-5486-MS, Los Alamos Scientific Laboratory, February 1974.

12. SCALE: A Modular Code System for Performing Standardized Computer Analyses for Licensing Evaluation, Vols. 1-3, NUREG/CR-0200, U.S. Nuclear Regulatory Commission (Draft, February 1990).

13. D. R. Vondy, T. B. Fowler, and G. W. Cunningham, VENTURE: A Code Block for Solving Multigroup Neutronics Problems Applying the Finite-Difference DiffusionTheory Approximation to Neutron Transport, Version II, ORNL-5062/R1, Union Carbide Corp., Nucl. Div., Oak Ridge Natl. Lab., November 1977.

14. I. I. Bondarenko, Ed., Group Constants for Nuclear Reactor Calculations, Consultants Bureau, New York, 1964.

15. T. B. Fowler and D. R. Vondy, Nuclear Reactor Depletion and Dynamics Code: CITATION, ORNL-TM-2496, Union Carbide Corp., Nucl. Div., Oak Ridge Natl. Lab., July 1969.

16. N. M. Greene and F. R. Mynatt, "The AMPX Modular Code System for Generating Coupled Neutron-Gamma Multigroup Cross-Section Sets," Trans. Am. Nucl. Soc. 15, 568 (1972); see also: N. M. Greene et al., AMPX: A Modular Code System for Generating Coupled Multigroup Neutron-Gamma Libraries from ENDF/B, ORNL-TM-3706, Union Carbide Corp., Nucl. Div., Oak Ridge Natl. Lab., March 1976.

\section{OVERVIEW}




\section{INPUT INSTRUCTIONS FOR AMPX-77 MODULES}

Input instructions for an AMPX-77 module consist, in general, of subsections that give a discussion of the module, the input data it requires, the input/output assignments, and references. Instructions for the modules are arranged in the following order:

$\underline{\text { Subsection }}$

3.AIM

3.AJAX

3.ALE

3.ALPO

3.BONAMI

3.CASTROL

3.CLAROL

3.COLLINS

3.COMAND

3.COMET

3.CONTAC

3.CORECTOL

3.CREST

3.FRESH

3.GERITOL

3.ICE

3.JERGENS

3.LAPHNGAS

3.LAVA

3.MAD

3.MALOCS

3.NITAWL-II

3.NPTXS

3.PAL
Subsection

3.PERFUME

3.POLIDENT

3.PRELL

3.PRUDE

3.RADE

3.ROLAIDS

3.RUFFLES

3.SALVAGE

3.SMILER

3.SMUG

3.TABU

3.TIDE

3.UNITAB

3.VASELINE

3.VEL

3.WAX

3.WINE

3.WISK

3.WORKER

3.WORM

3.XLACS-77

3.XSDRNPM

3.ZEST

Other than title cards, the FIDO input method is used to enter data into the AMPX modules. The FIDO system is described in Sect. 7.FIDO.

Many individuals other than the authors of this document either wrote or made major contributions to the modules in AMPX-77. The following chart identifies the primary authors of these modules: 
Module Responsible person(s)

\begin{tabular}{ll} 
AIM & N. M. Greene \\
AJAX & N. M. Greene \\
ALE & N. M. Greene \\
ALPO & N. M. Greene \\
BONAMI & N. M. Greene \\
CASTROL & N. M. Greene \\
CLAROL & N. M. Greene \\
COLLINS & N. M. Greene \\
COMAND & N. M. Greene \\
COMET & N. M. Greene \\
CONTAC & L. M. Petrie \\
CORECTCL & L. M. Petrie \\
CREST & N. M. Greene \\
FRESH & N. M. Greene \\
GERITOL & M. A. Bjerke \\
ICE & S. K. Fraley \\
JERGENS & N. M. Greene \\
LAPHNGAS & W. E. Ford, III, and J. L. Lucius \\
LAVA & N. M. Greene \\
MAD & N. M. Greene \\
MALOCS & N. M. Greene \\
NIIAWL-II & L. M. Petrie and N. M. Greene \\
NPTXS & R. Q. Wright \\
PAL & N. M. Greene \\
PERFUME & L. M. Petrie \\
POLIDENT & N. M. Greene and L. M. Petrie \\
PRELL & L. M. Petrie \\
PRUDE & N. M. Greene \\
RADE & N. M. Greene \\
ROLAIDS & R. M. Westfall \\
RUFFLES & L. M. Petrie \\
SALVAGE & N. M. Greene \\
SMILER & N. M. Greene \\
SMUG & J. L. Lucius \\
TABU & N. M. Greene \\
TIDE & L. M. Petrie \\
UNITAB & L. M. Petrie and N. M. Greene \\
VASELINE & N. M. Greene \\
VEL & N. M. Greene \\
WAX & N. M. Greene \\
WINE & N. M. Greene \\
WISK & N. M. Greene \\
& \\
\hline
\end{tabular}


3

Module $\quad \underline{\text { Responsible person(s) }}$

WORKER L. M. Petrie

WORM

N. M. Greene

XLACS-77

N. M. Greene

XSDRNPM

L. M. Petrie and N. M. Greene

ZEST

N. M. Greene 


\section{AIM - MODULE TO CONVERT MASTER CROSS-SECTION LIBRARIES FROM BCD TO BINARY FORMÁT (OR VICE VERSA)}

AIM ( to be able to pass AMPX master cross-section libraries between different computer hardware.

All AMPX cross-section files are written in binary formats, which are not movable between machines of different manufacturers. AIM has the capability to either read a binary-formatted master library and to "unload" it to the card-image (BCD) format or to read a file written in this same card-image format and create a binary master library. Because all machines can read card-image files, this file serves to transmit data between different computers. Alternatively, it is possible, though not necessarily efficient, to use AIM to create a master library using card-image input, because a user can independently create data in the special card-image format.

In the following descriptions, the term 1-D refers to group-averaged cross sections; $2-\mathrm{D}$ refers to group-averaged scattering matrices.

\section{AIM.1 AIM Input Data}

\section{Block 1.}

0\$ Logical Unit Assignments [2]*

1. MBIN - Binary master (1) $\dagger$

2. $\mathrm{MBCD}-\mathrm{BCD}$ master (18)

$1 \$$ Option Selection Trigger [1]

1. IOPT

0 - Binary to BCD conversion

$1-\mathrm{BCD}$ to binary conversion

$2 \$$ Data Edit Triggers [4]

1. IP1D (0)

0 - No 1-D edit

1 - Edit 1-D cross sections

2. IP2D $(-1)$

-1 - No 2-D edit

L - Edit through $P_{L}$ of 2-D arrays

3. IPRES (0)

0 - FIDAS print master library data as processed

1 - FIDAS print suppressed [WARNING - the FIDAS output from converting a $B C D$ file to a binary file can be a very voluminous amount of information for even a modest cross-section library. The user may want to consider suppressing this information.]

\footnotetext{
* The number of entries in the array is given in square brackets.
}

tDefault values are given in parentheses. 
4. IPBOND - Not used, enter zero $(0)$

T Terminate Block 1.

If, and only if, the BCD data are to be read from cards, continue with the following input; otherwise, the input to AIM is complete. Note that the following input corresponds closely to the AMPX master interface described in Sect. 4.MISCELLANEOUS.1 of this document.

\section{Block M1.}

$2 \$$ Tape Identification [10]

1. IID - Library identification number

2. NNUC - Number of nuclides in this library

3. IGM - Number of neutron energy groups

4. IFTG - First thermal group

5. MSN - Nordheim treatment revision level required for this library

6. IPM - Number of gamma-ray energy groups

7. 17 - Zero

8. 18 - Zero

9. I9 - Zero

10. 110 - Zero

T Terminate Block M1.

Five title cards, each in (20A4) format. The five cards of Hollerith information are used to describe the library.

\section{Block M2.}

Repeat Block M2 NNUC times.

Title card in (18A4) format. This information is the title for the nuclide.

$3 . \$ \operatorname{ID}(19-50)$ Refer to the following table for a description of the $\operatorname{ID}(19), \operatorname{ID}(20), \ldots$, ID $(50)$ entries. Note that floating-point entries can be entered using the $3^{*}$ designation, whereas the integers are input in a $3 \$$ array. Input both $3 \$$ and $3^{*}$ arrays and use the "skip" or "address" options to make the input to the proper place in the array when it is necessary to specify both integer and floating-point values. 


\begin{tabular}{|c|c|}
\hline Word $(s)$ & Item \\
\hline $1-18$ & 18 words of text describing the set \\
\hline 19 & Identifier of the set \\
\hline 20 & Number of 6-parameter sets of resolved resonance data \\
\hline 21 & Zero \\
\hline 22 & Number of $1-D$ neutron processes (temperature-independent) \\
\hline 23 & Number of $2-D$ neutron processes \\
\hline 24 & Zero \\
\hline 25 & Number of $1-D$ gamma processes \\
\hline 26 & Number of 2-D gamma processes \\
\hline 27 & Number of 2-D neutron-to-gamma processes \\
\hline 28 & $\begin{array}{l}\text { (Maximum order of scattering) } 32768^{*}+(\text { total number } \\
\text { of separate } 2-D \text { arrays for this set) }\end{array}$ \\
\hline 29 & A - neutron equivalent mass number \\
\hline 30 & $\mathrm{ZA}-1000^{*} \mathrm{Z}+\mathrm{A}$ \\
\hline 31 & Zero \\
\hline 32 & Zero \\
\hline 33 & Zero \\
\hline 34 & Power per fission in watt-s/fission \\
\hline 35 & Energy release per capture in watt-s/capture \\
\hline 36 & Maximum length of any $2-D$ record in the set \\
\hline 37 & Number of sets of Bondarenko data \\
\hline 38 & Number of $\sigma_{0}$ 's in Bondarenko data \\
\hline 39 & Number of T's in Bondarenko data \\
\hline 40 & Maximum number of groups in Bondarenko data \\
\hline 41 & Zero \\
\hline 42 & Zero \\
\hline 43 & $\sigma_{p}-$ potential scattering cross section \\
\hline 44 & Zero \\
\hline 45 & ENDF MAT for fast neutron data \\
\hline 46 & ENDF MAT for thermal neutron data \\
\hline 47 & ENDF MAT for gamma data \\
\hline 48 & ENDF MAT for gamma production data \\
\hline 49 & Nuclide symbol (text) \\
\hline 50 & Number of records in this set \\
\hline
\end{tabular}

T Terminate Block M2.

* 32768 is $2^{32}$, which makes this entry easy to split into its two constituents if one is examining an IBM "hex" dump, because the first two hex characters will be the maximum order of scattering, and the last two, the total number of 2-D arrays. 
Block M3.

7* Neutron Energy Group Boundaries [IGM+1]

The group boundaries are input high to low in $\mathrm{eV}$. This array can be ignored if a "standard" AMPX group structure is being used.

8* Gamma-Ray Group Boundaries [IPM+1]

The group boundaries are input high to low in $\mathrm{eV}$. This array can be ignored if a "standard" AMPX group structure is being used.

T Terminate Block M3.

Follow with NNUC sets of Blocks M4-M15 data, as described below:

\section{Block M4.}

Title card in (18A4) format. Use the same title card entered in data block M2 above for the nuclide title.

$3 . \$$ ID (19-50) Enter the same data for the appropriate nuclide as described in block M2 above.

T Terminate Block M4.

\section{Blocks M5 and M6. Bondarenko data}

If $\operatorname{ID}(37)>0$, enter the Bondarenko data.

In the following arrays, the array dimensions are taken from the ID values ( $3 \$$ array).

$\mathrm{ID}(37) \equiv \mathrm{NBOND}$, number of Bondarenko processes

$\mathrm{ID}(38) \equiv \mathrm{NSIG0}$, number of $\sigma_{0}$ values

ID $(39) \equiv$ NTEMP, number of temperatures

\section{Block M5.}

9* Bondarenko Table Ordinates [NTEMP+NSIG0+2]

Ir clude NSIG0 $\sigma_{0}$ ialues (high to low), followed by NTEMP temperatures in $\mathrm{K}$ (low to high), followed by ELO and EHI, the lower and upper limits of the energy range for which Bondarenko factors apply.

$10 \$$ Bondarenko Table Directory [6*NBOND]

The directory array consists of six strings of numbers, arranged one after the other. The first NBOND numbers are the process identifiers (MT numbers) (e.g., 2 for elastic scattering, 18 for fission, etc.). The second string of numbers are the first energy groups for which Bondarenko factors are given for each of the processes. The third string of numbers are the last energy group for which the factors are given. The fourth string will contain all zeroes, except for the case of self-shielding transfer matrices, in which case it contains the order of scattering for the process (i.e., 0 for $P_{0}$ terms, 1 for $P_{1}$, etc.). The fifth string will contain all zeroes except for transfer matrix shielding, in which case it contains the position of the term to be 
shielded, relative to the "magic word" (see Block M9) in the transfer matrix array (e.g., for the case of only downscatter, a 1 would normally point to the within-group term, a 2 to the first downscatter term, etc.). The sixth string contains zeroes.

T Terminate Block M5.

\section{Block M6.}

This data block will be repeated NBOND times. It consists of two arrays: the infinite dilution values for the process and the corresponding Bondarenko factors. NF is the first group for which Bondarenko data are given, and NL is the last group. (These items are taken from the second and third strings in the directory array discussed earlier.)

11* Infinite Dilution Cross Sections [NL-NF+1] $\left(\sigma_{\mathrm{ID}}(\mathrm{i}), \mathrm{i}=\mathrm{NF}, \mathrm{NL}\right)$

$12 *$ Bondarenko Factors [NSIG0*NTEMP*(NL-NF+1)] $(((B F(i, j, k), i=1, N S I G 0), j=1, N T E M P), k=N F, N L)$

T Terminate Block M6.

Block M7. Resonance Parameter and Other Neutron Cross-Section Data In this block, the following definitions are used:

NRES $\equiv \operatorname{ID}(20)=$ number of resolved resonances

$\mathrm{N} 1 \mathrm{D} \equiv \mathrm{ID}(22)=$ number of temperature-independent averaged neutron cross sections

$\mathrm{N} 2 \mathrm{D} \equiv \mathrm{ID}(23)=$ number of neutron-neutron scattering processes

13* Resonance Data Array; enter if NRES is nonzero [6*NRES+9]

The first nine words contain parameters used in both the resolved and unresolved resonance calculations:

1. A, the mass ratio for the isotope or mixture of isotopes

2. $\sigma_{p}$, the potential scattering cross section

3. $g$, the average statistical factor in the unresolved region

4. NRES, the number of six-parameter resonance sets

5. $\mathrm{s}$, a factor used in the Nordheim calculation to determine the range over which the calculation will be made

6. Zero

7. Zero

8. Zero

9. Zero

The next $6 *$ NRES words consist of six-word sets of data used in the Nordheim caiculation. The first six-word sets are

1. Zero 
2. NBLK, the number of blocks of resolved data

3. Zero

4. Zero

5. TREF, the reference temperature in Kelvin at which the infinite dilution arrays on this library were calculated

6. Zero

Following these six-word sets are NBLK six-word groups that are used to specify information concerning blocks of resonance data that apply to, for example, different isotopes, different energy regions, different kinds of data (s-wave or pwave resonances), etc. These are stacked as follows:

1. AWRI, the mass ratio associated with the block of data

2. ABUN, the abundance for the block of data

3. NRE, the number of resonances in the block

4. $\ell$, the value of spin for the resonances in the block

5. EL, the low-energy cutoff in $\mathrm{eV}$ for resonances in the block

6. EH, the upper-energy cutoff in $\mathrm{eV}$ for resonances in the block

After these NBLK six-word groups, the six-word sets of resonance parameters are arranged as follows:

1. $E_{0}$, the resonance energy

2. $\Gamma_{n}$, the neutron width of the resonance

3. $\Gamma_{\gamma}$, the gamma width of the resonance

4. $\Gamma_{f}$, the fission width of the resonance

5. $r$, a factor used in the Nordheim treatment for determining the range of calculation

6. $\mathrm{g}_{\mathrm{J}}$, the statistical factor for the resonance

The following chart schematically illustrates the structure just described:

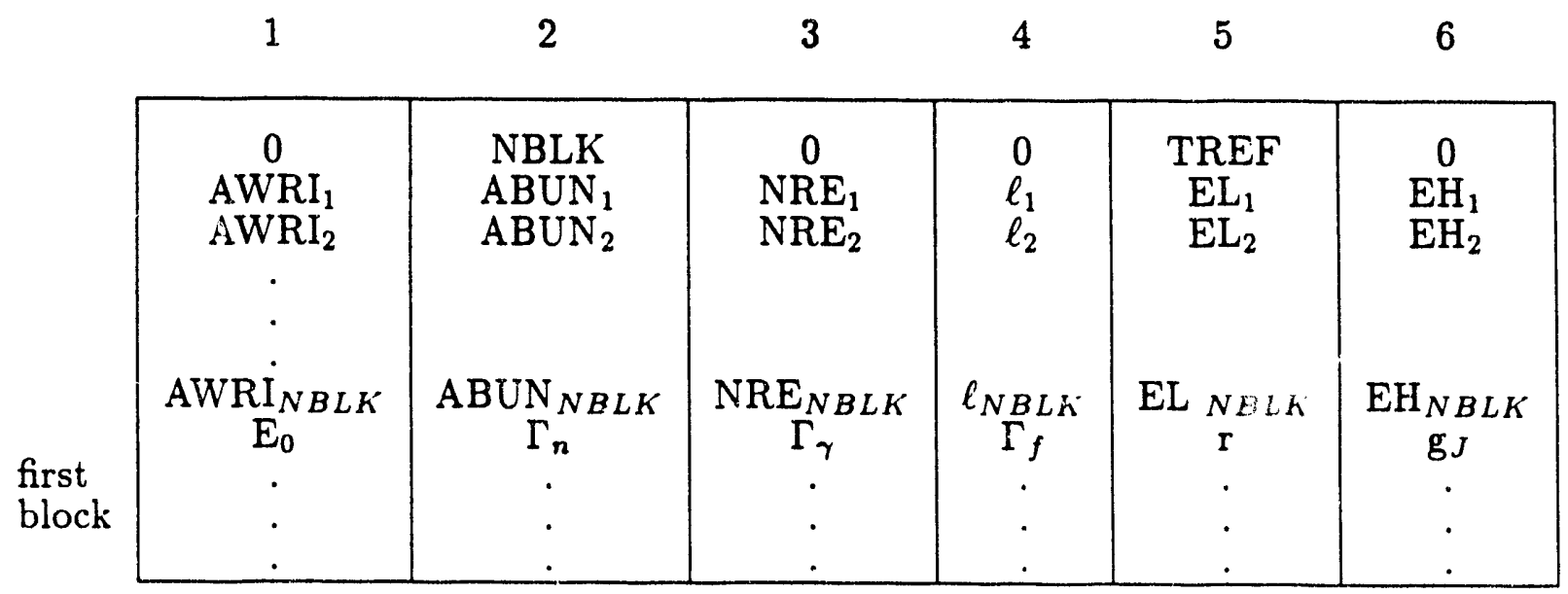


14* Temperature-Independent Averaged Neutron Cross Sections [N1D*(IGM+1)]

Enter process identifier for first process followed by averaged cross sections for all groups (highest energy group to lowest). Enter second process identifier followed by its cross sections, etc.

$16 \$$ Directory for Neutron-Neutron Scattering Data [N2D*4]

Enter the process identifiers for all processes, followed by the corresponding length of the "magic-word" (see Block M9) compressed arrays, followed by the order of the Legendre fit to each process, followed by the number of temperatures at which the cross sections for each process are given:

$$
\begin{aligned}
& \left(M T_{i}, \mathrm{i}=1, \mathrm{~N} 2 \mathrm{D}\right) \\
& \left(L_{i}, \mathrm{i}=1, \mathrm{~N} 2 \mathrm{D}\right) \\
& \left(N L_{i}, \mathrm{i}=1, \mathrm{~N} 2 \mathrm{D}\right) \\
& \left(N T_{i}, \mathrm{i}=1, \mathrm{~N} 2 \mathrm{D}\right)
\end{aligned}
$$

T Terminate Block M7.

\section{Blocks M8 and M9. Neutron-Neutron Scattering Matrices}

Each scattering process has an M8 and M9 block. Processes where NL is greater than zero have $N L+1$ repeats. When $N T>1$, th. nur aber of repeats is $N T^{*}(N L+1)$. Data for all arrays for the first temperature are given first, etc.

\section{Block M8. Parameters and Temperatures}

$17^{*}$ Temperatures in units of $\mathrm{eV}$ at which the cross c ctions are given [NT]

$18 \$$ Paraneters [2]

1. NMW - number of magic words required in ine transfer matrix

2. LPL - length of the compressed scattering matrix (including space for the magic words)

T Terminate Block M8.

\section{Block M9. Scattering Matrix}

$19 \$$ Magic Words [NMW]

Specify the magic words that are included in the transfer matrix.

20* Scattering Matrix [LPL]

Specify the transfer matrix as in the master library format described below. Include zeroes in the positions that will contain inagic words.

T Terminate Block M9. 


\section{AIM.2 Scattering Matrix Structure}

This record type is used to store scattering matrix data (sometimes called 2-D data). As will be illustrated, it has provisions for truncating zero and/or impossible elements from the array. It exists in two forms: (1) a self-defining form used for gamma production data on a master library, and (2) a form that is not self-defining. The only difference is that the self-defining form has the array length as the first word in the record, while the other does not, that is,

or

$$
\mathrm{L},(\mathrm{X}(\mathrm{I}), \mathrm{I}=1, \mathrm{~L})
$$

$$
(\mathrm{X}(\mathrm{I}), \mathrm{I}=1, \mathrm{~L}) \text {. }
$$

The structure of the $\mathrm{X}$-array is as follows:

magic word for a group

terms for scattering to the group

magic word for the next group

terms for scattering to this group

etc., etc.

A negative or zero magic word can also be used to specify the end of data in the record in the event that the array is shorter than the length specified. This situation occurs, for example, when the lengths of the matrices of Legendre coefficients for the 'scattering terms vary.

A magic word contains the following terms:

1. the sink group number, III

2. the first group number, JJJ, which scatters to group III

3. the last group number, $\mathrm{KKK}$, which scatters to group III

The magic word is defined as

$$
\mathrm{MW}=1000000^{*} \mathrm{JJJ}+1000^{*} \mathrm{KKK}+\mathrm{III}
$$

such that it is composed of three 3-digit integers:

MW : JJJKKKIII

The scatterin , terms below a magic word are in reverse ordering (following typical practice for transport theory programs) (i.e., the scattering term for scattering from the last group is first, etc.).

$$
\begin{gathered}
\text { MW for group III } \\
\sigma(K K K \rightarrow I I I) \\
\sigma(K K K-1 \rightarrow I I I) \\
\cdot \\
\cdot \\
\sigma(J J J \rightarrow I I I)
\end{gathered}
$$

The scattering matrix record will contain one $P_{\ell}$ matrix for a process. 
Consider an elastic-scattering matrix for hydrogen where all groups can scatter to any lower-energy group and assume three energy groups. The scattering matrix will look as follows:

$$
\begin{gathered}
1001001 \\
\sigma(1 \rightarrow 1) \\
1002002 \\
\sigma(2 \rightarrow 2) \\
\sigma(1 \rightarrow 2) \\
1003003 \\
\sigma(3 \rightarrow 3) \\
\sigma(2 \rightarrow 3) \\
\sigma(1 \rightarrow 3)
\end{gathered}
$$

Note that the record contains a mixture of integer and floating-point terms.

\section{Blocks M10, M11, and M12. Gamma Production Data}

These blocks are required only when $\mathrm{N} 2 \mathrm{DY}[\equiv \mathrm{ID}(27)]$ is greater than zero, where N2DY is the number of processes requiring gamma-production data.

\section{Block M10. Gamma Production Data Directory}

$21 \$$ Enter the gamma production process identifiers, followed by the maximum length of the scattering matrices for a process, followed by the order of scattering for the processes, followed by a trigger (0/1-yields/cross sections) that specifies whether the gamma-production matrices are in cross-section or yield units.

$$
\begin{aligned}
& \left(M T Y_{i}, \mathrm{i}=1, \mathrm{~N} 2 \mathrm{DY}\right) \\
& \left(L Y_{i}, \mathrm{i}=1, \mathrm{~N} 2 \mathrm{DY}\right) \\
& \left(N L Y_{i}, \mathrm{i}=1, \mathrm{~N} 2 \mathrm{DY}\right) \\
& \left(N T Y_{i}, \mathrm{i}=1, \mathrm{~N} 2 \mathrm{DY}\right)
\end{aligned}
$$

T Terminate Block M10.

\section{Blocks M11 and M12. Gamma Production Data}

These blocks are repeated NLY+1 times for each gamma production (N2DY) process. (Note that NLY is a function of the process.)

\section{Block M11. Parameters}

$22 \$$ Gamma Production Matrix Parameters [2]

1. NMWY - Number of magic words in the scattering matrix

2. LPLY - Length of the scattering matrix

T Terminate Block M11. 
Block M12. Scattering Matrix

$23 \$$ Magic Words [NMWY]

Specify the magic words that will be part of the scattering matrix.

$24^{*}$ Scattering Matrix [LPLY]

Specify the scattering matrix. Use zeroes in the positions that will contain magic words.

T Terminate Block M12.

\section{Blocks M13, M14, and M15. Gamma-Ray Cross Sections}

These blocks will only be used if either of the following two parameters is nonzero.

$\mathrm{N} 1 \mathrm{DZ} \equiv \mathrm{ID}(25)=$ number of gamma-ray averaged cross sections

$\mathrm{N} 2 \mathrm{DZ} \equiv \mathrm{ID}(26)=$ number of gamma-gamma scattering processes

\section{Block M13. Gamma Cross Sections and Directories}

25* Gamma-Ray Averaged Cross Sections [N1DZ*(IPM+1)]

Specify the identifier for the first process, followed by its cross sections, followed by the second process identifier and its cross sections, etc.

26* Directory for Gamma-Ray Scattering Data [N2DZ*4]

Specify the identifiers of all scattering processes, followed by the lengths of the scattering matrices for the corresponding processes, followed by the orders of scattering for the processes, followed by a zero for each process.

$$
\begin{aligned}
& \left(M T Z_{i}, \mathrm{i}=1, \mathrm{~N} 2 \mathrm{DZ}\right) \\
& \left(L Z_{i}, \mathrm{i}=1, \mathrm{~N} 2 \mathrm{DZ}\right) \\
& \left(N L Z_{i}, \mathrm{i}=1, \mathrm{~N} 2 \mathrm{DZ}\right) \\
& \left(N T Z_{i}, \mathrm{i}=1, \mathrm{~N} 2 \mathrm{DZ}\right)
\end{aligned}
$$

$\mathrm{T}$ Terminate Block M13.

\section{Blocks M14 and M15. Gamma-Scattering Matrices}

These blocks are repeated NLZ+1 times for each of the gamma-ray-scattering processes.

\section{Block M14. Gamma Parameters}

$27 \$$ Gamma-Gamma Matrix Size and Order

1. NMW2 - Number of magic words in the scattering matrix

2. LPL2 - Length of scattering matrix

T Terminate Block M14. 
Block M15. Gamma-Scattering Matrix

$28 \$$ Magic Words [NMW2]

Specify the magic words from the scattering matrix.

29* Scattering Matrix [LPL2]

Specify the scattering matrix with zeroes in the magic word positions.

$\mathrm{T}$ Terminate Block M15.

\section{AIM.3 AIM Input/Output Specifications}

The following devices are typically needed to execute AIM:

\begin{tabular}{|c|c|}
\hline Logical No. & Purpose \\
\hline 5 & Standard input \\
\hline 6 & Standard output \\
\hline 7 & Standard punch \\
\hline 17 & Scratch device \\
\hline 47 & $\begin{array}{l}\text { Built-in group structure library (not required for } \\
\text { binary-to-BCD conversion) }\end{array}$ \\
\hline MBIN(1) & Binary master \\
\hline $\operatorname{MBCD}(18)$ & BCD master \\
\hline
\end{tabular}




\section{AJAX - MODULE TO MERGE, COLLECT, ASSEMBLE, REORDER, JOIN, AND/OR COPY SELECTED DATA SETS FROM AMPX MASTER INTERFACES}

AJAX ( $\underline{\text { Automatic }}$ Joining of $\underline{\mathrm{A} M P X} \underline{\mathrm{X}}$-Sections) is a module to combine data from AMPX master interfaces. Options are provided to allow merging from any number of files in a manner as to allow the user to determine the final nuclide ordering. Any form of master interface (neutron, gamma-ray, or neutron-gamma) can be accessed. (AJAX always copies all the data for each nuclide selected, whereas UNITAB can be used to copy specific pieces.)

\section{AJAX.1 AJAX Input Data}

\section{Block 1.}

$-1 \$$ Core Size [1]

1. LENG - Number of words to allocate to AJAX (100000)

$0 \$$ Logical Unit Assignments [2]

1. MMT - Logical number of new library $($ default $=1)$

2. NMAX - Not used

$1 \$$ Number of Files [1]

1. NFILE - Number of files to be used in constructing the new library (When "reordering" operations are performed which required the same file to be accessed several times, each access is counted in determining the value of NFILE.)

T Terminate Block 1.

Blocks 2 and 3 are stacked, one after the other, NFILE times.

\section{Block 2.}

$2 \$$ File and Option Selection [2]

1. NF - Logical unit number of file considered

2. IOPT - Nuclide selection option

$-\mathrm{N}$ Delete $\mathrm{N}$ nuclides from those on logical $\mathrm{NF}$ and add the remainder to the new file

0 Add all nuclides on logical NF to the new file

$+\mathrm{N}$ Add $\mathrm{N}$ nuclides from logical $\mathrm{NF}$ to the new file

$\mathrm{T}$ Terminate Block 2.

Block 3. $\{$ Enter only when IOPT $\neq 0$ \}

$3 \$$ Nuclides Selected [|IOPT $\mid$ ]

Identifiers of nuclides which are to be added or deleted from NF. 
$4 \$$ New Identifiers [|IOPT|] Enter only if an identifier is to be changed

This array allows changing the identifier given in the $3 \$$ array for the set of data on the new library.

T Terminate Block 3.

Note that AJAX operates in a sequential manner to construct the new library. Once an identifier is selected for a nuclide on the new file, the occurrence of a set of data with that identifier will be ignored. Normally, the identifiers will remain the same as those used on the libraries from which the data are selected. The $4 \$$ array provides a way to override these identifiers.

\section{AJAX.2 AJAX Input/Output Specifications}

The following devices are normally needed to execute AJAX:

\begin{tabular}{c} 
Logical No. \\
\hline 5 \\
6 \\
15 \\
16 \\
18 \\
19 \\
MMT(1) \\
NF
\end{tabular}

Purpose

Card input

Standard output

Scratch device

Scratch device

Scratch device

Scratch device

Master file to be created

File(s) to be merged onto MMT 


\section{ALE - MODULE TO LIST INFORMATION FROM MASTER OR WORKING LIBRARIES}

ALE (AMPX Library Editor) is a module that will produce listings of information contained in an AMPX master or working library. It offers all the capabilities of the DIAL module, which it replaces, in addition to considerable improvement in the manner in which data for a particular nuclide can be selected. It also has provisions for controlling the spacing of the output by allowing the user to specify carriage control characters. In the following description, 1-D refers to group-averaged cross sections, and 2-D refers to scattering matrices.

\section{ALE.1 ALE Input Data}

\section{Block 1.}

$-1 \$$ Core Allocation [1]

1. ICORE - Number of words allocated to ALE (default $=100000$ )

0\$ Logical Assignments [2]

1. MMT - Master library (default $=1$ )

2. MWT - Working library (default $=0$ )

\section{$1 \$$ Selection Option [1]}

1. NEDIT - Number of nuclides for which listings are to be produced (a zero selects all nuclides on a library)

$2 \$$ Block Edit Option [10]

(A nonzero value selects editing the data.)

1. N1DN - 1-D neutron arrays

2. N1DG - 1-D gamma arrays

3. NRES - Resonance data (Use this option to select both resonance parameter and Bondarenko factor edits.)

4. IOPT4 - Not used

5. IOPT5 - Not used

6. IOPT6 - Not used

7. IOPT7 - Not used

8. IOPT8 - Not used

9. IOPT9 - Not used

10. ICORE - Not used

$3 \$$ Block Carriage Control Characters [25]

The entries have the following effect on how the header lines for particular kinds of data are printed - all values are 0 , by default, which says to skip a line before printing the data block. A 1 is used to start the block on a new page.

1. Nuclide directory information

2. 1-D cross sections (absorption, fission, etc.)

3. 2-D cross sections (scattering matrices) 
4. Bondarenko data

5. Unassigned

6. Unassigned

7. Unassigned

8. Unassigned

9. Unassigned

10. Unassigned

11. Unassigned

12. Unassigned

13. Unassigned

14. Unassigned

15. Unassigned

16. Unassigned

17. Unassigned

18. Unassigned

19. Unassigned

20. Unassigned

21. Unassigned

22. Unassigned

23. Unassigned

24. Unassigned

25. Unassigned

$4 \$$ Scattering Matrices to be Edited [100]

En er the process identifiers for which edits are desired.

$5 \$$ Orr..r of Scattering Matrices to be Edited [100]

The $4 \$$ and $5 \$$ arrays are entered in one-to-one correspondence. The entries in the $5 \$$ array give the maximum order of Legendre coefficient to be listed (e.g., a value of 2 would cause the $P_{0^{-}}, P_{1^{-}}$, and $P_{2^{-}}$scattering matrices to be listed).

T Terminate Block 1.

Block 2. $\quad$ Enter only if NEDIT $>0$ \}

$11 \$$ Nuclide Identifiers [NEDIT]

These are the identifiers for the nuclides for which listings are to be made.

T Terminate Block 2. 


\section{ALE.2 ALE Input/Output Specifications}

$A L E$ requires the following input/output devices:

\begin{tabular}{cll} 
Logical No. & & Purpose \\
\cline { 1 - 1 } 5 & & Standard input \\
6 & & Standard output \\
MMT & & Master library \\
MWT & & Working library
\end{tabular}




\section{ALPO - MODULE FOR PRODUCING ANISN LIBRARIES FROM AMPX WORKING LIBRARIES}

ALPO ( ANISN Library Production Option) is a module for producing ANISN libraries from AMPX working libraries. Several working libraries can be accessed in a given run. The ANISN library can be produced in either binary or BCD format.

\section{ALPO.1 ALPO nput Data}

\section{Block 1.}

- $1 \$$ Process Identifiers by Position in the ANISN Library [100]

This array can be used to override the normal arrangement of cross-section types in ANISN libraries or can be used to produce special cross-section sets (e.g., dose factor sets). The identifier for the process to be placed in row 1 of the ANISN tables is input first, etc. Note that nuclides that do not contain the processes noted in this table will revert to the normal ANISN ordering (viz., $\sigma_{T}$ in position IHT, $\overline{\nu \sigma_{f}}$ in position IHT-1, etc.).

$0 \$$ Logical Assignments [2]

1. MAN - Logical unit for the ANISN library (use a 7 when a punched card output is desired) (default $=20$ )

2. MAX - Logical unit of one of the working libraries which will be accessed (4).

$1 \$$ Primary Options [9]

1. NFILE - Number of working libraries to be accessed

2. IHT - Position of the total cross section in the ANISN tables (3)

3. IHS - Position of the within-group cross section in the ANISN tables, IHT + IGM - IFTG + 1, where IGM is the number of neutron energy groups; IFTG is the first thermal group

4. ITL - Table length of the ANISN tables, IHS + IGM + IPM - 1, where IPM is the number of gamma-ray groups

5. MAXPL - The maximum order of scattering to be written on the ANISN library (20)

6. IOPT1D - Option to print label with each block of ANISN cross sections (0) A "1" selects printing; a " 0 " selects no printing

7. IOPT2D - Option to print scattering matrices (0) A " 1 " selects printing

8. ITRANS - Transport correction option (0)

$-\mathrm{N}$, truncate $P_{N}$ and above matrices and correct all lower ordered within-group terms by subtracting $(2 \ell+1)^{*} \sigma_{N}\left(\mathrm{~g} \rightarrow \mathrm{g}^{\prime}\right) 2 /$ $(2 N+1)$

0 , no transport correction

1 , replace $\sigma_{t}$ with $\sigma_{t r}=\sigma_{a}+(1-\mu) \sigma_{s}$ where $\mu$ is calculated by summing the $P_{1}$ matrix and dividing by the $P_{0}$ sum, or by $2 /\left(3^{*} \mathrm{~A}\right)$, when $P_{1}$ is not given. The within-group term is also adjusted.

9. ICORE - Number of words to allocate to ALPO (50000) 
T Terminate Block 1.

Data Blocks 2 and 3 are repeated NFILE times.

\section{Block 2.}

$2 \$$ File Selection Options [2]

1. NF - Logical number of the working library

2. IOPT - Nuclide selection

$-\mathrm{N}$, accept all nuclides from the working library except the $\mathrm{N}$ designated in the $3 \$$ array below

0 , accept all nuclides from the working library

$+\mathrm{N}$, accept all nuclides that are designated in the $3 \$$ array below

\section{T Terminate Block 2.}

Block 3. $\{$ IOPT $\neq 0\}$

$3 \$$ Nuclides to be Selected or Ignored [|IOPT $\mid]$

T Terminate Block 3.

\section{ALPO.2 ALPO Input/Output Specifications}

The following devices are normally needed to execute ALPO:

\begin{tabular}{cll} 
Logical No. & & \multicolumn{1}{c}{ Purpose } \\
\cline { 1 - 1 } 5 & & Card input \\
6 & & Standard output \\
7 & & Punched card output \\
14 & & Scratch device \\
MAN(20) & & ANISN binary library \\
MAX(4) & & AMPX working library
\end{tabular}




\section{BONAMI - MODULE TO PERFORM BONDARENKO RESONANCE SELF-SHIELDING}

BONAMI (BONdarenko AMPX Interpolator) is an upgraded version of the BONAMI module. It uses Bondarenko factor data in a self-shielding calculation. The output is a master library that contains the self-shielded cross sections produced in the calculation.

The 9 ridarenko $^{1}$ method is basically an "infinite medium" method that parameterizes cross sections for a nuclide as a function of temperature, $\mathrm{T}$, and the "background" cross section, $\sigma_{0}$, of all the other nuclides mixed with the nuclide. Simplistically, given the temperature and background cross-section values, one determines self-shielded cross sections by interpolating in tables. Since self-shielding causes the "background" values that a nuclide sees to change, an iterative procedure involving all nuclides is used.

To account for two region systems, the $\sigma_{0}$ value is augmented by an escape cross section, $\sigma_{e}$. This escape cross-section value is a function of the total cross-section value in the medium and its geometry. Even though many implementations of this approach use the Wigner rational approximation or a modified form, BONAMI uses exact values of escape probabilities, developed for slabs, cylinders and spheres, by Case, de Hoffman, and Placzek. ${ }^{2}$ These "exact" values are also approximations, because they are based on assuming that the flux in the fuel is spatially flat and that the scattering is isotropic.

Multizone situations, such as reactor lattices, are accounted for by the use of Dancoff factors, which, in effect, modify the escape probability and, hence, the value of $\sigma_{e}$.

Certainly, several of the approximations used in the Bondarenko method break down in some situations. At low energies, many nuclides have resonances that are wide when compared with the scattering ranges for the mixtures in a particular configuration. If the Bondarenko factors used in the calculation are based on the narrow resonance approximation, this can produce cross-section values that are too high. Systems with nuclides whose resonances overlap also cause inaccuracies.

In AMPX, the treatment is used primarily for the unresolved energy region where the resonances are so narrow that they cannot be (have not been) resolved into sets of resonance parameters, etc. The Bondarenko method contains approximations, such as the flat flux and isotropic scattering assumptions, for determining escape probabilities that may have an effect on calculated results. However, there is probably more potential error in the final group-averaged values due to the procedures used to generate the original Bondarenko factor tables, which may or may not have included resonance resonance overlap terms, etc. In short, it is felt that the treatment is very good for the "unresolved" region.

BONAMI is an improvement over earlier versions in several respects, including:

- Several code errors have been corrected.

- It handles any number of Bondarenko processes for a nuclide.

- It can self-shield transfer arrays (e.g., elastic removal effects can be explicitly treated). 
- It will edit Bondarenko factors and/or self-shielded cross sections as a function of nuclide and process.

- It has more options for treating lattice effects.

- It has an improved scheme ${ }^{3}$ for interpolating within the Bondarenko factors which guarantees monotonicity and avoids other problems encountered with Lagrangian schemes widely used for this process.

- It uses a correction scheme developed by John Otter ${ }^{4}$ to use with the Wigner rational approximation that effectively determines exact escape probabilities for slabs, spheres, and cylinders.

- It allows the user to select temperatures by nuclide (i.e., all nuclides in the same zone need rot have the same temperature).

- It uses a reciprocity relationship to determine an effective "escape cross section" for moderator regions and uses this in the determination of a $\sigma_{0}$ for these regions.

BONAMI requires a mockup of the problem geometry in either 1-D slab, cylindrical, or spherical geometry or in a homogeneous representation. The mockup can include an arbitrary number of spatial regions, though all of the Dancoff options assume either a two- or a three-region system. To accomplish this, the code allows the user to "mark" the regions as to their type (fuel, moderator, or cladding) and will take care of collecting and adjusting parameters to fit the option.

For the homogeneous crse, the user can mock a multiregion case and have an arbitrary number of independent homogeneous calculations performed in the same run.

BONAMI produces a separate set of cross sections for every zone in which a nuclide occurs. The identifier of the individual sets can be specified by the user or, by default, the code assigns one of the form: original ID-0-zone number. For example, if a nuclide with identifier 1111 occurred in zones 1 and 3 of a problem, two sets would be produced with identifiers 111101 and 111103 , respectively.

A unique, and sometimes confusing, feature of BONAMI is that it copies every set of data on the input master library, including nuclides that are not required in the problem. The user who desires only those nuclides in the problem to be output should first run a service module, such as AJAX, and select the nuclides to be used.

BONAMI can be triggered to make either an iterative or noniterative calculation. In the iterative case, values for the macroscopic total cross sections (including heterogeneity effects) are iterated to convergence. From these values, nuclide values for $\sigma_{0}$ by 'roup can be determined and used to interpolate in Bondarenko factor tables. In the noniterative case, macroscopic cross sections are determined based on the potential scattering cross sections for the nuclides, and only one pass is made through the data. The interpolation in the Bondarenko data is analogous to the iterative case. The basis for the argument against iteration is that most resonances of most nuclides may not really overlap; hence, what any one resonance of a nuclide typically sees is the potential cross section of another nuclide and not an averaged value that contains added contributions from resonances that fall within a group. 
The Dancoff options in BONAMI were largely taken from options given in the SPHINX code and include:

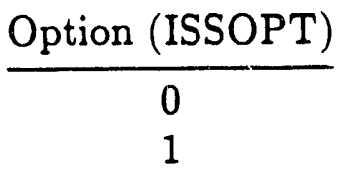

2

3

4

5

6

7

8

9
Situation

Homogeneous infinite medium

Sauer's approximation for cylindrical cell in a hexagonal lattice

Sauer's approximation for cylindrical cell in a square lattice

Symmetric slab cell

Asymmetric slab cell

Isolated rod

Bell approximation for cylindrical cell

Bell approximation for symmetric slab cell

Sauer's approximation for a cylindrical cell with cladding included in a hexagonal lattice

Sauer's approximation for a cylindrical cell with cladding included in a square lattice

Provisions are made to allow the user to input an "extra" escape cross section that will be used io augment the escape cross sections that are determined by the schemes mentioned above. In this case, the input values by zone are directly added to those determined by the Dancoff option. A typical use of this option is to mock up a homogeneous problem (ISSOPT $=0$ ) and to use an escape cross section determined by some external means or select cross sections for a particular value of $\sigma_{0}$.

\section{BONAMI.1 BONAMI Input Data}

\section{Block 1.}

$0 \$$ Logical Unit Assignments [4]

1. MMT - Input master library (default $=23$ )

2. MWT - Scratch for Bondarenko factors (default $=24$ )

3. MSC - Scratch device (default $=18$ )

4. NEW - Output master library (default $=22$ )

$1 \$$ Case Description [6]

1. IGR - Geometry $\begin{cases}0 & \text { homogeneous } \\ 1 & \text { slab } \\ 2 & \text { cylinder } \\ 3 & \text { sphere }\end{cases}$

2. IZM - Number of zones or material regions

3. MS - Mixing table length. This is the total number of entries needed to describe the concentrations of all constituents in all mixtures in the problem.

4. IBL - Cross-section edit option $(0 / 1-$ no/yes $)$ 
5. IBR - Bondarenko factor edit option $(0 / 1-$ no/yes $)$

6. ISSOPT - Dancoff factor

\begin{tabular}{ll}
$\frac{1}{\text { ISSOPT }}$ & \multicolumn{1}{c}{ Option } \\
\cline { 2 - 2 } 0 or 1000 & Homogeneous $\sigma_{e}=0.0$ \\
1 or 1001 & Cylindrical cell (Sauer's method) hex lattice \\
2 or 1002 & Cylindrical cell (Sauer's method) square lattice \\
3 or 1003 & Symmetric slab cell \\
4 or 1004 & Asymmetric slab cell \\
5 or 1005 & Isolated lump (Dancoff $=0.0)$ \\
6 or 1006 & Cylindrical cell (Bell approximation) \\
7 or 1007 & $\begin{array}{l}\text { Symmetric slab (Bell approximation) } \\
\text { Cylindrical cell (Sauer's method) } \\
\text { hex lattice w/clad } 1008\end{array}$ \\
9 or 1009 & $\begin{array}{c}\text { Cylindrical cell (Sauer's method) } \\
\text { square lattice w/clad }\end{array}$
\end{tabular}

Note that when ISSOPT $\geq 1000$, the code will use potential scattering values in place of total cross section in determining $\sigma_{0}$ values and will not iterate.

2* Floating-Point Constants [2]

1. EPS - Convergence criteria for the Bondarenko iteration (default $=0.001$ )

2. A - Geometrical escape probability adjustment factor. See notes below on this parameter. (default $=0.0$ )

T Terminate Block 1.

\section{Block 2.}

$3 \$$ Mixture Number in the Mixing Table [MS]

$4 \$$ Component (Nuclide) Identifiers in the Mixing Table [MS]

$5^{*}$ Concentrations (atoms/barn-cm) in the Mixing Table [MS]

$6 \$$ Mi:tures by Zone [IZM]

$7^{*}$ Outer Radii $(\mathrm{cm})$ by Zone [IZM]

$8^{*}$ Temperature (K) by Zone [IZM]

9* Extra Cross Section $\left(\mathrm{cm}^{-1}\right)$ by Zone [IZM]

$10 \$$ New Identifiers for Components in the Mixing Table [MS]

$11 \$$ Zone Type Identifiers

0 - fuel zone

1 - moderator zone

2 - cladding zone

12* Temperature $(\mathrm{K})$ of the Nuclide in a One-to-One Correspondence With the Mixing Table Arrays 
T Terminate Block 2.

This concludes the input data required by BONAMI.

\section{BONAMI.1.1 Notes on input}

In the $1 \$$ array, IGR specifies the geometry. This notation is used in conjunction with the $7^{*}$ array to calculate the volumes of each zone.

IZM, the number of zones, may or may not mock a real situation. It may, for example, be used to specify IZM independent infinite media when IGR, ISSOPT, and the entries in the $11 \$$ array are properly selected. It can also be used to perform a cell calculation in parallel with one or more infinite medium calculations, if properly directed. In this case, the entries in the $11 \$$ array for the infinite medium zones can be anything other than 0,1 , or 2 .

In the $2^{*}$ array, EPS is used to specify the convergence expected on all macroscopic total values by zone, that is, each $\Sigma_{t}(g, j)$ in group $g$ and zone $j$ is converged such that

$$
\frac{\left|\Sigma_{t}^{i}(g, j)-\Sigma_{t}^{i-1}(g, j)\right|}{\Sigma_{t}^{i}(g, j)} \leq \mathrm{EPS}
$$

where $\mathrm{i}$ is the iteration number.

The " $\mathrm{A}$ " factor in the $2^{*}$ array is the parameter used to adjust the Wigner rational approximation to give a more correct value for the escape probability. It has been suggested that if one wishes to use one constant value, A should be 1.0 for slabs and 1.35, otherwise. In the ordinary case, BONAMI defaults $\mathrm{A}$ to zero and uses a prescription ${ }^{4}$ developed by Otter to determine a cross-section geometry-dependent value of $\mathrm{A}$ which makes the escape probability "exact." The user who wishes the constant value can, however, use it by inputting a value other than zero.

The $3 \$, 4 \$$, and $5^{*}$ arrays are used to specify the concentrations of the constituents of all mixtures in the problem as follows:

\begin{tabular}{|c|c|c|c|}
\hline Entry & $\underline{3 \$ \text { (Mixture Number) }}$ & $4 \$$ (Nuclide ID) & $\underline{5^{*} \text { (Concentrations) }}$ \\
\hline 1 & 1 & 92235 & 0.040 \\
\hline 2 & 1 & 8016 & 0.035 \\
\hline$\cdot$ & & & \\
\hline MS & 10 & 92238 & 0.025 \\
\hline
\end{tabular}

The entries in these three arrays are in a one-to-one correspondence among themselves and also with the $10 \$$ and $12^{*}$ arrays. The $10 \$$ array provides a means of changing the identifier of any nuclide used in the problem. In the event that the 
number of zones, IZM, is more than one and when an entry in the $10 \$$ array is zero, BONAMI will automatically form a new identifier that is

$$
\text { original ID - } 0 \text { - zone number. }
$$

For example, a uuclide in zone 5 identified by 1111 would become 111105 on the output master library.

Because of the manner in which BONAMI references the nuclides in a calculation, each nuclide in the problem must have a unique entry in the mixing table. This says that one cannot specify a mixture and subsequently load it into more than one zone, such as can be the case with many modules requiring this type of data.

The $12^{*}$ array is used to allow varying the temperatures by nuclide within a zone. In the event this array is omitted, the $12^{*}$ array entries will default to the temperatures of the zone containing the nuclide.

The mixture numbers in each zone are specified in the $6 \$$ array. Mixture numbers are arbitrary and need only match up with those used in the $3 \$$ array.

The radii in the $7^{*}$ array are referenced to a zero value at the left boundary of the system.

In the event that the temperatures in the $8^{*}$ array are outside the temperature values in the Bondarenko tables, BONAMI will select the "bound" closest to the value. For example, a request for $273 \mathrm{~K}$ for a nuclide with Bondarenko sets at 300,900 , and $2100 \mathrm{~K}$ would use the $300 \mathrm{~K}$ values. No attempt will be made to extrapolate.

The extra cross sections in the $9^{*}$ array allow an extra value to be specified by zone. (This array can usually be ignored.) It is used in the calculation of $\sigma_{0}$ as follows:

$$
\sigma_{0}=\frac{\sum_{n \neq i} N_{n} \sigma_{t}^{n}+\mathrm{SIGH}+\sigma_{\text {extra }}}{N_{i}} .
$$

In the event one wishes to select cross sections at a fixed $\sigma_{0}$, a mixture with one nuclide with a number density of unity and

$$
\sigma_{\text {extra }}=\text { desired } \sigma_{0}
$$

will accomplish the request.

The zone-type identifiers in the $11 \$$ array are used in conjunction with the Dancoff option (ISSOPT) to determine which zones should be combined, etc., in determining a Dancoff factor. 


\section{BONAMI.2 BONAMI Input/Output Specifications}

BONAMI requires the following input/output assignments during a normal calculation:

\begin{tabular}{l} 
Logical No. \\
\hline 5 \\
6 \\
8 \\
9 \\
17 \\
18 \\
19 \\
$\operatorname{MMT}(23)$ \\
$\operatorname{NEW}(22)$
\end{tabular}

\begin{tabular}{l} 
Purpose \\
\hline Card input \\
Standard output \\
Scratch file \\
Scratch file \\
Scratch file \\
Scratch file \\
Scratch file \\
Input master cross-section library \\
Shielded master cross-section library
\end{tabular}

\section{REFERENCES}

1. I. Bondarenko, Ed., Group Constants for Nuclear Reactor Calculations, Consultants Bureau, New York, 1964.

2. K. M. Case, F. DeHoffman, and G. Placzek, Introduction to the Theory of Neutron Diffusion, U.S. Government Printing Office, Washington, D.C., 1953.

3. N. M. Greene, "A Simple Monotonic Interpolation Scheme," Trans. Am. Nucl. Soc. 34, 299 (June 1980).

4. J. M. Otter, Escape Probability Approximations in Lumped Resonance Absorbers, NAA-SE-9744, Atomics International, a division of North American Aviation, Inc., August 1964.

5. W. J. Davis, M. B. Yarbrough, and A. B. Bortz, SPHINX-A One Dimensional Diffusion and Transport Nuclear Cross Section Processing Code, WARD-XS-304517, Westinghouse Electric Corp., August 1977. 


\section{CASTROL - MODULE TO WRITE "TAB1" RECORDS WITH CROSS SECTIONS INPUT FROM CARDS}

CASTROL ( reate $\underline{A}$ STRing Ordered Library) allows one to specify a function on cards and have it written out in "TAB1" format, so that it can be used in the JERGENS, VEL, VASELINE, CREST, ZEST, or other AMPX modules where a "point" cross-section library is required.

\section{CASTROL.1 CASTROL Input Data}

\section{Block 1.}

0\$ Logical Unit Assignment [1]

1. LOG - Unit onto which the cross sections are to be written (31)

$1 \$$ Problem Size [2]

1. NS - Number of functions to be written

2. ICORE - Number of words to allocate to CASTROL (25000)

$\mathrm{T}$ Terminate Block 1.

Data Blocks 2 and 3 are repeated NS times.

\section{Block 2.}

$2 \$$ Function Description [9]

1. MAT - The material number to be assigned to the function

2. MF - The file number to be used

3. MT - The process identifier

4. $\mathrm{C} 1$ - Constant associated with the function

5. C2 - Second constant associated with the function

6. L1 - First special flag associated with the function

7. L2 - Second special flag associated with the function

8. N1 - Number of interpolation regions

9. N2 - Number of points (energy-value pairs)

$\mathrm{T}$ Terminate Block 2.

\section{Block 3.}

$3 \$$ Interpolation Table $[2 * \mathrm{~N} 1]$

$\left(\mathrm{NBT}_{i}, \mathrm{JNT}_{i}, i=1, \mathrm{~N} 1\right)$

NBT is the point up through which the corresponding interpolation type, JNT, is to be used. 
Allowed interpolation types are

\begin{tabular}{|c|c|}
\hline JNT & Type \\
\hline 1 & Histogram \\
\hline 2 & Linear $\mathrm{E}$, linear $\sigma$ \\
\hline 3 & Log $E$, linear $\sigma$ \\
\hline 4 & Linear $\mathrm{E}, \log \sigma$ \\
\hline 5 & $\log E, \log \sigma$ \\
\hline
\end{tabular}

$4^{*}$ Function $[2 * \mathrm{~N} 2]$

$\left(\mathrm{E}_{i}, \sigma_{i}, i=1, \mathrm{~N} 2\right.$ ), where $\mathrm{E}$ is the energy in $\mathrm{eV}$, with $\sigma$ being the cross section (or flux, etc.) value.

T Terminate Block 3 .

\section{CASTROL.2 CASTROL Input/Output Specifications}

CASTROL uses the following input/output assignments:

\begin{tabular}{|c|c|}
\hline Logical No. & Purpose \\
\hline$u$ & Card input \\
\hline $\begin{array}{c}6 \\
\mathrm{LOG}(31)\end{array}$ & $\begin{array}{l}\text { Printed output } \\
\text { "TAB1" library }\end{array}$ \\
\hline
\end{tabular}




\title{
3.CLAROL - A MODULE TO REPLACE CROSS SECTIONS ON AN AMPX MASTER INTERFACE
}

\author{
Only AMPX Knows for Sure
}

CLAROL ( Correct Libraries $\underline{A}$ nd $\underline{\text { Replace }} \underline{\text { Old }} \underline{\text { Labels})}$ ) is a module that replaces or adds data in an AMPX master library at the lowest level (e.g., it can replace individual elements in either 1-D or transfer arrays). It also has provisions for modifying entries in the "Table of Contents" on a master library and for overriding the title cards that are associated with each data set on a master library.

Because this module operates at such a detailed level, it is recommended that the user be familiar with the idiosyncracies of the AMPX master interface format (Sect. 4.MISCELLANEOUS.1) before attempting to use CLAROL.

Another capability of CLAROL is to merge ROLAIDS-produced "superzone" weighted cross sections* (logical NIN) and data on an existing AMPX master library (logical NLD) to create an updated resonance-shielded AMPX master library (logical NEW). Although the CLAROL input for this operation is straightforward, care must be taken to ensure that data sets in the existing AMPX master library have identifiers that are compatible with the data sets in the ROLAIDS library.

CLAROL input data and input/output logical unit assignments are given in Sects. 3.CLAROL.1 and 3.CLAROL.2, respectively. Hints for executing special CLAROL cases are given in Sect. 3.CLAROL.3.

\section{CLAROL.1 CLAROL Input Data}

Block 1.

$-1 \$$ Special Vector Operations [100]

See Sect. 3.CLAROL.3, "Special CLAROL Cases," Items 4 and 5

0\$ Logical Unit Assignments [3]

1. NEW - New master library (1)

2. NLD - Old master library (28)

3. NIN - This parameter is both a trigger and a logical unit assignment. (0) NIN's function depends on its values as follows:

0 CLAROL is to replace or add data-blocks 2 and 3 are read in the standard input stream (no ROLAIDS-produced data are to be used).

$\mathrm{N}$ Blocks 2 and 3 are read from the ROLAIDS-produced "CLAROL data file" mounted on logical $\mathrm{N}$.

$6 \$$ Direct-Access Parameters for Logical Unit 8 [2]

The data from block(s) 3 are placed on logical unit 8 . Efficient utilization of the direct-access device may require changes in the following parameters:

*The ROLAIDS-produced library of "superzone" weighted cross sections is also called a "CLAROL data file." 
1. NBK - Number of physical records on logical unit $8^{*}(200)$

2. NWB - Number of words/block on logical unit $8^{\dagger}(880)$

$7 \$$ Print Suppression Trigger [3]

1. NOUT - Logical unit for printing CLAROL data set instructions. Assign a dummy unit to suppress printing. (6)

2. NOP - Logical unit for input data from ROLAIDS-produced CLAROL data file. Assign a dummy unit to suppress print. (6)

3. NCM - Logical unit for "status statements" that monitor progress of CLAROL calculations. Assign a dummy unit to suppress printing. (6)

$8 \$$ Core Allocation [1]

1. NWORD - Number of words allocated to CLAROL (100000)

T Terminate Block 1.

If $\mathrm{NIN}=0$, blocks 2 and 3 are repeated until a set with an identifier II $=0$ is specified. If $\mathrm{NIN} \neq 0$, the input to CLAROL is complete.

\section{Block 2.}

$1 \$$ Data Ser Identifier on the Output Master Library [1]

1. IDOUT - The identifier of the set of data which CLAROL will create on the output master library. If IDOUT $=0$, CLAROL will use II from the $2 \$$ array. If less than 100, CLAROL will form an identifier of II - 0 - IDOUT. (Note that this parameter can be used to create multiple copies of the set identified by II.)

$2 \$$ Set and Process Selection [4]

1. II - Data set identifier on the master library (on NLD)

2. MT - Process identifier (ENDF MT numbers are used)

3. NF - First group to be replaced or added

4. NL - Last group to be replaced or added

$3 \$$ Transfer Matrix Pointers [3]

1. NSINK - Sink group corresponding to transfer elements for groups NF to NL $($ default $=0)$

2. L - Order of transfer matrix to be modified (default $=0$ )

3. NTEMP - Temperature number of the matrix to be modified (default $=1$ )

T Terminate Block 2.

\footnotetext{
* Each set of Block 3 data is placed on a separate record.

t The actual number of words/block is the number of entries in the Block 3 being written.
} 
Block 3.

4* Cross-Section Values [NL $-N F+1]$

If $\mathrm{NSINK}=0$, this is:

$[\sigma$ (process MT, group i), $\mathrm{i}=\mathrm{NF}, \mathrm{NL}]$ for the Lth order transfer matrix given at the NTEMPth temperature number.

$\uparrow$ Terminate Block 3.

\section{CLAROL.2 CLAROL Input/Output Specifications:}

CLAROL uses the following input/output devices:

\begin{tabular}{c} 
Logical No. \\
\hline 5 \\
6 \\
16 \\
18 \\
NEW $(1)$ \\
NLD $(28)$ \\
NIN
\end{tabular}

\begin{tabular}{l} 
Purpose \\
\hline Card input \\
Standard output \\
Scratch device \\
Scratch device \\
New master interface \\
Old master interface \\
As explained in the input instruction for the 0\$ array, \\
NIN is both a trigger and a logical unit assignment. If NIN \\
is input >0, it is the logical unit for the ROLAIDS-produced \\
CLAROL data file.
\end{tabular}

\section{CLAROL.3 Special CLAROL Cases}

Several helpful observations for executing special CLAROL cases are given below.

\section{CLAROL.3.1 Title card replacement}

Enter IDOUT $=0$

Enter $\mathrm{MT}=0$, with NF and NL entered as the relative position of the first and last characters to be replaced in the title. To replace the entire title card, NF and NL should be 1 and 72, respectively. Block 3 is entered in the following manner:
$4 / 1 \mathrm{~N} \mathrm{~T} \quad$ where $\mathrm{N}$ is the number of four-character words required to hold the characters being replaced. (The " $\mathrm{T}$ " is the $\mathrm{T}$ which is used to indicate the end of the block.)
( Title $_{\mathrm{i}}, \mathrm{i}=1, \mathrm{~N}$ ) is written on a separate card in 18A4 format. Characters NF through NL of the 72-character title are replaced by the first NF - NL +1 characters read in.




\section{CLAROL.3.2 Resonance parameter replacement}

Enter $\mathrm{MT}=151$ with $\mathrm{NF}=1, \mathrm{NL}=6$ and $\mathrm{L}$ specified as the resonance number to be replaced. The six parameters are then given in the $4^{*}$ array.

\section{CLAROL.3.3 Creating new cross-section arrays}

New 1-D cross-section arrays can be placed on a master library. The MT number assigned to the new data must be different from any MT numbers in the original library. New 1-D arrays cannot be placed on a coupled neutron-gamma library with CLAROL. For a coupled master library, the UNITAB module should be used to split the coupled data into separate neutron and gamma-ray libraries, CLAROL should be run to add the new data to the libraries separately, and UNITAB should be run to recouple the modified libraries.

\section{CLAROL.3.4 Special vector commands}

These commands are provided to reduce the number of commands that must be specified to modify a library. For example, if one modifies the neutron-gamma (MT = $102)$ cross section, changes are also required to the capture $(\mathrm{MT}=101)$, absorption $(\mathrm{MT}=27)$, and total $(\mathrm{MT}=1)$ cross sections. These changes can, of course, be specified singly, but a "vector" command can be given which says to modify the latter three values (which are already consistent with the old neutron-gamma values) by the changes to the neutron-gamma values. follows:

The $-1 \$$ array can contain up to 100 entries which are arranged in strings as

$$
\text { Primary MT—one-or-more secondary MTs-zero }
$$

A string says to change the values of the primary process every time a change is made to any one of the secondary processes. Up to 20 primary processes (strings) can be specified, and the strings are any length which fit into the 100 words. Zeroes are used as string delimiters. As an example, if one specifies new values for MT $=102$ values, the $\mathrm{MT}=1,27,101$ values will be modified if the following three strings are input:

$$
\begin{array}{lllllllll}
1 & 102 & 0 & 27 & 102 & 0 & 101 & 102 & 0 .
\end{array}
$$

Whenever NIN is input greater than zero ( $0 \$$ array), the following vector string is automatically inserted:

$\begin{array}{rrrrr}1 & 2 & 18 & 102 & 0 \\ 27 & 18 & 102 & 0 & \\ 101 & 102 & 0 & & \end{array}$

which says to modify the total values when the elastic or fission or neutron-gamma values change, modify the absorption values when the fission or neutron-gamma values change, and modify the capture values when the neutron-gamma values change. 


\section{CLAROL.3.5 Replacement of entries in the master library directories}

Included in the AMPX master library is a set of records that is repeated for each data set in the library. The first record in the set, record R1, is called the data set "directory." To change any of the 50 words in a directory, enter MT $=-1$ and denote the number of the first and last words to be changed with NF and NL, respectively. Using block 3 , enter the new [NL - NF +1 ] integers in a $4 \$$ array; or if floating-point values are to be changed, use a $4^{*}$ array.

\section{CLAROL.3.6 Replacement of values in the Bondarenko reference cross sections}

Enter the nuclide identifier in II, $10000+\mathrm{MT}$ in MT (this is the flag that Bondarenko data are being replaced), the first group in NF and the last group in NL. The cross-section values are entered in the $4^{*}$ array. For the case where values of scattering matrices are being modified, the particular set is designated by entering $(100+\mathrm{NOFF}+\ell)$, where NOFF is the "offset" and $\ell$ is the order in the $\mathrm{L}$ value in the $3 \$$ array.

\section{CLAROL.3.7 Replacement of Bondarenko factor values}

Enter the nuclide identifier in II, $10000+$ MT in MT, the first group in NF, and the last group in NL. Enter the relative position of the $\sigma_{0}$ value in NSINK and the relative position of the temperature set in NTEMP. The factor values are entered in the $4^{*}$ array. For the case of factors for scattering matrices, the particular set is designated by entering $(100 \times \mathrm{NOFF}+\ell)$ in the $\mathrm{L}$ value of the $3 \$$ array, where NOFF is the "offset" and $\ell$ is the scattering order. 


\section{COLLINS - MODULE TO COLLECT INELASTIC SCATTERING INTO A SINGLE MATRIX}

COLLINS (COLLect INelastic Scattering) adds up the matrices for $\mathrm{MT}=51$ through $\mathrm{MT}=91$ into a single $\mathrm{MT}=4$ matrix and rewrites the master library. The matrices for $\mathrm{MT}=51$ through $\mathrm{MT}=91$ are not carried. Necessary adjustments are made to the directories on the master library. This module is very useful for compressing a master library, thereby reducing the input/output penalty for reading the detailed arrays for inelastic scattering transfer matrices.

\section{COLLINS. 1 COLLINS Input Data}

\section{Block 1.}

$0 \$$ Logical Assignments [2]

1. MMT - Logical number of new library (default $=1$ )

2. NMAX - Logical number of file to be accessed in creating MMT buffer requirements (this is an input unit, not logical MMT)

$1 \$$ Number of files [2]

1. NFILE - Number of files which will have data selected from them

2. NWORD - Number of words of core to allocate to COLLINS (50000)

$\mathrm{T}$ Terminate Block 1.

Blocks 2 and 3 are stacked, one after the other, NFILE times.

\section{Block 2.}

$0 \$$ File and Option Selection [2]

1. NF - Logical number of file considered

2. $\mathrm{IOPT}^{*}=-\mathrm{N}$ Delete $\mathrm{N}$ nuclides from $\mathrm{NF}$ to create the new file on MMT

0 Add all nuclides to the new file on MMT

$\mathrm{N}$ Add $\mathrm{N}$ nuclides from NF to create the new file on MMT

T Terminate Block 2.

\section{Block 3. $\{$ Enter only when IOPT $\neq 0\}$}

$3 \$$ Nuclides Selected [|IOPT $\mid]$

Identifiers of nuclides which are to be added or deleted from NF.

$4 \$$ New Identifiers [|IOPT $]$ ]

This array allows changing the identifier given in the $3 \$$ array when it is selected for the new library.

*Sets with duplicate identifiers will not be entered on MMT. The first occurrence of an identifier selects that set for the new library. 
T Terminate Block 3.

\section{COLLINS.2 COLLINS Input/Output Specifications}

The following devices are usually needed to execute COLLINS:

Logical No.

5

6

14

15

16

17

18

19

MMT(1)

NF

$\mathrm{NF}_{2}$

$\mathrm{NF}_{3}$

$\mathrm{NF}_{N F I L E}$
Purpose

Card input

Standard output

Scratch device

Scratch device

Scratch device

Scratch device

Scratch device

Scratch device

Master file to be created

Files to be merged onto MMT(1) 


\section{COMAND - MODULE TO COLLAPSE ANISN CROSS- SECTION LIBRARIES}

COMAND (COllapse Multigroup ANISN Data) is a module for collapsing ANISN libraries. It accepts ANISN libraries in BCD or binary format, truncates upscatters and/or downscatters, combines the ANISN data from up to four libraries, and allows for stripping groups (neutrons or gammas) from the libraries. Several weighting spectra can be read in and used in the same computer run. COMAND collapses all sets of data on the input library.

\section{COMAND.1. COMAND Inpu' Data}

\section{Block 1.}

$1 \$$ ANISN Library Parameters [8]

1. IGM - Number of groups

2. IHT - Position of $\sigma_{\mathrm{T}}$

3. IHS - Position of $\sigma_{g \rightarrow g}$ Input ANISN library

4. IHM - Table length

5. IGHF -Number of groups

6. IHTF -Position of $\sigma_{\mathrm{T}}$

7. IHSF -Position of $\sigma_{g \rightarrow g}$

8. IHM - Table length

$2 \$$ More Data [8]

1. IEDIT - 0, almost no edit (0)

1 , edit the output collapsed data

2 , edit the input fine-group and the output collapsed data

2. NCARDS - 0 , no BCD-formatted input cross sections (0)

$\mathrm{N}$, use the Nth spectrum to weight $\mathrm{BCD}$-formatted input cross sections

3. MODE - 0, BCD input is free-form FIDO

$1, \mathrm{BCD}$ input is formatted FIDO

4. NPNCH -0 , no punch $(0)$

1 , punch collapsed cross sections

5. NSPEC - Number of weighting spectra that will be read in the $4^{*}$ array (1)

6. NSCRTH - A collapsed ANISN library is written on this logical number (2)

7. NUNIT - BCD-formatted data are input on this logical unit (5)

8. NCHI - Position of fission spectrum $(\chi)$ in fine-group tables - if these data are included in the ANISN library

9. NWORD - Number of words to allocate to COMAND (50000) 
$3 \$$ Logical Units of ANISN Binary Libraries and Weighting Spectra Selectors [8]

1. NT1 - Logical number of first ANISN binary library to be collapsed (0)

2. NT2 - Logical number of second ANISN binary library to be collapsed (0)

3. NT3 - Logical number of third ANISN binary library to be collapsed (0)

4. NT4 - Logical number of fourth ANISN binary library to be collapsed (0)

5. NS1 - Weighting spectrum to associate with NT1 (1)

6. NS2 - Weighting spectrum to associate with NT2 (1)

7. NS3 - Weighting spectrum to associate with NT3 (1)

8. NS4 - Weighting spectrum to associate with NT4 (1)

(Note that NT2 can equal NT1 and allow weighting the same cross sections over different spectra, etc.)

$\mathrm{T}$ Terminate Block 1.

Block 2.

4* Few-Group Weight Spectra [NSPEC*IGM]

Stack NSPEC sets of IGM numbers in this array. The value for the highest energy of the first spectrum is first.

5* Broad-Group Numbers by Few Group [IGM]

This group corresponds to the $28 \$$ array in ANISN and $51 \$$ array in XSDRNPM. A zero entry will delete a group. This feature allows stripping neutron or gamma-ray groups.

T Terminate Block 2.

\section{COMAND.2 COMAND Input/Output Specifications}

COMAND generally requires the following input/output devices to be available:

Logical No.

5

6

7

$\operatorname{NT} 1(0)$

NT2(0)

NT3(0)

NT4 $(0)$

NUNIT(5)

NSCRTH(2)
Purpose

Card input

Standard output

Punched output

ANISN binary library input

ANISN binary library input

ANISN binary library input

ANISN binary library input

BCD ANISN library

Collapsed ANISN binary library 


\section{COMAND.3 COMAND Special Notes}

A. To assume a flat (unity) weighting spectrum, enter $\Delta E_{g}$ in the $4^{*}$ array, where $\Delta E_{g}$ is the energy width of group in eV's. To assume a $1 / \mathrm{E}$ weighting spectrum, enter the lethargy widths of the energy groups.

B. BCD-formatted cross sections, input either on cards or on tape, are read in subroutine COLANS with the following statements:

DIMENSION A(12),D(NA)

-

150

READ(NUNIT,10400)NOG,ITL,ICT,ID,A

IF(ICT.EQ.7)GO TO 170

CALL READSG(D(LFINE),NOG*ITL,NUNIT,MODE)

10400 FORMAT $(416,12 \mathrm{~A} 4)$

GO TO 150

170 CONTINUE

where

$\mathrm{NA}$ is the dimension of the D array,

NOG is the number of groups in the ANISN library on NUNIT,

ITL is the cross-section table length,

ICT is the trigger to signal the end of the library,

ID is the identification number of the ANISN-formatted data set,

$A$ is the title card array (12A4 format), and

$\mathrm{D}$ is the array for the cross sections. Appropriate space is allocated in another subroutine.

READSG is an AMPX library routine. A "T" should not end the BCD-formatted cross sections [i.e., (NOG*ITL) cross sections are read in subroutine READSG]. Note that the final card of the BCD-formatted cross sections must be a "NOG, ITL, ICT, ID, A" card with ICT equal to seven. See statement 10400 above for the format. 
C. COMAND does not retain the original identification numbers on the input ANISN library or libraries. It numbers the identifiers in the collapsed ANISN library (on NSCRTH) sequentially (i.e., $1,2, \ldots, \mathrm{NT}$ ), where NT equals the total number of data sets on NT1, NT2, NT3, and NT4. 


\section{COMET - MODULE TO FORCE CONSISTENCY BETWEEN AVERAGED VALUES AND SCATTERING MATRICES ON AMPX MASTER LIBRARIES}

COMET ( COrrect Master Excessive Tolerance) is a module to correct selected portions of an AMPX master interface. A complete library can be easily corrected. Operations are provided to ensure that transfer matrices normalize to the proper 1-D values, or vice versa. Note that COMET copies the whole library, not just selected data sets. Although coupled neutron-gamma libraries can be read in, COMET only "operates" on the neutron data on a master library.

\section{COMET.1 COMET Input Data}

\section{Block 1.}

$-1 \$$ Special Vector Manipulations [200] (default = all zeroes)

These commands are used when it is desired that certain groupings of cross sections sum to a "total" value. For example, it is desirable that $\sigma_{a}=\sigma_{c}+\sigma_{f}$ or that all inelastic partial values sum to the total inelastic values. This array is made up of strings of MT numbers as follows:

Primary MT number-Secondary MT numbers-Zero

This string directs COMET to ensure that the use of the cross sections identified by the secondary MT numbers sums to the cross section identified by the primary identifier. Zeroes serve as "string delimiters." A positive primary identifier says to sum the secondaries and replace (or form) the primary values. A negative primary identifier says to normalize the secondaries such that they sum to the primary. Up to 20 strings can be specified. A negative secondary says to subtract this process so that one can form, for example, $\sigma_{c}=\sigma_{a}-\sigma_{f}$.

$0 \$$ Logical Unit Assignments [3]

1. MMTO - Old master library

2. MMTN - New master library

3. MSC - Scratch device

$1 \$$ Options [2]

1. IOPT

-1 - Correct the "Table of Contents" of the master library on MMTO. (MMTN is not used.)

0 - "Correct" the complete master library on MMTO, and write a new one on MMTN.

$\mathrm{N}$ - Correct $\mathrm{N}$ sets from IMTO and write on MMTN.

2. ICORE - Number of words to allocate to COMET (100000)

$2 \$$

MT - Numbers of processes to be corrected [200] 
Place up to $200 \mathrm{MT}$ numbers into the $2 \$$ array. A negative MT number says to force the $1-D$ cross section to agree with the appropriate transfer matrix sums; a positive MT number forces the transfer matrix to be normalized to the 1-D value. After entering the MT numbers of interest, complete the arrays by filling with zeroes.

$6 \$$ Direct-Access File Characteristics [2]

1. NBLK - Number of blocks to use in the direct-access file on logical N9 (400)

2. LRECL - Number of words in each block on the direct-access file on logical N9 (788)

T Terminate Block 1.

Block 2. $\quad\{$ Input if IOPT $>0$ \}

$3 \$$ Identifiers for Master Data Sets to be corrected [IOPT]

$\mathrm{T}$ Terminate Block 2.

\section{COMET.2 COMET Input/Output Specifications}

COMET requires the following input/output devices:

Logical No.

5

6

9

17

$\operatorname{MMTO}(28)$

$\operatorname{MMTN}(1)$

$\operatorname{MSC}(18)$
Purpose

Card input

Standard output

Scratch device (randorn access)

Scratch device

Old master library

New master library

Scratch device 


\section{CONTAC - MODULE TO PRODUCE ANISN OR CCCC ISOTXS LIBRARIES FROM AMPX WORKING LIBRARIES}

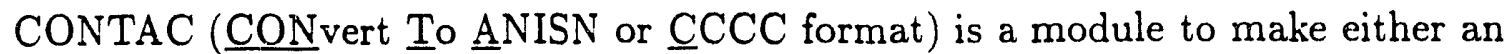
ANISN or a CCCC ISOTXS cross-section library from an AMPX working library. The complete working library is converted to either punched cards or a binary library if the ANISN format is selected. If a CCCC ISOTXS library is selected, the nuclides to be placed on the interface and their new names are specified in the input.

\section{CONTAC.1 CONTAC Input Data}

\section{Block 1.}

$-1 \$$ Core Allocation [1]

1. ICORE - Number of words to allocate to CONTAC (100000)

O\$ Logical Unit Assignments [4]

1. NT3 - AMPX working or weighted library (3)

2. NT2 - ISOTXS or ANISN binary library (20)

3. NT7 - Scratch device (18)

4. NPU - ANISN punched output

$1 \$$ Output Control Options [2]

1. ITP - 1 - Make a CCCC ISOTXS library (0)

2 - Punch ANISN cards

3 - Make ANISN binary library

2. IMP - Number of nuclides to be put on NT2 when an ISOTXS library is made (0)

$2 \$$ ANISN Output Control Parameters [6]

1. MSCM - Number of extra 1-D cross sections to be placed on ANISN output.

(0) If the value of this parameter is input as -1 and a CCCC ISOTXS library is being made (ITP =1), it signals that the fission spectrum to be written on that library will be specified in the $7^{*}$ array and velocities will be specified in the $8^{*}$ array below.

2. IHT - Position of total cross-section ANISN formats (0)

3. IDS - Position of within-group-scattering cross section in ANISN formats (0)

4. ITL - Table length for cross sections in ANISN formats (0)

5. IPRT - Print control for ANISN output (0)

(ANISN arrays will be printed through order of scattering equal IPRT. A -1 will suppress the printing of all ANISN arrays.)

6. IOLD - 0 - A new ANISN library will be written (0)

1 - Data will be added to an existing ANISN library

T Terminate Block 1 . 


\section{Block 2.}

$3 \$$ Nuclide Identifiers of Sets to be Placed on ISOTXS. [IMP]

$4 \$$ MT Number(s) of the Transport Cross Sections to be Placed on ISOTXS (filled with 1001) [IMP]

5/ Six-Character Hollerith Names to be Placed on ISOTXS

The names correspond to nuclides in the $3 \$$ array. [2*IMP] (The / is followed by an integer identifying the first location to be filled and then an integer identifying the last location to be filled [i.e., ( 1 and $2 *$ IMP)]. Starting on the next card, the names are read with an $18 \mathrm{~A} 4$ format, but the six-character Hollerith names should be considered as being read with a $9(A 6,2 X)$ format.

$6 \$$ Extra Cross-Section MT Numbers [MSCM]

For neutron data, these are the ENDF MT numbers of the cross sections which are to be placed in the ANISN cross-section tables in the first MSCM positions. For photon-interaction data (SMUG-produced data), these are the AMPX MT identifiers minus 500 (e.g., 102 is the identifier for the photoelectric cross section, which has an AMPX identifier of 602).

$7^{*}$ Fission Spectrum [IGM] $\{$ ITF $=1$ and MSCM $=-1\}$

Input the group-integrated values for the fission spectrum - highest-energy group to the lowest. Use this array to provide a fission spectrum to be written on an ISOTXS file, when the input library is a working library (not a weighted library).

8* Velocities [IGM] $\{$ ITF $=1$ and $\mathrm{MSCM}=-1\}$

Input weighted velocities for each neutron group. Use this to supply velocities for an ISOTXS file when the input library is a working library.

$9 \$$ Maximum Order of Scatter by Nuclide [IMP]

Use this array to specify the maximum order of scatter, which will be written on the ISOTXS file.

T Terminate Block 2.

Note: If the 5/ array is the last array in Block 2, an " $E \mathrm{~T}$ " is entered to end the data block. 


\section{CONTAC.2 CONTAC Input/Output Specifications}

CONTAC requires the following input/output devices:

Logical No.

Purpose

5

Standard card input

6

NPU(7)

NT2(20)

NT3(3)

NT7(18)

Standard printed output

Punched output

ISOTXS or ANISN binary library

Input AMPX working (or working/weighted)

library

Scratch device 


\section{CORECTOL - MODULE TO CONVERT PRE-AMPX-77 MASTER LIBRARIES TO THE AMPX-77 FORM}

Prior to AMPX-77, the implementation of the Nordheim Integral Treatrnent in the NITAWL module assumed that the cross sections in the master library would be split into two cases:

1. cross sections for the bodies of the resonances (weighted over a reference flux); and

2. wing cross sections, which included correction values, values for $\ell=1,2$, etc., resonances (i.e., everything in the cross sections which the Nordheim calculation was rot going to calculate)

This arrangement allowed one to simply add these pieces together if an infinite dilution set was desired, or to perform a resonance self-shielding calculation and add to the second set noted above to obtain self-shielded values.

This procedure required that the basic processing code, such as XLACS, be required to know exactly where the bodies of the resonances would be calculated, and, thereby, precluded the use of the Nordheim treatment with libraries not based on this knowledge.

It was recognized that a better way to implement the Nordheim treatment would be to reference the self-shielded values to infinite dilution values, that is, the Nordheim calculation would really be two calculations: (1) the regular calculation, just as before, and (2) a calculation where the cross-section values are simply weighted over a $1 / \mathrm{E}$ flux. The difference in the two values could then simply be added to the reference set to obtain shielded values. This method has several obvious advantages: (1) it allows the Nordheim calculation to be performed with any library that used $1 / \mathrm{E}$ weighting in the resonance range, a commonly used scheme; and (2) it allows one to always refine (or unrefine) a calculation by adding (for example, $\ell=1$ ) or deleting resonance parameters. This method has other advantages from an accuracy viewpoint, which we will not discuss here.

Clearly, the base library needed by the two approaches is different. The task of taking the earlie: AMPX master libraries and converting them to the new format by adding together the resonance body values and the wing values and discarding the wing values is the purpose of the CORECTOL (CORrECT Old Libraries) module. It is a very simple module to use and requires essentially that the user define where the files are located. Obviously, CORECTOL has no counterpart module for converting newer libraries to the older form, because it is this complicated task that the newer procedures have been designed to eliminate.

In the event you do not know if your library is in the older or newer format, the ALE module can be run (the header information contains a parameter called the "Nordheim Processing Level," which will be " 0 " on older libraries and will be " 2 " on the new ones). 


\section{CORECTOL.1 CORECTOL Input Data}

Block 1.

$-1 \$$ Core Assignment [1]

1. ICORE - Number of words of core to assign to this module (100000)

o\$ Logical Assignments [4]

1. NT1 - Old AMPX master

2. NT2 - New AMPX master

3. NS - Scratch device (18)

4. NT - Scratch device (19)

T Terminate Block 1.

\section{CORECTOL.2 CORECTOL Input/Output Specifications}

CORECTOL uses the following input/output devices:

\begin{tabular}{l} 
Logical No. \\
\hline 5 \\
6 \\
NT1(1) \\
NT2(2) \\
NS(18) \\
NT(19)
\end{tabular}

\begin{tabular}{l} 
Purpose \\
\hline Card input \\
Printed output \\
Old AMPX master library \\
New AMPX master library \\
Scratch device \\
Scratch device
\end{tabular}




\section{CREST - A MODULE TO COLLECT INFORMATION FROM ENDF/B, MASTER, AND WORKING LIBRARIES}

CREST (Collect and REname STrings) is a module that will scan ENDF/B and AMPX master and working libraries and collect information from them to write out in ENDF/B "TAB1" format (string format). The file, produced by CREST, can be used by a variety of modules, including JERGENS, VEL, and VASELINE.

The binary form of the "TAB1" format consists of three records per process:

$$
\begin{aligned}
& \text { Record } 1 \text { - MAT, MF, MT, NZ, NZ, NZ, NZ, NZ, NZ, } \\
& \text { Record } 2 \text { - MAT, MF, MT, NZ, NZ, NZ, NZ, N1, N2,(NBT }, \mathrm{JNJ}_{\mathrm{i}}, \mathrm{i}=1 \text {, } \\
& \text { N1), (X, } \left.\mathrm{X}_{\mathrm{i}}, \mathrm{i}=1, \mathrm{N2}\right), \\
& \text { Record } 3 \text { - MAT, MF, NZ, NZ, NZ, NZ, NZ, NZ, NZ, }
\end{aligned}
$$

where MAT is the MAT-number, MF is the file number (always 3 for CREST), MT is the process identifier, NZ is a zero, N1 is the number of interpolation regions, N2 is the number of energy value pairs, NBT,JNT are the interpolation table parameters, and $X, Y$ define the function. Values taken from a master or working library specify that histogram interpolation is to be used with the group-averaged values.

\section{CREST.1 CREST Input Data}

\section{Block 1.}

$1 \$$ Case Description [2]

1. MP - Number of "commands" given in Block(s) 2

2. IP - This is the logical number of the file to be written (default $=31$ )

$2 \$$ Options [10]

1. IOPT1 - Not used

2. IOPT2 - Not used

3. IOPT3 - Not used

4. IOPT4 - Not used

5. IOPT5 - Not used

6. IOPT6 - Not used

7. IOPT7 - Not used

8. IOPT8 - Not used

9. IOPT9 - Not used

10. IOPT10 - Number of words allocated to CREST (50000)

$\mathrm{T}$ Terminate Block 1.

Block 2 is repeated MP times. 
Block 2.

$3 \$$ Process Selection [5]

1. MATNO - New "MAT" number to be given the string of information

2. MT - Process identifier

3. IDM - Identifier of the nuclide on the master file

4. IDW - Identifier of the nuclide on the working file

5. IDE - MAT number for the nuclide on the ENDF file (only one of the parameters IDM, IDW, and IDE should be specified nonzero in a command)

$4 \$$ File Locations and Modes [5]

1. MMTN - Logical number of the master file (default $=1$ )

2. MWTN - Logical number of the working file (default $=4$ )

3. MENDF - Logical number of the ENDF library (default =11)

4. MODE - "Mode" of the ENDF library (default = 1 for binary formatted libraries; a "2" should be used for BCD formatted libraries)

5. MFILE - ENDF "file" containing the data (default = 3)

T Terminate Block 4.

\section{CREST.2 CREST Input/Output Specifications}

CREST may require the following input/output devices:

\begin{tabular}{c} 
Logical No. \\
\hline 5 \\
6 \\
IP(31) \\
MMTN \\
MWTN \\
MENDF
\end{tabular}

\begin{tabular}{l}
\multicolumn{1}{c}{ Purpose } \\
\hline Card input \\
Standard output \\
Created file \\
Master library \\
Working library \\
ENDF/B library
\end{tabular}

Note that several files may be required in a single execution of MMTN, MWTN, and MENDF. 


\section{FRESH - AMPX MODULE TO RENORMALIZE THERMAL SCATTERING MATRICES}

FRESH (Fix RESonance Hangups) is a module that allows one to post-process the thermal-scattering matrices for many resonance nuclides produced by XLACS-77 in order to normalize them to user-specified values. Note that only resonance nuclides need this operation, and only those with strong resonance effects at thermal energies.

A problem arises from the procedure used in XLACS-77 to generate these data. This procedure generates $S_{\alpha, \beta}$ data consistent with a free-gas model that assumes the thermal-scattering cross section is a flat value at $0 \mathrm{~K}$. This value is the so-called freeatom cross section. It is not part of the ENDF/B file and, therefore, must be acquired from somewhere else. At one point, XLACS used the value for elastic scattering in the first group above thermal. The present XLACS-77 scheme uses the scattering radius from File 2 on the ENDF library to calculate a scattering cross section. Neither of these schemes is very satisfactory, because they both generate a smooth variation in the thermal region, which does not reflect the true shape.

FRESH provides three options for dealing with nuclides:

Option 0 - do not adjust thermal matrices,

Option 1 - normalize the matrices to the averaged values produced by the resonance calculation in XLACS,

Option 2 - normalize the matrices such that the value in the first thermal group is equal to that in the first group above thermal.

Option 1 will probably ultimately be incorporated into XLACS-77, thereby precluding the need for this exercise. It certainly is the most reasonable approach. However, until this time, the FRESH module should be used. It is wise to keep in mind that the effect from this repair is negligible in most cases. Resonance nuclides are generally heavier nuclides, and, for this case, thermal-scattering kinematics do not greatly affect most analyses, because the result of a scattering is to shift the energy of the neutron only slightly.

\section{FRESH.1 FRESH Input Data}

\section{Block 1.}

$-1 \$$ Core Allocation [1]

1. ICORE - Number of words to allocate to FRESH (100000)

$0 \$$ Logical Assignments [1]

1. MMT - Output AMPX master library (1)

$1 \$$ Files to Process [1]

1. NFILE - Number of master libraries that will be accessed

T Terminate Block 1.

Blocks 2 and 3 are repeated NFILE times. 
Block 2.

$2 \$$ File Selection [12]

1. NF - Logical number of the file to process

2. IOPT - Number of nuclides to accept from $t$. : file on logical NF

3. II

4. I2

- $\quad$ Reserved for special applications that should be

. $\int$ of no interest to users

12. $\mathrm{I} 10$

T Terminate Block 2.

\section{Block 3.}

$3 \$$ Nuclide Selection [IOPT]

Input the identifiers for the nuclides selected from logical NF.

$4 \$$ Nuclide Identifier Override [IOPT]

Input a nonzero new identifier value in the position corresponding to an identifier in the $3 \$$ array to change the nuclides identified on the output file.

$5 \$$ Normalization Option [IOPT]

Enter 0 to bypass normalization, 1 to normalize to average to resonance values or 2 to normalize to the first scattering value above thermal. These values are given in one-to-one correspondence with the $3 \$$ array.

$6 \$$ Combination of $3 \$$ and $5 \$$ array [ $2 *$ IOPT]

This is a convenience array and recognizes that long lists in separate arrays, such as the $3 \$$ and $5 \$$ arrays, are sometimes difficult to keep value associations correct. If one chooses the alternative array, input the identifier followed by the option, etc., until IOPT pairs are specified. Do not input the $3 \$$ or $5 \$$ arrays. (The $4 \$$ array still must be given in correspondence with the doublets. It was elected not to include it in the $6 \$$ array in recognition that the need to override identifiers is not that common.)

T Terminate Block 3. 


\section{FRESH.2 FRESH Input/Output Specifications}

FRESH uses the following input/output assignments:

\begin{tabular}{c} 
Logical No. \\
\hline 5 \\
6 \\
15 \\
16 \\
18 \\
19 \\
MMT \\
NF
\end{tabular}

\begin{tabular}{l}
\multicolumn{1}{c}{ Purpose } \\
\hline Card input \\
Standard output \\
Scratch file \\
Scratch file \\
Scratch file \\
Scratch file \\
Output master file \\
Input master file \\
(there will be NFILE of these)
\end{tabular}




\section{GERITOL - AMPX MODULE TO PLACE GROUP CONSTAN'TS INTO A "STRING" LIBRARY}

Given a set of group fluxes

$$
\left(\phi_{i}=\int_{E_{i}+1}^{E_{i}} \phi(E) d E\right)
$$

or group cross sections

$$
\left(\sigma_{i}=\frac{1}{E_{i}-E_{i+1}} \int_{E_{i+1}}^{E_{i}} \sigma(E) d E\right),
$$

GERITOL (GEneRate InexacT Oscillating Lines) uses the interpolation allowed in ENDF and a least-squares fitting routine to generate a set of $(E, F(E))$ pairs to place the generated data into a set of ENDF HEAD, TAB1, and SEND records - a "string" library.

For each group in the input energy structure, GERITOL first calculates the value of $F(E)=\phi_{i} /\left(E_{i}-E_{i+1}\right)$ for input fluxes or $F(E)=\sigma_{i}$ for input cross sections. Then, the module fits the $F(E)$ 's to the following three forms: $F(E)=A+b \times \ln (E)$, $\ln [F(E)]=A+b \times E$, and $\ln [F(E)]=\ln [A+b \times E]$. (For those fitting functions linearly dependent on energy, the value of $F(E)$ is taken at the group midpoint energy. For those fitting functions dependent on the logarithm of energy, the value is taken at the lethargy midpoint.) The parameters $\mathrm{A}$ and $\mathrm{b}$ for each fitting function are then used to calculate the integral in the group, and the function yielding the integral with the smallest deviation from the known integral is assigned to that group.

If the integral calculated by the "best" function is not within an input fraction, DVMIN, of the true integral, this function is discarded and a linear-linear function is assumed. Likewise, if the integral computed by the linear-linear function is not within DVMIN of the true integral, or if the function at either group boundary is negative, this function is discarded, and a constant function is chosen to make the integral correct.

Once a best-fitting function is determined, the values of $F(E)$ at the group boundaries are computed and stored. When all groups have been processed, one has a TAB1 record, with the number of points equal to twice the number of groups. The number of points is then thinned by discarding the value of $F(E)$ at the lower boundary of a group if it is within an input fraction, EPS, of the value of $F(E)$ at the upper boundary of the previous group. Some thinning is also achieved by discarding points in successive regions whose parameters are equal within the same fraction, EPS.

GERITOL output on logical unit LUN consists of the three ENDF records (HEAD, TAB1, and SEND) in binary format with the MAT, MF, and MT identifiers specified in the input. If flux values are input (NTYPE $=0$ ), then the output values have units of flux per unit energy. If cross sections are input (NTYPE $=1$ ), the output has the same units as the input. Positions N1 and N2 in the HEAD record contain the input option variables NTYPE and IPLOT. The series of three ENDF records are repeated on LUN for each input group function (i.e., each set of Blocks 2 and 3 data). After the 
final series is written in LUN, the logical unit is rewound and read as a final check on the validity of the data. The integrals by group are recomputed and compared with the true integrals.

Two particularly useful applications of the string libraries on LUN are (1) for use as pointwise weighting functions in the neutron- and photon-production crosssection modules XLACS-77 and LAPHNGAS, respectively, and (2) for input to VEL, the AMPX module that converts pointwise data to multigroup data. Note that a GERITOL-VEL sequence could be used to change multigroup data from one group structure to another group structure. One must exercise caution, however, to ensure that the application of the pointwise function does not exceed the limitations of the input group function. If the input group values represent an integral of a function over an energy range in which the function has significant variation, then the subsequent use of this point function to calculate integrals over a much smaller energy range may not be valid.

An option is provided for producing point and multigroup data libraries for the subsequent plotting of the data with the VASELINE module. If the option is triggered (IPLOT = 1), a string library is produced on logical unit ISCR similar to the string library described above. The difference between the LUN and ISCR libraries is that, if fluxes are input, the units of the data on ISCR are flux times energy and, if cross sections are input, the units are the same as the data input in $4^{*}$ data. Thus, the data on ISCR are in the units required for plotting point data with VASELINE. Also, the Blocks 2 and 3 multigroup data are changed to the appropriate units, described below, and placed in AMPX working library format on logical unit KSCR - a forma.t which can be input to VASELINE. If only one set of Blocks 2 and 3 data is inI the abbreviated AMPX working library is placed on KSCR1. If more than one set of Blocks 2 and 3 data is input, GERITOL places all multigroup sets of data with the number of groups equal to IGM of the first Block 2 input on KSCR1. Multigroup data for the next value of IGM are placed on KSCR2, etc. GERITOL has provisions for a maximum of five KSCR units (i.e., has provisions for a maximum of five different values of IGM). If fluxes are input, the data on KSCR1 have units of flux/lethargy width; if cross sections are input, the units are the same as the $4^{*}$ data. In each case, the data on KSCR1 are in the units required for plotting multigroup data with VASELINE.

\section{GERITOL.1 GERITOL Input Data}

Input to GERITOL is made in the free-form FIDO schemes employed throughout AMPX. 
Block 1.

0\$ Logical Assignments [7]

1. LUN - String Library (31)

2. ISCR

3. KSCR1

4. KSCR2

5. KSCR3

$(0)(0)(0)(0)(0)(0)$

If the plot preparation option is triggered (IPLOT = 1), the string library for input to VASELINE is placed on ISCR.

6. KSCR4

7. KSCR5

The abbreviated working library is placed

on KSCR1 and, if required, other working libraries are placed on KSCR2, .., KSCR5.

T Terminate Block 1.

Blocks 2 and 3 are repeated for each set of group constants for which a point function is desired, and the run is terminated by specifying a zero for the number of groups. Redundant information, such as the group boundaries, does not need to be repeated in successive cases.

\section{Block 2.}

$1 \$$ General Information [6]

1. IGM - Number of groups

2. MAT3. MF4. MT ENDF identifiers given to output point function

5. NTYPE -0 , Group constants are fluxes $(0)$

6. IPLOT 1 , Group constants are cross sections

2* Tolerances [2] -0 , No plot tapes are desired $(0)$ 1, Plot tapes should be generated

1. DVMIN - Fractional difference allowed in group reconstruction $(0.05)$

2. EPS - Fractional difference allowed in thinning. This applies to function values reconstructed at group boundaries from parameters in adjacent regions and to the parameters themselves. $(0.05)$

$T$ Terminate Block 2.

\section{Block 3.}

$3^{*}$ Energy group boundaries, high to low in eV [IGM+1]

$4^{*}$ Group constants [IGM]

$\mathrm{T}$ Terminate Block 3.

\section{GERITOL.2 GERITOL Input/Output Specifications}

GERITOL requires the following devices during a normal calculation: 
Logical No.

Purpose

\begin{tabular}{cll}
\cline { 1 - 1 } 5 & & Card input \\
6 & & Standard output \\
LUN(31) & & Output string library \\
47 & & Built-in group structure library*
\end{tabular}

If plotting output is generated (IPLOT=1), the following devices are also required:

$\begin{array}{lc}\text { Logical No. } & \text { Purpose } \\ \text { ISCR(0) } & \begin{array}{c}\text { Output string library for VASELINE } \\ \text { KSCR1(0) }\end{array} \\ \text { Abbreviated working library for first } \\ \text { KSCR2(0) } & \text { AGM structure } \\ & \text { IGM structure } \\ \text { KSCR3(0) } & \text { Abbreviated working library for third } \\ & \text { IGM structure } \\ \text { KSCR4(0) } & \text { Abbreviated working library for fourth } \\ & \text { IGM structure } \\ \text { KSCR5(0) } & \text { Abbreviated working library for fifth } \\ & \text { IGM structure }\end{array}$

${ }^{*}$ See Sect. 4.MISCELLANEOUS.4. 


\section{ICE - MODULE TO MIX DATA ON A WORKING LIBRARY TO PRODUCE MACROSCOPIC LIBRARIES}

ICE (Initiate Compression Exercise) is a module that uses an AMPX working library in conjunction with a user-supplied set of instructions on how to mix cross sections to produce macroscopic cross sections which can be written in any of several popular library formats, including:

- an AMPX working library,

- an ANISN library, or

- a library for the KENO program.

Any or all of these libraries can be made in the same execution of ICE.

\section{ICE.1 ICE Input Data}

Card A (20A4) Title Card

\section{Block 1.}

$-1 \$$ Direct-Access Specifications [4]

1. NB8 - Number of blocks for the first direct-access unit (200)

2. NL8 - Length in units of 4-byte words of a block for the first direct-access unit (880)

3. NB9 - Number of blocks for the second direct-access unit (400)

4. NL9 - Length of a block for the second direct-access unit (880)

0\$ Logical Unit Specifications for Various Cross-Section Libraries [5]

1. INTAPE - Input AMPX working library unit (4)

2. IOT1 - Output AMPX working library unit (3)

3. IOT2 - Output ANISN library unit $(20)^{*}$

4. IOT3 - Output ANISN group-independent library urit 21)

5. IOT4 - Output Monte Carlo processed cross-section libsay unit (14)

If punched output is desired, the unit should be set to 7 .

$1 \$$ Problem Size and Major Options [7]

1. MIX - Number of cross-section mixtures to be made

2. NMIX - Number of mixing operations (elements times density operations) to be performed

3. IFLAG(1) - Set greater than zero if AMPX working Gbrary output desired.

4. IFLAG(2) - Set greater than zero if ANISN format ontrut desired.

\footnotetext{
*If punched output is desired, the unit should be set to 7 .
} 
5. IFLAG(3) - Set greater than zero if group-independent ANISN output desired.

6. IFLAG(4) - Set greater than zero if Monte Carlo processed cross-section library output desired.

7. KOPT - Option to be used to calculate $\chi$ of the mixture (2)

$\mathrm{KOPT}=0, \chi$ of element with the largest sum of $\nu \Sigma_{f} \phi$ over all groups is used

$\mathrm{KOPT}=1, \chi$ calculated based on an input flux distribution

$\mathrm{KOPT}=2, \chi$ calculated based on a default thermal reactor flux distribution

$\mathrm{KOPT}=3, \chi$ calculated based on a default fast reactor flux distribution

$\mathrm{KOPT}=4, \chi$ calculated based on the fluxes provided in the input tape for each element in the working library

$2 \$$ Core Allocation* [1]

1. NWORD - Number of words allocated to ICE (100000)

T Terminate Block 1.

The following table contains the options which can be used in the $1 \$$ array:

IFLAG options

Print options

0

No cross-section printing

1

Print 1-D cross sections (available only in AMPX working library format)

2

Print 1-D cross sections and $P_{0}$ cross sections (not available in ANISN group-independent format)

3

Complete cross-section printing

Output options

00

No tape or punch output

10

Binary tape output

20

Free-form FIDO punch output (available only in ANISN and group-independent ANISN formats)

30

Fixed-form FIDO punch output (available only in

ANISN and group-independent ANISN formats)

Note that IFLAG should be set to the sum c je print option and output option desired. 
Block 2.

$2 \$[N M I X]$

1. $(\mathrm{KM}(\mathrm{I}), \mathrm{I}=1, \mathrm{NMIX})$

Mixture numbers in the mixture specification table - values range from 1 to MIX

$3 \$$ [NMIX]

1. $(\mathrm{KE}(\mathrm{I}), \mathrm{I}=1, \mathrm{NMIX})$

Element identifiers for the mixture specification table

4* [NMIX]

1. $(\mathrm{RHO}(\mathrm{I}), \mathrm{I}=1, \mathrm{NMIX})$

Atom densities for the mixture specification table

$5 \$[\mathrm{MIX}]$

1. (NCOEF(I), I=1,MIX) Number of Legendre coefficients, including $P_{0}$, to be mixed for each mixture

6* $[\mathrm{NG}+4]$

1. $(\operatorname{FLUX}(\mathrm{I}), \mathrm{I}=1, \mathrm{NG})$

Flux to be used in calculation of $\chi$ if $\mathrm{KOPT}=1 ;$ default $(\mathrm{FLUX}(\mathrm{I})=1.0, \mathrm{I}=1, \mathrm{NG})$ where $\mathrm{NG}=$ number of neutron groups

Note: The four following parameters are required only if $\mathrm{KOPT}=2$ (i.e., a standard thermal reactor flux is calculated)

2. TEMP Temperature in Kelvin to be used for Maxwellian distribution (300)

3. THETA Temperature in $\mathrm{eV}$ to be used for fission spectrum $\left(1.3 \times 10^{6}\right)$

4. AKT Number of $\mathrm{kT}$ at which $1 / \mathrm{E}$ distribution and Maxwellian distribution join (5)

5. FCUT Energy in $\mathrm{eV}$ at which fission spectrum and $1 / \mathrm{E}$ distribution join $\left(67.5 \times 10^{3}\right)$

$7 \$ \quad[10]$

1. IHT Position of $\sigma_{T}$ in ANISN format output (3)

2. IHS Position of within-group scattering in ANISN format output (IHT plus number of thermal groups)

3. IHM Table length in ANISN format output (IHS plus total number of groups)

4. LOPT Set greater than zero if truncations of cross sections defined by the above parameters are to be applied to the AMPX working library and Monte Carlo processed cross-section library $(0)$ 
5. NNGA The number of neutron groups for which Monte Carlo cross sections will be processed (the number of neutron energy groups on the input library)

6. NGGA The number of gamma groups for which Monte Carlo cross sections will be processed (the number of gamma energy groups on the input library)

7. NOPT Option to be used if energy groups are truncated

0 downscatters to missing groups kept

1 downscatters to missing groups are summed into last downscatter cross section present

2 downscatters to missing groups are dropped (0)

8. NSCT Number of discrete scattering angles calculated (0)

9. IADJM Set greater than zero to produce a library for an adjoint problem (0)

10. NNGTP Set greater than zero if a completely coupled crosssection set is desired (0)

T Terminate Block 2.

\section{Block 3.}

$8 \$$ [MIX] \{Required only if IFLAG (1) $>0$ \}

1. (MID)(I),I=1,MIX) Mixture numbers for AMPX working library

$9 \$[N]$ Required only if IFLAG (2) or $\operatorname{IFLAG}(3)>0$ \}

1. $(M A D)(I), I=1, N)$ ANISN mixture ID numbers. NCOEF (I) ID's are required for each mixture for a total of

$$
\mathrm{N}=\sum_{I=1}^{\mathrm{MIX}} \mathrm{NCOEF}(\mathrm{I})
$$

Default as follows:

$\begin{array}{ll}P_{0} \text { for 1st mixture } & \text { ID }=109 \\ P_{1} \text { for 1st mixture } & \text { ID }=101 \\ P_{2} \text { for 1st mixture } & \text { ID }=102\end{array}$

$P_{0}$ for 2 nd mixture $\quad$ ID $=200$

$P_{1}$ for 2 nd mixture $\quad \mathrm{ID}=201$

$10 \$[\mathrm{IHT}]$ Required only if IFLAG (2) or IFLAG(3) $>0\}$ 
1. (IMT(I),I=IHT) ENDF-MT number of the ID cross sections to be used in the ANISN cross-section tables in inverted order (first five positions):

$1\left(\sigma_{T}\right), 1452\left(\nu \sigma_{f}\right), 27\left(\sigma_{a}\right), 18\left(\sigma_{f}\right)$, and $1018(\chi)$.

All other positions: 0

$11 \$[1+\mathrm{MIX}]$ Required only if IFLAG $(4)>0\}$

1. LID

Monte Carlo processed cross-section library identification number (1)

2. (MUD(I),I=1,MIX) Mixture ID numbers for Monte Carlo processed crosssection library $(\mathrm{MUD}(\mathrm{I})=\mathrm{I}, \mathrm{I}=1, \mathrm{MIX})$

T Terminate Block 3.

\section{ICE.2 ICE Input/Output Specifications}

An ICE calculation may require the following input/output devices:

Logical No.

5

6

7

8

9

INTAPE(4)

$\operatorname{IOT} 1(3)$

IOT2

IOT3

IOT4
Purpose

Card input
Printed output
Punched output
Direct-access scratch
Direct-access scratch

Input AMPX working library

Output AMPX working library

ANISN library

ANISN library in group-independent format

Monte Carlo (KENO) library 


\section{JERGENS - MODULE TO GENERATE WEIGHT FUNCTIONS AND TO COMBINE ENDF/B TAB1 RECORDS}

JERGENS (Just an Excellent Routine to GENerate $\underline{\text { Strings) }}$ is the module to use when it is necessary to construct a weighting spectrum, such as is needed by XLACS77, VEL, or LAPHNGAS. It can be used for a large variety of operations involving the combination of more than one ENDF/B TAB1 record. The TAB1 record might, for example, contain the energy-value pairs for a cross section for a particular process. All the arithmetic operations in JERGENS are performed to a user-controllable accuracy. The output from the module is a file containing the new functions written in "TAB1" format.

\section{JERGENS.1 JERGENS Input Data}

\section{Block 1.}

$-1 \$$ Options $[20]$

1. IOPT1(2) Used in conjunction with interpolation values

2. IOPT2(2) specified below

3. IOPT3-Maximum number of interpolation regions which can be generated (100)

4. IOPT4 - MAT number for all the functions to be produced by this JERGENS run (99)

5. IOPT5 - MF (file) number for all the functions to be produced by this JERGENS run (3)

6. INT1 - First interpolation scheme used (1)

7. INT2 - Second interpolation scheme used (2)

8. INT3 - Third interpolation scheme used (3)

9. INT4 - Fourth interpolation scheme used (4)

10. INT5 - Fifth interpolation scheme used (5)

11. ICORE - Number of words to allocate to JERGENS (100000)

12. Not used

13. Not used

14. Not used

15. Not used

16. Not used

17. Not used

18. Not used

19. Not used

20. Not used

The combination of IOPT1, IOPT2 with INT1, INT $2, \ldots$ INT 5 allows a very flexible way of selecting the kinds of interpolation schemes allowed in the functions produced. 
Recall that the interpolation schemes are the following:

\begin{tabular}{cll} 
Code & & \multicolumn{1}{c}{ Type } \\
\cline { 3 - 3 } 2 & & Histogram \\
3 & & Linear $x-\operatorname{linear} y$ \\
4 & & Log $x-\operatorname{linear} y$ \\
5 & & Log $x-\log y$
\end{tabular}

IOPT1 points to the word in INT1... INT5 which contains the first interpolation code to be tried. IOPT2 points to the last word in the string containing the code to be tried. JERGENS cycles through the codes specified in INT(IOPT1) to INT (IOPT2) to determine the "best code" to use. By default, IOPT1 and IOPT2 are both 2, indicating a linear-linear function is being constructed. IOPT $=1$ and IOPT2 $=5$ would try types $2,3,4$, and 5 in exactly that order. Making IOPT1 $=1$ and IOPT2 = 2 , and INT1 $=5$ with INT2 $=2$ would cycle between a $\log$-log and a linear-linear scheme, etc. Attempting INT = 1 (histogram) would be fruitless, because one would never satisfy accuracy specifications; therefore, it should be avoided.

0\$ Logical Assignments [3]

1. NDFB - All external functions required by JERGENS must reside here (The CREST module can be used to collect multiple functions onto a single file.)

2. MWT - The library produced by JERGENS is written here

3. MSC - Scratch file (default $=18)$

$1 \$$ Problem Information [1]

1. NMWT - Number of functions to be written on MWT

2* Energy Range [2]

1. ELO - Low-energy cutoff of fussctions to be generated (in eV) (default = $0.00001)$

2. EHI - High-energy cutoff of functions to be generated (in $\mathrm{eV})$ (default $=2.0 \times$ $10^{7}$ )

T Terminate Block 1. Repeat the following two blocks NMWT times.

\section{Block 2.}

$3 \$$ Identifier and Option Selectors [3]

1. IDWT - Identifier for function to be created. This is equivalent to the MT number in ENDF/B

2. NC - Number of "commands" associated with the construction of this function

3. IW - Special function generator option. This is used when $\mathrm{NC}=0$.

$0-1 / \mathrm{E}$

1 - flat

2 - Maxwellian-1/E-fission spectrum 
$3-\mathrm{E}$

4 - Maxwellian-1/E-fission spectrum-1/E above $10 \mathrm{MeV}$

5 - Neutron dose factors per ANSI/ANS 6.1.1-1977

6 - Gamma-ray dose factors per ANSI/ANS 6.1.1-1977

$7-1 / \mathrm{V}$ (normalized to 1.0 at $2200 \mathrm{~m} / \mathrm{s}$ )

4* Special Constants [6]

1. TMAX - "Temperature" of the Maxwellian spectrum $(\mathrm{K})($ default $=300)$

2. $\mathrm{AKT}$ - Multiplier on $\mathrm{KT}$ to determine Maxwellian to $1 / \mathrm{E}$ join point (default = 5)

3. THETA - Effective temperature in $\mathrm{eV}$ of the fission spectrum (default $=1.27 \times$ $10^{6}$ )

4. FCUT - Point at which to join $1 / \mathrm{E}$ to fission spectrum (default $=67.4 \times 10^{3}$ )

5. SIGD - Not used

6. EPS - Accuracy to which functions are to be generated (default $=0.001)$

T Terminate Block 2.

\section{Block 3. "Commands"}

This block is only required when $\mathrm{NC}>0$. It is written in free form in columns 1-72 of a card and should not be terminated by a T. Each "command" consists of six words:

1. IDCREATE - The identifier of a set "created" by the operation. Use any unique integer

2. IDINTACT - The identifier of a set previously created which is required by this command

3. OPERATION - An alphanumeric word describing the command

4. A - Constant which may be needed by the command (default $=1.0$ )

5. IDUSE - The identifier (MAT number) of the set of data on logical NDFB which is needed for this command

6. MTUSE - The MT number of the set of data on logical NDFB which is needed for this command

The commands, in groups of six items, are stacked one after the other until NC commands are specified.

As a general rule, the following type of operation is performed:

IDCREATE=IDINTACT $\{$ OPERATION $\}$ A*IDUSE .

That is to say, IDCREATE is a string of data points created by combining the string IDINTACT in conjunction with some form of operation with A times the string IDUSE.

Before two strings input on logical NDFB can be used in a command, one of them must be moved to the "intact" file by issuing a "MOVE" or an "ADD" command.

At least one IDCREATE should equal IDWT. This command will cause the string to be written to logical MWT; otherwise, strings are written onto MSC.

The possible commands and their actions are described as follows: 
4

\begin{tabular}{|c|c|c|c|c|c|}
\hline IDCREATE & IDINTACT & OPERATION & $\underline{A}$ & IDUSE & ACTION \\
\hline$\overline{\mathrm{IDC}}$ & $\overline{0}$ & $\overline{\text { MOVE }}$ & $\overline{\mathrm{A}}$ & $\overline{\mathrm{IDU}}$ & $\overline{\mathrm{IDC}}=\mathrm{A} \times \mathrm{IDU}$ \\
\hline IDC & IDI & $\mathrm{ADD}$ & A & IDU & $\mathrm{IDC}=\mathrm{IDI}+\mathrm{A} \times \mathrm{IDU}$ \\
\hline IDC & IDI & SUB & A & IDU & $\mathrm{IDC}=\mathrm{IDI}-\mathrm{A} \times \mathrm{IDU}$ \\
\hline IDC & IDI & DIV & A & IDU & $\mathrm{IDC}=\mathrm{IDI} /(\mathrm{A} \times \mathrm{IDU})$ \\
\hline IDC & IDI & MUIT & A & IDU & $\mathrm{IDC}=\mathrm{IDI} \times \mathrm{A} \times \mathrm{IDU}$ \\
\hline IDC & IDI & $\mathrm{ADD}$ & A & 0 & $\mathrm{IDC}=\mathrm{IDI}+\mathrm{A}$ \\
\hline IDC & IDI & SUB & A & 0 & $\mathrm{IDC}=\mathrm{IDI}-\mathrm{A}$ \\
\hline IDC & IDI & MULT & A & 0 & $\mathrm{IDC}=\mathrm{IDI} \times \mathrm{A}$ \\
\hline IDC & IDI & DIV & A & 0 & $\mathrm{IDC}=\mathrm{IDI} / \mathrm{A}$ \\
\hline IDC & 0 & $A D D$ & A & IDU & $\mathrm{IDC}=\mathrm{IDU}+\mathrm{A}$ \\
\hline IDC & 0 & SUB & $\mathrm{A}$ & IDU & $\mathrm{IDC}=\mathrm{IDU}-\mathrm{A}$ \\
\hline IDC & 0 & DIV & A & IDU & $\mathrm{IDU} \times \mathrm{A}$ \\
\hline IDC & 0 & MULT & $A$ & IDU & IDU $/ \mathrm{A}$ \\
\hline IDC & IDI & NORM & A & 0 & $\mathrm{IDC}=\int_{E L O}^{E H I} \mathrm{IDI}$ \\
\hline 0 & IDI & PRT & 0 & 0 & Print IDI \\
\hline IDC & IDI & MAX & 0 & 0 & $\begin{array}{l}\text { IDC = Maxwellian joined to } \\
\text { function IDI }\end{array}$ \\
\hline IDC & IDI & FISS & 0 & 0 & $\begin{array}{l}\text { IDC = IDI joined to a fission } \\
\text { spectrum }\end{array}$ \\
\hline 0 & IDI & $\mathrm{PNCH}$ & 0 & 0 & Punch IDI in ENDF TAB1 format \\
\hline IDC & IDI & FUNC & A & IDU & $\begin{array}{l}\text { Use a special function generator } \\
\text { as described in the following table. } \\
\text { In all cases the function } y(x) \text { is } \\
\text { identified by IDI, and the option is } \\
\text { selected by IDU }\end{array}$ \\
\hline
\end{tabular}

Note that in the above table, IDC is an abbreviation for IDCREATE, IDI for IDINTACT, and IDU for the combination of IDUSE and MTUSE. 
The following is a table of options for the "FUNC" operation:

$$
\begin{aligned}
& \text { IDU (option) Function to be constructed } \\
& 1 \quad \mathrm{G}(\mathrm{x})=\mathrm{A} \mathrm{y}(\mathrm{x}) \\
& 2 \quad \mathrm{G}(\mathrm{x})=\mathrm{A} / \mathrm{y}(\mathrm{x}) \\
& 3 \quad \mathrm{G}(\mathrm{x})=\mathrm{xAy}(\mathrm{x}) \\
& 4 \quad \mathrm{G}(\mathrm{x})=\frac{A}{\mathrm{xy}(\mathrm{x})} \\
& 5 \quad \mathrm{G}(\mathrm{x})=\frac{1.0}{\mathrm{y}(\mathrm{x})+\mathrm{A}} \\
& 6 \quad \mathrm{G}(\mathrm{x})=\frac{1.0}{\mathrm{x}[\mathrm{y}(\mathrm{x})+\mathrm{A}]} \\
& 7 \quad \mathrm{G}(\mathrm{x})=\mathrm{A} x / \mathrm{y}(\mathrm{x})
\end{aligned}
$$

\section{JERGENS.2 JERGENS Input/Output Specifications}

JERGENS requires the following input/output devices:

\begin{tabular}{cll} 
Logical No. & & \multicolumn{1}{c}{ Purpose } \\
\cline { 1 - 1 } 5 & & Card input \\
6 & & Standard output \\
NDFB & & Input functions \\
MWT & & Output functions \\
MSC & & Scratch device
\end{tabular}




\section{LAPHNGAS - MODULE FOR GENERATING MULTIGROUP SECONDARY GAMMA-RAY PRODUCTION CROSS SECTIONS}

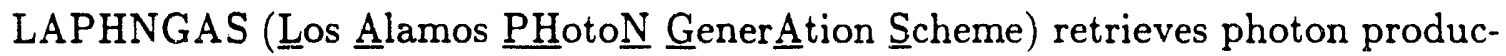
tion data from the ENDF data file and calculates either multigroup secondary gammaray production cross sections (SGRPXS) and/or multigroup secondary gamma-ray yields (multiplicities) that will be written to an AMPX master interface. To form coupled neutron-gamma cross sections, the SGRPXS or yields are passed to other AMPX modules, CHOX and NITAWL, to be combined with multigroup neutron and photon interaction cross sections. When used as a stand-alone code, LAPHNGAS can calculate either fine-neutron-group or broad-neutron-group SGRPXS for use with other codes.

D. J. Dudziak et al. of the Los Alamos Scientific Laboratory wrote a series of codes to prepare multigroup photon-production matrices from ENDF data. ${ }^{1}$ These codes have evolved from an early code called $\mathrm{LAPH}^{2}$ through a series of codes that incorporated various ENDF format changes to the LAPHAN0 $0^{3}$ code, which served as the starting point for the development of LAPHNGAS. The following modifications were made to LAPHAN0 to produce LAPHNGAS:

- The code was flexibly dimensioned.

- The capability of computing anistropic photon-production matrices was added.

- Various weighting options were provided.

- The input format was restructured to incorporate the FIDO (ANISN) input system described in Sect. 7.FIDO.

- A cross section or yield matrix for each reaction type processed is uniquely identified and made available to other AMPX modules. Yield matrices are calculated for the fission and capture reactions of resonance materials. This allows "self-shielded" gamma production matrices to be formed.

- Data management changes were made to reduce input/output operations and to allow processing of both unformatted (binary) and formatted (BCD) ENDF libraries.

- Cross-section and yield matrices are printed and punched only when indicated via input data (see $2 \$$ array, items 7 and 8 ).

- Binary search techniques were implemented to locate specific energy points within energy arrays arranged in ascending order.

- Capabilities were included to process both ENDF/B-IV and ENDF/B-V data.

The LAPHANO report $^{3}$ should be used as the theoretical reference for LAPHNGAS.

\section{LAPHNGAS.1 LAPHNGAS Input Data}

With the exception of the ENDF data and title cards, all data for LAPHNGAS are input in the FIDO (ANISN) format. Input data for the code are as follows:

A. Title Card (format 18A4) - Problem Title 
B. $1 \$$ Array - General Problem Information [4 entries]

1. MCR - Number of materials to be processed

2. NFG - Number of neutron groups

3. NGG - Number of gamma groups

4. IM - Neutron reaction cross section $(\mathrm{MF}=3)$ and/or photon-production crosssection ( $M F=13$ ) point-weighting option:

$=1$, constant weighting

$=2,1 / \mathrm{E}$ weighting

$=3$, fission spectrum weighting for $E_{n}>6.74 \times 10^{4} \mathrm{eV}$ and $1 / \mathrm{E}$

weighting for $E_{n} \leq 6.74 \times 10^{4} \mathrm{eV}$, where the fission spectrum equation used is

$$
W(E)=\sqrt{\frac{4 E}{\pi \theta^{3}}} e^{-E / \theta},
$$

where $\theta=1.273 \mathrm{MeV}$.

$=4$, exact $1 / \mathrm{E}$. The analytic solution of

$$
\bar{\sigma}=\frac{\int \sigma(E) W(E) d E}{\int W(E) d E}
$$

is programmed in LAPHNGAS. The equation used for the analytic solution depends on the interpolation scheme defined for adjacent $\sigma(E)$ values. The equations are defined in ref. 4, pp 29-31.

$=5,1 /\left(E \sigma_{T}\right)$ weighting

$=6$, arbitrary function input in $11 \$$ and $13^{*}$ arrays (enter a -6 to suppress the listing of the arbitrary weight function)

$1 /\left(E \sigma_{T}\right)$ weighting where $\sigma_{T}$ is obtained from the NPTXS module on logical unit 31

$\geq 100$, the arbitrary weighting function identified by $|I M|$ will be read from the weighting function library located on logical unit 46. IM $<0$ will suppress printing of the function.

Note: When either weighting option 5 or 7 is selected, a constant, $\sigma_{0}$, may be entered in the $13^{*}$ array, which is added to $\sigma_{T}$. This provides the capability for Bondarenko weighting, i.e.,

$$
W(E)=\left(\frac{1}{\sigma_{T}+\sigma_{0}}\right)\left(\frac{1}{E}\right) .
$$

$2 \$$ Array - General Problem Information [10 entries]

All parameters in this array have default values set by the code. For problems that can run with these default values, the $2 \$$ array may be omitted.

\section{LAPHNGAS}


1. LCSM - If $|I M| \neq 6$, enter zero

If $|I M|=6$, enter the number of interpolation regions used to specify the arbitrary weighting spectrum (default value is 0 )

2. $\mathrm{MCSM}$ - If $|I M| \neq 6$, enter zero

If $|I M|=6$, enter the number of points in the arbitrary weighting spectrum (default value is 0 )

3. IZM - $=0$, calculate photon-production cross sections (SGRPXs)

$=1$, calculate photon-energy-production cross sections (units will be $\mathrm{eV} \cdot \mathrm{cross}$ section)

4. MS - thermal capture cross-section representation

$=0$, no effect (i.e., weighting scheme identified by IM is used over the entire neutron energy range)

$=1$, use a Maxwellian weighting on the energy region lower than EUP (see $3^{*}$ array). The temperature for the Maxwellian spectrum is given in the $3^{*}$ array (default value is 1 )

5. MM - maximum number of reaction types (MT numbers) to be entered in the $14 \$$ array for this case. Default value is 1 to facilitate use of the $\mathrm{MT}=-1$ option (see $14 \$$ array)

6. IWO $=1$, photon effective average energy for each gamma group is assumed to be the group midpoint energy (track 1 calculation in ref. 2)

$=2$, yield function is photon energy weighting function (track 2 calculation in ref. 2)

$=3$, photon effective average energy for each gamma group is input in the $9^{*}$ array (track 1 calculation in ref. 2) (default value is 1 )

7. $100=-9$, prints SGRPXS matrix for total only (default value is -9)

0 , print yield or SGRPXS matrix for each reaction

8. $\mathrm{IAM}=0$, no effect (default value is 0 )

$=1$, punch the yield or SGRPXS matrix for each reaction

The punched data are in a format for use in the ASCC coupling code.* The punched output will not include the resonance yield data.

9. $\mathrm{ICK}=0$, no effect (default value is 0 )

$=1$, print a detailed output

10. NNBDGP - Not used

End Block B with a T.

C. Thermal information, group structures, and weighting function data. Arrays not applicable to problem should be omitted.

${ }^{*}$ The Sample Coupling Code (ASCC) is documented is Appendix B of ref. 5. 
3* Array \{ Enter only if $\mathrm{MS}=1$ and default values are unacceptable

1. TEMP - Mean neutron temperature of Maxwellian distribution (K)

(Default value is 293.6)

2. EUP - Upper neutron energy for application of the thermal $(n, \gamma)$ correction (eV) (Default value is $5 \mathrm{kT}=0.125$ )

3. VNON - Not used

7* Array (NFG+1 entries)

Neutron energy mesh in decreasing magnitude of energy (eV units). Many "standard" group structures are built into AMPX; when one of these group structures is desired, this array is omitted. (See Sect. 4.MISCELLANEOUS.4.1 for a list of the standard structures.)

$8^{*}$ Array (NGG+1 entries)

Gamma-group energy mesh in decreasing magnitude of energy ( $\mathrm{eV}$ units). If one of the "standard" gamma-gro! : structures will suffice, this array may be omitted. See Sect. 4.MISCELLANEOUS.4.2 for a list of the standard structures.

9* Array (NGG entries)

Enter only if IWO $=3$. Effective average gamma energy $\bar{E}_{g}$, for each group. (Same for all materials and reaction types. Code checks that $E_{g}<\bar{E}_{g} \leq E_{g+1}$.) Energies are in units of $\mathrm{eV}$, high to low.

$\left\{11 \$\right.$ and $13^{*}$ are entered only if $|\mathrm{IM}|=6$. $\}$

$11 \$$ Array $(2 *$ LCSM entries)

Interpolation data for arbitrary weighting spectrum (NBT(I), JNT(I), I=1, LCSM).

$13^{*}$ Arbitrary weighting spectrum (per unit energy), (E(I),W(I), I=1,MCSM) lowto-high energy. Units of $\mathrm{E}(\mathrm{I})$ are $\mathrm{eV}$.

End Block $\mathrm{C}$ with a $\mathrm{T}$.

D. $14 \$$ Array (5+MM entries), Material-Dependent Data

Enter a $14 \$$ array for each material to be processed.

1. Material identification (ENDF MAT number)

2. IQA - Order of $P_{n}$ (default value is 0 )

3. NDFB - Logical unit for the ENDF/B cross-section library (default value is 11)

4. $\mathrm{MODE}=1, \mathrm{ENDF} / \mathrm{B}$ library in unformatted (binary) mode (default value is 1 )

$=2$, ENDF $/ \mathrm{B}$ library in formatted (BCD) mode

5. MTSL - Number of reaction types to be processed. Default value is 1 to accommodate the normal case where MT $=-1$. MTSL must be $\leq \mathrm{MM}$ (see $2 \$$ array).

\section{LAPHNGAS}


6. MT - Enter the ENDF/B MTs, reaction-type identifiers, to be processed. If the default is not being used, MM (item $5,2 \$$ array, this section) entries are required. $\mathrm{MT}=-1$ means use all $\mathrm{MT}$ numbers for which photonproduction data exist. (default value is -1 .)

7. NDFTYP $=4$, the cross-section library on logical unit NDFB is in ENDF/BIV format $=5$, the library is in ENDF/B-V format (default value is 5 )

End each Block D with a $\mathrm{T}$.

This concludes the input to LAPHNGAS.

\section{LAPHNGAS.2 LAPHNGAS Input/Output Specifications}

Input/output devices required for LAPHNGAS are as follows:

\begin{tabular}{cll} 
Logical No. & & \multicolumn{1}{c}{ Purpose } \\
\cline { 1 - 2 } 5 & & Input \\
6 & & Printed output \\
7 & & Punched card output \\
14 & & Scratch device \\
16 & & Scratch device \\
17 & & Scratch device \\
18 & & Scratch device \\
19 & & Scratch device \\
24 & & AMPX devere \\
31 & & Total cross section from the \\
& NPTXS module, used only when \\
32 & & weighting option 7 is selected \\
33 & & Scratch device \\
35 & Scratch device \\
36 & Scratch device \\
46 & Scratch device \\
& Weighting function library, \\
& used only when selected by \\
NDFB (14\$ array) & input option \\
& ENDF cross-section library \\
& (default value is 11)
\end{tabular}

The logical unit numbers for the input, printed output, and punched card output IS0, ISI, and NPU, respectively - are "set" in LAPHNGAS subroutine LAPHOR. These numbers can be easily redefined for LAPHNGAS by changing the appropriate statements in LAPHOR. 


\section{LAPHNGAS.3 LAPHNGAS Error Messages}

Various error stops are included in LAPHNGAS which cause the module to stop computing and return control to the DRIVER module when an error condition is encountered. The fatal error returns are as follows:

\begin{tabular}{|c|c|c|}
\hline $\begin{array}{l}\text { Error } \\
\text { No. }\end{array}$ & $\begin{array}{l}\text { Detecting } \\
\text { subroutine }\end{array}$ & Explanation \\
\hline 50 & LAPHOR & Input error, see code output for an explanation \\
\hline 100 & PROB1 & $\begin{array}{l}\text { Input error, the neutron fine- and broad-group energy } \\
\text { boundaries must be given in order of decreasing } \\
\text { magnitude in energy }\end{array}$ \\
\hline 110 & PROB1 & $\begin{array}{l}\text { Input error, arbitrary weighting function energies must } \\
\text { be input in ascending order }\end{array}$ \\
\hline 133 & TERP1 & Interpolation code not in range $1-5$ \\
\hline 134 & TERP1 & $X \leq 0$ cannot be interpolated by logs \\
\hline 150 & MUSH & $\sigma_{T}$ not found, check ENDF library file $3, \mathrm{MT}=1$ \\
\hline 200 & INTG & $\begin{array}{l}\text { The flag indicating dependence of } Y(X) \text { on } X \text { is } 0 \text {, } \\
\text { but must be in range } 1-5 \text {; error is probably a program } \\
\text { bug }\end{array}$ \\
\hline 400 & MUSH & $\begin{array}{l}\text { Number of points at which } \sigma_{T} \text { is defined plus number } \\
\text { of neutron groups exceeds } 5000 \text { plus some additional } \\
\text { energy points to ensure at least ten energies within } \\
\text { each fine neutron group. Increase dimension of IBIG } \\
\text { in PROB1 }\end{array}$ \\
\hline 501 & PR15 & $\begin{array}{l}\text { The neutron energy must not lie outside the range } \\
\text { of energies for which the photon yield is tabulated or } \\
\text { photon distribution given, check ENDF data }\end{array}$ \\
\hline 502 & PR15 & Interpolation table incorrect - probable program bug \\
\hline 504 & PR15 & $\begin{array}{l}\text { File } 15 \text { data incompatible with mesh formed by } \\
\text { merger of energy points at which } \sigma_{T} \text { is defined and } \\
\text { neutron energy group boundaries. See subroutine } \\
\text { PR15, Statement } 606 \text {, for additional information }\end{array}$ \\
\hline 506 & PR15 & $\begin{array}{l}\text { Gamma group structure and file } 15 \text { data incompatible. } \\
\text { Check gamma group structure for input error and } \\
\text { then ENDF } / B \text { file } 15 \text { data files }\end{array}$ \\
\hline 507 & PR15 & See error message 506 \\
\hline 550 & MATT & $\sigma_{T}$ not found, check ENDF library file $3, \mathrm{MT}=1$ \\
\hline 600 & TRC2 & $\begin{array}{l}\text { Error in determining from which level in a cascade } \\
\text { a transition occurs. If an MT number for inelastic } \\
\text { scattering is input in the } 2 \$ \text { array, the MT numbers } \\
\text { for all lower levels must also be included }\end{array}$ \\
\hline 603 & $\mathrm{TRC} 2$ & $\begin{array}{l}\text { Interpolation table incorrect; see code output for } \\
\text { additional information }\end{array}$ \\
\hline 604 & TRC2 & $\begin{array}{l}\text { Subsequent cascades for file } 12, \mathrm{LO}=2 \text { data exceeded } \\
\text { the core allocation parameter MNC (set in MATT). } \\
\text { Increase MNC and resubmit case }\end{array}$ \\
\hline 610 & TRC2 & $\begin{array}{l}\text { The energy range of the arbitrary weighting function } \\
\text { read in may be incomplete. Check the } 13^{*} \text { array }\end{array}$ \\
\hline
\end{tabular}


620 TRC2 Interpolation tabie for arbitrary weighting function read in is probably incorrect. Check the $11 \$$ array

630 TRC2 Denominator was $\leq$ zero in weighting calculation. Check group structures and $3^{*}$ array input

640 TRC2 Interpolation table for $\sigma_{T}$ incorrect; check ENDF library $\mathrm{MF}=3, \mathrm{MT}=1$

701 XSEC Interpolation table probably in error. See code output for additional information

702 XSEC Cross sections in file 3 cannot be found corresponding to the MT number written on unit 15 or unit 17 .

Check ENDF data

710 XSEC The energy range of the arbitrary weighting function read in may be incomplete. Check the $13^{*}$ array

720 XSEC Interpolation table for arbitrary weighting function read in is probably incorrect. Check the $11 \$$ array

730 XSEC Interpolation table for $\sigma_{T}$ may be incorrect. Check ENDF library $\mathrm{MF}=3, \mathrm{MT}=1$

740 XSEC Denominator was $\leq 0$ in weighting calculation. Check group structures and $3^{*}$ array input

750 XSEC Code is attempting to execute an impossible path. Check all input relevant to the weighting options

1001 PHOTON First card on unit IEN is not head record. Ensure that the proper library was mounted on unit IEN

1002 PHOTON Files 12 and 13 both missing from ENDF library.

1200 CRAVE LAPHNGAS cannot process this material

1210 CRAVE

1220 CRAVE XL $>\mathrm{X} 1$, attempting to integrate over a range where Interpolation code not in range $1-5$ $\mathrm{X} 1>\mathrm{X} 2$, attempting to integrate over a range where no data are given - probable program bug no data are given - probable program bug

1230 CRAVE $\mathrm{XU}>\mathrm{X} 2$, see error message 1220

1250 CRAVE $\mathrm{XL}>\mathrm{XU}$, expects limits of integration to be in ascending order - probable program bug

1300 INGATE Lower limit of integral > upper limit of function probable program bug

1310 INGATE Upper limit of integral $\leq$ lower limit of function probable program bug

1320 INGATE No data available within integration limits - probable program bug

1330 INGATE Interpolation code not in range 1-5

1400 LEGMOM LI flag from file 14 data was not 0 or 1

1410 LEGMOM Could not find file 14 data

1420 LEGMOM Isotropic calculation requested via input parameter IQA, attempting anisotropic calculation - probable program bug

1440 LEGMOM Interpolation table incorrect. Check ENDF file 14 data

1450 LEGMOM LTT flag from file 14 was not 1 or 2 
1460 LEGMOM ILO > NP TS, data not available for processing; see subroutine LEGMOM in the vicinity of statement 755 for details

1510 BRDGP Broad group numbers not in increasing order

1520 BRDGP Process ID numbers must be 3,18 , or 102

1530 REDUCE NBG > NFG, requesting an impossible operation; check input data

\section{REFERENCES}

1. D. J. Dudziak, Ed., ENDF Formats and Procedures for Photon Production and Interaction Data, LA-4549, Los Alamos Scientific Lab., 1971.

2. D. J. Dudziak, A. H. Marshall, and R. E. Seamon, LAPH, A Multigroup Photon Production Matrix and Source Vector Code for ENDF/B, LA-4337, Los Alamos Scientific Lab., 1969.

3. D. J. Dudziak, R. E. Seamon, and D. V. Susco, LAPHANO: A P Multigroup Photon-Production Matrix and Source Code for ENDF/B, LA-4750-MS, Los Alamos Scientific Iuab. 1972.

4. R. Q. Wright, N. M Greene, J. L. Lucius, and C. W. Craven, Jr., SUPERTOG: A Program to Gensiate Fine Group Constants and $P_{n}$ Scattering Matrices from ENDF/B, ORNL-TM-2679, Union Carbide Corp., Nucl. Div., Oak Ridge Natl. Lab., September 1969.

5. W. E. Ford, III, and J. S. Gillen, The Testing of ${ }^{238} \mathrm{U}$ Secondary Gamma-Ray Production Data Sets From the POPOP 4 Library, ORNL-TM-3482, Union Carbide Corp., Nucl. Div., Oak Ridge Natl. Lab., February 1972. 


\section{LAVA - AMPX MODULE TO MAKE AN AMPX WORKING LIBRARY FROM AN ANISN LIBRARY}

LAVA (Let $\underline{A}$ NISN Visit $\underline{A} M P X)$ is a module that can convert an ANISN library (neutron, gamma, or coupled neutron-gamma) to an AMPX working library such as is used in XSDRNPM. ANISN cross sections can be input on cards (fixed or free-form FIDO format) or on a binary library.

\section{LAVA.1 LAVA Input Data}

Block 1.

$-1 \$$ Core Allocation [1]

1. NWORD - Number of words to allocate to LAVA (50000)

$0 \$$ Logical Definitions [6]

1. N1 - ANISN library (20)

2. N2 - AMPX working library (4)

3. N3 - Scratch (18)

4. N4 - Scratch (19)

5. N5 - Input (5)

6. N6 - Output (6)

$1 \$$ ANISN Library Parameter Data [8]

1. NNUC - Number of isotopes to be put on new library

2. IGM - Number of neutron groups

3. IHT - Position of $\sigma_{t}$

4. IHS - Position of $\sigma_{g \rightarrow g}$

5. IHM - Table length

6. IFTG - First thermal group

7. IPM - Number of gamma groups

8. IFM - Format, of ANISN library

$$
\begin{aligned}
& \text {-1, binary } \\
& 0, \text { free-form BCD } \\
& \text { 1, formatted BCD }
\end{aligned}
$$

9. IFLAG - Flag that selects the method for calculating scattering cross sections from scattering matrices -0 or 1 (default $=1$ )

ANISN matrices are the sum of all the individual scattering matrices (elastic, inelastic, $\mathrm{n} 2 \mathrm{n}, \mathrm{n} 3 \mathrm{n}$, etc.) for processes possible for the particular nuclide. LAVA attempts to arbitrarily determine values for an elastic $(\mathrm{MT}=2)$ and an $\mathrm{n} 2 \mathrm{n}(\mathrm{MT}=$ 16) cross section, recognizing that elastic scattering is generally the most dominant scattering process, and that $\mathrm{n} 2 \mathrm{n}$ is the most common scattering process that yields more than a single exit neutron. In order to accomplish this, the absorption cross section in the ANISN data must be the true absorption value (not an energy absorption cross section as in some older gamma-ray sets, or whatever alternative 
value). When IFLAG $=1$, requiring the correct absorption, the elastic value is taken as

$$
\sigma_{e \ell}^{g}=\sigma_{t}^{g}-\sigma_{a}^{g}
$$

while the $\mathrm{n} 2 \mathrm{n}$ is taken from

$$
\sigma_{n 2 n}^{g}=\sum_{g^{\prime}} \sigma_{0}\left(g \rightarrow g^{\prime}\right)-\sigma_{e \ell}^{g} .
$$

When IFLAG $=0$, no attempt is made to calculate an $n 2 n$ value, and the elastic value is simply

$$
\sigma_{e \ell}^{g}=\sum_{g \prime} \sigma\left(g \rightarrow g^{\prime}\right)
$$

T Terminate Block 1.

\section{Block 2.}

$2 \$$ Identifiers of $P_{0}$ Block of Data for the Nuclide on the ANISN Library [NNUC]

$3 \$$ Order of Scattering for the Nuclide on the ANISN Library [NNUC]

If an order of scattering for a nuclide is negative, the $P_{\ell}(\ell>0)$ matrices for the nuclide will be multiplied by $(2 \ell+1)$ to account for differences in the way different computer programs expect these to be normalized.

4\$ AMPX Identifiers for the Nuclides Selected From ANISN Library [NNUC]

$5 \$$ Process Identifiers for the Top Positions in the ANISN Cross-Section Tables [IHT] The order is from position IHT to position 1 (i.e., it is backwards from the way it is in the cross-section tables). Note, MT numbers of frequently used data appearing in the 1-D positions are as follows:

\begin{tabular}{crr} 
Reaction & & MT \\
\cline { 1 - 1 }$\sigma_{T}$ & & No. \\
$\nu \bar{\sigma}_{f}$ & & 1452 \\
$\sigma_{a}$ & 27 \\
$\bar{\nu}$ & 452 \\
$\chi$ & & 1018 \\
$\sigma_{f}$ & & 18
\end{tabular}

ANISN always expects $\sigma_{T}$ in position IHT, with $\nu \bar{\sigma}_{f}$ above that, and $\sigma_{a}$ above that. The contents of the other positions are arbitrary.

6* Fission Spectrum [IGM]

If a nuclide has a nonzero fission cross section, and either no fission spectrum $(\mathrm{MT}=1018)$ is specified in the ANISN library, or the fission spectrum (CHI) flag 
for that nuclide has been set in the $9 \$$ : ay, then the fission spectrum specified in the $6^{*}$ array is used for that nuclide

7* Neutron Energy Group Boundaries* [IGM+1]

8* Gamma-Ray Energy Group Boundaries* [IPM+1]

$9 \$$ Nuclide CHI Flags [NNUC]

$0 / 1$ - Use the fission spectrum from the ANISN library/use the fission spectrum from the $6^{*}$ array.

T Terminate Block 2.

\section{LAVA.2 LAVA Input/Output Specifications}

The following devices are usually needed tia running the LAVA module:

Logical No.

$\mathrm{N} 2$

N5

N6

N3

$\mathrm{N} 4$

N1

47
Purpose

AMPX working library written by LAVA (4)

Card input (5)

Standard output (6)

Scratch device (18)

Scratch device (19)

ANISN library (20)

Standard AMPX Group Boundary Library

* Read high to low in energy $(\mathrm{eV})$. 


\section{MAD - MODULE TO MAKE CHANGES IN THE NUCLIDE DIRECTORY ON A MASTER INTERFACE}

MAD (Modify AMX Directory) is a module to make changes to individual entries in the nuclide directory of an AMPX master cross-section library. In this respect, it offers no new capabilities other than simplification over the capabilities provided in CLAROL.

Recall that the directory of an AMPX master library contains 50 words for e nuclide arranged as shown below:

\begin{tabular}{cll} 
Words & \multicolumn{1}{c}{ Contents } & Type \\
\cline { 1 - 3 } $1-18$ & Nuclide description & Text \\
19 & Nuclide identification number & Intrger \\
20 & Number of resolved resonance sets & Iner \\
21 & Number of unresolved resonance energy points & Integer \\
22 & Number of temperature-independent 1-D neutron & Integer \\
& cross sections & Integer \\
23 & Number of 2-D neutron processes & \\
24 & Number of temperature-dependent 1-D neutron & Integer \\
& cross sections & Integer \\
25 & Number of 1-D gamma cross sections & Integer \\
26 & Number of 2-D gamma processes & Integer \\
27 & Number of gamma production processes & Integer \\
28 & (Maximum order of L) $\times 32768+$ total number of 2-D arrays & Floating \\
29 & Mass number (neutron equivalent) & Floating \\
30 & ZA & Integer \\
31 & Neutron weighting option & \\
32 & Not used & Integer \\
33 & Gamma weighting option & Floating \\
34 & Power per fission (watt-s/fission) & Floating \\
35 & Capture en rgy release (watt-s/capture) & Integer \\
36 & Maximum length of 2-D record in this set & Integer \\
37 & Number of Bondarenko processes & Integer \\
38 & Number ct of 's in Bondarenko data & Integer \\
39 & Number of lemperatures in Bondarenko data & Integer \\
40 & Maximum number of groups in Bondarenko data & \\
41 & Not used & Floating \\
42 & Not used & Integer \\
43 & Potential scattering cross section & Integer \\
44 & Gamma production wei rhting option & Integer \\
45 & Source of fast neutron data & Integer \\
46 & Source of thermal neutron data & Integer \\
47 & Source of gamma data & Integer \\
48 & Source of gamma production data & Integer \\
49 & Standard CITATION identifier & \\
50 & Number of records in this set of data &
\end{tabular}




\section{MAD.1 MAD Input Data}

\section{Block 1.}

0\$ Logical Unit Assignments [2]

1. MOLD - Original master library (default $=1$ )

2. MNEW - New master library (default $=2$ )

$1 \$$ Options [2]

1. IORDER - 0, Input data are blocked by word position in the string of 50 numbers by nuclide; 1 , Input data by nuclide

2. NWORD - Number of words of core to allocate to MAD (50000)

$\mathrm{T}$ Terminate Block 1.

\section{Block 2.}

$2 \$$ Input Block Description [2]

1. IIB -0 , Terminate reading input Blocks 2 and 3

$\mathrm{N}$, Word position this data block applies to if IORDER (1\$ array) is input as zero, or nuclide identifier for this block if the nuclide block option (IORDER =1) is selected.

2. LB - Length of arrays in Block 3

$3 \$$ Overrides on First Record on Master Library [10]

1. Enter a new value for the first word in the first record

2. Enter a new value for the second word

10. Enter a new value for the tenth word.

$\mathrm{T}$ Terminate Block 2.

\section{Block 3.}

$4 \$$ Nuclide Identifier or Word Positions Depending on IORDER [LB]

1. $(\mathrm{NN}(\mathrm{i}), \mathrm{i}=1, \mathrm{LB})$

$5 \$$ or $5^{*}$ or $5 \mathrm{U}$ Values to be Replaced in Directory-corresponding in one-to-one ordering to entries in $4 \$$ array [LB]

1. (Value(i), $i=1, L B)$

$\mathrm{T}$ Terminate Block 3 . 


\section{MAD.2 MAD Input/Output Specifications}

MAD requires the following $I / O$ assignments:

\begin{tabular}{|c|c|}
\hline Logical No. & Purpose \\
\hline 5 & \\
\hline 6 & Standard output \\
\hline MOLD(1) & Original master cross-section library \\
\hline MNEW(2) & New master cross-section library \\
\hline
\end{tabular}

\section{MAD.3 MAD Examples}

The input description for MAD is confusing at best. Its structure is designed to cover two cases:

1. the case wherein one wants to modify a single type of variable for many nuclides, and

2. the case wherein one wants to modify many parameters for a nuclide.

An example of the first case might be to input a potential scattering cross section for many nucides. In this case, one would choose IORDER $=0$ and would set IIB $=43$ $\left(\sigma_{p}\right.$ position), after which the $4 \$$ array would contain the nuclide identifiers and the $5^{*}$ array the corresponding cross-section values.

If, on the other hand, one had a series of more random changes to make for several nuclides, IORDER $=1$ would seem more appropriate, with IIB set to the nuclide identifier, after which the $4 \$$ array would specify the word position to change and the $5 \$, 5^{*}$, or $5 \mathrm{U}$ array would be used to specify the changes. 


\section{MALOCS - MODULE TO COLLAPSE AMPX MASTER CROSS-SECTION LIBRARIES}

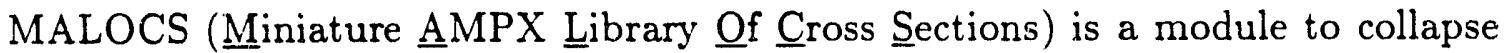
AMPX master cross-section libraries. The module can be used to collapse neutron, gamma-ray, or coupled neutron-gamma master libraries.

\section{MALOCS.1 MALOCS Input Data}

\section{Block 1 .}

$-1 \$$ Core Allocation [1]

1. NWORD - Number of words of core to allocate to MALOCS (100000)

$1 \$$ Case Description [6] $]^{*}$

1. NNEUT - Number of neutron fine groups

2. IGMF - Number of neutron broad groups

3. NGAMM - Number of gamma-ray fine groups

4. IPMF - Number of gamma-ray broad groups

5. IWN - Neutron weighting option $(0)^{\dagger}$

-N1 - Use the 1-D data identified with an MT number of IOPT2 (see $3 \$$ array) from the IOPT1 master data set on logical unit $\mathrm{N} 1$ for the neutron weighting spectrum for all neutron data sets being collapsed

0 - Input neutron weighting spectrum in the $5^{*}$ array

1 - Use MT=1099 1-D neutron data from each fine-group master data set for the neutron weighting spectrum

6. IWG - Gamma-ray weighting spectrum option (0)

-N2 - Use the 1-D data identified with an MT number of IOPT6 from the IOPT5 master data set on logical unit N2 for the gamma-ray weighting spectrum for all gamma-ray data sets being collapsed

0 - Input gamma-ray weighting spectrum in the $7^{*}$ array

1 - Use MT=1099 1-D gamma-ray data from each master data set for the gamma-ray weighting spectrum

$2 \$$ Library Logical Unit Numbers [2]

1. NOLD - Logical number of device containing fine-group AMPX master library (1)

2. NNEW - Logical number of device containing broad-group AMPX master library (22)

$3 \$$ Option Triggers [10]

1. IOPT1 - Auxiliary neutron weighting spectrum trigger $(\mathrm{IWN}=-\mathrm{N} 1)(0)$

\footnotetext{
* Number of entries in array.
}

${ }^{\dagger}$ Default value. 
0 - No effect

ID - Identification number of master data set from which the neutron weighting spectrum (IOPT2 data) will be obtained

2. IOPT2 - Process identifier (MT number) of neutron weighting spectrum in IOPT1 master data set (used when IWN = -N1) (0)

3. INPT3 - Trigger to print broad-group 1-D cross section (1)

0 - Print the data

1 - Do not print

4. IOPT4 - Trigger to print broad-group transfer matrices (1)

0 - Print the data

1 - Do not print

5. IOPT5 - Auxiliary gamma-ray weighting spectrum trigger (used when IWG $=-\mathrm{N} 2)(0)$

0 - No effect

ID - Identification number of master data set from which the gamma-ray weighting spectrum (IOPT6 data) will be obtained

6. IOPT6 - Process identifier (MT number) of gamma-ray weighting spectrum in IOPT5 master data set (IWN $=-\mathrm{N} 2)(0)$

7. IOPT7 - Trigger to collapse out upscatter terms if nonzero (0)

8. IOPT8 - Trigger to truncate downscatters to a maximum of IOPT8 terms below the within group if IOPT8 is nonzero (0)

9. IOPT9 - Not used. Enter zero (0)

10. IOPT10 - Not used

T Terminate Block 1.

\section{Block 2.}

$4 \$$ Neutron broad-group numbers by fine group* [NNEUT]

$5^{*}$ Neutron weighting spectrum [NNEUT] \{Enter only if IWN=0

$6 \$$ Gamma-ray broad-group numbers by fine group ${ }^{\dagger}$ [NGAMM]

7 * Gamma-ray weighting spectrum [NGAMM] \{Enter only if IWG $=0$ \}

T Terminate Block 2.

"A zero "suppresses" a fine group.

tWhen collapsing the gamma groups in a coupled master library, the $6 \$$ entries are the actual group numbers and do not need to include the number of neutron groups.

MALOCS 


\section{MALOCS.2 MALOCS Input/Output Specifications}

MALOCS generally requires the following input/output assignnents:

Logical No.

Purpose

NOLD(1) Fine-group AMPX master library

5 Card input

$6 \quad$ Standard output

17 Scratch device

18 Scratch device

19 Scratch device

NNEW(22) Broad-group AMPX master library produced by MALOCS

N1(0) Logical number of master library containing data that are to be used as a weighting spectra for neutron cross sections

N2(0) Logical number of master library containing data that are to be used as weighting spectra for gamma-ray cross sections 


\section{NITAWL-II - MODULE TO CONVERT MASTER LIBRARIES TO WORKING LIBRARIES AND TO PERFORM RESONANCE SELF-SHIELDING CALCULATIONS}

An AMPX master library is not in a form where it can be directly used in most analysis programs. Instead of containing the "summed" transfer matrices, such as one might use in a flux calculation, it carries the matrices for each of the constituent processes. It also carries resonance parameters, which must be used in order to obtain self-shielded cross-section values. It is the role of the NITAWL (Nordheim Integral

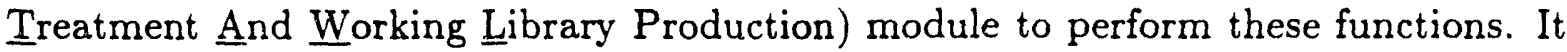
and the WORKER module provide the capability for converting from AMPX master to working format that many of the other modules use.

The optional resonance self-shielding calculation is for the resolved energy range and employs the Nordheim Integral Treatment.

The Nordheim Integral Treatment is a two-region integral transport theory method, a fuel lump surrounded by a moderator region, in which the moderator region is assumed to have an asymptotic $(1 / E)$ flux everywhere and at all energies. Escape probabilities are used to account for coupling between the two regions. NITAWL uses tabulations of exact probabilities for slabs, cylinders, and spheres as developed by Case, de Hoffman, and Placzek, but which are based on assuming the flux in the fuel lump is flat and that the scattering is isotropic. To account for lattice effects, a Dancoff factor can be specified, though it is a single value, which must be used for all energies of all resonances.

Aside from the fact that this treatment considers only one resonance nuclide in its calculation (i.e., the overlap between resonances of different nuclides is not treated), it performs a separate calculation for each resonance, which also assumes the resonances of a nuclide do not overlap.

Certainly, resonance-resonance overlap effects can be important. For nuclides such as ${ }^{233} \mathrm{U}$ and ${ }^{241} \mathrm{Pu}$, which have several large and important overlapping low-energy resonances, one would not expect the treatment to be very accurate. The same is true for multinuclide systems that contain many overlapping resonances. Examples are

1. systems containing mixed oxides of plutonium and uranium, such as solutions in LMF'BRs, and

2. advanced thorium-converter systems.

For LMFBR systems, the effects are not as severe as one might expect for criticality studies, because the most important energy region is high relative to the resolved energy regions for most nuclides.

For systems involving primarily ${ }^{235} \mathrm{U}$ and ${ }^{238} \mathrm{U}$, such as the light-water reactor, the treatment is quite good, because the resonances of these two nuclides do not overlap that much.

An area in which the treatment is particularly weak is that of calculating small, tightly packed lattices, wherein the flux in the moderator is significantly different from the asymptotic value. This is the case with the analysis of the HTGR fuel pellet, which uses tiny fuel pellets coated with a graphite moderator.

NITAWL-II 
The use of escape probabilities based on flat flux and isotropic scattering assumptions has been shown to lead to errors in some cases. Even fixups involving making transport corrections to the scattering have not proved that satisfactory.

The fact that the treatment only treats free-atom elastic scattering may introduce errors in some cases where inelastic sources may be important or for very low-energy resonances where upscattering effects should be included.

In general, however, the Nordheim treatment does quite well on a variety of criticals and is certainly superior to many treatments based on the narrow resonance approximation, such as the Bondarenko method (see the BONAMI module description).

\section{NITAWL-II.1 NITAWL-II Input Data}

$-1 \$$ Core Assignment [1]

1. NWORD - Number of words to allocate to NITAWL (200000)

$0 \$$ Logical Assignments [9]

This array is input only if a user needs to modify default values (shown in parentheses).

1. N1 - Input master cross-section interface (1)

2. N2 - Input working/weighted cross-section interface (2)

3. N3 - Input working/weighted cross-section interface (3)

4. N4 - Working cross-section interface produced by NITAWL (4)

5. N5 - Scratch (18)

6. N6 - Scratch (19)

7. N7 - Random access scratch (9)

8. N8 - Not used

9. N9 - Master sensitivity interface produced by NITAWL (20)

$1 \$$ Integer Parameters [12]

1. MSCM - Not used (0)

2. MMT - Number of nuclides to read from the master cross-section iibrary mounted on N1. Resonance calculations can be made with "resonance" nuclides from a master library, but not from a working/weighted library (0)

3. MWT - Number of nuclides to read from the working/weighted library on logical N2 (0)

4. MXT - Number of nuclides to read from the working/weighted library on logical N3 (0)

5. MCR - Sensitivity output trigger (0) $=0$, do not produce master sensitivity library $>0$, produce master sensitivity library

Note - Master sensitivity library will contain only nuclides selected from the master cross-section library on logical N1.

6. MXX - Not used (0)

7. MS - Not used (0) 
8. IRES - Number of resonance calculations to be performed (0)

Resonance is required in the $3^{*}$ array discussed below.

9. IQM - Not used (0)

10. IPM - Not used (0)

11. IPP - Output option trigger (0)

0 - no cross-section edits

1 - edit reaction cross sections

$>1$ - edit reaction cross sections and transfer arrays through expansion order IPP-2 (e.g., five edits through $\mathrm{P}_{3}$ cross sections)

12. IFG - Print for resonance mesh generation (0)

If IFG is 10 , then debug messages from resonance mesh generator will be printed.

$6 \$ \$$ Direct-Access Device Space [6]

1. NBLK 8 - Number of direct-access blocks on lugical 8 (not used in NITAWL)

2. LBLK8 - Length of direct-access blocks in words on logical 8 (not used in NITAWL)

3. NBLK9 - Number of direct-access blocks on logical 9 (NITAWL typically determines this parameter from the cross-section library directory, but uses this value, if input)

4. LBLK9 - Length of direct-access blocks in words on logical 9 (as above, NITAWL calculates a value for this parameter, but will use this value, if input)

5. NBLK10 - Number of direct-access blocks on logical 10 (not used in NITAWL)

6. NBLK10 - Length of direct-access blocks in words on logical 10 (not used in NITAWL)

T Terminate Block 1.

\section{Block 2.}

$2 \$$ Identification Numbers of Nuclides to be Placed on the Working Library $[\mathrm{MMT}+\mathrm{MWT}+\mathrm{MXT}]$

NITAWL can combine data from three sources to make a working library on logical unit N4. This working library contains only those nuclides selected and is intended for use by another module, such as XSDRNPM/S or KENO V.a. The nuclide identifications are input in this order:

1. MMT identifications for nuclides from the master library on N1,

2. MWT identifications for nuclides from the working/weighted library on $\mathrm{N} 2$, and

3. MXT identifications for nuclides from the working/weighted library on N3.

Within each group, the identifications can be in any order.

A facility is provided for creating duplicate sets for a particular nuclide. For example, a resonance nuclide might occur in different compositions, thereby requiring different resonance calculations. To create a new set, place a unique negative number in the identification string after the nuclide's identification that is 
to be duplicated. The new set will use this as its identifier. As many sets as needed can be had, merely by stringing out negative numbers. All subsequent references to the new sets of data should use a positive identification. The values MMT, MWT, and MXT must be increased to reflect the inclusion of these sets.

3* Resonance Data [IRES*15] (IRES $>0$ )

For each resonance calculation, 15 items are required. These blocks of data are placed, one set after the other, in any order in the $3^{*}$ array. Note that only one $3^{*}$ array is input. Resonance calculations can only be performed on resonance nuclides from a master library. The 15 items are

1. Nuclide's identification number.

2. Temperature (K).

3. Geometry for Nordheim treatment:

0 - homogeneous,

1 - slab,

2 - cylindrical, and

3 - spherical.

4. Absorber lump dimension in centimeters (i.e., the thickness of the slab or radius of a sphere or cylinder). A zero is used for the homogeneous case.

5. Dancoff correction factor. This factor corrects for shadowing effects caused by the presence of other absorber lumps (e.g., fuel elements) in the neighborhood of the lump being calculated. It is zero for the homogeneous and the single lump case.

6. $a_{i}$, the inner radius of the spherical or cylindrical shell, if a "shell" geometry is required. (Note that a value of zero specifies a solid sphere or cylinder. If the inner region does not contain a light-mass moderating material, the absorber should be treated as a solid.) (0.0)

7. $\mathrm{N}_{\mathrm{a}}$, absorber number density in the lump.

8. Treatment of absorber's contribution to the collision density:

1 - Nordheim integral method,

2 - narrow resonance approximation, and

3 - infinite mass approximation.

9. Atomic mass of the first moderator in the lump. (The resonance routines have provisions for treating two moderators. Note that these absorbers are in the lump mixture and are not the external moderator.)

10. $\sigma_{\mathrm{s} 1}$, first moderator scattering cross section per absorber atom,

$$
\sigma_{\mathbf{s} 1}=N_{1} \sigma_{s}(\text { moderator } 1) / N_{\mathrm{a}}
$$

where $\mathrm{N}_{1}$ is the number density of the first moderator.

11. Treatment of the first moderator's contribution to the collision density:

0 - no first moderator,

1 - Nordheim's integral method, and

2 - asymptotic approximation.

12. Atomic mass of the second moderator in the lump.

13. $\sigma_{s 2}$, the second moderator scattering cross section per absorber atom. 
14. Treatment of the second moderator's contribution to the collision density:

0 - no second moderator,

1 - Nordheim's integral method, and

2 - asymptotic approximation.

15. Volume fraction of the absorber lump in the cell. This parameter is used for "cell-averaging" the resonance cross sections. If a value other than 1.0 is input, the output cross sections are appropriate for use in a smeared cell calculation. If your cross sections are meant for use in an explicit mock-up of the cell (e.g., in XSDRNPM), input 1.0.

4* Thermal-Scattering Kernel Temperatures for Nuclides Selected (K) [MMT]

Scattering kernels are sometimes provided at several temperatures for a nuclide. NITAWL will pick the one closest to the temperature input in this array. This array has no effect for sets of data with zero or one thermal kernel.

$6 \$$ MT Number of the Incoherent Thermal-Scattering Kernel [MMT]

These allow selecting thermal-scattering kernels with MT (identifying) numbers other than the default (1007).

T Terminate Block 2.

\section{NITAWL-II.2 NITAWL-II Input/Output Specifications}

NITAWL typically requires the following input/output devices during an execution:

Logical No.

Purpose

NT1 (1)

NT2 (2)

NT3 (3)

NT4 (4)

NT5 (18)

NT6 (19)

NT7 (9)

NT9 (20)

5

6

\begin{tabular}{l} 
Purpose \\
\hline Master cross-section library \\
Previously prepared working/weighted library \\
Previously prepared working/weighted library \\
New working library \\
Scratch unit \\
Scratch unit \\
Random access scratch unit \\
Sensitivity-produced master library \\
Card input \\
Printed output
\end{tabular}




\section{NPTXS - MODULE TO PRODUCE POINT DATA FOR RESONANCE NUCLIDES}

NPTXS (Neutron Poin $\underline{\underline{X}}$ - $\underline{\text { Section) }}$ is provided to "preprocess" resonance or nonresonance ENDF nuclides and make point files containing the total, fission, elastic scattering, and $(n, \gamma)$ cross sections. It is a predecessor of the POLIDENT module, which also performs this function.

The point cross-section library written by NPTXS on logical unit 31 looks very much like file 3 of an ENDF library. (See Sect. 3.NPTXS.3 for the format of the pointwise file.) One of the important uses of the library is to provide $\sigma_{T}$ data for the $1 / \mathrm{E} \sigma_{T}$ weighting of a resonance nuclide. This information can be input to JERGENS for appropriate processing into the inverse form.

Other features of NPTXS are as follows:

1. The module can process single-level Breit-Wigner (SLBW) and multilevel BreitWigner (MLBW) resolved resonance data. The resonance data can be Dopplerbroadened using the numerical integration techniques developed by Cullen (subroutine BROADN) or the PSI-CHI method (subroutine VOGAM). Note that the background ENDF file 3 data will be Doppler-broadened if subroutine BROADN is used, but will not be Doppler-broadened if the PSI-CHI method is used.

2. Unresolved processing uses the same techniques employed in XLACS. These techniques were originally developed for the MC-2 code.

3. ENDF MAT numbers are used as identifiers for data sets on the point cross-section library. When NSIGP $>1$, the first $\sigma_{0}$ in the $4^{*}$ array is used to calculate the unresolved component of the point data set placed in the point cross-section library. Unresolved cross-section data for the second through NSIGP $\sigma_{0}$ 's are punched on cards.

The resolved resonance treatment used in NPTXS is described in refs. 1 and 2; the unresolved resonance treatment, in refs. 3 and 4 ; and the Doppler-broadening, in refs. 5-10.

\section{NPTXS.1 NPTXS Input Data}

\section{Block 1.}

0\$ Doppler-Broadening Option [1]

1. IDPL - $0 / 1$ use BROADN/VOGAM method (0)

$1 \$$ Case Description [1]

1. NNUC - Number of ENDF nuclides to be processed

2. NVER - $4 / 5$ version of ENDF being processed (4)

$\mathrm{T}$ Terminate Block 1.

Repeat blocks 2 and 3 for NNUC nuclides. 


\section{Block 2.}

$2 \$$ Nuclide Selection Array [5]

1. MATNO - ENDF MAT number for nuclide

2. NDFB - Logical unit containing the ENDF data (11)

3. IDTAP - Check the library label (0/1: no/yes) (1)

4. MODE - ENDF library mode (0/2: binary/BCD) (0)

5. NSIGP - Number of $\sigma_{0}$ values * (1)

3* Nuclide Options [4]

1. $\mathrm{RFACT}^{\dagger}-$ The resolved resonance region energy mesh is based directly on this parameter. Points are chosen such that the ratio of total cross section from point to point is roughly RFACT (0.9)

2. SFACT - Number of practical widths over which the RFACT scheme is used for a particular resonance (10)

3. SIGP $-\sigma_{0}$ for the unresolved calculation $\left(10^{8}\right)$

4. TDEGIC - Temperature in Kelvin for Doppler-broadening in the resolved and unresolved resonance regions (0)

T Terminate Block 2.

Block 3. $\{$ NSIGP $>1\}$

$4^{*} \sigma_{0}$ array for the unresolved calculation. [NSIGP**] $\{$ NSIGP $>1\}$ ( $\mathrm{SIGNL}(\mathrm{N}), \mathrm{N}=1, \mathrm{NSIGP}$ )

The $\sigma_{0}$ values should be input high to low and NSIGP must not exceed 7 .

T Terminate Block 3.

*The NPTXS $\sigma_{0}$ and the XLACS potential scattering cross section, $\sigma_{p}$, are identical.

${ }^{\dagger} A$ reasonable range for $R F A C T$ is $0.70 \leq R F A C T \leq 1.0$. If the input RFACT is not within the reasonable range, NPTXS resets the parameter to the default value.

${ }^{* *}$ If NSIGP $=1$, the desired $\sigma_{0}$ should be input in the $3^{*}$ array, and the $4^{*}$ array is not required. 


\section{NPTXS.2 NPTXS Input/Output Specifications}

The following ir, at/output assignments are required by NPTXS:

\begin{tabular}{cll} 
Logical No. & & \multicolumn{1}{c}{ Purpose } \\
\cline { 1 - 1 } 5 & & Card input \\
6 & & Standard output \\
7 & & Standard punch (required if NSIGP > 1) \\
14 & & Scratch device \\
15 & & Scratch device \\
16 & & Scratch device \\
17 & & Scratch device \\
18 & & Scratch device \\
19 & Scratch device \\
31 & Point files produced by NPTXS \\
NDFB $(11)$ & ENDF library
\end{tabular}

\section{NPTXS.3 NPTXS Output Format}

The NPTXS poini cross-section library, written on logical unit 31 , is in essentially the same format as file $3(\mathrm{MF}=3)$ of an ENDF library. The only difference is that the NPTXS output files are not restricted to a maximum of 5000 points as required for ENDF file 3. The NPTXS output files are unformatted. For a given material, MAT, and a given reaction type, MT, the structure of a section is

(MAT, 3, MT/ZA, AWR, LIS, LFS, 0, 0)HEAD

(MAT, 3, MT/S , Q , LT, LR, NR, NP/E

(MAT, 3, $0 / 0.0,0.0,0,0,0,0)$ SEND

See ref. 1 for definitions of the variables used and for additional details concerning the format.

\section{REFERENCES}

1. D. Garber, C. Dunford, and S. Pearlstein, Data Formats and Frocedures for the Evaluated Nuclear Data File, ENDF, BNL-NCS-50496, Brookhaven National Lab., October 1975 .

2. D. R. Mathews, FSDP : A Computer Program for Computing Cross Sections from Resonance Parameters, Gulf-GA-AL2052, Gulf General Atomics, pp. 1-13, April 1972.

3. N. M. Greene et al., XLACS: A Program to Produce Weighted Multigroup Neutron Cross Sections from ENDF/B, ORNL/TM-3646, Union Carbide Corp., Nucl. Div., Oak Ridge Natl. Lab., pp. 21-28, April 1972. 
4. G. L. Singer and R. A. Grimesey, C-Sure: Cross Sections for Unresolved Resonance from ENDF/B, ANCR-1068, Aerojet Nuclear Company, Idaho Falls, Idaho, pp. 115, May 1972.

5. D. E. Cullen, O. Ozer, and C. R. Weisbin, "Exact Doppler Broadening of Evaluated Neutron Cross Section," Trans. Am. Nucl. Soc. 16, 320 (1973).

6. D. E. Cullen, "Conservation During Doppler Broadening," Nucl. Sci. Eng. 52, 498-500 (1973).

7. G. W. Hinman et al., "Accurate Doppler Broadened Absorption," Nucl. Sci. Eng. 16, 202-207 (1963).

8. R. Q. Wright, ADLER-III: Program to Calculate Cross Sections from Adler-Adler Resonance Parameters, ORNL/TM-4257, Union Carbide Corp., Nucl. Div., Oak Ridge Natl. Lab., June 1973.

9. D. R. Mathews, FSDP9: A Computer Program for Computing Cross Sections from Resonance Parameters, Gulf-GA-AL2052, Gulf General Atomics, pp. 14-18, April 1972.

10. G. I. Bell and S. Glasstone, Nuclear Reactor Theory, Van Nostrand Reinhold Co., New York, 1970, pp. 401-406. 


\section{PAL - MODULE TO PUNCH 1-D CROSS SECTIONS FROM AMPX LIBRARIES}

PAL (Punch AMPX Libraries) is provided to allow punching the 1-D cross-section arrays on either an AMPX master or an AMPX working library. Data can be punched in a fixed-form (1P,6E12.5) or the free-form FIDO format.

\section{PAL.1 PAL Input Data}

\section{Block 1.}

$0 \$$ Logical Unit Assignments [1]

1. MMT - Master working library logical unit (0)

2. MWT - Working library logical unit (0)

3. N7 - Punch device (7)

$1 \$$ Options [3]

1. NPUNCH - Number of nuclides for which data are to be punched. A zero causes data for all nuclides on the library to be punched. (0)

2. IFORM - 0, Fixed format (i.e., 1P,6E12.5 format)

1, Free-form FIDO format (1)

3. ICORE - Number of words to allocate to PAL (50000)

$2 \$$ MT Numbers to be Punched [100]

Stack up to $100 \mathrm{MT}$ numbers for the processes to be punched in this array [e.g., to punch the $(\mathrm{n}, \gamma)$ data]. $2 \$ \$ 102 \mathrm{~F} 0$. (Note that $\mathrm{MT}=999$ can be used to punch the energy group boundaries from the library.)

T Terminate Block 1.

\section{Block 2. $\{$ Input if NPUNCH $>0$ \}}

$3 \$$ Identifiers for Nuclides Whose Cross Sections are to be Punched $\{$ NPUNCH\}

T Terminate Block 2.

A card is punched between each set of cross sections which identifies the data set and process.

\section{PAL.2 PAL Input/Output Specifications}

PAL requires the following input/output devices:

\begin{tabular}{cll} 
Logical No. & & \multicolumn{1}{c}{ Purpose } \\
\cline { 1 - 1 } 5 & & Standard input \\
6 & & Printed output \\
7 & & Punched output \\
MMT & & Master library \\
MWT & & Working library
\end{tabular}




\section{PERFUME - MODULE TO CORRECT LEGENDRE POLYNOMIAL FITS TO SCATTERING MATRICES}

PERFUME (PERmute FaUlty Moment Expansions) is a module that examines scattering matrices to locate those that yield nonphysical fits and adjusts them to produce acceptable distributions. The procedure involves an examination of the moments of the cross section, as described in Appendix F9.D.2 of the SCALE documentation. With these prescriptions, a range of possible coefficients is determined, after which PERFUME arbitrarily picks a value in the range.

\section{PERFUME.1 PERFUME Input Data}

$-1 \$$ Core Assignment [1]

1. NWORD - Number of words to allocate to PERFUME (100000)

O\$ Logical Assignments [3]

1. NTP - Input master library (23)

2. NOT - Output master library (1)

3. NER - Error message output file (6) Assign this to a unit other than 6 to suppress the voluminous error message listing that may be produced by some libraries, if yo' 1 do not wish to view this information.

$1 \$$ Output Suppression Factor [1]

1. EPS - Information conceriling bad moments is edited when the zeroeth moment of the transfer term (i.e., $\sigma_{0}$ ) satisfies the following expression $($ default $=0.0001)$ :

$$
\sigma_{0}>\epsilon * N^{3}
$$

where $\mathrm{N}$ is the order of Legendre fit to the cross section. [Warning: For a library with a large number of energy groups and nuclides, the amount of output can be substantial.]

T Terminate Block 1.

\section{PERFUME.2 PERFUME Input/Output Specification}

PERFUME uses the following input/output assignments:

\begin{tabular}{c} 
Logical No. \\
\hline 5 \\
6 \\
$\operatorname{NTP}(23)$ \\
$\operatorname{NOT}(1)$
\end{tabular}

$\frac{\text { Purpose }}{\text { Card input }}$
Printed output
Input master library
Output master library




\section{POLIDENT - MODULE TO PRODUCE POINT DATA FROM RESONANCE DATA}

POLIDENT (POint LIbraries of Data from ENDF/B Tapes) is a module that accesses the resonance parameters in File 2 of an ENDF/B library, generates point cross sections from them, and combines these with the point data in File 3. As such, it is intended to replace NPTXS.

POLIDENT has several characteristics that distinguish it from the earlier NPTXS module:

- It outputs essentially all point data on File 3 of the ENDF/B library, not just the total, elastic, fission, and neutron-gamma values, as does NPTXS.

- It processes Adler-Adler parameters.

- It can process multi-isotope nuclides that have different resonance ranges.

- It will produce more accurate representations of cross sections in the resolved region than NPTXS because of a restructuring of the way it combines strings of cross sections.

\section{POLIDENT.1 POLIDENT Input Data}

Block 1.

$-1 \$$ Core Allocation [1]

1. NWORD - Number of words to allocate to POLIDENT (100000)

$0 \$$ Output Library [1]

1. LOGP - Output from POLIDENT written here (31)

$1 \$$ Number of Cases [1]

1. NNUC - Number of nuclides to process

$\mathrm{T}$ Terminate Block 1.

Repeat Block 2 NNUC times.

\section{Block 2.}

$2 \$$ ENDF/B Data Source [3]

1. MATNO - Material identifier for the nuclide on the ENDF/B library

2. NDFB - Logical unit on which the ENDF/B library is mounted (11)

3. MODE - Format of the ENDF/B library (1-binary/2-BCD) (default =1)

3* Parameters for Resonance Processing [2]

1. SIG0 - Background cross section, $\sigma_{0}$, value to use in processing unresolved resonance data, if appropriate

2. $\mathrm{T}$ - Temperature in Kelvin at which resonances are to be Doppler-broadened

4* Floating-Point Parameters [14] 
1. EPS - To construct a final function, POLIDENT must combine strings of point data from File 3 with resolved and unresolved data arrays. The final function contains enough points such that any intermediate value is accurate to EPS $(0.001$ or $0.1 \%)$.

2. $\mathrm{R}$ - The ratio factor used in a cross-section energy mesh.

3. XNP - The number of points taken equally spaced in lethargy in between "resonance bodies" (50.0).

4. XGT - The multiplier on the total width above and below a resonance over which the ratio mesh scheme is used (50.0).

5. OPT(1) - Not used.

14. OPT (10) - Not used.

$5 \$$ Options [8]

1. IOPT1

2. IOPT2

These are used in conjunction with IOPT4 through

IOPT8 to select the ENDF/B interpolation options

3. IOPT3 -

4. IOPT4

5. IOPT5

6. IOPT6

7. IOPT7

8. IOPT8 to be tried. These are both set to 1 by default.

Maximum number of interpolation regions allowed in the output ENDF/B function.

Interpolation types to be tried. IOPT 4 is a 2 by default, while the others are zero.

IOPT4 through IOPT8 are used to specify the interpolation types and their order which will be tried in combining two or more ENDF/B functions. The types are as follows:

$\begin{array}{cll}\text { Value } & & \text { Type of interpolation } \\ 1 & & \text { Histogram } \\ 2 & & \text { Linear } x, \operatorname{linear} y \\ 3 & & \text { Linear } x, \log y \\ 4 & \text { Log } x, \operatorname{linear} y \\ 5 & \text { Log } x, \log y\end{array}$

IOPT1 and IOPT2 specify which entries in the five-position table are to be used (e.g., the default values of 1 say to use only the first entry in the table, or linearlinear interpolation by default).

\section{POLIDENT}


$6 \$$ Input Function Limits [1]

1. N1MAX - Maximum number of interpolation regions in any "input" ENDF/B function (20)

2. N2MAX - Maximum number of points in any input ENDF/B TAB1 array (5000)

$\mathrm{T}$ Terminate Block 2.

\section{POLIDENT.2 POLIDENT Input/Output Specifications}

POLIDENT requires the following input/output units:

Logical No.

Purpose

5 Card input

$6 \quad$ Printed output

LOGP(31) Functions produced by POLIDENT are written here

14

Scratch device

15

Scratch device

16

Scratch device

17

Scratch device

18 Scratch device 


\section{PRELL-MODULE TO PRODUCE AND MANIPULATE AN ENERGY LIMITS LIBRARY}

PRELL (Produce Reordered Energy Limits Library) is an AMPX module to create, copy, modify, punch, or list an AMPX energy-group-limits library. The new library will be reordered in increasing number of groups for neutron structures, then in order of increasing groups for gamma structures. The new library may be printed. Modification features include adding new structures to an existing library and changing boundaries in an existing structure.

\section{PRELL.1 PRELL Input Data}

\section{Block 1.}

$0 \$$ Logical Unit Assignments [3]

1. NO - Logical unit number of the old library (47)

2. NW - Logical unit number of the new library (18)

3. NS - Logical unit number of a random-access scratch unit

$1 \$$ Options [2]

1. NOPT - Option flag NOPT $<0$ - punch new library (0) Option flag $\mid$ NOPT $\mid<1-$ print new library (0)

2. NSETS - Number of sets to be added and modified (0)

$\mathrm{T}$ Terminate Block 1.

If NSETS $=0$, no more data are input.

\section{Block 2.}

$3 \$$ Flags [3*NSETS]

1. IG - Number of groups in set

2. ITYPE - A flag

ITYPE $=$ ITP $+10 *$ NUP

where,

ITP $=0$ for a neutron-group-structure library

$=1$ for a gamma-group-structure library

NUP $=0$ if the NSETSth entry is new group structure

$>0$ if the NSETSth entry is an existing structure where NUP modifications will be made to the structure

3. IVER - Version number (only 0 can be used at present)

Repeat above three entries NSETS times.

$\mathrm{T}$ Terminate Block 2. 
Block 3. \{Repeat NSETS times

$7^{*}[\mathrm{IG}+1]$ if $\mathrm{NUP}=0$

E - Group boundaries (in decreasing order) of a new group structure to be added to the library. Boundaries should be in eVs.

$8^{*}$ [NUP]

IBND - Indices of boundaries to be changed

$9^{*}$ [NUP]

ENEW - New values of boundaries to be changed

T Terminate Block 3.

End of Block 3.

\section{PRELL.2 PRELL Logical Assignments}

PRELL requires the following input/output assignments:

\begin{tabular}{c} 
Logical No. \\
\hline 5 \\
6 \\
NO(47) \\
NW(18) \\
NS(8)
\end{tabular}

Purpose
Card input
Stardard output
Old AMPX energy-group-limits library
New AMPX energy-group-limits library
Random access scratch unit




\section{PRUDE - AMPX MODULE TO CREATE CROSS SECTIONS FOR THE UNRESOLVED RESONANCE ENERGY REGION}

PRUDE (PRocess Unresolved Data on ENDF/B) is a module that accesses the unresolved resonance data in file 2 of an ENDF/B library and writes out a file which gives the energy variation of "average" cross sections for several important processes as a function of temperature and the weighting parameters, $\sigma_{0}$. Its primary use is to pass these data to the TABU module, which creates Bondarenko factors that ultimately become part of an AMPX master interface. The Bondarenko factors are used by the BONAMI module for performing self-shielding in the unresolved region.

Recall that in the development of the Bondarenko treatment, a narrow-resonance weighting of the form

$$
\bar{\sigma}\left(\sigma_{0}, T\right)=\frac{\int_{q} \frac{\sigma(E, T) \phi(E) d E}{\sigma_{T}(E)+\sigma_{0}}}{\int_{g} \frac{\phi(E) d E}{\sigma_{T}(E)+\sigma_{0}}}
$$

is used, where $\phi(E)$ is a smooth weighting function (generally $1 / E$ in the unresolved region), $\mathrm{T}$ is the temperature at which the cross sections were Doppler-broadened, and $\sigma_{0}$ is the cross section that accounts for the cross sections of other nuclides in the mix with the resonance nuclide.

PRUDE accepts an arbitrary number of temperatures and $\sigma_{0}$ values as input. At each pair of values, $\mathrm{T}$ and $\sigma_{0}$, it makes a calculation to determine "shielded" cross sections. The energy mesh is picked to be either the energy mesh at which the unresolved parameters are specified in the ENDF/B library or at 100 points equally spaced in lethargy over the unresolved region, when the parameters are constant.

The output from PRUDE is a file of records written in the ENDF/B "TAB1" format, as follows:

Record 1: MAT, MF, MT, 0, 0, 0, 0, 0, 0

Record 2: MAT, MF, MT, T, $\sigma_{0}, 0,0, \mathrm{NR}, \mathrm{NP}$,

$\left(\mathrm{NBT}_{i}, \mathrm{JNT}_{i}, \mathrm{i}=1, \mathrm{NR}\right)$,

$\left(E_{i}, \sigma_{i}\left(\mathrm{~T}, \sigma_{0}\right), \mathrm{i}=1, \mathrm{NP}\right)$

Record 3: MAT, MF, 0, 0, 0, 0, 0, 0, 0

where MAT is the material identifier,

$M F$ is the "file" number,

MT is the process identifier,

$\mathrm{T}$ is the temperature in Kelvin,

$\sigma_{0}$ is the background cross section,

$\mathrm{NR}$ is the number of interpolation regions,

$\mathrm{NBT}_{i}, \mathrm{JNT}_{i}$ comprise the interpolation table, 
$\mathrm{E}, \sigma$ are the energy cross-section values.

Each T, $\sigma_{0}$ pair will generate the triplet of records shown above for each of six processes:

$\mathrm{MT}=1$, total cross section

$\mathrm{MT}=2$, elastic scattering

$\mathrm{MT}=102,(\mathrm{n}, \gamma)$

$\mathrm{MT}=18$, fission

$\mathrm{MT}=1000$, transport cross section

$\mathrm{MT}=4$, inelastic scattering

\section{PRUDE.1 PRUDE Input Data}

\section{Block 1.}

$-1 \$$ Core Allocation [1]

1. NWORD - Number of words to allocate to PRUDE (50000)

$0 \$$ Point File Assignment [1]

1. LOGP - Logical unit where point data are to be written (31)

$1 \$$ Processing Option [1]

1. NNUC - Number of materials to process

$\mathrm{T}$ Terminate Block 1.

Repeat blocks 2 and 3 NNUC times.

\section{Block 2.}

$2 \$$ Data Source and Problem Options [5]

1. MATNO - Material number for the ENDF/B data

2. NSIGO - Number of $\sigma_{0}$ values

3. NTEMP - Number of temperatures

4. NDFB - Logical unit containing the ENDF/B data (11)

5. MODE - Format of ENDF/B data (1-binary, 2-BCD) (default =1)

T Terminate Block 2.

\section{Block 3.}

3* Background Cross Sections [NSIGO]

$\left(\sigma_{0}, \mathrm{i}=1, \mathrm{NSIGO}\right)$ Specify these values in descending order.

$4^{*}$ Temperatures [NTEMP]

$\left(T_{i}, \mathrm{i}=1\right.$, NTEMP) Specify these in ascending order in Kelvin.

$\mathrm{T}$ Terminate Block 3. 


\section{PRUDE.2 PRUDE Input/Output Specifications}

PRUDE requires the following input/output assignments:

\begin{tabular}{l} 
Logical No. \\
\hline 5 \\
6 \\
LOGP $(31)$ \\
NDFB(11) \\
14 \\
15 \\
16 \\
17 \\
18
\end{tabular}

\begin{tabular}{l}
\multicolumn{1}{c}{ Purpose } \\
\hline Card input \\
Printed output \\
Point data file is written here \\
ENDF/B library \\
Scratch device \\
Scratch device \\
Scratch device \\
Scratch device \\
Scratch device
\end{tabular}




\section{RADE - MODULE TO CHECK AMPX MASTER CROSS- SECTION LIBRARIES}

RADE (ㅁancid AMPX Data Exposer) is provided to check AMPX- and ANISNformatted multigroup libraries. It will check neutron, gamma, or coupled neutrongamma libraries.

Some of the more important checks are made to ensure that:

1. $\sigma_{t}=\sigma_{a}+\sigma_{s}$

2. $\sigma_{i n}=\Sigma \sigma_{i n}^{\text {partial }}$

3. $\sigma_{a}=\sigma_{c}+\sigma_{f}$

4. $\sigma_{c}=\sigma_{n \gamma}+\sigma_{n \alpha}+\sigma_{n p}+\sigma_{n d}+\ldots$

5. $\sigma_{e l}^{g}=\sum_{g^{\prime}} \sigma_{e l, 0}\left(g \rightarrow g^{\prime}\right)$ (also made on any process with a scattering matrix)

6. $\sigma_{0\left(g \rightarrow g^{\prime}\right)}>0$

7. $\sigma_{T}, \sigma_{a}, \sigma_{f}, \sigma_{n \gamma}, \sigma_{n p}, \ldots>0$

8. $f_{\ell}^{\min } \leq f_{\ell}\left(g \rightarrow g^{\prime}\right) \leq 1.0$,

where $f_{\ell}\left(g \rightarrow g^{\prime}\right)=\frac{\sigma_{\ell}\left(g \rightarrow g^{\prime}\right)}{(2 \ell+1) \sigma_{0}\left(g \rightarrow g^{\prime}\right)}$

and $f_{\ell}^{\min }=-1.0$ for all odd $\ell$, and is given in the following table for even $\ell$

\begin{tabular}{ll}
$\ell$ & $f_{\ell}^{\min }$ \\
\hline 2 & -0.5 \\
4 & -0.433 \\
6 & -0.419 \\
8 & -0.414 \\
\hline
\end{tabular}

In addition to these checks, the code will compute an estimate of the capturebinding energy for each neutron group in a coupled neutron-gamma set. On option, one can request a display of differential cross sections.

\section{RADE.1 RADE Input Data}

\section{Block 1.}

$-1 \$$ Core Assignment [1]

1. NWORD - Number of words to allocate to RADE (100000)

$1 \$$ Checking Commands [4]*

1. MMT - Check the AMPX master interface on logical MMT. This can be a neutron, gamma, or a coupled neutron-gamma library

2. MWT - Check the AMPX working/weighted interface on logical MWT

3. MAN - Check the ANISN binary-formatted library on logical MAN

4. IFM - -1, ANISN library is binary formatted

0 , ANISN library is BCD free form

1 , ANISN library is BCD fixed form

${ }^{*}$ Number of entries in the array. 
$2 \$$ Options [20]

1. IOPT1 - Number of angles at which a display of differential cross sections is desired. These angles will be equally spaced in the cosine range, -1 to +1 . These edits are for the group-integrated cross sections and not for each group-to-group transfer.

2. IOPT2 - The $\epsilon$, in 1/1000's of a percent, to which checks are made (e.g., IOPT2 = 1) is equivalent to $0.001 \%$ checking. This is the default value when IOPT2 is not input or when a zero value is input.

3. IOPT3 -Enter a 1 to suppress printing the estimated binding energy tables for processes with gamma production data.

. Future checking options; skip or input zeroes

20. IOPT20

$3 \$$ ANISN Options [7] $\{\mathrm{MAN} \neq 0\}$

1. NSET - Number of ANISN sets to check

2. IHT - Position of $\sigma_{T}$

3. IHS - Position of $\sigma_{g g}$

4. ITL - Table length

5. NL - Maximum order of scattering

6. IGM - Number of neutron groups

7. IPM - Number of photon groups

T Terminate Block 1.

\section{Block 2. \{Input only when MAN $>0$ \}}

$4 \$$ Identification Numbers of $P_{0}$ Sets on ANISN Binary Library on Logical MAN [NSET]

$5 \$$ Order of Scattering for Sets of ANISN Data on Logical MAN [NSET]

7* Neutron Group Structure, high to low in eV [IGM+1]

8* Gamma Group Structure, high to low in eV [IPM+1]

T Terminate Block 2. 
3

3.RADE.2 RADE Input/Output Specifications

RADE requires the following input/output assignments:

\begin{tabular}{|c|c|}
\hline Logical No. & Purpose \\
\hline 5 & Card input \\
\hline 6 & Printed output \\
\hline 18 & Scratch device \\
\hline 19 & Scratch device \\
\hline MMT & Master library \\
\hline MWT & Working library \\
\hline MAN & ANISN library \\
\hline
\end{tabular}




\section{ROLAIDS - MODULE FOR TREATING RESONANCE SHIELDING IN MULTIREGION GEOMETRIES}

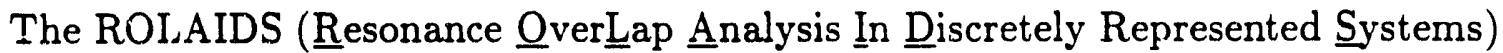
module is provided in AMPX for treating problems in which either resonance overlap causes mutual shielding or severe spatial attenuation of the neutron flux indicates the need for spatially dependent resonance cross sections. Given a point cross-section library and an AMPX master cross-section library, ROLAIDS performs an integral transport calculation for the energy-pointwise, slowing-down flux distribution in each zone of a 1-D multiregion geometry. (The ROLAIDS geometry and boundary condition specifications are fully compatible with those of XSDRNPM. ${ }^{1,2}$ ) ROLAIDS provides resonance-shieided fine-group constants (on logical unit N10) to be folded into the existing AMPX master data sets. The folding is subsequently done with the CLAROL module. The modified AMPX master library can be used in NITAWL to generate a working library for use in XSDRNPM discrete-ordinates analyses (which yield the higher order transport effects over the entire energy range) or to generate other muligroup libraries for use with other transport codes. Detailed documentation of the neutronics theory and numerical methods applied in ROLAIDS is given in ref. 3. Included here is a brief description of the operations performed by ROLAIDS.

The first operation that ROLAIDS performs in executing a problem is to calculate and store the firs'-flight escape and transmission probabilities needed for the transport arialysis. For spherical and cylindrical geometries, these parameters are functions of Doth curvature and optical thickness. Therefore, the user should be cognizant of the extra container ("D") array storage ( $~ 80 \mathrm{~K}$ bytes) required by these geometries relative to the siorage required for the homogeneous and slab geometries. Also, evaluation of the probabilities for eylindrical geometry requires about 2 additional minutes of CPU91 time (compared with the execution time for other geometries) due to the large number of numerical integrations performed. (CPU time is central processing unit time on the ORNL IBM $3030 / 91$ computing system.)

Pointwise energy files and corresponding unshielded cross sections prepared by the NPTXS module (N1 and N2) and the energy boundaries in the existing AMPX master library (MASTU) are input to ROLAIDS. An energy array is formed for the ROLAIDS transport calculation from all of the NPTXS pointwise files, the multigroup energy structure in the AMPX mas'er library, and 99-point equal-lethargy-based structure inserted for optional fine-group boundaries. Also inserted is an energy grid determined by successive neutron downscatterings, with the heaviest nuclide to be placed on the data file. This set of minimum slowing-down intervals ensures an adequate coverage of all energy ranges. Then follows a determination of slowing-down parameters for each nuclide at each energy point. ("Slowing-down parameters" are described in ref. 3.) This determination provides the array sizes used to flexibly dimension the slowingdown sequence. A ROLAIDS data file containing the pointwise cross sections and slowing-down parameters for all nuclides is then created (N7).

A ROLAIDS data file is created for a class of problems (e.g., light-water-reactor analysis) by combining NPTXS data for all the pertinent nuclides. Once created, a ROLAIDS data file cannot be altered. Therefore, it is advisable to include any nuclides that might eventually be required for analyzing similar systems. The ROLAIDS data 
file is geometry independent and can be used for any problem for which the fine-group boundaries and nuclide information have been included. Creation of the file requires approximately CPU91 $(\mathrm{min})=($ the total number of NPTXS energy points $) \times 10^{-4}$.

The slowing-down calculation performed by ROLAIDS is based on isotropic elasticscattering theory with an assumed linear variation of the scattering density between energy points. All downscattered source terms are explicitly calculated and stored. Since ROLAIDS does not treat inelastic scattering, the maximum problem energy, EHI, should be set below any significant inelastic thresholds $(\sim 20 \mathrm{keV})$, and the minimum energy, ELO, should be above the range of upscattered sources $(\sim 2 \mathrm{eV})$. Neutron fluxes in the source range above EHI are assumed to vary asymptotically as (energy) $\exp (-\mathrm{EZP})$, EZP being an input parameter with a default value of 1 . Execution of a ROLAIDS slowing-down calculation requires approximately CPU91 $(\min )=T_{0}+$ No. energies $\times$ No. zones $\times$ No. densities $\times 4.2 \times 10^{-7}$, where $T_{0}=3$ for cylindrical geometry and $T_{0}=1$ for slab, spherical, and homogeneous geometries. In this expression, the number of densities is the total number of densities in the total number of nonzero atom densities for all materials in all zones. The expression does not hold for cases with periodic boundaries that require a full matrix solution for the interface currents and, therefore, run much longer.

Where applicable, an effort was made to maintain commonality between ROLAIDS and XSDRNPM problem descriptions. Thus the input parameters IGE, IZM, IM, IBL, IBR, MXX, and MS have identical functions in the two modules. However, for many applications, the integral transport solution applied in ROLAIDS does not require as fine a spatial mesh as the finite-difference calculation performed by XSDRNPM. When a ROLAIDS-CLAROL-...-XSDRNPM sequence is being executed, the user may set the number of mesh intervals ROLAIDS and XSDRNPM to be equal [i.e., $\mathrm{IM}(\mathrm{ROLAIDS})=\mathrm{IM}(\mathrm{XSDRNPM})]$ and input the XSDRNPM radii $(\mathrm{IM}+1)$. Then, the number of zones, IZM, can be specified in the range IM $\leq$ IZM (ROLAIDS) $\geq$ IZM (XSDRNPM). The ROLAIDS output of fine-group cross sections is averaged over contiguous ROLAIDS zones of the same material specification ("superzones"). For most problems, these ROLAIDS superzones will correspond to the XSDRNPM zones.

The ROLAIDS superzone-weighted cross sections are written on logical unit N10, a CLAROL data file. These cross sections include scattering terms expanded to Legendre order specified by ISCT ( $3 \$$ array). The identification numbers from the NPTXS data sets are used by ROLAIDS as identifiers for the data sets on the CLAROL data file. CLAROL should be executed to update the AMPX master library (MASTU) with the ROLAIDS-produced library (N10). As noted above, an AMPX working library is produced by using NITAWL to process the CLAROL-updated AMPX master library. Master data sets that have been processed with ROLAIDS-CLAROL will not contain resonance parameters. (This fact must be considered when determining the value of IRES for the NITAWL $1 \$$ array.)

Printed output options in ROLAIDS include fine-group cross sections averaged according to standard cell, zone, and region definitions and according to the superzone specification discussed above. For comparison purposes, the $1 / E$ weighted fine-group cross sections and infinitely dilute and effective resonance integrals (by zone) for the

ROLAIDS 
entire problem energy range are also printed. The user has the option of selectively suppressing printed output.

An option is provided (ICOM = 6) to save the energy-pointwise flux distributions in lieu of producing a CLAROL data file on N10. In this instance, ROLAIDS joins a fission spectrum and a thermal Maxwellian to the appropriate ends of the flux distributions as follows:

\begin{tabular}{|c|c|}
\hline Energy range & Spectrum \\
\hline $20 \mathrm{MeV}$ to $\mathrm{EHI}$ & $\begin{array}{l}\chi(\mathrm{E}), \text { with a spectrum temperature of } 1.29 \mathrm{MeV} \\
\text { at } 100 \text { energies }\end{array}$ \\
\hline EHI to ELO & ROLAIDS energies and fluxes \\
\hline $\mathrm{ELO}$ to $1.0 \times 10^{5} \mathrm{eV}$ & $\begin{array}{l}\text { Maxwellian, with a spectrum temperature } \\
\text { of TK (input), at } 100 \text { energies }\end{array}$ \\
\hline
\end{tabular}

No AMPX module uses the energy-pointwise flux distributions per se when saved on N10. However, the data have been used to prepare point-to-fine-group weighting spectra for use with XLACS-2 and LAPHNGAS calculations. Format of the data is as follows:

\begin{tabular}{|c|c|}
\hline Record & Variable \\
\hline 1 & $\begin{array}{l}\text { 1. } \mathrm{IZM} \text { - number of zones } \\
\text { 2. } \mathrm{NB} \text { - number of energies }\end{array}$ \\
\hline $2, \mathrm{NB}+1$ & $\begin{array}{l}\text { 1. E- energy (eV) } \\
\text { 2. PHI(IZM) - fluxes, zone } 1 \\
\left.\text { through IZM (neutrons } / \mathrm{cm}^{2} \cdot \mathrm{s} \cdot \mathrm{eV}\right)\end{array}$ \\
\hline
\end{tabular}

Provision has been made to generate a problem restart file on an output device for problems that run longer than an input time estimate (TE). When CPU time used equals TE, ROLAIDS "dumps" pertinent data onto N10. To continue the calculation, the ROLAIDS data files N7 and N10 from the first case are remounted, a value of -2 is entered for IPRB, and the ROLAIDS case is resubmitted to continue. To use the restart feature, the ROLAIDS calculation must have passed the point where the phrase "DATA TAPE COMPLETE" is written on the case printout. Execution time required to prepare the ROLAIDS data file is not included in the time-check algorithm. Therefore, the user should have his job request time exceed his restart time estimate by at least the estimated time required to create a ROLAIDS data file (see above).

\section{ROLAIDS.1 ROLAIDS Input Data}

\section{Block 1.}

$-1 \$$ Core Assignment [1]

1. NWORD - Number of words to allocate to ROLAIDS (200000) 
0\$ Array [10]

1-10. Logical number of devices Ni-N10

See Sect. 3.ROLAIDS.2, ROLAIDS Input/Output Specifications

$1 \$$ Array [6]

1. IGE - Problem geometry

0 - homogeneous

1 - slab

2 - cylinder

3 - sphere

2. IZM - Number of zones for the integral transport calculation

3. IM - Number of spatial intervals

4. IBL - Boundary condition at the left-hand boundary of the system 0 - vacuum (slab geometry only)

1 - isotropic reflection (always for cylinders and slabs)

2 - periodic (slab geometry only)

5. IBR - Boundary condition at the right-hand boundary of the system

0 - vacuum

1 - isotropic reflection

2 - periodic (only when $\mathrm{IBL}=2$ )

6. IPRB - Problem type

1 - create a ROLAIDS data file and perform a slowing-down calculation

0 - create a ROLAIDS data file

-1 - use a previously made ROLAIDS data file and perform a slowing-down calculation

-2 - use a previously made ROLAIDS data file and a problem restart file and perform a slowing-down calculation

$2 \$$ Array [8]

1. NDF1 - Number of nuclides to be picked from NPTXS library on device N1

2. NDF2 - Number of nuclides to be picked from NPTXS library on device N2

3. NMAST - Not used, enter zero

4. NFLAT - Number of nonresonance nuclides treated with specified flat scattering and $1 / \mathrm{v}$ absorption cross sections (if NFLAT $>0$, the $16^{* *}-19^{* *}$ arrays are required)

5. MXX - Number of compositions used in the problem mockup

6. MS - Number of entries in the mixing table which specify the makeup of the MXX compositions

7. ICON - Print control for weighted 1 -D cross sections (e.g., ICON $=0$ triggers the printing of both superzone and zone-weighted 1-D cross sections)

7 - superzone

ROLAIDS 


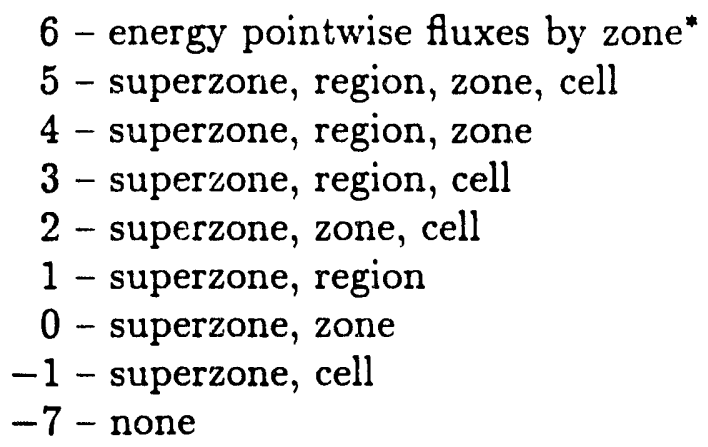

8. MASTU - Unit number of the AMPX master library. ROLAIDS will search the library energy group structure for boundaries between EHI and ELO, thus determining the number of resonance groups (28)

$3 \$$ Array [2]

1. ISCT - The $P_{N}$-scattering cross-section expansion order $(-1)^{\dagger}$

2. $\mathrm{MM}-$ Not used in this version. Enter unity

$4 *$ Array [5]

1. ELO - Minimum problem energy (eV)

2. EHI - Maximum problem energy $(\mathrm{eV})$

3. EZP - Magnitude of exponent of assumed flux shape above EHI (1.0)

4. TK - Temperature (K) for thermal Maxwellian, used only if ICON $=6$

5. TE - Time estimate (CPU min) corresponding to the requested time for the job. Used for terminating a slowing-down calculation and generating a restart file. Used only if $\mathrm{TE}>0$

T Terminate Block 1.

\section{Block 2.}

$5^{*}$ Array $[\mathrm{IM}+1]$

Mesh interval boundaries from left to right $(\mathrm{cm})$

$6 \$$ Array [IM]

Zone numbers by interval

$7 \$$ Array [IZM]

Mixture numbers by zone

9* Array $[\mathrm{NMT}=\mathrm{NDF} 1+\mathrm{NDF} 2+\mathrm{NFLAT}]$

${ }^{*} \mathrm{ICON}=6$ produces the same printed cross sections as ICON $=5$. In addition, the pointwise fluxes by zone are written on the output file (N10). An update file for CLAROL is not produced when $\mathrm{ICON}=6$.

${ }^{\dagger}$ ISCT $=-1$ bypasses the calculation of multigroup transfer arrays; thus, a CLAROL data file is not generated. For a "typical" ROLAIDS calculation, ISCT $\geq 0$. ROLAIDS uses the elasticscattering cross-section data from the NPTXS-produced point library to prepare the transfer arrays. 
Temperature of each nuclide $(K)$. These values are checked against those on libraries N1 and N2. This check provides the capability of using NPTXS data for a single ENDF MAT number at more than one temperature.

$11 \$$ Array [NMT]

Nuclide identification numbers in order of NDF1, NDF2, NFLAT. These may have any order within NDF1 and NDF2. NFLAT nuclide ID numbers must be specified in the same sequence with which the mass numbers are input in the $16^{* *}$ array.

$12 \$$ Array [NMT]

These are in one-to-one correspondence with the $11 \$$ array and give the identifier of the nuclide on the AMPX master library, if it is different from that on the point library.

$13 \$$ Array [MS]

Mixture numbers in mixing table ranging from one to MXX

$14 \$$ Array [MS]

Nuclide identification numbers in the mixing table

15* Array [MS]

Nuclide atom densities (atoms/b-cm) in the mixing table

16* Array [NFLAT] Entries

Atomic weights for the NFLAT nuclides (e.g., example values for oxygen and flourine would be $16.0,19.0$ )

17* Array [NFLAT] Entries

Constant microscopic scattering cross sections (b) for the NFLAT nuclides (e.g., $3.75,3.6)$

18* Array [NFLAT] Entries

Thermal $(0.0253 \mathrm{eV})$ fission cross sections (b) for the NFLAT nuclides (e.g., 2E-4, 9E-3)

19* Array [NFLAT] Entries

Thermal $(0.0253 \mathrm{eV})$ fission cross sections $(\mathrm{b})$ for the NFLAT nuclides (e.g., 0.0, 0.0)

T Terminate Block 2.

\section{ROLAIDS.2 ROLAIDS Input/Output Specifications}

ROLAIDS generally requires the following input/output devices: 
Logical No.

Purpose

N1 (31) First NPTXS library (required if IPRB $\geq 0$, and NDF1 $>0$ )

N2 (32) Second NPTXS library (required if IPRB $\geq 0$, and NDF2 $>0$ )

N3 (16) Scratch device (required if IPRB $\geq 0$ )

N4 Scratch device. Default logical number depends on IPRB as follows:

Default

IPRB Logical No.

$\begin{array}{ll}\geq 0 & 18 \\ <0 & 29\end{array}$

N5 (5) Card input device

N6 (6) Printed output device

N7 (29) ROLAIDS data file (note that this file is written if IPRB $\geq 0$, and is read if IPRB <0)

N8 (17) Scratch device (required if ICON $=6$, and IPRB $<0$ )

N9 (9) Direct access scratch device (required if either IPRB $\geq 0$ or ISCT $\geq$ $0)$

N10 (26) Requirement for and use of N10 depends on IPRB and ICON as follows:

$\frac{\text { IPRB }}{0} \cdot \frac{\text { ICON }}{-} \frac{\text { Use }}{\text { Device required for possible restart file }}$

$\neq 0 \quad \neq 6 \quad$ CLAROL data file

$\neq 0 \quad 6 \quad$ Energy-pointwise flux distribution file

MASTU (28) Existing AMPX master library

\section{ROLAIDS.3 ROLAIDS Error Messages}

ROLAIDS makes various checks during problem execution and terminates jobs for inconsistent data or insufficient storage. Printed error messages bear a close resemblance to the following.

1. INPUT DATA NOT IN REQUIRED ORDER.

2. PERIODIC BOUNDARY CONDITION IS ONLY VALID FOR SLAB GEOMETRY.

3. SEASON COULD NOT DETERMINE ZONE BOUNDARIES FROM INPUT DATA.

4. PLUCK COULD NOT FIND NUCLIDE $\underline{X}$, AT TEMPERATURE $\underline{Y}$, ON TAPE NO. $\underline{\mathrm{N}}$.

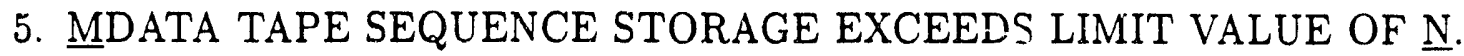


is M POINTWISE ARRAY EXCEEDS LMDUM VALUE OF $\underline{\mathrm{N}}$.

7. N ENERGY POINTS EXCEEDED LIME AT NUCLIDE $\underline{I}, M T=\underline{M}$.

8. ATTEMPT TO WRITE A NEW DATA TAPE WITH NO NUCLIDE FROM ENDF1 SPECIFIED.

9. M DOWN-SCATTERING SEQUENCE STORAGE EXCEEDS LIMIT VALUE OF $\underline{N}$, DUM DIMENSIONED AT $\underline{N 1}$.

10. X EV MAX PROBLEM ENERGY EXCEEDS MAX NDATA ENERGY OF $\underline{Y}$.

11. $\underline{X}$ EV MIN PROBLEM ENERGY LESS THAN MIN NDATA ENERGY OF $\underline{Y}$.

12. POSITION I NUCLIDE, TAPE ID $\underline{N}$ DISAGREES WITH PROBLEM ID $\underline{\mathrm{M}}$.

13. NUMBER OF NDATA NUCLIDES $\underline{\mathbf{N}}$, DISAGREES WITH NUMBER OF PROBLEM NUCLIDES $\underline{\mathrm{M}}$.

14. $\underline{X}$ EV VALUE OF EHI NOT ON NDATA.

15. BOUNDARY OF FINE GROUP $\underline{I}, \mathrm{BEG}=\underline{\mathrm{X}}$ NOT INCLUDED ON NDATA

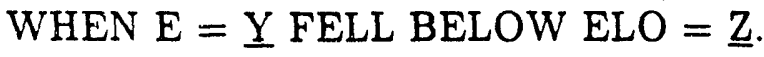

16. $\underline{\mathrm{M}}$ OUTPUT SEQUENCE STORAGE EXCEEDS LIMIT VALUE OF $\underline{\mathrm{N}}$.

\section{REFERENCES}

1. N. M. Greene et al., AMPX: A Modular Code System for Generating Coupled Multigroup Neutron-Gamma Libraries from ENDF/B, ORNL/TM-3706, Union Carbide Corp., Nucl. Div., Oak Ridge Natl. Lab., March 1976; see Sect. 8.0, "XSDRNPM: AMPX Module with One-Dimensional $S_{n}$ Capability for Spatial Weighting."

2. N. M. Greene and C. W. Craven, Jr., XSDRNPM: A Discrete Ordinates Spectral Averaging Code, ORNL-TM-2500, Union Carbide Corp., Nucl. Div., Oak Ridge Natl. Lab., July 1969.

3. R. M. Westfall, Theory and Validation of ROLAIDS: AMPX Module for Treating Resonance Shielding in Multiregion Geometries, ORNL/CSD/TM-63, Martin Marietta Energy Systems, Inc., Oak Ridge Natl. Lab. (to be published).

ROLAIDS 


\section{RUFFLES - MODULE TO CHANGE THE ENERGY GROUP STRUCTURE FOR AN ANISN OR XSDRNPM SCALAR FLUX TAPE}

RUFFLES (Regroup, Undulate, and Fix Fluxes in Your Library's Energy Structure) is a module to change the energy-group structure for an ANISN or XSDRNPM scalar flux tape. The output group structure is independent of the input structure. The group structures may be read from cards, or default neutron and/or gamma-ray structures from the AMPX group structure library may be used. Where an input group is split between two or more output groups, the flux is divided by assuming a constant flux in lethargy within the input group. If there are flux moments on the tape, the same assumption is applied to the moments.

\section{RUFFLES. 1 RUFFLES Input Data}

\section{Block 1.}

$0 \$$ Array [2]

1. N1 - Logical unit for input scalar flux tape (14)

2. N2 - Logical unit for output scalar flux tape (15)

$1 \$$ Array (IGE, ISCT, IM, IGM1, and IGG1 are obtained from the XSDRNPM or ANISN case that produced the flux tape on N1)

1. IGE - Geometry type: $0 / 1 / 2 / 3$ - homogeneous/slab/cylinder/sphere

2. ISCT - Order of scattering

3. IM - Number of mesh points

4. ISN - Order of $S_{n}$ quadrature

5. IGM1 - Number of neutron energy groups for input scalar flux tape:

6. IGM2 - Number of neutron energy groups for output scalar flux tape

7. IGG1 - Number of gamma-ray energy groups for input scalar flux tape

8. IGG2 - Number of gamma-ray energy groups for output scalar flux tape

$2 \$$ Array [2]

1. ITYPE - Type of scalar flux tape $0 / 1$ - ANISN/XSDRNPM (0)

2. IPRT - Print control flag: $0 / 1 / 2 / \ldots / \mathrm{N}$ - Minimum printout $/ 0$. Print plus scalar flux printout/1 print plus current. Printout/.../ (N-1) print plus Nth moment printout. (0)

$\mathrm{T}$ Terminate Block 1.

Block 2.

$4^{*}$ Array [IGM1 + 1]

Energy boundaries (high to low in eVs) of neutron group structure used in XSDRNPRM or ANISN case that produced scalar flux tape on N1. (Defaults to AMPX neutron-group-structure library.) 
5* $[$ IGM2 + 1]

Energy boundaries of output neutron group structure (defaults to AMPX neutron group structure library)

6* $[\mathrm{IGG} 1+1]$

Energy boundaries of input gamma-ray group structure (defaults to AMPX gamma-ray group structure library)

$7^{*}[\mathrm{IGG} 2+1]$

Energy boundaries of output gamma-ray group structure (defaults to AMPX gamma-ray group structure library)

T Terminate Block 2.

\section{RUFFLES.2 RUFFLES Input/Output Specifications}

RUFFLES generally requires the following input/output devices:

Logical No.

Purpose

$5 \quad$ Card input device

$6 \quad$ Printed output device

$47 \quad$ "Built-in" group structure library

8 Random access scratch

N1 (14) Input scalar flux tape

N2 (15) Output scalar flux tape 


\section{SALVAGE - AMPX MODULE TO RECOVER USABLE POR- TIONS OF MASTER LIBRARIES WHICH ARE INCOMPLETE}

There are at least two abnormal occurrences that result in the actual number of data sets contained in an AMPX master library being less than the number specified by the NNUC parameter in the first record of the library. The most prevalent occurrence is the AMPX master library tape which "goes bad" somewhere in the middle (due to age, mishandling, etc.). Another occurrence is when there is an abortion during the execution of an XLACS case (code failure, job pulled by operator, machine error, etc.).

Because of the great expense in creating a large nuclide master library, it is highly desirable to be able to "SALVAGE" any portion of the library that can be accessed. The SALVAGE ( $\underline{\text { Save }} \underline{\mathrm{A}} \underline{\text { Library }} \underline{\mathrm{V} i t c h}$ 'A $\mathbf{s}$ Gone Escew) module was written expressly for this purpose. The user first determines the number of data sets in the library which can be read (by running RADE or DIAL, for example), inputs this information to SALVAGE, and makes a new master library containing the good portion of the old library. The SALVAGE-produced master library is a complete library (i.e., the NNUC parameter, the master library table of contents, and the number of data sets contained in the library agree).

\section{SALVAGE.1 SALVAGE Input Data}

One data block (two arrays) of data is required by this module.

\section{Block 1.}

0\$ Logical Unit Assignments [2]

1. MIN - Old master library (23)

2. MOU - New master library (1)

$1 \$$ Set Selector [2]

1. NEWNUC - Number of data sets which can be copied

2. NWORD - Number of words to allocate to SALVAGE (50000)

$\mathrm{T}$ Terminate Block 1.

\section{SALVAGE.2 SALVAGE Input/Output Specifications}

SALVAGE requires the following input/output devices:

Logical No.

5

6

17

18

MIN (23)

MOU (1)
Purpose

Card input

Standard output

Scratch

Scratch

Old master

New master 


\section{SMILER - AMPX MODULE TO CONVERT NJOY GENDF FILES TO AMPX MASTER LIBRARIES}

The SMILER module (Second MILER*) was written in response to a need to circumvent inefliciencies observed in the use of the original code.

MILER ${ }^{1}$ provides a means of converting group-averaged cross sections from the $\mathrm{NJOY}^{2}$ system for use by modules written for the AMPX system. By default, NJOY writes these data in a format called the GENDF format, which is an ENDF/B-like format.

SMILER is not, in any sense, a revision of MILER. Rather, it is a response to the observation that many situations will cause MILER to require exorbitant input/output operations in order to convert between GENDF format and the AMPX master library format. SMILER uses procedures that take advantage of the current large-computer memories, which allow one to liberally use core-size allocations, as opposed to the situation which existed only a few years ago, wherein one had to shuttle data in and out of core to accommodate many problems. Because of this change in programming style, SMILER uses simpler procedures than might have been previously employed, thereby making it more compact and easier to maintain.

As with MILER, SMILER requires little input over simply specifying the GENDF files that are to be combined and converted. Like MILER, a SMILER run produces cross sections for only one nuclide. These one-nuclide master libraries can be easily collected by the AJAX (see Sect. 3.AJAX) module. SMILER accepts the BCD or binary formats of GENDF files.

Unlike MILER, SMILER produces a master interface that is compatible with the Nordheim Integral Treatment for resonance self-shielding, when these are used with the NITAWL-II module from AMPX-77 or from SCALE-4. This feature would require the addition of resonance parameters, for example, by using the UNITAB (see Sect. 3.UNITAB) module, to the master library produced by SMILER.

Note that no code which prepares an AMPX master library should include an array identified by $1452\left(\overline{\nu \sigma_{f}}\right)$ in the 1-D arrays. SMILER does not, and should never be modified to do this, as it will result in completely erroneous results, when used in some code combinations.

\section{SMILER.1 SMILER Input Data}

SMILER uses the FIDO input schemes employed in the AMPX system. It requires one block of data.

\section{Block 1.}

$-1 \$$ Core Allocation [1]

1. ICORE - Number of words of core to allocate to SMILER (300000)

$0 \$$ Logical Assignments (4)

1. MMT - Logical unit of AMPX master interface (1)

${ }^{*} \mathrm{MILER}^{1}$ is an acronym for Master Interface Library MakER. 
2. MG1 - First GENDF file (0)

3. MG2 - Second GENDF file (0)

4. MG3 - Third GENDF file (0)

(Note that because photo-only GENDF files do not strictly follow the GENDF format specifications and specify the number of photon groups in the word designated for the number of neutron groups, we have reserved MG3 as the location for this type of file. Logical units MG1 and MG2 can both contain either neutron-only or coupled neutron-gamma data. Borrowing an idea from MILER, positive values for MG1, MG2, MG3 are used for BCD files, whereas negative values are used for binary files.)

$1 \$$ Nuclide Identifier and Direct-Access File Status [2]

1. ID19 - Identifier of the set of data produced by SMILER (1)

2. N9STAT - Status of direct-access scratch file (0/1 - create new file/use existing file) (0)

N9STAT is a provision for minimizing the number of input/output operations associated with direct-access files. At least on IBM systems using standard FORTRAN features, the initialization of a new direct-access file requires two input/output operations for every block in the file. Saving and reusing this file reduces the penalty to a negligible number.

$2 \$$ NJOY/AMPX Thermal Identifier Correspondence List [100]

(Up to 50 doublets which give the NJOY identifiers for a thermal-scattering process followed by its corresponding AMPX identifier. By default, this array contains 221 1007, followed by 98 zeroes, which says to use an AMPX identifier of 1007 on the arrays which NJOY identifies with 221.)

$6 \$$ Direct-Access Storage Parameters [2]

1. NBLK - Number of blocks to initialize in the direct-access scratch file $(500)$

2. LRECL - Length of direct-access file blocks in words (1000)

$\mathrm{T}$ Terminate Block 1.

\section{SMILER.2 SMILER Logical Assignments}

SMILER uses the following $\mathrm{I} / \mathrm{O}$ assignments:

\begin{tabular}{c} 
Logical No. \\
\hline 5 \\
6 \\
9 \\
$\operatorname{MMT}(1)$ \\
$\operatorname{MG1}(0)$ \\
MG2(0) \\
MG3(0)
\end{tabular}

Purpose

Standard input

Standard output

Direct-access scratch file

AMPX master interface

First GENDF file

Second GENDF file

Third GENDF file 


\section{SMILER.3 SMILER Procedures}

Converting between different multigroup cross-section formats is a very common requirement. That it is not trivial is due to the wide variety of choices that one can make in designing a format.

The differences in GENDF and AMPX formats clearly demonstrate areas that can be different.

- There is a difference in the ordering of energy groups. Traditionally, group 1 is the highest energy group, as it is in the AMPX master interface. On a GENDF file, group 1 is the lowest energy group.

- The Legendre coefficients in scattering matrices in the AMPX master interface include the $(2 \ell+1)$ multiplier following conventions established for the ANISN and DOT programs in the mid-1960s. GENDF does not.

- The matrices for reactions that produce multiple secondary particles, such as $\mathrm{n} 2 \mathrm{n}$, contain the multiplicity on GENDF files. In AMPX, they do not.

- The units of the temperatures associated with scattering matrices are in $\mathrm{eV}$ in AMPX, versus $\mathrm{K}$ in GENDF. Definitely, Kelvin units are preferable.

- Various process identifiers for averaged cross sections need to be carefully monitored in order to interact properly with various AMPX modules. For example, the GENDF-scattering matrices for fission are identified by $\mathrm{MT}=18$, which is perfectly logical and as it should be; however, use of this identifier on the AMPX master interface would lead to undesirable results, and it is redefined to be 9018. Likewise, $M T=221, \ldots$ for thermal-scattering processes are converted to $\mathrm{MT}=1007,1008, \ldots$ to interface with the AMPX procedures.

- The fission spectrum on the GENDF file is in scattering-matrix form (a more correct form), whereas it is generally expected to be a single array on the AMPX interface.

The basic procedure in SMILER is very simple. Note that even though a GENDF file can contain many (up to the number of groups) collections of records for a process at a single temperature, these can be collected into a single record, before they are shuttled to a direct-access scratch file. Furthermore, if one chooses a procedure that constructs all of the matrices for Legendre coefficients of scattering processes in-core, prior to writing to the direct-access file, the requisite $1 / O$ operations are minimized.

As each process is finalized and written, a directory is constructed which consists of the following attributes: 
MAT - the ENDF/B MAT number

MT - the ENDF/B process identifier

$\mathrm{L}$ - the length of the record in the direct-access file

NREC - the block number in the direct-access file

TYPE - the ENDF/B "file" number

ORDER - the order of the Legendre coefficient

$\mathrm{T}$ - the temperature associated with the process

The up-to-three GENDF files are read and their contents noted in the above directory as they are written to the direct-access file.

After this, the direct-access directory contains all the information necessary to determine the structure of the AMPX master.

In a subroutine called MASTER, this structure is determined, along with performing most of the other operations necessary to create the AMPX master interface. For example, to create Bondarenko factors (which will derive from averaged cross sections as a function of $\sigma_{0}$ and temperature on the GENDF files), MASTER first finds the lowest temperature for a process. This set is denoted as the reference infinite dilution set, after which all nonzero temperatures are located and the Bondarenko factors determined by dividing the averaged values by corresponding values in the reference set.

In a subroutine called CHI1D, the fission-scattering matrix is combined with the fission cross section, the $\bar{\nu}$ value, and the flux to create a fission spectrum array appropriate for use in codes used at Oak Ridge and elsewhere.

A subroutine called YIELD calculates photon yields for $\mathrm{MT}=18$ and $\mathrm{MT}=102$ by dividing the photon cross-section production matrices by averaged cross sections for the appropriate process.

Test cases comparing SMILER to MILER clearly demonstrate the efficacy of the alternative approach. Converting a 201-neutron/42-gamma-group library for ${ }^{55} \mathrm{Mn}$ required around 70,000 I/Os with MILER. SMILER required slightly over $2000 \mathrm{I} / \mathrm{Os}$ for this case.

\section{REFERENCES}

1. G. C. Panini and A. Barbieri, MILER: A Computer Code for Interfacing NJOY/THEMIS With SCALE System, PSR-271, Radiation Shielding Information Center, Oak Ridge National Laboratory, 1989.

2. R. E. McFarlane, D. W. Muir, and R. M. Boicourt, The NJOY Nuclear Data Processing System, Volume I: User's Manual, LA-9303-M, Vol. I (ENDF-324), May 1982. 


\section{SMUG - MODULE FOR GENERATING MULTIGROUP PHOTON CROSS SECTIONS}

SMUG (Simple MUltigroup Gammas) is a revision of the MUG ${ }^{1}$ code incorporating the necessary programming changes to create a functional module in the AMPX system. It calculates multigroup photon cross sections, with transfer coefficients represented by a Legendre approximation of an arbitrary order. The scattering moments are computed from the Klein-Nishina ${ }^{2}$ equation. The photoelectric and pair-production cross sections are obtained from ENDF $/ \mathrm{B}^{3}$ Three sets of dose factors ${ }^{4}$ are built in and can be averaged over the problem group structure. The MUG report is the reference for the theory used in SMUG, and the primary programming differences are outlined below.

MUG solves the Klein-Nishina equation by treating each moment independently. In SMUG the programming is restructured to take advantage of the recursive properties of the Legendre polynomial. This reduces the impact of the order of $P_{n}$ as a factor in calculation time. Reductions by a factor of 5 to 10 have been observed.

SMUG is flexibly dimensioned, permitting efficient use of core storage. Problem size is limited only by total core storage available rather than the size of any single parameter.

SMUG produces an averaged energy absorption coefficient, denoted by MT = 1527, which has units of MeV-b.

\section{SMUG.1 SMUG Input Data}

A. Title Card (20A4)

\section{Block 1.}

$-1 \$$ Core Assignment [1]

1. NWORD - Number of words to allocate to SMUG (3500)

$0 \$ \$$ Logical Assignments [3]

1. NEWS - Master library (26)

2. N14 - Scratch file (14)

3. N15 - Scratch file (15)

$1 \$$ Array [3]

1. NGG - Number of gamma groups

2. NNUC - Number of nuclides to process

3. LMAX - Order of $P_{n}$

$2 \$$ Array [8]

Default values are set for all parameters in this array. If all of the default values are acceptable for the calculation, the array may be omitted.

1. $\mathrm{MF}=1$, Flat weighting $($ default $=1)$

$=2,1 /$ E weighting

$=6$, Arbitrary weighting spectrum to be entered in $6 \$$ and $7^{*}$ arrays 
2. $N W R=$ Number of interpolation regions used to specify the arbitrary weighting spectrum (default $=0$ )

3. NWP $=$ Number of points in the arbitrary weighting spectrum (default $=$ 0 )

4. $N U F=0$, Energy-dependent flux, $=1$, Photon number flux (default $=1$ )

5. $\mathrm{NSP}=$ Number of intervals in outer integral (default $=31$ )

6. $\mathrm{NPHI}=$ Number of intervals in inner integral (default $=51$ )

7. NDS $=$ Number of downscatters required (default $=N G G-1$ )

8. $\mathrm{NDF}=0$, No print

$=1$, Print a set of factors for the problem group structure (default $=1$ )

$=2$, Print and punch the dose factors

T Terminate Block 1.

Block 2. $\left\{6 \$\right.$ and $7^{*}$ arrays are entered only if $\left.M F=6\right\}$

$6 \$\left(2^{*} \mathrm{NWR}\right.$ entries): Interpolation table for arbitrary weighting spectrum (NBT(I),JNT(I),I=1,NWR)

$7^{*}(2 *$ NWP entries): Arbitrary weighting spectrum (per unit energy), $(E(I), W(I), I=1, N W P)$, ascending in energy. Units of energy are $\mathrm{eV}$.

8* Array (NGG + 1 entries): Gamma group energy mesh in decreasing magnitude of energy. Energies are in units of eV. Several "standard" group structures are built into AMPX and when one of these is desired this array is omitted. See Sect. 4.MISCELLANEOUS.4.2 for a list of the standard structures.

T Terminate Block 2.

The following block is repeated NNUC times (i.e., input a $10 \$$ array for each nuclide).

\section{Block 3.}

$10 \$$ Array [5]

1. LEMNO - ENDF/B material number

2. LORDER - Highest order of $P_{n}$ to be printed/punched for this nuclide $($ default $=0)$

3. ILT - Logical number of cross-section library for this material (default $=11$ )

4. $\mathrm{MODE}=0$, Indicates cross-section library is unformatted (binary) $($ default $=0)$

$=2$, Indicates cross-section library is formatted (BCD)

5. LPUN - Controls printing and punching of $P_{n}$ cross-section arrays $=0$, print, no punch

$=1$, print and punch 


$$
\begin{aligned}
& =-1, \text { no print, no punch }(\text { default }=-1) \\
& =2, \text { punch, no print }
\end{aligned}
$$

Note: Punched cards are free-form format.

T Terminate Block 3.

\section{SMUG.2 SMUG Input/Output Specifications}

SMUG requires the following input/output devices during an execution:

\begin{tabular}{cll} 
Logical No. & \multicolumn{1}{c}{ Purpose } \\
\cline { 1 - 1 } 5 & & Input \\
6 & & Printed output \\
7 & Punched card output \\
14 & Scratch \\
26 & Scratch \\
& AMPX interface which contains \\
ILT (supplied by user & Cross-section library \\
in $10 \$$ array of input) &
\end{tabular}

\section{SMUG.3 SMUG Error Messages}

When errors are encountered which SMUG cannot overcome, one of the following messages will be printed and the control returned to the AMPX DRIVER.

Message: CORE USED XXXXXXXX LIMIT XXXXXXXX

Detecting Routine: SMUG

Cause: Inadequate space available. Reduce problem complexity or increase space allotted in SMUG main program.

Message: MATERIAL XXXXXX NOT ON ENDF TAPE

Detecting Routine: FINDXS

Cause: Self-explanatory. Check input data and contents of ENDF library requested.

Message: FILE 23 OF MATERIAL XXXXXX NOT ON ENDF TAPE

Detecting Routine: FINDXS

Cause: Self-explanatory. Check contents of ENDF library requested.

Message: SECTION XXXX OF FILE 23 OF MATERIAL XXXXXX NOT ON ENDF TAPE

Detecting Routine: FINDXS

Cause: SMUG expects to find reaction types 501, 502, 504, 516, 602 in file 23. The calculation cannot be performed if these data are not available. 
Message: STOP IN FINDXS, NRI, XXXXXX NPI XXXXXX

Detecting Routine: FINDXS

Cause: Programming error; ENDF data more voluminous than anticipated. Programming changes are required in subroutines SMUG and FINDXS.

Message: DATA FOR MATERIAL XXXXXX SECTION XXX ARE TEMPERATURE DEPENDENT

Detecting Routine: FINDXS

Cause: This version of SMUG does not accept temperature-dependent file 23 data.

Message: ENERGY ARRAY OUT OF SEQUENCE MATERIAL XXXXXX SECTION XXXX

Detecting Routine: FINDXS

Cause: Energies not in increasing order; check ENDF data.

\section{SMUG.4 SMUG Warning Messages}

When data are not defined by ENDF to cover the entire energy range required by the problem, SMUG defines the cross sections in the undefined range as zero, prints a warning message, and continues the calculation. If any of these warnings should occur, the user must assess the validity of the calculation.

\section{REFERENCES}

1. J. R. Knight and F. R. Mynatt, MUG: A Program for Generating Multigroup Photon Cross Sections, CTC-17, Union Carbide Corp., Nucl. Div., Oak Ridge Gaseous Diffusion Plant, January 1970.

2. R. D. Evans, The Atomic Nucleus,Chapter 23, McGraw-Hill, 1555.

3. D. J. Dudziak, ENDF Formats and Procedures for Photon Production and Interaction Data, LA-4549 (ENDF-102), Rev. Vol. II, Los Alamos Scientific Lab., July 1971.

4. B. J. Henderson, Conversion of Neutron or Gamma Ray Flux to Absorbed Dose Rate, XDC-59-8-179, General Electric Co., Cincinnati, Ohio, August 1959. 


\section{TABU - AMPX MODULE TO CREATE BONDARENKO FACTORS FOR THE UNRESOLVED RESONANCE ENERGY REGION IN ENDF/B}

TABU (Tailor AMPX Bondarenko Factors for the Unresolved Region) is a module that is intended to prepare an AMPX master cross-section file which only contains Bondarenko factor data for the unresolved energy regions of ENDF/B nuclides. Because the unresolved energy range in ENDF/B can vary from nuclide to nuclide, the same sort of generality is required for TABU and, of course, the formats of the AMPX master file.

TABU does not process the ENDF/B files directly, but assumes that these files have been preprocessed into point "average" values by some other code, such as PRUDE. These average values are expected to be available in ENDF/B TAB1 formats, separated out according to MAT (material), MT (process), T (temperature), and SIG0 (sigma-0, the "background" cross section associated with Bondarenko data).

In order to convert the point data to group-averaged form, TABU requires a smooth weighting spectrum. This can be supplied in the same TAB1 formats as used for the cross-section data on logical MWT, though it is probably reasonable to use the default $1 / E$ weighting in the unresolved region.

Input to TABU is particularly brief and requires little knowledge on the part of the user as to details of the ENDF/B unresolved data or on how many processes, temperatures, sigma-0's, etc., went into the point file.

\section{TABU.1 TABU Input Data}

\section{Block 1.}

$-1 \$$ Core Assignment [1]

1. LLLL - Number of words of core allocated to this module (50000)

0\$ Logical Unit Assignments [3]

1. MMT - Logical unit number on which the master file will be written (default $=1$ )

2. MXS - Logical unit number containing point cross sections (default $=31$ )

3. MWT - Logical unit number containing weighting spectra (for $1 / \mathrm{E}$ weighting spectrum, input MWT $=0$ along with $\mathrm{MATW}=99, \mathrm{MFW}=3$, and $\mathrm{MTW}=1$ in $11 \$ \$$ array $)($ default $=0)$

$1 \$$ Problem Size [3]

1. NNUC - Number of materials which will be processed

2. IGM - Number of energy groups

3. IFTG - First thermal group 
$2 \$ \$$ Options [10]

1. IOPT1 - 0 - Cross sections are calculated in PRUDE and input on logical MXS

$\mathrm{N}$ - Cross sections are from the UNRESR module of NJOY and are input on logical $\mathrm{N}$

2. IOPT2 - Not used

4. IOPT4 $-0 / 1-$ do not/do print energy-group boundaries $($ default $=0)$

10. IOPT10 - Not used

$\mathrm{T}$ Terminate Block 1.

\section{Block 2.}

7* Energy Group Boundaries (high to low in eV)

(Omission of this input will select a standard AMPX structure.)

T Terminate Block 2.

\section{Block 3. \{This block will be repeated NNUC times.\}}

Title Card for the Material

$10 \$$ Material Designation [1]

1. MATN - ENDF/B material number (i.e., MAT number)

$11 \$$ Smooth Weighting Function [3]

1. MATW - MAT number of weighting spectrum (default $=99)$

2. MFW - MF number of weighting spectrum (default = 3)

3. MTW - MT number of weighting spectrum (default $=1$ ) (for $1 / \mathrm{E}$ weighting spectrum, these three parameters must be 99,3 , and 1 , respectively)

T Terminate Block 3.

\section{TABU.2 TABU Input/Output Specifications}

TABU requires the following logical unit assignments:

Logical No.

5

6

MMT (1)

MXS (31)

MWT (46)

17

18
Purpose

Standard input

Standard output

AMPX master file written here

Point cross-section file

Smooth weighting spectra file

Scratch file

Scratch file 


\section{TIDE - MODULE TO CORRECT AN AMPX MASTER LIBRARY DIRECTORY}

TIDE (TIdy AMPX Master Library Directories Easily) is a module to correct the table of contents of an AMPX master library, including an accounting of the number of records for each nuclide. The input consists of specifying the logical unit number that the master library resides on. Optionally, two scratch units may be overridden.

\section{TIDE.1 TIDE Input Data}

\section{Block 1.}

$1 \$$ Master Logical Unit [1]

1. Logical unit number of the master library

$2 \$$ Other Input Iterns [3]

1. NS - Logical unit number of the first scratch unit (default $=18$ )

2. NT - Logical unit number of the second scratch unit (default $=19)$

3. NWORL - Number of words to allocate to TIDE (100000)

$\mathrm{T}$ Terminate Block 1.

\section{TIDE.2 TIDE Input/Output Specifications}

The following are typical I/O assignments in TIDE:

\begin{tabular}{c} 
Logical No. \\
\hline 5 \\
6 \\
18 \\
19 \\
NT1
\end{tabular}

\begin{tabular}{l}
\multicolumn{1}{c}{ Purpose } \\
\hline Card input \\
Standard output \\
Scratch unit \\
Scratch unit \\
Master library
\end{tabular}




\section{UNITAB - AMPX MODULE TO MERGE SELECTED PORTIONS OF MASTER LIBRARIES INTO HYBRID MASTER SETS}

UNITAB (UNITe Anything Briskly) is provided to allow merging data at the cross-section-set level from up to 12 different sources (master libraries). UNITAB is so general that it practically allows constructing any sort of "hybrid set," so long as energy-group structures match. For example, one can use it as CHOX is presently used, where neutron, gamma-ray interaction, and gamma-ray production data are input on separate libraries and end up in a single library, or, more interestingly, the coupled neutron-gamma library can be split apart into its separate components. Better yet, selected individual components of the data can be placed into a new data set. For example, the new data set can have resonance data (Bondarenko factors, etc.) from one data set, 1-D data from another, elastic transfer data from another, etc. A user can create a mess if he/she is not careful.

\section{UNITAB.1 UNITAB Input Data}

\section{Block 1.}

$-1 \$$ Core Assignment [1]

1. NWORD - Number of words to allocate to UNITAB (100000)

$0 \$$ Logical Assignments [16]

1. INEW - New master library (1)

2. MSC - Scratch device (18)

3. MDA - Direct-access scratch (9)

4. MDB - Direct-access scratch (8)

5. MM1 - First master library (23)

6. MM2 - Second master library (24)

7. MM3 - Third master library (26)

8. MM4 - Fourth master library (0)

9. MM5 - Fifth master library (0)

10. MM6 - Sixth master library (0)

11. MM7 - Seventh master library (0)

12. MM8 - Eighth master library (0)

13. MM9 - Ninth master library (0)

14. MMA - Tenth master library (0)

15. MMB - Eleventh master library (0)

16. MMC - Twelfth master library (0)

$1 \$$ Coupling Information [2]

1. NNNC - Number of sets to be created

2. NCOM - Number of "coupling" commands 
$6 \$$ Direct-Access Parameters for MDA * [2]

1. NBLK - Number of direct-access blocks (200)

2. LREC - Length of direct-access blocks (880)

T Terminate Block 1 .

\section{Block 2.}

This block contains one array of information (the coupling commands) which tells how the data are to be combined.

\section{$2 \$$ Coupling Commands (NCOM*4)}

The "commands" are in groups of four entries, stacked one after the other. The entries are

1. Identifier of the data set on the new rnaster library

2. Logical number of the device containing data wanted in the new master data set

3. Identifier of the master data set on the old library containing the desired data

4. "Type" of data desired (allowed types are specified below):

\begin{tabular}{c} 
"Type" selectors \\
\hline 1111 \\
2222 \\
3333 \\
4444 \\
5555 \\
6666 \\
7777 \\
$\mathrm{MT}$ \\
$-\mathrm{MT}$
\end{tabular}

Function

All neutron data
All gamma-ray production data
All gamma-ray interaction data
Bondarenko data
Nordheim resonance data
1-D neutron data
1-D gamma data
Selects transfer matrix for process MT
When used in conjunction with a
select all option, allows excluding
transfer matrix for process MT

Note that individual portions of the "all" selectors can be overridden with the other selection options. The selection of a specific component (e.g., Bondarenko data) takes precedence over a global request.

T Terminate Block 2.

The title of the new set is determined in a hierarchal manner. It is the title from the set containing the first neutron data request; or, if none, it is the title from the set containing the first gamma request; or, if still none, it is the title from the first gamma production request.

"The new master library is "built" on MDA prior to finally being placed on NEW. Therefore, NBLK $\times$ LREC must be $\geq$ the number of words in the new master library. 


\section{UNITAB.2 UNITAB Input/Output Specifications}

The following input/output specifications are required by UNITAB.

$\frac{\text { Logical No. }}{5}$
6
$\operatorname{MDB}(8)$
$\operatorname{MDA}(9)$
$\operatorname{MSC}(18)$
NEW(1)
MM1(23)
MM2(24)
MM3(0)
MM4(0)
$\cdot$
$\cdot$
MMC $(0)$

\begin{tabular}{l}
\multicolumn{1}{c}{ Purpose } \\
\hline Card input \\
Standard output \\
Direct-access scratch \\
Direct-access scratch \\
Scratch space \\
New master library \\
First existing master library \\
Second existing master library \\
Third existing master library, if any \\
Fourth existing master library, if any \\
$\qquad \cdot$ \\
. \\
Twelfth existing master library, if any
\end{tabular}




\section{VASELINE - AMPX MODULE TO PLOT ENDF POINT AND AMPX GROUP-AVERAGED CROSS SECTIONS}

VASELINE (Visual Aids SELected IN Ecstasy) is a plotting module for AMPX. It is written to plot point-versus-point data, point-versus-multigroup data, or multigroupversus-multigroup data. Any ENDF array from files 3, 13, or 23 can be plotted as can any 1-D group-averaged neutron or gamma cross sections on a master or working/weighted cross-section interface. Selections can be made from any combination of ENDF or multigroup sources. The number of curves on any plot is arbitrary.

\section{VASELINE.1 VASELINE Input Data}

\section{Block 1.}

$-1 \$$ Core Assignment [1]

1. ICORE - Number of words to allocate to VASELINE (100000)

$1 \$$ Plot Selection

1. MPLOT - Number of plot commands given below

2. IPLOT* -What to plot

$$
\begin{aligned}
& 0 \text { - points only } \\
& 1 \text { - lines between points } \\
& 2 \text { - points and lines } \\
& 3 \text { - histogram }
\end{aligned}
$$

$2 \$$ Plot Options [10]

1. IOPT1 - Plot type (default $=4)$

$$
\begin{aligned}
& 1 \text { - linear-linear } \\
& 2 \text { - x log - y linear } \\
& 3 \text { - x linear - y log } \\
& 4 \text { - log-log }
\end{aligned}
$$

2. IOPT2 - Number of special plot titles (default $=0$ )

3. IOPT3 - Number of special ordinate labels (default $=0$ )

4. IOPT4 - Not used

5. IOPT5 - Not used

6. IOPT6 - Not used

7. IOPT7 - If greater than 0 , plots will be subtitled with an identifier selected by the MT of the first executed command for that plot

8. IOPT8 - Not used

9. IOPT9 - Points that are plotted will be printed, if greater than 0

10. IOPT10 - Not used

T Terminate Block 1.

\footnotetext{
* This parameter applies to data taken from ENDF libraries only.
} 


\section{Block 2.}

This block of three arrays will be repeated MPLOT times.

$3 \$$ Identifiers of Data to be Plotted [5]

1. NPLOT - Plot number on which the data selected by this block will be plotted

2. MT - Process to be plotted. ENDF process identifiers are employed where applicable

3. IDM - The identification number of a set of data on an AMPX master interface which contains the "MT" data. A zero indicates that this comr 'and does not select from a master interface.

4. IDW - The identification number of a set of data on an AMPX working interface which contains the "MT" data. A zero indicates that this command does not select from a working interface.

5. IDE - The ENDF "MAT" number for the set of data which contains "MT"

$4 \$$ Library Selectors [5]

Omit this array if default values are acceptable.

1. MMTN - Logical number of AMPX master interface (default =1)

2. MWTN - Logical number of AMPX working interface (default $=4)$

3. MENDF - Logical number of the ENDF library (default $=11$ )

4. MODE - Tape mode of MENDF (default = 1)

1 - binary

$2-\mathrm{BCD}$

5. MFILE - File number containing ENDF data (default $=3$ )

(Note that data can only be plotted from files 3,13 , and 23 in the present version of VASELINE.)

5* Plot Range [2]

1. ELO - Low-energy limit of plot $(\mathrm{eV})$

2. EHI - High-energy limit of plot $(\mathrm{eV})$

This array can be omitted, in which case the range is automatically determined to be the full range of the data selected.

T Terminate Block 2.

Special plot titles [IOPT2] (I6,A52)

1. IPLOT - The plot number for which this title is to be used

2. PTITL - The plot title (52 characters)

Special labels [IOPT3] (I6,A36/6X,A36)

1. IPLOT - The plot number for which this label is to be used

2. OLABL - The $x$-axis label ( 36 characters)

3. ALABL - The $y$-axis label (36 characters) 


\section{VASELINE.2 VASELINE Input/Output Specifications}

VASELINE generally requires the following I/O assignments:

\begin{tabular}{c} 
Logical No. \\
\hline 5 \\
6 \\
18 \\
MMTN(1) \\
MWTN $(4)$ \\
MENDF(1i) \\
70 \\
71 \\
72 \\
73 \\
74 \\
75
\end{tabular}

\begin{tabular}{l}
\multicolumn{1}{c}{ Purpose } \\
\hline Card input \\
Standard output \\
Scratch device \\
1MPX master interface \\
AMPX working interface \\
ENDF library \\
DISSPLA DVSD file \\
DISSPLA VSCR file \\
DISSPLA POPF file \\
DISSPLA ISC1 file \\
DISSPLA ISC2 file \\
DISSPLA ISC3 file
\end{tabular}

Note that the DISSPLA files are required, depending on the particular situation. Logical 72 is the metafile and will always be required, since this is the file which will be passed to a postprocessing routine that actually produces the plots. In our experience with the present version of DISSPLA (version 10.5), the other files have not been required by VASELINE and need not be allocated. 


\section{VEL - A MODULE FOR GROUP-AVERAGING POINT DATA}

VEL (VEctor Libraries) is a module for producing group-averaged cross sections from point cross-section data strings. It expects its point cross sections to be input in the library formats such as are written by NPTXS (module to process ENDF/B files 2 and 3 data into point data) or CREST (module to collect together point data from several ENDF/B libraries, AMPX master or working libraries), or CASTROL (module to create a binary point library from card input). The weighting spectrum used in VEL is also expected to be input in the same format as the point data. The JERGENS module contains a wide variety of options for creating weighting spectra and outputs its data in this format. The output from VEL is a master library containing the group-averaged data. Either neutron or gamma data can be processed.

\section{VEL.1 VEL Imput Data}

\section{Block 1.}

$0 \$$ Logical Assignments [3]

1. MMT - Master library is written here (1)

2. MXS - Point data are input here (31)

3. MWT - Weighting spectra are input here (46)

$1 \$$ General Case Data [5]

1. NC - Number of weighting commands specified in Block 3

2. IGM - Number of neutron energy groups

3. IPM - Number of gamma energy groups

4. ICORE - Number of words of core to use (50000)

5. IS - Not used at present

T Terminate Block 1.

\section{Block 2. (Energy Boundaries)}

7* Neutron Energy Group Boundaries - high to low in eV (IGM + 1)

8* Gamma Energy Group Boundaries - high to low in eV (IPM + 1) (The $7^{*}$ and $8^{*}$ arrays can be omitted if standard AMPX group structures are being used; however, the " $T$ " which ends Block 2 must be specified.)

T Terminate Block 2.

\section{Block 3. (Weighting Commands)}

This block is repeated NC times. One "command" is required for each process to be weighted, and specifies the identifier on the master library under which several groupaveraged functions may be collected and the identifiers of process and the weighting spectrum. 
$10 \$$ Process and Set Description [3]

1. ID19 - The identifier on the master library for the set containing the process

2. NP - The identifier of the process on the master library

3. NORG- Not used

$11 \$$ Point Data and Weighting Spectrum Selection Parameters [6]

1. MATX - The "MAT" number of the point data to be read from logical MXS

2. MTX - The "MT" number of the point data

3. MFX - The "MF" number (normally 3) of the point data

4. MATW - The "MAT" number of the weighting spectrum to be read from logical MWT

5. MTW - The "MT" number of the weighting spectrum

6. MFW - The "MF" number (normally 3) of the weighting spectrum

$12 \$$ Multigroup Weighting Spectrum Identifier for the Set of Data Identified by ID19 [1]

1. NPW - The process identifier for the group-integrated weighting spectrum values (normally 1099). (Note that the $12 \$$ array should be used in only one of the commands that describe processes to be collected under the identifier, ID19. Multiple entries will cause the weighting spectrum to be included that number of times on the master library.)

T Terminate Block 3.

This is the end of the data input typically required for VEL; however, in the event you wish to supply a title for any of the sets of cross sections VEL produces, you can include them at this point in an (I12/18A4) format, where you put the set identifier (ID19) right-justified in the first 12 characters of one line, followed by a line containing the new title. Stack as many of these two-line overrides as you wish.

\section{VEL.2 VEL Input/Output Specifications}

The following devices are required by VEL:

Logical No.

5

6

7

8

$\operatorname{MMT}(1)$

$\operatorname{MXS}(31)$

$\operatorname{MWT}(46)$
Purpose

Standard input

Standard output

Punched output

Direct-access scratch space

Master interface written here

Point cross-section library

Weighting function library 


\section{WAX - MODULE TO MERGE, COLLECT, ASSEMBLE, REOR.DER, JOIN, COPY SELECTED NUCLIDES FROM AMPX WORKING INTERFACES}

WAX (Working Library AJ $\underline{\mathrm{AX}}$ ) is a module to combine data on AMPX working libraries. Options are provided to allow merging from any number of files in a manner that will allow the user to determine the final nuclide ordering, if desired. WAX is equivalent to the AJAX module, except that it deals with working libraries instead of master libraries.

\section{WAX.1 WAX Input Data}

\section{Block 1.}

$-1 \$$ Core Assignment [1]

1.NWORD - Number of words to allocate to WAX (50000)

$0 \$$ Logical Assignments [2]

1. MWT - Logical number of new library (default $=1$ )

2. NWAX - Not used

$1 \$$ Number of Files [1]

1. NFILE - Number of files from which data will be selected

T Terminate Block 1.

Blocks 2 and 3 are stacked, one after the other, NFILE times.

\section{Block 2.}

$2 \$$ File and Option Selection [2]

1. NF - Logical number of file considered

2. IOPT $^{*}=-\mathrm{N}$ Delete $\mathrm{N}$ nuclides from NF to create the new file on MWT $=0$ Add all nuclides to the new file on MWT

$=\mathrm{N}$ Add $\mathrm{N}$ nuclides from $\mathrm{NF}$ to create the new file on MWT

T Terminate Block 2.

Block 3. (Enter only when IOPT $\neq 0$ )

$3 \$$ Nuclides Selected [|IOPT $\mid$ ]

Identifiers of nuclides that are to be added or deleted from NF

$4 \$$ New Identifiers [|IOPT|]

*Sets with duplicate identifiers will not be entered on MWT. The first occurrence of an identifier selects that set for the new library. 
This array allows changing the identifier given in the $3 \$$ array when it is selected for the new library.

T Terminate Block 3.

\section{WAX.2 WAX Input/Output Specifications}

The following devices are typically needed to execute WAX:

Logical No.

5

6

15

16

18

19

MWT(1)

$\mathrm{NF}$
Purpose

Card input

Standard output

Scratch device

Scratch device

Scratch device

Scratch device

Working file to be created

File(s) to be merged onto MWT 


\section{WINE - AMPX MODULE TO FORCE NORMALIZATION OF SELECTED PORTIONS OF AMPX WORKING LIBRARIES}

WINE (WorkIng NormalizEr) is a module to manipulate data in an AMPX working library. The module can create new 1-D arrays in a working data set by algebraically summing existing 1-D arrays, replace data set titles, and change the data set identification numbers. WINE performs the specified 1-D manipulations on all data sets in the input working library.

The characteristics of an AMPX working library impose limitations to data manipulations. Because a working data set has a single 2-D transfer array that cannot be separated into components by reaction type, WINE includes no provisions for manipulating data in the 2-D array. Cross sections for a specific reaction may be a separate 1-D array and may also be included in the 2-D array. Consequently, the use of WINE to manipulate 1-D data for such reactions (and the corresponding manipulation of the 1-D total cross section) should be approached with caution because there is no way to manipulate the $2-\mathrm{D}$ array.

\section{WINE.1 WINE Input Data}

\section{Block 1.}

$-1 \$$ Vector Commands [200] (Default $=$ all zeros)

Simple "vector commands" are used to specify the 1-D data manipulations. A "vector command" is composed of a primary MT number, followed by one or more secondary MT numbers, and a zero that serves as a delimiter on the command string, that is,

Primary MT number-Secondary MT numbers-Zero.

A positive primary MT number says that the 1-D array to be created is an algebraic sum of the 1-D arrays identified by the secondary MT numbers. A negative primary MT number triggers the uniform normalization of the 1-D arrays identified by the secondary MT numbers such that they sum to the reaction cross sections identified by the absolute value of the primary MT number. A negative value for a secondary MT number is taken as a directive to subtract the process.

0\$ Logical Unit Assignments and Core Assignment [3]

1. N1 - Logical unit number for existing working library (20)

2. N2 - Logical unit number for new working library (4)

3. NWORD - Number of words to allocate to WINE (50000)

$1 \$$ Option Trigger [1]

1. NTIT - Number of data set titles to be replaced (0)

$\mathrm{T}$ Terminate Block 1. 
If NTIT is zero, the WINE input is complete; otherwise, enter NTIT data block 2's. Note that the data block 2's are used to change the identification numbers and/or titles of data sets in the working library.

\section{Block 2.}

$2 \$$ ID - Identification number of data set (on N1) [1]

$3 \$$ IDNEW - New identification number of the set. If the ID number is not to be changed, omit the $3 \$$ array. [1]

4U Array - The new title card for the data set-(18A4) format. (Any time a data block 2 is entered, there must be a $4 U$ array. If the objective is to only change the data set identification number, the old title card must be entered in the $4 U$ array.) The alphanumeric data are entered as follows: $4 \mathrm{U}$

-Title Card-

\section{WINE.2 WINE Input/Output Specifications}

WINE requires the following logical unit devices:

\begin{tabular}{c} 
Logical No \\
\hline 5 \\
6 \\
N1 $(20)$ \\
N2 (4) \\
17 \\
18
\end{tabular}

\begin{tabular}{l} 
Purpose \\
\hline Standard input \\
Standard output \\
Input working library \\
Output working library \\
Scratch device \\
Scratch device
\end{tabular}




\section{WISK - A MODULE TO CREATE A SENSITIVITY LIBRARY FROM AN AMPX MASTER INTERFACE}

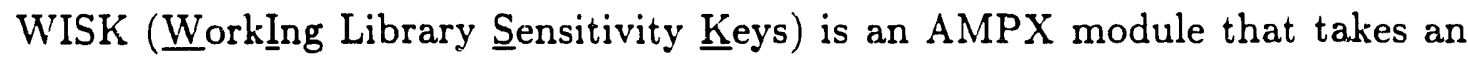
AMPX master interface and separates each process into a set of data on an AMPX working interface. Each process contains all the necessary cross-section information for cross-section sensitivity calculations. For applications requiring an ANISN-formatted library (as per NITAWL), the working library from WISK can be input to the CONTAC module, which makes the necessary format conversion.

The identifiers of the sets of data on the working library start at 1 and range to $\mathrm{N}$, where $\mathrm{N}$ is the total number of unique processes for all nuclides found on the master interface. The structure of each set is standard and generally consists of the following:

1. The title record.

2. A record with a single vector of cross sections for the process identified by the proper ENDF MT number.

3. Records containing the transfer matrices for all orders required to represent the angular variation of the process. Note that these arrays contain any "multiplicity" of secondary particles [e.g., the $P_{0}(n, 2 n)$ matrix will sum to twice the $(n, 2 n)$ reaction values].

WISK constructs "total" sets for inelastic scattering, capture, fission, absorption, and total neutron cross sections. The structure is as above except that the fission, absorption, and total cross sections contain several vectors in the 1-D array. The fission set contains $\mathrm{MT}=18, \mathrm{MT}=1018$, and no transter matrices, even though the absorption and total contain these processes, in addition to the absorption and total vectors, and can contain transfer matrices.

\section{WISK.1 WISK Input Data}

\section{Block 1.}

$-1 \$$ Core Allocation [1]

1. ICORE - Number of words to allocate to WISK (100000)

$1 \$$ Logical Unit Assignments [2]

1. MMT - Master library to be converted to sensitivity form (default $=1$ )

2. MWT - Sensitivity library in working library format (default $=4$ )

$6 \$$ Direct Access Space Parameters [4]

1. NBLK8 - Number of blocks to assign to logical unit 8 (350)

2. LBLK 8 - Length of blocks in words on logical unit $8(500)$

3. NBLK9 - Number of blocks to assign to logical unit 9 (350)

4. LBLK9 - Length of blocks in words on logical unit 9 (500)

T Terminate Block 1. 


\section{WISK.2 WISK Input/Output Specifications}

WISK requires the following input/output devices:

Logical No.

5

6

8

9

17

18

$\operatorname{MMT}(1)$

MWT(4)
Purpose

Card input

Standard output

Direct access scratch

Direct access scratch

Scratch device

Scratch device

Mater library (read)

Sensitivity library in working format (written) 


\section{WORKER - A MODULE TO CONVERT FROM AMPX MASTER FORMAT TO AMPX WORKING FORMAT}

WORKER (WORKing Library MakER) is a module that will read nuclides from an AMPX master library and write them in an AMPX working library. As such, its capabilities are similar to those of NITAWL, which also allows Nordheim Integral Treatment calculations for nuclides with resonance parameters.

WORKER does not provide the resonance calculational option. It is primarily intended for use with those libraries that use Bondarenko methods for self-shielding and do not use the Nordheim method. In addition, it allows one to linearly interpolate within thermal-scattering matrices to obtain cross sections for intermediate temperatures. This temperature interpolation is only performed within the range of temperatures on the library. Requests for values outside the range will use the end sets.

\section{WORKER.1 WORKER Input Data}

\section{Block 1.}

$-1 \$$ Core Allocation [1]

1. ICORE - Number of words to allocate to WORKER (200000)

$0 \$$ Logical Assignments [2]

This array is input only if a user needs to modify default values (shown in parentheses).

1. N1 - Input master cross-section interface (1)

2. N2 - Output working cross-section interface (4)

$1 \$$ Integer Parameters [2]

1. MMT - Number of nuclides to read from the master cross-section library mounted on $\mathrm{N} 1$.

2. IPP - Output option trigger (-2)

-2 - no cross-section edits

-1 - edit reaction cross sections

$>-1$ - edit reaction cross sections and transfer arrays through order IPP

T Terminate Block 1.

\section{Block 2.}

$2 \$$ Identification Numbers of Nuclides to be Placed on the Working Library [MMT] MMT identifications for nuclides from the master library on N1.

$3 \$$ Thermal Scattering Kernel Temperatures for Nuclides Selected (K) [MMT]

$4 \$$ MT Number of the Incoherent Thermal Scattering Kernel $[M M T]($ default $=1007)$

T Terminate Block 2. 


\section{WORKER.2 WORKER Input/Output Specifications}

WORKER typically requires the following I/O devices:

\begin{tabular}{cll} 
Logical No. & & Purpose \\
\cline { 1 - 1 } NT1 (1) & & Master cross-section library \\
NT4 (4) & & New working library \\
NT5 (18) & & Scratch unit \\
NT6 (19) & & Scratch unit \\
NT7 (9) & & Random access scratch unit \\
5 & & Card input \\
6 & & Printed output \\
97 & & Binary input
\end{tabular}




\section{WORM - AMPX MODULE TO CONVERT AN AMPX WORKING LIBRARY TO AN AMPX MASTER LIBRARY}

WORM (WORking to Master Converter) is an AMPX module to convert a binary AMPX working library into a binary AMPX master library. WORM works with any working library to produce a master library containing neutron and/or gamma and/or gamma-production information. In the case of the working library containing more than one of the above types of data, WORM automatically splits the transfer matrices such that all neutron data are carried together and identified by $\mathrm{MT}=1$, gamma production data are carried together and identified by $\mathrm{MT}=1$, and, likewise, gamma data are carried together and identified by $\mathrm{MT}=501$. One-dimensional (reaction averages) cross sections are carried on a process-by-process basis, exactly as in the master library. Only the "total" transfer matrices are available, since it is impossible to split out individual transfer processes, in a general manner, once they are added together to produce the working library.

\section{WORM.1 WORM Input Data}

\section{Block 1.}

$-1 \$$ Core Allocation [1]

1. ICORE - Number of words to allocate to WORM (50000)

$0 \$$ Logical Unit Assignments [2]

1. MMT - Master library is written on this logical unit (default $=1)$

2. MWT - Working library is mounted on this logical unit (defa:lt $=4$ )

$\mathrm{T}$ Terminate Block 1.

\section{WORM.2 WORM Input/Output Specifications}

WORM requires the following $I / O$ devices:

Logical No.

5

6

17

18

$\operatorname{MMT}(1)$

MWT(4)
Purpose

Card input

Standard output

Scratch file

Scratch file

Master file (written)

Working file (read) 


\section{XLACS - MODULE TO PRODUCE GROUP-AVERAGED NEUTRON CROSS-SECTION LIBRARIES ON AN AMPX MASTER LIBRARY}

XLACS-77 (EX $\underline{x}$ tract a Library of Averaged Cross Sections) is the latest of the $\mathrm{XLACS}^{1}$ series of programs and produces a multigroup library in the AMPX master library format by averaging point-neutron cross-section data taken from ENDF/B libraries.

XLACS-77 has several distinguishing features from its predecessors (through XLACS-II):

1. It is written in FORTRAN-77.

2. It makes use of the dynamic core allocations in AMPX which makes it easier to increase the sizes of working data arrays.

3. It contains a new thermal processing package taken from the Australian version of XLACS-II, which effectively circumvents processing problems of other versions of XLACS.

4. It does not produce data for older versions of NITAWL, thereby requiring NITAWL-II, a new and improved resonance processor.

5. It does not require external resonance processing to produce elastic scattering arrays that are used to produce elastic transfer matrices (internal schemes are provided which should perform this task as well or better than external processors).

6 . It contains a new treatment ${ }^{2}$ for generating elastic matrices for hydrogen.

7. The evaporation model processing procedures are new and much more accurate, general, and efficient than those from other versions of the program.

8. It allows for processing file 6 ENDF/B data (at present this treatment is limited to the thermal energy region).

Perhaps the most notable improvement in the new program is its increased accuracy that resulted from its use to generate cross sections for a 620-group processing codes comparison study. This led to many improvements, particularly in resonance parameter processing.

\section{XLACS.1 XLACS-77 Input Data}

As with earlier versions, the initial input to XLACS-77 consists of five title cards that are used to provide a title for its multigroup library.

Five Title Cards (80A1)

\section{Block 1.}

This data block is used to describe general data (such as the number of energy groups) for the problem.

0\$ Major Logical Assignments [3]

1. NDFBFF - Primary source of regular ENDF/B data (default =11)

2. NDFBTT - Not used 
3. MASTER - Master library (default $=23$ )

$1 \$$ Major Problem Parameters [3]

1. NUMNUC - Number of nuclides processed by this run

2. NEGF - Total number of energy groups

3. NEGT - Number of thermal energy groups (These are the bottom groups and upscatter extends to the first of these.)

$2 \$$ Working Array Sizes [3]

1. MAXN1 - Maximum number of interpolation regions allowed in a TAB1 function (default $=50$ )

2. MAXN2 - Maximum number of points allowed in a TAB1 function (default $=5000$ )

3. MAXA - Number of words to allocate to XLACS (default $=150000)$

3\$\$ Special Calculational Options [25]

1. IFORCE(1) - MLBW calculation option (0/1 - treat as specified/force MLBW even when SLBW is specified in the ENDF file) (default $=0$ )

25. IFORCE (25) - Not used

T Terminate Block 1.

\section{Block 2.}

This data block is given one time for a problem and contains the energy group boundaries. The " $T$ " which ends this block must be specified, even when one of the default group structures is selected.

$7^{*}$ Energy Group Boundaries (NEGF + 1)

Specify the energy group boundaries in $\mathrm{eV}$ (high to low). If the group structure is a standard AMPX set, do not input the array. See Sect. 4.MISCELLANEOUS.4.1 for standard neutron group structures.

$\mathrm{T}$ Terminate Block 2.

The following two data blocks are to be repeated NUMNUC times.

\section{Block 3.}

This data block selects the ENDF/B materials and options to be used in producing multigroup cross sections.

Title Card for the Nuclide (18A4). This card must always be present and must always be the first card in block 3 .

$10 \$$ Nuclide Selection and Identification [3]

1. ID19 - Identifier of the multigroup set of data on the master library. 
2. MATF - ENDF/B material identifier for the data for fast and epithermal cross sections.

3. MATT - ENDF/B material identifier for therma-scattering low data (or File 6 thermal data) to be used in the thermal range (This is specified as zero when free gas thermal values are desired.)

$11 \$$ Weighting Function Selection Parameters [4]

1. IWC - Weighting option

$$
\begin{aligned}
0-\phi(\mathrm{E}) & =1 / \mathrm{E} \\
1-\phi(\mathrm{E}) & =1.0 \\
2-\phi(\mathrm{E}) & \sim \mathrm{Ee}^{\mathrm{E} / \mathrm{kTMAX}} \quad(\mathrm{E}<\mathrm{EMAX}) \\
& \sim 1 / \mathrm{E} \quad(\text { EMAX }<\mathrm{E}<\mathrm{EFISS}) \\
& \sim \sqrt{\mathrm{E}} \mathrm{e}^{-\mathrm{E} / \mathrm{TFISS}}\left(\mathrm{EFISS}<\mathrm{E}<10^{7}\right) \\
& \sim 1 / \mathrm{E} \quad\left(10^{7}<\mathrm{E}\right)
\end{aligned}
$$

where $\mathrm{c}$ and $\mathrm{d}$ are determined to make these functions

join smoothly with the $1 / \mathrm{E}$ function and the other

3 - Not used

parameters can be specified in the $18^{*}$ array.

$4-\phi(E)$ is input in the $20 \$$ and $21^{*}$ arrays

5 - Not used

$6-\phi(E)$ is input on logical 46

(Note: The JERGENS module is expected to be employed to form other special weighting functions, such as $1 / \mathrm{E} \sigma_{\mathrm{T}}$.)

2. N1W - Number of points in the interpolation table of the arbitrary spectrum to be input as card images (this is only needed when IWC $=4$ ).

3. N2W - Number of points in the arbitrary spectrum (this is only needed when IWC = 4).

4. MTW - The "MT" number of the weight function from logical 46 (this is only used when IWC $=6)$. Note that the weight function must have $\mathrm{MAT}=8000$ and $\mathrm{MF}=3$.

$12 \$$ Scattering Matrix Calculational Parameters [4]

1. NLF - The order of Legendre fit to be made to scattering matrices in the fast and epithermal energy ranges. This order is used for elastic scattering and discrete level inelastic scattering.

2. NLT - The order of Legendre fit to thermal-scattering matrices. This order is used, for example, with the free gas treatment or with thermalscattering law data.

3. MMME - The number of angles to be used in the treatment that calculates elastic-scattering matrices. This parameter is automatically set and will be of no interest to most users.

4. MMMI - The number of angles to be used in calculating inelastic-scattering matrices. This parameter is automatically set and will be of no interest to most users. 
$13 \$$ ENDF/B Data Libraries [5]

1. NDFBF - The logical unit of the file containing the ENDF/B data to be used in the epithermal and fast energy ranges (default $=11$ ).

2. MODEF - The "mode" ( 1 for binary and 2 for BCD) of the files on logical NDFBF (default = 1).

3. NDFBT - The logical unit of the file containing the ENDF/B data (scattering law or File 6 data) to be used in the thermal energy range (default $=12$ ).

4. MODET - The mode of the thermal data (default = 1).

5. NATOM - The number of atoms in the molecule when scattering law data are used (default $=1$ ). In the case of $\mathrm{H}_{2} \mathrm{O}$ and $\mathrm{D}_{2} \mathrm{O}$, the scattering law data are for $\mathrm{H}_{2}$ and $\mathrm{D}_{2}$, such that this should be input as 2 .

$14 \$$ ENDF/B Versions [2]

1. IVERF - Version of the fast ENDF/B data from NDFBF (default = 5) XLACS-77 is presently only able to process versions 4 or 5 .

2. IVERT - Version of the thermal ENDF/B data from NDFBT (default =4)

$15 \$$ Thermal Kernel Count [1]

1. MTEMP - The number of temperatures at which thermal scattering matrices are to be calculated.

$17 \$$ Print Options [10]

The print options in XLACS-77 are generally in one-to-one correspondence with the particular ENDF/B "file" that is being processed. For example, File 2 is used for resonance data; option 2 controls the printing of various parameters related to resonance processing. As a rule, a higher value for a particular option also selects all the output generated by any lower number. For example, if a 3 specifies a certain kind of output, a 4 and any higher value also selects this output. In the following table, a 3 is the lowest value used to select sutpur. Values 1 and 2 are reserved for output options of interests primarily for code-checking purposes and are not documented here.

1. IOPT1 - File 1 - Not used

2. IOPT2 - File 2 - 0 - No effect

3 - Print the parameters written on the AMPX master interface

10 - List the ENDF/B resonance data

3. YOPT3 - File $3-0$ - No effect

3 - List the averages for File 3

4. I0PT4 - File $4-0$ - No effect

3 - List $P_{0}$ scattering matrices for processes with File 4 data

4 - List $\mathrm{P}_{\ell}$ scattering matrices for processes with File 4 data

5. IOPT5 - File 5-0 - No effect

3 - List scattering matrices derived from File 5 data

6. IOPT6 - File $6-0$ - No effect

3 - List $P_{0}$ scattering matrices derived from File 6 data

$4-$ List $P_{\ell}$ scattering matrices derived from File 6 data 
7. IOPT7 - File 7 - 0 - No effect

3 - List $\mathrm{P}_{0}$-scattering matrices derived from scattering law data or from the free-gas treatment

8. I0PT8 - Not used

4 - List $P_{\ell}$ matrices

9. IOPT9 - Not used

10. I0PT10 - Weighting spectrum -0 - No effect

3 - List weighting spectrum

18* Weighting Spectrum and Other Floating-Point Parameters [10]

1. TMAX - The temperature of the Maxwellian spectrum ( $300 \mathrm{~K})$

2. EMAX - The upper energy limit on the Maxwellian spectrum $(0.125 \mathrm{eV})$

3. TFISS - The temperature of the fission spectrum $\left(1.273 \times 10^{6} \mathrm{eV}\right)$

4. EFISS - The lower energy limit of the fission spectrum $(67.4 \mathrm{keV})$

5. SIGP - Not used

6. EFISSH - The upper limit of the fission spectrum $\left(1 \times 10^{7} \mathrm{eV}\right)($ a $1 / \mathrm{E}$ spectrum is tacked on above this energy)

7. Not used

8. RFACT - The ratio factor used in constructing the cross-section mesh in the resolved range $(0.8)$

9. ANDEC - The number of points taken equally spaced in lethargy per decade in between resonance bodies (50)

10. XFACT - The multiple of $\Gamma_{t}$ on either side of a resonance for which the ratio mesh scheme is used (50.0)

$19 \$$ Triggers to Control Processing of ENDF/B Files [25]

This array can be used to turn on or off the processing of the various ENDF/B files. It should not be used unless the user is very familiar with both the structure of an ENDF/B neutron cross-section library and the processing details of the XLACS module. There is one exception to this directive, as will be noted below. The reason for the precautionary notes just given is best evident when one realizes that it would make no sense to tell the code to process File 4 or File 5 ENDF/B data without telling the code to also process File 3 data (i.e., point data are required to be used with either angular or energy distribution data, etc.) In any event, the dimension of the array (25) is clearly more files than XLACS uses, is a tipoff that this is the code developer's array for testing various options, without requiring the program to always exercise every nuance of its capabilities. It is, unfortunately, required in order to process File 6 data into thermal scattering matrices. In this case, the code user will want to "turn off" the regular File 7 scattering law processing and to "turn on" the File 6 processing. This will require a $19 \$$ array specified as follows:

$19 \$ \$ 6 R 1 \quad 0 \quad \mathrm{E}$

which says to process the first $6 \mathrm{ENDF} / \mathrm{B}$ files and ignore the 7th.

$\mathrm{T}$ Terminate Block 3 . 
Block 4. \{Required only when MTEMP $>0$ or when $\mathrm{IW}=4$ \}

$20 \$$ Interpolation Table for Arbitrary Weighting Function [N1W*2]

$\left(\mathrm{NBTW}_{\mathrm{i}}, \mathrm{JNTW}_{\mathrm{i}}, \mathrm{i}=1, \mathrm{~N} 1 \mathrm{~W}\right)$

where $\mathrm{NBTW}_{\mathrm{i}}$ is the energy point up to which the ith interpolation type is to be used and $\mathrm{JNTW}_{\mathrm{i}}$ is the interpolation type:

\begin{tabular}{cll} 
JNTW & & Interpolation \\
\cline { 3 - 3 } 1 & & Histogram \\
2 & & Linear $x-\operatorname{linear} y$ \\
3 & & Linear $x-\log y$ \\
4 & & Log $x-\operatorname{linear} y$ \\
5 & & Log $x-\log y$
\end{tabular}

21* Arbitrary Weighting Function [N2W*2]

$\left(X_{i}, Y_{i}, i=1, N 2 W\right)$

where these energy-value pairs specify the arbitrary weighting function.

22* Temperatures at Which to Calculate Thermal Scattering Matrices [MTEMP] $\left(T_{i}, \mathrm{i}=1\right.$, MTEMP $)$ - units are Kelvin

T Terminate Block 4.

\section{XLACS.2 XLACS-77 Input/Output Specifications}

An XLACS-77 execution will generally require some combination of the following I/O devices:

Logical No.

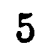

$\operatorname{NDFBF}(11)$

$\operatorname{NDFBT}(12)$

MASTER(23) 46

47
Purpose

\begin{tabular}{l}
\hline Standard input \\
Standard output \\
Scratch device \\
Scratch device \\
Scratch device \\
Scratch device \\
Scratch device \\
ENDF/B library (fast data) \\
ENDF/B library (thermal data) \\
AMPX master library produced by XLACS-77 \\
Weighting function library \\
Built-in group structures
\end{tabular}




\section{XLACS.3 XLACS-77 Error Message}

During execution, XLACS-77 makes many checks to determine if a calculation is proceeding correctly. If difficulties are encountered, a message is printed, and the problem may be terminated. Some of the messages are printed by the routine where they are detected; others are "numbered" messages from the error subroutines, ERROR.

There are several places in the module wherein checks are made in order to determine if enough core is available to perform a procedure. As a general rule, a "header" line telling what file is being processed will be produced, and, in the case where there is insufficient core, a message will be issued in the form:

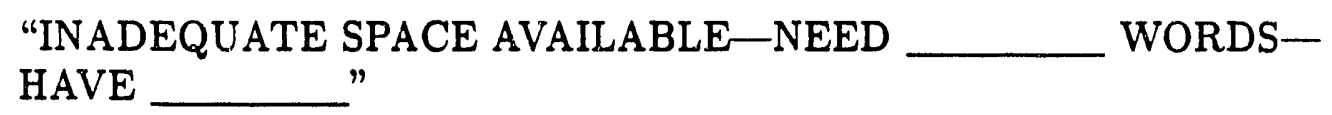

In this case, the user should check his input for obvious errors (too many groups, etc.), and either correct any errors or allocate more space and resubmit. The places where checks are made are

FILE6 - Main File 6 processing routine,

FLANGE - Thermal processing,

INELAS - File 5 processing,

MAIN - Overall control routine, and

XLACS - Primary control routine.

Another error message that can originate from several places is that caused by a bad interpolation parameter. Except for the case where the user inputs a bad specification in an arbitrary weighting function or on a library input to XLACS-77, which was improperly prepared in an auxiliary module, these are usually due to program problems having to do with the particular problem. In the event these messages occur, if a review of the user's input fails to locate problems, personnel responsible for this software should be contacted. Interpolation problems are generally well described and will be flagged by the following routines:

ECSI - Integration module

GRATE - Integration module

$\mathrm{KERC}$ (error 232) $-\mathrm{S}_{\alpha, \beta}$ processing

KERC2 (error 232) - $S_{\alpha, \beta}$ processing

PROF7 (error 230) - $S_{\alpha, \beta}$ processing

TERPN - Interpolation routine

TERPO - Interpolation routine

TERP1 (error 133) - Interpolation routine

XTWO - Production of free-atom scattering matrices for hydrogen

In another error related to interpolation, a message is produced that indicates that a negative or zero value has been improperly used with a scheme which requires taking the $\log$ of the value. This requires the same attention as with the previous error. This error may be written by TERP1 as error 134 or by XTWO.

Both of the above errors can arise when the interpolation table for a function is incomplete (e.g., when interpolation specifications are not given for all energy points). 
Another type of error involves the detection of an improper record sequence in the input file. Even though this certainly can arise from a bad ENDF library, it is more commonly due to a coding problem. Keep in mind that the ENDF/B formats are very general, such that all combinations of options and situations have not been (and may never be) used. When these errors occur, the user is generally stuck with notifying the personnel responsible for XLACS-77.

Examples of these error messages are given below.

A routine named CONT is used to read various "control" records on ENDF/B. It may write the following line:

\section{TPID RECORD IS INCORRECT OR MISSING,}

indicating that the "tape identification" record on the ENDF/B library is not correct. This may be due to the user referencing a file that is not an ENDF library or which is in improper mode (BCD when a binary library was designated, for example).

It will also issue messages exactly as above, except with the record type HEAD, SEND, FEND, MEND, or TEND, indicating errors in trying to read a head, section end, file end, material end, or tape end record, respectively.

The primary routine for processing File 3 data is called FILE3 and produces an error message if it does not find a proper file end record for File 2:

\section{LIBRARY POSITIONING ERROR AT THE END OF FILE2}

This message probably indicates problems in the resonance processing routines.

Some processing routines use a routine, RDENDF, to read ENDF/B records. This routine produces messages of the form:

\section{TAPE POSITIONING ERROR-WANTED MAT=} $-\mathrm{AT}$ MAT $=$

or the same sort of line for MF or MT, indicating a record for the wrong material, file, or process, respectively, has been read.

Another error that can occur arises when a particular kind of data cannot be found. In some cases, it is due to referring to the wrong ENDF/B library, or simply because the data are not on the file. In some cases, it is of no consequence, because the processing code can take alternative action or ignore the fact that it doesn't exist.

When one requests thermal scattering matrices and $S_{\alpha, \beta}$ data are requested, if the data are not found, messages are written by PROF7. If it is found, FLINK1 tries to find File 3 thermal data. A message is produced if it is not present, but the execution continues, expecting the requisite values to have been produced when the fast data were processed.

If data for a nuclide cannot be found, TMAT produces a message:

DESIRED MATERIAL NUMBER IS NOT ON TAPE OR IS OUT OF ORDER. TAPE HAS BEEN SEARCHED TO MATERIAL NUMBER

In this case, the user should determine if the material really should be on the library that is referred to in the input.

In some cases, the routine, TPOS, may produce a message indicating the data for a nuclide has been read to its end without finding the necessary data: 
DATA FOR MATERIAL EXHAUSTED WHILE SEARCHING FOR

$\mathrm{MF}=$ , $\mathrm{MT}=$

A nonfatal TPOS error message is of the form:

NO MF $=$ DATA FOR MAT $=$ POSITIONED AT MAT = $\mathrm{MF}=$ $\mathrm{MT}=$ , $\mathrm{MT}=$ , LIBRARY

This message generally just indicates the data are not present, such that no action is necessary.

A message from TMF1,

ERROR LNU = BUT MUST BE 1 OR 2,

indicates the ENDF/B evaluation is improper as this parameter, showing how $\nu(\mathrm{E})$ is presented, is improper. Processing may be adequate, however, if values for $\nu(\mathrm{E})$ are not needed in the particular application.

If the REATS routine produces error message 224 , it probably indicates the temperature dependence of the $S_{\alpha, \beta}$ data was improperly descr.bed. If it writes

ALPHA VALUE EXPECTED = ACTUAL $=$

it also indicates inconsistencies in the evaluation. Because these may be of little consequence, ten of these errors are allowed before the program aborts.

If the user asks for $S_{\alpha, \beta}$ data to be processed outside the range it is specified, REATS issues ERROR 223. The user should correct his request and resubmit.

Also in thermal processing, PROF7 may issue ERROR 231 which says the mass ratio is zero. This is a probable error in the data evaluation. It may also write out a message

\section{FREE ATOM CROSS SECTION $=0.0$}

\section{IT IS RESET TO 1.0,}

and continue processing, indicating a problem in the evaluation or problems set-up. (The library produced from this run can be properly adjusted, when a good ralue for the free-atom value is determined, using the FRESH module, which is not part of this release.)

If the TMF5 routine writes

\section{FILE 5 PROCESSING NO LONGER ALLOWED FOR LF =}

it indicates the data evaluator has improperly used an archaic dats born, or that the user is trying to process data earlier than Version 4.

Another message that sometimes is written by the MASH routine when it processes SLBW or MLBW resonance parameters is

SPACING PARAMETER ADJUSTMENT-MESH = $\mathrm{R}=$ , $S=$ $\mathrm{EO}=$ 
indicating a problem in assigning enough core for cross-section arrays for resonance processing. In most cases, this message will be of little consequence unless it is issued several times for a resonance, which means that resonance may have a poor energy mesh. Increasing the core allocated for the job will automatically increase the space that can be assigned.

\section{XLACS.4 XLACS-77 Procedures}

XLACS-77 is a fairly complicated program involving many steps and procedures. Several persons have noted the lack of any detailed documentation on the code. Certainly it should be thoroughly documented, and will be, provided adequate funding and time are made available. In the interim, it is hoped that this section will serve to at least describe in a macroscopic sense what data are processed, where they are processed, and how they communicate with each other. In parallel with how the data are presented on ENDF/B, the subsections will be named according to the ENDF/B file.

\section{XLACS.4.1 File 1 processing}

File 1 is primarily information that describes an evaluation. The only data in the file processed by XLACS are the three $\bar{\nu}$ 's:

1. $\mathrm{MT}=452$, total $\bar{\nu}$

2. $\mathrm{MT}=455, \bar{\nu}$ for delayed neutrons

3. $\mathrm{MT}=456, \bar{\nu}$ for prompt neutrons

These data may be tabulated or given in a parameterized fit. In the latter case, XLACS constructs a 50-poin mesh equally spaced in lethargy from $10^{-5}$ to $2 \times 10^{7} \mathrm{eV}$. The values for the three functions are written to scratch unit ITP4 for later processing with File 3 data by the FILE3 routine.

\section{XLACS.4.2 File 2 processing}

The processing of File 2 data is controlled by RESN, which starts by calling TMF2 to read all of the resonance data into core. This includes both resolved and unresolved data for one or more isotopes. These data are indexed into "blocks," with a block consisting of a particular kind of data for an individual isotope (e.g., the unresolved data for an isotope or the Adler-Adler, etc.). These blocks will later be processed in a manner completely independent of each other, which means one can accommodate multiple representations for data-Adler-Adler, SLBW, MLBW, etc--and can have overlapping energy rariges, if these are necessary for the evaluation.

Optionally, EDF2 is called to produce a listing of all the resonance parameter data.

The primary control of the resonance calculations is by RESN1, which is called next. RESN1 is blocked into sections, which call a calculational routine for the type of data in a block.

In the case of SLBW or MLBW parameters, the RESS and RESR routines are called; RESS, for absorption and fission processing, and RESR, for elastic scattering. 
In RESS, it is recognized that the sum of averages is the average of sums and that the absorption cross sections of Breit-Wigner resonance fits are independent of each other. This allows one to take each resonance and construct an energy mesh over the entire resonance range for the single resonance, calculate the cross section using the VOGAM routine that is based on $\psi$ and $\chi$ techniques for Doppler broadening, and then average the resonance pieces into the group structure. The energy mesh itself is constructed using the following schemes:

1. Inside the range $E_{0} \pm 4 \Gamma_{t}$ a mesh is constructed such that the ratio of cross-section values is 0.92 , except at low energies for very broad resonances $\left(\frac{\Gamma_{t}}{E_{0}}>0.075\right)$ where the ratio is taken at 0.999 . The ratio scheme is described below.

2. In the two surrounding regions, out to $E_{0} \pm 300 \Gamma_{t}$, the ratio scheme uses 0.9 (or 0.98 for broad resonances).

3. In the regions outside this, a mesh equally spaced in lethargy with 200 points per energy decade is used.

The ratio scheme is simple and is based on solving the $1 /\left(1+x^{2}\right)$ SLBW shape, where $x \equiv\left(E-E_{0}\right) / 2 \Gamma_{t}$, for the energy points. This quadratic equation has two solutions, one applicable above $E_{0}$ and the other below.

There is nothing elegant about these methods to establish a resonance mesh. Rather, they have developed over the years in response to practical observations on real problems. In most cases, they probably assign more points than necessary to yield resonance fits that are generally more accurate than $1 \%$. While there are more correct and efficient schemes that have been developed, these generally require considerably more computer time because of extra points that are calculated to ensure an adequate mesh.

Two special integration routines are used in RESS, XTWO, and JTWO: (1) the former for averaging cross sections over the problem-weighting spectrum and (2) the latter to produce reference infinite dilution values that are needed by the NITAWL-II formulation of the Nordheim integral treatment.

Elastic-scattering processing for Breit-Wigner resonances is handled by RESR. This routine takes all the resonances for a single $\ell$-state of a single isotope and calls a routine called SMESH to determine an energy mesh over the resolved energy range, after which it calculates the cross sections, using VOGAM for Doppler-broadening. Routines ATWO and BTWO are called for averaging the cross sections, ATWO corresponding to XTWO discussed earlier and BTWO for JTWO. The point-elastic cross sections are also written to scratch unit, ITP2, for later processing by RESA for averaging the data and X2D to make elastic transfer matrices. SMESH uses the ratio scheme for resonances within $E_{0} \pm \Gamma_{t}$ where the ratio is 0.8 and uses a lethargy spacing of the $\ell \mathrm{n}$ of 0.1 divided by 50 outside this region. In cases where the scheme generates more points than can be accommodated by the space assigned, RESR calculates cross sections for a string, determines a new mesh above the old mesh, calculates more, etc., until the entire resonance range is covered.

For Adler-Adler parameters, routine ADLER calls SMESHA to perform an analogous function to SMESH, except that in this case both absorption and scattering processes are calculated. ATWO and BTWO are used as in RESR. Multiple strings may be written to ITP2. Doppler-broadening uses VOGAM. 
For unresolved resonance data, RESU is called to control the calculation. It calls RESN1 to determine an energy mesh over which to calculate cross sections. If the parameters are given as a function of energy, the mesh is the same as that of the parameters, consistent with the directives for processing the data in the ENDF/B procedures manual. When the parameters are constant, an equal lethargy spacing of 100 points over the unresolved resonance range is taken. Subsequently, the RESU2 routine is called to calculate cross sections. As with previous routines, ATWO and BTWO are used to produce averaged data. Point values are written to logical ITP2 for later processing by the X2D routine to make elastic-scattering transfer matrices.

Next, RESD is called to select the resonance parameters to be passed for processing by the NITAWL-II module. At present, this means all $\ell=0$ resonances whose energies are positive. These data are written to scratch unit IO8.

\section{XLACS.4.3 File 3 processing}

File 3 processing is relatively straightforward and consists of reading the data from File 3 and performing one of the following operations:

1. All processes with MTs between 2 and 50 and 101 to 900 .

ATWO is called to average the data over the selected weighting spectrum. BTWO is also called for $\mathrm{MT}=2,18$, and 102 to produce infinite dilution averages.

2. Processes that generate scattering matrices $(\mathrm{MT}=2,6,7,8,9,16,17$ have their cross sections written to logical ITP2 for later processing.

3. Process $\mathrm{MT}=1$ and those with MT $>900$ are ignored.

4. A special section is included for averaging the $3 \nu$ 's. This parameter is passed to FILE3 on logical ITP1.

The proper weighting of $\nu$, the number of neutrons produced per fission, requires that it be weighted over $\sigma_{f} \phi$, not $\phi$ like most other cross sections. When the fission cross section (MT $=18$ ) is read, logical ITP1 is also read to access the $\nu$ functions. The routine ATWO is called to obtain the integral of the product of the fission cross section times the flux, which is subsequently to be divided by the integral of the flux to produce the averaged fission cross section. ATHREE is called to obtain the integral of the product of $\nu$ times $\sigma_{f}$ times $\phi$, and this is divided by the result from ATWO to produce $\bar{\nu}_{\boldsymbol{g}}$.

\section{XLACS.4.4 File 4 processing}

The processing of this file is used to produce transfer matrices for elastic scattering $(\mathrm{MT}=2)$ and for discrete level inelastic scattering $(\mathrm{MT}=51-90)$. It is also used for the special " $n 2 n$ " processes $(\mathrm{MT}=6-9)$ for ${ }^{9} \mathrm{Be}$. In these cases the distribution of the ai.gles a neutron will scatter through at a particular source energy is given in the ENDF/B file, either tabulated in angles or as a Legendre fit in angles. These representations are generally in the center-of-mass system (CM), though the laboratory system may be used. In all cases, the kinematics deals with conserving energy and 
momentum and is such that, when an initial energy and scattering angle is specified, there is only one possible energy at which the neutron can emerge from the collision.

These calculations are all controlled by X2D, which reads the data on ITP2, after which it calls TMG4 and TMH4 to try to find any File 4 data that may be present on the ENDF/B file. GDDD is then called which generates $\sigma_{s}\left(E, \mu_{m}\right)$ (i.e., the differential scattering cross section for the process at a set of angles in the laboratory system). The angles are predetermined by calling ANGL, which has all even-ordered Lobatto quadratures up through order 96. GDDD may have to use tabulated data or Legendre fits to determine data and may have to convert from the CM system to the laboratory system.

The angles picked are in Lobatto quadrature schemes which are Gaussian quadrature sets that force one to always pick the $\mu=-1.0$ and +1.0 angles for inclusion in the set. These sets are built into XLACS through order 96 and have the very nice property that, if the functional to be integrated is a polynomial of order $2 n-1$, or less, where $n$ is the number of angles taken, then the integration is exact.

The subroutine XFER is called which uses the differential cross sections at each angle in the quadrature to generate transfer matrices for the angle. These are subsequently summed to produce a "total" transfer matrix for the process (level). This summing is performed in the subroutine called DRUG.

The procedure used in XFER is particularly simple. The expression to be evaluated is

$$
\sigma_{\ell}\left(g \rightarrow g^{\prime}\right)=\frac{\frac{1}{2 \ell+1} \int_{-1}^{1} d \mu P_{\ell}(\mu) \int_{g} d E \sigma_{s}(E, \mu) \phi(E) \int_{g^{\prime}} d E^{\prime} f\left(E \rightarrow E^{\prime}, \mu\right)}{\int_{g} d E \phi(E)},
$$

where for the discrete processes the "transfer" function, $f\left(E \rightarrow E^{\prime}, \mu\right)$, is a $\delta$ function, $\delta\left(E \rightarrow E^{\prime}, \mu\right)$.

First we choose to perform the angular integral using a simple quadrature that is based on the angles and weights for the Lobatto quadratures mentioned above. This reduces our integral:

$$
\sigma_{\ell}\left(g \rightarrow g^{\prime}\right)=\frac{1}{2 \ell H} \frac{\sum_{m=1}^{M M} W_{m} P_{\ell}\left(\mu_{m}\right) \int_{g} d E \sigma_{s}\left(E, \mu_{m}\right) \phi(E) \int_{g^{\prime}} d E^{\prime} \delta\left(E \rightarrow E^{\prime}, \mu_{m}\right)}{\int_{g} d E \phi(E)} .
$$

The averaged cross section for scattering through an angle whose cosine is $\mu_{m}$ is

$$
\sigma\left(g \rightarrow g^{\prime}, \mu_{m}\right)=\frac{\int_{g} d E \sigma_{s}\left(E, \mu_{m}\right) \phi(E) \int_{g^{\prime}} d E^{\prime} \delta\left(E \rightarrow E^{\prime}, \mu_{m}\right)}{\int_{g} d E \phi(E)},
$$

such that

$$
\sigma_{\ell}\left(g \rightarrow g^{\prime}\right)=\frac{1}{2 \ell+1} \sum_{m=1}^{M M} W_{m} P_{\ell}\left(\mu_{m}\right) \sigma\left(g \rightarrow g^{\prime}, \mu_{m}\right) .
$$

If we examine the expression for $\sigma\left(g \rightarrow g l, \mu_{m}\right)$, it is immediately apparent that what the $\delta$ function does is to delimit a region out of group $g$ which can scatter to 
group $g /$ for the angle, $\mu_{m}$. Since the integral over a $\delta$ function is unity by definition, we need merely to use the $\delta$ function to determine the appropriate part of $g$ and make the integration:

$$
\sigma\left(g \rightarrow g^{\prime}, \mu_{m}\right)=\frac{\int_{E_{L}\left(g \rightarrow g^{\prime}, \mu_{m}\right)}^{E_{H}\left(g \rightarrow g l, \mu_{m}\right)} d E \sigma\left(E, \mu_{m}\right) \phi(E)}{\int_{g} d E \phi(E)} .
$$

This is precisely what is programmed in XFER. A closer examination of the procedure will note that all required integrations are very simple, that the computer time requirements vary linearly with the number of energy groups, that it can accommodate any process wherein the differential behavior is characterized by a $\delta$ function.

There are two areas, however, where this simple procedure produces disturbing, though probably usable, results. These are related and are (1) for very light nuclides, such as hydrogen, and (2) for very fine group structures.

Both of these situations will frequently miss many transfer cross sections for practical angular quadratures (say 8 to 50 angles). However, though the individual transfers are missed, the procedure does account for them by putting their contributions into the closest transfers that the procedure does "see." This effect is not observed by other methods which calculate group-to-group values because they, in essence, take each transfer and use the $\delta$ function to explicitly determine necessary integration limits, etc.

In summary, the above procedure has been successfully employed to produce multigroup libraries here at Oak Ridge. It is not only much faster and simpler than most alternative approaches, yielding results that almost always seem to fall within the accuracy limits of those approaches, but it has a very interesting possible new application. Several people have experimented with schemes that avoid Legendre fits to cross sections. This approach calculates the transfer matrices needed by many of these schemes (i.e., the group-to-group terms for scattering through a particular angle) and does that essentially without approximation.

After the summing is complete over all angles, CMPR is called to compress out zero and impossible values. The final matrices are written to scratch unit IO8.

Hydrogen is treated as a special case in File 4 processing. First, it is recognized that elastic scattering in hydrogen is isotropic in the CM except at energies in the $\mathrm{MeV}$ range. Because of this, it is elected to ignore the File 4 data and to call SIGH, which performs an analytic calculation to determine the scattering matrices. The treatment accounts for the energy variation in both the cross section and flux as opposed to earlier analytic treatments in XLACS, which assumed the flux was $1 / E$ and that the cross section had a very smooth variation.

Given these assumptions, a routine called XTWO, which calls XSMUD, which calls $\mathrm{XBEAX}$ and XEALNC in some cases, is used to perform the integrations. Subroutines DRUG and CMPR are used, as described above, except that there is no requirement to sum over angles. 


\section{XLACS.4.5 File 5 processing}

In cases where the inelastic levels are so closely spaced that they cannot be resolved, the secondary energy distribution of neutrons may be expressed in terms of a smooth distribution as a function of scattering energy. Note that a situation of this sort always implies a large number of levels and will generally ignore any angular variation in the scattering (i.e., the scattering is assumed isotropic in the laboratory system).

Subroutine INELAS controls the generation of scattering matrices for these processes. The data on ITP2 are read, and those which have File 5 data are processed.

The routine called TMF5 is called to read File 5 data.

File 5 processing makes use of an observation which greatly simplifies the problem (viz., that the Cartesian interpolation schemes in ENDF/B allow one to perform the integrations over its distributions in any order). The basic problem is stated as follows:

$$
\sigma\left(g \rightarrow g^{\prime}\right)=\frac{\int_{g} d E \phi(E) \sigma(E) \rho(E) \int_{g^{\prime}} d E^{\prime} f\left(E \rightarrow E^{\prime}\right)}{\int_{g} d E \phi(E)},
$$

where $\sigma\left(g \rightarrow g^{\prime}\right)$ is the group-to-group scattering term, $\phi(E)$ is the weighting flux, $\sigma(E)$ is the cross section, $\rho(E)$ is an ENDF/B option which allows the evaluator to include multiple distributions for describing the scattering, and $f\left(E \rightarrow E^{\prime}\right)$ is any one of the distributions defined in File 5.

It is recognized that one can simplify the above equation by integrating the File 5 function over the secondary group structure and using these, which are now like "vectors" that vary as a function of initial energy, in the final calculation:

$$
\sigma(g \rightarrow g \prime)=\frac{\int_{g} d E \phi(E) \sigma(E) \rho(E) F\left(E \rightarrow g^{\prime}\right)}{\int_{g} d E \phi(E)} .
$$

To further simplify the processing, it is recognized that $F\left(E \rightarrow g^{\prime}\right)$ is linear in $E$ per the recommendations of ENDF/B specifications, and that it is a smoothly varying function in an energy mesh which is taken as the union of energy points in $F(E \rightarrow g \prime)$ and the problem group structure.

If we look at the $i$ th panel in the mesh, which is inside the two panel energy boundaries $E_{a}$ and $E_{b}$,

$$
\begin{array}{llll}
F\left(E_{a} \rightarrow g^{\prime}\right) & & & F\left(E_{b} \rightarrow g^{\prime}\right) \\
E_{a} & E_{i} & E_{i+1} & E_{b},
\end{array}
$$

and we note that since the group boundaries are always part of the mesh, the panel $i$ must be totally contained in some group, $g$, then the contribution for the panel for the group $g$ can be written as

$$
\sigma\left(i \epsilon g \rightarrow g^{\prime}\right)=\frac{\int_{g} d E \phi(E) \sigma(E) \rho(E) \bar{F}\left(i \rightarrow g^{\prime}\right)}{\int_{g} d E \phi(E)},
$$


where $\bar{F}\left(i \rightarrow g^{\prime}\right)$ is the simple arithmetic average of $F(E \rightarrow g \prime)$ over the $i$ th panel:

$$
\bar{F}\left(i \rightarrow g^{\prime}\right)=\frac{\int_{i} d E F(E \rightarrow g \prime)}{E_{i t h}-E_{i}},
$$

where

$$
F\left(E \rightarrow g^{\prime}\right)=F\left(E_{a} \rightarrow g^{\prime}\right)+\frac{E-E_{a}}{E_{b}-E_{a}}\left[F\left(E_{b} \rightarrow g^{\prime}\right)-F\left(E_{a} \rightarrow g^{\prime}\right)\right] .
$$

When all substitutions are made, and the integration is performed,

$$
\begin{aligned}
\bar{F}\left(i \rightarrow g^{\prime}\right) & =F\left(E_{a} \rightarrow g^{\prime}\right)\left[1-\frac{E_{i+1}+E_{i}}{2\left(E_{b}-E_{a}\right)}+\frac{E_{a}}{E_{b}-E_{a}}\right] \\
& +F\left(E_{b} \rightarrow g^{\prime}\right)\left[\frac{E_{i+1}+E_{i}}{2\left(E_{b}-E_{a}\right)}-\frac{E_{a}}{E_{b}-E_{a}}\right] \\
& \equiv F\left(E_{a} \rightarrow g^{\prime}\right) W_{i a}+F\left(E_{b} \rightarrow g^{\prime}\right) W_{i b}
\end{aligned}
$$

Note that since $F\left(E_{a} \rightarrow g^{\prime}\right)$ and $F\left(E_{b} \rightarrow g^{\prime}\right)$ sum to 1 , and since $\bar{F}\left(i \rightarrow g^{\prime}\right)$ must sum to 1 , the sum of $W_{i a}$ and $W_{i b}$ will always sum to 1 .

INELAS is the control routine for generating cross sections with the above procedure. As with the X2D procedure, an "outer" loop in the code reads cross sections from logical ITP2. As each process is read, a call is made to TMF5 to determine if evaporation data are present and, if they are, to read the data. Processes with no data are skipped.

The next consideration is to obtain tabulated distributions. When the data are presented in tabular form, it will be used directly. Otherwise, MLF1 is called, which determines an energy mesh equally spaced in lethargy over the possible range of values prescribed in the $\rho(E)$ array. After this, INT5 is called, which takes each of the tabular distributions and integrates them to produce the $F(E \rightarrow g \prime)$ functions.

Next, SETPAN is called to determine the panel energy mesh and to set arrays that set the energy group for the panel and the weights $\left(W_{i a}\right.$ and $\left.W_{i b}\right)$ to be used with the panel.

Next, IMAT1 is called, which has an outside loop that reads in each of the $F(E \rightarrow$ $g \prime)$ vectors. For each of these, another loop is made over the panels determined by SETPAN. For the panels marked to use the particular $F(E \rightarrow g \prime)$, the code then calls ATHREE to integrate the product of $\sigma$ times $\phi$ times $\rho$. This product is multiplied by each of the $F\left(E \rightarrow g^{\prime}\right)$ times the weight determined in SETPAN and put into the transfer matrix for the process. After the processing is complete, CMPR is called to compress out zero and impossible transfer terms, after which the scattering matrix is written to logical 108.

The fission cross section $(M T=18)$ receives a special treatment. In this case, a transfer matrix for fission is generated as described above. The terms in the matrix are then multiplied by $\bar{\nu}$ to create a fission neutron scattering matrix in a routine called CHIMAT. Th.is matrix is output on logical 108 in two parts, each identified with MT = 1452. This splitting is done to reduce the size of the arrays, because fission generally requires a matrix diniensioned by the number of groups squared. A routine called CHI 
is also called to produce the $\chi$ function required by most analysis codes which assumes that the neutrons produced by fission are not a function of energy. For this function, the full transfer matrix is used and $\chi(g \rightarrow g \prime)$ is weighted over the product of $\nu$ times $\sigma_{f}$ times $\phi$, as should be done.

\section{XLACS.4.6 File 6 processing}

In XLACS-77, at present, the processing of File 6 data is strictly limited to several in-house sets of specialized thermal-scattering data which use this presentation form, though it could be easily modified to handle other situations. File 6 is not used in any of the standard ENDF/B evaluations for Version 5 and earlier.

The procedure to process these data closely parallels that for processing File 5 data and makes use of the same observation that the order of the integrations can be taken in any order. The equation to be processed is

$$
\sigma_{\ell}\left(g \rightarrow g^{\prime}\right)=\frac{\frac{1}{2 \ell+1} \int_{-1}^{1} d \mu P_{\ell}(\mu) \int_{g} d E \sigma_{s}(E) \phi(E) \int_{g \prime} d E^{\prime} f\left(E \rightarrow E^{\prime}, \mu\right)}{\int_{g} d E \phi(E)}
$$

The tabular function $f\left(E \rightarrow E^{\prime}, \mu\right)$ is given as a series of tabulations at discrete values of initial energy, $E$, and cosine angle, $\mu$.

If we note that the variation of the tabulations is linear in cosine of angle and use the same procedure for initial energy as was used for File 5 data, the above equation simplifies to

$$
\sigma_{\ell}\left(g \rightarrow g^{\prime}\right)=\frac{\frac{1}{2 \ell+1} \sum_{m=1}^{M} W_{\ell m} \sum_{i \epsilon g} W_{i} \bar{F}_{i}(i \rightarrow g \prime) \int_{g} d E \sigma_{s}(E) \phi(E)}{\int_{g} d E \phi(E)}
$$

where, as before, the $W_{i}$ are the "weights" associated with panels in group $g$, and $W_{\ell m}$ is determined by integrating the product of a linear function times a Legendre polynomial of order $\ell$

$$
f(\mu)=f_{m}+\frac{\mu-\mu_{m}}{\mu_{m+1}-\mu_{m}}\left(f_{m+1}-f_{m}\right) .
$$

If we integrate this variation times the Legendre polynominal for all intervals in the angular mesh, a point $m$ will contribute to two panels. The terms for the two panels are combined to form the $W_{\ell m}$ for the various orders.

FILE6 uses TMF6 to read the File 6 data. As the panels are read, they are integrated over the secondary energy into the problem group structure and are then written onto direct-access File N9, where they can be randomly accessed based on indexing by the angle and the particular source energy.

WT6 is called to calculate the $W_{\ell m}$ discussed above, after which SETPAN is called to calculate the $W_{i}$ using exactly the same procedures as used with File 5 data.

Following this, IMAT6 is called to perform the final calculation. The outer loop in this rse is over the order of the matrix to be calculated. Inside this is a loop over the angivs in the tabulation. Inside this is a loop over the source energies, and on the 
inside of this is a loop over the "panels" in a group. The parameters set in SETPAN specify the energy range for the integration and the group to which a panel belongs. Using these, ATWO is called to calculate

$$
\int_{\text {panel }} d E \sigma_{s}(E) \phi(E)
$$

which is, in turn, multiplied by $W_{\ell m}$ and $W_{i}$ and then by $\bar{F}_{i} \rightarrow g$ to sum into the transfer matrix.

After all panels are processed, CMPR is called to compress the scattering matrix, which is then written to logical unit $\mathrm{IO8.}$

\section{XLACS.4.7 File 7 processing}

The processing of File 7 involves using a procedure developed by Mr. G. S. Robinson of the Australian Atomic Energy Commission which he graciously supplied to us.

Mr. Robinson noted glaring deficiencies for many cases arising from using the original procedures in XLACS-II. His new procedure is essentially a total rewrite of the schemes used in the original program and, based on our experiences in using it, is significantly more accurate, while requiring much less computer time.

This calculation is controlled by a routine named FLANGE.

FLANGE starts by calling FLINK1, which tries to access any File 3 and File 4 data supplied with the thermal file for describing the coherent scattering data. If present, these are averaged and are introduced to the within-group scattering terms of the thermal-scattering matrices, based on the assumption that the ccherent scattering is that form arising from lattice coupling of the atoms in such a manner as to make them appear to the neutron as an atom with a very large mass.

Next, MESH is called to determine an energy mesh over which the differential scattering cross sections will be calculated.

PROF7 is then called to control the processing of the $S_{\alpha, \beta}$ data in File 7 . For nuclides (most nuclides) which don't have these data, PROF7 calls routines that generate $S_{\alpha, \beta}$ data consistent with a free-gas thermal-scattering treatment.

When $S_{\alpha, \beta}$ data are present, PROF7 calls REATS to read these data. A routine named FENT contrcls the computation of scattering kernels and cross sections. For the case where no $S_{\alpha, \beta}$ data are given, FENA is the controlling module.

FENT calls KERC and KERC2 to calculate differential cross sections, $\sigma(E \rightarrow$ $\left.E^{\prime}, T\right)$, from $S_{\alpha, \beta}$ data. These are stored in a packed-core array using a routine namea STORK.

Both KERC and KERC2 call a variety of routines (GNCRA, GNCRB, CLAM, KERAS) for performing the necessary transformations, moment fits, etc.

FENA makes extensive use of a routine called KERA for calculating free-gas differential-scattering cross sections. As with FENT, STORK is called to stuff these into a packed-core array. 
After all differential data are calculated, REKON is called to read the data back from the packed-core array (using STORK) and to form the final scattering matrices. These are compressed using CMPS and are written to logical unit IO8.

\section{XLACS.4.8 Collecting data for the AMPX master interface}

At the end of the loop over nuclides in the XLACS routine, there is a call to REC4, whose function is to read all the information from logical unit IO8 and to reassemble it into the formats needed on the master interface written on logical unit ITXN. Optionally, routines such as PRT1D and MWLS may be called to produce edits of the data as they are collected.

\section{REFERENCES}

1. N. M. Greene, J. L. Lucius, J. E. White, R. Q. Wright, C. W. Craven, Jr., and M. L. Tobias, XLACS: A Program to Produce Weighted Multigroup Neutron Cross Sections from $E N D F / B$, ORNL/TM-3646, Union Carbide Corp., Nucl. Div., Oak Ridge Natl. Lab., April 1972.

2. J. A. Bucholz, A Method of Generating Multigroup Transfer Matrices Using an Analytic Angular Integration Free of Truncation Error, NUREG/CR-0295, (ORNL/NUREG/CSD-8), U.S. Nuclear Regulatory Commission, September 1978. 


\section{XSDRNPM - MODULE WITH ONE-DIMENSIONAL $S_{n}$ CAPABILITY FOR SPATIAL WEIGHTING}

The XSDRNPM ( $\underline{X}$ - $\underline{\text { Section }}$ Dynamics for Reactor $\underline{\text { Nucleonics with }}$ Petrie Modifications) module is provided in AMPX for two purposes:

1. to provide a 1-D transport calculation capability for calculating reaction rates, eigenvalues, and critical dimensions, and

2. to allow spatial cross-section weighting to be performeu.

XSDRNPM* differs from its predecessor XSDRN, ${ }^{1}$ in several important respects.

- It will do coupled neutron-gamma calculations.

- It allows any mixture to be represented to an arbitrary order of anisotropic rer resentation.

- It will do an adjoint calculation.

- The resonance calculation is removed and is provided in AMPX in the NITAWL module.

- It employs improved thermal flux scaling techniques for better problem convergence.

- Input specifications have been reordered, and more defaults have been provided to make the use of this module easier.

- It will calculate $S_{n}$ constants for any even order for any of the 3 1-D geometries available.

- Mixture-dependent fission spectra are calculated and used in XSDRNPM, which takes into account all fissionable nuclides in a problem.

The collapsed cross sections from XSDRNPM are written as an AMPX-weighted library on logical 3 which can be used directly by the module to do transport calculations (an AMPX-weighted library uses the same format as a working library). They can also be output on cards or in a binary format suitable for the ANISN, DOT, or MORSE codes.

\section{XSDRNPM.1 Cross-Section Weighting Options}

XSDRNPM weights cross sections according to the following four options:

1. "cell" weighting

2. "zone" weighting

3. "region" or "vein" weighting

4. "inner-cell" weighting

In all cases, the "averaged" cross sections are defined in a manner that conserves reaction rates, that is,

$$
\bar{\sigma}_{G} \int_{\text {Space }} \overrightarrow{d r} N_{D}(\vec{r}) \int_{G} d E \psi(E, \vec{r})=\int_{\text {Space }} \overrightarrow{d r} N(\vec{r}) \int_{G} d E \sigma(E, \vec{r}), \psi(E, \vec{r})
$$

*XSDRN with $\underline{\text { Petrie }}$ Modifications. The original acronym for XSDRN was suggested by W. A. Rhoades. 
where

$\bar{\sigma}_{G} \equiv$ average cross section in group $\mathrm{G}$,

$N_{D}(\vec{r}) \equiv$ number density used in the definition for the weighting option selected,

$\psi(E, \vec{r}) \equiv$ weighting spectrum

$N(\vec{r}) \equiv$ real number density as a function of spatial position,

$\sigma(E, \vec{r}) \equiv$ cross section in unreduced form.

If we convert to multigroup notation and use $W$ for the weighting spectrum (instead of $\psi$ ), Eq. (1) becomes

$$
\bar{\sigma}_{G} \sum_{j}^{\substack{\text { applicable } \\ \text { spatial } \\ \text { regions }}} N_{D}^{j} \sum_{g \in G} W_{g}^{j}=\sum_{j}^{\substack{\text { applicable } \\ \text { spatial } \\ \text { regions }}} N^{j} \sum_{g \in G} \sigma_{g}^{j} W_{g}^{j},
$$

where

$$
W_{g}^{j} \equiv \int_{j} \overrightarrow{d r} \int_{g} d E \psi(E, \vec{r})
$$

\section{XSDRNPM.1.1 "Cell" weighting}

Cell weighting is consistent with homogenizing the cross sections in a heterogeneous cell. This is the recommended option to prepare cross sections for a real reactor calculation that will be made with a 2- or 3-D model of the reactor. Most of these codes have no provisions for explicitly representing individual fuel elements which are interspersed in a moderator region. Hence, regions of this type are mocked in a homogeneous representation.

Cell-weighted cross sections are defined in a manner that attempts to preserve the reaction rates that occur in a representative cell from the reactor. In Eq. (2) the weighting involves the following substitution:

$$
N_{D}^{j} \equiv \bar{N}=\frac{\sum_{j}^{\text {cell }} V^{j} N^{j}}{\sum_{j}^{c e l l} V^{j}},
$$

where

$$
V^{j} \equiv \text { volume of zone } j \text {. }
$$

\section{XSDRNPM.1.2 "Zone" weighting}

Zone weighting is the simplest of the three XSDRNPM weighting options. Each zone produces a unique set of cross sections which preserves reaction rates for the zone. In Eq. (2), the spatial sum is over the zone considered, and $N^{j}$ and $N_{D}^{j}$ are unity.

\section{XSDRNPM}


Zone weighting is used very frequently, especially for problems whose collapsed cross sections are to be used in a problem whose geometrical and material layout is similar to that in the weighting problem.

\section{XSDRNPM.1.3 "Region" weighting}

"Region-" or "vein-" weighted cross sections are veighted "where the nuclide is." In most problems, there are nuclides of secondary importance which do not need a separate "zone-weighted" set for every region in which the nuclide occurs. Examples are the components of stainless steel. Stainless steel is encountered in a variety of locations and flux environments, but generally one set of cross sections for iron, chromium, manganese, nickel, etc., will suffice for most reactor calculations. with

In Eq. (2), the spatial sum is over all zones that contain the nuclide of interest

$$
N_{D}^{j}=N^{j}
$$

\section{XSDRNPM.1.4 "Inner-cell" weighting}

For inner-cell weighting, cell weighting is performed over specified innermost regions in the problem. Nuclides outside these regions are not weighted.

This option is generally employed as follows: A "cell" is described in exactly the same manner as for cell weighting except that in this case it is surrounded by a homogeneous representation for the remainder of the core and by blankets, reflectors, etc. The flux calculation is made over this complete system, which should have a more realistic treatment of the leakage across the outer boundary of the interior cell. The cell weighting is subsequently made only over the interior cell.

\section{XSDRNPM.1.5 Multigroup weighting equations}

The following are the multigroup forms of the weighting equations used in XSDRNPM:

1. Cell weighting

$$
\bar{\sigma}_{G} \equiv \frac{\sum_{j}^{I Z M} N^{j} \sum_{g \in G} \sigma_{g}^{j} W_{g}^{j}}{\bar{N} \sum_{j}^{I Z M} \sum_{g \in G} W_{g}^{j}},
$$


where

$$
\begin{gathered}
\bar{N} \equiv \frac{\sum_{j}^{I Z M} V^{j} N^{j}}{\sum_{j}^{I Z M} V^{j}}, \\
W_{g}^{j} \equiv \psi_{g}^{j}=\int_{j} \psi_{g}(\vec{r}) \overrightarrow{d r} .
\end{gathered}
$$

2. Zone weighting

$$
\bar{\sigma}_{G}^{j} \equiv \frac{\sum_{g \in G} \sigma_{g}^{j} W_{g}^{j}}{\sum_{g \in G} W_{g}^{j}} .
$$

3. Region weighting

$$
\bar{\sigma}_{G} \equiv \frac{\sum_{j} N^{j} \sum_{g \in G} \sigma_{g}^{j} W_{g}^{j}}{\sum_{j} N^{j} \sum_{g \in G} W_{g}^{j}} .
$$

\section{XSDRNPM.1.6 Transfer matrices}

Collapsing transfer matrices is not quite as simple as collapsing cross sections with a single value per group. A group-to-group term in the broad group sense conserves the scattering rate from one group to the other, that is,

$$
\bar{N}^{*} \bar{\sigma}\left(G \rightarrow G^{\prime}\right) \psi_{G} \equiv \int_{\text {Space }} \overrightarrow{d r} N(\vec{r}) \int_{g} d E \psi(E, \vec{r}) \int_{g^{\prime}} d E^{\prime} \sigma\left(E \rightarrow E^{\prime}\right),
$$

where the asterisk $\left(^{*}\right)$ denotes that the number density on the left side of the equation is consistent with the weighting desired. Therefore, the multigroup forms of the weighting equations for components of the transfer matrices are as follows:

1. Cell weighting

$$
\bar{\sigma}_{G \rightarrow G^{\prime}} \equiv \frac{\sum_{j}^{I Z M} N^{j} \sum_{g \in G} W_{g}^{j} \sum_{g^{\prime} \in G^{\prime}} \sigma^{j}\left(g \rightarrow g^{\prime}\right)}{\bar{N} \sum_{j}^{I Z M} \sum_{g \in G} W_{g}^{j}}
$$


2. Zone weighting

$$
\bar{\sigma}_{G \rightarrow G^{\prime}} \equiv \frac{\sum_{g \in G} W_{g}^{j} \sum_{g \in G^{\prime}} \sigma^{j}\left(g \rightarrow g^{\prime}\right)}{\sum_{g \in G} W_{g}^{j}} .
$$

3. Region weighting

$$
\bar{\sigma}_{G \rightarrow G^{\prime}} \equiv \frac{\sum_{j} N^{j} \sum_{g \in G} W_{g}^{j} \sum_{g / \epsilon G^{\prime}} \sigma^{j}\left(g \rightarrow g^{\prime}\right)}{\sum_{j} N^{j} \sum_{g \in G} W_{g}^{j}} .
$$

Theoretically, the higher-than-zero order $\sigma_{l}(g \rightarrow g \prime)$ should be weighted over $\psi_{l}$. Since these functions are generally positive-negative, $\psi_{l}$ weighting does not always work in practice, and XSDRNPM weights the $\sigma_{l}\left(g \rightarrow g^{\prime}\right), \ell>0$, by the scalar flux, which is positive. This procedure gives usable values for most cases.

\section{XSDRNPM.1.7 Weighting of $\bar{\nu}$}

In weighting parameters such as $\bar{\nu}$, the average number of neutrons produced per fission, one is interested in preserving the fission source; therefore, the weighting is over $\sigma_{f} \psi$ instead of just $\psi$. The weighting procedure in XSDRNPM is to calculate $\left(\overline{\nu \sigma_{f}}\right)_{G}$ and $\left(\overline{\sigma_{f}}\right)_{G}$ using the appropriate choice from Eq. (12), (13), or (14). Then,

$$
\bar{\nu}_{G}=\frac{\left(\overline{\nu \sigma_{f}}\right)_{G}}{\left(\overline{\sigma_{f}}\right)_{G}}
$$

\section{XSDRNPM.1.8 Transport cross sections}

Transport cross sections are not as directly related to the physical properties of a material as other group-averaged values. Instead of a reaction rate, these numbers must attempt to preserve a "flux gradient," which not only depends on the cross sections of the material, but is also very strongly influenced by the geometry and the other nuclides in the vicinity of a material.

Two options are provided in XSDRNPM to generate transport cross sectionsoptions based on the "consistent" and "inconsistent" methods for solving the $P_{1}$ transport equations. These approximations are referred to as the "outscatter" and "inscatter" approximations because of the nature of the equations used.

Outscatter approximation (inconsistent method). In the outscatter approximation, the assumption is made that

$$
\sigma_{t r}^{g}=\sigma_{t}^{g}-\bar{\mu}^{g} \sigma_{s}^{g}
$$


When one notes that

$$
\bar{\mu}^{g} \equiv \frac{\sigma_{1}^{g}}{3 \sigma_{0}^{g}}
$$

and that

$$
\sigma_{1}^{g}=\sum_{g^{\prime}} \sigma_{1}\left(g \rightarrow g^{\prime}\right)
$$

where the $\sigma_{1}(g \rightarrow g \prime)$ terms are the $P_{1}$ coefficients of the scattering matrix, the origin of the term "outscatter" to designate the approximation is evident.

Inscatter approximation (consistent method). In the "consistent" solution of the $P_{1}$ point transport equations, it can be shown that

$$
\sigma_{t r}(E)=\sigma_{t}(E)-\frac{1}{3 J(E)} \int_{0}^{\infty} d E^{\prime} \sigma_{1}\left(E^{\prime} \rightarrow E\right) J\left(E^{\prime}\right),
$$

where $J\left(E^{\prime}\right)$ is the current.

If one multiplies the equation by $J(E)$, integrates over group $g$, and converts to group-averaged form by dividing by $\int_{g} J(E) d E$, the following expression is derived:

$$
\sigma_{t r}^{g}=\sigma_{t}^{g}-\frac{1}{3 J_{g}} \sum_{g^{\prime}} \sigma_{1}\left(g^{\prime} \rightarrow g\right) J_{g^{\prime}} .
$$

This is the "inscatter" approximation. It is consistent because the transport values are explicitly derived from the $P_{0}$ and $P_{1}$ equations. As a general rule, the transport values from this treatment are "better" than those from the "inconsistent" treatment. However, in some cases (notably hydrogen at lower energies), negative numbers may be calculated which are unusable, and the more approximate approach must be used.

Weighting function for transport cross section. Unfortunately, the matter of choosing a current to use in the "transport" weighting is not simple. In real problems, currents are positive-negative as a function of energy and space. When cross sections are averaged over positive-negative functions, the "law-of-the-mean" no longer holds and the average value can take on any value. These nonphysical values are unusable in diffusion calculations.

Approximations that inherently guarantee positive currents are generally used in other codes that circumvent the positive-negative weighting problem. For example, in $B_{n}$ theory, the current is given by

$$
j \sim B \psi
$$

where $B$ and $\psi$ are both positive.

In XSDRNPM, more direct routes that ensure positivity are taken (e.g., one might set $\left.W_{g} \equiv\left|W_{g}\right|\right)$. This is crudely supported by the following argument.

Consider a 1-D cylindrical calculation. In two dimensions, the current is a vector combination, that is,

$$
\bar{J}=\bar{J}_{r}+\bar{J}_{z} .
$$


In XSDRNPM, the $z$ direction is treated by using a buckling approximation, that is,

$$
\bar{J}_{z}=B \psi \text {. }
$$

In the weighting calculation, we want to weight over the magnitude of the current. In XSDRNPM, the $z$-current is imaginary, since we are not calculating a $z$-direction:

$$
\bar{J}=\bar{J}_{r}+\bar{i} B \psi \text {. }
$$

The magnitude of a complex quantity is

$$
J=\frac{\left(\bar{J}_{r}+\bar{i} B \psi\right)\left(\bar{J}_{r}-\bar{i} B \psi\right)}{\sqrt{\left(\text { Value }_{r}\right)^{2}}+B^{2} \psi^{2}},
$$

which is always positive.

In a discrete-ordinates calculation, the current is easily obtained because it is the first flux moment. XSDRNPM has the following options for calculating the current:

$$
\begin{gathered}
J_{g}=\sqrt{\left(\psi_{l}^{g}\right)^{2}+\left(D B \psi_{g}\right)^{2}}, \\
J_{g}=\left|\psi_{l}^{g}\right|, \\
J_{g}=D B^{2} \psi_{g}+\int_{0}^{1} d \mu \mu \psi\left(g, r_{\text {out side }}, \mu\right), \\
J_{g}=\frac{\psi_{0}^{g}}{\sum_{t}^{g}}, \\
J_{g}=D B \psi_{g} .
\end{gathered}
$$

The first option is the recommended option. Option 2 treats only the current in the primary direction. Option 3 will always be positive and is a weighting over the total leakage from the system. Option 4 is sometimes referred to as a "bootstrap" approximation. Option 5 is equivalent to that used in codes that employ $B_{n}$ theory.

Once the currents are determined, the transport values are determined as set forth in the equations discussed above. For example, consider cell weighting and the "inscatter" approximation,

$$
\sigma_{t r}^{G}=\frac{\sum_{j} N^{j} \sum_{g \in G}\left[J_{g} \sigma_{t}^{g}-\frac{1}{3} \sum_{g^{\prime}} \sigma_{1}\left(g^{\prime} \rightarrow g\right) J_{g^{\prime}}\right]}{\bar{N} \sum_{j}^{I Z M} \sum_{g \in G} J_{g}} .
$$

For cell weighting and the "outscatter" approximation,

$$
\sigma_{t r}^{G}=\frac{\sum_{j} N^{j} \sum_{g \in G} J_{g}\left[\sigma_{t}^{g}-\frac{1}{3} \sum_{g^{\prime}} \sigma_{1}\left(g \rightarrow g^{\prime}\right)\right]}{\bar{N} \sum_{j}^{I Z M} \sum_{g \in G} J_{g}} .
$$




\section{XSDRNPM.2 XSDRNPM Input Data}

Title Card-Format (20A4)

This is the title card for the problem. It will be used to label the problem output.

\section{Block 1.}

This block contains information to set up various array dimensions and most calculational and editing options. Various convergence criteria and special constants can be input.

$-1 \$$ Core Allocation [1]

1. NWORD - Number of words of core to use for the calculation (100000)

$0 \$$ Logical Assignments [11]

1. LPUN - Logical number for punched card output (7)

2. LRSF - Random access scratch for fluxes (10)

3. LAWL - Input AMPX working library (4)

4. LANC - ANISN binary or CCCC ISOTXS library (20)

5. LOWL - Output weighted library (3)

6. LANG - Angular flux scratch file (16)

7. LSF1 - Sequential scratch space (17)

8. LSF2 - Sequential scratch space (18)

9. LSF3 - Sequential scratch space (19)

10. LRSM - Random access scratch for macroscopic cross sections (8)

11. LRSX - Random access scratch for macroscopic cross sections (9)

$1 \$$ General Problem Description [15]

1. IGE - Problem geometry (1)

0 - homogeneous (this causes a $B_{N}$ calculation to be made for all zones)

1 - slab

2 - cylinder

3 - sphere

2. IZM - Number of separate material regions or zones (1)

3. IM - Number of spatial intervals in the problem (1)

4. IBL - Boundary condition at the left-hand boundary of the system (1)

0 - vacuum boundary

1 - reflected boundary

2 - periodic boundary

3 - white/albedo boundary

5. IBR - Boundary condition at the right-hand boundary of the system (1)

0 - vacuum boundary

1 - reflected boundary

2 - periodic boundary

3 - white/albedo boundary

6. $\mathrm{MXX}-$ Number of compositions used in the problem mockup 
7. MS - Number of entries in the mixing table which specifies the makeup of the MXX compositions

8. ISN - Order of angular quadrature to be used. If ISN $>0$, XSDRNPM will calculate an angular quadrature for the appropriate geometry. If ISN $<0$, the calculation is bypassed, and the user can supply a set in the $42^{*}$ and $43^{*}$ arrays.

9. ISCT - Order of scattering. Flux moments will be calculated through this order.

10. IEVT - Type of calculation (1)

0 - fixed source

1 - $\mathrm{k}$ calculation

$2-\alpha$ calculation (flux is assumed to have an $e^{-\alpha t}$ time variation)

3 - inoperable in present version

4 - zone width search

5 - outer radivis search

6 - buckling sich

7 - direct buckling search

11. IIM - Inner iteration maximum used in an $S_{n}$ calculation (10)

12. ICM - Outer iteration maximum (10)

After ICM outer iterations, the problem will be forced into the termination phase, and the program will continue as if full convergence was attained. A message to this effect is printed.

13. ICLC - Theory option (0)

0 - use $S_{n}$ theory always

$\mathrm{N}$ - use alternative theory (diffusion, infinite medium, or $B_{n}$ for $N$ outer iterations, after which change to $S_{n}$ theory

$-\mathrm{N}$ - always use alternative theory

14. ITH - Forward/adjoint selector (0)

0 - solve the forward Boltzmann equation

1 - solve the adjoint Boltzmann equation

15. IFLU - Not used (0)

$2 \$$ Editing and Special Options [10]

1. IPRT - Fine-group mixture cross-section edits $(-1)$

-2 - no edits

-1 - edit 1-D cross sections

0 - edit through $P_{N}$ cross sections

$N$ - 1-D edits are also made

2. ID1 - Flux editing options (0) 
The flux editing options are:

$$
\text { Print Print Punch }
$$

ID1 angular fluxes scalar fluxes scalar fluxes

$\begin{array}{cccc}-1 & \text { No } & \text { No } & \text { No } \\ 0 & \text { No } & \text { Yes } & \text { No } \\ 1 & \text { Yes } & \text { Yes } & \text { No } \\ 2 & \text { No } & \text { Yes } & \text { Yes } \\ 3 & \text { Yes } & \text { Yes } & \text { Yes }\end{array}$

Note that |IDI| $>3$ fluxes are written on |IDI| in binary format. Fluxes will be punched in a format suitable for restarting an XSDRNPM calculation.

3. IPBT - Balance table edits (0)

$$
\begin{aligned}
& -1 \text { - none } \\
& 0 \text { - make fine-group balance tables } \\
& 1 \text { - make fine- and broad-group balance tables }
\end{aligned}
$$

4. ISX - Broad-group flux edit as a function of interval ( 0 )

$$
(0 / 1=\text { no/yes })
$$

5. ISEN - Outer iteration acceleration. Input a zero.

6. IBLN - Logical unit on which to output activity file (0)

7. NBANDS - Number of flux rebalance bands

$<0$, then the number of bands in the thermal range is the absolute value of NBANDS.

8. IFSN - If $>0$, means no fission source if IEVT $=0$

9. NBL - Logical unit on which to output balance table file (0)

10. IDM4 - Not used

The structure of the "activity" and the "balance table" files is described in Sect. 3.XSDRNPM.2.2.

$3 \$$ Various Options [12]

1. IFG - Cross-section weighting (0)

0 - none required

1 - collapse cross sections

2. IQM - Volumetric sources (0)

0 - none

$\mathrm{N}-\mathrm{N}$ volumetric source spectra will be input in the $31^{*}$ array

3. IPM - Boundary sources ( 0 )

0 - none

$\mathrm{N}-\mathrm{N}$ boundary source spectra will be input in the $32^{*}$ array

4. IFN - Starting guess ( 0$)$

0 - flux guess (33\# array)

1 - fission density guess (34\# array)

$\mathrm{N}>3$ - unit on which fluxes are stored in a binary format 
The binary flux tape is written by XSDRNPM at the end of the problem in a routine called WRTF. The format is as follows, with one record per energy group, beginning with group 1:

$(\mathrm{XN}(\mathrm{i}), \mathrm{i}=1, \mathrm{IM})$,

$(\mathrm{XJ}(\mathrm{i}), \mathrm{i}=1, \mathrm{IMT})$,

(B(i), $i=1, M M)$,

where $\mathrm{XN}$ is the scalar flux, IM is the number of intervals, $\mathrm{XJ}$ contains the flux moments (higher than order zero) stacked one after the .... er for IM intervals, with IMT moments, and B is the boundary angular Hux for the $M M$ angles in the quadrature for the problem. In the case of a $P_{0}$ calculation, only the XN array is written.

5. ITMX - Maximum time allowed for the flux calculation in minutes. A value of zero specifies that the calculation should $\boldsymbol{\Gamma}$. be terminated because of time; otherwise the problem will bi corced into the termination phase when ITMX is exceeded (0). Bear in mind that this is an internal timing check and has no connection with operator or system terminations due to excessive times.

6. IDAT1 - External data storage. If IDAT1 is input as zero, XSDRNPM will automatically select the most efficient means of storing data.

0 - keep all arrays in core if possible

1 - store mixture cross sections externally on a direct access device

2 - store cross sections and fixed sources externally on direct access devices

3 - store cross sections, fixed sources, and flux moments externally on direct access devices

7. IPN - Diffusion coefficient option for transverse leakage corrections (0)

0 - determine a transport cross section for each zone using $P_{0}$ and $P_{1}$ cross sections and, hence, a diffusion coefficient from $1 / 3 \sum_{t r}$

1 - spatially average the diffusion coefficients determined as for the above option and use it for all zones

2 - spatially average the $\mathrm{tr}^{-}$. sport cross sections for all zones and determine a diffusion ( efficient to be used in all zones by taking one over three times this value

Typically, the first option (IPN =0) is adequate; however, in cases involving regions of low concentration (near void) and, hence, very low-transport cross sections, the very large diffusion coefficients lead to nonphysical behavior. In this case, the IPN $=2$ option has been used satisfactorily.

8. IDFM - Density factors (0)

0 - none

1 - read in density factors in the $38^{*}$ array

9. IAZ - Activity calculation trigger (0)

0 - none 
$\mathrm{N}$ - calculate the reaction rates by material zone for $\mathrm{N}$ different processes specified in the $49 \$$ and $50 \$$ arrays

10. IAI - Spatially dependent activity rates $(0)$

0 - none

1 - calculate reaction rates in each interval for IAZ processes

11. IFCT - Thermal upscatter scaling (0)

0 - none

1 - use upscatter scaling for accelerated problem convergence

12. IPVT - Parametric eigenvalue search (0)

0 - none

1 - a search calculation will be made for an eigenvalue other than 1.0

$4 \$$ Cross-Section Weighting Options [9] $\{$ IFG $=1\}$

1. ICON - Type of weighting

$-\mathrm{N}$ - inner cell (with $\mathrm{N}$ zones in the cell). Cell weighting is performed over the $\mathrm{N}$ innermost regions in the problem.

Nuclides outside these regions are not weighted.

$$
-1 \text { - cell }
$$

0 - zone

1 - region or vein

2. IGMF - Number of energy groups in the collapsed sets

3. ITP - Collapsed output format desired

0-19- cross sections are written only in the AMPX weighted library formats on logical 3. A weighted library is always written when $I F G=1$.

20-29 - output in the CCCC ISOTXS version 3 format

30-39 - output in the ANISN/DOT/MORSE card format

40-49 - output in the ANISN/DOT/MORSE binary format on logical 20

The various values of ITP (module 10) are used to select the different transport cross-section weighting options mentioned earlier. The options are

$\mathrm{ITP}=0,10,20, \ldots \sqrt{\left(\psi_{1}^{g}+\left(D B \psi_{g}\right)^{2}\right.}$

ITP $=1,11,21, \ldots$ absolute value of current

ITP $=2,12,22, \ldots D B^{2} \psi_{g}+$ outside leakage

ITP $=3,13,23, \ldots \psi / \sum_{t}^{g}$

ITP $=4,14,24, \ldots D B \psi_{g}$

ITP $=$ other values are reserved for future development and should not be used

4. IPP - Weighted cross-section print option $(-1)$

-2 - none

-1 - print 1-D data

$\mathrm{N}$ - print through $P_{N}$ cross-section arrays (1-D edits are given) 
5. IHTF - Total cross-section position in the ANISN format or the number of $\mathrm{CCCC}^{5}$ formatted cross-section sets to be output (3)

6. NDSF - Within-group scattering cross-section position in the ANISN format (4)

7. NUSF - Table length in the ANISN formats (IGMF + NDSF - 1)

8. IAP - ANISN cross-section edit option or the scattering order to be output on a CCCC ISOTXS file $(-1)$

-1 - ANISN (do not produce edits of ANISN formatted cross sections)

CCCC (output all orders on the ISOTXS file)

$$
\left\{\frac{0}{N}\right\}-\left(\frac{\text { edit }- \text { ANISN }}{\text { output }- \text { CCCC }}\right) \text { through the } P_{N} \text { arrays. }
$$

9. MSCM - Extra "activity" cross sections in ANISN formats. MSCM extra cross-section types are put above the absorption cross-section position in the ANISN formats. The types are specified in the $12 \$$ array.

5* Convergence Criteria and Assorted Constants [12]

1. EPS - Overall problem convergence $\left(10^{-4}\right)$

2. PTC - Scalar flux convergence $\left(10^{-4}\right)$

3. XNF - Normalization factor (1.0)

When IEVT $=0$, the fixed sources are normalized to XNF

For IEVT $>0$, the fission source is normalized to XNF

When XNF $=0.0$, no normalization is made

4. EV - Starting eigenvalue guess for search calculations

5. EVM - Eigenvalue modifier used in a search calculation

The following is a tabulation of recommended values for $\mathrm{EV}$ and EVM:

\begin{tabular}{|c|c|c|c|}
\hline IEVT & Calculation type & EV & EVM \\
\hline 0 & Fixed source & 0 & 0 \\
\hline 1 & k-calculation & 0 & 0 \\
\hline 2 & Direct $\alpha$-search & 0 & 0 \\
\hline 3 & Not used & - & - \\
\hline 4 & Zone width search & 0 & -0.1 \\
\hline 5 & Outer radius search & $\begin{array}{c}\text { Starting } \\
\text { outer radius }\end{array}$ & $-0.1 * \mathrm{EV}$ \\
\hline 6 & Buckling search & 1.0 & -0.1 \\
\hline 7 & Direct buckling search & 0.0 & 0.0 \\
\hline
\end{tabular}

6. BF - Buckling factor (1.420892)

This parameter is two times the multiplier on the "extrapolation" distance used to determine where a linearly extrapolated line from 
the asymptotic flux shape would go to zero (e.g., for slabs, the extrapolation distance is $\simeq 0.71 \lambda_{\text {ir }}$ and, hence, $\mathrm{BF} \simeq 1.42$ ).

7. DY - First transverse dimension in centimeters used in buckling correction to calculate leakage normal to the principal calculation direction (i.e., the height of a slab or a cylinder)

8. DZ - Second transverse dimension in centimeters used for a buckling correction (i.e., the width of a slab)

9. VSC - Void streaming correction

This is the height of a void streaming path in a cylinder or slab in centimeters.

10. PV - Parametric eigenvalue When IPVT $=1$ and IEVT $>1$, this is the value of k-effective on which the search calculation is to be made.

11. EQL - Eigenvalue convergence for a search $\left(10^{-3}\right)$

12. XNPM - New parameter modifier used in search calculations (0.75)

T Terminate Block 1.

\section{Block 2.}

This block contains information about the composition of the materials used in the calculation. Also included is an array to select special cross sections for ANISN and an array to identify cross sections written on the CCCC ISOTXS library.

$10 \$$ CCCC Transport Cross-Section Selection [IHTF]

This array contains the process identifier for the transport cross section which is to be used on the CCCC ISOTXS library. It is defaulted to 1001 for all nuclides, which selects the "inscatter" or "consistent" transport values. The other option is 1000 , which selects the "outscatter" values. Use of this array can become necessary when negative transport values are encountered, such as is the case in some problems with hydrogen in its lower energy groups. This array is input in a one-to-one correspondence with the $16 \$$ and $18 \mathrm{U}$ arrays.

$12 \$$ Special Cross-Section ENDF/B Identifiers for Inclusion in ANISN Libraries [MSCM]

This option allows the placement of "activity" cross sections in positions 1 through MSCM of ANISN formatted data. The user must ensure that MSCM is consistent with other positional parameters given in the $4 \$$ array. Use ENDF MT numbers to select processes.

The next arrays (13\$, $14 \$$, and $15^{*}$ ) comprise the mixing table in XSDRNPM. The entries in the arrays go together in a one-to-one correspondence to form triplets:

$$
\frac{13 \$}{\mathrm{M}} \frac{14 \$}{\mathrm{~N}} \frac{15 *}{\mathrm{X}}
$$

This command instructs that nuclide $\mathrm{N}$ is to be added into mixture $\mathrm{M}$ with a concentration (atoms $/ \mathrm{b}-\mathrm{cm}$ ) of $\mathrm{X}$.

$13 \$$ Mixture Numbers in Mixing Table [MS] 
The values range from 1 to $\mathrm{MXX}$.

$14 \$$ Isotope Identifiers in Mixing Table [MS]

A set of data with this identification must be on logical 4, the XSDRNPM working

library, though the code will not make checks to ensure this is the case.

15* Isotope Concentrations in Mixing Table [MS]

$16 \$$ CCC Set Selection Identifiers [IHTF]

These are the identifiers for the sets of cross sections on the AMPX weighted library that are to be put on the CCCC ISOTXS library.

$18 \mathrm{U}$ CCCC Set Identifiers [IHTF]

Cross-section sets on the CCCC ISOTXS library are identified by six-character Hollerith strings (e.g., PU239 could be used for ${ }^{239} \mathrm{Pu}$, etc.). Since IBM singleprecision word lengths will only hold four characters, this array will require the reading of two times IHTF words. One way to accomplish this is to use the "variable format" (U) option, whereby, the user specifies a two-word format for each six-character string. For example, one can use the following scheme to read these data:

$18 \mathrm{U}$

$((12(\mathrm{~A} 4, \mathrm{~A} 2)))$

Enter 12 identifiers per card as if they were read in a " $12 \mathrm{~A} 6$ " format.

A second way to accomplish reading this array is to use the Hollerith field option, where one could use:

$18 \#$ \# 6Hxxxxxx 6Нyyyyyy .......

where $\operatorname{xxxxxx}$, yyyyyy, etc., are the identifier strings. This array is input in a one-to-one ordering with the $10 \$$ and $16 \$$ arrays.

$\mathrm{T}$ Terminate Block 2.

Block 3. $\quad\{$ IEVT $=0\}$

This block is used to specify fixed sources.

$30 \$$ Source Spectrum Number by Interval [IM]

31* Volumetric Source Spectra [IQM*IGM]

32* Surface Source Spectra [IPM*IGM*MM]

Each of the IQM or IPM spectra is specified in the $31^{*}$ or $32^{*}$ array and are stacked one after the other in that array. If both volumetric and surface sources are used in the same problem, the surface source number is multiplied by (IPM + 1) when entered in the $30 \$$ array.

A volumetric spectrum will consist of IGM (number of energy groups) entries that are the relative integrated values of the source in each group.

A surface source is always assumed to be on the right-hand side of a spatial interval. It is input as was the volumetric source, except that each group contains 
entries for the MM angles in the $S_{n}$ quadrature chosen for the problem. Note that a surface source is an integrated value and is actually a flux condition in the $S_{n}$ equations.

In the $30 \$$ array, a zero entry specifies that no source is in an interval.

T Terminate Block 3 .

\section{Block 4.}

This data block contains starting guesses for fluxes and fission densities. If fluxes are read from an external device (IFN $>3$ ), this data block is omitted. The binary flux tape is written by XSDRNPM at the end of the problem in a routine called WRTF. The format is as follows, with one record per energy group, beginning with group 1:

$$
\begin{aligned}
& (\mathrm{XN}(\mathrm{i}), \mathrm{i}=1, \mathrm{IM}), \\
& (\mathrm{XJ}(\mathrm{i}), \mathrm{i}=1, \mathrm{IMT}), \\
& (\mathrm{B}(\mathrm{i}), \mathrm{i}=1, \mathrm{MM}),
\end{aligned}
$$

where $\mathrm{XN}$ is the scalar flux, IM is the number of intervals, $\mathrm{XJ}$ contains the flux moments (higher than order zero) stacked one after the other for IM intervals, with IMT moments, and B is the boundary angular flux for the MM angles in the quadrature for the problem. In the case of a $\mathrm{P}_{0}$ calculation, only the $\mathrm{XN}$ array is written. Both arrays in this block are double-precision arrays, which will require the use of the "\#" array designator; otherwise, the number of entries read into the arrays will be incorrect or may contain nonsensical values for the starting guess.

33 沛 Flux Guess [IM*IGM] $\{$ IFN $=0\}$

A guess for the scalar flux is specified in the order:

( $(F L U X(I, J), I=1, I M), J=1, I G M)$, where IM is the number of spatial intervals and IGM is the total number of energy groups. For fixed-source problems, without better information, use zeroes. For eigenvalue problems, a nonzero flux guess must be used. The fluxes punched by using the ID1 parameter in the $2 \$$ array can use $d$ here in restart calculations.

34 \# Fission Density Guess [IM] $\{$ IFN $=1\}$

This is a guess at the number of fission neutrons produced in an interval. When IFN $=1$, XSDRNPM uses diffusion theory for the first outer iteration, after which it reverts to the normal mode.

T Terminate Block 4.

\section{Block 5.}

This block contains the remaining data needed for an XSDRNPM calculation.

35* Interval Boundaries [IM+1] $(\mathrm{cm})$

This array describes the spatial quadrature into which the problem mock-up is divided. The boundaries are nonnegative and in increasing order. Usually they will start with a zero value, though this is not necessary.

$36 \$$ Zone Number for Each Spatial Interval [IM]

Spatial zones should be contiguous.

\section{XSDRNPM}


38* Density Factors by Interval [IM] \{IDFM $\neq 0\}$ (1.0)

These factors are used to effect a density variation in a mixture as a function of spatial interval. Zero for a density factor affords a convenient way for mocking a void region.

$39 \$$ Mixture Numbers by Zone [IZM]

The mixture that is in a zone is specified here.

$40 \$$ Order of Scattering by Zone [IZM] (ISCT)

This is the order, $l$, of the $S_{n} P_{l}$ calculation, which is desired in a zone. This number should be no larger than the ISCT.

$41^{*}$ Radius Modifiers by Zone [IZM] $\{$ IEVT $=4\}$

These parameters specify the relative movement of the width of a zone in a zone width search. A zero indicates that a zone's width is fixed.

42* Weights of the Angles in the Discrete-Ordinates Quadrature [MM*]

Input this array if you wish to override those provided by XSDRNPM.

43* Cosines of the Angles in the Discrete-Ordinates Quadrature [MM]

Input this array if you wish to override those provided by XSDRNPM.

$46 \$$ Calculational Option by Group [IGM] \{ICLC $>0\}$

0 - Perform discrete-ordinates calculation for this group

1 - Perform a diffusion calculation for this group for ICLC outer iterations and then use discrete-ordinates theory

2 - Perform a homogeneous calculation for this group for ICLC outer iterations and then use discrete-ordinates theory

3 - Perform a homogeneous calculation using $B_{n}$ theory for this group for ICLC outer iterations and then use discrete-ordinates theory

47* Right-Boundary Albedos by Group [IGM] \{IBR = 3\} (1.0)

A right-boundary albedo is specified for each fine group. The return current is distributed isotropically in angle.

48* Left-Boundary Albedos by Group [IGM] $\{\mathrm{IBL}=3\}$ (1.0)

As for the $47^{*}$ array but for the left boundary. Note that if IBR or IBL is 3 and the corresponding $47^{*}$ or $48^{*}$ array is omitted, XSDRNPM fills the array with 1.0 's, effecting a boundary with zero net current and with isotropic neutron return.

$49 \$$ Material Number for Activities [IAZ] $\{$ IAZ $\neq 0\}$

$50 \$$ Process Number for Activities [IAZ] $\{$ IAZ $\neq 0\}$

${ }^{*} \mathrm{MM}=\mathrm{ISN}+1$ for slabs and spheres; MM $=1 S N^{*}(\mathrm{ISN}+4 / 4)$ for a cylinder. 
The $49 \$$ and $50 \$$ arrays provide a means of obtaining the activity (reaction rate) for any process for which cross sections are available in the XSDRNPM calculation. A representative activity table entry is shown below:

$\begin{array}{cc}\text { ACTIVITY } & \text { TABLE ENTRY } \\ 49 \$ & 50 \$ \\ \mathrm{M} & \mathrm{N}\end{array}$

This entry specifies that the activity $\mathrm{N}$ for material $\mathrm{M}$ be calculated for all parts of the system which contain that material.

If $\mathrm{N}$ is $<0$, a density of 1.0 is used to calculate activities instead of densities in the mixing table. Allowable process identifiers are given in Sect. 4.MISCELLANEOUS.6.

$51 \$$ Broad Group Numbers [IGM] $\{$ IFG $\neq 0\}$

This array contains the broad-group numbers into which the fine groups are collapsed in a flux-weighting calculation. For example, if the first five fine groups are to be collapsed to the first broad group, the first entries in the $51 \$$ array are 1 , etc. A zero value can be used to ignore (or truncate) a group.

$52 \$$ Lower Band Group Numbers [NBANDS]

Group numbers giving the last group in a flux rebalance band. Overrides the default set supplied by XSDRNPM.

\section{XSDRNPM.2.1 Abbreviated XSDRNPM input description}

After several problem setups have been made for XSDRNPM, the user will probably establish a familiarity, such that the more detailed notes given above are not necessary or desirable. Because of this, the following abbreviated list is given which will, hopefully, benefit many users.

Title card - (18A4)

DATA BLOCK 1

$0 \$$ - Logical assignments [11]

1. Punched cards (7)

2. Direct access - fluxes (10)

3. Working library (4)

4. ANISN or ISOTXS (20)

5. Weighted library (3)

6. Angular fluxes (16)

7. Scratch (17)

8. Scratch (18)

9. Sratch (19)

10. Direct access (8)
1\$- General description [15]

1. IGE - geometry

2. IZM - number of zones

3. IM - number of intervals

4. IBL - left boundary condition

5. IBR - right boundary condition

6. MXX - number of mixtures

7. MS - mixing table length

8. ISN - angular quadrature

9. ISCT - order of scattering

10. IEVT - problem type

\section{XSDRNPM}


11. Direct access (9)

$2 \$$ - Editing and control options [10]

1. Fine-group mixture edit

2. Fine-group flux edit

3. Balance table edit

4. Broad-group flux edit

5. Zero

6. Logical unit for activities

7. Number of bands

8. Suppress fixed source fission

9. Logical unit for balance tables

10. Zero

3\$- Other options [12]

1. IFG - weighting option

2. IQM - volumetric sources

3. IPM - boundary sources

4. IFN - starting guess

5. ITMX - time shut-off

6. IDAT1 - storage scheme

7. IPN - diffusion coefficient option

8. IDFM - density factors

9. IAZ - activities by zone

10. IAI - activities by interval

11. IFCT - thermal scaling

12. IPVT - search on $\mathrm{k} \neq 1$

$5^{*}$ - Floating point values [12]

1. EPS - overall convergence

2. PTC - point flux convergence

3. XNF - normalization

4. $\quad E V$ - starting guess for search

5. EVM - modifier for search

6. BF - buckling factor

7. DY - height

8. $\mathrm{DZ}$ - width

9. VSC - void streaming height

10. $\mathrm{PV}-\mathrm{k}$ for search
11. IIM - inner-iteration maximum

12. ICM - outer-iteration maximum

13. ICLC - optional theory

14. ITH - forward or adjoint

15. IFLU - zero
4\$- Weighting options [9]

1. Type of weighting

2. Number of broad groups

3. Output format

4. Edit option

5. $\sigma_{T}$ position or number CCCC

6. $\sigma_{g g}$ position

7. Table length

8. ANISN edit option

9. Extra cross sections 
11. EQL - search convergence

12. XNPM - search modifier

T Terminate Block 1.

\section{DATA BLOCK 2}

$10 \$$ CCCC transport cross-section selector [IHTF]

$12 \$$ Additional processes to be put on ANISN library [MSCM]

$13 \$$ Mixture numbers [MS]

$14 \$$ Isotope identifiers [MS]

$15 \$$ Isotope concentrations [MS]

$16 \$$ CCCC identifiers from working library [IHTF]

$18 \mathrm{U}$ or 18 \# CCCC identifiers on ISOTXS [IHTF]

T Terminate Block 2.

\section{DATA BLOCK 3}

Required only when IQM or IPM is nonzero.

$30 \$$ Spectrum number by interval (IQM $>0$ ) or

right-hand interval boundary (IPM $>0)$ [IM]

$31^{*}$ Volumetric sources [IQM*IGM]

32* Boundary sorsices [IPM*IGM*MM]

T Terminate Block 3.

\section{DATA BLOCK 4}

When fluxes are read from an external device-IFN $>3$-this block is omitted.

$33 \#$ Flux guess [IM*IGM] $\{\mathrm{IFN}=0\}$

$34 \#$ Fission density guess [IM] $\{$ IFN $=1\}$

T Terminate Block 4.

\section{DATA BLOCK 5}

35* Interval boundaries [IM+1]

$36 \$$ Zone numbers by interval [IM]

38* Density factors [IM]

$39 \$$ Mixture number by zone [IZM]

$40 \$$ Order of scattering by zone [IZM]

$41^{*}$ Radius modifier by zone [IZM] IEVT=4

42 * Discrete-ordinates cosines [MM]

$43^{*}$ Discrete-ordinates weights [MM] 
$46 \$$ Alternate theory selection [IGM] ICLC $>0$

$47^{*}$ Right boundary albedos [IGM]

$48^{*}$ Left boundary albedos [IGM]

$49 \$$ Activity material or nuclide numbers [IAZ]

$50 \$$ Activity process numbers [IAZ] (see Appendix B).

$51 \$$ Broad group numbers [IGM] IFG $>0$

$52 \$$ Lower band group numbers [NBANDS]

$\mathrm{T}$ Terminate Block 5.

\section{XSDRNPM.2.2 Special XSDRNPM files}

Two special files that can be optionally produced by XSDRNPM are described in this section. (See Sect. 3.XSDRNPM.2 and the discussion of parameters in the $2 \$$ array.)

\section{XSDRNPM.2.2.1 Activity file}

The following is the record structure of the activity file which can be produced on logical unit IBLN.

Record 1 - (Title(I),I=1,18),IGM,NNEUT,NGAMM,IM,IAZ,IAI

1. Title -

2. IGM -

3. NNEUT -

4. NGAMM -

5. IM -

6. IAZ -

7. IAI -

Record $2-(\mathrm{EN}(\mathrm{I}), \mathrm{I}=1, \mathrm{NNEUT}+1)$

When NNEUT $>0$, this record contains the energy boundaries for the neutron group structure (high to low).

Record 3 - (EG(I),I=1,NGAMM+1)

When NGAMM $>0$, this record contains the energy boundaries for the gamma group structure (high to low).

Record 4 - (R(I),I=1,IP)

Radii corresponding to all interval boundaries in the system starting at the left side of the system.

Record 5 - (MA(I),I=1,IM)

Zone numbers by interval.

Record 6 - (J3(I),I=1,IAZ)

"Material" numbers for activities.

(This is the $49 \$$ array.)

Record 7 - (J4(I),I=1,IAZ) 
"Process" identifiers for activities. (This is the $50 \$$ array.)

These seven records are followed by IAZ records which have the following structure and contain the activities as a function of interval:

$$
(\mathrm{ACT}(\mathrm{I}), \mathrm{I}=1, \mathrm{IM})
$$

\section{XSDRNFM.2.2.2 Balance table file} $>0$ is:

The structure of the "balance table file" to be written on logical NBL when NBL

Record 1 - AKEFF, SP, IZP, IGP

1. AKEFF - $k_{\text {eff }}$ for problem

2. SP - search parameter for case

3. IZP - number of zones plus one

4. IGP - number of groups plus one

Record 2

\section{XSDRNPM.3 XSDRNPM Input/Output Specifications}

The following logical units can be required in an XSDRNPM calculation:

\begin{tabular}{cll} 
Logical No. & \multicolumn{1}{c}{ Purpose } \\
\cline { 1 - 1 } 3 & & Weighted library (produced by XSDRNPM) \\
4 & & Working library (input) \\
5 & & Card input \\
6 & & Standard output \\
7 & & Punch fluxes or ANISN libraries \\
8 & Scratch direct access device for external \\
& cross-section storage \\
9 & Scratch direct access device for mixing and \\
10 & weighting operations \\
& Scratch direct access device for external \\
16 & Alux moment storage \\
17 & Scratch device \\
18 & Scratch device \\
19 & Scratch device \\
20 & ANISN binary library or CCCC ISOTXS \\
& interface
\end{tabular}




\section{XSDRNPM.4 Error Messages}

During the course of a problem, XSDRNPM makes many checks to determine if input data is in the required form. If inconsistencies are spotted, a message is printed, and the problem may be terminated. Some of these messages are listed below, along with a brief description of their possible cause.

CORE $\mathrm{N}$ Insufficient core storage, $\mathrm{N}=$ required storage locations

DATA $N \quad N$ arrays have been input with incorrect length. See the messages produced as arrays are read to determine specific arrays.

SN-1 N The Nth entry in the $S_{n}$ quadrature direction is zero (43* array)

SN-2 $0 \quad$ The $S_{n}$ weights do not sum to 1.0 (42* array)

SN-3 0 The sum of the products of $S_{n}$ weights and directions is not 0.0 [i.e., the directions are not symmetric ( $42^{*}$ and $43^{*}$ arrays)]

Cause: Self-explanatory. Increasing IDT1 may correct this difficulty.

SUMMARY TABLE STORAGE INSUFFICIENT SYSTEM TREATED AS ONE ZONE

Cause: Insufficient core is available to set up all the arrays needed for balance tables. Increasing IDT1 may correct this difficulty.

TAPE $N$ Insufficient storage is available to produce a library tape for another code in the form specified. $\mathrm{N}$ locations needed.

***** CAUTION ${ }^{* * * * *}$ CAUTION ${ }^{* * * * *}$ CAUTION

THIS CELL HAS ZERO LEAKAGE-THE SCALAR FLUX WILL BE USED TO WEIGHT CROSS SECTIONS

Cause: The definition of a transport cross section requires a leakage spectrum. A completely reflected cell has been specified.

\section{REFERENCE}

1. N. M. Greene and C. W. Craven, Jr., XSDRN: A Discrete Ordinates Spectral Averaging Code, ORNL/TM-2500, Union Carbide Corp., Nucl. Div., Oak Ridge Natl. Lab., July 1969. 


\section{ZEST - A MODULE TO MANAGE STRING LIBRARIES}

ZEST ( Zipp Ensembler of STrings) is a module that is analogous to AJAX, except ZEST uses "strıng" libraries, suci as the libraries produced by NPTXS. A string is a TAB1 record in ENDF nomenclature. Options are provided to allow merging from any number of files in a manner to allow the user to determine the final nuclide ordering, if desired.

\section{ZEST.1 ZEST Input}

\section{Block 1.}

$-1 \$$ Core Assignment [1]

1. NWORD - Number of words to allocate to ZEST (50000)

$0 \$$ Logical Assignnents [2]

1. LOG - Logical number of library to be written (31)

2. LBIG - Logical number of string library (input) with largest block size (0)

$1 \$$ Library Selector [1]

1. NLOG - Number of "commands" (or libraries) required to create LOG

$\mathrm{T}$ Terminate Block 1 .

Blocks 2 and 3 are stacked, one after the other, NLOG times.

\section{Block 2.}

$2 \$$ Input Library Selection

1. NLIN = Logical number of input library

2. $\mathrm{NC}=-\mathrm{N}$ Delete $\mathrm{N}$ strings from NLIN to create LOG

0 Accept all strings from NLIN which have not been placed on LOG by a previous command

$\mathrm{N}$ Add $\mathrm{N}$ strings from NLIN to create LOG

T Terminate Block 2.

Block 3. $\{$ Required only if $\mathrm{NC} \neq 0)$ \}

$3 \$$ MAT Numbers From NLIN (0 selects all materials)

$4 \$$ MF Numbers From NLIN (0 selects all files)

$5 \$$ MT Numbers From NLIN (0 selects all processes)

Note that a triplet of values in the 3,4 , and $5 \$$ arrays selects a string from NLIN. If the identifiers are to be changed, enter the new numbers in the corresponding locations in the 6,7 , and $8 \$$ arrays:

$6 \$$ New MAT Numbers

$7 \$$ New MF Numbers 
$8 \$$ New MT Numbers

Note that a zero value or the omission of values from the 6,7 , and $8 \$$ arrays will leave the old identifiers intact.

T Terminate Block 3.

\section{ZEST.2 ZEST Input/Output Specifications}

The following devices are normally needed to execute ZEST:

\begin{tabular}{cll}
$\frac{\text { Logical No }}{5}$ & & \multicolumn{1}{c}{ Purpose } \\
6 & & Pard input \\
Printed output \\
NLG (31) & String library to be created \\
NIIN & String libraries to be merged onto LOG
\end{tabular}




\section{AMPX-77 MISCELLANEOUS USEFUL INFORMATION}

This section contains descriptions of several AMPX features, including:

Subsection

4.INFORMATION.1

4.INFORMATION.2

4.INFORMATION.3

4.INFORMATION.4

4.INFORMATION.5

4.INFORMATION.6

4.INFORMATION.7
Topic

AMPX Master Library Format

AMPX Working Library Format

ANISN Library Format

Standard Energy Group Structures

Weighting Function Library

Reaction Type Identifiers

Glossary of Terms

\section{INFORMATION.1 AMPX MASTER LIBRARY FORMAT}

An AMPX master library is not structured for direct use in a multigroup particle transport calculation. The intent is to provide a means to present a library that has the potential of being problem independent, which can be tailored at run time for a particular problem. The library carries data at a sufficient level of detail to allow satisfying many of the less common, but very useful, analyses such as cross-section sensitivity studies and coupled neutron-gamma transport calculations. The library can contain data to allow resonance self-shielding to be performed using the Nordheim method, the Bondarenko method, or an integral transport calculation. Temperature dependence of data is allowed. Any number of scattering processes can be included to an arbitrary order of anisotropic representation.

The AMPX master format has been designed to allow a generality paralleling that of the ENDF/B point libraries, for example:

1. The formats can accommodate neutron libraries, gamma libraries, or coupled neutron-gamma libraries.

2. An arbitrary number of reaction cross sections can be included that generally use ENDF/B identifiers for processes (see Sect. 4.INFORMATION.6).

3. An arbitrary order of anisotropy can be presented which can vary from nuclide to nuclide or from process to process.

4. Temperature dependence is allowed.

5. Resonance parameters may be included for use in Nordheim resolved rfsonance calculations.

6. The library can contain Bondarenko data for resonance self-shielding.

7 . The library can include scattering matrix data for an arbitrary number of processes.

In the case of the resonance data, partial energy range data can be specified. For example, in CSRL-V the Bondarenko data are only for the unresolved region which will vary from nuclide to nuclide.

Potentially, the most space-consuming data on a cross-section library are the transfer matrix data. AMPX uses so-called "magic-word" arrays for these data which help to eliminate zero and/or impossible data elements. This is especially important for 
the master library, where the library may contain data for $\sim 50$ separate processes represented to an arbitrary level of anisotropy.

The master format is written using a combination of seven kinds of information, each of which has one or more record types associated with it:

1. Header information - written on the front of the library to specify the number of neutron and/or gamma groups, the number of nuclides, etc., contained in the library (record type 1).

2. Energy structure information - contains the group boundaries (record type 2).

3. Nuclide directory information - fifty words which give a title for the nuclide along with other parameters which specify the kinds of information included for the nuclide, such as number of records in the library for the nuclide and how much neutron and gamma data are given (record type 3 ).

4. Resonance parameter data - provisions for resolved resonance parameters in the SLBW or MLBW formats (record type 4).

5. Bondarenko data - four record types are used for this information:

a. A record that gives the values of $\sigma_{0}$ and temperature at which the factors are tabulated, along with cut-off energies for the Bondarenko calculation (record type 5).

b. A directory record containing information about the specific processes for which the Bondarenko factor data are given, such as the process, the energy groups for which data are given, etc. (record type 6).

c. A record containing infinite dilution values for a process (record type 7).

d. A record containing the Bondarenko factors for a process (record type 8).

6. A record containing average cross sections by process (record type 9 ).

7. Three record types are used to present transfer matrices:

a. A directory record that specifies the processes, orders of anisotropy, lengths, units, etc (record type 10).

b. A record to specify temperatures when the matrices are temperature-dependent (record type 11).

c. A "magic-word" record to store a transfer matrix (record type 12).

The structure of the various record types is now discussed.

\section{Record type 1 (header record)}

The header record is the first record on the library and contains 110 words:

1. IDTAPE - An identification number for the library

2. NNUC - The number of sets of data on the library

3. IGM - The number of neutron energy groups on the library

4. IFTG - The first thermal neutron group on the library (i.e., the first group that receives an upscatter source)

5. MSN - 0 - pre-NITAWL-II library

2 - library for use with NITAWL-II

6. IPM - The number of gamma-ray energy groups on the library

7. II - Zero 
8. I2 - $(0 / 1=$ no/yes $)$ A trigger that specifies that this library was produced by weighting a working library in the AMPX XSDRNPM module. This applies only to a working library, which will be discussed in

9. I3 - Zero Sect. 4.INFORMATION.2.

10. I4 - Zero

11.-110. ( $\operatorname{TITLE}(\mathrm{I}), \mathrm{I}=1,100)$ - One hundred words of text describing the crosssection library

\section{Record type 2 (energy boundaries)}

This record specifies the energy boundaries in $\mathrm{eV}$ of the neutron groups and/or gamma groups, followed by the corresponding lethargy boundaries. The energy boundaries are arranged in descending order, followed by the lethargy boundaries in ascending order. The "lethargy zero" is taken at $10 \mathrm{MeV}$. The structure is

$$
(\mathrm{EB}(\mathrm{I}), \mathrm{I}=\mathrm{I}, \mathrm{IGP}),(\mathrm{UB}(\mathrm{I}), \mathrm{I}=1, \mathrm{IGP})
$$

where IGP is the number of groups plus one.

\section{Record type 3 (cross-section set directory record)}

Each set of data has a 50-word directory record that specifies certain parameters needed to determine dimensions required to process the data and to describe the makeup of the set of data. The following table describes these data:

\begin{tabular}{|c|c|}
\hline Word(s) & Content \\
\hline $1-18$ & 18 words of text describing the set ( 72 characters) \\
\hline & Identifier of the set \\
\hline 20 & $\begin{array}{l}\text { Number of six-parameter sets of resolved } \\
\text { resonance data }\end{array}$ \\
\hline 21 & Zero \\
\hline 22 & $\begin{array}{l}\text { Number of } 1-\mathrm{D} \text { neutron processes (temperature } \\
\text { independent) }\end{array}$ \\
\hline 23 & Number of 2-D neutron processes \\
\hline 24 & Zero \\
\hline 25 & Number of 1-D gamma processes \\
\hline 26 & Number of 2-D gamma processes \\
\hline 27 & Number of 2-D neutron-to-gamma processes \\
\hline 28 & $\begin{array}{l}\text { (Maximum order of scattering) } * 32768+(\text { total } \\
\text { number of separate } 2-D \text { arrays for this set) }\end{array}$ \\
\hline 29 & A-neutron equivalent mass number \\
\hline 30 & $\mathrm{ZA}-1000 * \mathrm{Z}+\mathrm{A}$ \\
\hline 31 & Zero \\
\hline 32 & Zero \\
\hline 33 & Zero \\
\hline 34 & Power per fission in W-s/fission \\
\hline 35 & Energy release per capture in W-s/capture \\
\hline 36 & Maximum length of any 2-D record in the set \\
\hline
\end{tabular}


$37 \quad$ Number of sets of Bondarenko data

38 Number of $\sigma_{0}$ 's in Bondarenko data

39 Number of temperatures in Bondarenko data

40 Maximum number of groups in Bondarenko data

41 Zero

42 Zero

$43 \quad \sigma_{\mathrm{p}}$-potential scattering cross section

$44 \quad$ Zero

45 ENDF MAT for fast neutron data

46 ENDF MAT for thermal neutron data

47 ENDF MAT for gamma data

48 ENDF MAT for gamma production data

49 Nuclide symbol (4 characters of text)

50 Number of records in this set

Note that the 50-word records are made up of integer, character, and floating-point words. Words 1 to 18 and word 49 are character data. Words $29,30,34,35$, and 43 are floating point. All other words are integers. For both neutron and gamma libraries, many parameters may have no meaningful interpretation. Zero values are used when a parameter is not applicable.

\section{Record type 4 (resonance parameters)}

This record is present only when the number of six-word resonance sets, NRES, is nonzero. Its length is

$$
9+6^{*} \text { NRES } .
$$

All values are in floating point.

The first nine words contain parameters used in both the resolved and unresolved resonance calculations:

1. A, the mass ratio for the isotope or mixture of isotopes

2. $\sigma_{\mathrm{P}_{0}}$, the potential scattering cross section

3. Zero

4. NRES, the number of six-parameter resonance sets

5. S, a factor used in the Nordheim calculation to determine the range over which the calculation will be made

6. Zero

7. Zero

8. Zero

9. Zero

The next $6 *$ NRES words consist of six-word sets of data used in the Nordheim calculation.

1. Zero

2. NBLK, the number of blocks of resolved data

3. Zero

4. Zero

INFORMATION 
5. Temperature is Kelvin at which the infinite dilution cross sections were produced (if 0.0 , it defaults to $300 \mathrm{~K}$ ).

6. Zero

Following these six words are NBLK six-word groups, which are used to specify information concerning biocks of resonance data that apply to, for example, different isotopes, different energy regions, different kinds of data ( $\mathrm{s}$-wave or $\mathrm{p}$-wave resonances), etc. These word groups are stacked as follows:

1. AWR, mass ratio for this isotope

2. ABUN, the abundance for the block of data

3. NRE, the number of resonances in the block

4. $\ell$, the value of spin for the resonances in the block

5. EL, the lower energy cutoff for resonances in the block

6. EH, the upper energy cutoff for resonances in the block

After the NBLK groups of six words, the six-word sets of resonance parameters are arranged as follows:

1. $E_{0}$, the resonance energy

2. $\Gamma_{\mathrm{n}}$, the neutron width of the resonance

3. $\Gamma_{\gamma}$, the gamma width of the resonance

4. $\Gamma_{\mathrm{f}}$, the fission width of the resonance

5. $r$, a factor used in the Nordheim treatment for determining the range of calculation

6. $\mathrm{g}$, the statistical factor for the resonance

The following chart illustrates the structure just described: 


\begin{tabular}{|c|c|c|c|c|c|}
\hline 1 & 2 & 3 & 4 & 5 & 6 \\
\hline 0 & NBLK & 0 & 0 & TEMP & 0 \\
\hline $\mathrm{AWR}_{1}$ & $\mathrm{ABUN}_{1}$ & $\mathrm{NRE}_{1}$ & $\ell_{1}$ & $\mathrm{EL}_{1}$ & $\mathrm{EH}_{1}$ \\
\hline $\mathrm{AWR}_{2}$ & $\mathrm{ABUN}_{2}$ & $\mathrm{NRE}_{2}$ & $\ell_{2}$ & $\mathrm{EL}_{2}$ & $\mathrm{EH}_{2}$ \\
\hline $\mathrm{AWR}_{\mathrm{NBLK}}$ & $A B U N_{N B L K}$ & $N R E_{N B L K}$ & $\ell_{\text {NBLK }}$ & $\mathrm{EL}_{\mathrm{NBLK}}$ & $\mathrm{EH}_{\mathrm{NBLK}}$ \\
\hline$E_{0}$ & $\Gamma_{\mathrm{n}}$ & $\Gamma_{\gamma}$ & $\Gamma_{\mathrm{f}}$ & $r$ & g \\
\hline$\bullet$ & $\bullet$ & $\bullet$ & $\bullet$ & - & - \\
\hline$\bullet$ & $\bullet$ & $\bullet$ & $\bullet$ & $\bullet$ & $\bullet$ \\
\hline$\bullet$ & $\bullet$ & - & - & - & $\bullet$ \\
\hline
\end{tabular}

\section{Record type 5 (first record of Bondarenko block)}

This record is used to specify the $\sigma_{o}$ and temperature values at which all Bondarenko factors for the nuclide will be given. It also specifies the upper and lower energies that factors cover. The number of values, NSIG0, is specified in the 38th word in the set directory, and the 39 th word specifies the number of temperatures, NT. The record structure is

$$
\left(\sigma_{0}(\mathrm{I}), \mathrm{I}=1, \mathrm{NSIG} 0\right),(\mathrm{T}(\mathrm{I}), \mathrm{I}=1, \mathrm{NT}), \mathrm{ELO}, \mathrm{EHI} \text {. }
$$

The $\sigma_{0}$ values can either ascend or descend, but the temperatures are in Kelvin in ascending order.

\section{Record type 6 (directory for Bondarenko data)}

This record type is used to specify the processes that have Bondarenko data in the set. Its length is six times NBOND, the number of Bondarenko processes, specified in the 37 th word in the set directory. The structure is

$$
\begin{aligned}
& (\mathrm{MT}(\mathrm{I}), \mathrm{I}=1, \mathrm{NBOND}) \\
& (\mathrm{NF}(\mathrm{I}), \mathrm{I}=1, \mathrm{NBOND})
\end{aligned}
$$




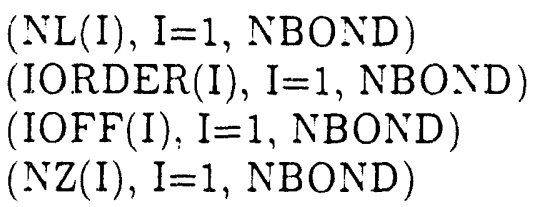

The parameters have the following interpretation: MT is the identifier of the process (e.g., $\mathrm{MT}=2$ is for elastic scattering as in ENDF/B). NF is the number of the first energy group for which parameters are given. NL is the last group for which parameters are given. IORDER is used to specify the order of the scattering matrix in the event it is to be self-shielded. IOFF is the offset from the "magic word" in the transfer matrices (e.g., for elastic scattering, the first word after the magic word is generally the within-group term such that IOFF $=1$ would specify the shielding for the within-group terms). (This parameter will be clearer in the discussion of AMPX transfer matrices given below.) NZ is presently unused and has a zero value.

\section{Record type 7 (infinite dilution values for Bondarenko data)}

Each process that has Bondarenko data has one of these records which contains the infinite dilution values or reference cross sections for the process. Its structure is

$$
\left(\sigma^{\infty}(\mathrm{I}), \mathrm{I}=\mathrm{NF}, \mathrm{NL}\right) \text {, }
$$

where NF and NL are the first and last groups with data for the process.

\section{Record type 8 (Bondarenko factors)}

This record is a 3-D array and contains the Bondarenko factors for a process. It $;$ structure is

$$
(((B F(I, J, K), I=1, N S I G 0), J=1, N T), K=N F, N L ; .
$$

\section{Record type 9 (temperature-independent average cross sections)}

This record type is used to present average cross sections (sometimes called 1-D cross sections) on the library.

Its structure is

$$
\begin{aligned}
& \mathrm{MT}_{1},\left(\sigma_{1}(\mathrm{I}), \mathrm{I}=1, \mathrm{IGM}\right), \\
& \mathrm{MT}_{2},\left(\sigma_{2}(\mathrm{I}), \mathrm{I}=1, \mathrm{IGM}\right), \\
& \cdot \\
& \cdot \\
& \mathrm{MT}_{\mathrm{LAST}},\left(\sigma_{\mathrm{LAST}}(\mathrm{I}), \mathrm{I}=1, \mathrm{IGM}\right),
\end{aligned}
$$

where the MTs are the process identifiers, and the cross sections, $\sigma$, are given for all groups. (Note that the MTs are given as floating-point numbers.)

Note that a process identified by $1452\left(\overline{\nu \sigma_{f}}\right)$ should never be used in this record to avoid the possibility that some module may pass these values without accounting for self-shielding. Use processes $18\left(\sigma_{f}\right)$ and $452(\nu)$ instead. 


\section{Record type 10 (scattering matrix directory)}

An AMPX master library can include a directory that identifies the scattering matrices that are given for a nuclide. The structure is

$$
\begin{aligned}
& (\mathrm{MT}(\mathrm{I}), \mathrm{I}=1, \mathrm{~N} 2 \mathrm{D}), \\
& (\mathrm{L}(\mathrm{I}), \mathrm{I}=1, \mathrm{~N} 2 \mathrm{D}), \\
& (\mathrm{NL}(\mathrm{I}), \mathrm{I}=1, \mathrm{~N} 2 \mathrm{D}), \\
& (\mathrm{NT}(\mathrm{I}), \mathrm{I}=1, \mathrm{~N} 2 \mathrm{D}),
\end{aligned}
$$

where N2D is the number of scattering (2-D) processes, MT is the process identifier, $L$ is the maximum length of any of the scattering matrices for the process, NL is the order of Legendre fit to the scattering matrix, and NT is a parameter whose definition depends on the type of data (whether neutron, gamma production, or gamma) given as follows:

1. For neutron-neutron data, NT is the number of temperatures at which scattering matrices are given.

2. For gamma production data, NT is zero if the data are in yield units and is unity if they are in cross-section units.

3. For gamma-gamma data, NT is zero.

\section{Record type 11 (scattering matrix temperatures)}

This record type specifies the temperatures (in $\mathrm{eV}$ ) of the scattering matrices. It is only used for neutron-neutron data and is given when NT $>0$ (see record type 10). The temperziures are in ascending order as follows:

$$
(\mathrm{T}(\mathrm{I}), \mathrm{I}=1, \mathrm{NT}) \text {. }
$$

\section{Record type 12 (scattering matrix)}

This record type is used to store scattering matrix data (sometimes called 2-D data). As will be illustrated, it has provisions for truncating zero and/or impossible elements from the array. It exists in two forms: (1) a self-defining form used for gamma production data and (2) a form that is not self-defining. The only difference is that the self-defining form specifies the length as the first word, while the other does not; that is,

or

$$
\mathrm{L},(\mathrm{X}(\mathrm{I}), \mathrm{I}=1, \mathrm{~L})
$$

$$
(\mathrm{X}(\mathrm{I}), \mathrm{I}=1, \mathrm{~L}) \text {. }
$$

The structure of the $\mathrm{X}$-array is as follows:

Magic word for a group

Terms for scattering to the group

Magic word for the next group

Terms for scattering to this group

Etc., etc.

In some cases, a negative or zero magic word is used to specify the end of data in the record. 
A "magic word" is used to define:

1. the sink group number, III;

2. the first group number, JJJ, which scatters to this group; and

3. the last group number, KKK, which scatters to this group.

The magic word is then defined as

$$
\mathrm{MW}=1000000^{*} \mathrm{JJJ}+1000 * \mathrm{KKK}+\mathrm{III}
$$

such that it is composed of three 3-digit integers:

$$
\text { MW : JJJKKKIII . }
$$

The scattering terms below a magic word are in reverse ordering (following typical practice for transport theory programs) (i.e., the scattering term for scattering from the last group is first, etc.):

$$
\begin{aligned}
& \text { MW for group III } \\
& \sigma(\mathrm{KKK} \rightarrow \mathrm{III}) \\
& \sigma(\mathrm{KKK}-1 \rightarrow \mathrm{III}) \\
& \cdot \\
& \cdot \\
& \cdot \\
& \sigma(\mathrm{JJJ} \rightarrow \mathrm{III})
\end{aligned}
$$

A scattering matrix record will contain one $\mathrm{P}_{\ell}$ matrix for a process.

Consider an elastic scattering matrix for hydrogen which will be a full triangular matrix and assume three energy groups. The scattering matrix will look as follows:

$$
\begin{aligned}
& 1001001 \\
& \sigma(1 \rightarrow 1) \\
& 1002002 \\
& \sigma(2 \rightarrow 2) \\
& \sigma(1 \rightarrow 2) \\
& 1003003 \\
& \sigma(3 \rightarrow 3) \\
& \sigma(2 \rightarrow 3) \\
& \sigma(1 \rightarrow 3)
\end{aligned}
$$

A characteristic of AMPX libraries which is of paramount importance to note is the higher ordered scattering matrices, $\sigma_{\ell}\left(g \rightarrow g^{\prime}\right), \ell>0$ include a $2 \ell+1$ multiplier. 
Note that the record is a mixture of integer and floating-point terms.

The overall structure of an AMPX master library is given below:

\begin{tabular}{|c|c|}
\hline & Record type \\
\hline Header record & 1 \\
\hline $\begin{array}{c}\text { Nuclide directory } \\
\text { (one record per nuclide) }\end{array}$ & 3 \\
\hline Neutron energy boundaries & 2 \\
\hline Gamma energy boundaries & 2 \\
\hline
\end{tabular}

Records for nuclide 1

Records for nuclide 2

Etc. 
The structure of the records of a nuclide is

\begin{tabular}{|l|c|}
\hline \multicolumn{2}{|c|}{ Record type } \\
\hline Nuclide directory record & 3 \\
\hline Bondarenko data & $5,6,7,8$ \\
\hline Resonance parameter data & 4 \\
\hline Temperature-independent group-averaged & 9 \\
\hline neutron cross sections & $10,11,12$ \\
\hline Scattering matrix data for neutrons & 10,12 \\
\hline Scattering matrix data for gamma production & 9 \\
\hline Group-averaged gamma cross sections & 10,12 \\
\hline Scattering matrix data for gammas & \\
\hline
\end{tabular}


The internal structure for Bondarenko data is

\begin{tabular}{|c|c|}
\hline & Record type \\
\hline$\left(\sigma_{0}(\mathrm{I}), \mathrm{I}=1, \mathrm{NSIG} 0\right),(\mathrm{T}(\mathrm{J}), \mathrm{J}=1, \mathrm{NT}), \mathrm{EL}, \mathrm{EH}$ & 5 \\
\hline $\begin{array}{c}\text { Bondarenko data directory } \\
\text { (MT(I), I=1, NB) } \\
\text { (NF(I),I=1, NB) } \\
\text { (NL(I),I=1, NB) } \\
\text { (ORDER(I), I=1, NB) } \\
\text { (IOFF(I),I=1, NB) } \\
\text { (NZ(I),I=1, NB) }\end{array}$ & 6 \\
\hline \multicolumn{2}{|l|}{$\begin{array}{l}\text { The following records are given in pairs for } \\
\text { all NB Bondarenko processes. }\end{array}$} \\
\hline $\begin{array}{l}\text { Infinite dilution values } \\
\left(\sigma^{\infty}(\mathrm{I}), \mathrm{I}=\mathrm{NF}, \mathrm{NL}\right)\end{array}$ & 7 \\
\hline $\begin{array}{l}\text { Bondarenko factors } \\
(((\mathrm{BF}(\mathrm{I}, \mathrm{J}, \mathrm{K}), \mathrm{I}=1, \mathrm{NSIG}), \mathrm{J}=1, \mathrm{NT}), \mathrm{K}=\mathrm{NF}, \mathrm{NL})\end{array}$ & 8 \\
\hline
\end{tabular}


The internal structure of the scattering matrix data for neutrons is

\begin{tabular}{|l|l|}
\hline \multicolumn{2}{|c|}{ Record type } \\
\hline $\begin{array}{l}\text { Scattering matrix directory } \\
(\mathrm{MT}(\mathrm{I}), \mathrm{I}=1, \mathrm{~N} 2 \mathrm{D}) \\
(\mathrm{L}(\mathrm{I}) \mathrm{I}=1, \mathrm{~N} 2 \mathrm{D}) \\
(\mathrm{NL}(\mathrm{I}), \mathrm{I}=1, \mathrm{~N} 2 \mathrm{D}) \\
(\mathrm{NT}(\mathrm{I}), \mathrm{I}=1, \mathrm{~N} 2 \mathrm{D})\end{array}$ & 10 \\
\hline The following structure is repeated N2D times. \\
\hline $\begin{array}{l}\text { Temperature values }(\mathrm{NT}>0) \\
(\mathrm{T}(\mathrm{I}), \mathrm{I}=1, \mathrm{NT})\end{array}$ \\
\hline \begin{tabular}{l} 
The following record is repeated $\mathrm{MA} \mathrm{X}(1, \mathrm{NT})^{*}(\mathrm{NL}+1)$ times. \\
\hline $\begin{array}{l}\text { Scattering matrix } \\
(\mathrm{X}(\mathrm{I}), \mathrm{I}=1, \mathrm{~L})\end{array}$
\end{tabular} \\
\hline
\end{tabular}

The internal structure for gamma production scattering is

\begin{tabular}{|l|l|}
\hline \multicolumn{2}{|c|}{ Record type } \\
\hline $\begin{array}{l}\text { Scattering matrix directory } \\
(\mathrm{MT}(\mathrm{I}), \mathrm{I}=1, \mathrm{~N} 2 \mathrm{D}) \\
(\mathrm{L}(\mathrm{I}), \mathrm{I}=1, \mathrm{~N} 2 \mathrm{D}) \\
(\mathrm{NL}(\mathrm{I}), \mathrm{I}=1, \mathrm{~N} 2 \mathrm{D}) \\
(\mathrm{NT}(\mathrm{I}), \mathrm{I}=1, \mathrm{~N} 2 \mathrm{D})\end{array}$ \\
\hline $\begin{array}{l}\text { For each process, I=1, N2D, the following record type } \\
\text { given NL }+1 \text { times corresponding to the } P_{0}, \mathrm{P}_{1}, \ldots \\
\mathrm{P}_{\mathrm{NL} \text { matrices. }}\end{array}$ \\
\hline $\begin{array}{l}\text { Self-defining scattering matrix length } \\
\text { LENGTH, (X(I), I=1, LENGTH) }\end{array}$ \\
\hline
\end{tabular}


The internal structure for gamma-gamma scattering is

\begin{tabular}{|l|c|}
\hline \multicolumn{2}{|c|}{ Record type } \\
\hline $\begin{array}{l}\text { Scattering matrix directory } \\
(\mathrm{MT}(\mathrm{I}), \mathrm{I}=1, \mathrm{~N} 2 \mathrm{D}) \\
(\mathrm{L}(\mathrm{I}), \mathrm{I}=1, \mathrm{~N} 2 \mathrm{D}) \\
(\mathrm{NL}(\mathrm{I}), \mathrm{I}=1, \mathrm{~N} 2 \mathrm{D}) \\
(\mathrm{NT}(\mathrm{I}), \mathrm{I}=1, \mathrm{~N} 2 \mathrm{D})\end{array}$ \\
\hline $\begin{array}{l}\text { For each process, } \mathrm{I}=1, \mathrm{~N} 2 \mathrm{D}, \text { the following record } \\
\text { type is given NL+1 times corresponding to the } \mathrm{P}_{0}, \\
\mathrm{P}_{1}, \ldots \mathrm{P}_{\mathrm{NL}} \text { matrices. }\end{array}$ \\
\hline $\begin{array}{l}\text { Scattering matrix } \\
(\mathrm{X}(\mathrm{I}), \mathrm{I}=1, \mathrm{~L})\end{array}$ \\
\hline
\end{tabular}

\section{INFORMATION.2 AMPX WORKING LIBRARY FORMAT}

The preceding section described the format of an AMPX mastes library with all its generality and detail. The other format used by AMPX modules is called the working, or weighted, library format. As contrasted with the master format, it has neither the detcil nor th 'complexity, since its data are derived by taking data from a master library and collecting them into forms ready for use in a flux calculation of some sort. For these needs it carries only three kinds of data for a nuclide:

1. a diıectory record describing the cross sections;

2. group-averaged data; and

3. a total (sum of all processes) group-to-group scattering matrix, which, of course, will consist of several matrices for anisotropic cases. 
The overall structure of a working library is given below:

\begin{tabular}{|c|c|}
\hline \multicolumn{2}{|c|}{ Record type } \\
\hline Header record & 1 \\
\hline Neutron energy boundaries & 2 \\
\hline Gamma energy boundaries & 2 \\
\hline Nuclide directory \\
(one record per nuclide) & 3 \\
\hline Records for nuclide 1 & \\
\hline Records for nuclide 2 \\
\hline
\end{tabular}

The structure of the records for a nuclide is

\begin{tabular}{|l|c|}
\hline & Record type \\
\hline Nuclide directory record & 3 \\
\hline Group averaged neutron cross sections & 9 \\
\hline Group averaged gamma cross sections & 9 \\
\hline $\begin{array}{l}\text { The } \mathrm{P}_{0}, \mathrm{P}_{1} \ldots, \mathrm{P}_{\mathrm{NL}} \text { total scattering matrices are } \\
\text { presented in self-defining records. }\end{array}$ & 12 \\
\hline
\end{tabular}

The record types are the same as those described in Sect. 4.INFORMATION.1 for the AMPX master, except for two cases: 
1. The directory records have a different structure, as shown below:

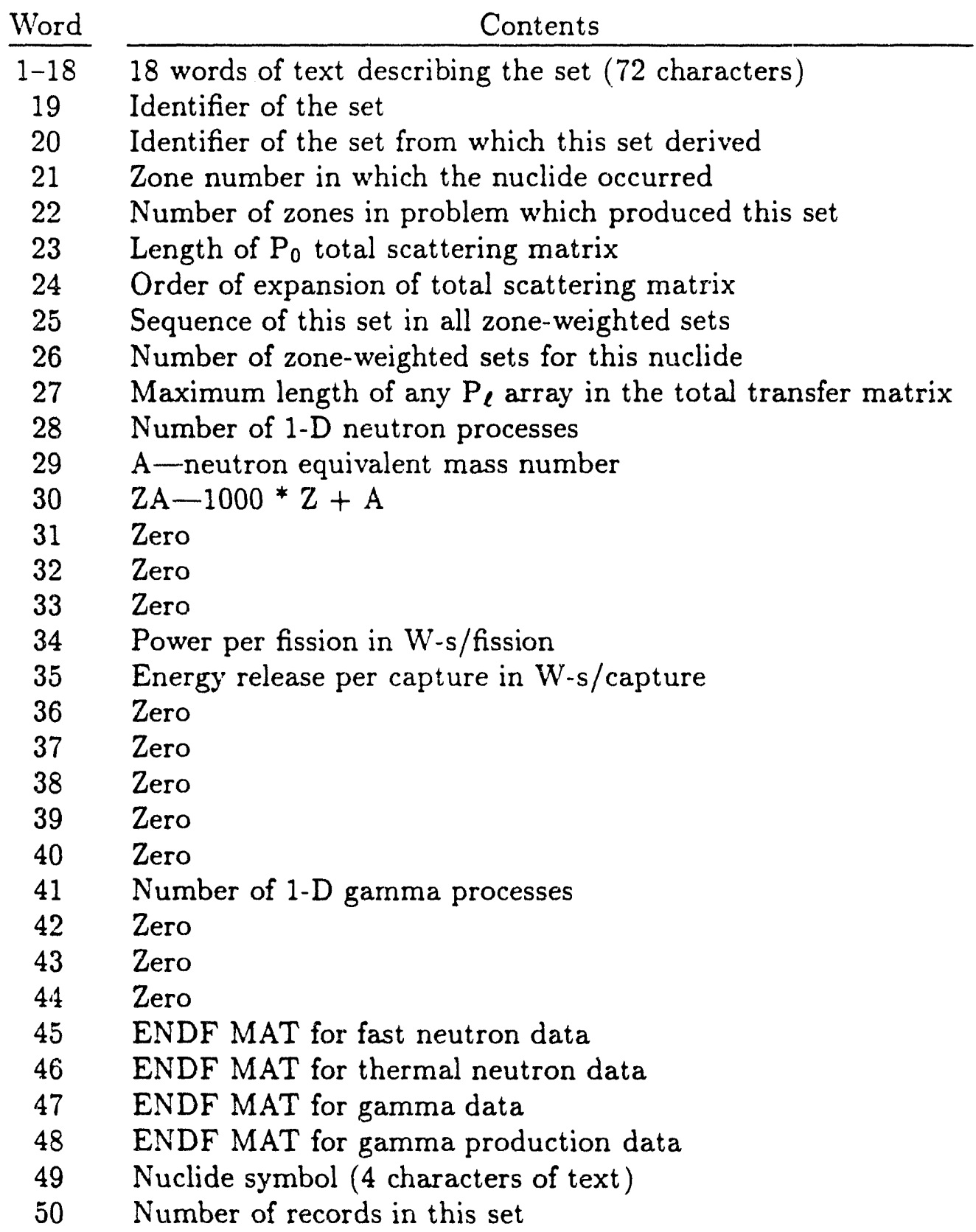

2. For the case of the weighted library only ( $\mathrm{I} 2=1$ in the library header record), the neutron group structure record contains the inverse velocities for each group ( $2200 \mathrm{~m} / \mathrm{s}$ units) followed by the fission spectrum from the problem which produced the weighted library. 


\section{INFORMATION.3 ANISN LIBRARY FORMAT}

The CONTAC, ALPO, and XSDRNPM modules in AMPX all can write a library in the ANISN format. These libraries can be output in two forms: (1) a BCD or card-image form, or (2) a binary form.

In both cases, "blocks" of cross sections are specified using two types of records. A "block" of data in the ANISN context consists of values for one $\mathrm{P}_{\ell}$ matrix of the cross sections of a nuclide or a mixture. The first record or card for a block contains 16 words as follows:

1. NOG - Number of energy groups in the block

2. IHM - Table length of the cross sections in the block

3. ICT - Control word which is 0 normally and is 7 for the last record in the cross-section library

4. ID - Numeric identifier for the cross-section block

5-16. (TEXT(I), I=1,12) - 48 characters of text to describe the cross-section block

In the case of the card-image format, the above is written:

$$
\left(1 \mathrm{H}^{\prime}, 2 \mathrm{I} 6, \mathrm{I} 4, \mathrm{I} 6,12 \mathrm{~A} 4\right)
$$

The cross sections in a block are in a rectangular array and are written:

$$
((\mathrm{XS}(\mathrm{I}, \mathrm{J}), \mathrm{I}=1, \mathrm{IHM}), \mathrm{J}=1, \mathrm{NOG})
$$

In the case of card output, the values are written using the free-form FIDO schemes discussed in Sect. 7.FIDO.

A schematic of the structure is shown below:

\section{GROUP NUMBER}

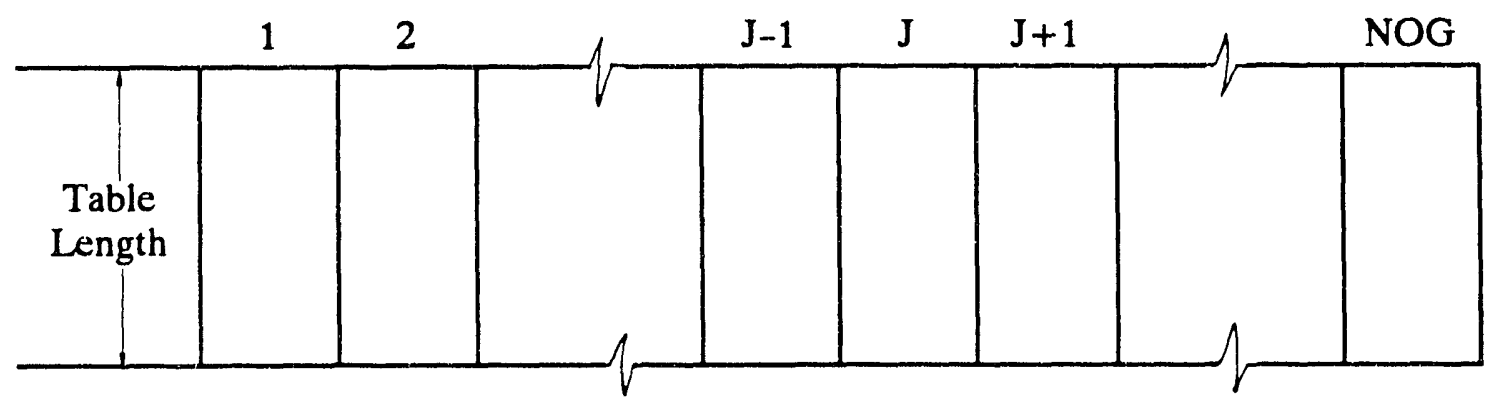

Within a column for a group, the group-averaged values for various processes along with the values for scattering to the group $G$ are given: 


\begin{tabular}{|c|c|}
\hline Position & Type of value \\
\hline 1 & (user specified) \\
\hline · & \\
\hline$\dot{.}$ & \\
\hline IHT -4 & $x$ \\
\hline $\mathrm{IHT}-3$ & $\hat{\Sigma}_{f}$ \\
\hline IHT -2 & $\Sigma_{\mathrm{a}}$ \\
\hline IH'T -1 & $\frac{a_{d}}{\nu \Sigma_{f}}$ \\
\hline $\mathrm{IHT}$ & $\Sigma_{t}$ \\
\hline - & . \\
\hline . & \\
\hline IHS -2 & $\Sigma(\mathrm{G}+2 \rightarrow \mathrm{G})$ \\
\hline IHS -1 & $\Sigma(\mathrm{G}+1 \rightarrow \mathrm{G})$ \\
\hline IHS & $\Sigma(\mathrm{G} \rightarrow \mathrm{G})$ \\
\hline IHS +1 & $\Sigma(\mathrm{G}-1 \rightarrow \mathrm{G})$ \\
\hline . & . \\
\hline - & . \\
\hline - & \\
\hline IHM & \\
\hline
\end{tabular}

where IHT is the position of the total cross-section values in the table, IHS is the within-group scattering position, and IHM is the table length. Note that positions that are undefined contain zeroes. For example, when IHT $=3$, there are no values for $\Sigma_{f}$ in the table, etc., or if IHT $=3$ and IHS $=4$, there are no upscatters in the table. When IHM is less than (IHS + total number of groups less one), some possible downscatters are truncated, etc.

Another characteristic to note concerning ANISN values is that the matrices are multiplied by $(2 \ell+1)$ (i.e., $\mathrm{P}_{0}$ terms are multiplied by unity, $\mathrm{P}_{1}$ terms by three, etc.).

In some analyses, especially for certain shielding problems, calculations are made with ANISN cross sections which contain both neutron and gamma cross-section information. These libraries contain three kinds of scattering data:

1. neutron-to-neutron

2. neutron-to-gamma

3. gamma-to-gamma 
For the forward (as opposed to adjoint) case, the cross sections will be arranged as in the following diagram:

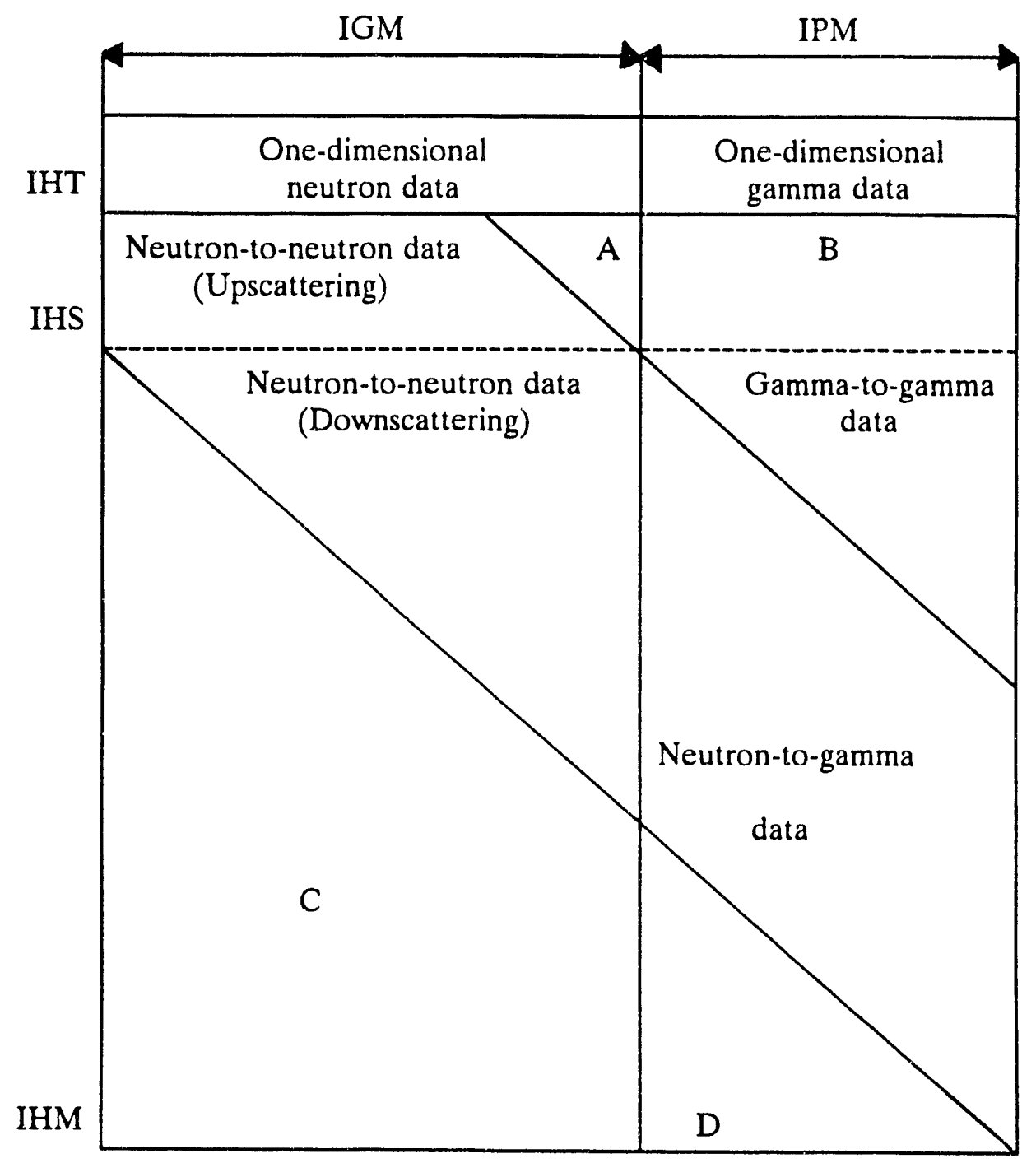

where IGM and IPM are the number of neutron and gamma groups, respectively, areas $C$ and $D$ are zero because they represent transfers from nonexistent groups, $A$ is zero because gammas do not generally transfer to neutron groups, through there are nuclides which do have reactions which do transfer, and B is zero because gammas do not upscatter.

\section{INFORMATION.4 STANDARD ENERGY GROUP STRUCTURES}

For convenience, a group boundary library is provided with the AMPX system which contains several structures that have been used at ORNL to make neutron and gamma cross-section libraries. AMPX modules requiring this input automatically access this library. The library is indexed by the data type (neutron or gamma) and 
the number of groups. To override a standard group structure, simply input your own structure in the appropriate input array.

\section{INFORMATION.4.1 "Standard" Neutron Boundaries}

The available neutron group structures are listed on the following pages. They include:

1. a 16-group Hansen-Roach structure

2. a 22-group structure designed for atmospheric transport calculations

3. a 27-group structure used in the SCALE system

4. a 37-group Defense Nuclear Agency structure

5. a 39-group structure selected for use in the design of the Advance Neutron Source Reactor

6. a 50-group structure derived from the GAM-II structure and intended for fast reactor applications

7. a 51-group structure identical to the 50-group structure, but having one extra thermal group

8. an 84-group structure for the thermal application

9. a 100-group structure consisting of the GAM-II structure with one thermal group

10. a 104-group structure formed by adding 4 groups to the 100-group structure to include important variations in the cross sections for major components of the atmosphere

11. a 105-group structure which is the 104-group structure with one group above $15 \mathrm{MeV}$

12. a 119-group structure tailored for fast reactor, air transport, and several shielding applications

13. a 123-group structure which consists of the GAM-II boundaries combined with a 30-group THERMOS structure below $1.86 \mathrm{eV}$

14. a 126-group structure for fast reactor and shielding applications

15. a 171-group structure for fast reactor and shielding applications,

16. a 174-group structure which is the 171-group structure with three additional groups

17. a 218-group structure developed for shipping cask analyses and other thermal applications

18. a 227-group structure developed from the 218-group structure

19. a 231-group structure based on the 238-group CSEWG structure but which has more thermal groups and no groups above $15 \mathrm{MeV}$

20. a 238-group structure developed by adding extra groups in the very low-energy thermal range to the 227 -group structure

These 20 structures are shown on the following pages.

INFORMATION 
16 NEUTRON GROUP STRUCTURE

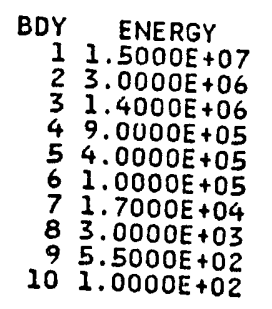

22 NEUTRON GROUP STRUCTURE

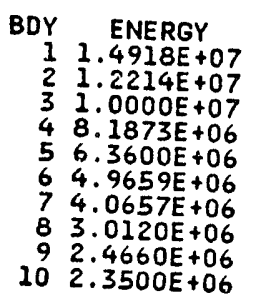

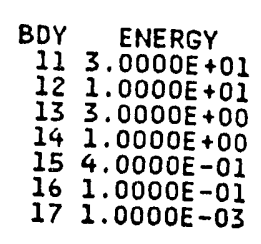

ENERGY

$123.0000 E+01$

$133.0000 E+00$

$171.0000 \mathrm{E}-03$

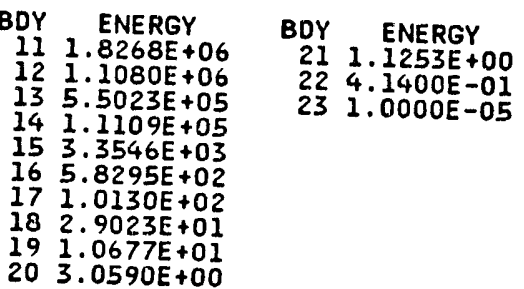

27 NEUTRON GROUP STRUCTURE

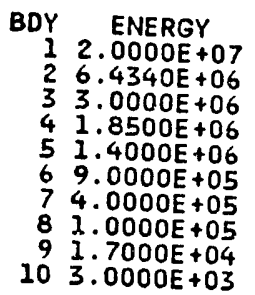

BDY ENERGY

$115.5000 E+02$

$121.0000 E+02$

$133.0000 E+01$

14 1. $0000 \mathrm{E}+01$

I5 $3.0000 \mathrm{E}+00$

$153.0000 E+00$

$161.7700 E+00$

$181.1300 \mathrm{E}+00$

191.0000

20 8.0000E-0I

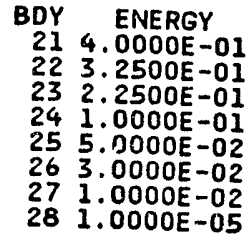

\section{NEUTRON GROUP STRUCTURE}
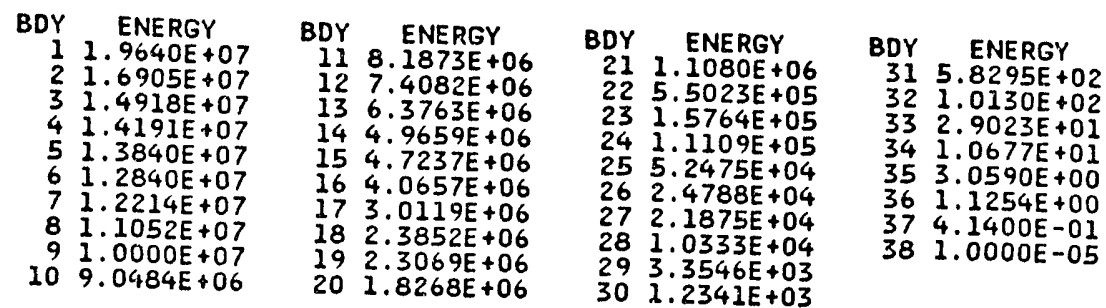
39 NEUTRON GROUP STRUCTURE

$\begin{array}{rcrc}\text { BDY } & \text { ENERGY } & \text { BDY } & \text { ENERGY } \\ 1 & 2.0000 E+07 & 11 & 5.5000 E+02 \\ 2 & 6.4340 E+06 & 12 & 1.0000 E+02 \\ 3 & 3.0000 E+06 & 13 & 3.0000 E+01 \\ 4 & 1.8500 E+06 & 14 & 1.0000 E+01 \\ 5 & 1.4000 E+06 & 15 & 3.0000 E+00 \\ 6 & 9.0000 E+05 & 16 & 1.7700 E+00 \\ 7 & 4.0000 E+05 & 17 & 1.3000 E+00 \\ 8 & 1.0000 E+05 & 18 & 1.0000 E+00 \\ 9 & 1.7000 E+04 & 197.6500 E-01 \\ 10 & 3.0000 E+03 & 205.8800 E-01\end{array}$

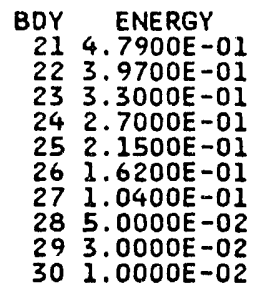

BNY ENERGY

$314.4500 E-03$

$323.2500 \mathrm{E}-03$

$332.6000 \mathrm{E}-03$

$342.1500 E-03$

$351.8000 \mathrm{E}-03$

$361.4500 E-03$

$371.1500 E-03$

$388.5000 E-04$

(39 $5.5000 \mathrm{E}-04$

$401.0000 E-05$
50 NEUTRON GROUP STRUCTURE

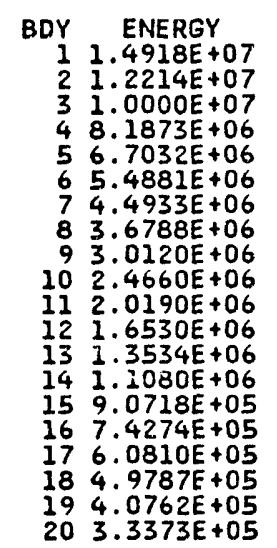

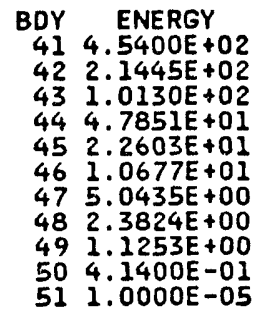

51 NEUTRON GROUP STRUCTURE

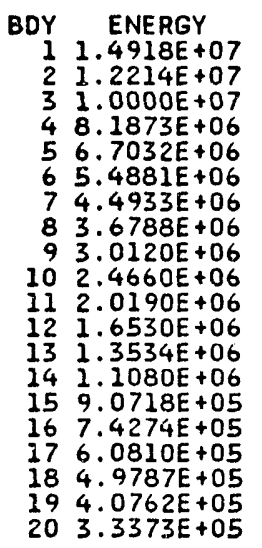

$$
\begin{array}{rc}
\text { BDY } & \text { ENERGY } \\
21 & 2.7324 E+05 \\
22 & 2.2371 E+05 \\
23 & 1.8316 E+05 \\
24 & 1.4996 E+05 \\
25 & 1.2277 E+05 \\
26 & 8.6517 E+04 \\
27 & 5.2475 E+04 \\
28 & 4.0868 E+04 \\
29 & 3.1828 E+04 \\
30 & 2.4788 E+04 \\
31 & 1.9305 E+04 \\
32 & 1.5034 E+04 \\
33 & 7.1018 E+03 \\
34 & 4.3074 E+03 \\
35 & 3.3546 E+03 \\
36 & 2.6126 E+03 \\
37 & 2.0347 E+03 \\
38 & 1.5846 E+03 \\
39 & 1.2341 E+03 \\
40 & 9.6112 E+02
\end{array}
$$

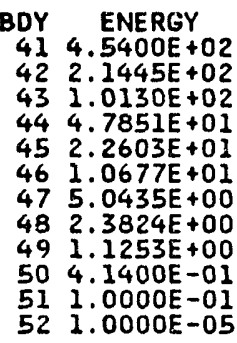

$521.0000 E-05$ 
84 NEUTRON GROUP STRUCTURE

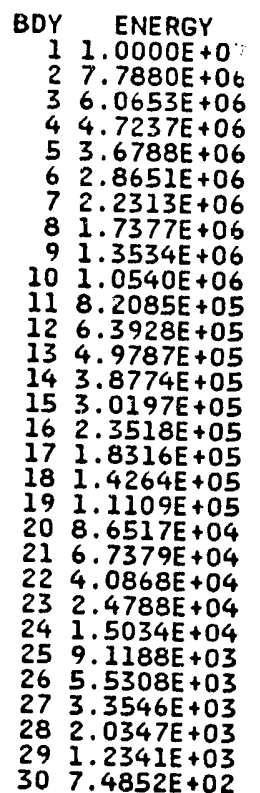

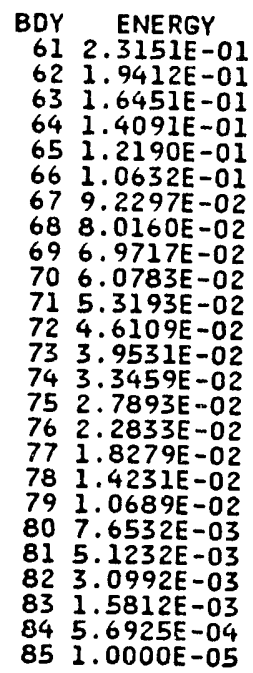

100 NEUTRON GROUP STRUCTURE

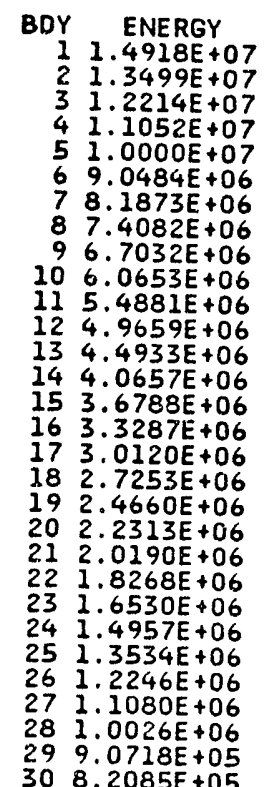

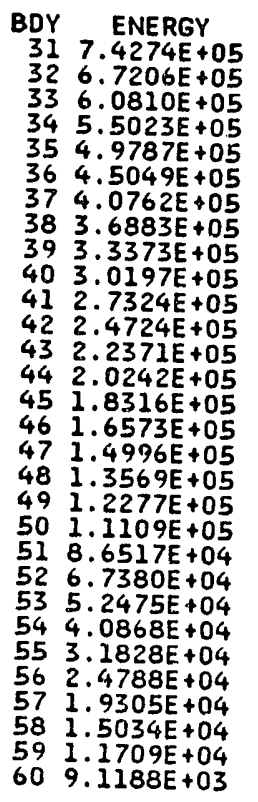

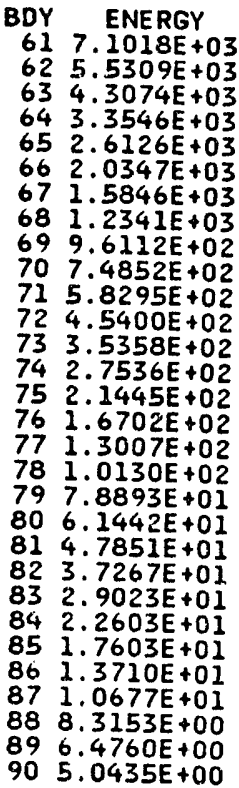

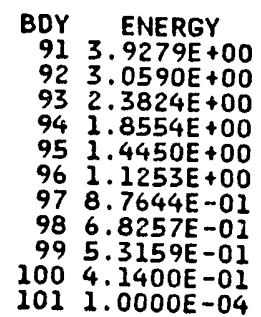

BDY ENERGY

$923.0590 \mathrm{E}+00$

$932.3824 \mathrm{E}+00$

45 I. $554 E+00$

96 1. $1253 E+00$

$8.7644 \mathrm{E}-01$

$1004.1400 \mathrm{E}-01$

$1011.0000 E-04$ 
104 NEUTRON GROUP STRUCTURE

$\begin{array}{cc}\text { BDY } & \text { ENERGY } \\ 1 & 1.4918 E+07 \\ 2 & 1.3499 E+07 \\ 3 & 1.2214 E+07 \\ 4 & 1.1052 E+07 \\ 5 & 1.0000 E+07 \\ 6 & 9.0484 E+06 \\ 7 & 8.1873 E+06 \\ 8 & 7.4082 E+06 \\ 9 & 7.0000 E+06 \\ 10 & 6.7032 E+06 \\ 11 & 6.3600 E+06 \\ 12 & 6.0653 E+06 \\ 13 & 5.4881 E+06 \\ 14 & 4.9659 E+06 \\ 15 & 4.7500 E+06 \\ 16 & 4.4933 E+06 \\ 17 & 4.0657 E+06 \\ 18 & 3.6788 E+06 \\ 19 & 3.3287 E+06 \\ 20 & 3.0120 E+06 \\ 21 & 2.7253 E+06 \\ 22 & 2.4660 E+06 \\ 23 & 2.3500 E+06 \\ 24 & 2.2313 E+06 \\ 25 & 2.0190 E+06 \\ 26 & 1.8268 E+06 \\ 27 & 1.6530 E+06 \\ 28 & 1.4957 E+06 \\ 29 & 1.3534 E+06 \\ 30 & 1.2246 E+06\end{array}$

$\begin{array}{rc}\text { BDY } & \text { ENERGY } \\ 61 & 1.9305 E+04 \\ 62 & 1.5034 E+04 \\ 63 & 1.1709 E+04 \\ 64 & 9.1188 E+03 \\ 65 & 7.1018 E+03 \\ 66 & 5.5309 E+03 \\ 67 & 4.3074 E+03 \\ 68 & 3.3546 E+03 \\ 69 & 2.6126 E+03 \\ 70 & 2.0347 E+03 \\ 71 & 1.5846 E+03 \\ 72 & 1.2341 E+03 \\ 73 & 9.6112 E+02 \\ 74 & 7.4852 E+02 \\ 75 & 5.8295 E+02 \\ 76 & 4.5400 E+02 \\ 77 & 3.5358 E+02 \\ 78 & 2.7536 E+02 \\ 79 & 2.1445 E+02 \\ 80 & 1.6702 E+02 \\ 81 & 1.3007 E+02 \\ 82 & 1.0130 E+02 \\ 83 & 7.8893 E+01 \\ 84 & 6.1442 E+01 \\ 85 & 4.7851 E+01 \\ 86 & 3.7267 E+01 \\ 87 & 2.9023 E+01 \\ 88 & 2.2603 E+01 \\ 89 & 1.7603 E+01 \\ 90 & 1.3710 E+01\end{array}$

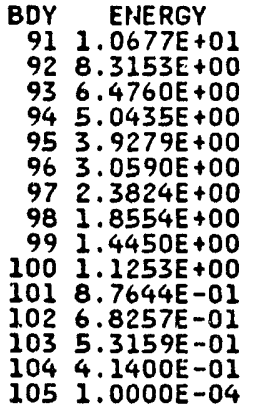

$1051.0000 E-04$

\section{NEUTRON GROUP STRUCTURE}

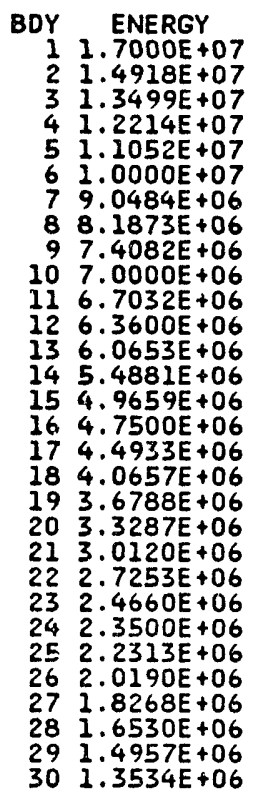

BDY ENERGY

$311.2246 \mathrm{E}+06$

$321.1080 E+06$

$331.0026 \mathrm{E}+06$

$349.0718 E+05$

$358.2085 \mathrm{E}+05$

$367.4274 \mathrm{E}+05$

$376.7206 E+05$

$386.0810 \mathrm{E}+05$

$395.0810 E+05$

$404.9787 E+05$

$404.9787 E+05$

$414.5049 E+05$

$424.0762 E+05$

$443.3373 E+05$

$453.0197 \mathrm{E}+05$

$462.7324 E+05$

$472.4724 E+05$

$482.23711+05$

$482.2371 E+05$

$492.0242 E+05$

$501.8316 \mathrm{E}+05$

$511.6573 E+05$

$531.3569 E+05$

$541.2277 E+05$

$551.2109 E+05$

$568.6517 E+04$

$576.7380 E+04$

$\begin{array}{ll}57 & 6.7380 E+04 \\ 58 & 5.2475 E+04\end{array}$

$594.0868 E+04$

$603.1828 E+04$
BDY ENERGY

$612.4788 E+04$ $621.9305 E+04$ $631.5034 \mathrm{E}+04$ $641.1709 E+04$ $659.1188 E+03$ $667.1018 E+03$ $675.5309 \mathrm{E}+03$ 68 \%. $3074 \mathrm{E}+03$ $693.3546 E+03$ $693.3546 E+03$ $702.6126 \mathrm{E}+03$
$712.0347 \mathrm{E}+03$ $721.5846 \mathrm{E}+03$ 73 I.2341E+03 $749.6112 E+02$ $757.4852 E+02$ $767.4852 E+02$

$774.5400 \mathrm{E}+02$

$774.5400 E+02$
$783.5358 E+02$ $783.5358 E+02$
79 $792.7536 E+02$ $802.1445 E+02$ 82 1.3007E+02 $831.0130 E+02$ $847.8893 E+01$ $856.1442 E+01$ $856.1442 E+01$ $864.7851 E+01$ $873.7267 E+01$ $882.9023 E+01$ 90 I. $7603 E+01$

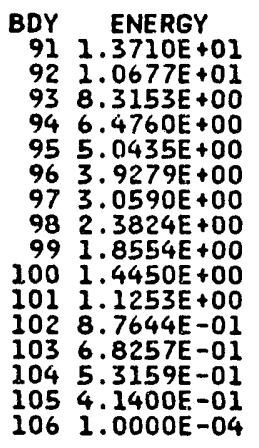

\section{INFORMATION}


119 NEUTRON GROUP STRUCTURE

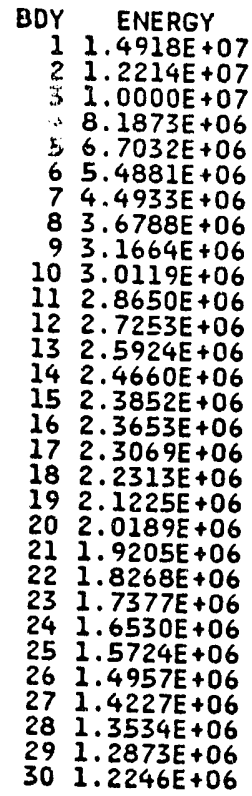

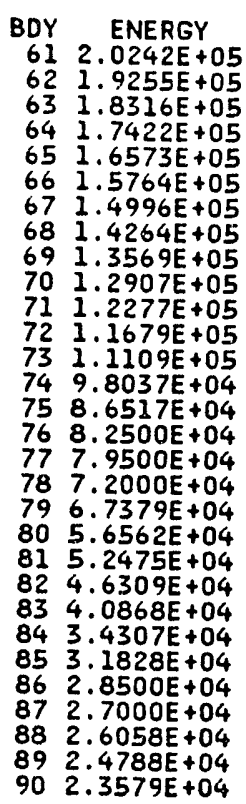

BDY ENERGY

$912.1875 \mathrm{E}+04$

$921.9305 \mathrm{E}+04$

$931.5034 \mathrm{E}+04$ 94 1. $1709 \mathrm{E}+04$ $959.1188 E+03$ $967.1017 E+03$ $975.5308 E+03$ $984.3074 E+03$ $993.7074 E+03$

$1003.3546 \mathrm{E}+03$

$1013.0354 \mathrm{E}+03$

$1022.7465 E+03$

$1022.7465 \mathrm{E}+03$

$\begin{aligned} & 103 \\ & 104\end{aligned} 2.6126 \mathrm{E}+03$

$1051.5846 \mathrm{E}+03$

$1061.2341 \mathrm{E}+03$

$1079.6112 E+02$

$1087.4852 \mathrm{E}+02$

$1094.5400 \mathrm{E}+02$

$1102.1445 \mathrm{E}+02$

$\begin{array}{ll}110 & 2.1445 E+02 \\ 111 & 1.0130 E+02\end{array}$

$1124.7851 \mathrm{E}+01$

$1132.2603 \mathrm{E}+01$

$1141.0677 \mathrm{E}+01$

$1155.0435 \mathrm{E}+00$

$116 \quad 2.3824 \mathrm{E}+00$

$1171.1254 \mathrm{E}+00$

$118 \quad 4.1399 \mathrm{E}-01$

1191.0000 -OI

1201.0000 E-05

\section{NEUTRON GROUP STRUCTURE}

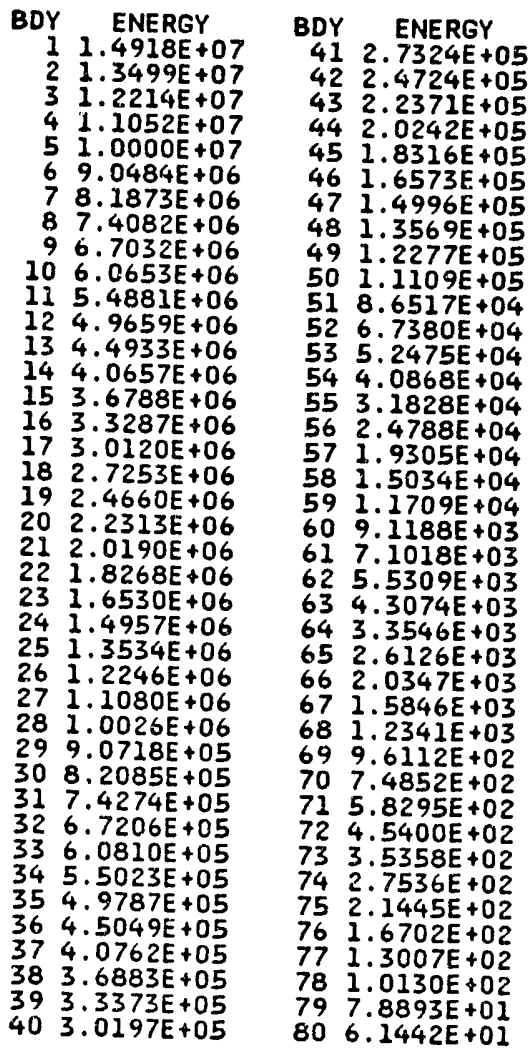
BDY "ENERIGY
$814.7851 . E+01$
$823.7267 E+01$
$842.2603 \mathrm{~B}+01$
$842.26033 E+01$
$\begin{array}{ll}85 & 1.7603 E+01 \\ 86 & 1.3710 E+01\end{array}$
87 1. 067 ' ' +01
$888.3153 E+00$
$896.4760 \mathrm{E}+00$
$905.043 .5 E+00$
913.927 GE +00
$923.0591)+00$
$932.3826 \mathrm{C}+00$
$941.8600 \mathrm{PE}+00$
$941.8601 \mathrm{JE}+000$
$951.71010 \mathrm{DE}+00$
$96 \frac{1}{1.5600 E+00}$
$981.2900 E+00$
$991.1250 E+00$ $1001.0000 E+00$ $1018.7600 \mathrm{E}-01$ $1027.7600 \mathrm{E}-01$ $102 \quad 7.7600 \mathrm{E}-01$
103 6.5000E-01
$1045.5000 E-0$
$1063.5000 E-01$
$1073.0000 E-01$
$1082.5000 \mathrm{E}-01$
$1092.0000 \mathrm{E}-01$
$1101.8000 \mathrm{E}-01$
I11 $1.6000 \mathrm{E}-01$
$1111.6000 \mathrm{E}-01$
$1131.2000 E-01$
$1141.000 D E-01$
$1158.00010 E-02$
$1167.0000 \mathrm{E}-02$
$1176.0000 \mathrm{E}-02$
$1185.0001 \mathrm{E}-02$
$1194.0000 \mathrm{E}-02$
$1203.0001 \mathrm{E}-02$

BDY ENERGY

1212.0000 E -02

$1221.5000 \mathrm{E}-02$

$1231.0000 E-02$

124 5.0000E-03 
126 NEUTRON GROUP STRUCTURE

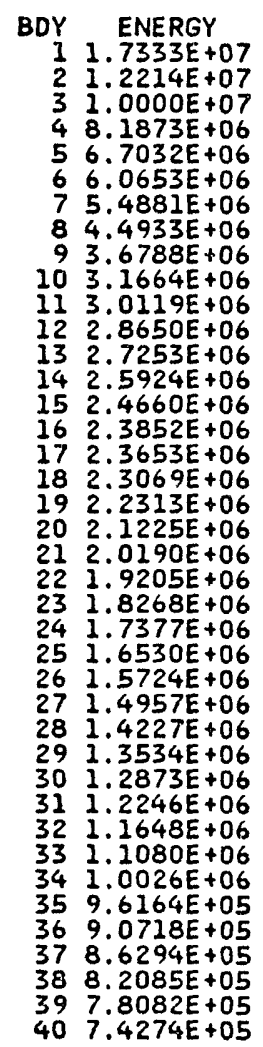

BDY ENERGY $417.0651 \mathrm{E}+05$ $426.7206 E+05$ $436.3928 E+05$ $446.0810 E+05$ $455.7844 E+05$ $465.5023 E+05$ $475.2340 E+05$ 48 4. $9787 E+05$ $494.5049 E+05$ $504.0762 E+05$ $513.6883 E+05$ $523.3373 E+05$ $533.0197 E+05$ $542.9850 E+05$ $552.9720 \mathrm{E}+05$ 56 2.9452E+05 $572.8725 E+05$ $582.7324 E+05$ $592.4724 \mathrm{E}+05$ $602.2371 E+05$ $612.1280 \mathrm{E}+05$ $612.1280 \mathrm{E}+05$ $622.0242 E+05$ $631.9255 E+05$ $641.8316 E+05$ $651.7422 E+05$ $661.6573 E+05$ $671.5764 E+05$ $681.4996 \mathrm{E}+05$ $691.4264 E+05$ $701.3569 \mathrm{E}+05$ 71 1.2907E+05 72 1.2277E+05 $731.1679 E+05$ 73 1.1679E+05 $741.3109 E+05$ $768.6517 E+04$ $87.2500 E+04$ $787.9500 E+04$ $797.2000 E+04$ $806.7379 E+04$

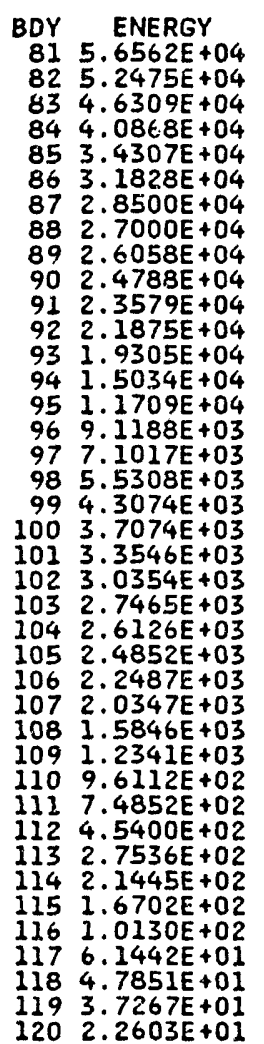

BOY ENEREY

$1211.0677 \mathrm{E}+01$

$1225.0435 \mathrm{E}+00$

$1232.3724 E+00$

124 1.1254E+00

125 4.1399E-01

$127 \begin{aligned} & 1.0000 E-05 \\ & 126\end{aligned}$ 
171 NEUTRON GROUP STRUCTURE

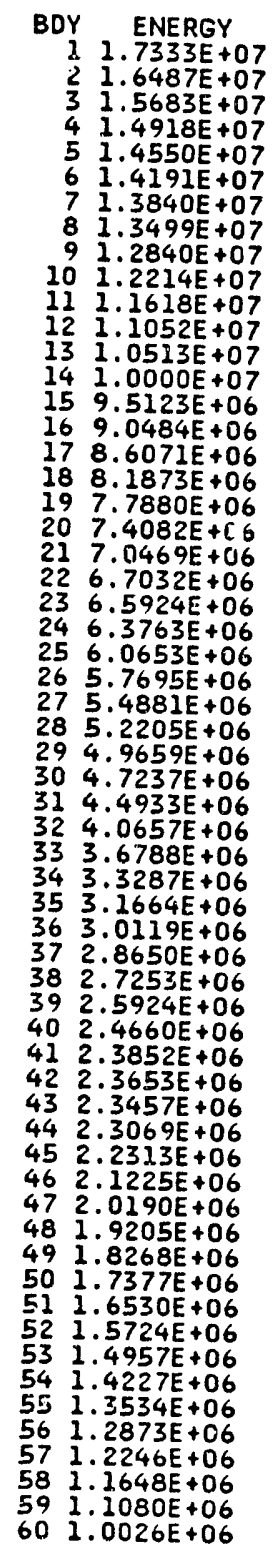

BDY ENERGY

$619.6164 \mathrm{E}+05$ $629.0718 E+05$ $638.6294 E+05$ $648.2085 E+05$ $657.8082 E+05$ $667.4274 E+05$ $677.0651 \mathrm{E}+05$ $686.7206 \mathrm{E}+05$ $696.3928 E+05$ $706.3928+05$ 70 . $0810 E+05$ $715.7844 E+05$ $725.5023 E+05$ $735.2340 E+05$ $744.9787 E+05$ $754.5049 E+05$ $764.0762 E+05$ $773.8774 E+05$ $783.6883 \mathrm{E}+05$ $793.3373 E+05$ $803.3373 E+05$ $813.0197 E+05$ $812.9850 E+05$ $822.9720 E+05$ $832.9452 E+05$ $842.8725 \mathrm{E}+05$ $852.7324 E+05$ 列 7 . 列 $892.1280 E+05$ $902.0242 E+05$ $911.9255 \mathrm{E}+05$ $921.8316 E+05$ $931.7422 \mathrm{E}+05$ $941.6573 \mathrm{E}+05$ $951.5764 \mathrm{E}+05$ $961.4996 \mathrm{E}+05$ $971.4264 \mathrm{E}+05$ $983.3569 \mathrm{E}+05$ 99 1. $2907 \mathrm{E}+05$ $1001.2277 \mathrm{E}+05$ $1011.1679 \mathrm{E}+05$ $1021.1109 E+05$ $1039.80375+04$ $1048.6517 \mathrm{E}+04$ . $2500 E+04$ $1067.9500 E+04$ $1077.2000 E+04$ $1086.7379 E+04$ $1095.6562 \mathrm{E}+04$ $1105.2475 \mathrm{E}+04$ 11] $4.6309 E+04$ II $4.6309 E+04$ 1) . $1143.1828 \mathrm{E}+04$ $2.8500 E+04$ $1162.7000 E+04$ $172.6058 \mathrm{E}+04$ $1182.4788 E+04$ $192.4176 \mathrm{E}+04$ $1202.3579 \mathrm{E}+04$

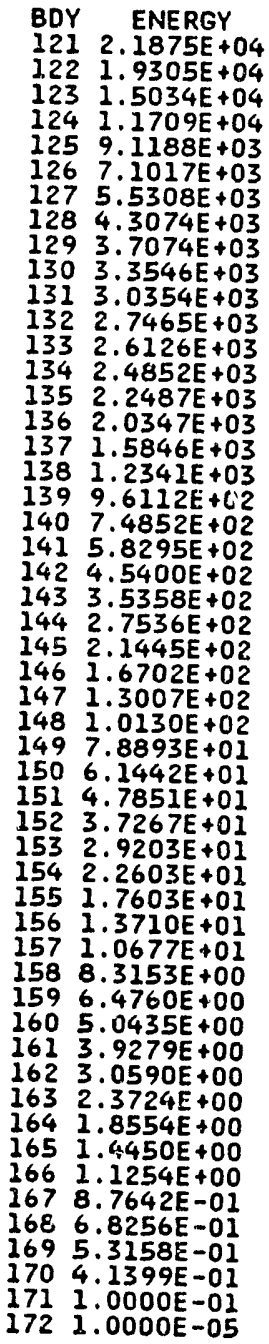

$1212.1875 E+04$ $1231.93034 \mathrm{E}+04$ $1709 E+04$ 促 $127.5108+03$ $1293.7074 \mathrm{E}+03$ $1303.3546 \mathrm{E}+03$ $1313.0354 E+03$ $1342.4852 E+03$ $1352.2487 E+03$ $1362.0347 \mathrm{E}+03$ $1371.5846 \mathrm{E}+03$ $1381.5341 \mathrm{E}+03$ $1399.6112 \mathrm{E}+\mathrm{C} 2$ $1407.4852 \mathrm{E}+02$ $5.8295 E+02$ $1452.1445 \mathrm{E}+02$ $1461.6702 E+02$ $1471.3007 \mathrm{E}+02$ $1497.8393 E+01$ $1506.1442 E+01$ $1514.7851 \mathrm{E}+01$ $1523.7267 \mathrm{E}+01$ $1532.9203 \mathrm{E}+01$ $542.2603 \mathrm{E}+01$ I. 158 . $596.4760 E+00$ . $5.0435 E+00$ $613.9279 E+00$ $1632.3724 \mathrm{~F}+00$ TE +00 1.4450E+00 $678.7642 E-01$ $1686.8256 E-01$ $1695.3158 \mathrm{E}-01$ $1711.0000 \mathrm{E}-01$ 172 1.0000E-05 
174 NEUTRON GROUP STRUCTURE

\begin{tabular}{|c|c|c|}
\hline 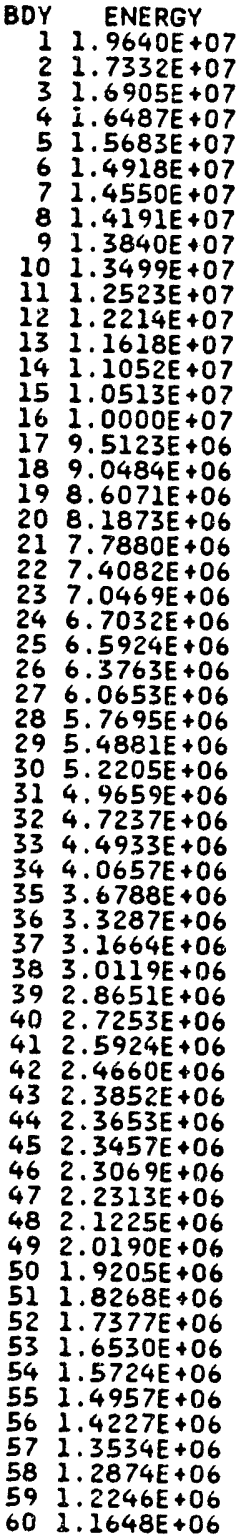 & 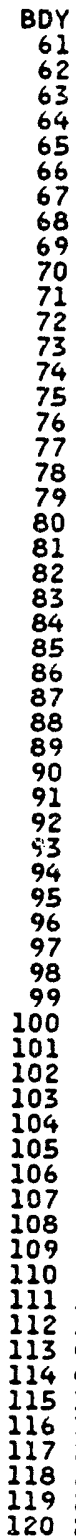 & 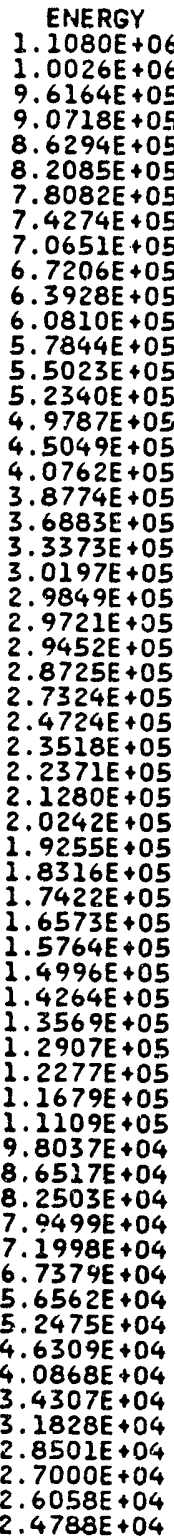 \\
\hline
\end{tabular}

BDY ENERGY

$2212.4176 E+04$

$1222.3579 E+04$

$1232.1875 E+04$

$1241.9305 E+04$

$1251.5034 \mathrm{E}+04$

126 1. $1709 E+04$

$1271.0333 E+04$

$1289.1188 E+03$

$1297.1017 E+03$

$1305.5308 E+03$

$1314.3074 \mathrm{E}+03$

132 $3.7074 \mathrm{E}+03$

133 . $3546 \mathrm{E}+03$

133 . $3546 E+03$

$1343.0354 E+03$

$1352.7465 E+03$

$1362.6126 E+03$

$1372.4852 E+03$

$1382.2487 E+03$

$1392.0347 E+03$

$1401.5846 \mathrm{E}+03$

$1429.6112 E+02$

$1437.4852 \mathrm{E}+02$

$1445.8295 \mathrm{E}+02$

$1454.5400 E+02$

$1463.5357 E+02$

$1472.7536 E+02$

$1482.1445 E+02$

$1491.6702 \mathrm{E}+02$

$1501.3007 \mathrm{E}+02$

$1511.0130 \mathrm{E}+02$

$1527.8893 \mathrm{E}+01$

$1536.1442 E+01$

$1544.7851 \mathrm{E}+01$

$1553.7266 \mathrm{E}+01$

$1562.9023 \mathrm{E}+01$

$1572.2603 E+01$

$158.2603 \mathrm{E}+01$

158 $1.7603 E+01$

$1591.3710 E+01$

$1601.0677 E+01$

$1618.3153 E+00$

$1626.4759 E+00$

$1635.0435 E+00$

$1643.9279 E+00$

$1653.0590 \mathrm{E}+00$

165 .

$16672.3824 E+00$

$1671.8554 \mathrm{E}+00$

$1681.4450 \mathrm{E}+00$

$1691.1253 \mathrm{E}+00$

$1708.7642 E-01$

$1716.8256 \mathrm{E}-0$

$1725.3158 E-01$

$1734.1399 E-01$

174 I.1399E 0

$1751.0000 E-05$

INFORMATION 
218 NEUTRON GROUP STRUCTURE

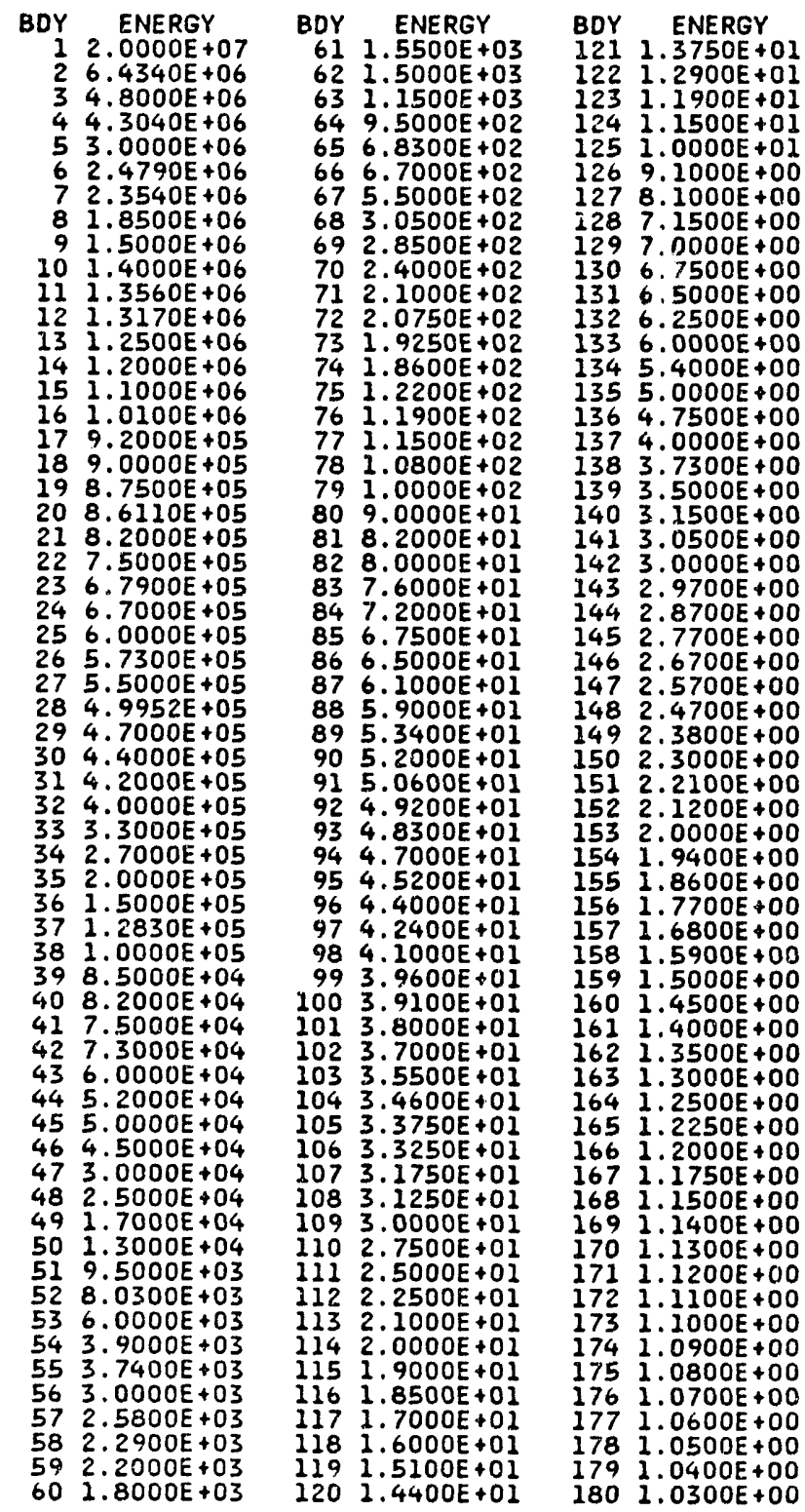

BDY ENERGY

$1811.0200 E+00$

$1821.0100 E+00$

$1831.0000 E+00$

$1849.7500 E-01$

$1859.5000 E-01$

$1869.2500 E-01$

$1879.0000 E-01$

188 8.5000E-0I

$1898.0000 \mathrm{E}-0 \mathrm{I}$

1898.0000 - 01

$1907.5000 \mathrm{E}-0 \mathrm{I}$

$1917.0000 E-01$

$1926.5000 \mathrm{E}-01$

1936.0000 E-OI

194 5.5000E-0I

$1955.0000 E-01$

$1964.5000 \mathrm{E}-01$

$1974.0000 E-01$

1983.750

$1983.7500 E-01$

$1993.5000 \mathrm{E}-01$

$2003.2500 E-01$

$2013.0000 \mathrm{E}-01$

$2022.7500 \mathrm{E}-01$

$2032.5000 E-01$

$2042.2500 E-01$

$2052.0000 E-01$

$2061.7500 E-01$

207 I.5000E-OI

$2071.5000 \mathrm{E}-01$

$2081.2500 E-01$

2091.0000 E -01

$2109.0000 E-02$

$2118.0000 \mathrm{E}-02$

2127.0000 E-02

$213 \quad 6.0000 E-02$

$2145.0000 E-02$

$2154.0000 \mathrm{E}-02$

$2163.0000 E-02$

$2172.5300 \mathrm{E}-02$

$2172.5300 \mathrm{E}-02$

$\begin{array}{ll}218 & 1.0000 E-02 \\ 219 & 1.0000 E-05\end{array}$ 
227 NEUTRON GROUP STRUCTURE

\begin{tabular}{|c|c|c|}
\hline 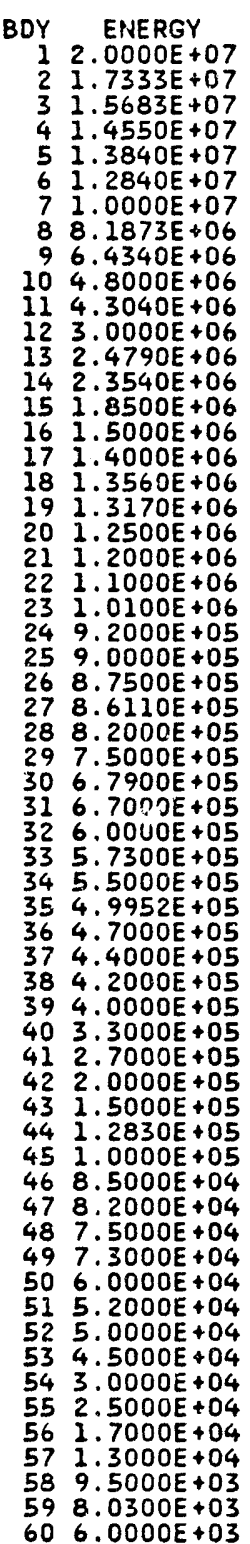 & $\begin{array}{rc}\text { BDY } & \text { ENERGY } \\
61 & 3.9000 E+03 \\
62 & 3.7400 E+03 \\
63 & 3.0000 E+03 \\
64 & 2.5800 E+03 \\
65 & 2.2900 E+03 \\
65 & 03 \\
66 & 2.2000 E+03 \\
67 & 1.8000 E+03 \\
68 & 1.5500 E+03 \\
69 & 1.5000 E+03 \\
70 & 1.1500 E+03 \\
71 & 9.5000 E+02 \\
72 & 6.8300 E+02 \\
73 & 6.7000 E+02 \\
74 & 5.5000 E+02 \\
75 & 3.0500 E+02 \\
76 & 2.8500 E+02 \\
77 & 2.4000 E+02 \\
78 & 2.1000 E+02 \\
79 & 2.0750 E+02 \\
80 & 1.9250 E+02 \\
81 & 1.8600 E+02 \\
82 & 1.2200 E+02 \\
83 & 1.1900 E+02 \\
84 & 1.1500 E+02 \\
85 & 1.0800 E+02 \\
86 & 1.0000 E+02 \\
87 & 9.0000 E+01 \\
88 & 8.2000 E+01 \\
89 & 8.0000 E+01 \\
90 & 7.6000 E+01 \\
91 & 7.2000 E+01 \\
92 & 6.7500 E+01 \\
93 & 6.5000 E+01 \\
94 & 6.1000 E+01 \\
95 & 5.9000 E+01 \\
96 & 5.3400 E+01 \\
97 & 5.2000 E+01 \\
98 & 5.0600 E+01 \\
994 & 4.9200 E+01 \\
100 & 4.8300 E+01 \\
101 & 4.7000 E+01 \\
102 & 4.5200 E+01 \\
103 & 4.4000 E+01 \\
104 & 4.2400 E+01 \\
105 & 4.1000 E+01 \\
106 & 3.9600 E+01 \\
107 & 3.9100 E+01 \\
108 & 3.8000 E+01 \\
109 & 3.7000 E+01 \\
1103 & 3.5500 E+01 \\
111 & 3.4600 E+01 \\
112 & 3.3750 E+01 \\
113 & 3.3250 E+01 \\
114 & 3.1750 E+01 \\
115 & 3.1250 E+01 \\
116 & 3.0000 E+01 \\
117 & 2.7500 E+01 \\
118 & 2.5000 E+01 \\
119 & 2.2500 E+01 \\
120 & 2.1000 E+01\end{array}$ & $\begin{array}{lc}\text { BDY } & \text { ENERGY } \\
121 & 2.0000 E+01 \\
122 & 1.9000 E+01 \\
123 & 1.8500 E+01 \\
124 & 1.7000 E+01 \\
125 & 1.6000 E+01 \\
126 & 1.5100 E+01 \\
127 & 1.4400 E+01 \\
128 & 1.3750 E+01 \\
129 & 1.2900 E+01 \\
130 & 1.1900 E+01 \\
131 & 1.1500 E+01 \\
132 & 1.0000 E+01 \\
133 & 9.1000 E+00 \\
134 & 8.1000 E+00 \\
135 & 7.1500 E+00 \\
136 & 7.0000 E+00 \\
131 & 6.7500 E+00 \\
138 & 6.5000 E+00 \\
139 & 6.2500 E+00 \\
140 & 6.0000 E+00 \\
141 & 5.4000 E+00 \\
142 & 5.0000 E+00 \\
143 & 4.7500 E+00 \\
144 & 4.0000 E+00 \\
145 & 3.7300 E+00 \\
146 & 3.5000 E+00 \\
147 & 3.1500 E+00 \\
148 & 3.0500 E+00 \\
149 & 3.0000 E+00 \\
150 & 2.9700 E+00 \\
151 & 2.8700 E+00 \\
152 & 2.7700 E+00 \\
153 & 2.6700 E+00 \\
154 & 2.5700 E+00 \\
155 & 2.4700 E+00 \\
156 & 2.3800 E+00 \\
157 & 2.3000 E+00 \\
158 & 2.2100 E+00 \\
159 & 2.1200 E+00 \\
160 & 2.0000 E+00 \\
161 & 1.9400 E+00 \\
162 & 1.8600 E+00 \\
163 & 1.7700 E+00 \\
164 & 1.6800 E+00 \\
165 & 1.5900 E+00 \\
166 & 1.5000 E+00 \\
167 & 1.4500 E+00 \\
168 & 1.4000 E+00 \\
169 & 1.3500 E+000 \\
170 & 1.3000 E+00 \\
171 & 1.2500 E+00 \\
172 & 1.2250 E+00 \\
173 & 1.2000 E+000 \\
174 & 1.1750 E+00 \\
175 & 1.1500 E+00 \\
176 & 1.1400 E+00 \\
1777 & 1.1300 E+000 \\
178 & 1.1200 E+000 \\
1779 & 1.1100 E+00 \\
180 & 1.1000 E+00\end{array}$ \\
\hline
\end{tabular}

BDY ENERGY

$1811.0900 E+00$

$1821.0800 E+00$

$1831.0700 E+00$

$1841.0600 E+00$

$185 ? .0500 \mathrm{E}+00$

$1861.0400 E+00$

$187 \div .0300 \mathrm{E}+00$

$1881.0200 E+00$

$1891.0100 E+00$

$1901.0000 E+00$

$1919.7500 E-01$

$1929.5000 \mathrm{E}-01$

$1939.2500 \mathrm{E}-0 \mathrm{I}$

$1939.2500 \mathrm{E}-\mathrm{O}$

$1949.0000 \mathrm{E}-01$

$1958.5000 E-01$

$1968.0000 E-01$

$1977.5000 \mathrm{E}-01$

$1987.0000 E-01$

$1996.5000 E-01$

$2006.0000 E-01$

$2015.5000 E-01$

$2025.0000 E-01$

202 5.

$2034.5000 E-01$

$2044.0000 E-01$

$2053.7500 E-01$

$2063.5000 E-01$

$2073.2500 E-01$

$2083.0000 E-02$

$2092.7500 E-01$

$2102.5000 E-01$

21 $2500 \mathrm{E}-\mathrm{O}$

1)

212 2.0000 -OI

$2131.7500 \mathrm{E}-01$

$2141.5000 E-01$

$2151.2500 E-01$

$2161.0000 E-01$

2179.0000 E-02

218 8.0000E -02

$2197.0000 E-02$

$2206.0000 E-02$

221 $5.0000 \mathrm{E}-02$

221 $5.0000 \mathrm{E}-02$

2224.0000 - 02

$2233.0000 \mathrm{E}-02$

$2242.5300 \mathrm{E}-02$

2251.0000 -02

$2261.0000 E-03$

$2271.0000 E-04$

$228 \mathrm{2} .0000 \mathrm{E}-05$ 
231 NEUTRON GROUP STRUCTURE

\begin{tabular}{|c|c|c|}
\hline $\begin{array}{rc}\text { BDY } & \text { ENERGY } \\
1 & 1.4918 E+07 \\
2 & 1.4550 E+07 \\
3 & 1.4191 E+07 \\
4 & 1.3840 E+07 \\
5 & 1.3499 E+07 \\
6 & 1.3165 E+07 \\
7 & 1.2840 E+07 \\
8 & 1.2523 E+07 \\
9 & 1.2214 E+07 \\
10 & 1.1912 E+07 \\
11 & 1.1618 E+07 \\
12 & 1.1331 E+07 \\
13 & 1.1052 E+07 \\
14 & 1.0779 E+07 \\
15 & 1.0513 E+07 \\
16 & 1.0253 E+07 \\
17 & 1.0000 E+07 \\
18 & 9.7531 E+06 \\
19 & 9.5123 E+06 \\
20 & 9.2774 E+06 \\
21 & 9.0484 E+06 \\
22 & 8.8250 E+06 \\
23 & 8.6071 E+06 \\
24 & 8.3946 E+06 \\
25 & 8.1873 E+06 \\
26 & 7.9852 E+06 \\
27 & 7.7880 E+06 \\
28 & 7.5957 E+06 \\
29 & 7.4082 E+06 \\
30 & 7.2253 E+06 \\
31 & 7.0469 E+06 \\
32 & 6.8729 E+06 \\
33 & 6.7032 E+06 \\
34 & 6.6476 E+06 \\
35 & 6.5924 E+06 \\
36 & 6.5377 E+06 \\
37 & 6.3763 E+06 \\
38 & 6.2189 E+06 \\
39 & 6.0653 E+06 \\
40 & 5.9156 E+06 \\
41 & 5.7695 E+06 \\
42 & 5.4881 E+06 \\
43 & 5.2205 E+06 \\
44 & 4.9659 E+06 \\
45 & 4.8432 E+06 \\
46 & 4.7237 E+06 \\
47 & 4.6070 E+06 \\
48 & 4.4933 E+06 \\
49 & 4.2741 E+06 \\
50 & 4.0657 E+06 \\
51 & 3.8674 E+06 \\
52 & 3.6788 E+06 \\
53 & 3.4994 E+06 \\
54 & 3.3287 E+06 \\
55 & 3.2465 E+06 \\
56 & 3.1664 E+06 \\
57 & 3.0882 E+06 \\
58 & 3.0119 E+06 \\
59 & 2.8650 E+06 \\
60 & 2.7253 E+06\end{array}$ & $\begin{array}{rc}\text { BDY } & \text { ENERGY } \\
61 & 2.5924 E+06 \\
62 & 2.4660 E+06 \\
63 & 2.4251 E+06 \\
64 & 2.3852 E+06 \\
65 & 2.3653 E+06 \\
66 & 2.3457 E+06 \\
67 & 2.3069 E+06 \\
68 & 2.2689 E+06 \\
69 & 2.2313 E+06 \\
70 & 2.1225 E+06 \\
71 & 2.0190 E+06 \\
72 & 1.9691 E+06 \\
73 & 1.9205 E+06 \\
74 & 1.8731 E+06 \\
75 & 1.8268 E+06 \\
76 & 1.7377 E+06 \\
77 & 1.6530 E+06 \\
78 & 1.6122 E+06 \\
79 & 1.5724 E+06 \\
80 & 1.5335 E+06 \\
81 & 1.4957 E+06 \\
82 & 1.4227 E+06 \\
83 & 1.3534 E+06 \\
84 & 1.2873 E+06 \\
85 & 1.2246 E+06 \\
86 & 1.1943 E+06 \\
87 & 1.1648 E+06 \\
88 & 1.1080 E+06 \\
89 & 1.0540 E+06 \\
90 & 1.0026 E+06 \\
91 & 9.7783 E+05 \\
92 & 9.6164 E+05 \\
93 & 9.5369 E+05 \\
94 & 9.0718 E+05 \\
95 & 8.6294 E+05 \\
96 & 8.2085 E+05 \\
97 & 7.8082 E+05 \\
98 & 7.4274 E+05 \\
99 & 7.0651 E+05 \\
100 & 6.7206 E+05 \\
101 & 6.3928 E+05 \\
102 & 6.0810 E+05 \\
103 & 5.7844 E+05 \\
104 & 5.5023 E+05 \\
105 & 5.3665 E+05 \\
106 & 5.2340 E+05 \\
107 & 5.1047 E+05 \\
108 & 4.9787 E+05 \\
109 & 4.7359 E+05 \\
110 & 4.5049 E+05 \\
111 & 4.2852 E+05 \\
112 & 4.0762 E+05 \\
113 & 3.8774 E+05 \\
114 & 3.6883 E+05 \\
115 & 3.5084 E+05 \\
116 & 3.3373 E+05 \\
117 & 3.1746 E+05 \\
118 & 3.0962 E+05 \\
119 & 3.0197 E+05 \\
120 & 2.9452 E+05\end{array}$ & $\begin{array}{lc}\text { BDY } & \text { ENERGY } \\
121 & 2.8725 E+05 \\
122 & 2.8015 E+05 \\
123 & 2.7324 E+05 \\
124 & 2.59911 E+05 \\
125 & 2.4724 E+05 \\
126 & 2.3518 E+05 \\
127 & 2.23711 E+05 \\
128 & 2.1280 E+05 \\
129 & 2.0242 E+05 \\
130 & 1.9255 E+05 \\
131 & 1.83166 E+05 \\
132 & 1.7422 E+05 \\
133 & 1.6992 E+05 \\
134 & 1.6573 E+05 \\
135 & 1.61633+05 \\
136 & 1.5764 E+05 \\
137 & 1.4996 E+05 \\
138 & 1.4264 E+05 \\
139 & 1.3569 E+05 \\
140 & 1.29077 E+05 \\
141 & 1.22777+05 \\
142 & 1.1679 E+05 \\
143 & 1.11099+05 \\
144 & 9.8037 E+04 \\
145 & 8.6517 E+04 \\
146 & 7.6351 E+04 \\
147 & 6.7379 E+04 \\
148 & 6.25111 E+04 \\
149 & 5.9462 E+04 \\
150 & 5.6562 E+04 \\
151 & 5.2475 E+04 \\
152 & 4.6309 E+04 \\
153 & 4.0868 E+04 \\
154 & 3.6066 E+04 \\
155 & 3.5175 E+04 \\
156 & 3.4307 E+04 \\
157 & 3.1828 E+04 \\
158 & 2.8088 E+04 \\
159 & 2.6058 E+04 \\
160 & 2.4788 E+04 \\
161 & 2.4176 E+04 \\
162 & 2.3579 E+04 \\
163 & 2.1875 E+04 \\
164 & 2.1335 E+04 \\
165 & 1.9305 E+04 \\
166 & 1.7036 E+04 \\
167 & 1.5034 E+04 \\
168 & 1.3268 E+04 \\
169 & 1.17099+04 \\
170 & 1.0333 E+04 \\
171 & 9.1188 E+03 \\
172 & 8.0473 E+03 \\
173 & 7.10177+03 \\
174 & 6.2673 E+03 \\
175 & 5.5308 E+03 \\
176 & 5.0045 E+03 \\
177 & 4.5283 E+03 \\
178 & 4.3074 E+03 \\
179 & 4.0973 E+03 \\
180 & 3.7074 E+03\end{array}$ \\
\hline
\end{tabular}

BDY ENERGY

$1813.3546 \mathrm{E}+03$

$1823.0354 \mathrm{E}+03$

$1832.8635 \mathrm{E}+03$

$1842.7465 E+03$

$1852.6126 E+03$

$1862.4852 E+03$

$1872.2487 E+03$

$1882.0347 E+03$

$1891.8411 E+03$

$1901.6659 E+03$

191 . $5846 E+03$

$1911.5846 \mathrm{E}+03$

$193.5073 E+03$

$1931.3639 E+03$

$1941.2341 \mathrm{E}+03$

$1956.1120 E+01$

$1967.4852 \mathrm{E}+02$

$1975.8295 E+02$

$1984.5400 \mathrm{E}+02$

$1993.5358 \mathrm{E}+02$

200 .

$2002.7536 \mathrm{E}+02$

$2012.1445 E+02$

$2021.6702 E+02$

$2031.3007 E+02$

$2041.0130 E+02$

$2057.8893 E+01$

$2066.1442 E+01$

$2074.7851 E+01$

$2083.7267 E+01$

$2092.9023 \mathrm{E}+01$

$2102.2603 E+01$

$2111.7603 E+01$

$2121.3710 E+01$ $2131.0677 \mathrm{E}+01$ $2148.3153 E+00$ $2156.4760 \mathrm{E}+00$ $2165.0435 E+00$ $2173.9279 \mathrm{E}+00$ $2183.0590 \mathrm{t}+00$ $2192.3824 \mathrm{E}+00$ $\begin{array}{ll}219 & 2.3824 \mathrm{E}+00 \\ 220 & 1.8554 \mathrm{E}+00\end{array}$ $2211.4450 E+00$ $2221.1254 \mathrm{E}+00$ $2238.7642 E-01$ $2248.3368 \mathrm{E}-01$ $2256.8256 \mathrm{E}-01$ $2266.2506 \mathrm{E}-01$ $2266.2506 E-01$ $227 \begin{aligned} & 5.3158 E-01 \\ & 228 \\ & 4.1399 E-0 I\end{aligned}$ $2284.2399 \mathrm{E}-01$ $2293.0000 E-01$ $2311.0000 E-01$ 2321.0000 E-05 
238 NEUTRON GROUP STRUCTURE

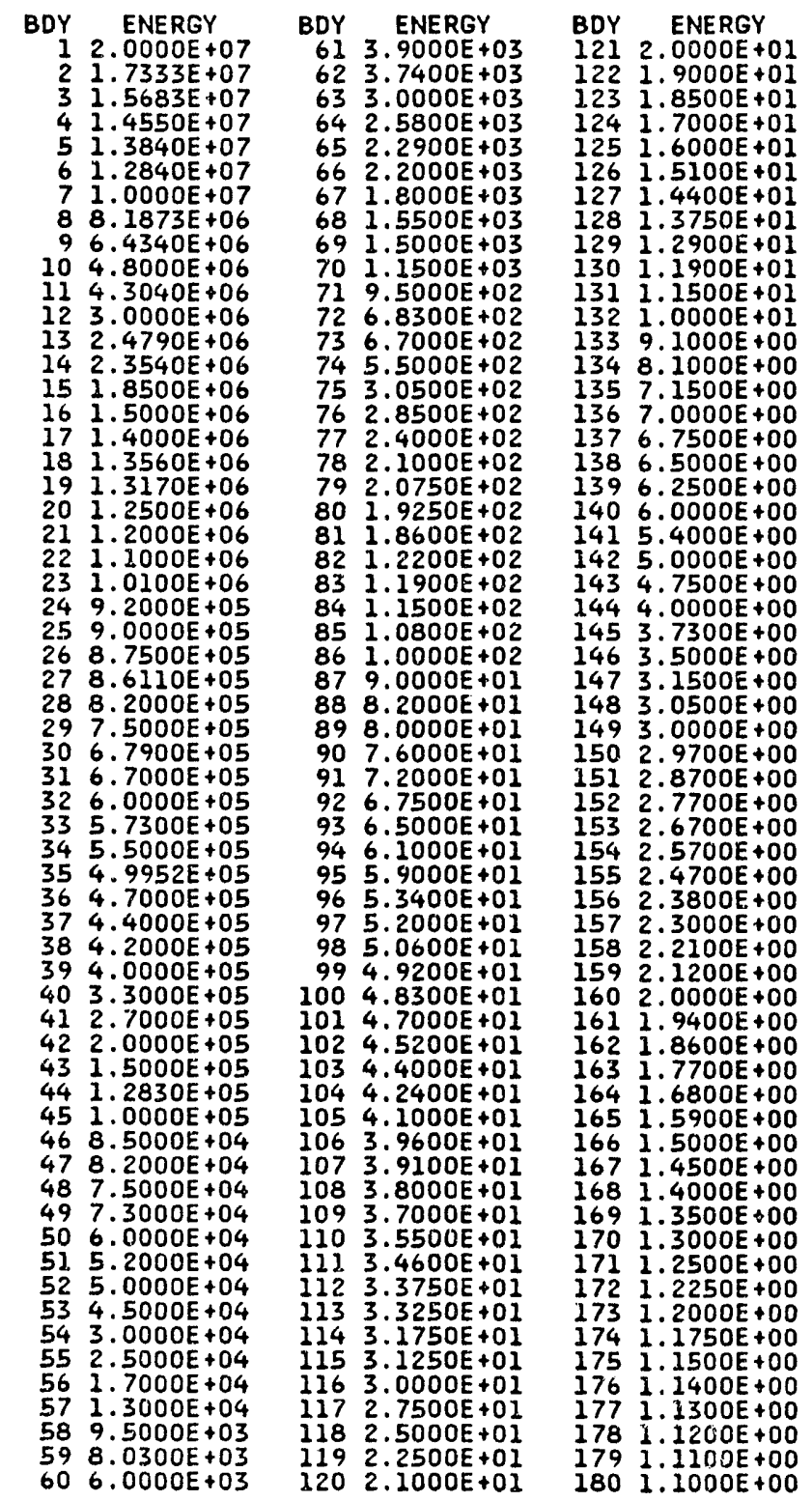

BDY ENERGY

$1811.0900 \mathrm{E}+00$

$1821.0800 E+00$

$1831.0700 E+00$

$1841.0600 E+00$

$1851.0500 E+00$

$1861.0400 E+00$

$1871.0300 E+00$

$1881.0200 E+00$

$1891.0100 E+00$

$1901.0000 E+00$

$1919.7500 \mathrm{O} 00$

$1919.7500 E-01$

$1929.5000 \mathrm{E}-01$

$1939.2500 E-01$

$1949.0000 \mathrm{E}-01$

$1958.5000 E-01$

$1968.0000 E-01$

$1977.5000 E-01$

$1987.0000 E-01$

$1996.5000 \mathrm{E}-01$

$1996.500 E-01$

$2006.2500 E-0 I$

$2016.0000 E-01$

$2025.5000 E-01$

203 5.0000E-01

204 4.5000E-OI

$2054.0000 E-01$

$2063.7500 E-01$

$2073.5000 E-0$

$2083.2500 E-01$

2093.0000 - 01

209 .

$2102.7500 \mathrm{E}-01$

$2112.5000 E-01$

$2122.2500 E-O 1$

2132.0000 - 01

2141.7500 - 01

2151 . $5000 E-01$

$2161.2500 E-01$

$2171.0000 \mathrm{E}-01$

217 . OOOE-OI

$2189.0000 \mathrm{E}-02$

$2198.0000 \mathrm{E}-02$

$2207.0000 \mathrm{E}-02$

$2216.0000 E-02$

222 5. 0000E - 02

$2234.0000 E-02$

$2243.0000 E-02$

$2252.5300 \mathrm{E}-02$

$2262.0000 E-02$

2261.0000 -02

$2277.5000 E-03$

$2285.0000 E-03$

$2294.0000 E-03$

$2303.0000 E-03$

$2312.5000 E-03$

232 2.0000E -03

$2331.5000 E-03$

$2341.2000 E-03$

$2351.0000 E-03$

$2367.0000 \mathrm{O}$

$2367.5000 E-04$

237 $5.0000 \mathrm{E}-04$

$\begin{array}{ll}238 & 1.0000 E-04 \\ 239 & 1.0000 E-05\end{array}$ 


\section{INFORMATION.4.2 "Standard" Gamma Boundaries}

The following gamma energy group structures are built into AMPX:

1. an 18-group structure, widely used for atmospheric transport calculations;

2. a 21-group structure, which is a Defense Nuclear Agency structure;

3 . a 25-group structure;

4. a 26-group structure; and

5. a 44-group structure.

These structures are defined on the following page. 
18 GAMMA GROUP STRUCTURE

$\begin{array}{rcrc}\text { BDY } & \text { ENERGY } & \text { BDY } & \text { ENERGY } \\ 1 & 1.4000 E+07 & 11 & 1.5000 E+06 \\ 2 & 1.0000 E+07 & 12 & 1.0000 E+06 \\ 3 & 8.0000 E+06 & 13 & 7.0000 E+05 \\ 4 & 7.0000 E+06 & 14 & 4.5000 E+05 \\ 5 & 6.0000 E+06 & 15 & 3.0000 E+05 \\ 6 & 5.0000 E+06 & 16 & 1.5000 E+05 \\ 7 & 4.0000 E+06 & 17 & 6.0000 E+04 \\ 8 & 3.0000 E+06 & 183.0000 E+04 \\ 9 & 2.5000 E+06 & 19 & 1.0000 E+04 \\ 10 & 2.0000 E+06 & & \end{array}$

21 GAMMA GROUP STRUCTURE

$\begin{array}{rcrc}\text { BDY } & \text { ENERGY } & \text { BDY } & \text { ENERGY } \\ 1 & 1.4000 E+07 & 11 & 1.5000 E+06 \\ 2 & 1.0000 E+07 & 12 & 1.0000 E+06 \\ 3 & 8.0000 E+06 & 13 & 7.0000 E+05 \\ 4 & 7.0000 E+06 & 14 & 4.5000 E+05 \\ 5 & 6.0000 E+06 & 153.0000 E+05 \\ 6 & 5.0000 E+06 & 16 & 1.5000 E+05 \\ 7 & 4.0000 E+06 & 17 & 1.0000 E+05 \\ 8 & 3.0000 E+06 & 18 & 7.0000 E+04 \\ 9 & 2.5000 E+06 & 194.5000 E+04 \\ 10 & 2.0000 E+06 & 203.0000 E+04\end{array}$

25 GAMTA GROUP STRUCTURE

$\begin{array}{rc}\text { BDY } & \text { ENARGY } \\ 1 & 1.3000 E+07 \\ 2 & 1.0197 E+07 \\ 3 & 7.9983 E+06 \\ 4 & 6.2737 E+06 \\ 5 & 4.9210 E+06 \\ 6 & 3.8599 E+06 \\ 7 & 3.0277 E+06 \\ 8 & 2.3748 E+06 \\ 9 & 1.8628 E+06 \\ 10 & 1.4611 E+06\end{array}$

BDY ENERGY

$212.0000 E+04$

22 I. $0000 E+04$

26 GAMPA GROUP STRUCTURE

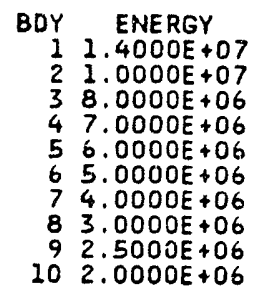

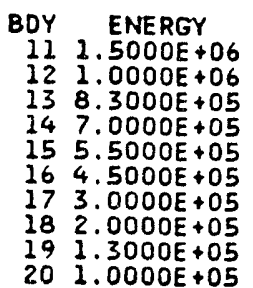

$112.5000 \mathrm{E}+06$ $121.0000 E+06$ (3000E +05 . $164.5000 E+05$ $173.0000 E+05$ $192.0000+05$ $201.0000 E+05$

44 GAMAA GROUP STRUCTURE

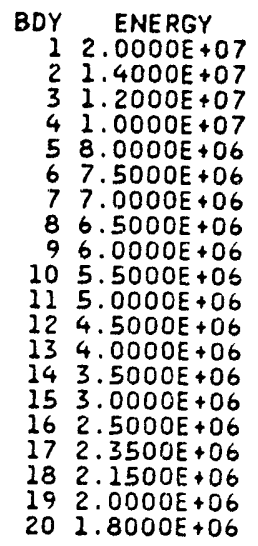

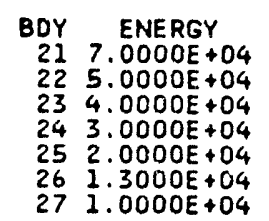

BDY ENERGY

$211.0104 E+05$ 22 $7.9252 \mathrm{E}+04$ 24 $4.2164 E+04$ 25 $3.8247 \mathrm{E}+04$ $263.0000 E+04$ 


\section{INFORMATION.5 AMPX WEIGHTING FUNCTION LIBRARY}

The XLACS and LAPHNGAS modules in AMPX can select from a library of weighting functions. The ENDF/B "TAB1" structure is used as a format. These are written on logical unit 46 as follows (a set of three binary records is required for each function):

Record $1-[8000,3, \mathrm{MT}, 0,0,0,0,0,0]$

Record $2-[8000,3, \mathrm{MT}, 0,0,0,0, \mathrm{~N} 1, \mathrm{~N} 2$, $\left(\mathrm{NBT}_{\mathrm{i}}, \mathrm{INT}_{\mathrm{i}}, \mathrm{i}=1, \mathrm{~N} 1\right)$, $\left.\left(\mathrm{X}_{\mathrm{j}}, \mathrm{Y}_{\mathrm{j}}, \mathrm{j}=1, \mathrm{~N} 2\right)\right]$

Record 3 - $[8000,3,0,0,0,0,0,0,0]$

where MT is the function identifier, N1 is the number of points in the interpolation table, N2 is is number of points in the function, NBT and INT comprise the interpulation as described below, and $\mathrm{X}$ and $\mathrm{Y}$ describe the function.

The interpolation table is defined as follows:

NBT is the point in the $\mathrm{X}, \mathrm{Y}$ table up to which the corresponding interpolation type, as specified by INT, is to be used where the following codes are used:

\begin{tabular}{cll}
\cline { 1 - 1 } Code & & Interpolation Type (INT) \\
2 & & Histogram \\
3 & & Linear X, linear Y \\
4 & & Log X, $\mathrm{X}, \log \mathrm{Y}$ \\
5 & & $\log \mathrm{X}, \log \mathrm{Y}$
\end{tabular}

Functions are stacked on logical unit 46 , one after the other.

\section{INFORMATION.6 REACTION TYPE IDENTIFIERS}

Reaction types in ENDF data are identified by integers called MT numbers. Within the AMPX system, the ENDF MT numbers are used where possible to identify the appropriate cross-section data. AMPX processed data that have no ENDF MT number counterpart are identified by integers outside the range of the ENDF MT numbers. The following definition of reaction types is taken from Appendix B of the ENDF manual, ${ }^{1}$ being augmented where necessary to describe identifiers assigned to AMPX processed data. 
The MT number generally refers to a specific neutron-nucleus interaction mechanism, but occasionally it indicates that a particular type of information is given. The general rules for assignment of MT numbers are as follows:

\begin{tabular}{|c|c|}
\hline MT (range) & Description of class of reactions \\
\hline $1-100$ & $\begin{array}{l}\text { Reaction types in which secondary particles of } \\
\text { the same type as the incident particles are } \\
\text { emitted }\end{array}$ \\
\hline $101-150$ & $\begin{array}{l}\text { Reaction types in which no secondary particles } \\
\text { of the same type as the incident particles } \\
\text { are emitted }\end{array}$ \\
\hline $151-200$ & Resonance region information \\
\hline $201-450$ & Quantities derived from the basic data \\
\hline $451-699$ & Miscellaneous quantities \\
\hline $700-799$ & $\begin{array}{l}\text { Excitation cross sections for reactions that } \\
\text { emit charged particles }\end{array}$ \\
\hline $800-999$ & (Not assigned) \\
\hline 1000-9999 & AMPX special identifiers \\
\hline
\end{tabular}

Within AMPX, different modules can assign the same MT number to a more or less inclusive set of data pertaining to the implied reaction. Specific MT assignments are given in the following table. Situations where different AMPX modules use the same MT number to identify the more or less inclusive data are identified below:

\begin{tabular}{|c|c|}
\hline MT & Description \\
\hline 1 & $\begin{array}{l}\text { Total cross section (redundant, equal to the sum of all partial cross } \\
\text { sections) }\end{array}$ \\
\hline 2 & Elastic scattering cross section \\
\hline 3 & $\begin{array}{l}\text { Nonelastic cross section (redundant, equal to the sum of all partial } \\
\text { cross sections except elastic scattering) }\end{array}$ \\
\hline 4 & $\begin{array}{l}\text { Total inelastic cross section (redundant, equal to the sum of } \mathrm{MT}= \\
51,52,53, \ldots, 90,91)\end{array}$ \\
\hline 5 & (To be assigned) \\
\hline 6 & $(n, 2 n)$ cross section for first excited state (describes first neutron) \\
\hline 7 & $(\mathrm{n}, 2 \mathrm{n})$ cross section for second excited state (describes first neutron) \\
\hline 8 & $(n, 2 n)$ cross section for third excited state (describes first neutron) \\
\hline 9 & $(n, 2 n)$ cross section for fourth excited state (describes first neutron) \\
\hline $10-15$ & (To be assigned) \\
\hline 16 & $\begin{array}{l}\text { Direct }(\mathrm{n}, 2 \mathrm{n}) \text { cross section [total }(\mathrm{n}, 2 \mathrm{n}) \text { cross section is sum of } \mathrm{MT}= \\
\quad 6,7,8,9, \text { and } 16]\end{array}$ \\
\hline
\end{tabular}


$(n, 3 n)$ cross section

Total fission cross section (sum of MT $=19,20,21,38$ )

$(\mathrm{n}, \mathrm{f})$ cross section (first chance fission)

$\left(\mathrm{n}, \mathrm{n}^{\prime} \mathrm{f}\right)$ cross section (second chance fission)

$(\mathrm{n}, 2 \mathrm{nf})$ cross section (third chance fission)

$\left(\mathrm{n}, \mathrm{n}^{\prime} \alpha\right)$ cross section

$\left(\mathrm{n}, \mathrm{n}^{\prime} 3 \alpha\right)$ cross section

$(\mathrm{n}, 2 \mathrm{n} \alpha)$ cross section

$(\mathrm{n}, 3 \mathrm{n} \alpha)$ cross section

$(\mathrm{n}, 2 \mathrm{n})$ isomeric state cross section

Absorption cross section (sum of $\mathrm{MT}=18$ and 101) includes particle reactions

$\left(\mathrm{n}, \mathrm{n}^{\prime} \mathrm{p}\right)$ cross section

$\left(\mathrm{n}, \mathrm{n}^{\prime} 2 \alpha\right)$ cross section

$(\mathrm{n}, 2 \mathrm{n} 2 \alpha)$ cross section

Used only in ENDF/B data as an LR flag to indicate that $\gamma$-emission is the mode of decay of the residual nucleus formed in the primary reaction $^{a}$

$\left(\mathrm{n}, \mathrm{n}^{\prime} \mathrm{d}\right)$ cross section

$\left(n, n^{\prime} t\right)$ cross section

$\left(\mathrm{n}, \mathrm{n}^{\prime 3} \mathrm{He}\right)$

$\left(\mathrm{n}, \mathrm{n}^{\prime} \mathrm{d} 2 \alpha\right)$ cross section

$\left(\mathrm{n}, \mathrm{n}^{\prime} \mathrm{t} 2 \alpha\right)$ cross section

$(\mathrm{n}, 4 \mathrm{n})$ cross section

(n,3nf) cross section (fourth chance fission)

Used only in ENDF/B data to indicate that internal conversion in the mode of decay of the residual nucleus formed in the primary reaction $^{a}$

Used only in ENDF/B data to indicate that electron-position pair formation is the mode of decay of the residual nucleus formed in the primary reaction ${ }^{a}$

(To be assigned)

Cross section for describing the second neutron from $(n, 2 n)$ reaction for first excited state

Cross section for describing the second neutron from $(n, 2 n)$ reaction for second excited state

Cross section for describing the second neutron from $(n, 2 n)$ reaction for third excited state 
Cross section for describing the second neutron from $(n, 2 n)$ reaction for fourth excited state [Note: $M T=46,47,48$, and 49 should not be included in the sum for the total $(n, 2 n)$ cross section]

50

51

52

90

91

$92-100$

101

102

103

104

105

106

107

108

109

110

111

112

113

114

$115-119$

120

$121-150$

151

$152-200$

201-202

203

204
(To be assigned)

$\left(n, n^{\prime}\right)$ to the first excited state

$\left(n, n^{\prime}\right)$ to the second excited state $\left(n, n^{\prime}\right)$ to the 40 th excited state

$\left(n, n^{\prime}\right)$ to the continuum

(To be assigned)

Neutron disappearance (sum of all cross sections in which a neutron is not in the exit channel), i.e.,

$$
\mathrm{MT}=101 \text { is } \sum_{\mathrm{i}=2}^{14}(\mathrm{MT}=100+\mathrm{i})
$$

$(\mathrm{n}, \gamma)$ radiative capture cross section

$(\mathrm{n}, \mathrm{p})$ cross section

$(\mathrm{n}, \mathrm{d})$ cross section

$(n, t)$ cross section

$\left(n,{ }^{3} \mathrm{He}\right)$ cross section

$(\mathrm{n}, \alpha)$ cross section

$(\mathrm{n}, 2 \alpha)$ cross section

$(n, 3 \alpha)$ cross section

(To be assigned)

$(\mathrm{n}, 2 \mathrm{p})$ cross section

$(\mathrm{n}, \mathrm{p} \alpha)$ cross section

$(\mathrm{n}, \mathrm{t} 2 \alpha)$ cross section

$(\mathrm{n}, \mathrm{d} 2 \alpha)$ cross section

(To be assigned)

Target destruction $=$ nonelastic less total $\left(n, n^{\prime} \gamma\right)$

(To be assigned)

General designation for resonance information

(To be assigned for specific resonance information)

(To be assigned)

Total hydrogen production

Total deuterium production 
205

206

207

208-250

251

252

253

254-300

301-450

451

452

453

454

455

456

457

458

459-500

501

502

503

504

505-514

515

516

517

518

519-526

527

528-531

532

533

Total tritium production

Total ${ }^{3} \mathrm{He}$ production

Total ${ }^{4} \mathrm{He}$ production

(To be assigned)

$\bar{\mu}_{L} ;$ the average cosine of the scattering angle (laboratory system) for elastic scattering

$\xi$, the average logarithmic energy decrement for elastic scattering

$\gamma$, the average of the square of the logarithmic energy decrement for elastic scattering, divided by twice the average logarithmic decrement for elastic scattering

(To be assigned)

Energy release rate parameters, $\overline{\mathrm{E} * \sigma}$, for total and partial cross sections. Subtract 300 from this number to obtain the specific reaction type identification. For example, $\mathrm{MT}=302=(300+2)$ denotes elastic scattering

Heading or title information (given only in file 1)

$\bar{\nu}$, average total (prompt plus delayed) number of neutrons released per fission event

Radioactive nuclide production

Fission-product yield data

Delayed neutrons from fission

Prompt neutrons from fission

Radioactive decay data

Energy release in fission

(To be assigned)

Total photon interaction cross section

Photon coherent scattering

(To be assigned)

Photon incoherent scattering

(To be assigned)

Pair production, electron field

Pair production, nuclear and electron field (i.e., pair plus triplet production)

Pair production, nuclear field

Photofission $(\gamma, \mathrm{f})$

(To be assigned)

Sum of all gamma-ray absorption processes

(To be assigned)

Photoneutron $(\gamma, n)$

Total photonuclear 


$\begin{array}{cl}534-601 & (\text { To be assigned) } \\ 602 & \text { Photoelectric } \\ 603-699 & (\text { To be assigned) } \\ 700 & \left(\mathrm{n}, \mathrm{p}_{0}\right) \text { cross section (cross section for leaving the residual nucleus in } \\ \text { the ground state) } \\ 701 & \left(\mathrm{n}, \mathrm{p}_{1}\right) \text { cross section for first excited state } \\ 702 & \left(\mathrm{n}, \mathrm{p}_{2}\right) \text { cross section for second excited state } \\ 703 & \left(\mathrm{n}, \mathrm{p}_{3}\right) \text { cross section for third excited state } \\ 704 & \left(\mathrm{n}, \mathrm{p}_{4}\right) \text { cross section for fourth excited state } \\ \cdot & \\ \cdot & \\ 718 & \left(\mathrm{n}, \mathrm{p}_{\mathrm{c}}\right) \text { cross section for continuum excited state } \\ 719 & \left(\mathrm{n}, \mathrm{p}_{\mathrm{c}}^{\prime}\right) \text { cross section for continuum specifically not included in total } \\ 720 & (\mathrm{redundant}, \text { used for describing outgoing proton) } \\ 721 & \left(\mathrm{n}, \mathrm{d}_{0}\right) \text { cross section for ground state } \\ 722 & \left(\mathrm{n}, \mathrm{d}_{1}\right) \text { cross section for first excited state } \\ . & \left(\mathrm{n}, \mathrm{d}_{2}\right) \text { cross section for second excited state } \\ . & \\ 738 & \left(\mathrm{n}, \mathrm{t}_{2}\right) \text { cross section for second excited state } \\ 739 & \left(\mathrm{n}, \mathrm{d}_{\mathrm{c}}\right) \text { cross section for continuum excited state } \\ 740 & \left(\mathrm{r}, \mathrm{t}_{0}\right) \text { cross section for ground state } \\ 741 & \left(\mathrm{n}, \mathrm{t}_{1}\right) \text { cross section for first excited state } \\ & \end{array}$
(redundant, used for describing outgoing triton) 
(n, ${ }^{3} \mathrm{He}_{\mathrm{c}}$ ) cross section for continuum

$\left(\mathrm{n},{ }^{3} \mathrm{He}_{\mathrm{c}}\right.$ ) cross section for continuum specifically not included in $\sigma$ total (redundant, used for describing outgoing ${ }^{3} \mathrm{He}$ ) $\left(\mathrm{n}, \alpha_{0}\right)$ cross section for ground state

. $\left(\mathrm{n}, \alpha_{1}\right)$ cross section for first excited state

$\left(\mathrm{n}, \alpha_{\mathrm{c}}\right)$ cross section for continuum

( $\mathrm{n}, \alpha_{\mathrm{c}}$ ) cross section for continuum specifically not included in $\sigma_{\mathrm{T}}$ (redundant, used to describe outgoing $\alpha$ )

$1000^{6}$

$1001^{b}$

1007

(To be assigned)

XSDRNPM-produced transport cross section based on the outscatter approximation, that is,

$$
\sigma_{\mathrm{tr}}=\sigma_{\mathrm{a}}+(1-\bar{\mu}) \sigma_{\mathrm{s}}
$$

(Note: $\sigma_{\mathrm{a}}$ is $\mathrm{MT}=27 ; \sigma_{\mathrm{s}}$ is $\mathrm{MT}=2$ )

XSDRNPM-produced transport cross section based on the inscatter approximation, that is,

$\sigma_{\mathrm{tr}} \equiv \sigma_{\mathrm{t}}-\frac{\int_{0}^{\infty} \sigma_{1}\left(\mathrm{E}^{\prime} \rightarrow \mathrm{E}\right) \mathrm{j}\left(\mathrm{E}^{\prime}\right) \mathrm{d} \mathrm{E}^{\prime}}{3 \mathrm{j}(\mathrm{E})}$

1008 Thermal scattering matrix for coherent data

1018 Fission spectrum, chi $(\chi)$. The fission spectrum is represented in ENDF file 5 data as a function of neutron energy. This array is a collapse of that data

1099 Group integral of the weighting function

1452 Average of the product of $\bar{\nu}$ times the fission cross section, $\overline{\nu \sigma_{f}}$

(Note that this "derived" parameter should never be used on an

AMPX master library, in order to avoid the possibility that it

may be processed in some modules without being self-shielded.)

1500-1501 Same as 1000-1001 except for gamma-ray cross sections

1527 Gamma-ray energy absorption coefficients. These data are also called kerma factors

$3002 \quad$ Infinite dilution averages for elastic scattering

3018

3102

9901-9999 Successive values of weighting spectra that were used to produce the

Infinite dilution averages for fission

Infinite dilution averages for $(n, \gamma)$ current version of this library

a The "primary" reaction could be, for example, an $\left(n, n^{\prime}\right),(n, p),(n, \alpha),\left(n, n^{\prime} p\right)$, etc., reaction.

${ }^{b}$ Transport cross sections are weighted with currents in XSDRNPM. All other cross sections are flux weighted. 


\section{INFORMATION.7 GLOSSARY OF TERMS}

Multigroup cross-section processing has its own jargon. In the following glossary, terms used to describe cross-section libraries, terms applicable to cross sections used in discrete-ordinates codes, ENDF terms, AMPX format terms, CCCC format terms, resonance region and temperature effects terminology, and terms applicable to thermal cross sections are defined. References cited in the glossary give more thorough discussions of the terms.

\section{INFORMATION.7.1 General Terms Used to Describe Cross-Section Libraries}

BCD

Used to denote that a computer code or data library is in a formatted form, which usually can be readily exchanged between installations with different computers (could be actually in BCD or EBCDIC characters), usually in the form of card records or print records.

\section{Binary}

Used to describe an unformatted library, which will probably only be useable on the brand of computing system on which it was written.

\section{Blocked tape}

A magnetic tape written with each physical record containing a fixed number of logical records. For example, computer codes in most RSIC packages are written with 40 card images (logical records) per block.

\section{Card image}

A record on a tape or disk which is a copy of an 80-column card. Most source programs and data libraries which the Radiation Shielding Information Center (RSIC) distributes are in card-image form. Card image tapes are "BCD" in the sense defined above.

\section{CCC}

The RSIC Computer Code Collection (CCC). ${ }^{1}$ These are packages associated with a computer code or system of codes usually designed to perform radiation transport calculations. The package consists of source program, sample input, and output data for executing one or more demonstration problems. Packages are assigned a CCC number (e.g., CCC-254/ANISN-ORNL).

\section{DD cards}

The DD (data definition) statement describes a data set that is to be used in a job step and specifies the input and output facilities required for use of the data set. 
DLC

The RSIC Data Library Collection (DLC). ${ }^{2}$ These are packages associated with a data library. A retrieval code is usually included for manipulating the data into a form suitable for use in one of the CCC's. Packages are assigned a DLC number (e.g., DLC-41/VITAMIN-C).

$\mathrm{JCL}$

The Job Control Language (JCL) refers to the statements containing the information required by the operating system to initiate and control the processing of jobs.

\section{PSR}

The RSIC Peripheral Shielding Routine Collection (PSR). ${ }^{3}$ These are packages associated with a code or code system designed to perform operations peripheral to an actual radiation transport calculation (e.g., multigroup cross-section generation). Some RSIC DLCs produced with PSR packages are assigned a PSR number (e.g., PSR-63/AMPX).

\section{INFORMATION.7.2 Discrete-Ordinate Terms}

\section{ANISN activity cross section}

An extra cross section inserted into an ANISN cross-section table for the purpose of calculating reaction rates, dose factors, etc., using the "activity" option in ANISN.4

\section{ANISN binary format}

An unformatted cross-section representation for use in ANISN. Each ANISN "material" is represented by two records, an identification record, and a record containing an ANISN cross-section table.

\section{ANISN card-image format}

A "BCD" form of cross-section representation which can be easily exchanged between installations. Most DLCs with ANISN cross sections are distributed in this form with a simple computer code to allow the user to convert to ANISN binary format.

\section{ANISN cross-section table}

A matrix of multigroup cross sections with $\mathrm{N}$ columns, one for each energy group, with each column containing terms describing $\sigma_{\mathrm{a}}, \overline{\nu \sigma_{\mathfrak{f}}}, \sigma_{\mathrm{t}}$ and cross sections for scattering into that group (including within-group scattering). Column 1 refers to the highest energy group and column $\mathrm{N}$ to the lowest energy group. More than $\mathrm{N}+$ 3 ) terms will be required if "activity" cross sections are included and/or if there are "upscattering" terms in the table. 


\section{ANISN material}

Each component of a Legendre expansion of the scattering processes for a given element (e.g., oxygen) is described by an ANISN cross-section table. These are referred to as a "material." Thus, for $\mathrm{P}_{3}$ representation, four materials are required $\left(\mathrm{P}_{0}, \mathrm{P}_{1}, \mathrm{P}_{2}\right.$, and $\left.\mathrm{P}_{3}\right)$.

\section{ANISN $(2 \ell+1)$ term}

The ANISN code does not internally multiply terms by $(2 \ell+1)$ during the course of the discrete-ordinates calculation. The factor ( 3 for $P_{1}, 5$ for $P_{2}$, etc.) can be provided by means of the mixing table but is quite often included in the cross sections prior to input to ANISN. DLCs in RSIC in "ANISN" format have the $(2 \ell+1)$ factor included. Note that the DTF series of codes do multiply by the $(2 \ell+1)$ internally. Thus, DLCs in "ANISN" format will cause trouble if used directly in DTF-IV, and vice versa.

\section{Coupled multigroup cross sections}

Coupled refers to a cross-section data set with information describing neutron interactions, gamma-ray production from neutron interactions, and gamma-ray interactions. For $\mathrm{N}$ neutron groups and $\mathrm{G}$ gamma-ray groups, the corresponding ANISN cross-section table would have $\mathrm{N}+\mathrm{G}$ columns, column 1 for the highest energy neutron group, column $\mathrm{N}$ for the lowest energy neutron group, column $N+1$ for the highest energy gamma-ray group, and column $\mathrm{N}+\mathrm{G}$ for the lowest energy gamma-ray group. A coupled cross-section set can be used to do neutron, secondary gamma-ray, and primary gamma-ray transport in a single computer run.

\section{FIDO input}

A scheme that allows many shorthand provisions for representing card input data. FIDO input is used for many codes developed at ORNL, including ANISN, DOT, and AMPX. See Sect. 7.FIDO.

\section{Fixed and free-form FIDO}

The original FIDO format was a fixed format that required rigid observance of the columns in which data were input. The later free-form formats allow the user to write his input without regard to column position, where he simply separates his input with one or more blanks.

\section{Group-ordered form}

An alternative form of cross sections for a discrete-ordinates code. All cross sections for all materials for a given energy group are located together. This reduces the amount of core storage required in running a discrete-ordinates code because data for all materials for only one energy group are needed in core at a given time. The term "group-independent" is sometimes used when referring to the group-ordered form.

\section{INFORMATION}




\section{INFORMATION.7.3 ENDF Terms}

\section{ENDF/B}

The Evaluated Nuclear Data File ${ }^{5}$ ENDF) is a library of cross-section data for a variety of materials. Each evaluated data set ("evaluation") represents a "best" judgment for a given material of how the cross sections and energy and angular distributions of scattered particles vary with incident particle energy considering all the available measured data, and, where necessary, nuclear model calculations. Each version of ENDF/B is approved and released by the Cross Section Evaluation Working Group (CSEWG) which has members from various laboratories, industry, and universities throughout the United States. The data are available from the National Nuclear Data Center (NNDC) at Brookhaven National Laboratory. The current version is ENDF/B-V.

\section{ENDF format (BCD and binary)}

The format ${ }^{2}$ for ENDF/B is a set of rules for representing cross sections and angular and energy distributions of neutrons and gamma rays. The BCD format is a card-image form used for transmittal between installations. The binary format is usually used to store data at a given installation. Most processing codes (including the AMPX system) will process either format. The binary format is a more efficient format that permits faster access to its data and requires considerably less space for its storage.

\section{CSEWG}

The Cross Section Evaluation Working Group (CSEWG) consists of representatives from various laboratories, universities, and industries who meet annually to discuss the status of ENDF/B. They are responsible for the evaluation, review, testing, and subsequent approval of cross sections for inclusion in the ENDF/B libraries.

\section{Resonance parameters}

ENDF/B evaluations can represent the energy region where many cross-section resonances occur with resonance parameters. For these cases, the evaluation may also have "background" point cross sections in the resonance region. The resonance parameters are used in specified formulae to generate point cross sections. These are then added to the "background" cross sections to reproduce the point cross-section representation which the evaluator intended for that material.

Resonance parameters provide a way of specifying the features of a cross section with sets of parameters, which, when used in a fitting procedure, allow one to determine an energy mesh and associated cross-section values. A variety of types of resonance parameters may be encountered, including single-level Breit-Wigner (SLBW), multilevel Breit-Wigner (MLBW), Adler-Adler, and Reich-Moore (RM). The latter two forms provide a more general means of fitting the data, but have rarely been used in earlier versions of ENDF/B. Parameters can also be found for what are referred to as unresolved resonances. In this case, the fits are for a region where the resonances are 
so closely spaced that it is impossible or impractical to "resolve" them and, thereby, be able to use normal resonance parameters. In this region, resonances are specified only in a statistical sense and require special processing procedures.

\section{Gamma-ray multiplicities}

An energy-dependent ratio of the gamma-ray production cross section (for a given neutron interaction) to the neutron interaction cross section. The ratio is the number of gamma rays (discrete or continuum) produced by a given neutron interaction.

\section{Gamma-ray production cross section}

The energy-dependent cross section for producing gamma rays due to a given neutron interaction. These are represented as "point" cross sections on ENDF/B. Gamma-ray production cross sections can be given in ENDF format for discrete gamma rays or for a continuum of gamma rays.

MAT

The ENDF ${ }^{6}$ identification number for a particular material's cross sections.

MF

An ENDF file identification for a particular type of data (e.g., MF $=3$ refers to neutron point cros. - ctions, $M F=4$ to neutron scattering angular distributions, etc.). These are denoted as different "files" of data but are not actually in a different physical tape file.

MT

An ENDF reaction-type identification for a particular cross section. Note that MAT and MT are sometimes used as input parameters to multigroup processing codes. MT numbers used by ENDF and in the AMPX system are defined in Sect. 4.INFORMATION.6.

\section{NNDC}

The National Nuclear Data Center (NNDC) (formerly NNCSC) at Brookhaven National Laboratory is responsible for distributing the ENDF/B data to requesters in the United States.

\section{Point cross sections}

ENDF/B evaluations normally have energy cross-section pairs to represent the energy dependence of a given reaction. This representation is termed "point cross sections." As indicated above, a point cross-section representation may be accompanied in the resonance region by a set of resonance parameters. An ENDF "resonance" evaluation may be processed by a code such as NPTXS ${ }^{1}$ in AMPX which converts the resonance parameters into point cross sections.

INFORMATION 
Resolved resonance region

The energy region where cross sections are fit by resonance parameters is called the resolved resonance region.

Unresolved resonance region

The energy region where information about specific resonances cannot be determined. Instead, parameters which describe families or sequences of resonances in a statistical sense are given.

\section{INFORMATION.7.4 Multigroup Cross-Section Terms}

\section{Collapsing}

The process of weighting a multigroup library over a multigroup flux spectrum. The weighting spectrum may be guessed at or calculated. The reduction is from a "fine-" group to a "broad-" group level. In general, the collapsing procedure preserves reaction rates for each energy range.

\section{Downscatter}

Terms that refer to multigroup cross sections for transferring from a given energy group to a group at a lower energy.

\section{Multigroup cross sections}

The cross sections which are appropriate for solving a multigroup representation of the Boltzmann transport equation. Multigroup reaction cross sections and transfer matrices are generated by averaging over a weighting function with an energy dependence that is (hopefully) close to the spectrum of particles in the problem to be solved. It is presumed that the more groups that are used to represent the energy region, the better the multigroup approximation becomes.

\section{Transfer matrix}

The multigroup cross sections representing scattering between energy groups. They can be a combination of upscatter, within-group, and downscatter terms. A transfer matrix may be provided for many processes on a general library, such as an AMPX master library.

\section{Weighting function}

The energy-dependent function used to calculate the average cross sections and transfer matrices for a multigroup cross-section library. Typical functions include a Maxwellian function for the thermal energy region, $1 / E$ in the slowing-down region above thermal and below the region where fission neutrons are born, a fission spectrum, a Gaussian distribution near $14 \mathrm{MeV}$, etc. For some applications, the reciprocal total 
cross section $\left(1 / \sigma_{t}\right)$ is used to handle situations where deep penetration problems in a pure material are to be solved.

\section{Within-group scattering}

The multigroup cross section for scattering and remaining in the same energy group.

\section{INFORMATION.7.5 AMPX Format Terms}

\section{AMPX interface} format.

A multigroup library in AMPX master or AMPX working library interface

\section{AMPX master library interface format}

A multigroup library format for representing reaction cross sections and transfer matrices for an arbitrary number of processes. Data can be provided for neutron reactions and scattering processes, gamma-ray production processes, and gamma-ray reactions and scattering processes. The formats are designed with a generality paralleling ENDF/B in that all reactions can be carried along with any associated scattering transfer matrices. In addition, resonance parameters can be carried on the interface, and/or Bondarenko factors can be provided. Identification records are included which describe all nuclides on the master library interface as well as other useful information, such as energy-group structures.

\section{AMPX weighted library}

XSDRNPM can be used to collapse an AMPX working library using a variety of options. The resulting library is referred to as a "weighted" library. It has the same format as an AMPX working library interface (see below).

\section{AMPX working library interface}

A master interface is not a convenient form for use in most nuclear calculations. NITAWL will combine cross sections, perform resonance self-shielding calculations, combine neutron and gamma-ray matrices, etc., to produce an AMPX working interface. This working library is analogous to (but more general than) an ANISN library.

\section{BCD AMPX library}

A BCD format is used to allow transmitting of AMPX libraries between installations with different computers. The AIM module of AMPX, which creates this library, can also be used to read it and convert it back to the binary form.

\section{INFORMATION}




\section{LAPHNGAS library}

An AMPX interface created by the LAPHNGAS module to describe gammaray production processes. Both gamma-ray production cross sections and multiplicities can be included. This is in the AMPX master library format.

\section{Magic word}

The AMPX interfaces use a "magic-word" scheme to compact the potentially lengthy transfer matrix arrays. This effectively eliminates zero and impossible elements. A magic word is a 9-digit integer consisting of three 3-digit integers defining the energy group and the range of energy groups that can scatter into the given group. The group numbers are in the sequence of highest-source, lowest-source and sink groups.

\section{SMUG library}

An AMPX interface created by the SMUG module to describe gamma-ray reaction and scat ering processes. This is in the AMPX master library format.

\section{INFORMATION.7.6 CCCC Format Terms}

\section{$\mathrm{CCCC}$}

The Committee on Computer Code Coordination (CCCC) has devised standards and procedures for facilitating the exchange of data between various reactor physics codes. The ISOTXS library produced by the AMPX system is in the CCCC format, which is a format for multigroup neutron cross sections..

\section{BRKOXS}

This is another CCCC file and is used with the ISPTXS file when the Bondarenko self-shielding method is to be used. The file $\mathrm{e}^{7}$ contains multigroup self-shielding factors for total (current weighted), capture, fission, transport, and elastic-scattering cross sections. Data can be provided for several values of $\sigma_{0}$ and temperature.

\section{ISOTXS}

A nuclide ordered file ${ }^{7}$ of neutron multigroup cross sections. Reaction cross sections and transfer matrices are provided for a selected number of reactions rather than all the possible reactions included on an ENDF tape. The reaction data are infinitely dilute cross sections and can be used in combination with the BRKOXS file in a code like SPHINX ${ }^{8}$ to provide problem-dependent self-shielding cross sections at the problem temperature. 
Total cross section (current weighted)

The calculation of diffusion coefficients requires an inverse weighting of the total cross section. These values are not equal to the sum of the multigroup partial cross sections that are produced with flux weighting.

\section{Transport cross section}

A transport cross section is needed in diffusion theory to determine a diffusion coefficient. In transport theory, it is often used to account for the effects of truncating the order of fit to the scattering matrices.

\section{INFORMATION.7.7 Resonance Region and Temperature Effects}

\section{Bondarenko factors}

Multigroup factors that are tabulated as a function of background cross section per atom $\left(\sigma_{0}\right)$ and temperature $(\mathrm{T})$. These factors can be used in an iterative procedure that uses infinite dilution cross sections, the Bondarenko ${ }^{9}$ factors, and the system description to produce self-shielded cross sections. Codes such as BONAMI and SPHINX provide these kinds of calculations.

\section{Dancoff correction factor}

A factor ${ }^{10}$ that accounts for shadowing effects caused by the presence of other absorber lumps in the neighborhood of the lump being calculated. For example, Dancoff factors $\operatorname{can}$ be used to account for the effects of locating fuel pins in lattice geometries in a simple calculation that only mocks up a single fuel pin and its surrounding moderator.

\section{Doppler broadening}

An accounting of the energy variation of cross sections as a function of temperature. Codes that process ENDF data usually perform Doppler broadening for a given temperature (or several temperatures) prior to the multigroup averaging.

\section{f-factors}

The same as Bondarenko factors.

\section{Nordheim integral treatment}

An integral transport theory method ${ }^{11}$ based on reducing a two-region (fuel/moderator) system to a single region by assuming the moderator flux is $1 / E$. Other notable characteristics of the method are in using escape probabilities for region coupling, $\psi$ and $\chi$ functions for Doppler broadening, and a particularly clever and efficient scheme for calculating slowing-down integrals. It has been very successfully used for a wide variety of thermal system analyses. 


\section{Self-shielding}

This is an accounting for the effects that a resonance has on the flux in the vicinity of the resonance. Self-shielding depends on the temperature, composition, and geometry of the particular situation. Various approaches are taken to account for this effect in multigroup libraries, including the Nordheim integral treatment, the Bondarenko method, and integral transport theory in the AMPX system.

\section{INFORMATION.7.8 Thermal Region}

\section{Free-gas model}

A thermal-scattering model that assumes the target nucleus has a Maxwellian thermal energy distribution and that atoms are unbound.

\section{Maxwell-Boltzmann distribution}

This function is often used as a guess for a weighting flux in the thermal range. It is based on slowing down in a pure scattering region whose temperature is characterized by the temperature of the medium.

$\mathbf{S}(\alpha, \beta)$

A representation used in ENDF/B to present thermal-scattering cross sections that can include chemical, molecular, and crystalline binding effects.

\section{REFERENCES}

1. B. McGill, B. F. Maskewitz, C. M. Anthony, H. E. Comolander, and H. R. Henrickson, Abstracts of the Computer Code Packages Assembled by the Radiation Shielding Information Center, ORNL/RSIC-13, Vols. I-IV, Union Carbide Corp., Nucl. Div., Oak Ridge Natl. Lab., January 1976.

2. R. W. Roussin and J. B. Wright, Abstracts of the Data Library Packages Assembled by the Radiation Shielding Information Center, ORNL/RSIC-31, Union Carbide Corp., Nucl. Div., Oak Ridge Natl. Lab., 1973.

3. B. F. Maskewitz, Abstracts of the Peripheral Shielding Code Packages Assembled by the Radiation Shielding Information Center, ORNL/RSIC-31, Union Carbide Corp., Nucl. Div., Oak Ridge Natl. Lab., 1973.

4. W. W. Engle, Jr., A User's Manual for ANISN-A One-Dimensional Discrete Ordinates Transport Code with Anisotropic Scattering, K-1693, Union Carbide Corp., Nucl. Div., Oak Ridge Gaseous Diffusion Plant, March 30, 1967.

5. D. Garber, ENDF/B Surnmary Documentation, BNL-17541 (ENDF-201), Brookhaven Natl. Lab., October 1975.

6. D. Garber et al., Data Formats and Procedures for the Evaluated Nuclear Data File, ENDF, BNL/NCS-50496 (ENDF 102), Brookhaven Natl. Lab., October 1975. 
7. B. M. Carmichael, Standard Interface Files and Procedures for Reactor Physics Codes, Version III, LA-5486-MS, Los Alamos Natl. Lab., February 1974.

8. W. J. Davis, M. B. Yarbrough, and A. B. Bortz, SPHINX: A OneDimensional Diffusion and Transport Nuclear Cross Section Processing Code, WARD-XS-3045-17, Westinghouse Electric Corp., August 1977.

9. I. I. Bondarenko, Ed., Group Constants for Nuclear Reactor Calculations Consultants Bureau, New York, 1964.

10. Reactor Physics Constants, ANL-5800, Argonne National Lab., July 1963, pp. 280-291.

11. L. W. Nordheim, "The Theory of Resonance Absorption," p. 58 in Proceedings of Symposia in Applied Mathematics, Vol. XI, G. Birkhoff and E. P. Wigner, Eds., American Mathematical Society, 1961. 


\section{AMPX-77 INSTALLATION GUIDE}

AMPX-77 is distributed on a standard label magnetic tape. The various files on this tape contain several types of information, including (1) the source decks for the various modules, (2) the source decks fc, various auxiliary programs, (3) a FORTRAN77 source file of routines shared by several of the modules, (4) a collection of IBM assembler language routines shared by the modules, (5) a collection of FORTRAN routines to override most of the assembler programming, (6) the system driver program for both IBM and Cray computers, (7) sample problem input and output, and (8) auxiliary files for use in creating a group-structure library, supplying basic cross-section data, etc.

The contents of the files are given in the following table:

\begin{tabular}{|c|c|c|c|c|}
\hline File & Contents & Language & Blocking & Entry \\
\hline 1 & AIM & $\overline{\text { FORTRAN }}$ & 80 & $\overline{\text { AIM }}$ \\
\hline 2 & AJAX & FORTRAN & 80 & AJAX \\
\hline 3 & ALE & FORTRAN & 80 & MAIN \\
\hline 4 & ALPO & FORTRAN & 80 & MAIN \\
\hline 5 & BONAMI & FORTRAN & 80 & BONAMI \\
\hline 6 & CASTROL & FORTRAN & 80 & MAIN \\
\hline 7 & CLAROL & FORTRAN & 80 & CLAROL \\
\hline 8 & COLLINS & FORTRAN & 80 & MAIN \\
\hline 9 & COMAND & FORTRAN & 80 & MAIN \\
\hline 10 & COMET & FORTRAN & 80 & MAIN \\
\hline 11 & CONTAC & FORTRAN & 80 & CONTAC \\
\hline 12 & CORECTOL & FORTRAN & 80 & CRCTOL \\
\hline 13 & CREST & FORTRAN & 80 & MAIN \\
\hline 14 & FRESH & FORTRAN & 80 & MAIN \\
\hline 15 & GERITOL & FORTRAN & 80 & MAIN \\
\hline 16 & $\mathrm{ICE}$ & FORTRAN & 80 & ICE2 \\
\hline 17 & JERGENS & FORTRAN & 80 & MAIN \\
\hline 18 & LAPHNGAS & FORTRAN & 80 & MAIN \\
\hline 19 & LAVA & FORTRAN & 80 & LAVA \\
\hline 20 & MAD & FORTRAN & 80 & MAIN \\
\hline 21 & MALOCS & FORTRAN & 80 & MALOCS \\
\hline 22 & NITAWL-II & FORTRAN & 80 & NITAWL \\
\hline 23 & NPTXS & FORTRAN & 80 & MAIN \\
\hline 24 & PAL & FORTRAN & 80 & MAIN \\
\hline 25 & PERFUME & FORTRAN & 80 & PERFUM \\
\hline 26 & POLIDENT & FORTRAN & 80 & NPTXS \\
\hline 27 & PRELL & FORTRAN & 80 & PRELL \\
\hline 28 & PRUDE & FORTRAN & 80 & MAIN \\
\hline 29 & RADE & FORTRAN & 80 & RADE \\
\hline 30 & ROLAIDS & FORTRAN & 80 & RELIEF \\
\hline
\end{tabular}




\begin{tabular}{|c|c|c|c|c|}
\hline File & Contents & Language & Blocking & Entry \\
\hline 31 & RUFFLES & FORTRAN & 80 & $\overline{\text { RUFFLES }}$ \\
\hline 32 & SALVAGE & FORTRAN & 80 & MAIN \\
\hline 33 & SMILER & FORTRAN & 80 & SMILER \\
\hline 34 & SMUG & FORTRAN & 80 & SMUG \\
\hline 35 & TABU & FORTRAN & 80 & TABU \\
\hline 36 & TIDE & FORTRAN & 80 & TIDE \\
\hline 37 & UNITAB & FORTRAN & 80 & UNITAB \\
\hline 38 & VASELINE & FORTRAN & 80 & MAIN \\
\hline 39 & VEL & FORTRAN & 80 & MAIN \\
\hline 40 & WAX & FORTRAN & 80 & WAX \\
\hline 41 & WINE & FORTRAN & 80 & MAIN \\
\hline 42 & WISK & FORTRAN & 80 & MAIN \\
\hline 43 & WORKEK & FORTRAN & 80 & WORKER \\
\hline 44 & WORM & FORTRAN & 80 & MAIN \\
\hline 45 & XLACS & FORTRAN & 80 & MAIN \\
\hline 46 & XSDRNPM & FORTRAN & 80 & XSDRN \\
\hline 47 & ZEST & FORTRAN & 80 & ZEST \\
\hline 48 & Subroutine library & FORTRAN & 80 & \\
\hline 49 & $\begin{array}{l}\text { Subroutine library } \\
\text { (IBM Assembler) }\end{array}$ & IBM Assembler & & \\
\hline 50 & $\begin{array}{l}\text { Cray FORTRAN replacements } \\
\text { for IBM assembler routines }\end{array}$ & $\begin{array}{l}\text { FORTRAN } \\
\text { for UNICOS }\end{array}$ & & \\
\hline 51 & $\begin{array}{l}\text { ALIAS, program to produce sub- } \\
\text { routine library from the decks }\end{array}$ & PL1 & & \\
\hline 52 & AMPX-77 driver program (IBM) & IBM Assembler & & \\
\hline 53 & AMPX-77 driver program (Cray) & $\begin{array}{l}\text { FORTRAN for } \\
\text { UNICOS }\end{array}$ & & \\
\hline 54 & $\begin{array}{l}\text { Program to translate IBM } \\
\text { FORTRAN to Cray FORTRAN }\end{array}$ & FORTRAN & & \\
\hline 55 & $\begin{array}{l}\text { AMPX procedure for IBM } \\
\text { MVS systems }\end{array}$ & & & \\
\hline 56 & $\begin{array}{l}\text { Thermal ENDF/B data for } \\
\text { Task } 1\end{array}$ & & & \\
\hline 57 & Input data for Task 1 & & & \\
\hline 58 & Input data for Task 2 & & & \\
\hline 59 & Input data for Task 3 & & & \\
\hline 60 & Input data for Task 4 & & & \\
\hline 61 & Input data for Task 5 & & & \\
\hline 62 & Input data for Task 6 & & & \\
\hline 63 & Input data for Task 7 & & & \\
\hline 64 & Output from Task 1 & & & \\
\hline 65 & Output from Task 2 & & & \\
\hline 66 & Output from Task 3 & & & \\
\hline
\end{tabular}




\begin{tabular}{|c|c|}
\hline File & Contents \\
\hline 67 & Output from Task 4 \\
\hline 68 & Output from Task 5 \\
\hline 69 & Output from Task 6 \\
\hline 70 & Output from Task 7 \\
\hline 71 & $\begin{array}{l}\text { AIM unloaded copy of library } \\
\text { from Task } 2\end{array}$ \\
\hline 72 & Group structures \\
\hline
\end{tabular}

Even though any of the modules in AMPX can be made to execute independently, the modular design makes it highly advantageous to build the system of codes that will then be accessed by the AMPX driver module (File 52 or 53).

The first task to initiate is that of building a subroutine library, containing the routines shared by many of the major computational modules. Although it is possible simply to compile these and to always link them with the program routines, this method will prove very wasteful, at least on IBM MVS systems, because no program will require all of them.

\section{INSTALLATION.1 CONSTRUCTION OF SUBROUTINE LIBRARY}

\section{INSTALLATION.1.1 IBM MVS System}

This involves compiling or assembling the routines on Files 48 and 49 of the tape. Once the hex decks are obtained they can be processed through the ALIAS routine (File 51) to yield the requisite subroutine library.

The procedure is illustrated below:

// EXEC FORTVC

//SYSIN DD Point to File 48

// EXEC ASMHC

//SYSIN DD Point to File 49

// EXEC PGM=ALIAS

//STEPLIB DD Point to a load module file containing ALIAS from File 51

//SYSLIN DD This is your subroutine library

//SYSIN DD Point to hex decks from the two compilations, above

\section{INSTALLATION.1.2 Cray UNICOS System}

This involves compiling the FORTRAN routines in Files 48 and 50 and then using the loader to create the requisite library.

1. Create, procure, or use some method to get your subroutines into a file. 
2. Execute "cft77" or other compiler specifying desired options and point to the file mentioned in the first step.

3. Execute "bld" specifying desired options and using the output from Step 2. The output from this operation is your subrovtine library, and will be one of the input files to the "segldr" when you create your load modules.

\section{INSTALLATION.2 AMPX DRIVER PROGRAM CONSTRUCTION}

This involves either assembling the program on File 52 (IBM) or compiling the program on File 53 (Cray) to produce the requisite load module.

\section{INSTALLATION.2.1 IBM MVS System}

1. Assemble the program in File 52.

2. Prepare a load module named AMPX using the linkage editor. You may need to use the "NCAL" parameter, which says to ignore unresolved references.

\section{INSTALLATION.2.2 Cray UNICOS System}

1. Compile the program in File 53 using cft 77 or alternative.

2. Execute the "segldr" and save the resulting load module.

Note that the driver in this case is really the driver program for the SCALE system and will absolutely require that the input data for the modules always be terminated by an "end" card. This driver also expects to access a file named "aliases," which can consist of one blank card, in order to make the program operate properly.

\section{INSTALLATION.3 AMPX-77 MODULE CONSTRUCTION}

This is the next step and involves the compilation of the source routines for any or all of the 47 modules in this release (Files 1-47) and linking with the subroutine library routines we discussed in Sect. 5.INSTALLATION.1 to produce a load module. (In the case of IBM systems, these modules can be made members of a program library that is part of the "GO" STEPLIB data collection.)

\section{INSTALLATION.2.1 IBM MVS System}

The procedure is as follows:

// EXEC FORTVCL

//SYSIN DD Point to the source deck in the appropriate file

//LKED.STEPLIB DD

$/ / \mathrm{DD}$

INSTALLATION 
// DD Point to the subroutine library from Sect. 5.INSTALLATION.1

//LKED.SYSLMOD DD Point to a partitioned dataset to be used

//LKED.SYSIN DD * as a program library

ENTRY Name of entry point for the module (see table at front of Sect. 5.INSTALLATION)

NAME Module $(R)$

\section{INSTALLATION.3.2 Cray UNICOS System}

1. Put the source for the module in a file.

2. Compile the file using "cft77" or an equivalent.

3. Execute "bld" on the file to create a library file for the subroutines in the module.

4. Execute "segldr," including the file from Step 3 and the subroutine library from Sect. 5.INSTALLATION.1 to create a load module. The entry point for the module is taken from the table at the front of Sect. 5.INSTALLATION and is part of the input to "segldr."

\section{INSTALLATION.4 EXECUTING A MODULE}

\section{INSTALLATION.4.1 IBM MVS System}

The panacea (real or imagined) for using an IBM system is that of dealing with the job control language (JCL), which is used to identify all files and to specify their interrelationships. Many of these problems can be minimized by using the IBM facility that allows one to write JCL procedures which can be accessed simply by referencing the procedure; however, this method may itself be very lengthy and complicated.

The procedure shown in Fig. 5.INSTALLATION.1 and given in File 55 of the tape is such a procedure. It was used to execute all the sample problems described in Sect. 6.SAMPLE PROBLEMS of this report. The discussion of the procedure given below will not attempt to discuss the coding aspects of the procedure, but will identify the AMPX-specific characteristics.

The simplest use of the procedure involves simply executing it:

\section{// EXEC AMPX77}

This activates the procedure, which is two steps: a linkage editor step that allows one to add on and modify modules (the LKED step), and a step (the GO step) which executes the modules selected for the problems.

When theie is no requirement for the linkage editor step, the card above will be immediately followed by a card pointing to a file that will be used by one of the modules. This card will generally be in the form:

$$
\text { //GO.FTnnF001 DD "information to point to file" }
$$

where $\mathrm{nn}$ is the logical number of the file. 


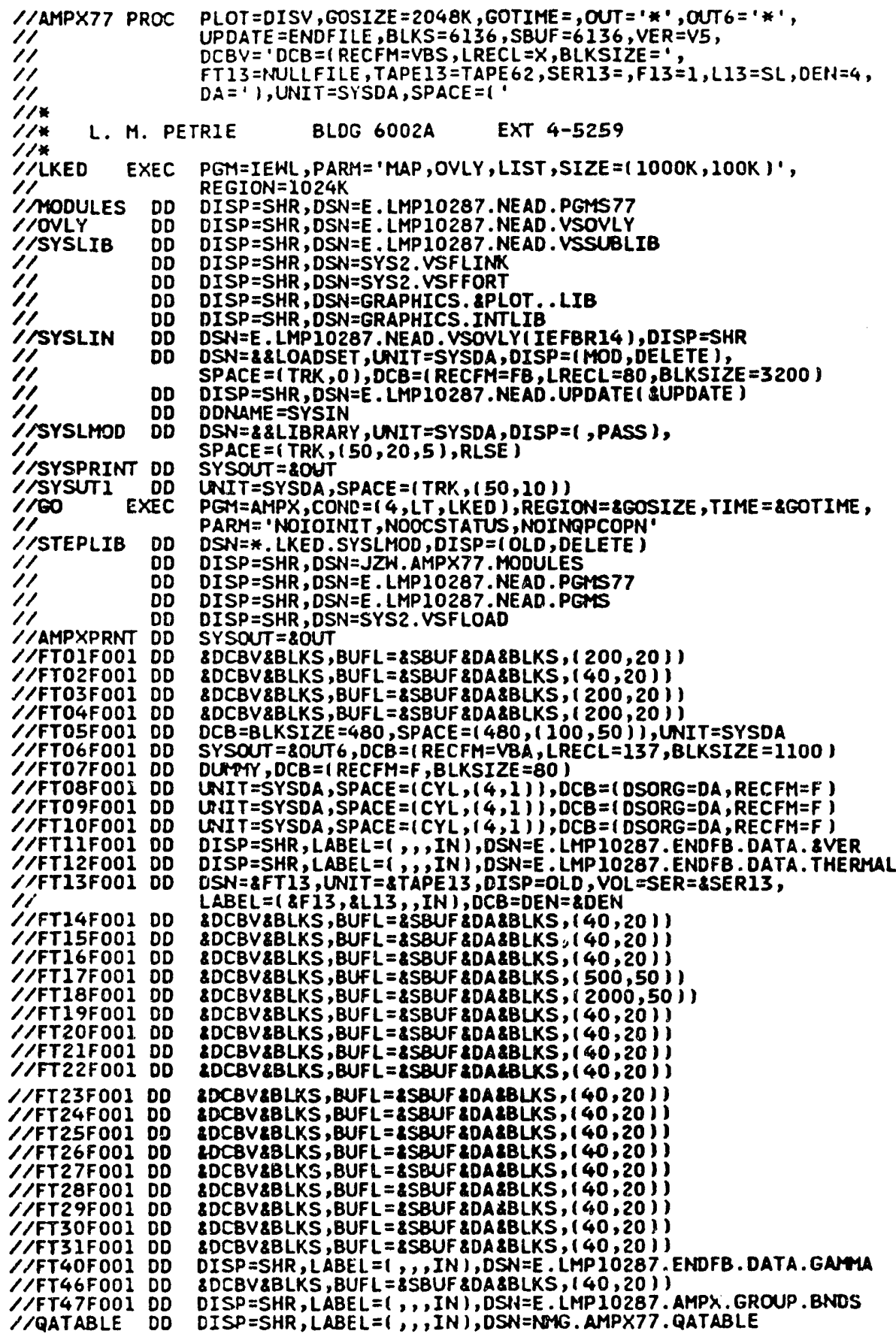

Fig. 5.INSTALLATION.1. IBM procedure to execute AMPX-77. 
The standard procedure has the following default assignments:

\begin{tabular}{|c|c|}
\hline Logical Nos. & Default file \\
\hline $1-4,14-31,46$ & System space \\
\hline 5 & Module data input \\
\hline 6 & Printed output \\
\hline 7 & Dummy (intended for punch output) \\
\hline $8-10$ & Direct access scratch space \\
\hline 11 & General-purpose ENDF/B library \\
\hline 12 & Thermal ENDF/B data library \\
\hline 13 & BCD ENDF/B data on tape \\
\hline 40 & ENDF/B gamma file \\
\hline 47 & Group boundary file \\
\hline
\end{tabular}

If a module reads or writes to one of the above assignments, the user may have to override the JCL in the procedure. Following IBM replacement requirements, the overridden JCL statements must be given as the first of the "GO" DD cards and must be in exactly the order in which they are used in the procedure (which is in simple numerical order in our case).

The input for the modules is given in the SYSIN file. If a user wanted only default assignments, an execution would consist of the following JCL:
// EXEC AMPX77
//GO.SYSIN DD *
problem data

\section{INSTALLATION.4.1.1 Adding or modifying a module using the IBM procedure}

The procedure for compiling and executing a new program using the procedure described above is as follows:

// EXEC FORTRAN compiler procedure

//SYSIN DD *

FORTRAN decks

// EXEC AMPX

//SYSIN DD *

NAME module

//GO.DD Cards

problem data

where "module" is the name assigned to the new module.

If it is necessary to replace a subroutine in an existing module, the subroutine must be compiled and "linked" with the other routines in the module. In this case, it is noted that the modules in AMPX are located in a file which has a DDNAME of MODULES. The procedure is the following: 
// EXEC FORTRAN compiler procedure

//SYSIN DD *

Subroutine cards

// EXEC AMPX77

//SYSIN DD *

INCLUDE MODULES (module)

ENTRY entry

NAME module

//GO.DD cards

problem data

In this case "module" is the name of the module to update, and "entry" is the name of the main program for the module.

\section{INSTALLATION.4.2 Cray UNICOS System}

1. Place the source into a separate file.

2. Use "cft77," or equivalent, to compile the file.

3. Run "bld" on the file from Step 2.

4. Run the "segldr" using the file from Step 3, the subroutine library from Sect. 5.INSTALLATION.1 to create a new program.

5. Execute the "SCALE" driver discussed in Sect. 5.INSTALLATION.2 with the input for the module placed in a file called "sysin." Note that the input for each module must be terminated by an "end" card. 


\section{SAMPLE PROBLEMS}

This section contains a discussion of several sample problems that use AMPX modules. They will perhaps serve to clarify some of the information presented in the discussion of options and inputs. Another obvious use of this section is for checking the codes as they are implemented on your computer system. The runs selected address several common tasks one must perform in producing and using multigroup cross sections. As such, a run may require executing many AMPX modules and, thereby, will serve as mini-tutorials to identify how the codes relate to each other. The tasks that will be demonstrated are designed to do the following:

1. create a multigroup neutron library,

2. create a multigroup coupled neutron-gamma-ray library,

3. do a resonance self-shielding calculation,

4. homogenize and collapse cross sections,

5. move multigroup libraries to another compu ${ }^{\wedge}$ ing system,

6. average point cross sections, and

7. convert the cross sections on an AMPX master library for use in the ANISN program.

\section{SAMPLE PROBLEMS.1 CREATE A MULTIGROUP NEUTRON LIBRARY}

This task is the most difficult, time-consuming, and expensive task one will encounter in dealing with multigroup cross sections. At least part of the problem is related to the codes and procedures one must use, but the very nature of the problem is complex, requiring several diverse and complicated developments to be vist to produce a complete set of data.

One is immediately confronted by several decisions:

1. A procedure must be selected. In some cases, the choice of a code or a technique will automatically select the procedure. In a modular programming system, however, this may invilve selecting between options, for example, to use for resonance self-shiel 2 . will need to consider the approximations as they relate to the particular application.

2. A group structure must be selected. Many times this will involve either picking an existing structure previously shown to be adequate for a similar application, or at least starting with an existing structure and adding or deleting boundaries, as deemed necessary. Generally speaking, the multigroup structure will not be fine enough to explicitly do resonance self-shielding calculations. This will require supplemental calculations to treat these effects. 
3. A spectrum must be picked to use in weighting the cross sections. If the energy group structure contains on the order of a hundred or more energy groups, divided roughly according to bins of equal lethargy, an "approximate" spectrum can be taken, such as a Maxwellian-shaped function at thermal, tied to a $1 / E$ spectrum in the slowing-down range above thermal and below a fission spectrum distribution, which may continue all the way to the upper energy boundary, although it is probably prudent to cut this distribution at 8-10 MeV, above which a $1 / \mathrm{E}$ variation is probably more reasonable. Note that by using this very simplistic approach, we are depending on the calculation used to collapse from the fine-group structure to a broad group (25-50 energy groups, or less), augmented by a resonance calculation, to refine the averaged values, to correct the scattering distributions, etc.

4. A resonance self-shielding method must be selected. If the application is for fast reactors, a treatment based on the narrow-resonance approximation, such as the Bondarenko method, may be adequate. Thermal systems may be adequately handled by the Nordheim integral treatment or even the Bondarenko method if the factors are prepared using weighting functions based on integral transport calculations. Any situation needing high accuracy or having complicated geometries may need a detailed integral transport treatment.

5. A decision must be made on the order of fit that will be made to the groupto-group scattering matrix terms. For many reactor applications, $\mathrm{P}_{1}$ fits are adequate; however, for accurately calculating leakage effects, at least a $\mathrm{P}_{3}$ fit should be taken. (Note that the cost of generating the highordered terms is small relative to that of generating the $P_{0}$ terms and that it is easy to throw away terms that may not be necessary. Therefore, it is prudent to generate termsat least to the highest order that one will need when making the original library.) Generally speaking, shielding applications require higher-ordered fits than do reactor applications.

6. Consideration should be given to thermal-scattering effects. If one is, for example, making a set of cross sections for hydrogen, its cross sections at thermal will be different, depending on whether the hydrogen atom is in gaseous form, bound in a water molecule or a polyethylene molecule, or part of $\mathrm{ZrH}$, etc. The physics of all these situations is presented in $\mathrm{ENDF} / \mathrm{B}$ as $\mathrm{S}_{\alpha \beta}$ (scattering law) data.

7. The basic cross sections must be located in ENDF/B-formatted files. Usually, one will use the standard libraries, though there are several files available from other evaluation efforts, such as the LENDL file (a file of evaluations from Lawrence Livermore Laboratory) and the JENDL file (a file of Japanese evaluations) - both in the ENDF/B structure.

The nuclides we will process in our sample cases are from the ENDF/B Version V "standards" library (see Table 6.SAMPLE PROBLEMS.1). Even though the extent 
of this collection is not sufficient to solve many practical problems, there are no restrictions on its distribution, such as exist with other ENDF/B-V files.

\begin{tabular}{llc}
\multicolumn{2}{l}{ Table 6.SAMPLE PROBLEMS.1. } & ENDF/B "standard" library \\
\hline MAT & Nuclide & Photon production data \\
\hline 1146 & He-3 & No \\
1301 & H & Yes \\
1303 & Li-6 & Yes \\
1305 & B-10 & Yes \\
1306 & C & Yes \\
1379 & Au-197 & No \\
1395 & U-235 & Yes \\
\hline
\end{tabular}

We will process ${ }^{235} \mathrm{U}$ and $\mathrm{C}$ for our sample cases.

For a group structure, we will use a 238-group structure originally developed for shipping cask studies. For weighting we will select a simple spectrum consisting of a Maxwellian spectrum at thermal extending to $0.125 \mathrm{eV}$, tied to a $1 / \mathrm{E}$ variation up to $67.4 \mathrm{keV}$, tied to a fission spectrum which extends up to $10 \mathrm{MeV}$, which in turn is tied to a $1 / \mathrm{E}$ variation up to the top of the group structure. This spectrum will be used on all nuclides.

If our application had included large regions containing a single mixture, we could have decided that it was important to refine our weighting spectrum by weighting the cross sections by a $1 / E \Sigma_{t}$ spectrum, where $\Sigma_{t}$ might be taken as the macroscopic point cross sections for important mixtures in the problem.

The processing procedure used for this task is shown in Fig. 6.SAMPLE PROBLEMS.1. Here the processing of a nuclide will involve taking ENDF/B data and processing it through XLACS to produce an AMPX master library, which is then scanned by the RADE module to look for inconsistencies or improper values. The library is also used in the VASELINE module, which plots group-averaged data. The results of RADE and VASELINE are studied, after which the COMET module may be run to repair any moderate inconsistencies that were observed.

This procedure may be augmented with other codes when the nuclide has unresolved resonance data that can be processed into Bondarenko factors (see Fig. 6.SAMPLE PROBLEMS.2). In this case, the XLACS run is made in parallel with runs of PRUDE, which makes point cross-section strings for unresolved cross sections, and TABU, which converts them to Bondarenko factors. Finally, UNITAB couples the XLACS output with the Bondarenko factors to produce a master that is then processed as in the previous procedure.

Figure 6.SAMPLE PROBLEMS.3 shows another procedure which would have been used had we elected to do some $1 / E \Sigma_{t}$ weighting. Here, POLIDENT is used to generate a set of point data for the nuclide, JERGENS uses it to form a $1 / E \Sigma_{t}$ function and to tie Maxwellian and fission spectrum to it, after which XLACS uses the spectrum to weight its cross sections. 


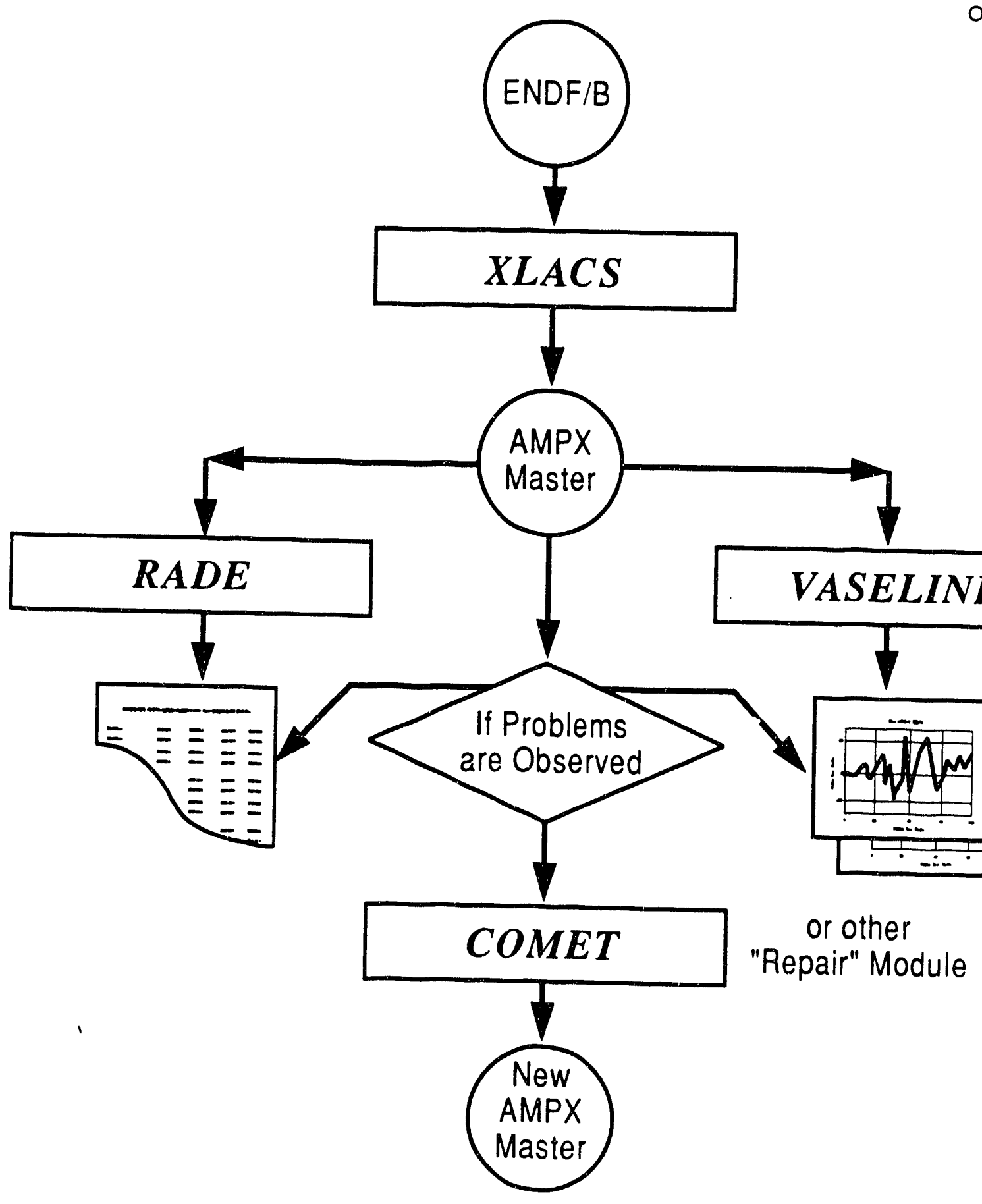

Fig. 6.SAMPLE PROBLEMS.1. Primary neutron library production procedure. 


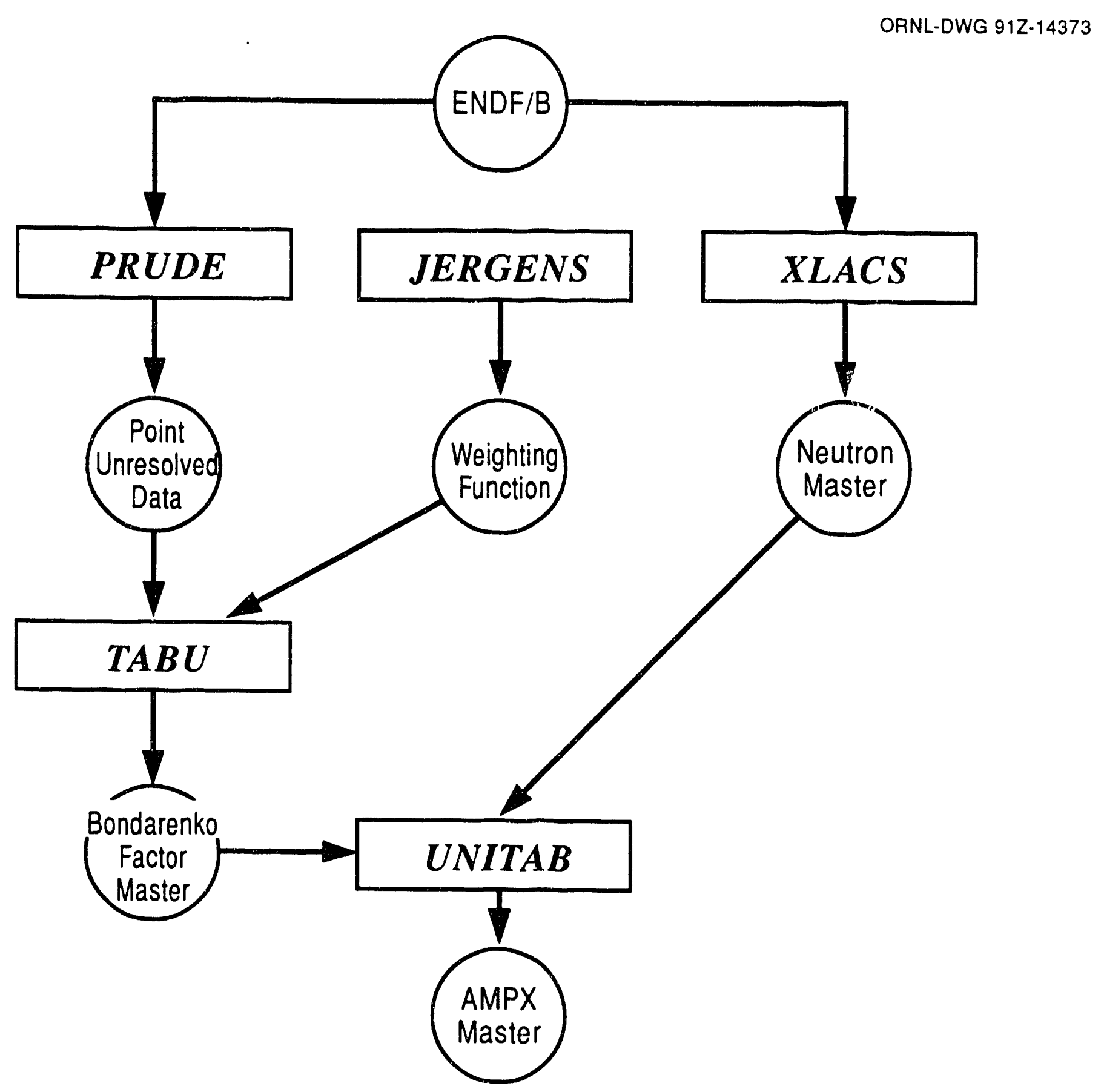

Fig. 6.SAMPLE PROBLEMS.2. Procedure to add Bondarenko factors to an AMPX master. 


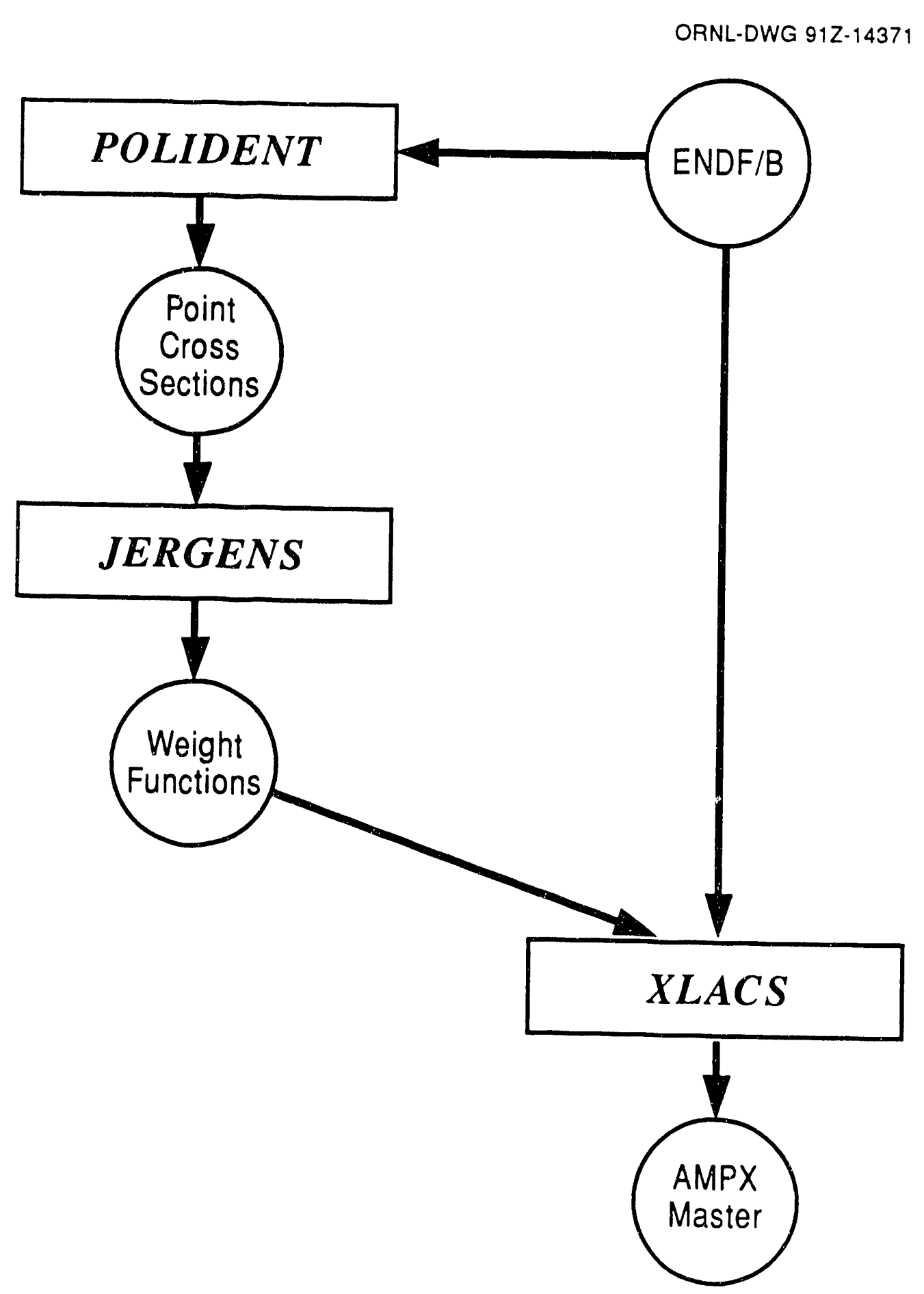

Fig. 6.SAMPLE PROBLEMS.3. Procedure to perform $1 / E \Sigma_{t}$ weighting. 
When all nuclides are processed, the individual master files are combined into a single master file using the AJAX module. This procedure is illustrated in Fig. 6.SAMPLE PROBLEMS.4.

ORNL-DWG $91 Z-14374$

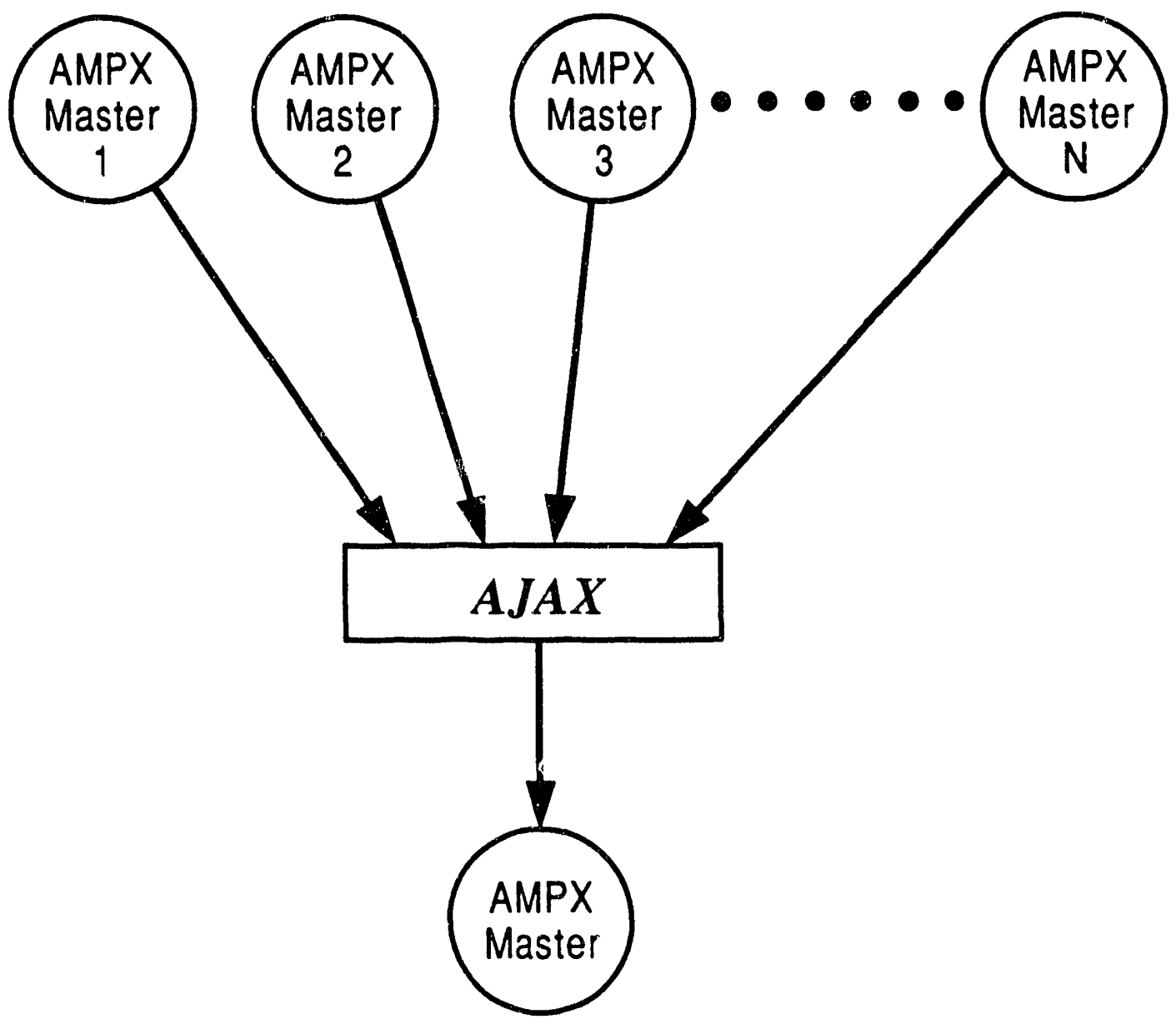

Fig. 6.SAMPLE PROBLEMS.4. Procedure to combine AMPX masters. 
The input for the ${ }^{235} \mathrm{U} X \mathrm{XACS}$ and RADE runs is shown below:

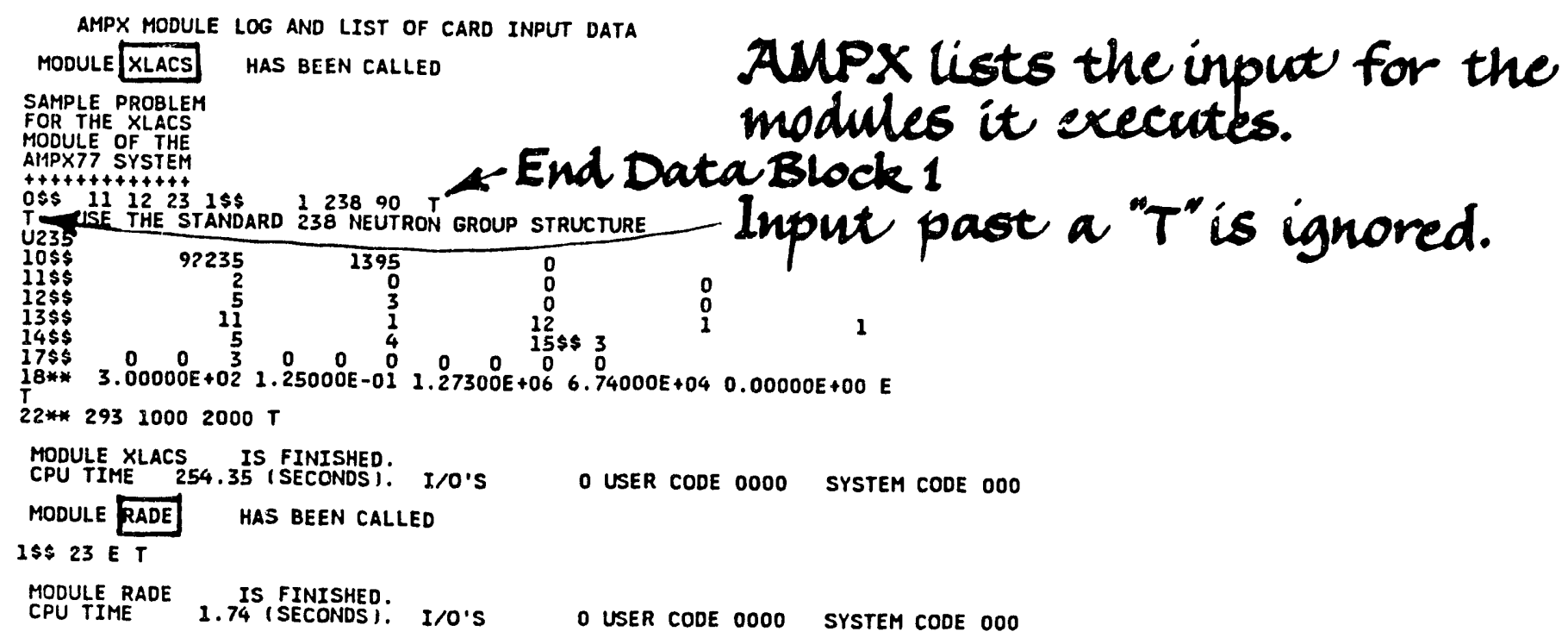

Portions of the output for ${ }^{235} \mathrm{U}$ follow: 


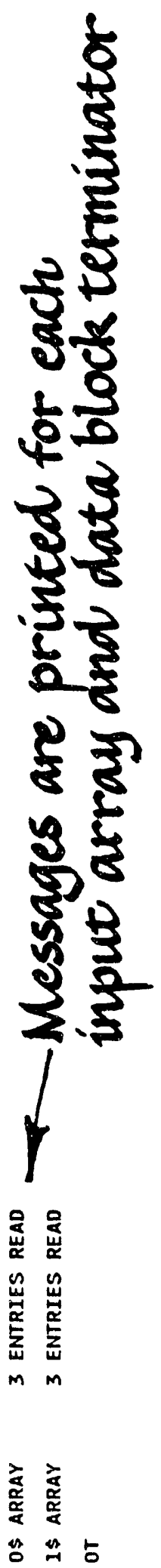



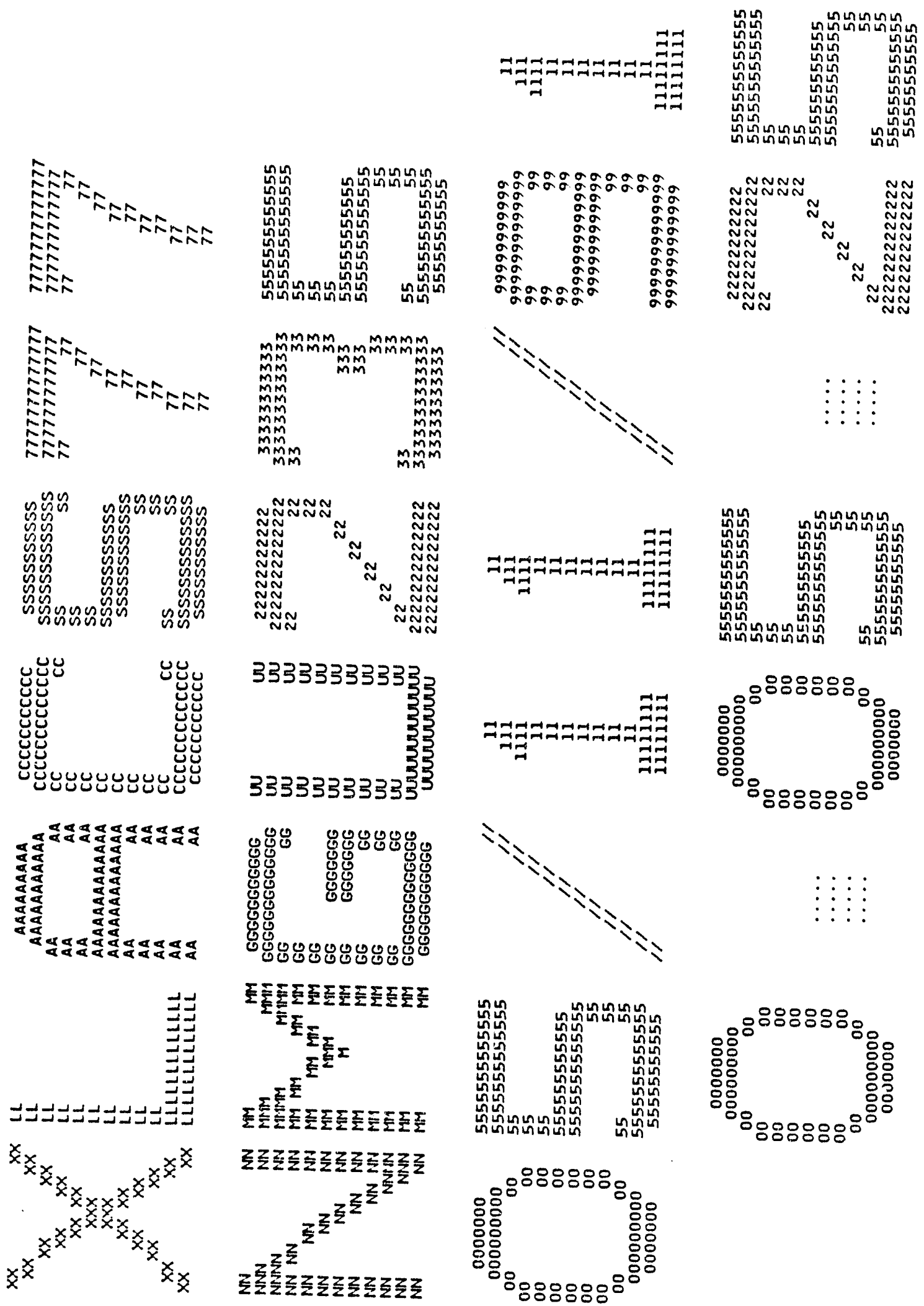


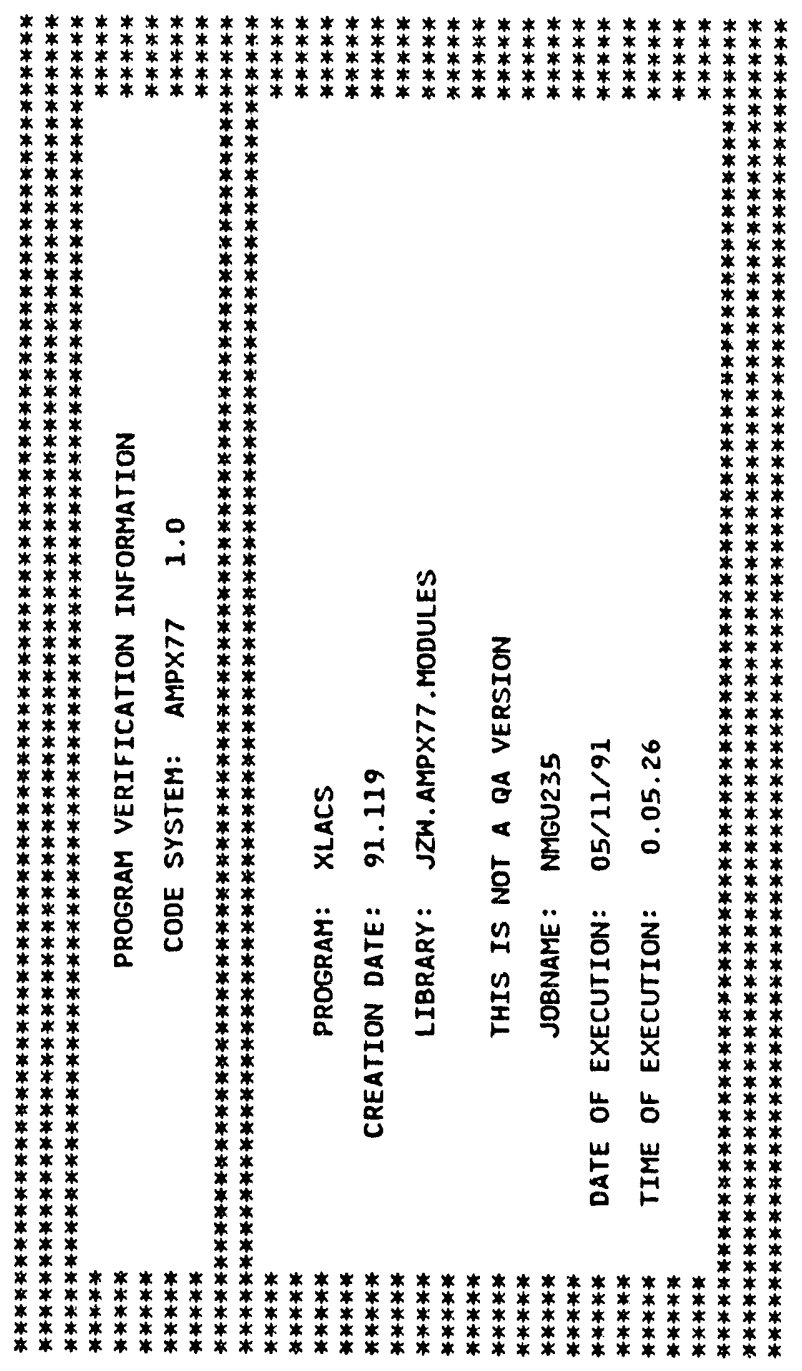

SAMPLE PROBLEMS 


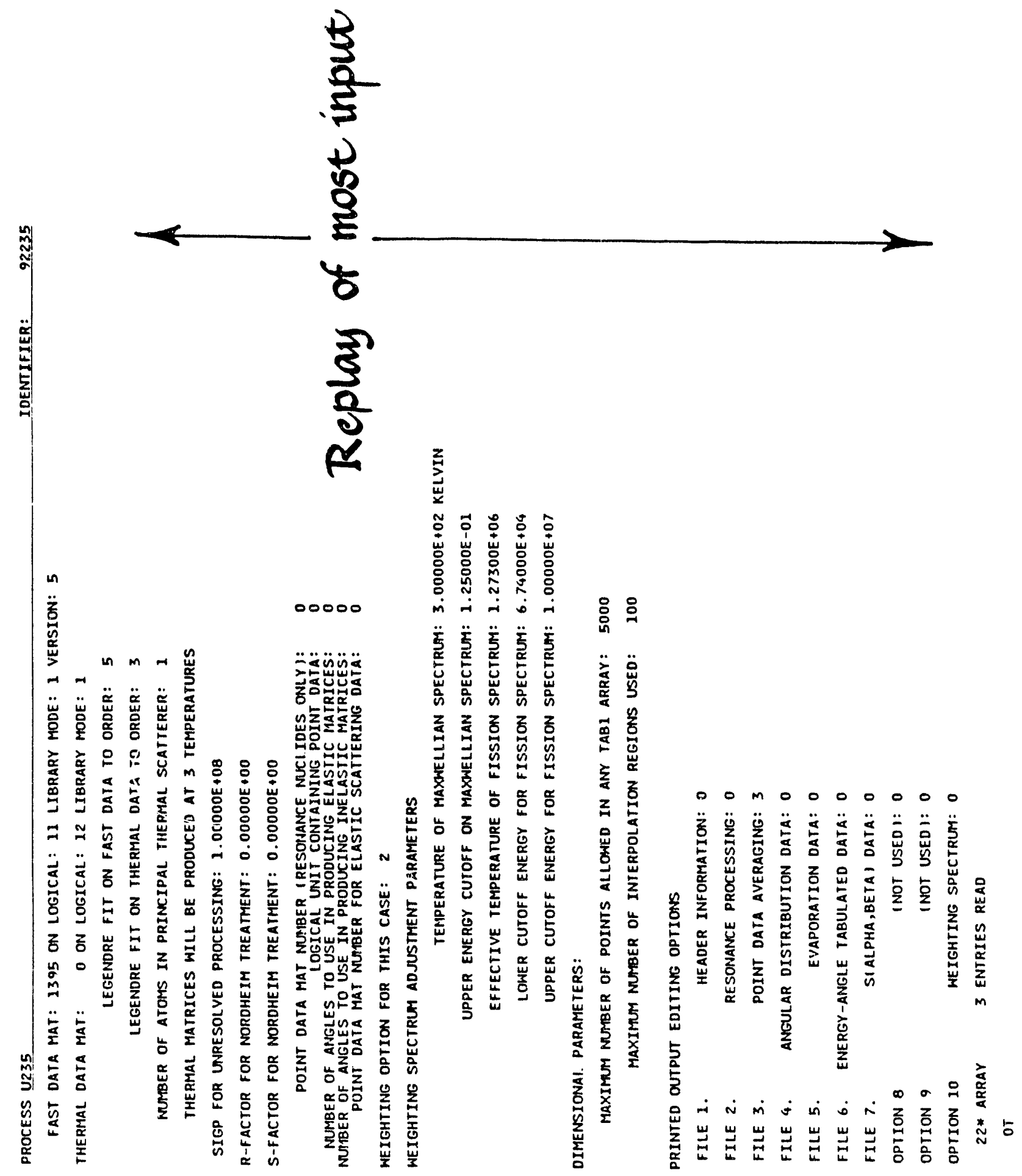



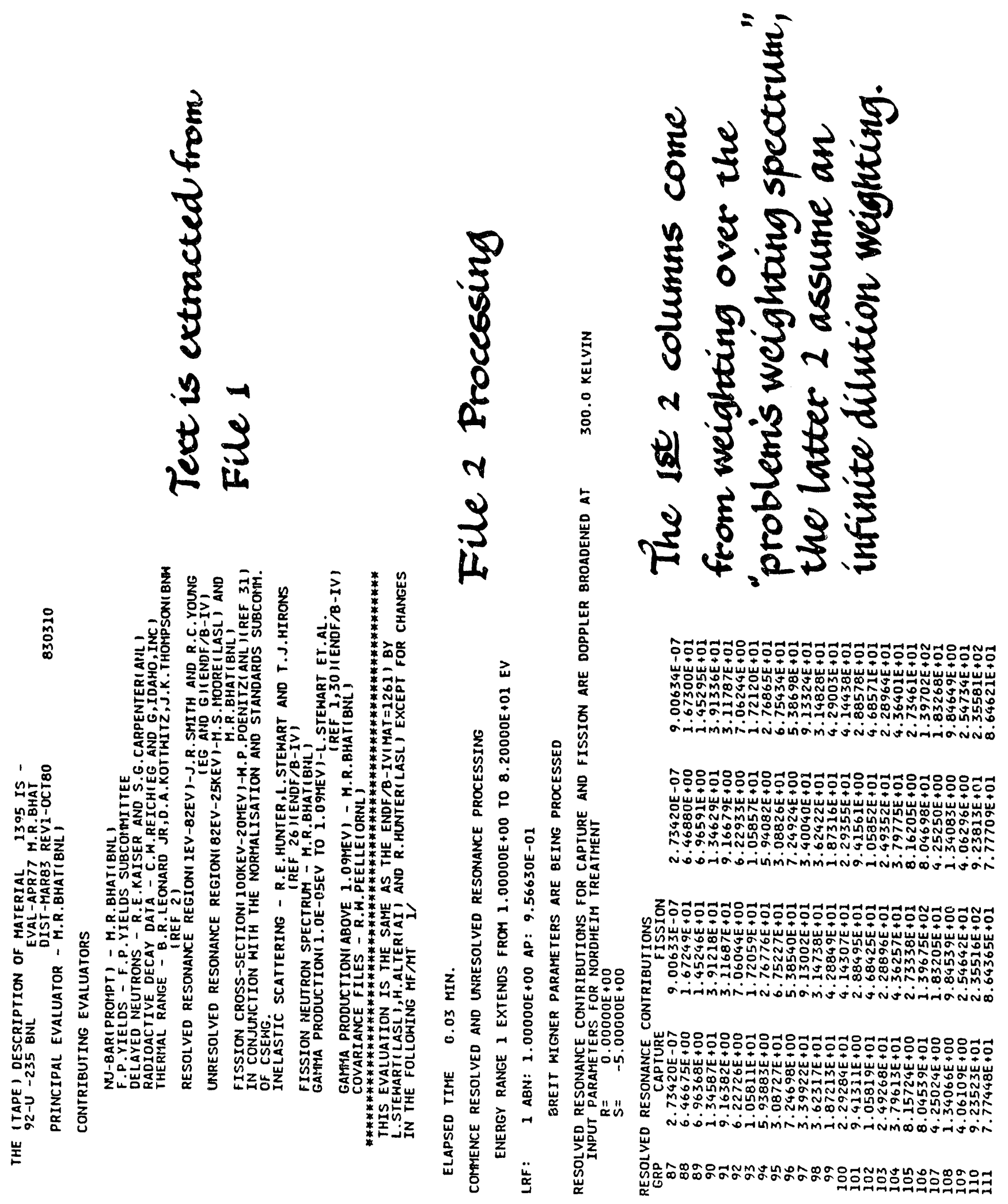

SAMPLE PROBLEMS 


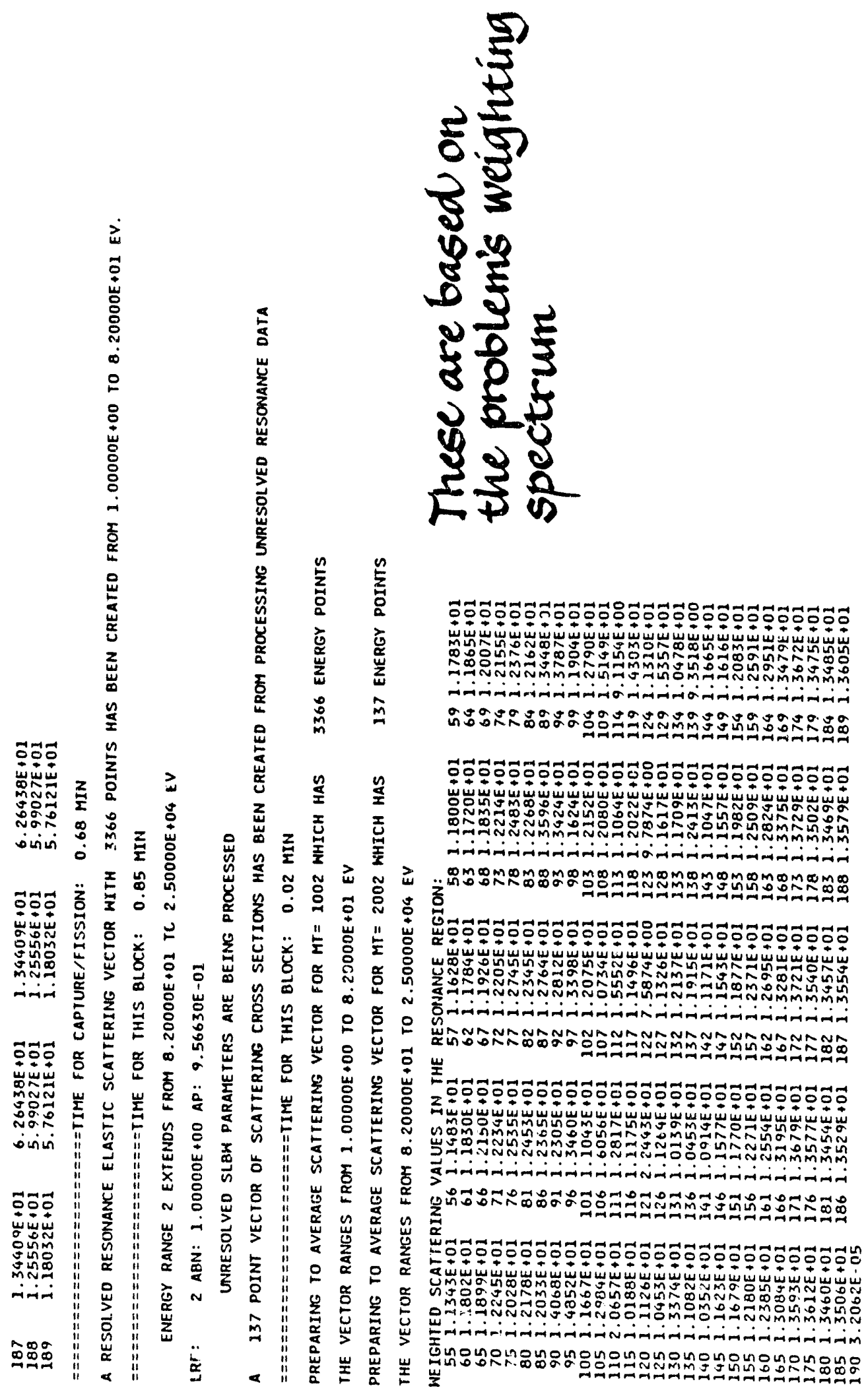

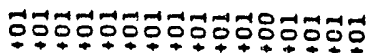

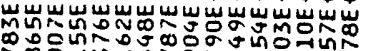

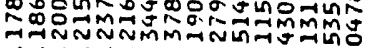

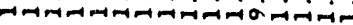

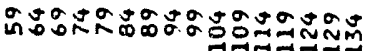

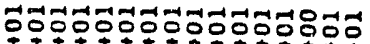

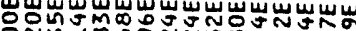
๑ñ

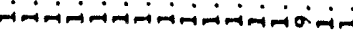

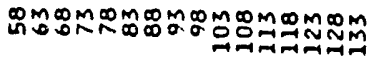

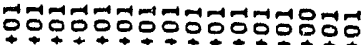

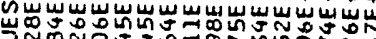

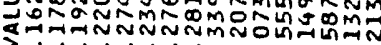

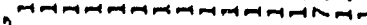

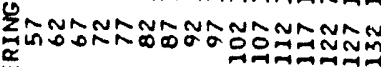

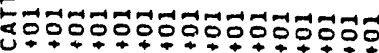

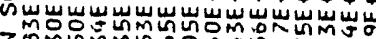

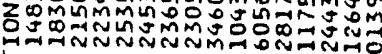

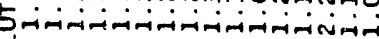

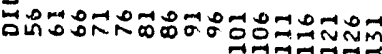
แ

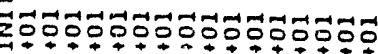

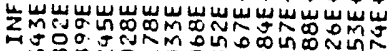
คำ

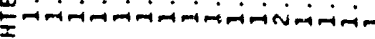

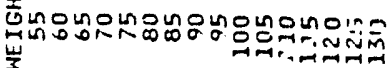

\section{SAMPLE PROBLEMS}




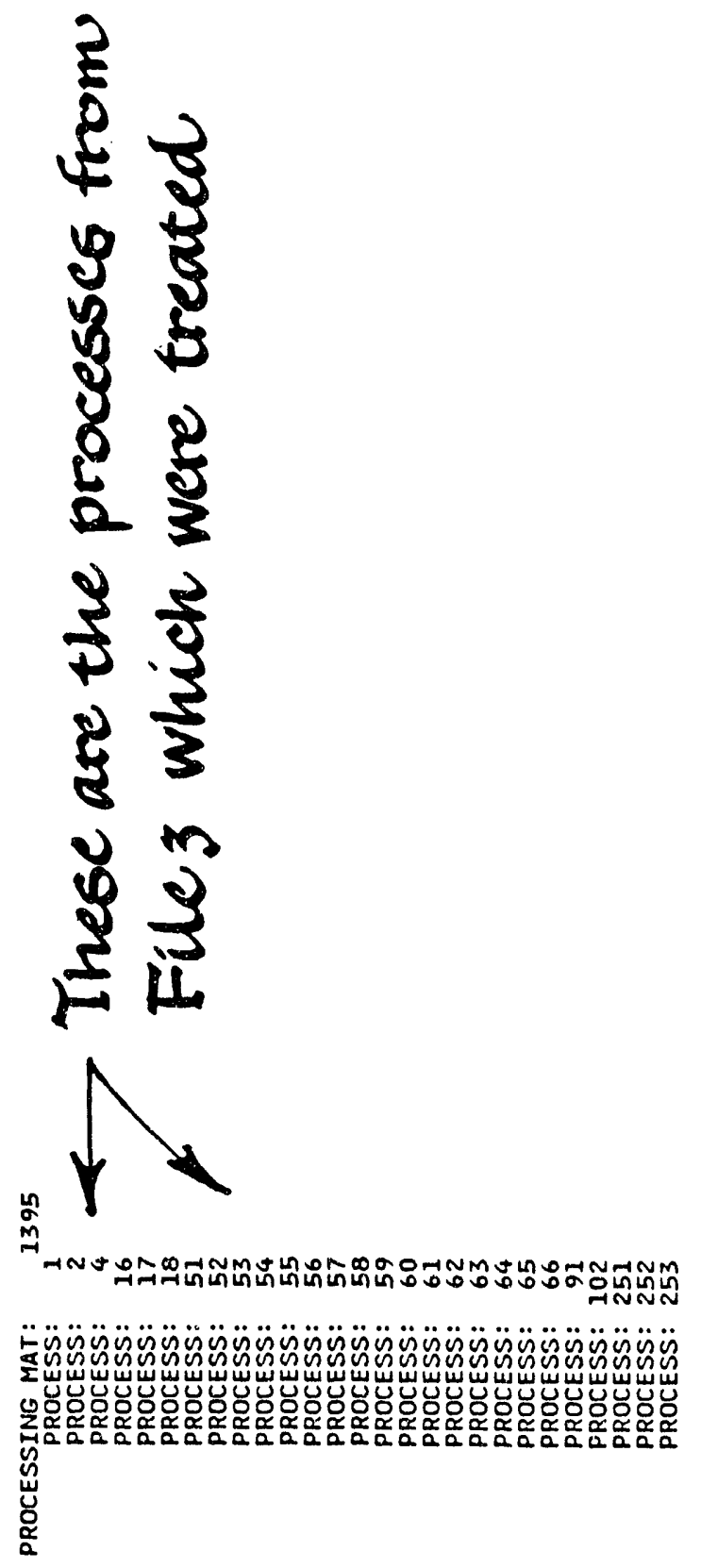




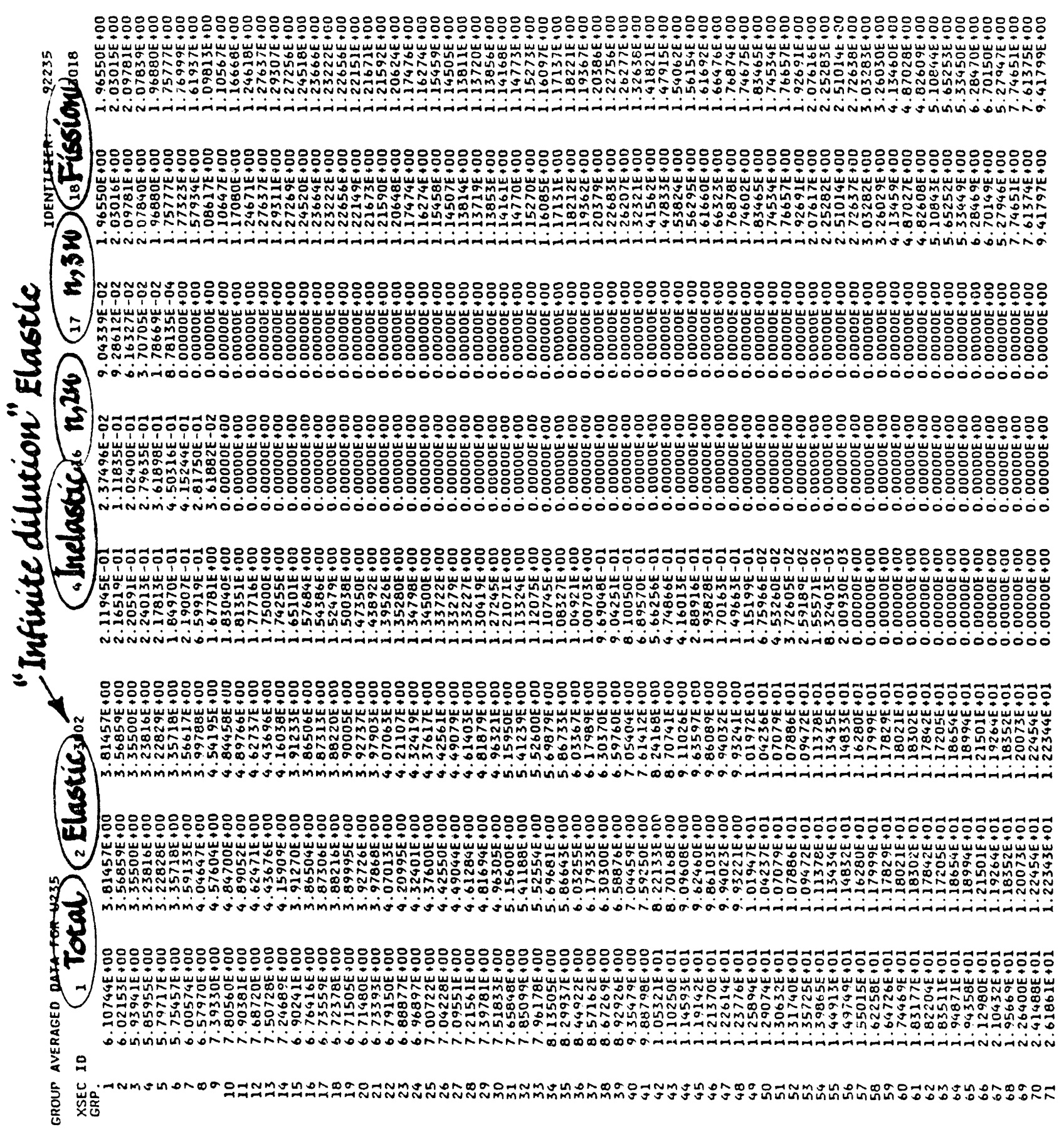

SAMPLE PROBLEMS 

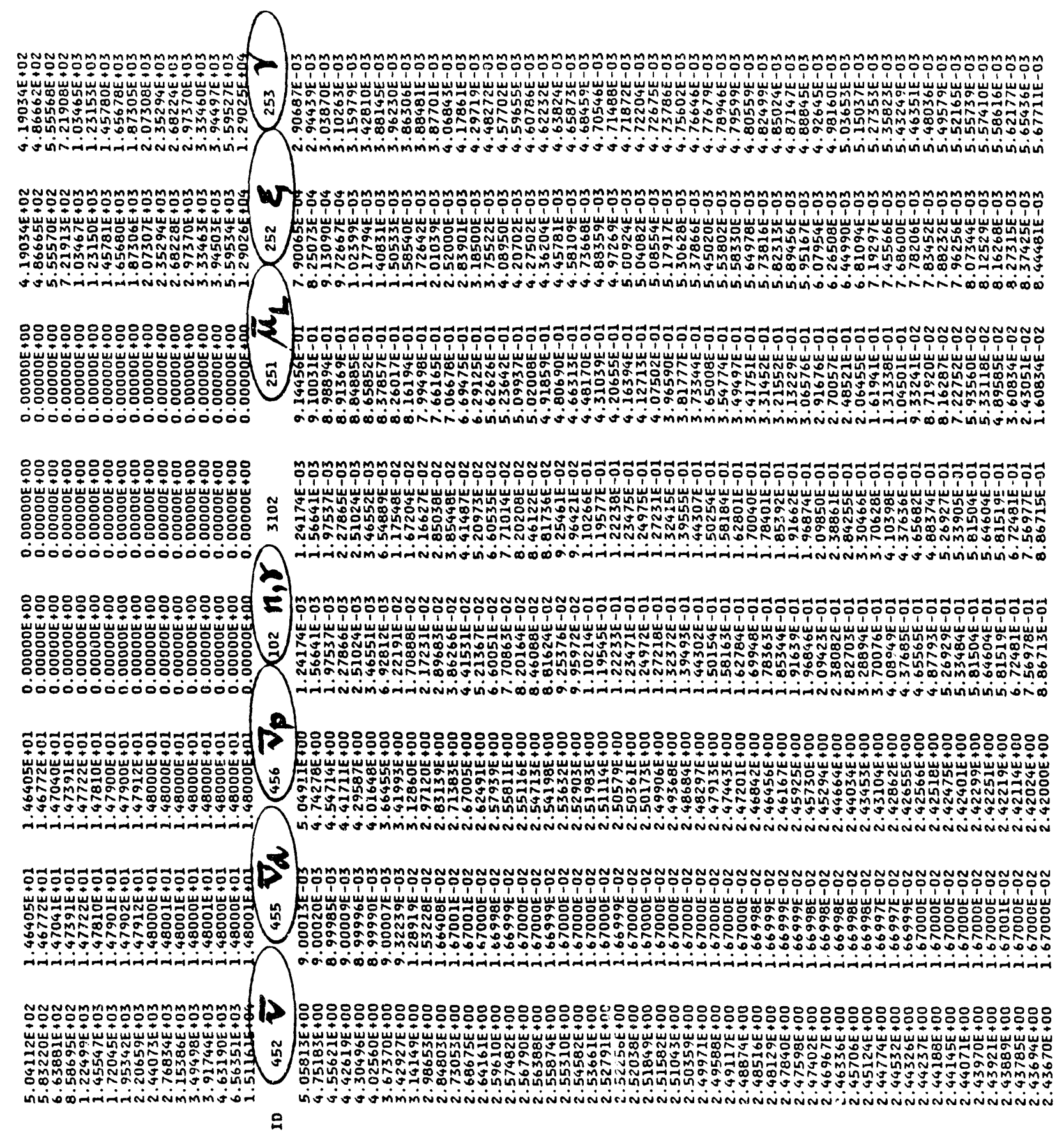

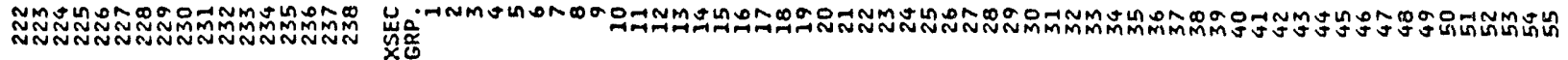




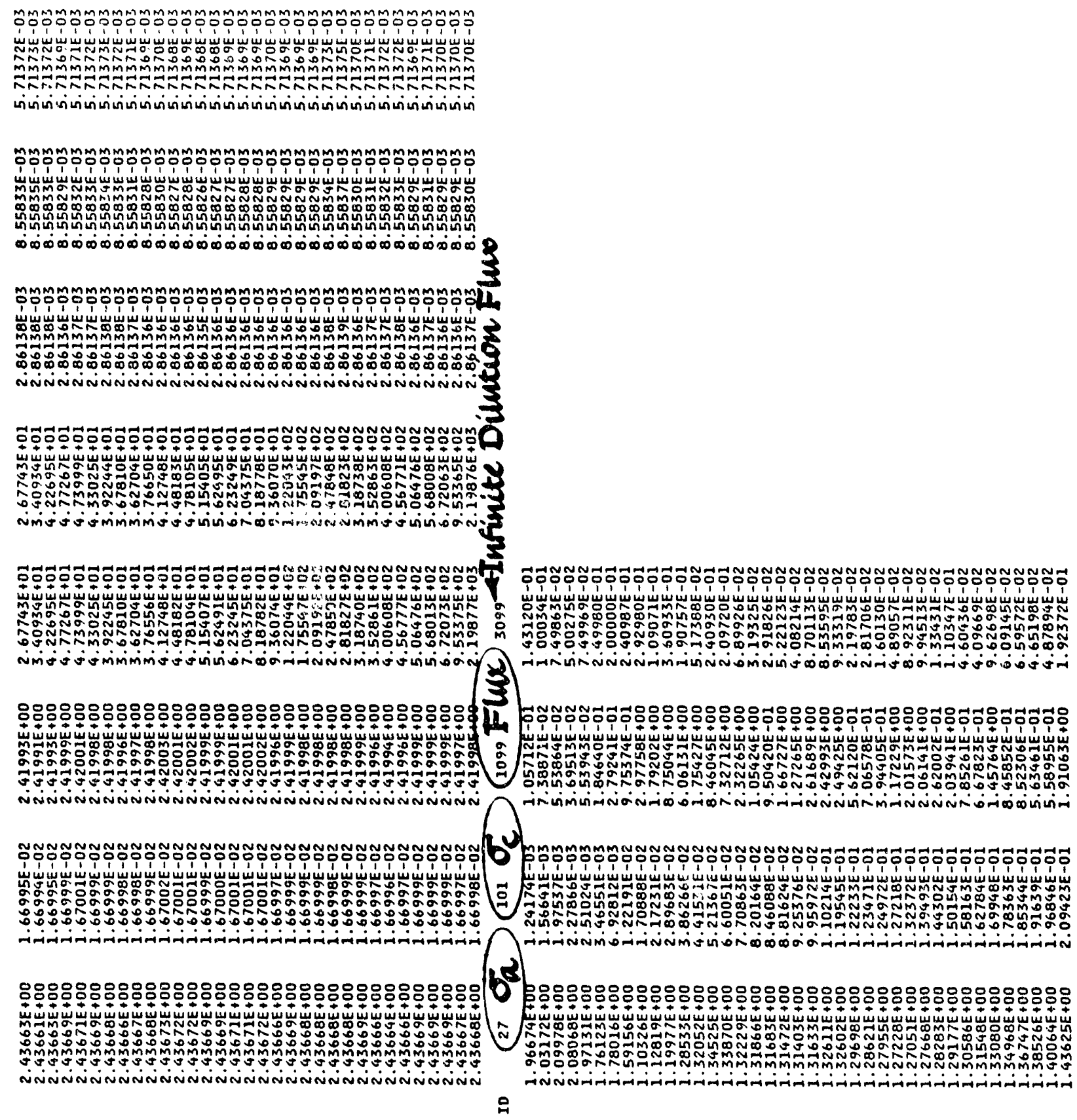

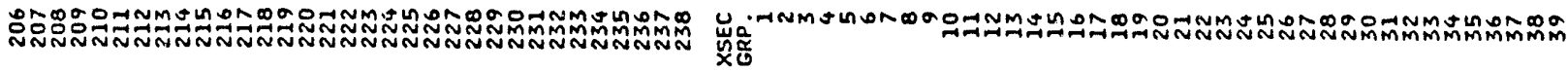




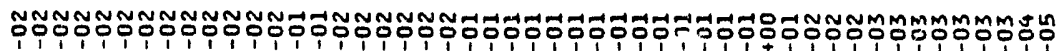

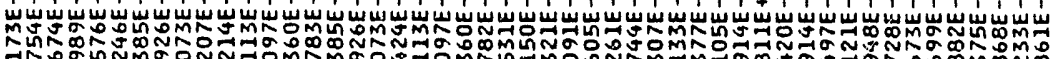
जिَ:

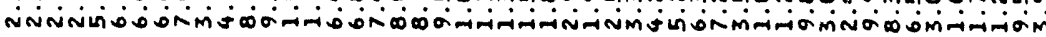

Nพ

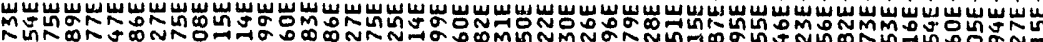

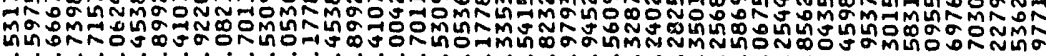

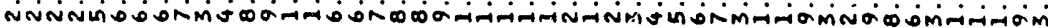

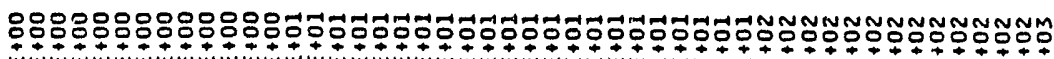

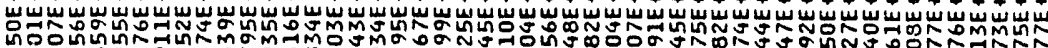
U.

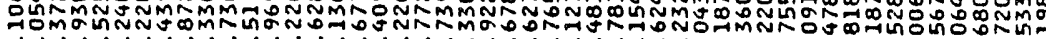

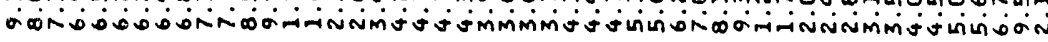

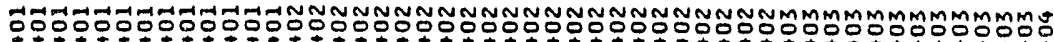

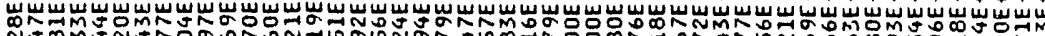
类

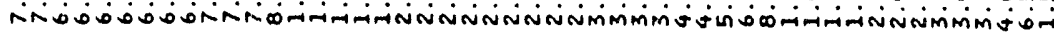

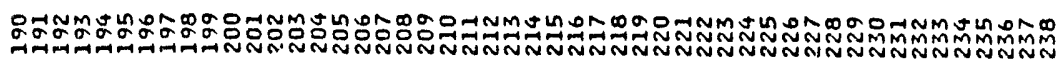

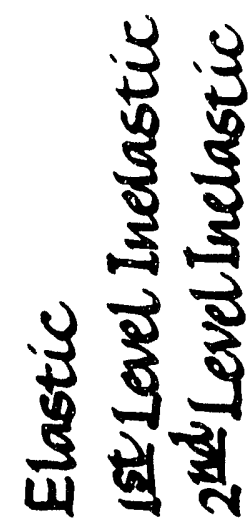

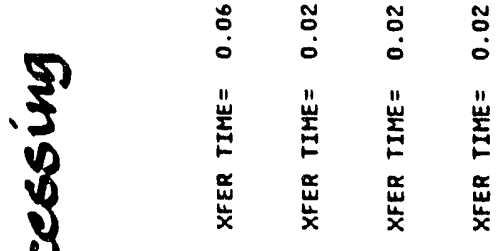

l. 3

Q.

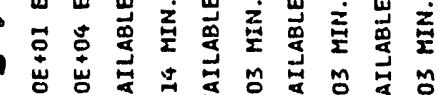

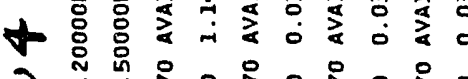

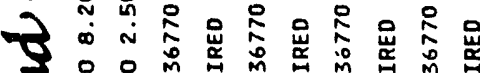
\$ $\left\{\begin{array}{l}0 \\ 0 \\ 0\end{array}\right.$

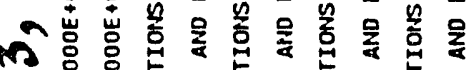

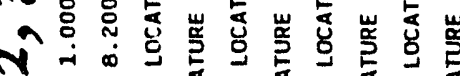

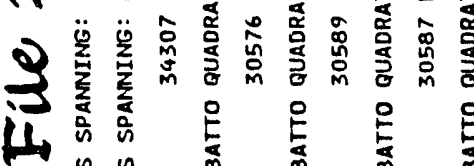

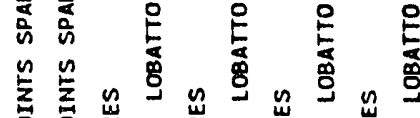

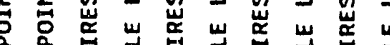

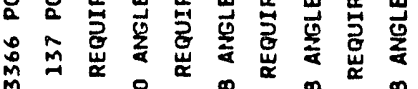

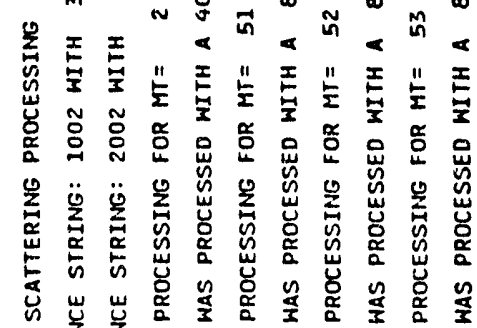

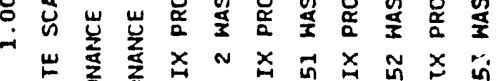

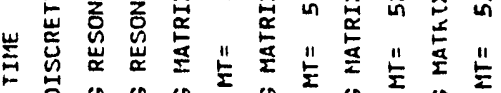

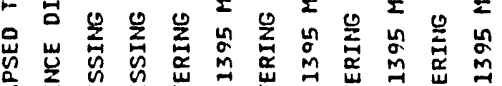

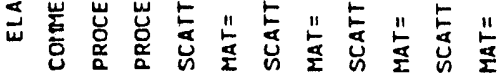




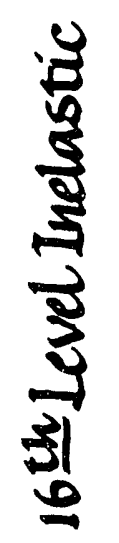

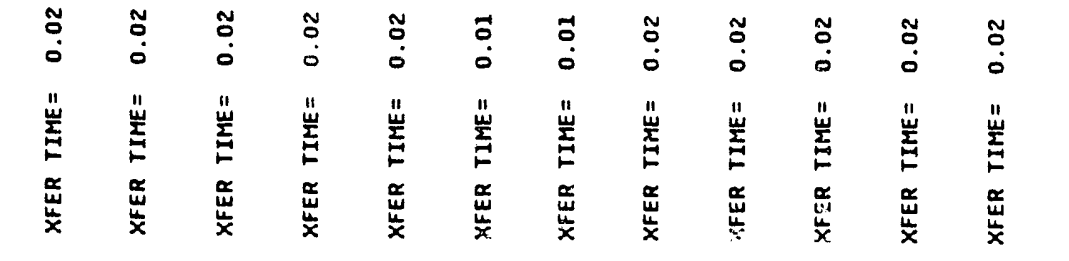

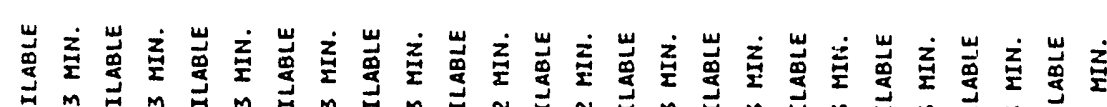

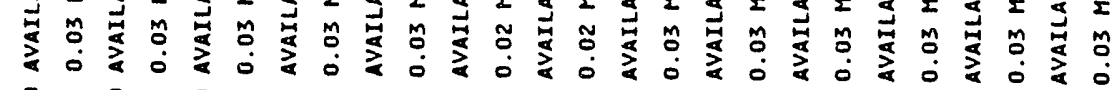

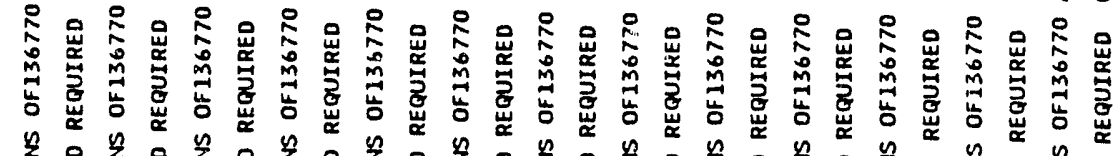

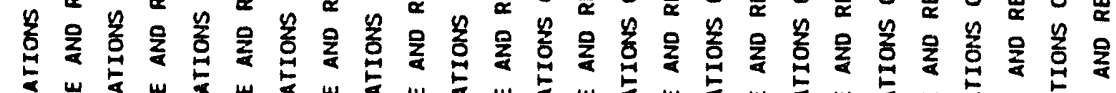

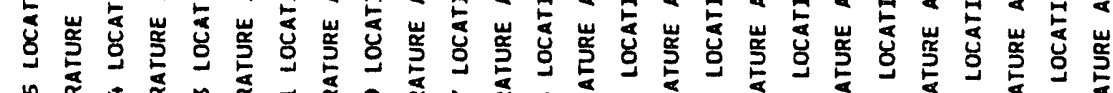

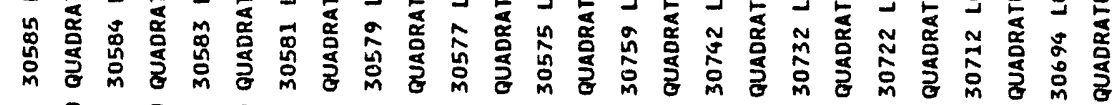

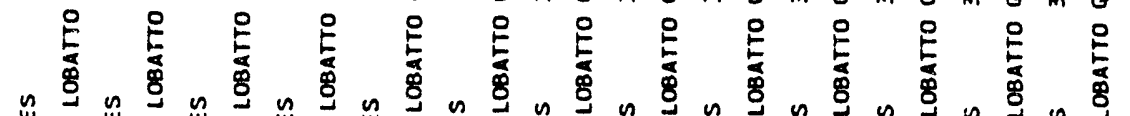

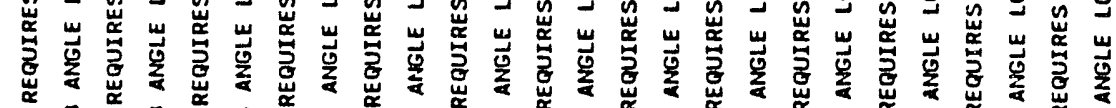

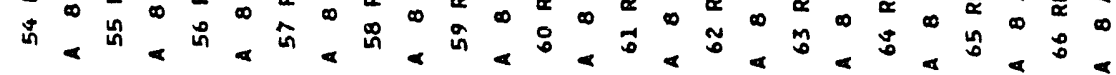

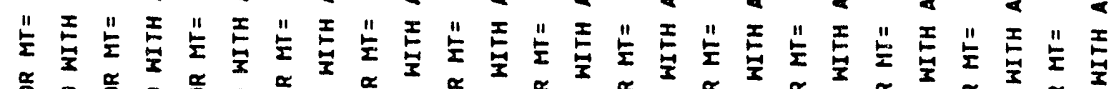

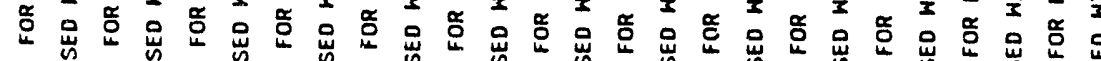

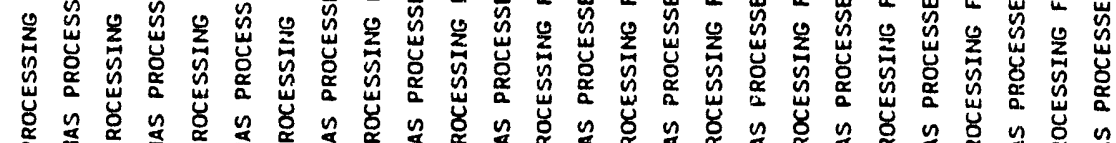

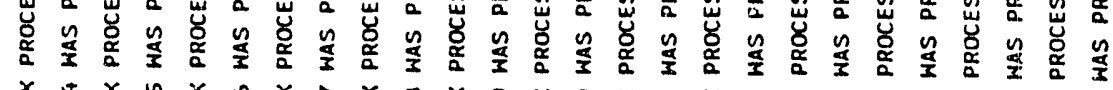

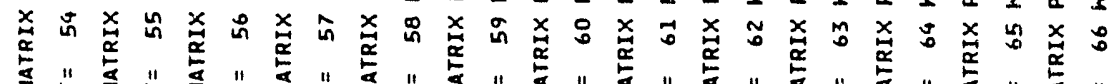

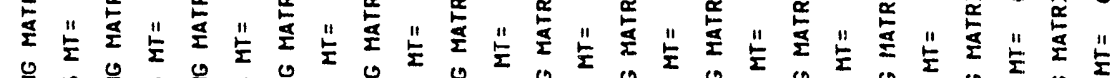

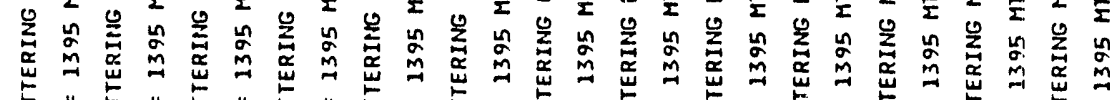

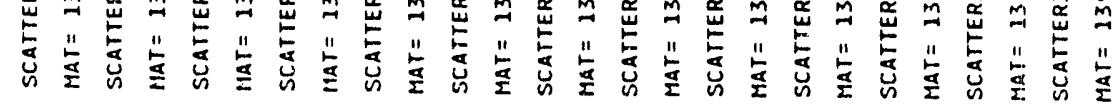




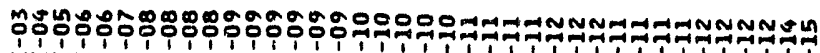

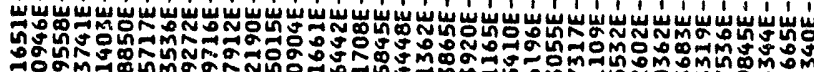
กัดกติ -inañ

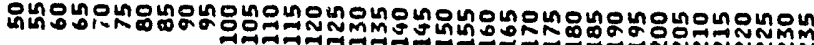

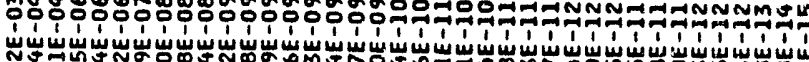

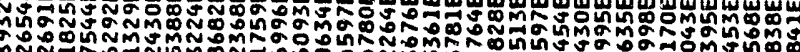

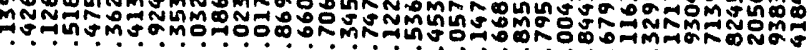

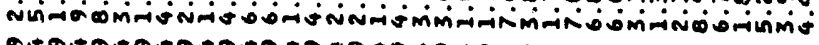

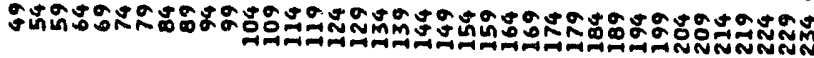

ธำดันน

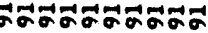

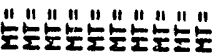
nninnunininumin "I" " " " " " " "

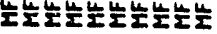

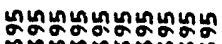
11

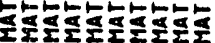

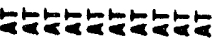

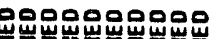
88608 бо 880

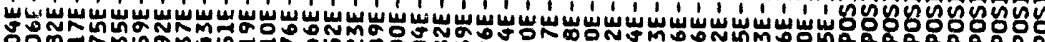

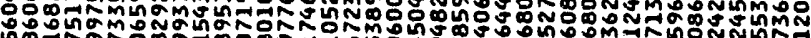

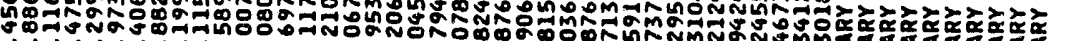

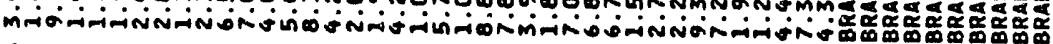
:

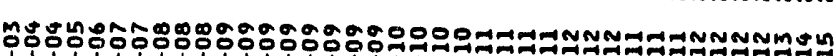

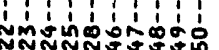

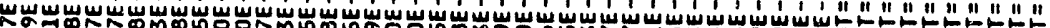

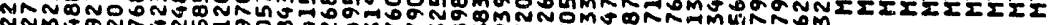

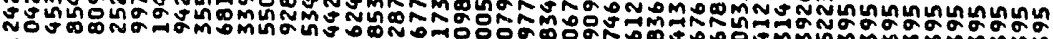

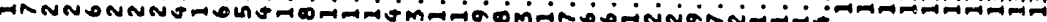

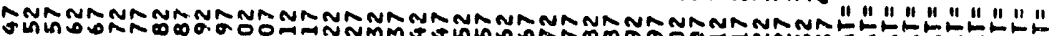

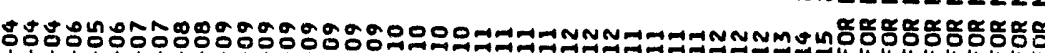

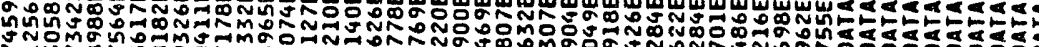
T) vinmo

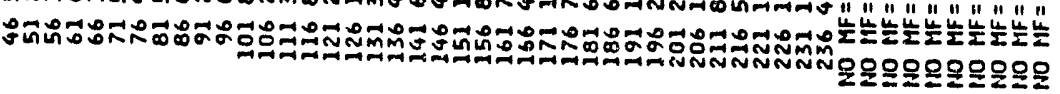

5
4
3
8
0
15
3

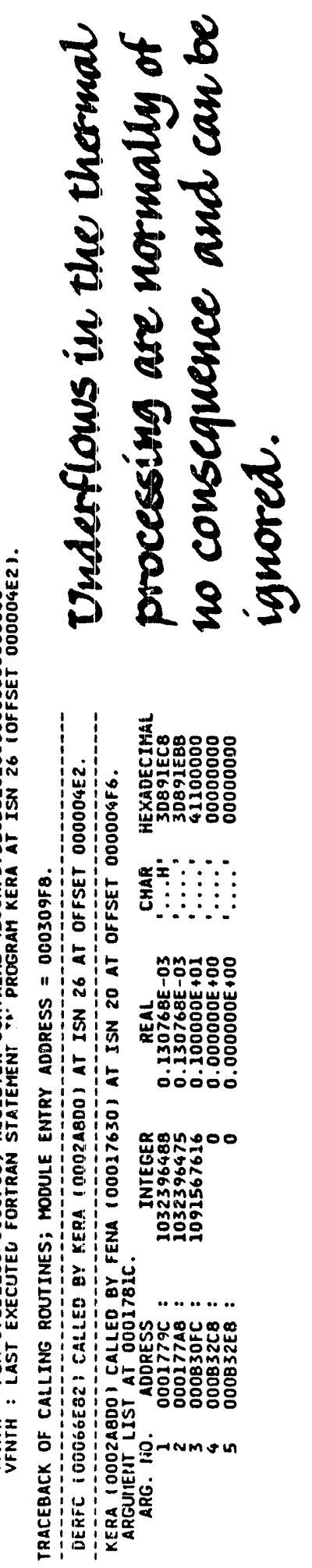

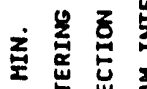

象实

: 5 w

i

支密 …...

焉

SAMPLE PROBLEMS 

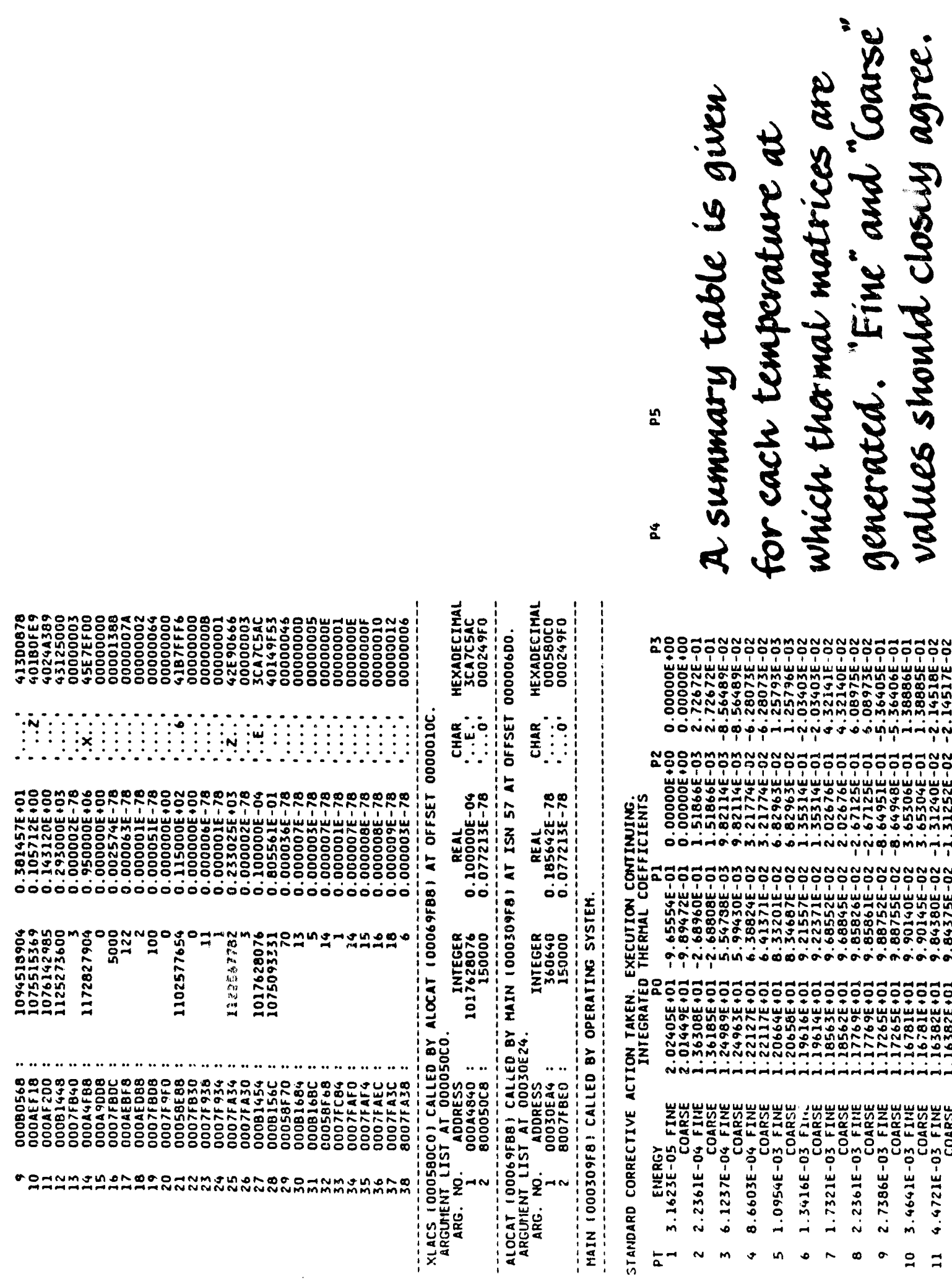

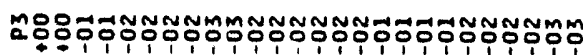

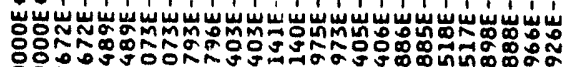

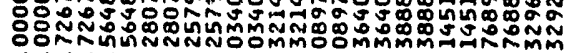
DonNip p.

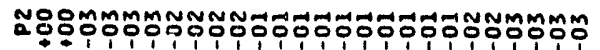

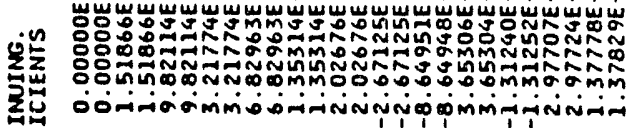

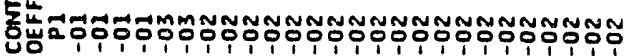

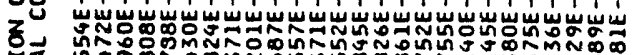
o 5 Н.

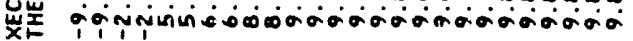

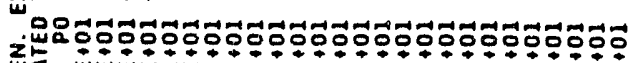

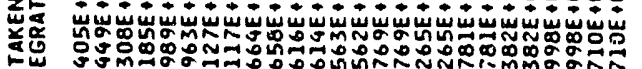

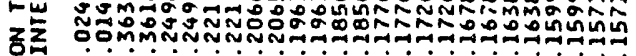

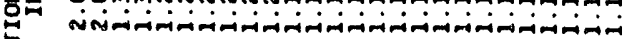
乡

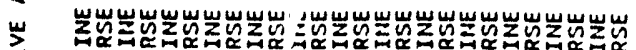

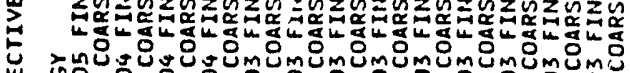
世

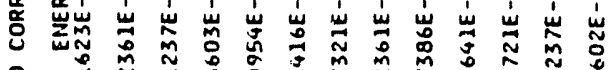

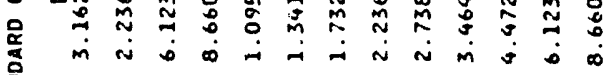

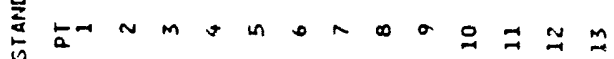



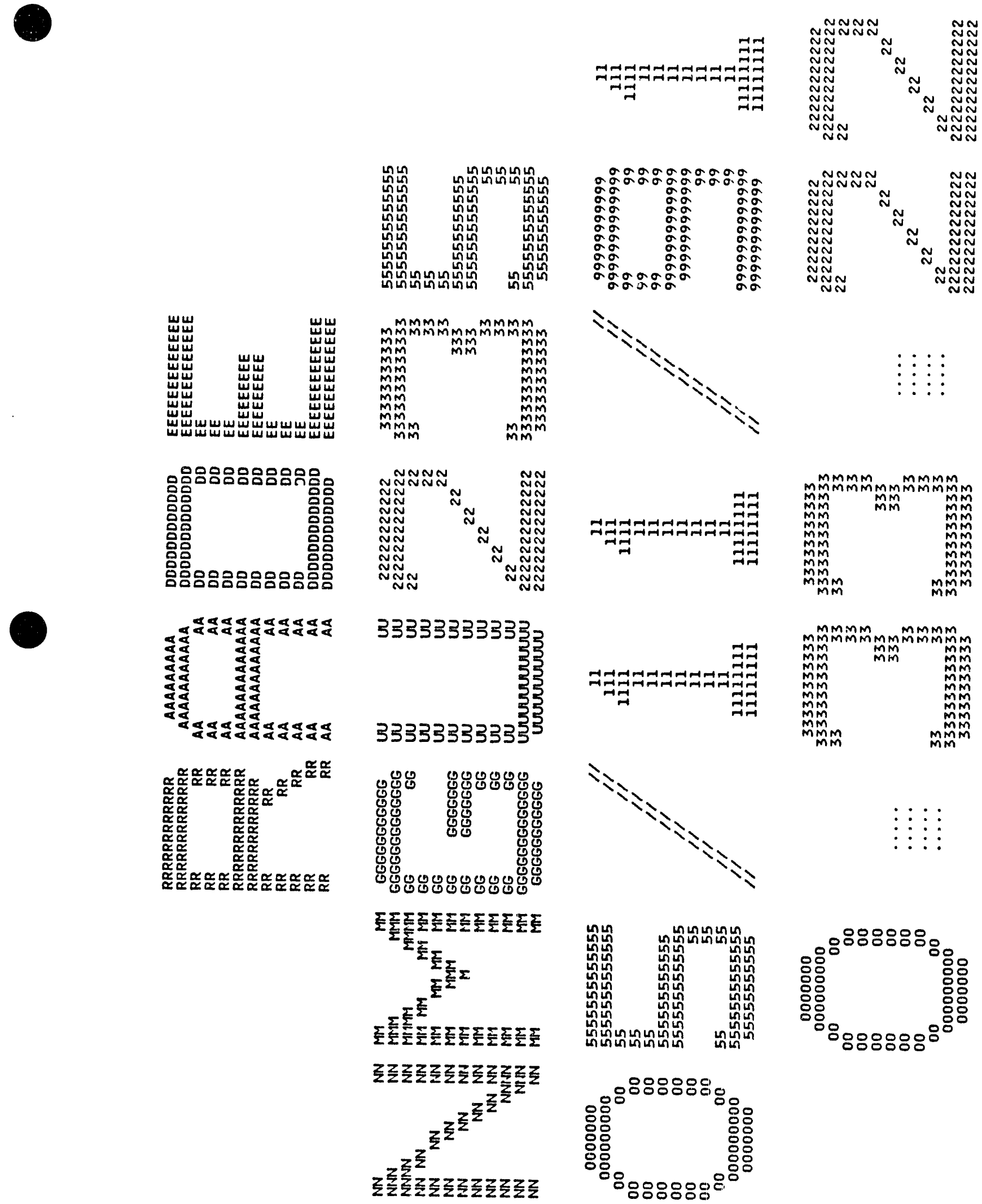


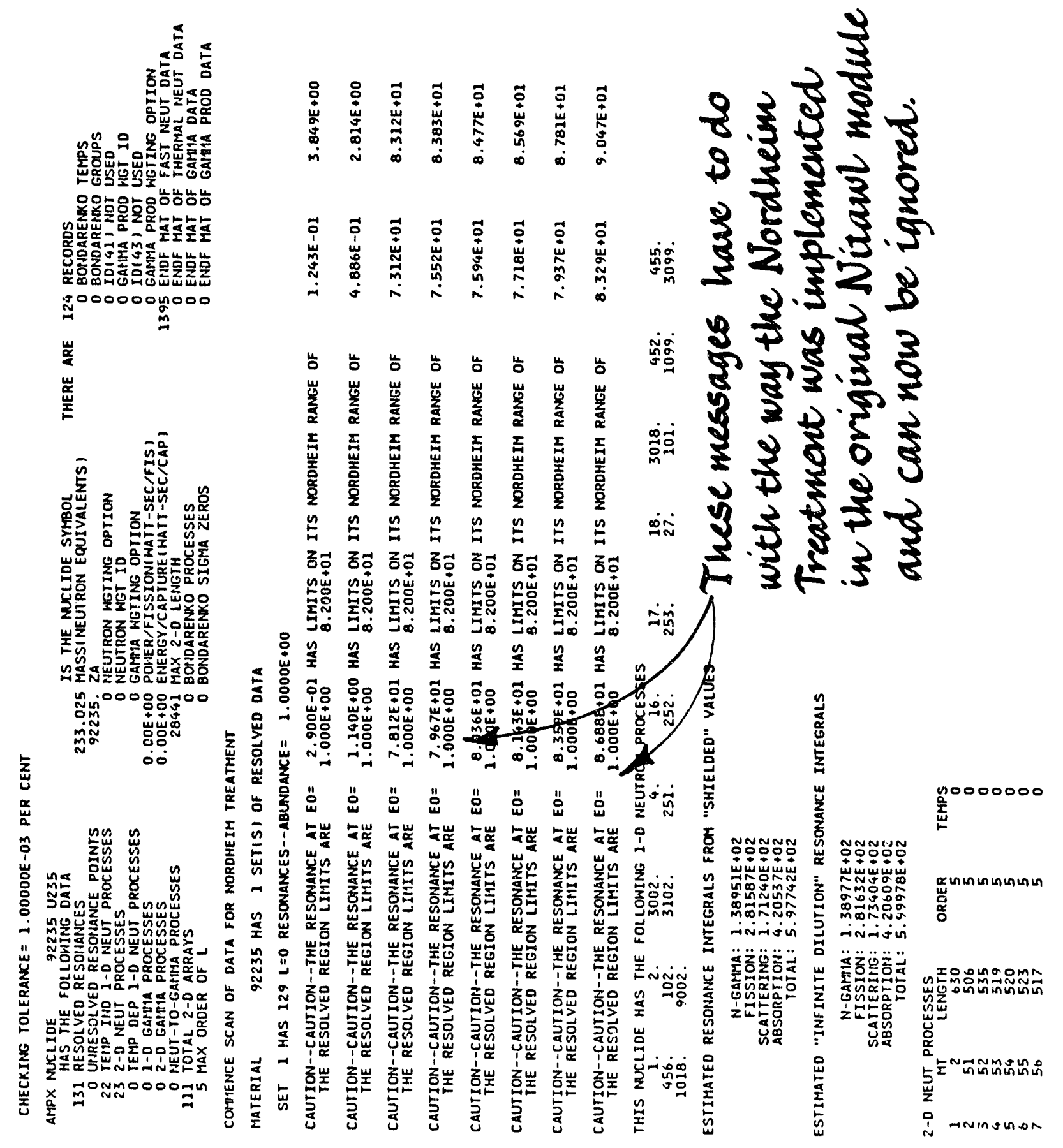




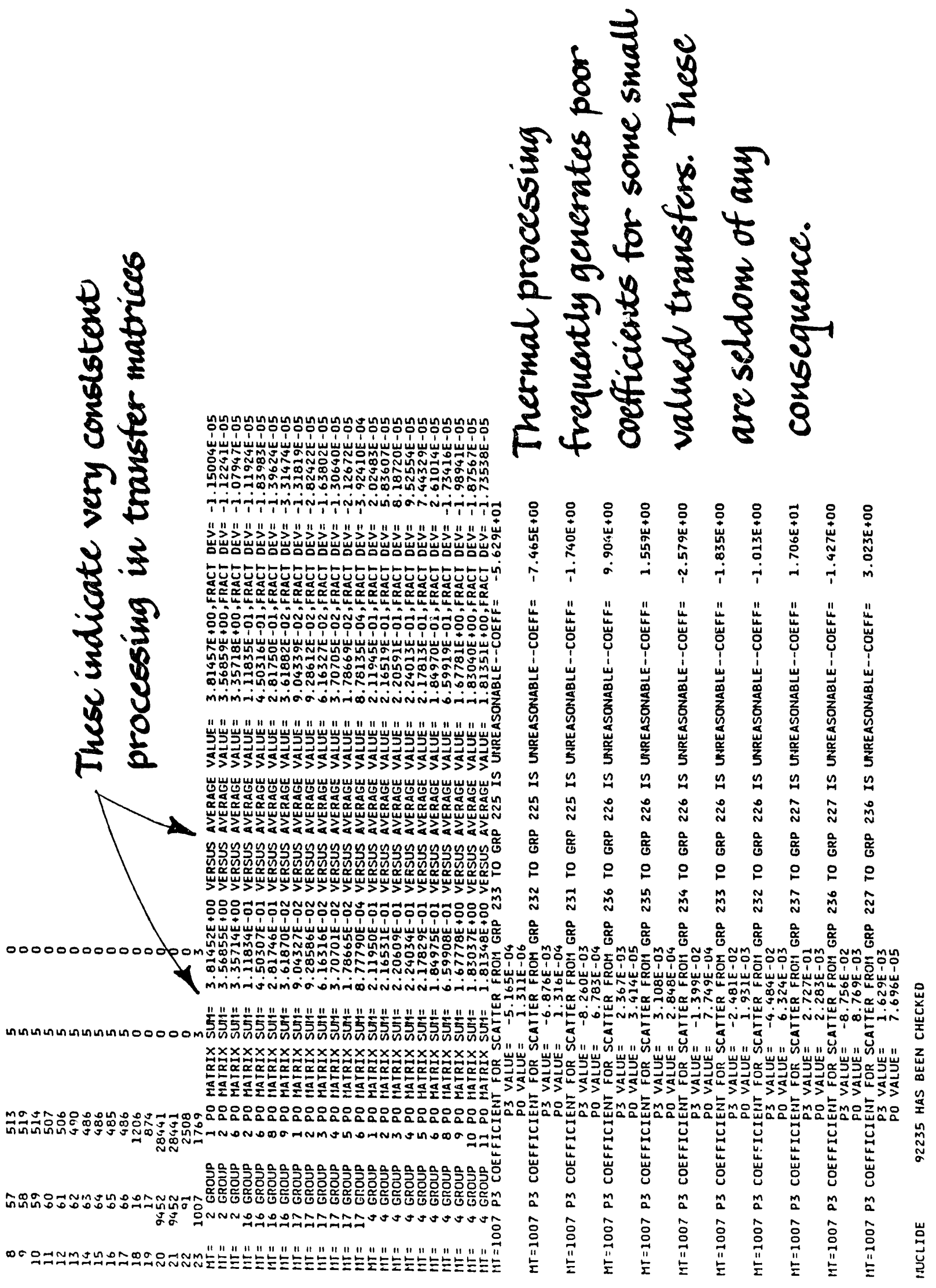


The input for the graphite case is given below. In the XLACS run, note that we are combining ENDF/B data from two evaluations: (1) the standard Version V data designated by MAT 1306 and (2) some thermal-scattering law data specifically for graphite (MAT 1065) which dates back to Version III of ENDF/B, or earlier. Note also that we have requested processing of all ten temperature sets in the thermal-scattering law data file. As it is programmed, XLACS will accept requests for processing at any temperature intermediate to those in the file; however, it has been demonstrated several time that the requisite interpolations produce very poor results, such that one should select only temperatures explicitly included in the files and use procedures which interpolate within cross-section values after the data are processed into multigroup form.

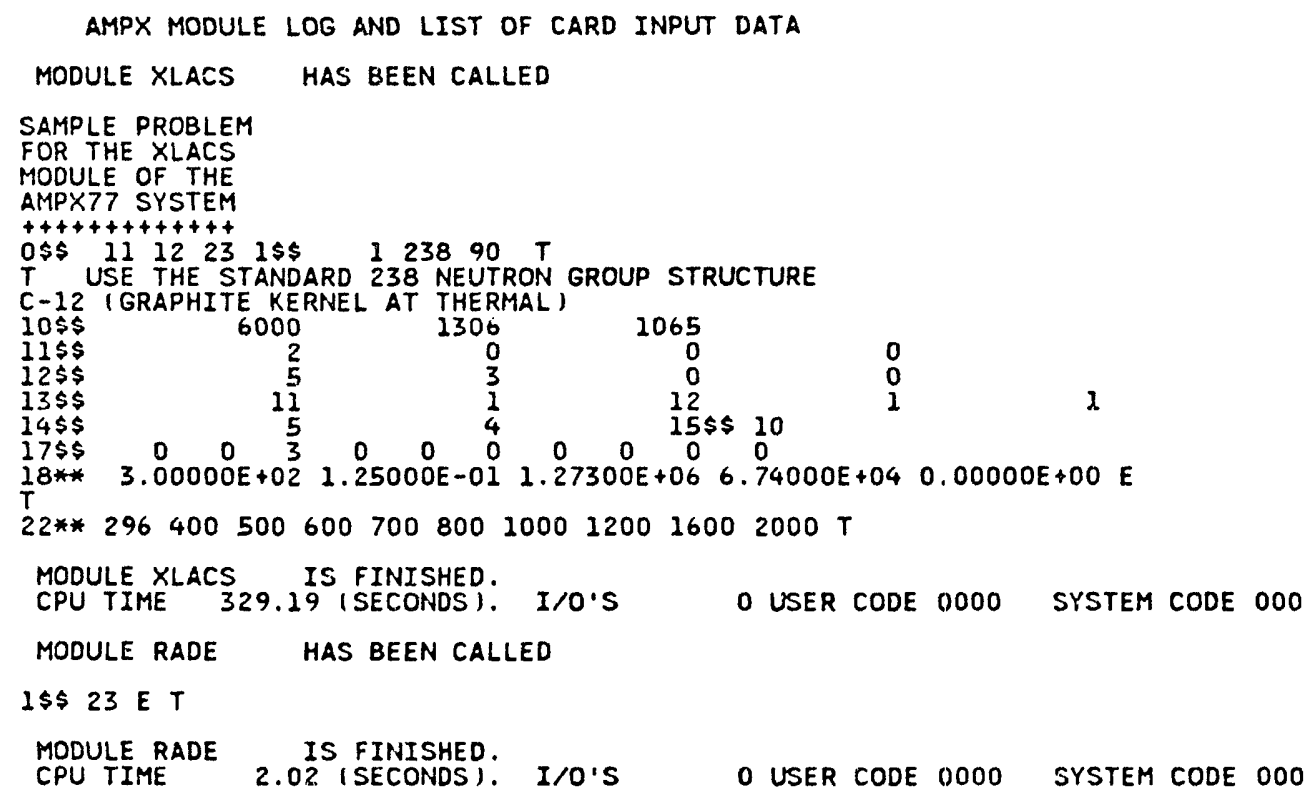

Portions of the output from XLACS and RADE are shown on the following pages. (The complete outputs for the codes used in Task 1 are given in File 65 on the tape discussed in Sect. 5.INSTALLATION of this report.) 

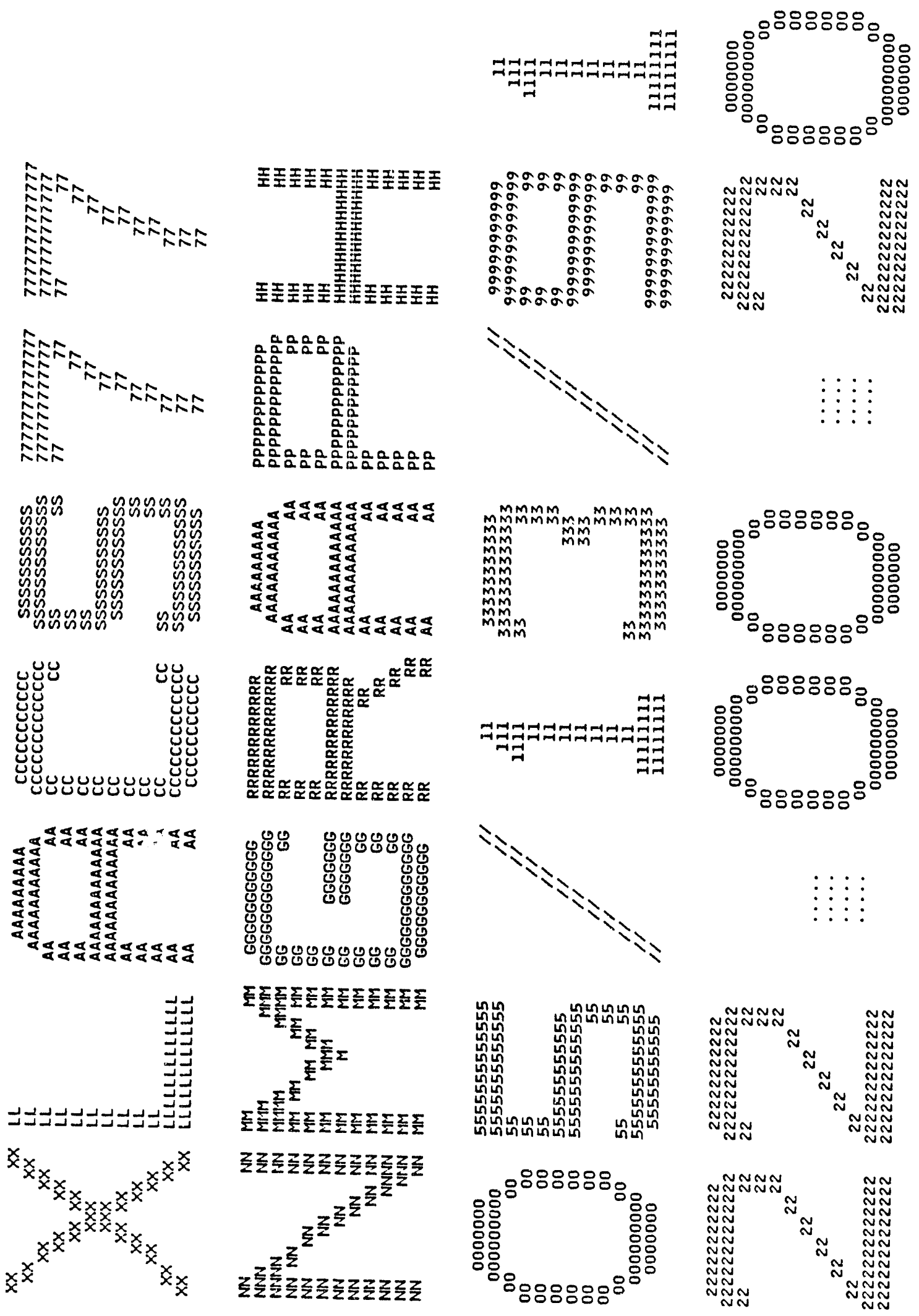

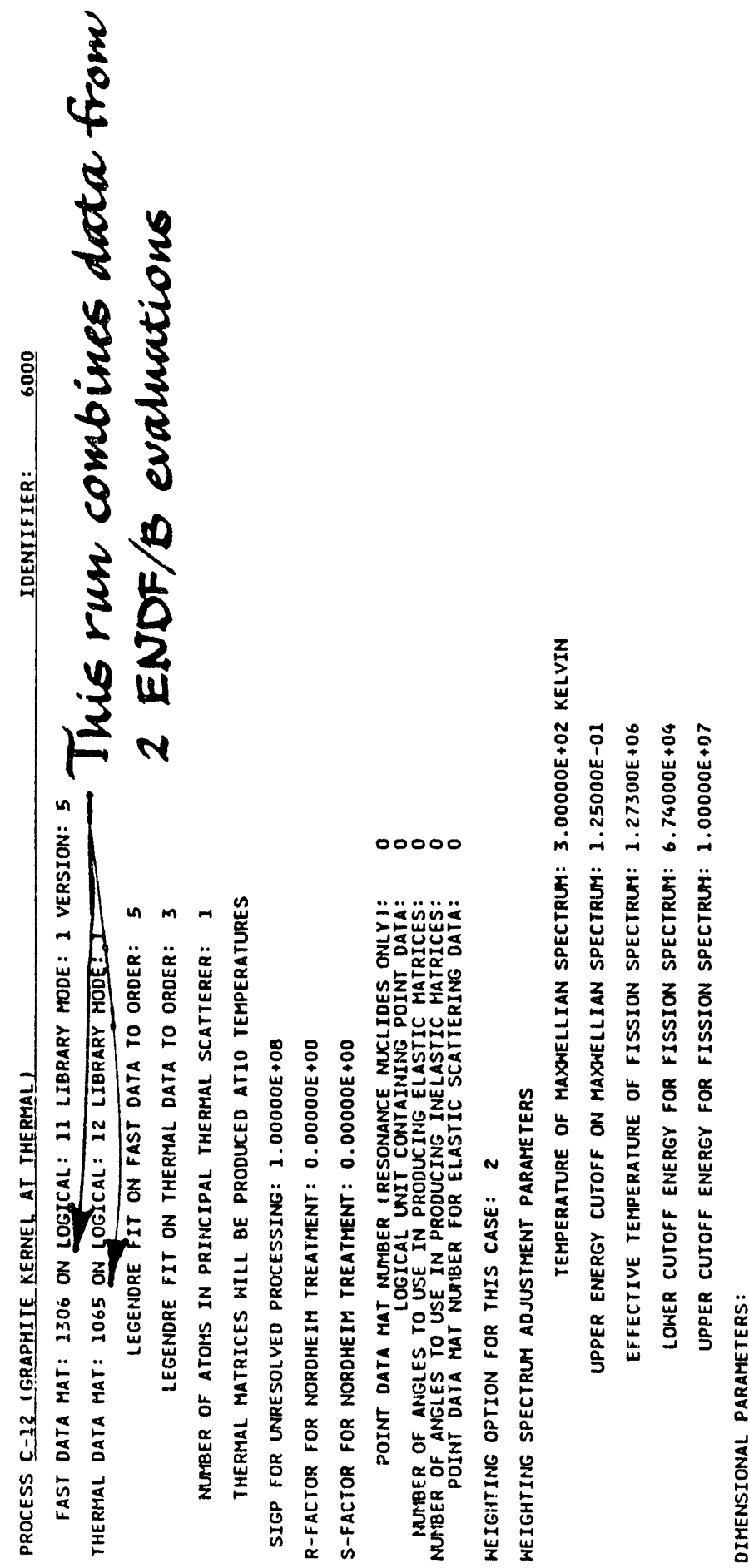

오ํ :

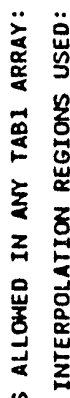

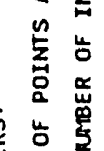

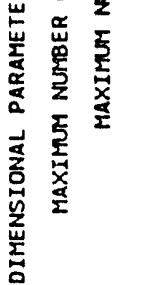

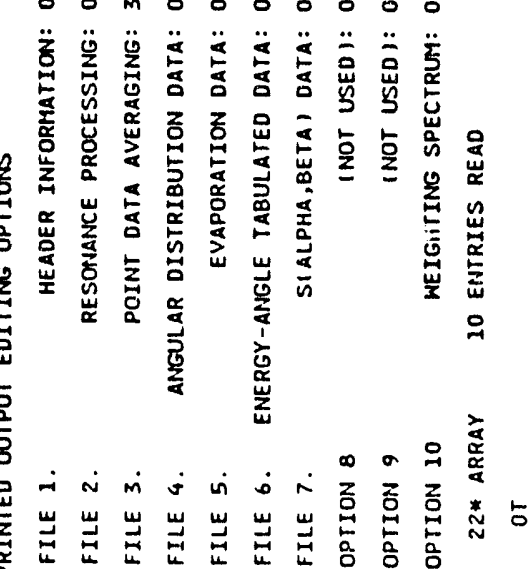




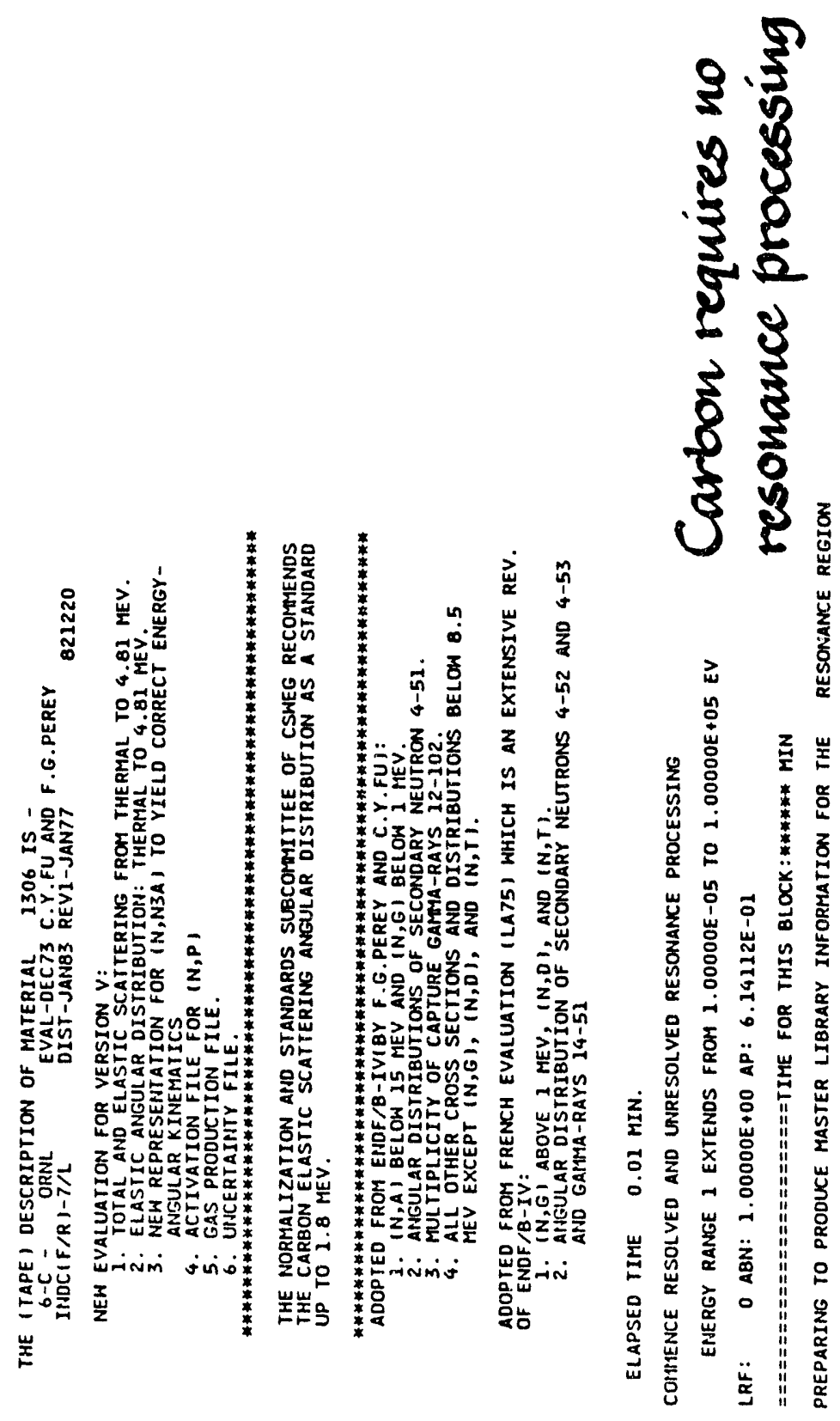

SAMPLE PROBLEMS 

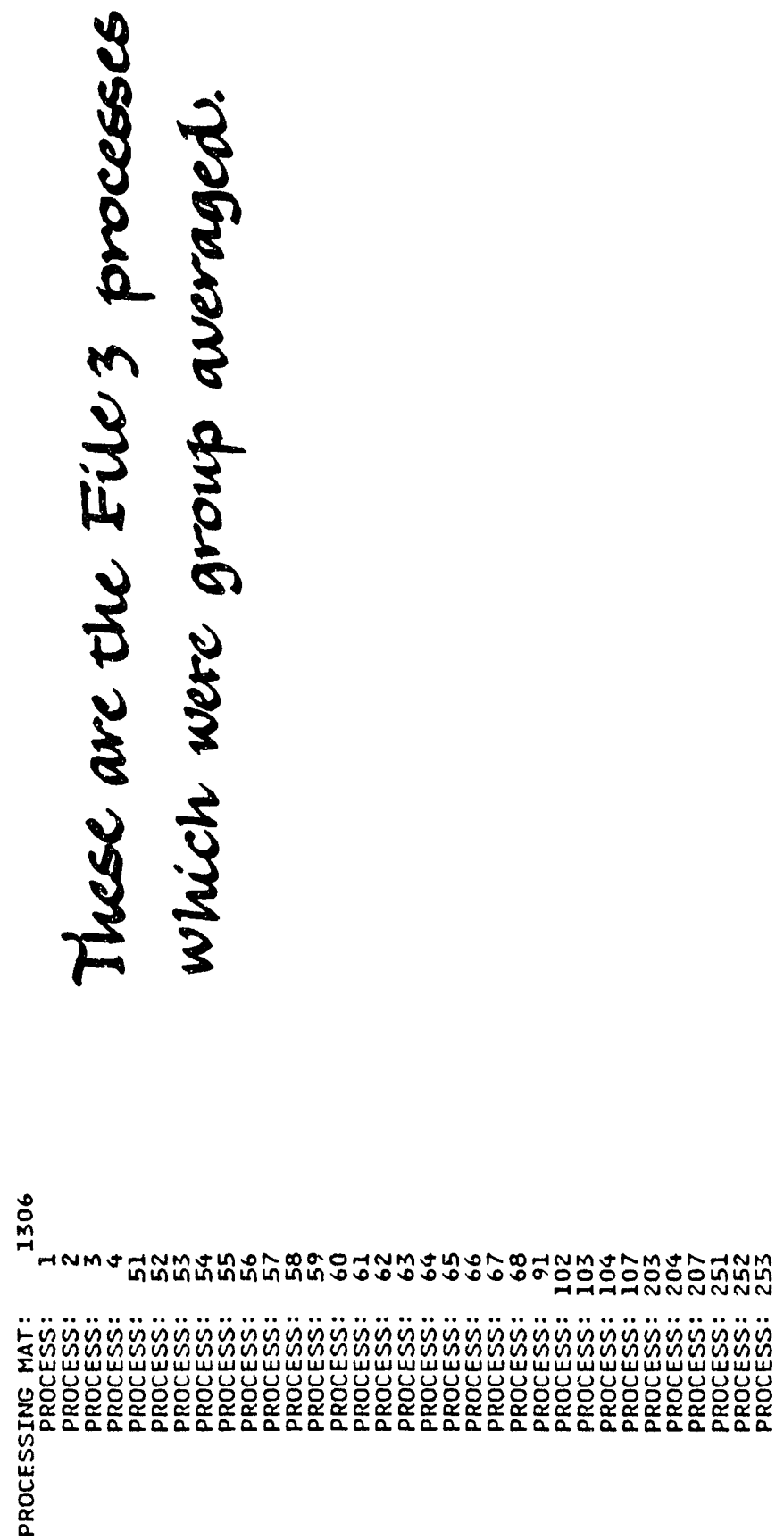


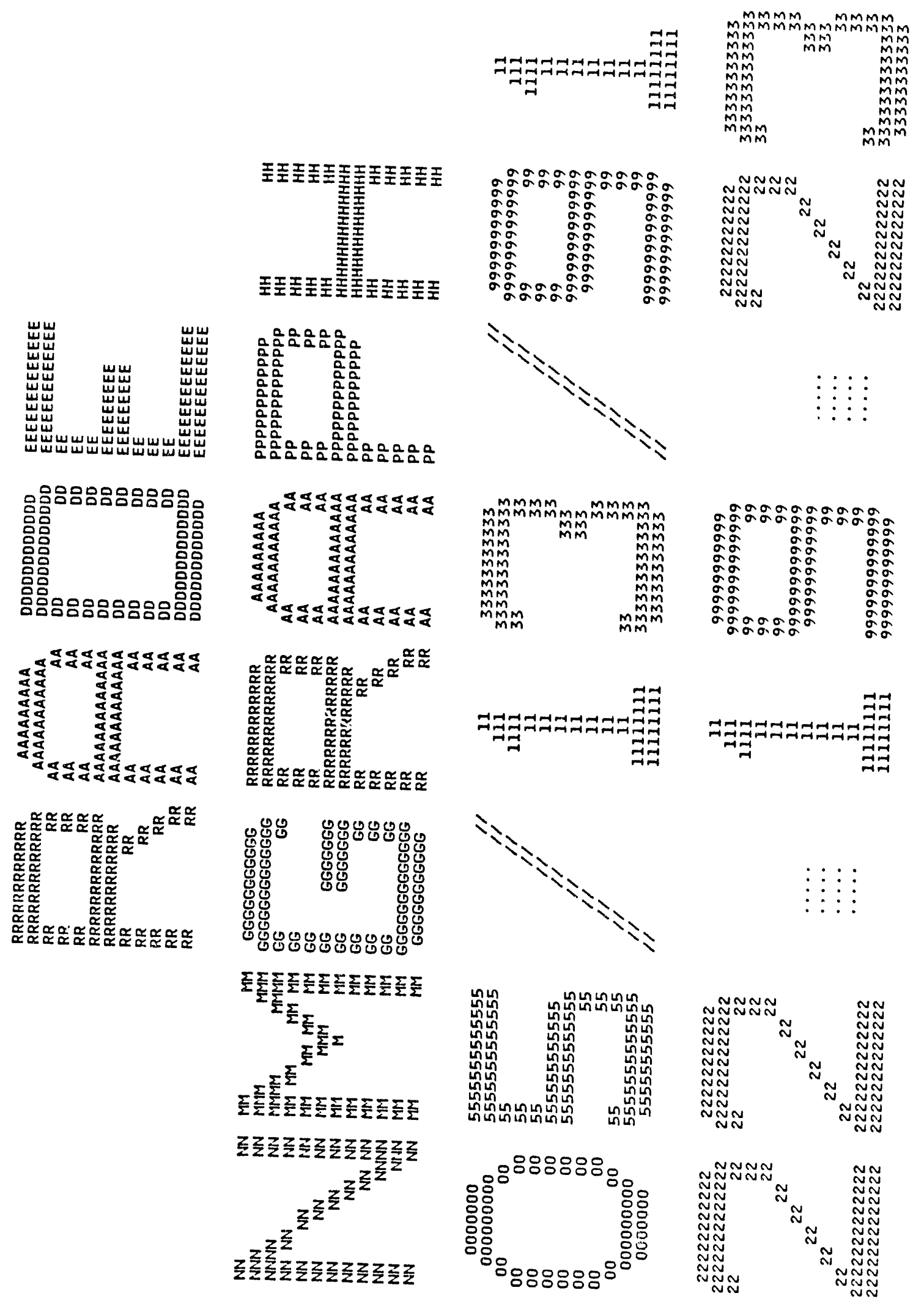




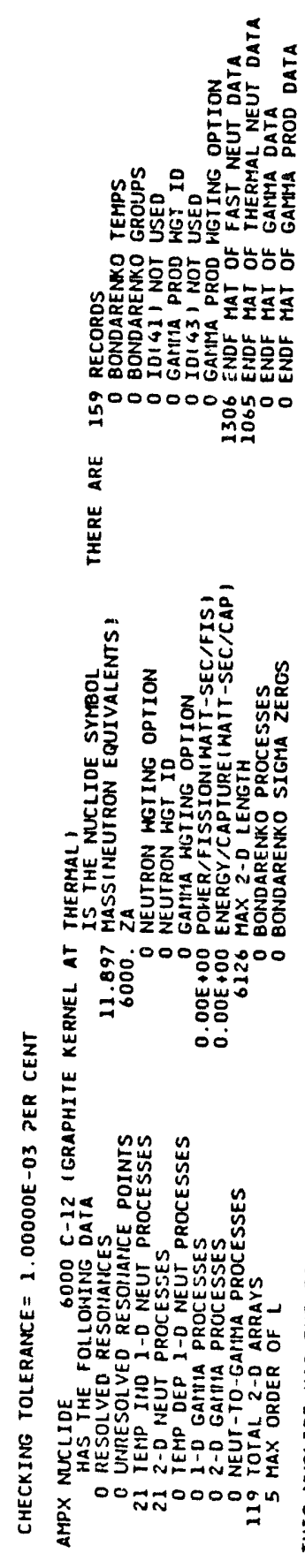

م。

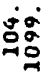

จิنَ

จุ่

ธักิบ
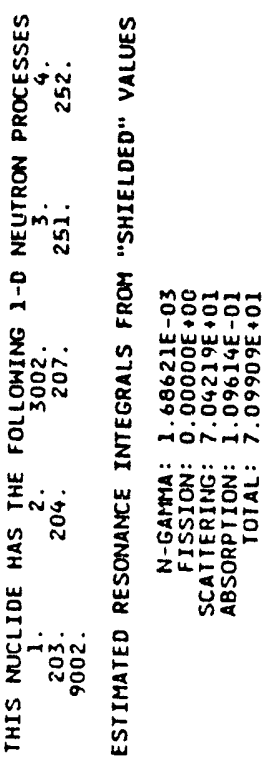

贻

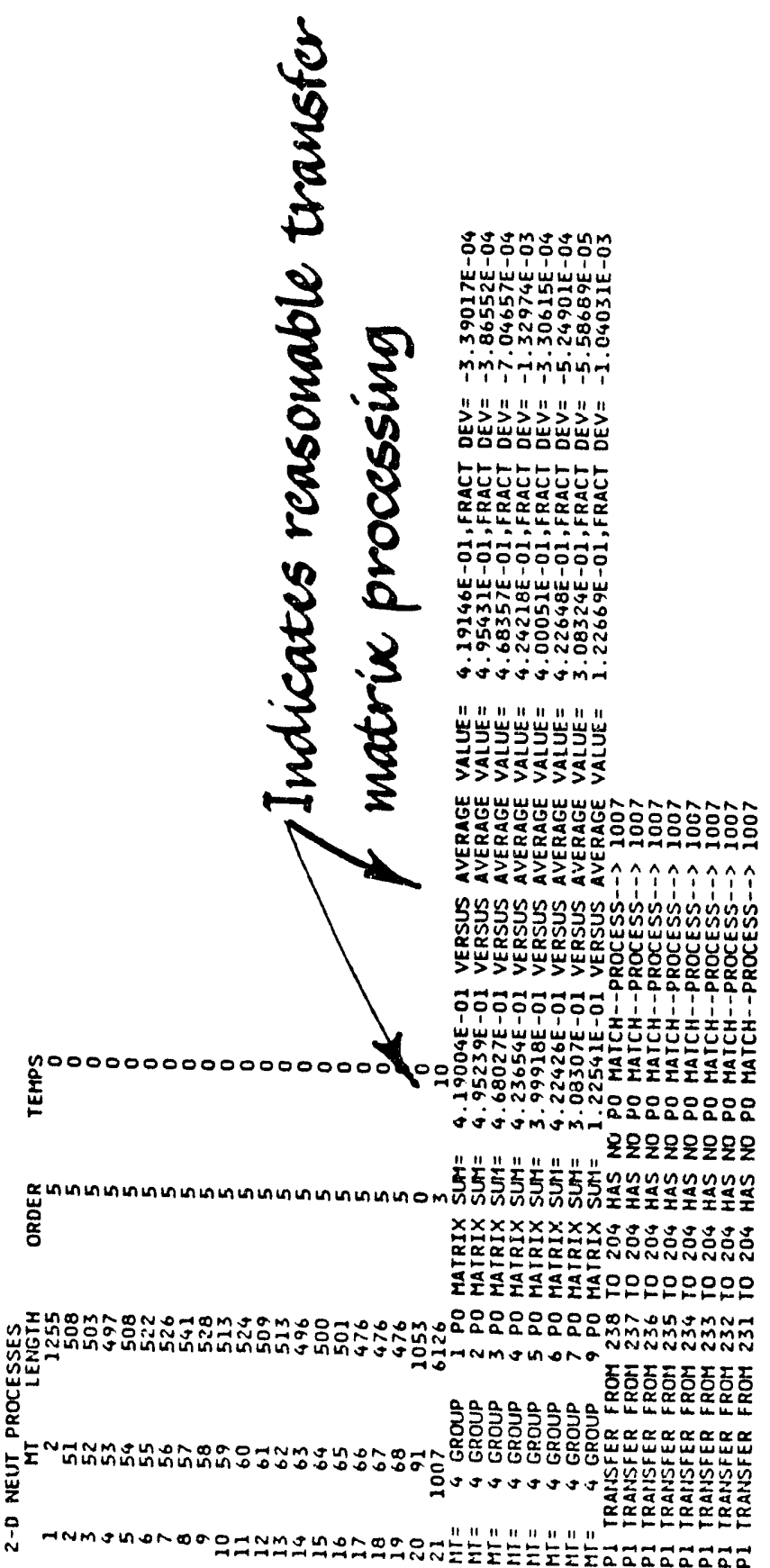

SAMPLE PROBLEMS 


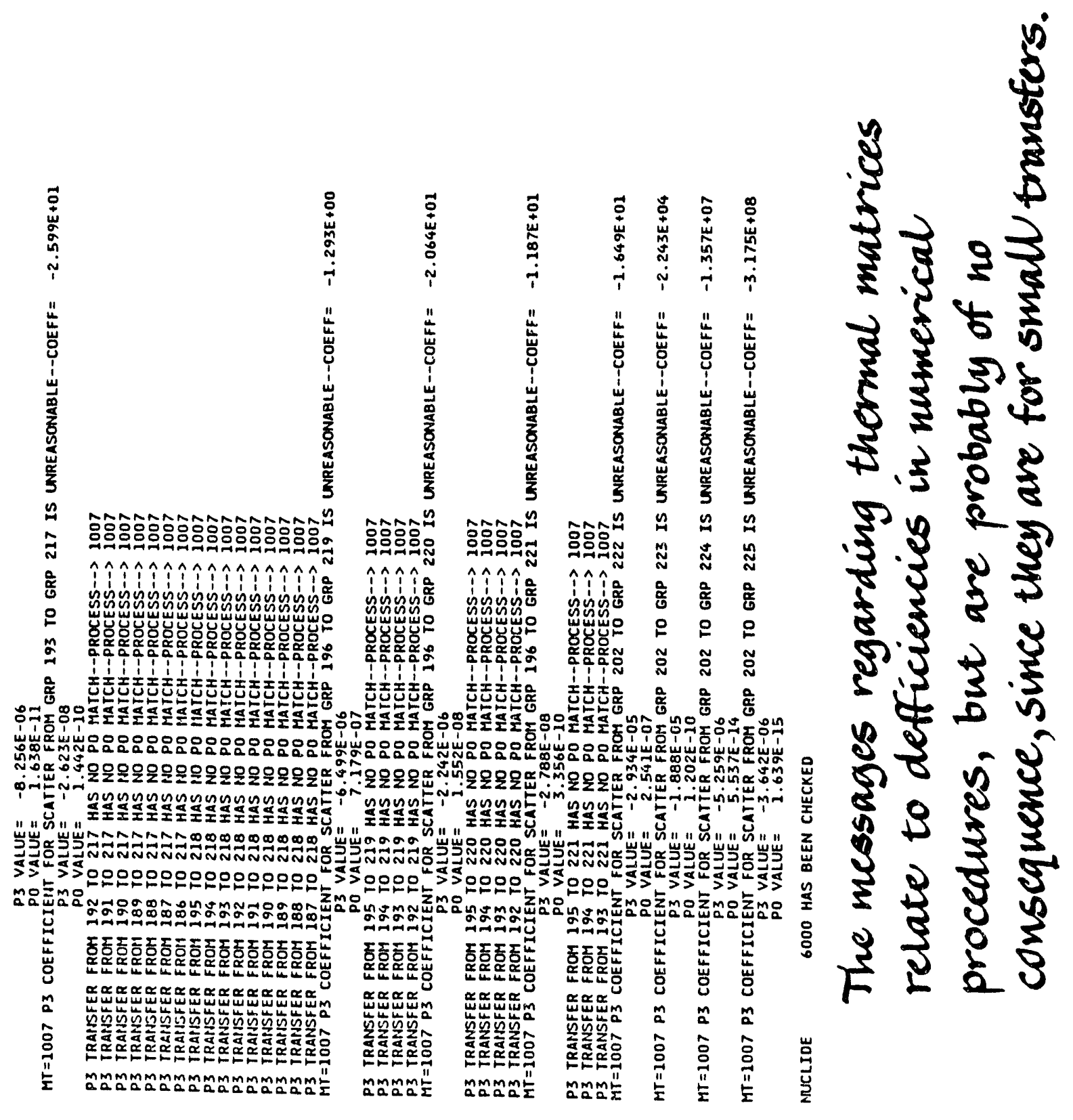


The final parts of Task 1 involve producing Bondarenko factors for ${ }^{235} \mathrm{U}$, coupling these with the ${ }^{235} \mathrm{U}$ data from XLACS, coupling this ${ }^{235} \mathrm{U}$ file with the carbon file, forcing transfer matrices to properly normalize on this file, and, finally, checking the library. This procedure will involve performing operations shown in Figs. 6.SAMPLE PROBLEMS.2 and 6.SAMPLE PROBLEMS.4. (Note that the modular capabilities of the AMPX system do not require a user to slavishly follow a set procedure, but, rather, allow one to tie together codes in any way that makes sense. In the example on the following pages, we have rearranged several operations previously discussed.) The input for the task is given, followed by portions of the output.

\footnotetext{
AMPX MODULE LOG AND LIST OF CARD INPUT DATA

MODULE PRUDE HAS BEEN CALLED

Make Untesolved Point Functions

MODULE PRUDE IS FINISHED: I/O'S O USER CCDE OODO SYSTEM CODE 000

MODULE JERGENS HAS BEEN CALLED

$0 \$ \$ A 225181 \$ \$ 1 T$

Make a Weighting Function

MODULE JERGENS IS FINISHED.

CPU TIME $\quad .60$ (SECONDS). I/O'S 0 USER CODE OOOO SYSTEM CODE OOO

MOdULE TABU has BEEN CALLED

058131251951238238258 o . Make Bondarenko Factors

U-235 BONDARENKO FACTORS IN THE UNRESOLVED RANGE
$10 \$ \$ 1395$ II\$\$ $9931 \mathrm{~T}$

MODULE TABU IS FINISHED.

CPU TIME 42.55 (SECONDSI. I/O'S 0 USER CODE 0000 SYSTEM CODE OOO

MODULE UNITAB HAS BEEN CALLED

$0 \$ \$ 65189859$ I O E I\$\$ $126 \$ \$ \$ \$ 5001000 \mathrm{~T}$
$2 \$ \$$

$2 \$ \$ \$ 2235 \quad 59 \quad 92235 \quad 1111$

92235113954444

Combine Bondarenko Factors

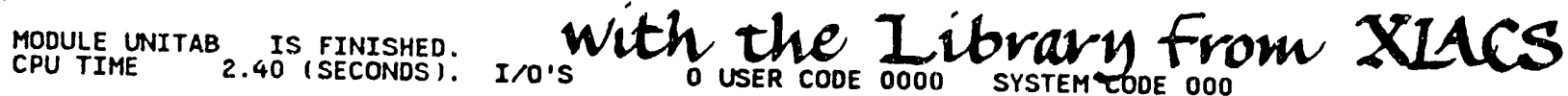

MODULE AJAX HAS BEEN CALLEO

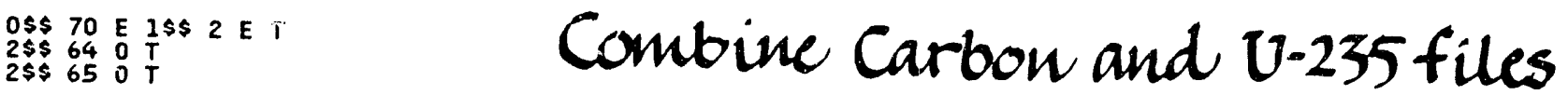

MODULE AJAX IS FINISHED.

CPU TIME $\quad 1.03$ (SECOHISI: I/O'S 0 USER CODE 0OOO SYSTEM CODE OOO

MODULE COMET HAS BEEN CALLED

0\$5 7071 E 15s 0 E 285241617 E 65800500 T Force Normalization

MODULE COMET IS FINISHED.

CPU TIME 16.48 (SECONDS): I/O'S O USER CODE DOOO SYSTEM CODE OOO

MODULE RADE HAS BEEN CALLED

$1 \$ \$ 1 E T$

MODULE RADE IS FINISHED.

CPU TIME $\quad 3.37$ (SECONDSI. I/O'S 0 USER CODE OOOO SYSTEM CODE DOO

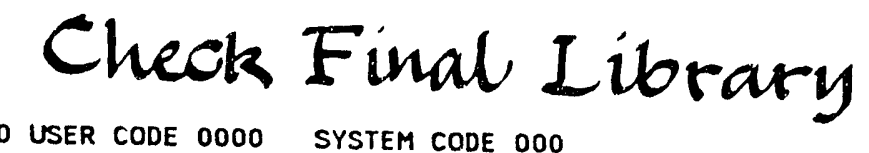




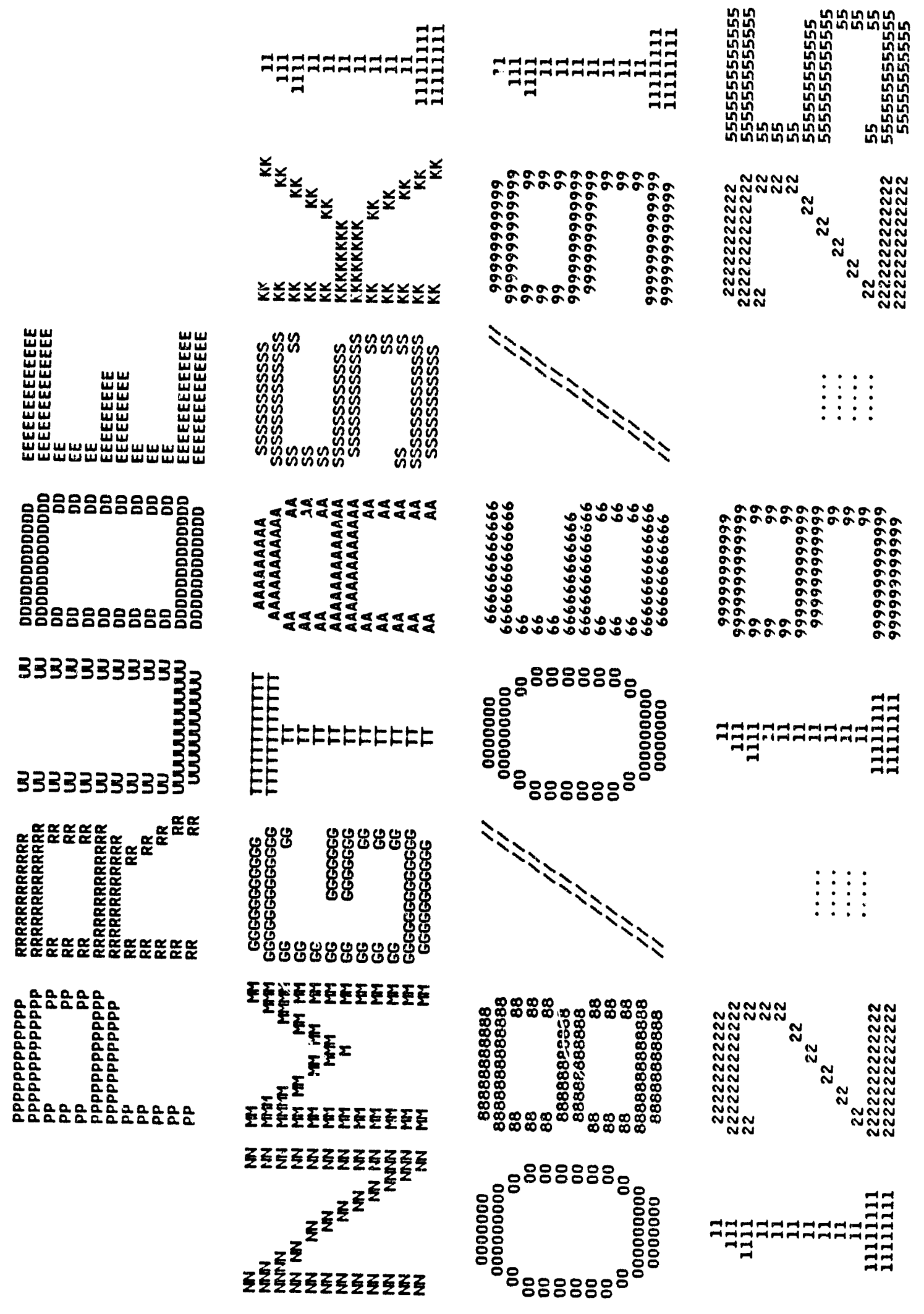




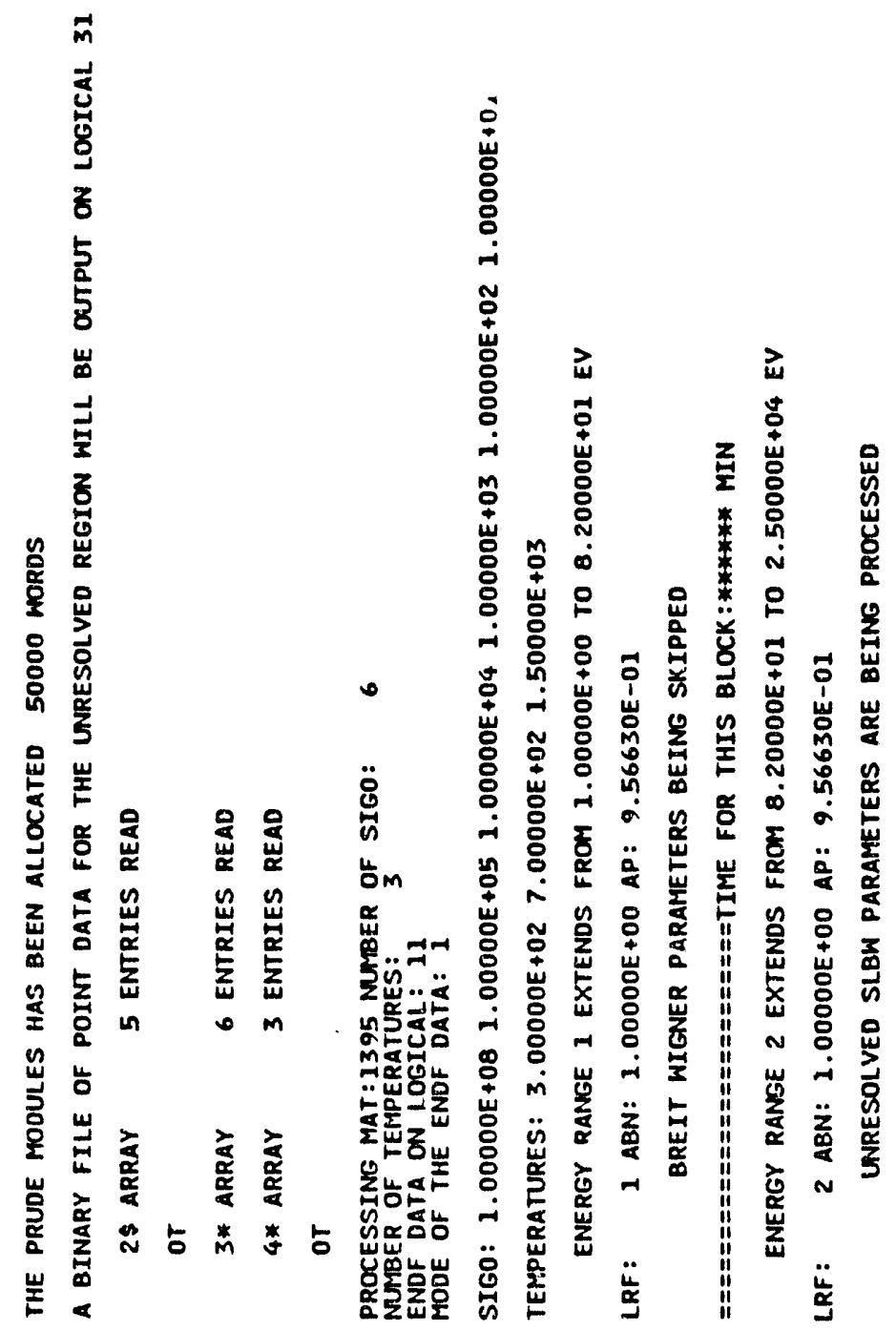



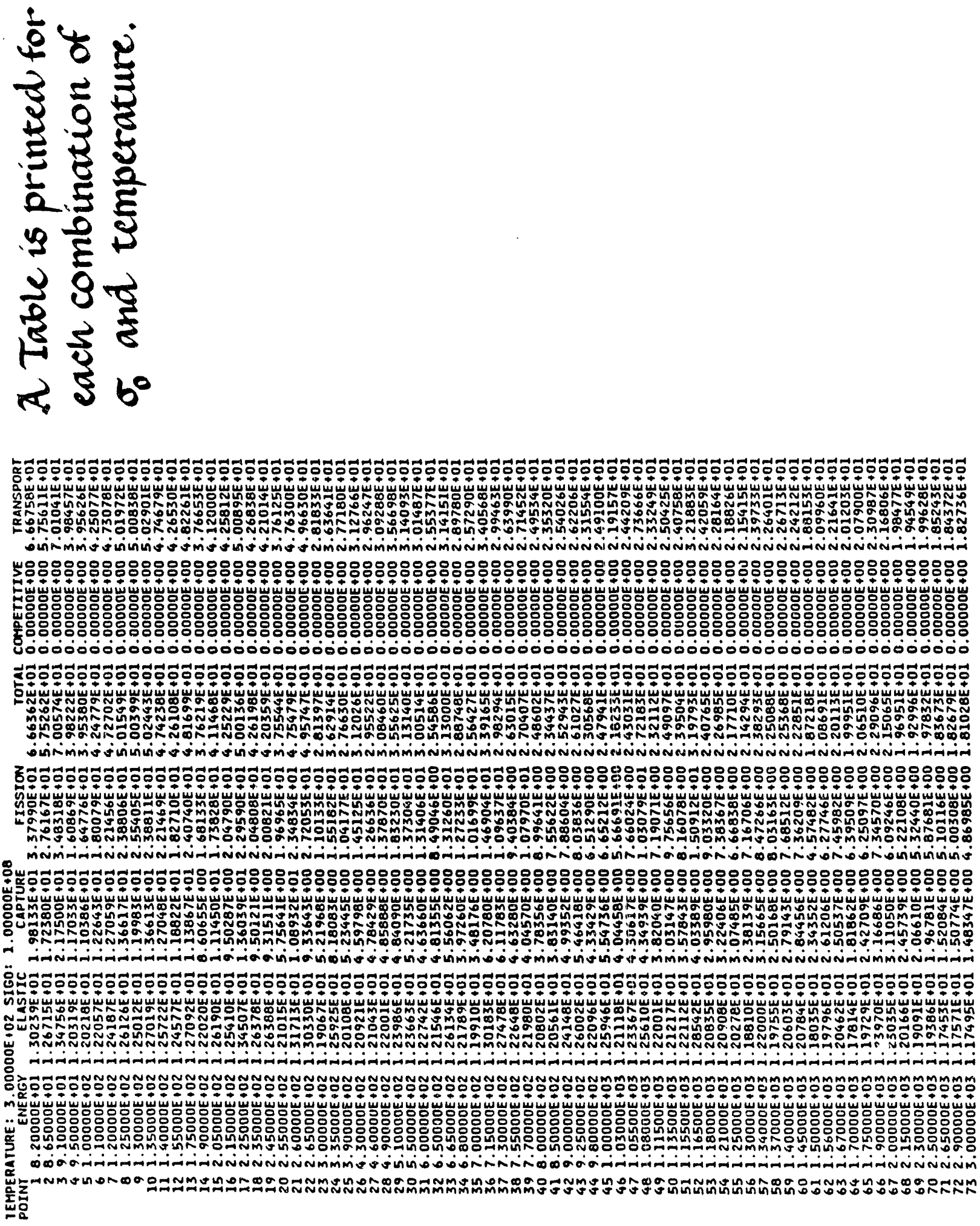

SAMPLE PROBLEMS 


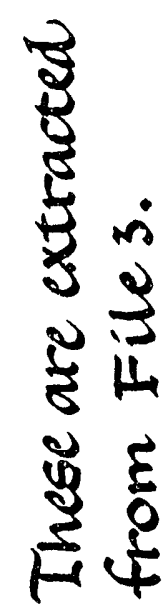

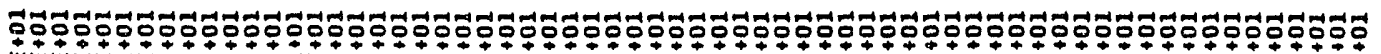

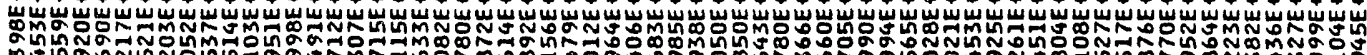
งूँ

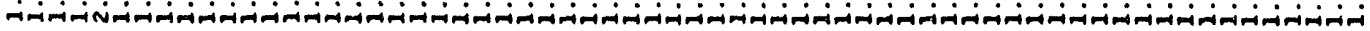

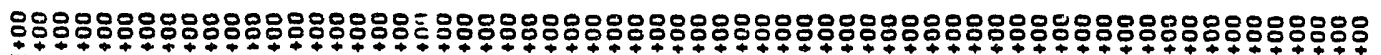

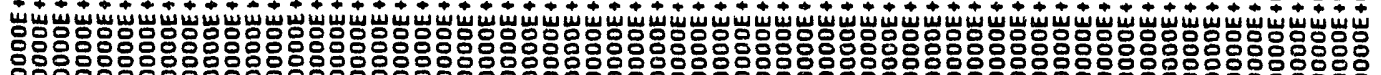

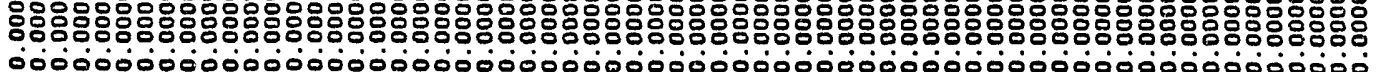

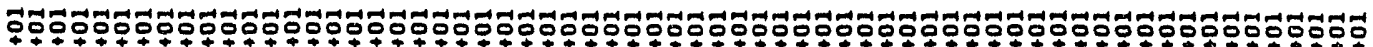

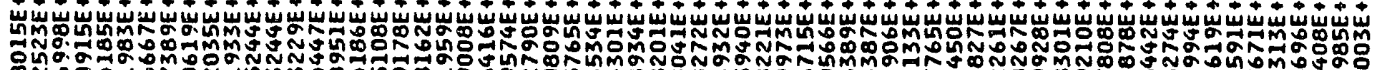
อิ

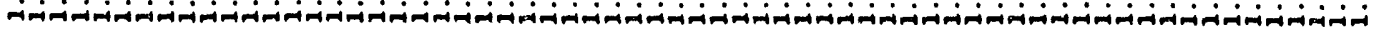

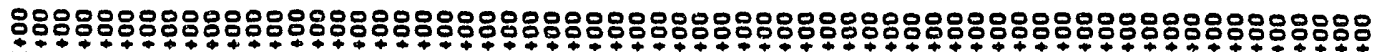

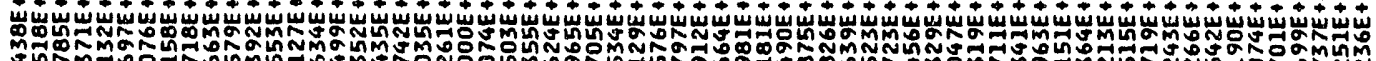

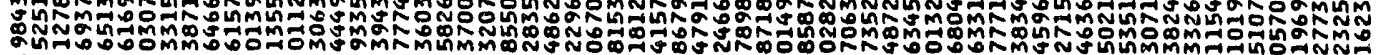

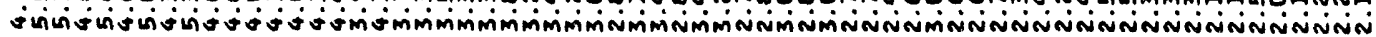

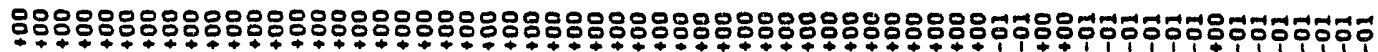

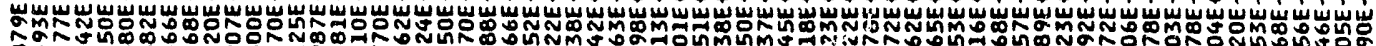
ỡ

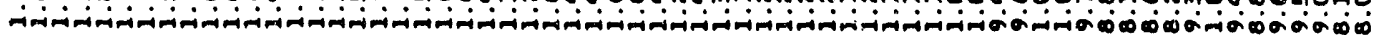

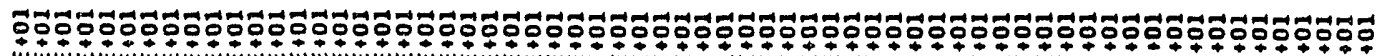

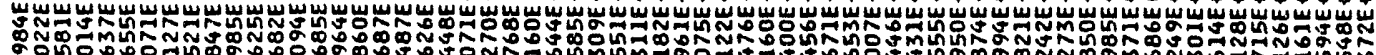
Don.

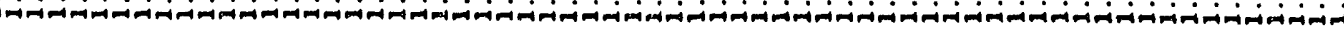

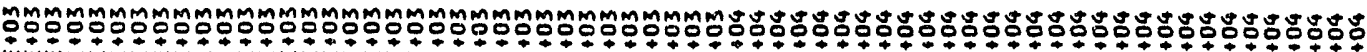

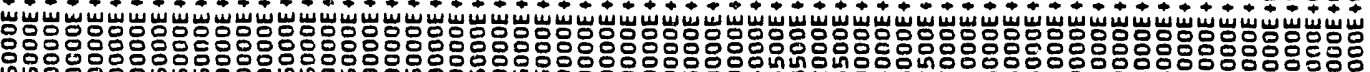

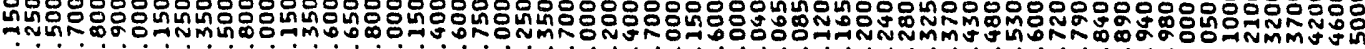

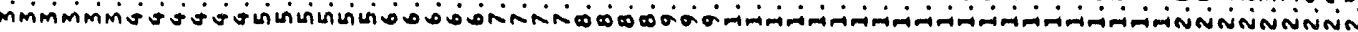

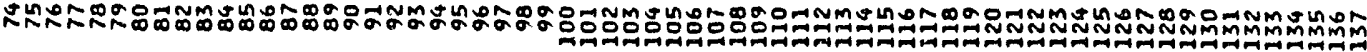

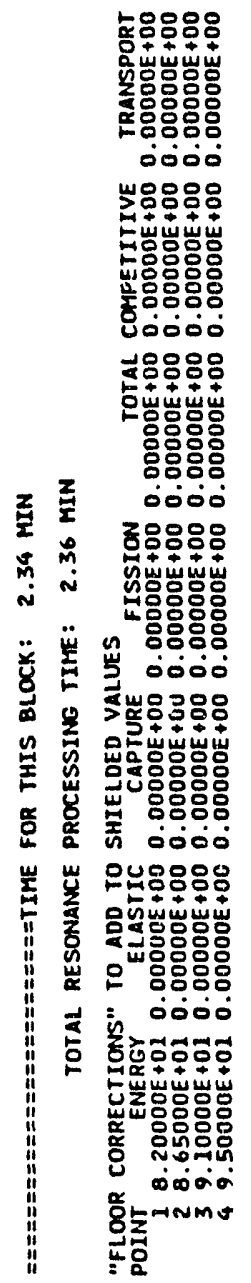




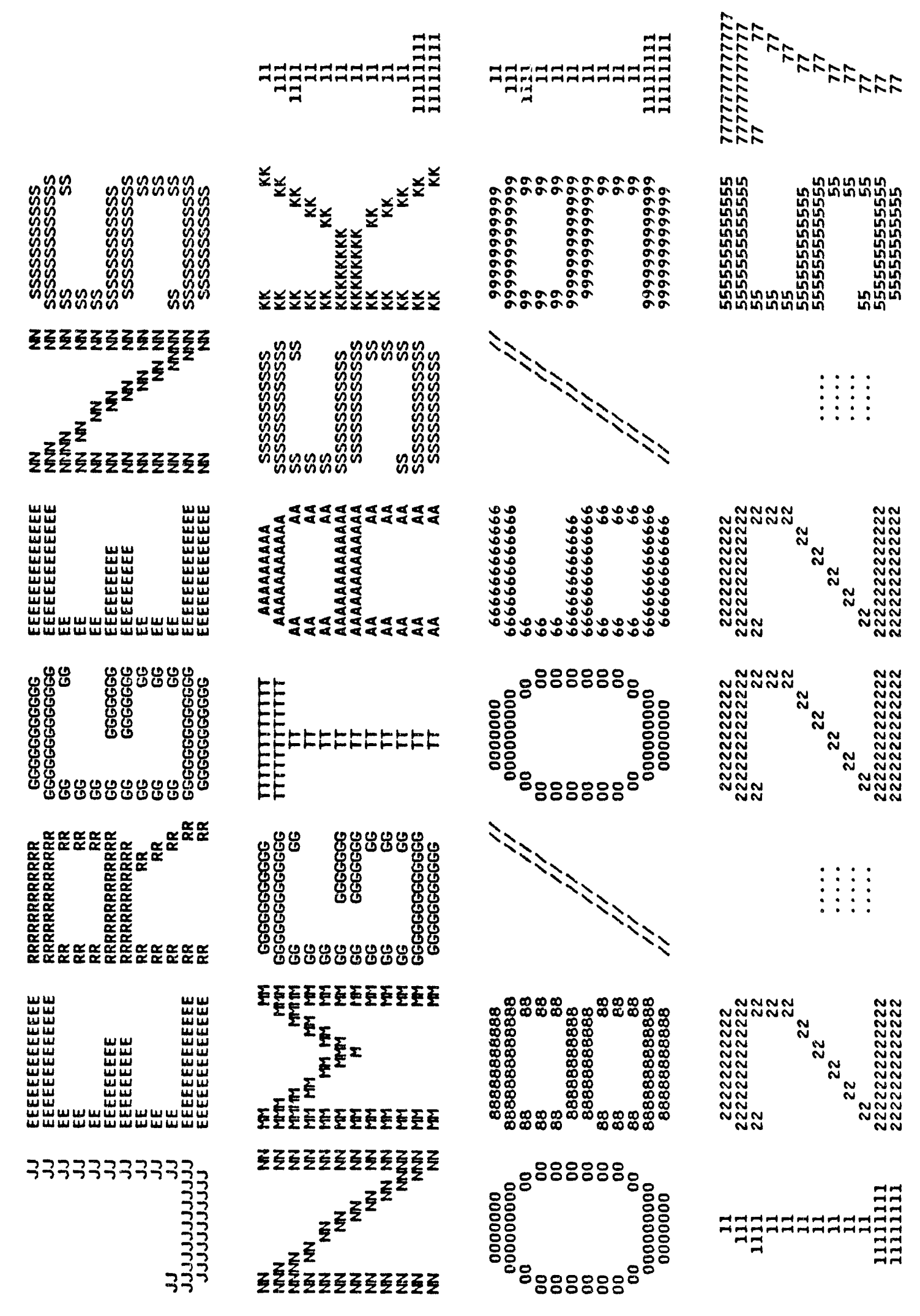

SAMPLE PROBLEMS 


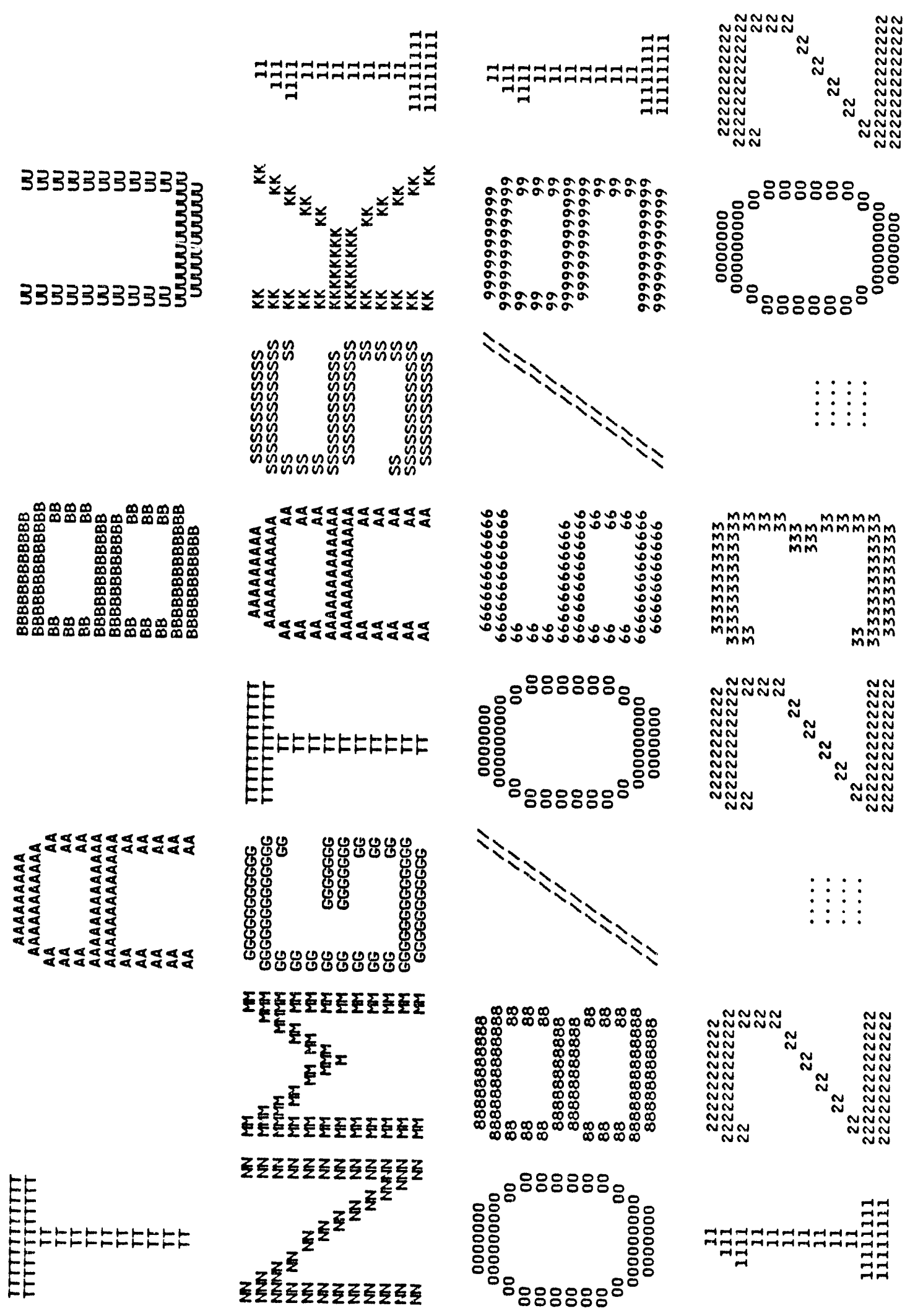




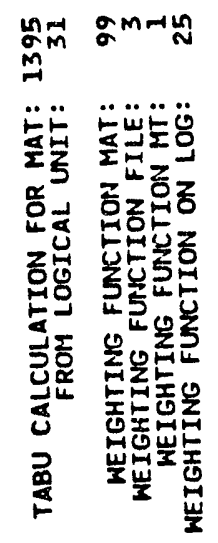




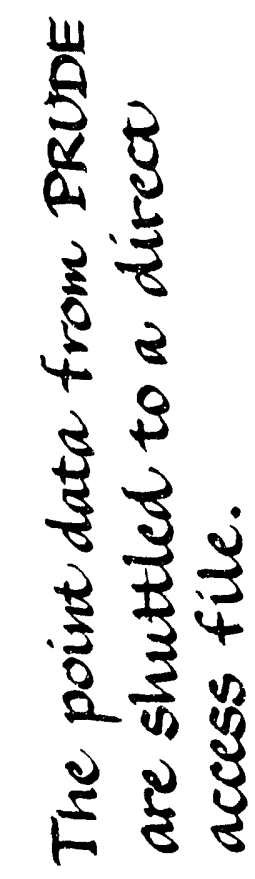

总

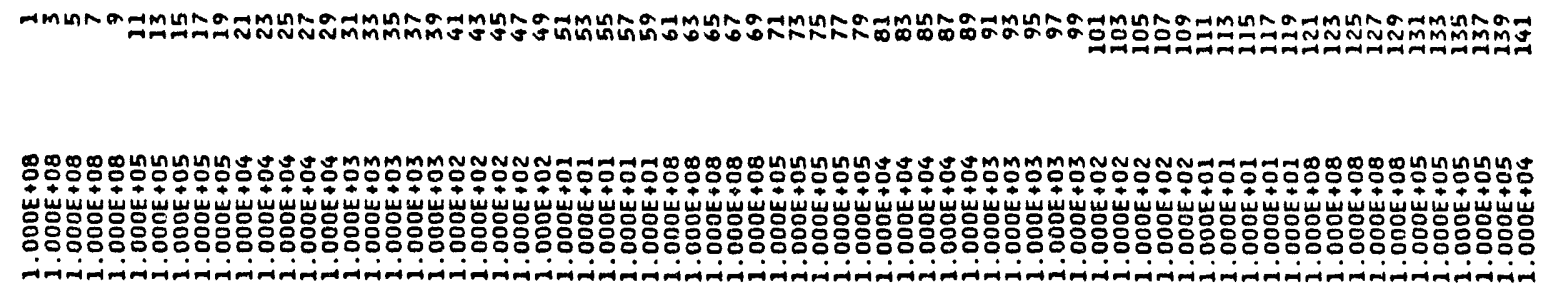

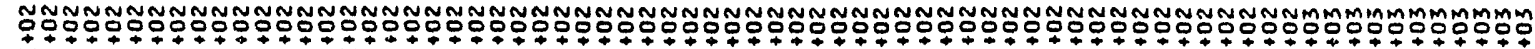

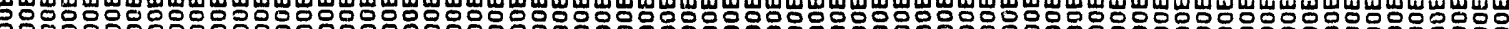

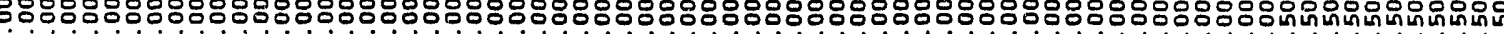

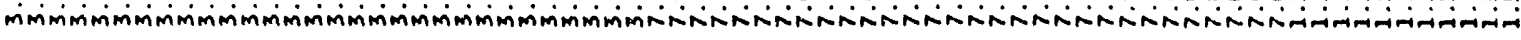

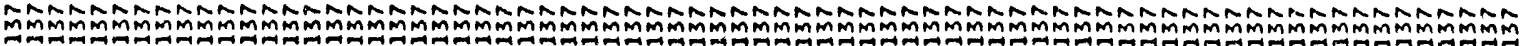

象要

:

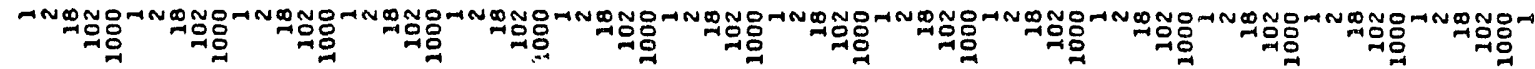

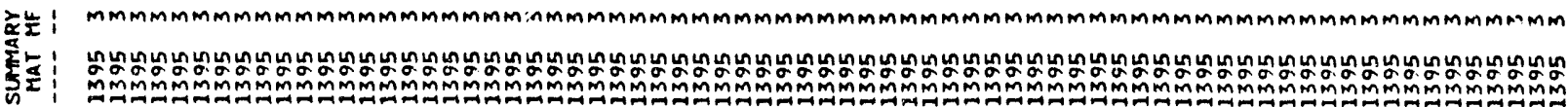

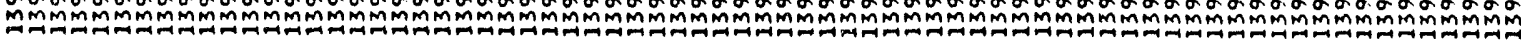




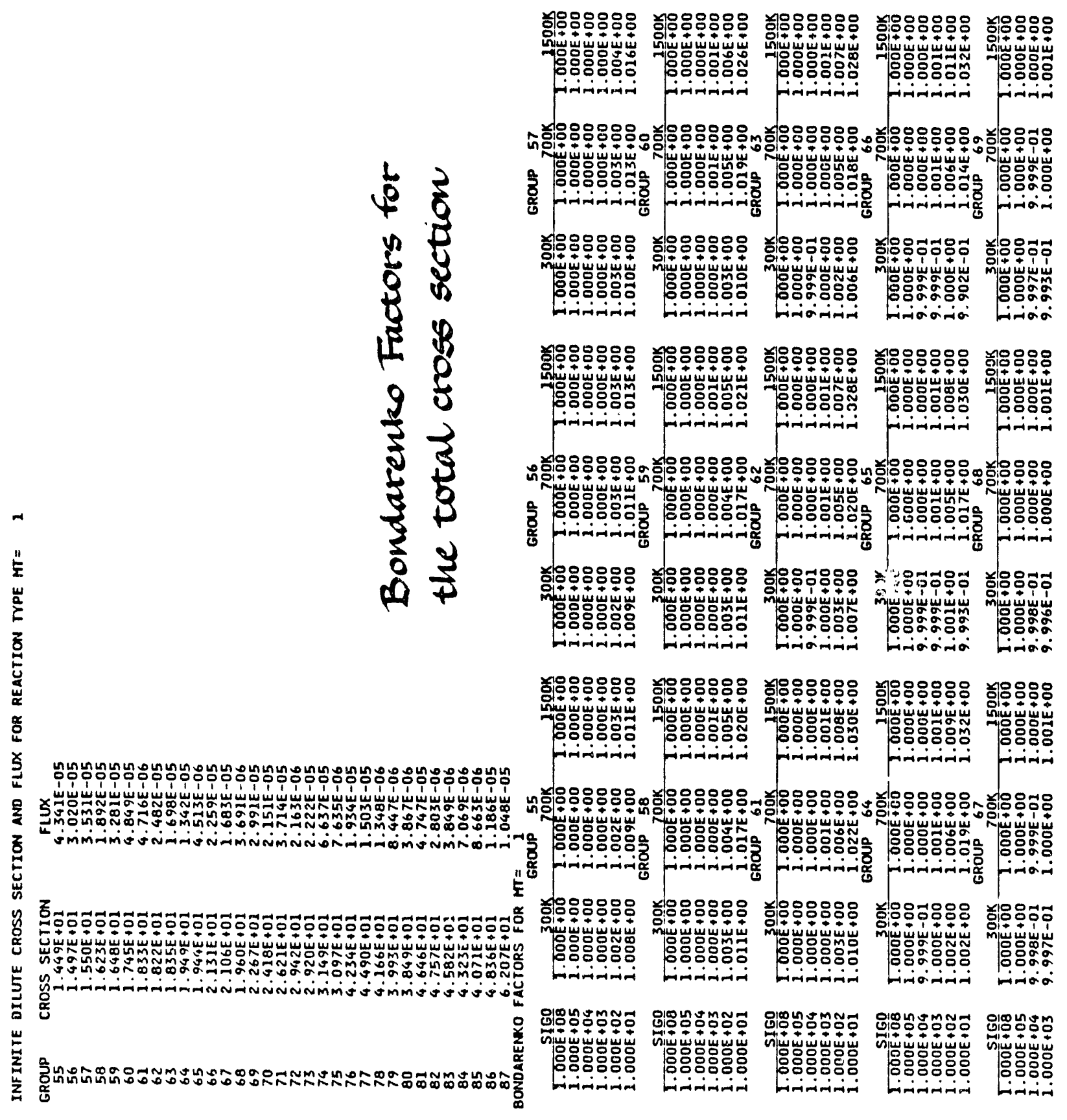

SAMPLE PROBLEMS 


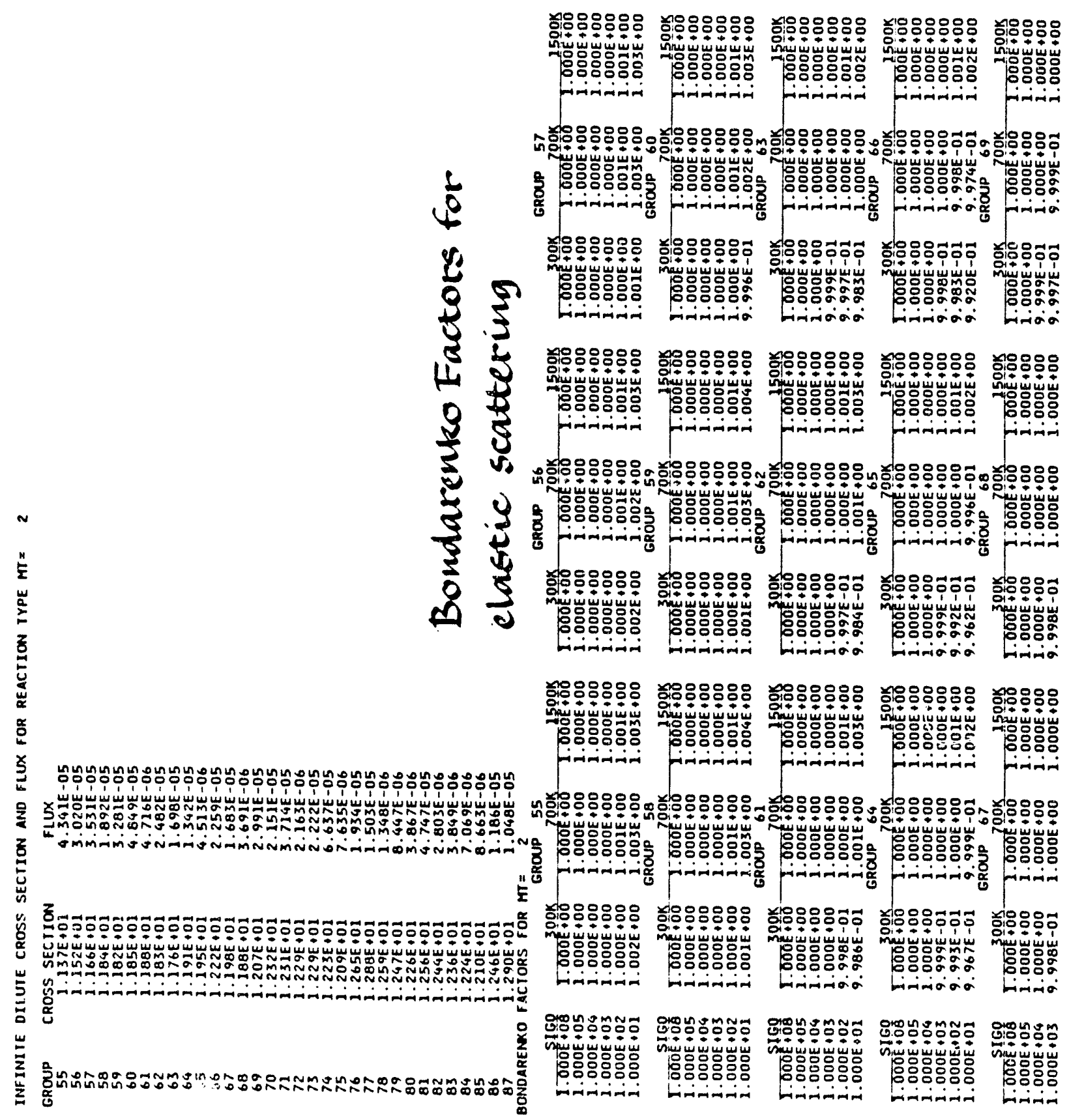

SAMPLE PROBLEMS 

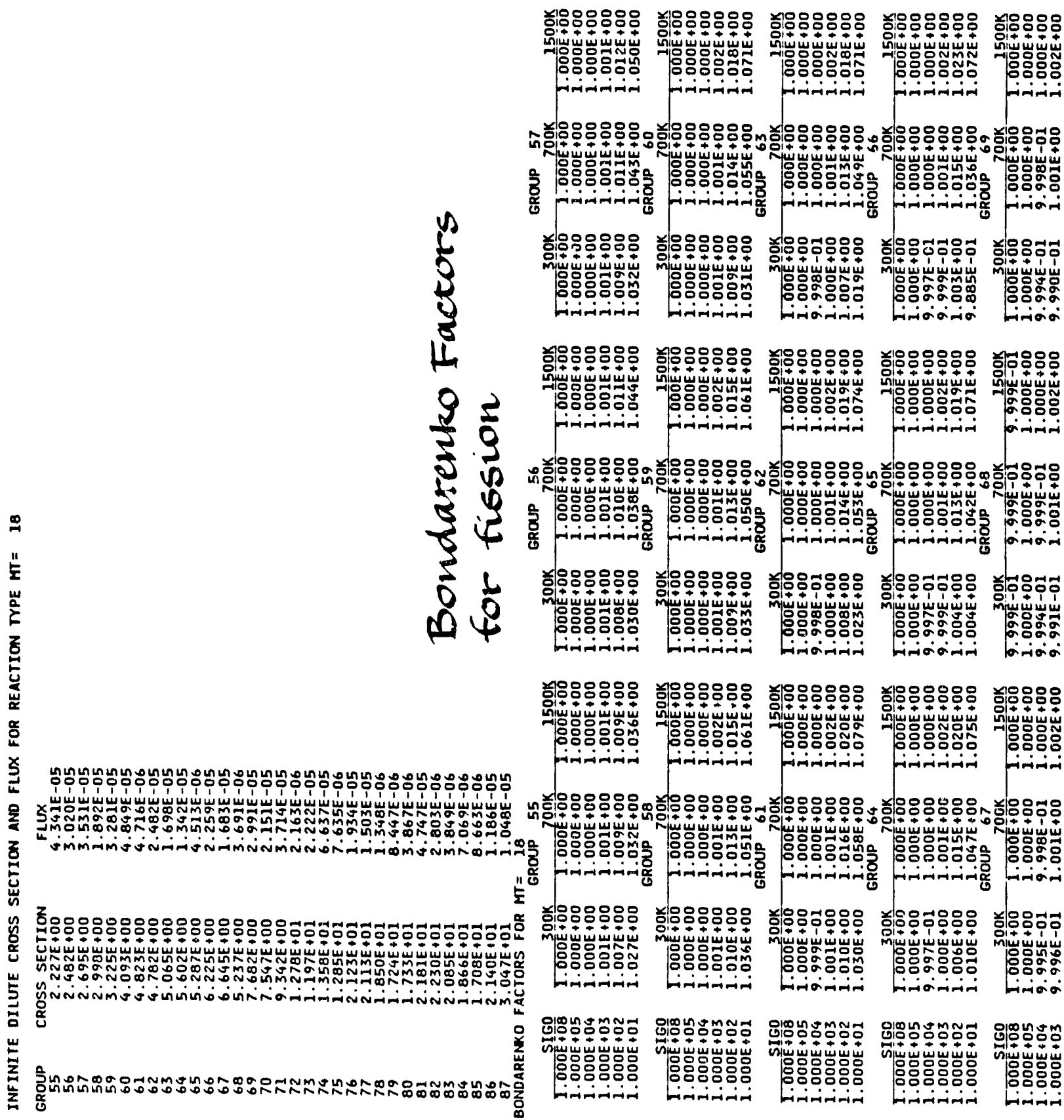

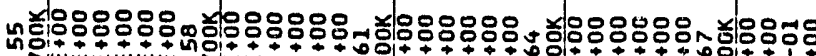

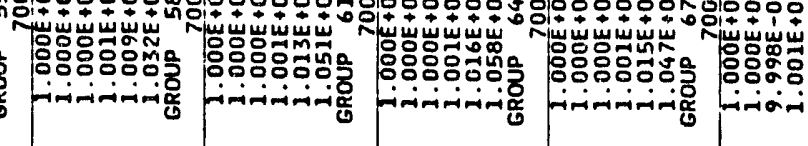

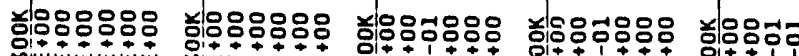

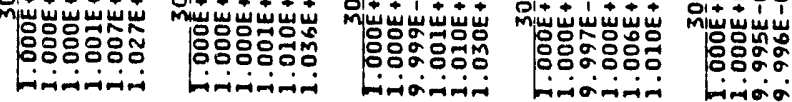

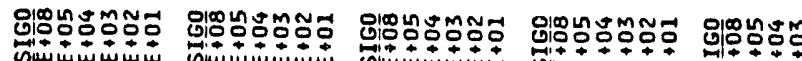

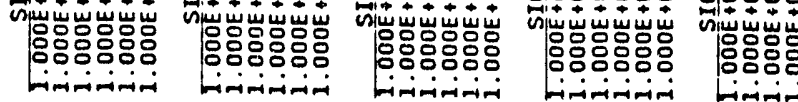




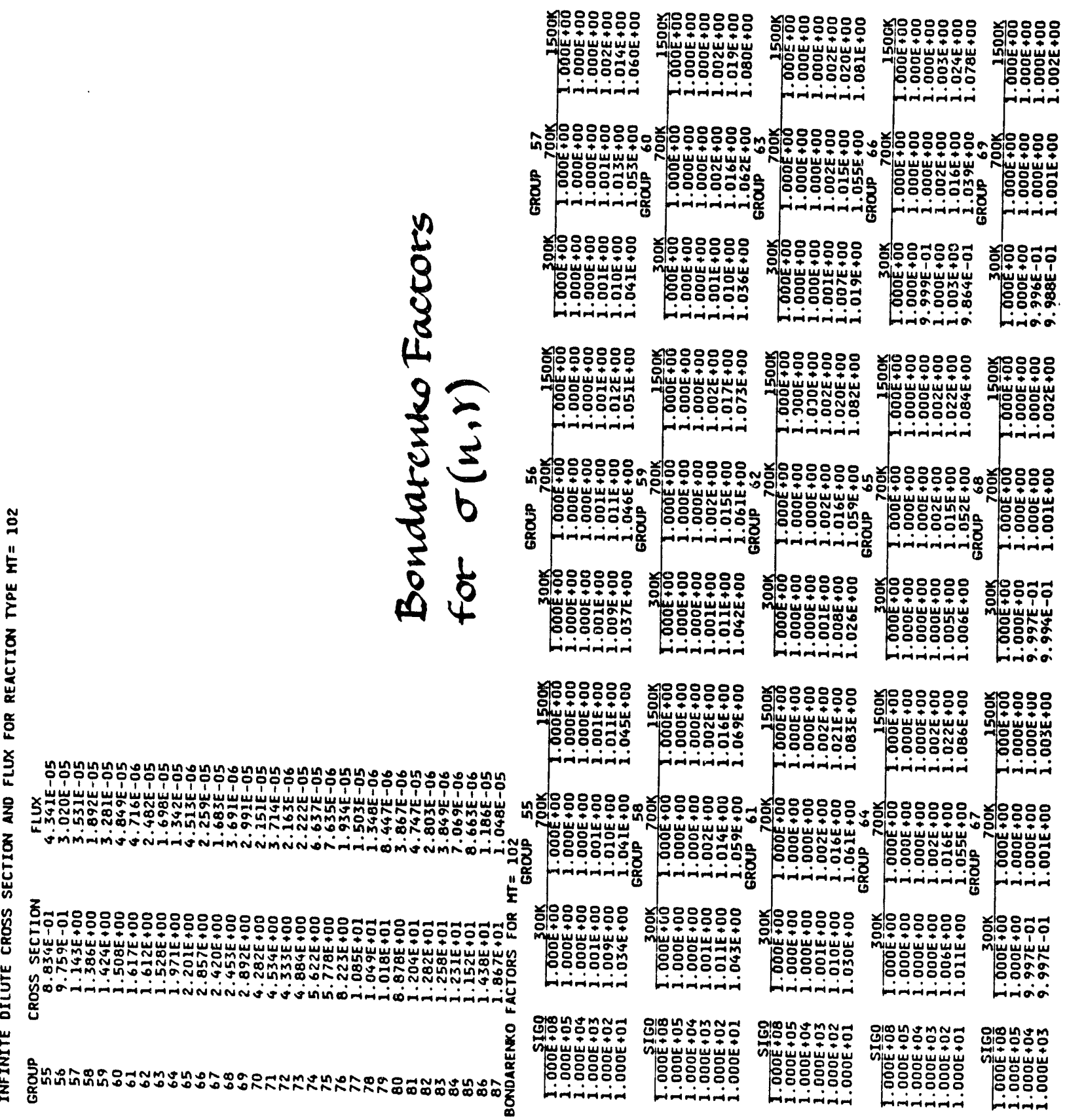

SAMPLE PROBLEMS 

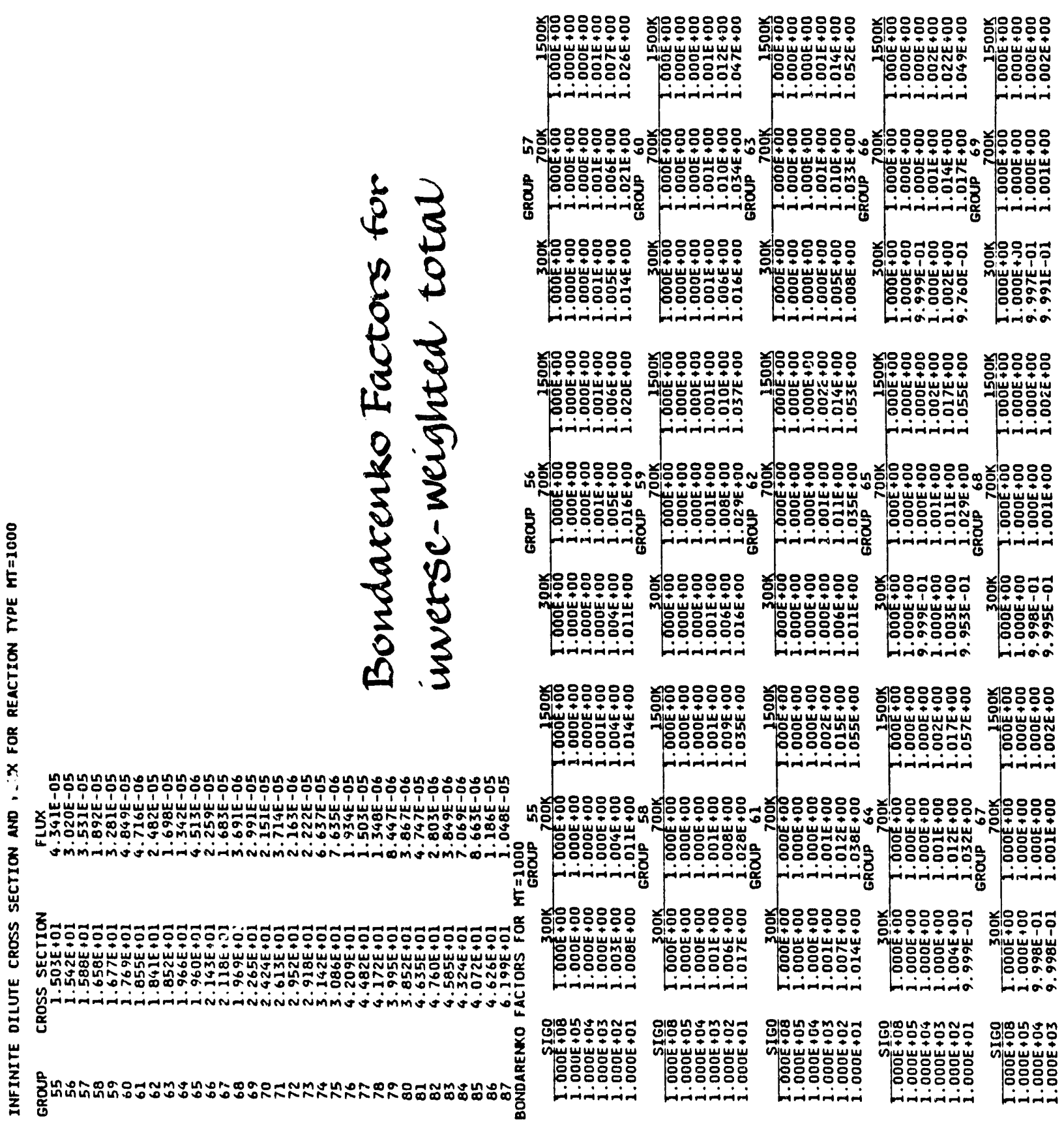

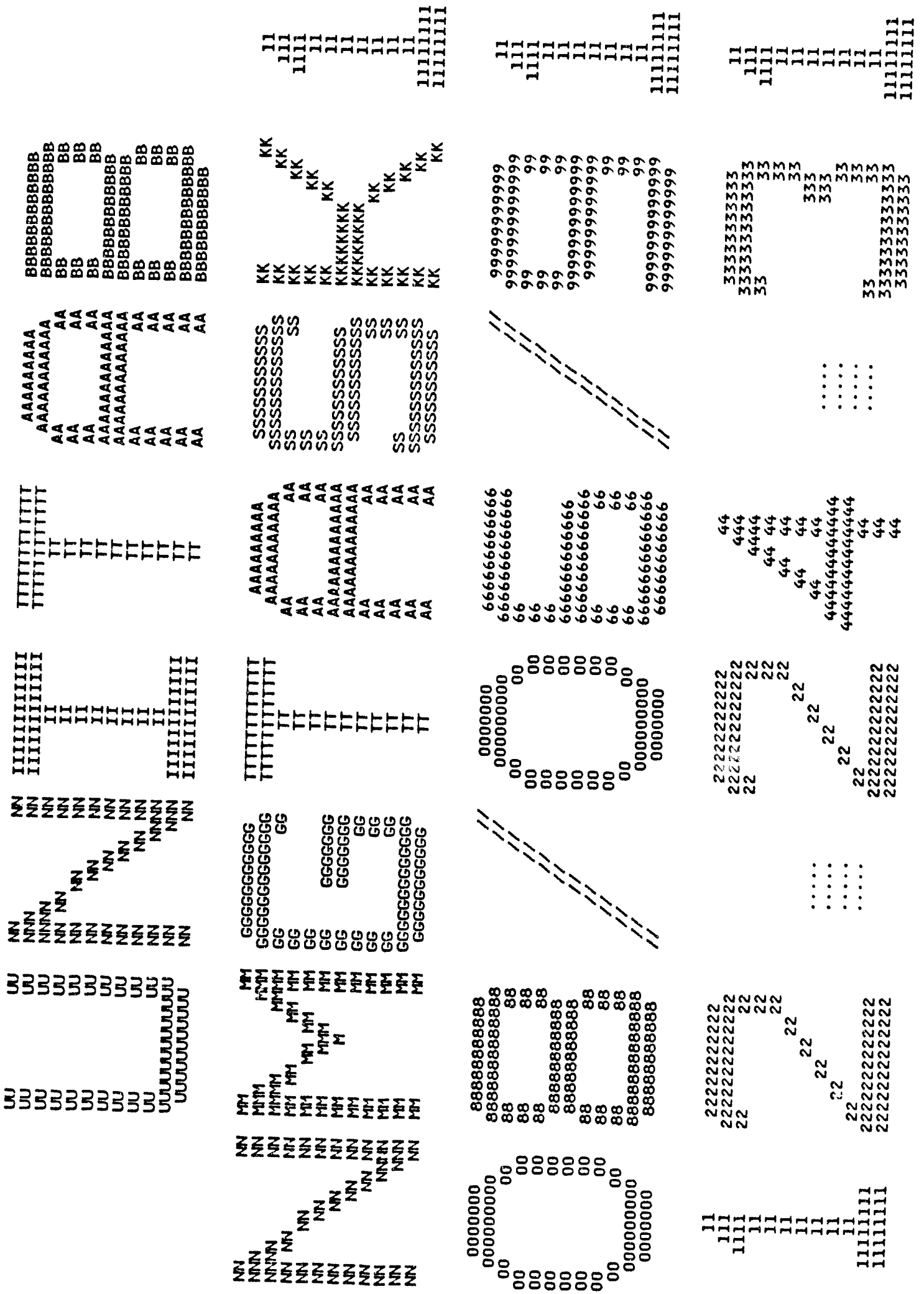

SAMPLE PROBLEMS 


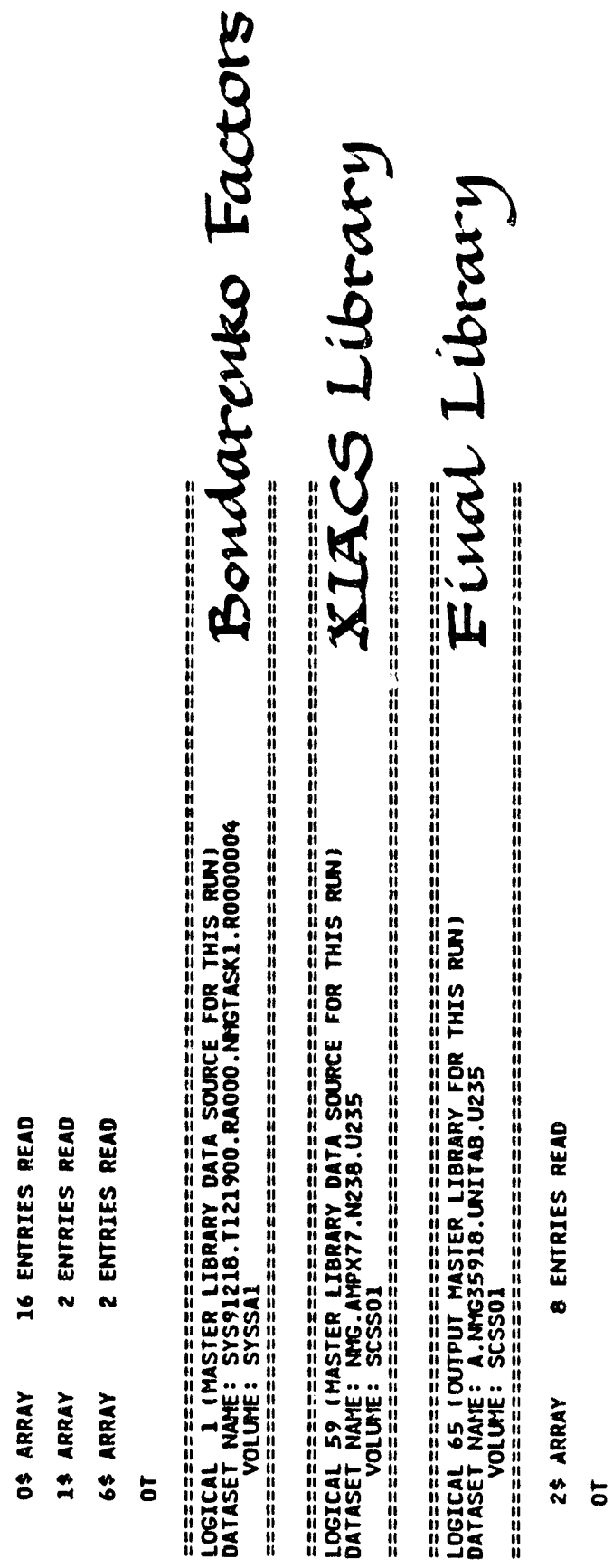




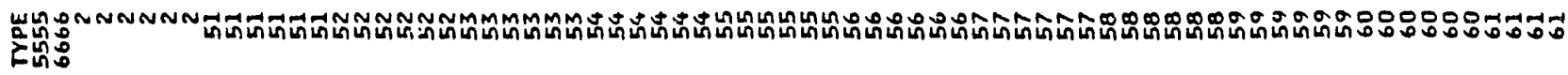

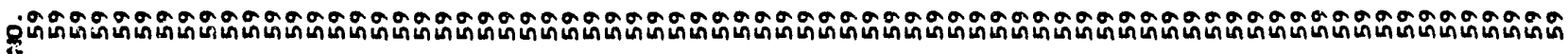

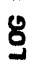

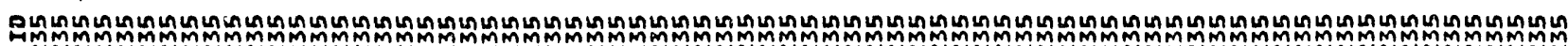

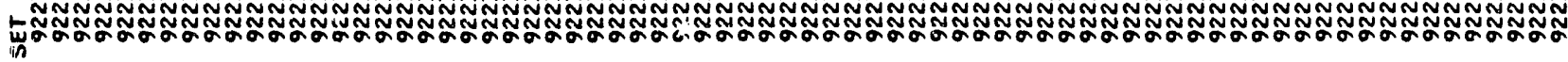

z.․․․

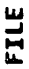

$\frac{x}{n}$

产

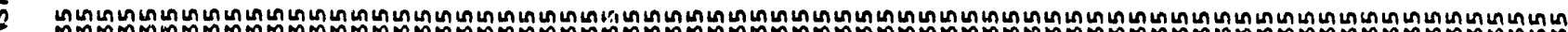

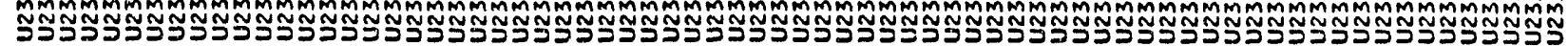

$\mathbf{z}$

岁

웅 突

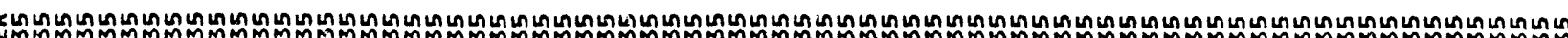

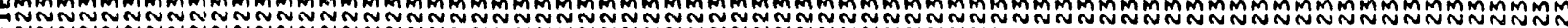

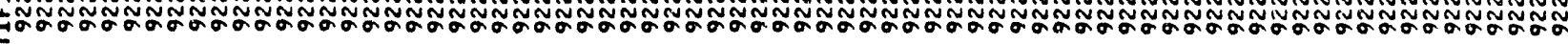

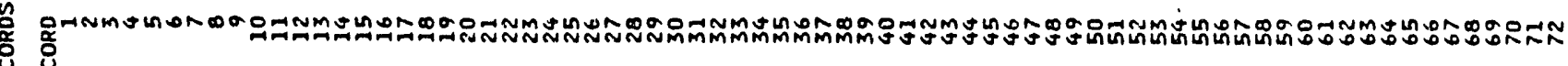

总 


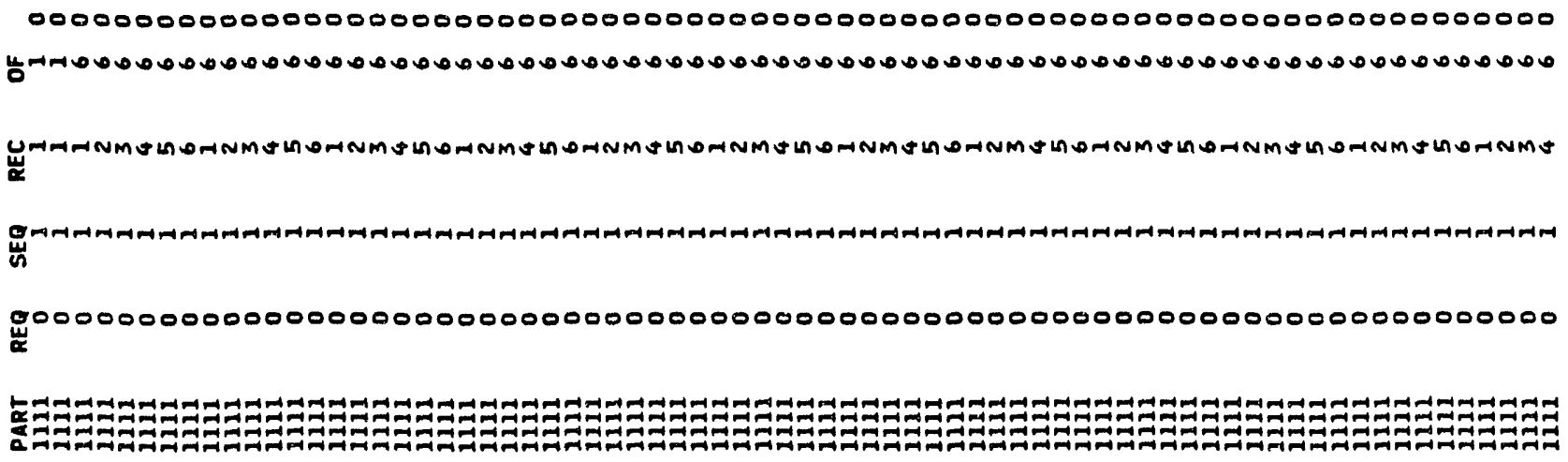

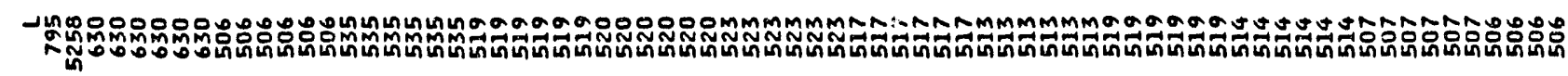
ํำ

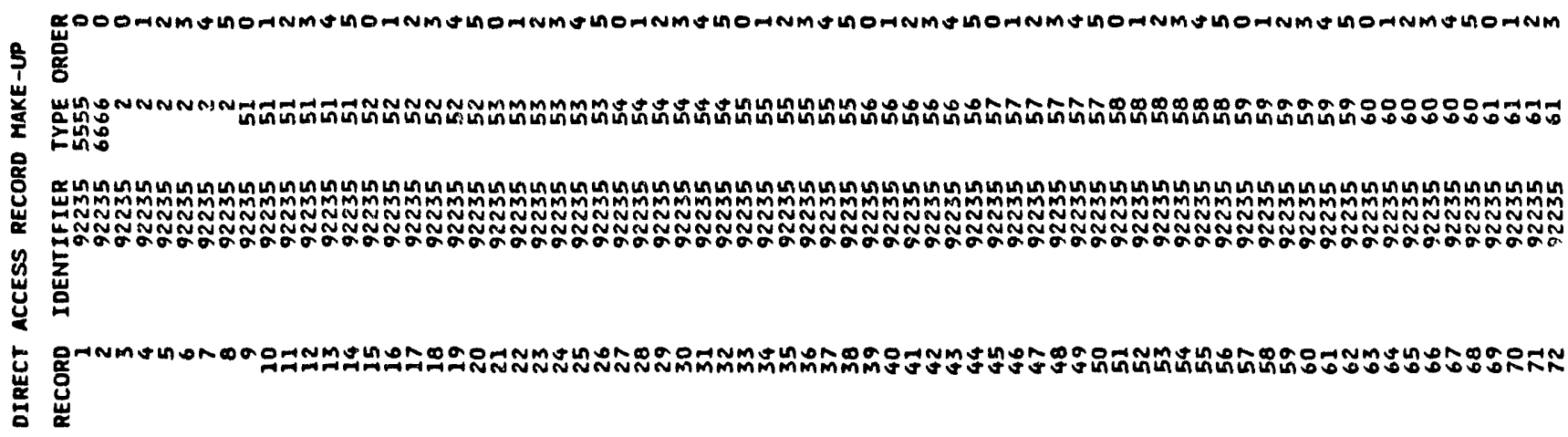




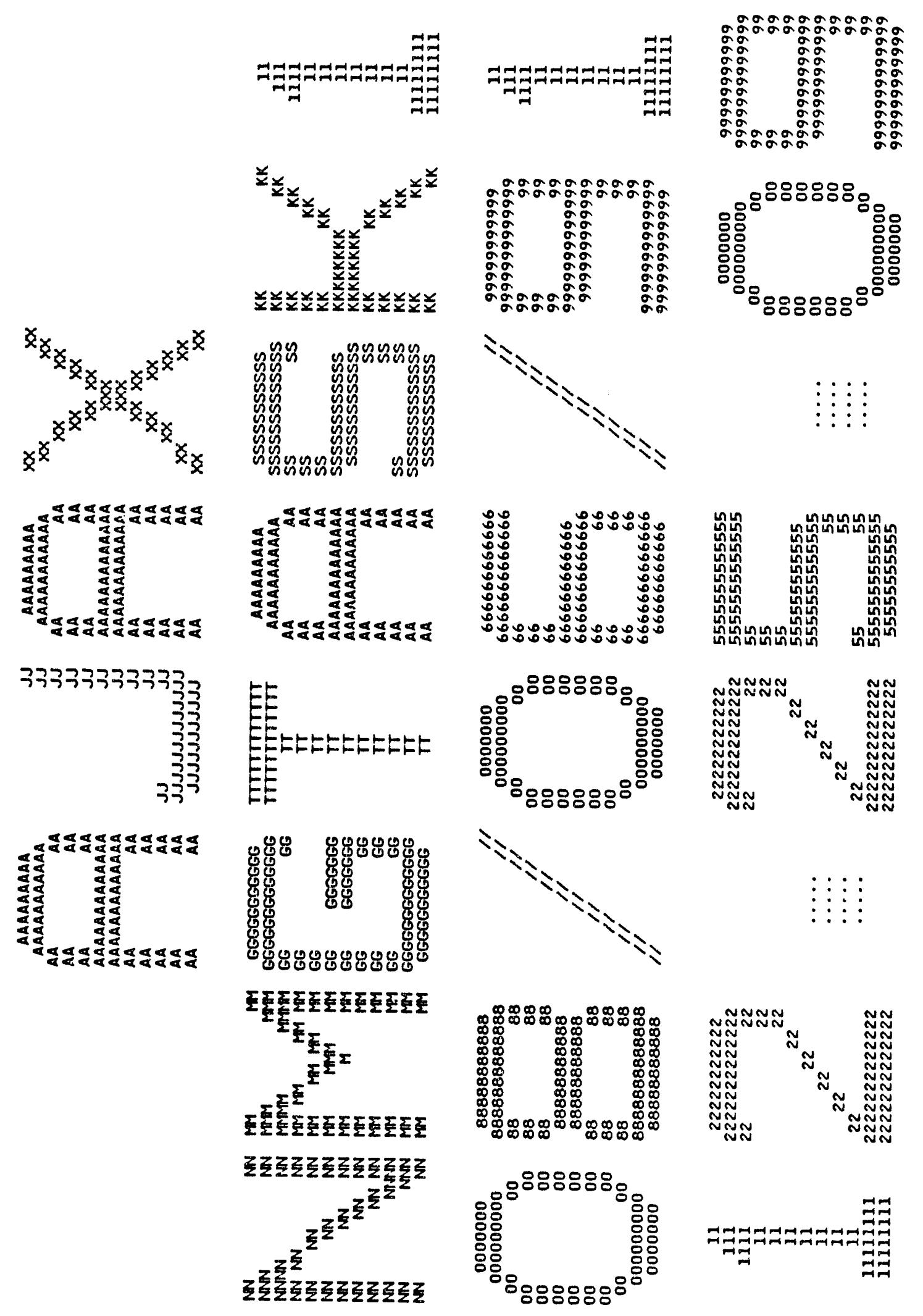




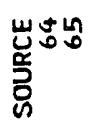

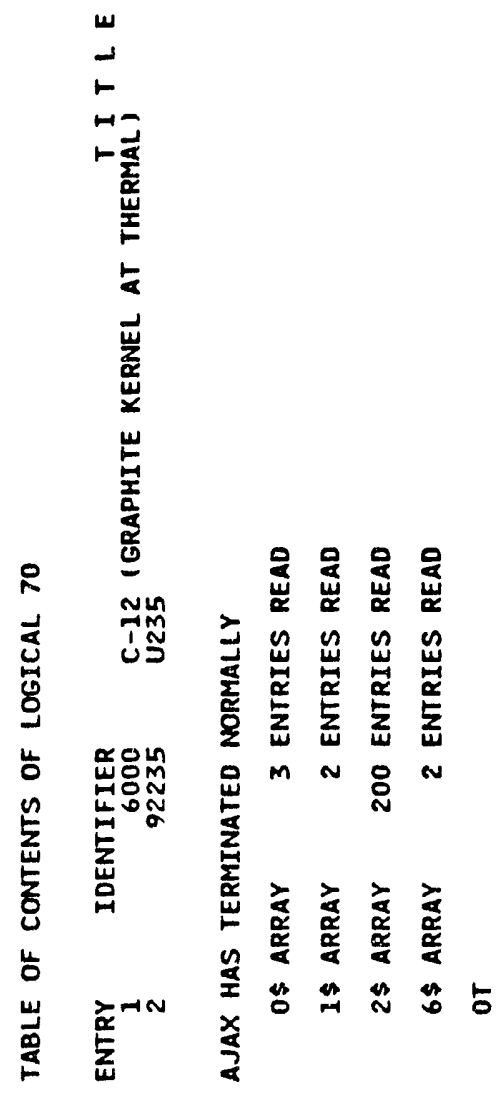



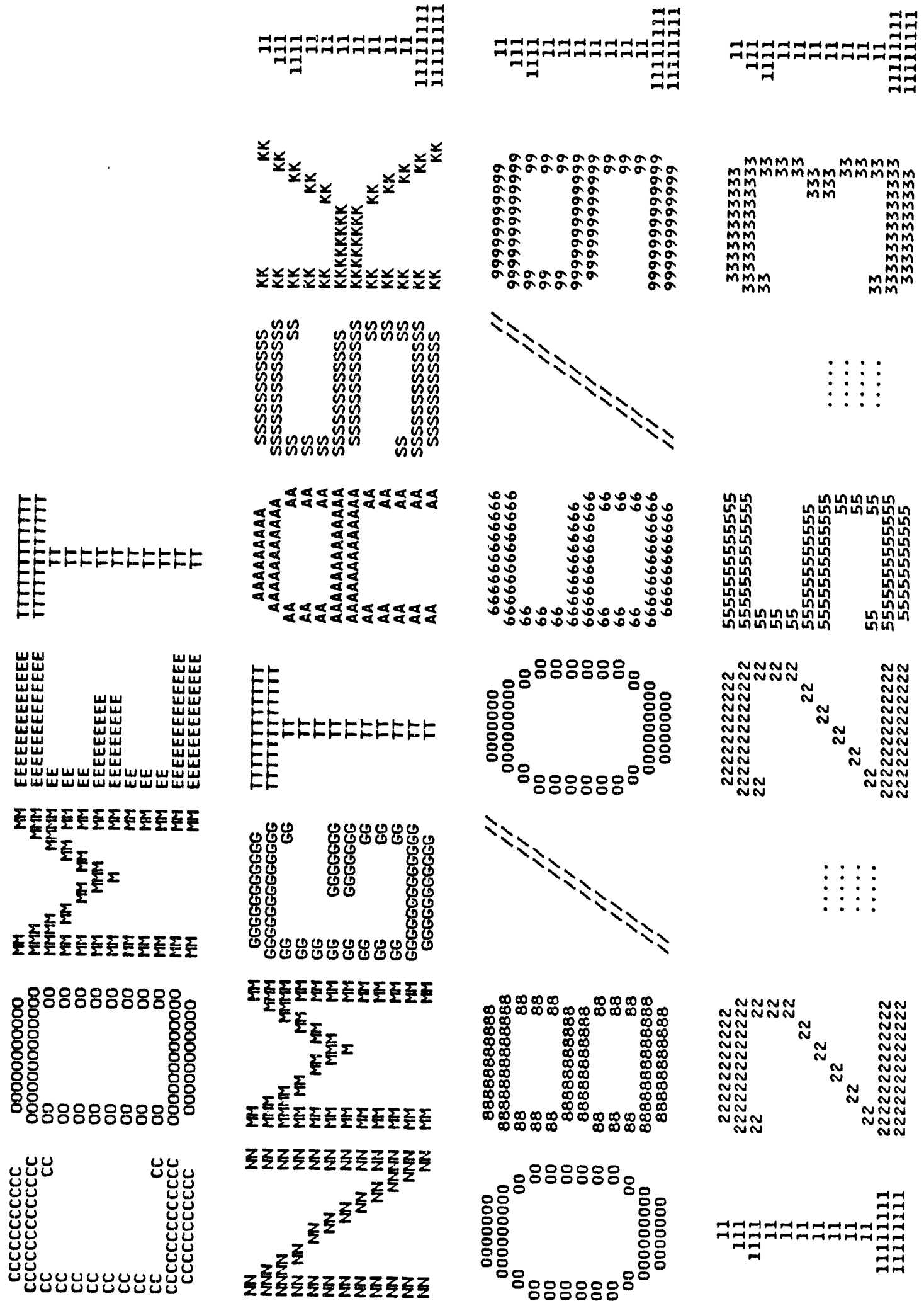

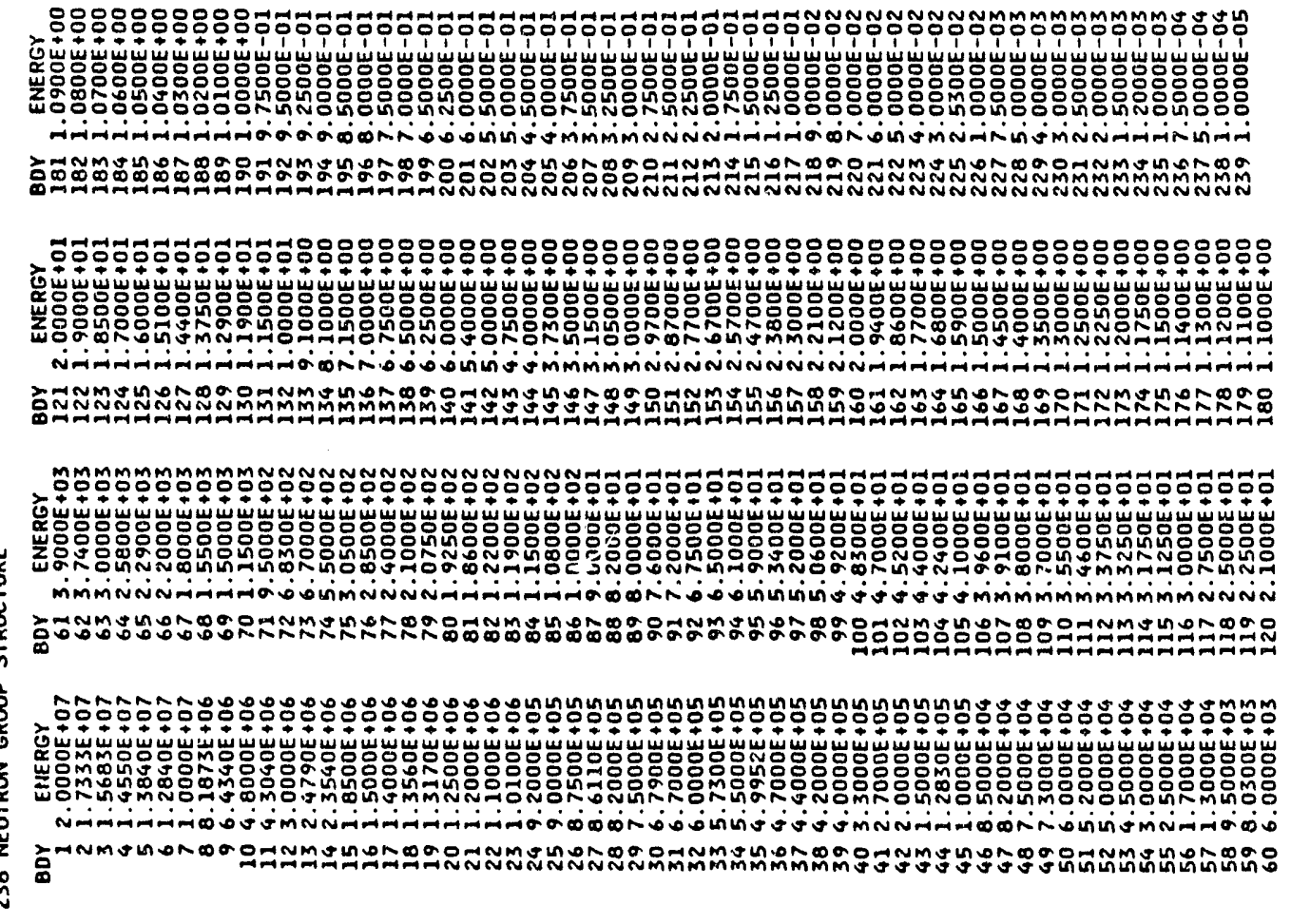

$\pi$

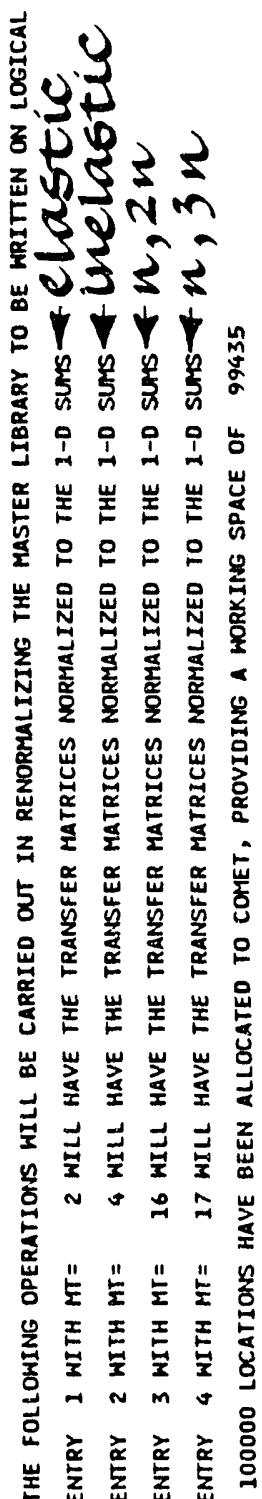




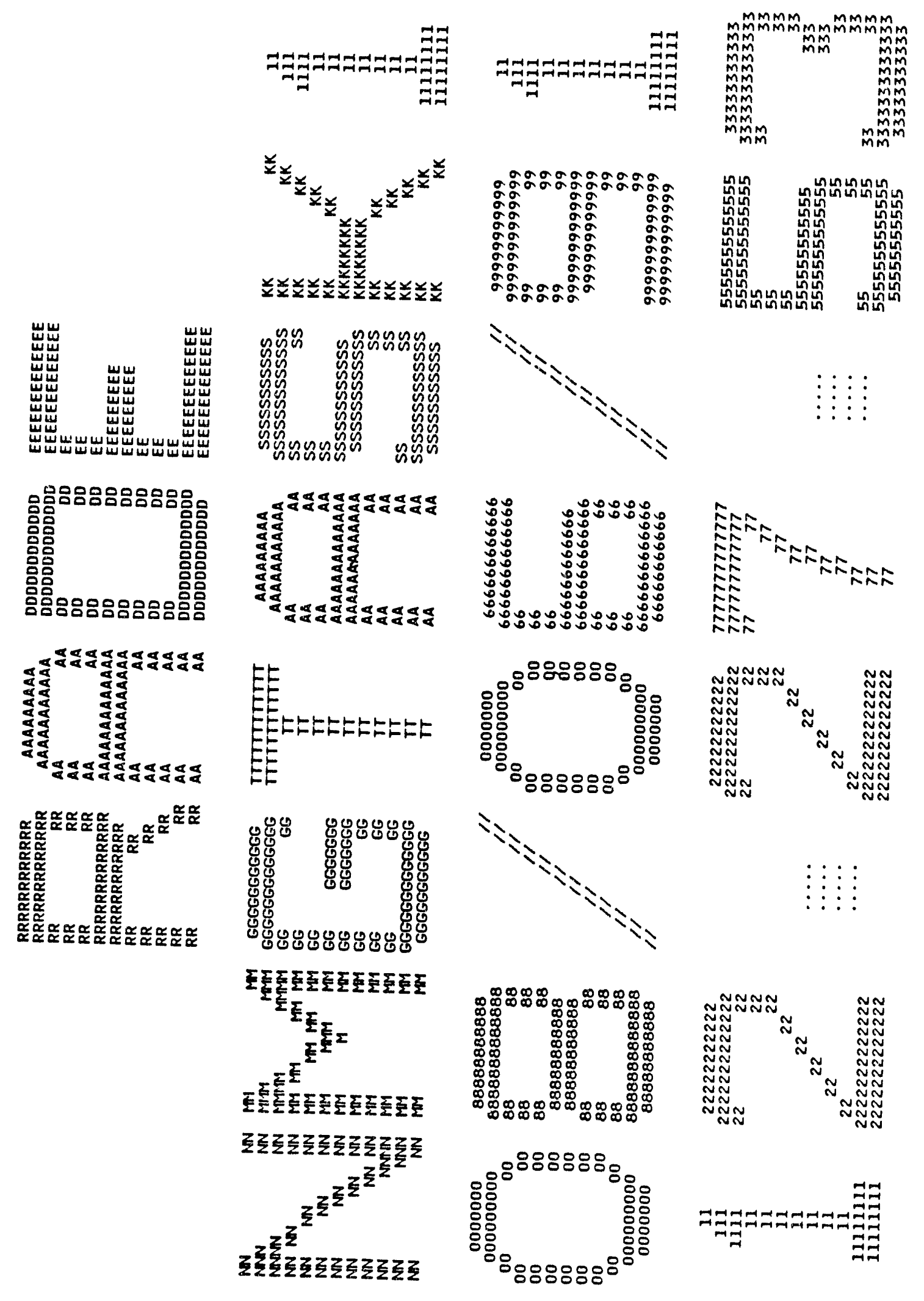



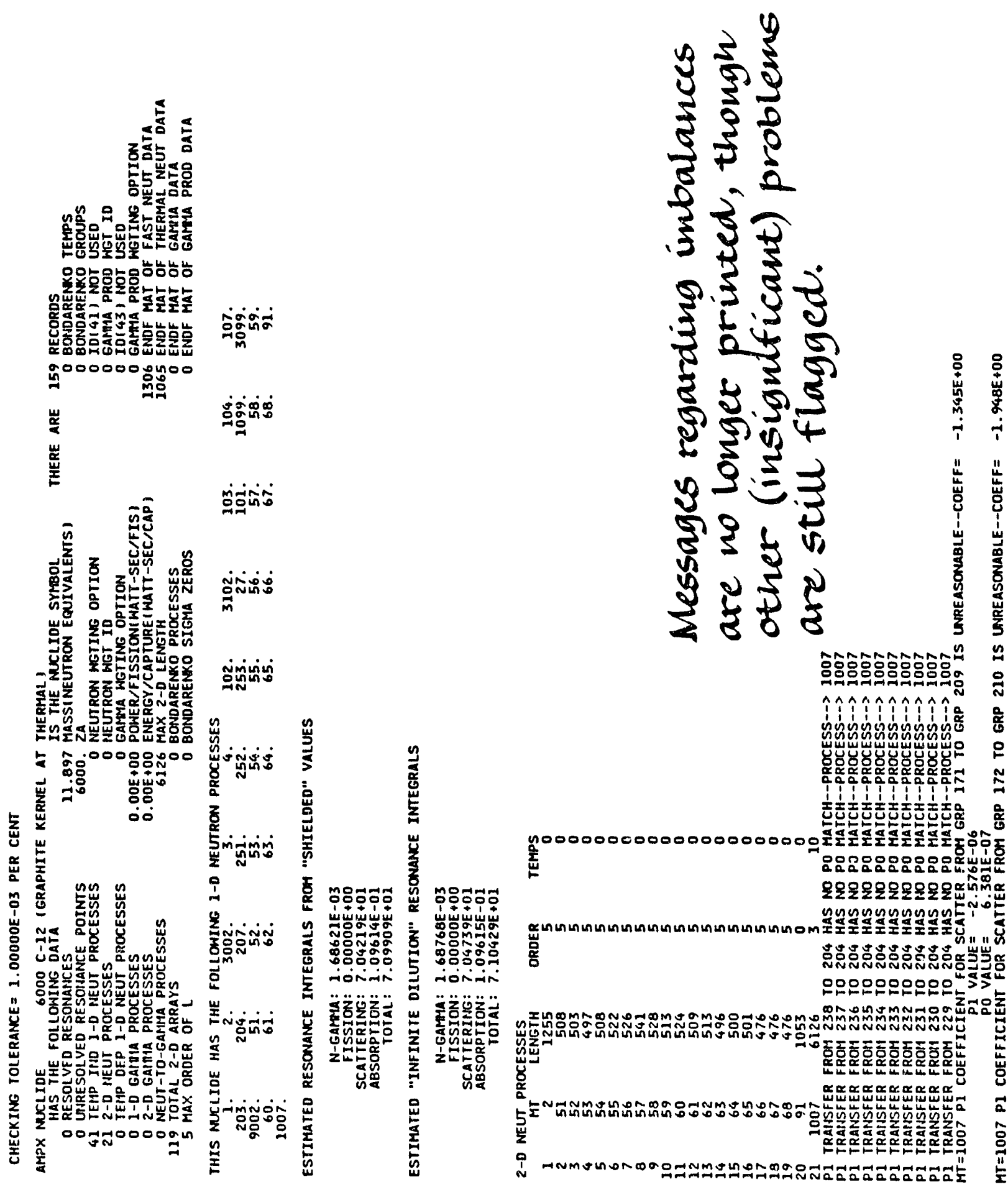


\section{SAMPLE PROBLEMS.2 CREATE A MULTIGROUP COUPLED NEUTRON-GAMMA-RAY LIBRARY}

In making a coupled library, one has exactly the same problems as for Task 1 , discussed earlier, because a neutron library is part of the coupled library. The considerations for making the neutron-to-gamma production cross sections are exactly the same as for the neutron library, since all the "weighting" is in the neutron realm. The additional concern is that of picking a gamma group structure. The major problem is simply that of being able to find evaluations that include gamma production data for all the nuclides of interest. In Table 6.SAMPLE PROBLEMS.1, one notes, for example, that two of the nuclides have no photon production data. Similar considerations are needed to make gamma-ray interaction cross sections. Generally speaking, this is a simpler problem, or at least is treated simpler. The weighting spectrum is frequently taken to be flat, though calculational results suggest a $1 / \mathrm{E}$ variation is more reasonable. The group structure is selected by taking uniform bins in an $\mathrm{MeV}$ sense with groups added to encompass important gamma source energies for the application. The SMUG program treats only Klein-Nishina scattering, which is for scattering on a free electron. Cross sections for absorption, pair-production, etc., do not vary as a function of the isotope of a nuclide, which greatly simplifies the problem of locating gamma-ray data.

The gamma group structure used in the sample cases is a 44-group structure developed for shipping cask studies.

The procedure selected for this task is shown in Fig. 6.SAMPLE PROBLEMS.5. The neutron path is a combination of any of the procedures discussed in the previous task description. The running of the checking and fix-up modules can (and should be) used on the coupled master library.

For reasons of simple convenience, we split Task 2 into three subtasks:

1. a task to produce a master library containing only the gamma production data,

2. a task to produce a master library containing only the gamma interaction data, and

3. a task to couple the libraries from the two above subtasks with the neutron library from Task 1.

The input for Subtask 1 is shown below, followed by portions of the output. 


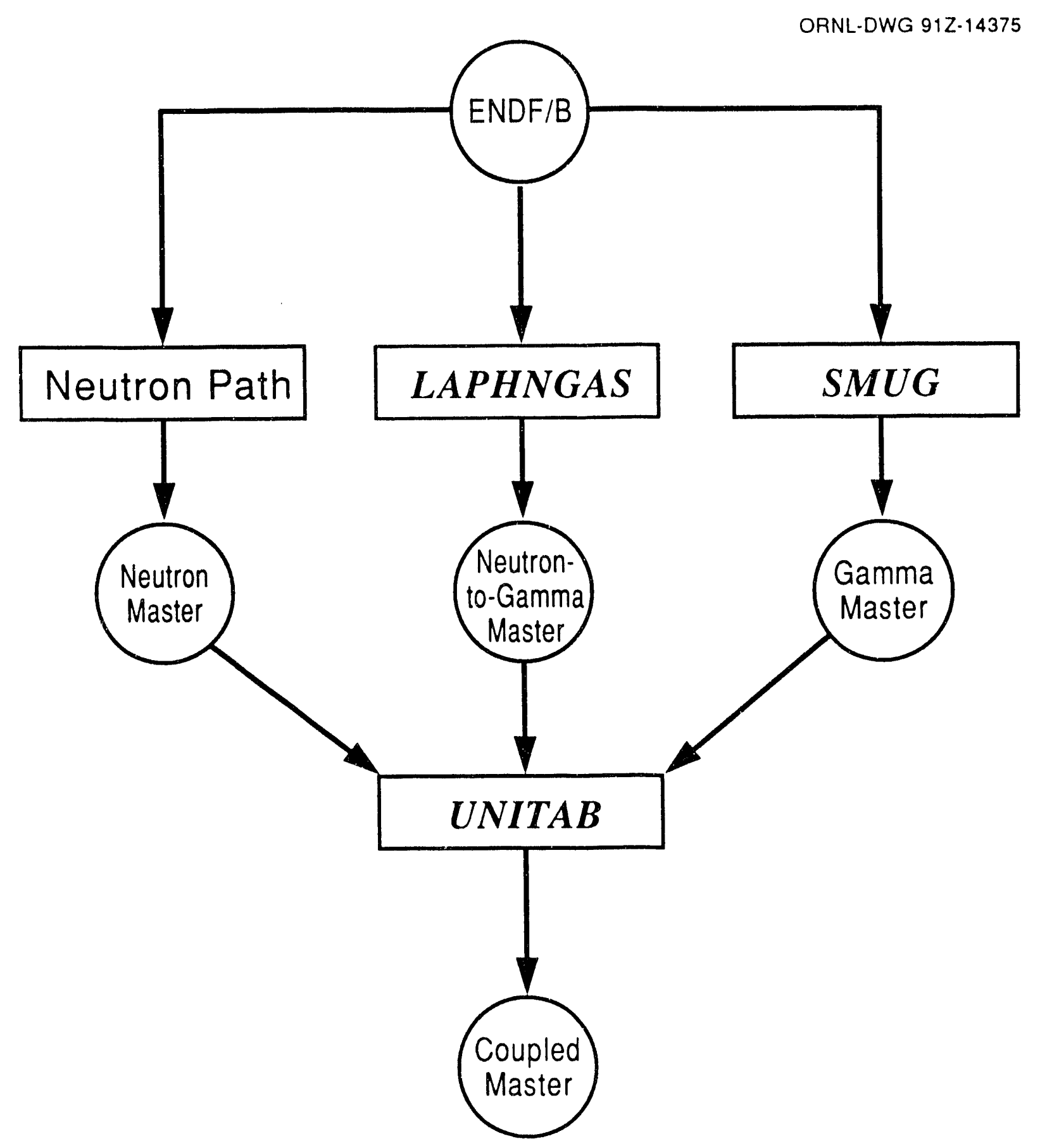

Fig. 6.SAMPLE PROBLEMS.5. Procedure to produce a coupled neutron-gamma library. 


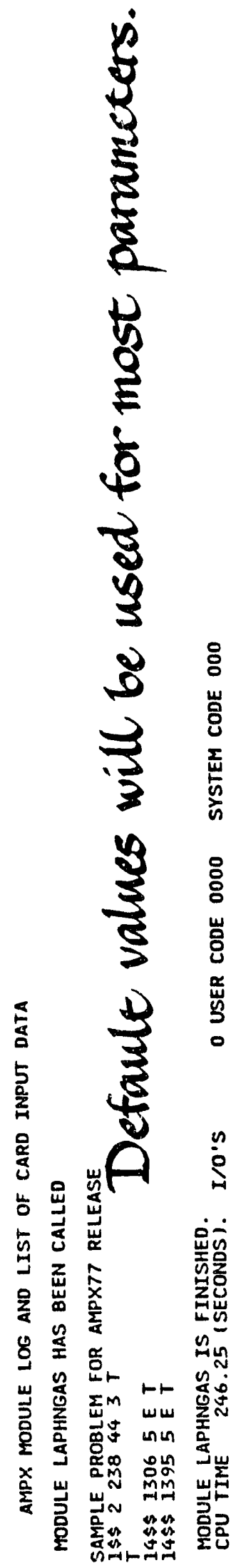

SAMPLE PROBLEMS 


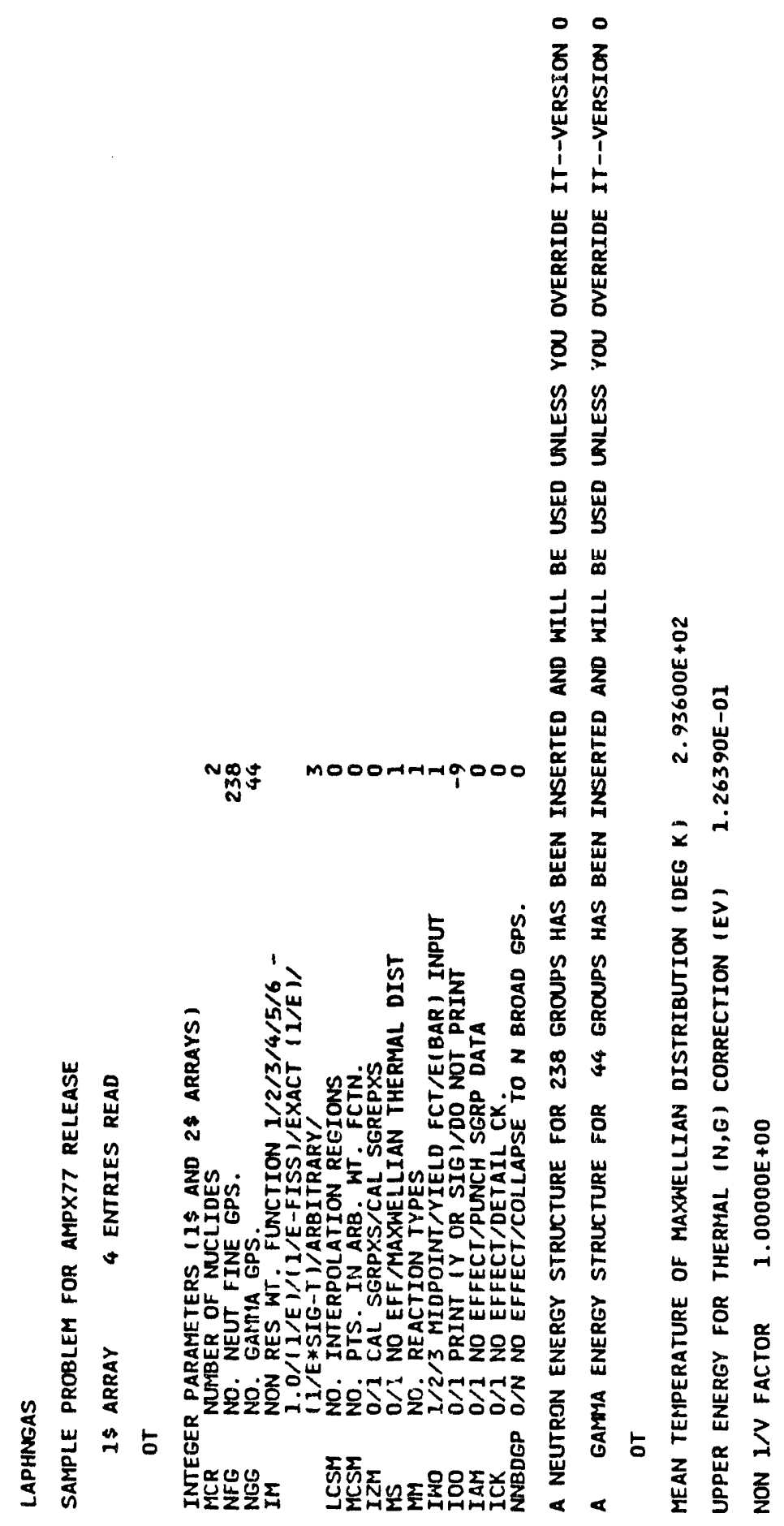




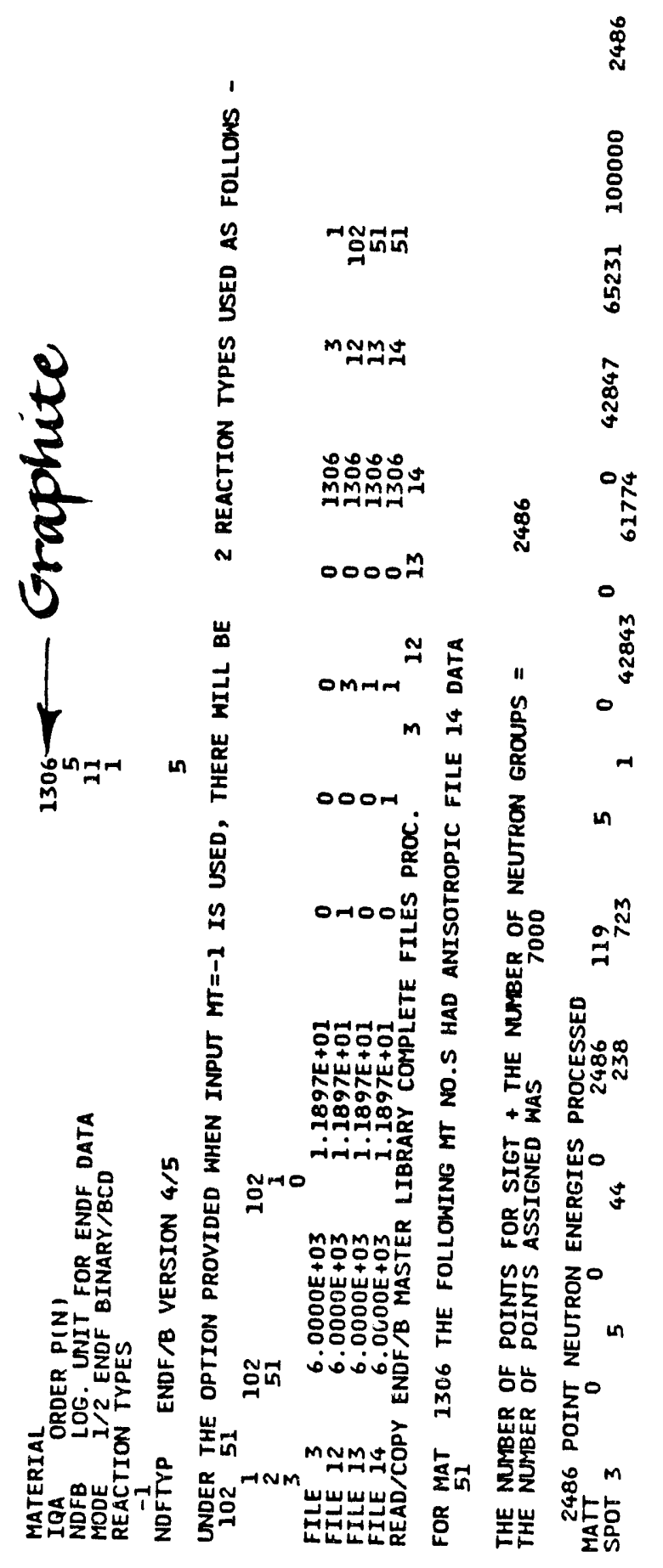




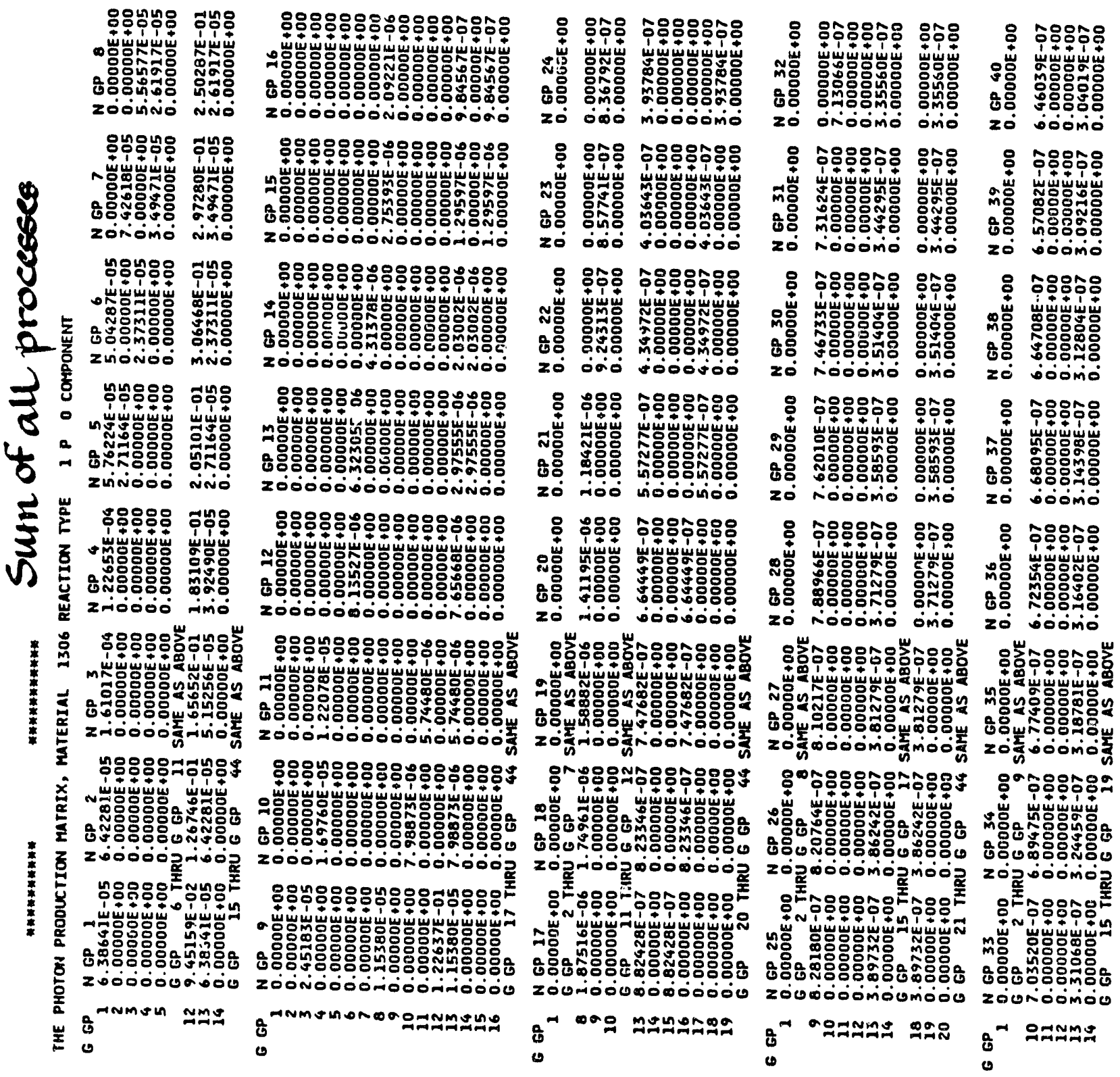




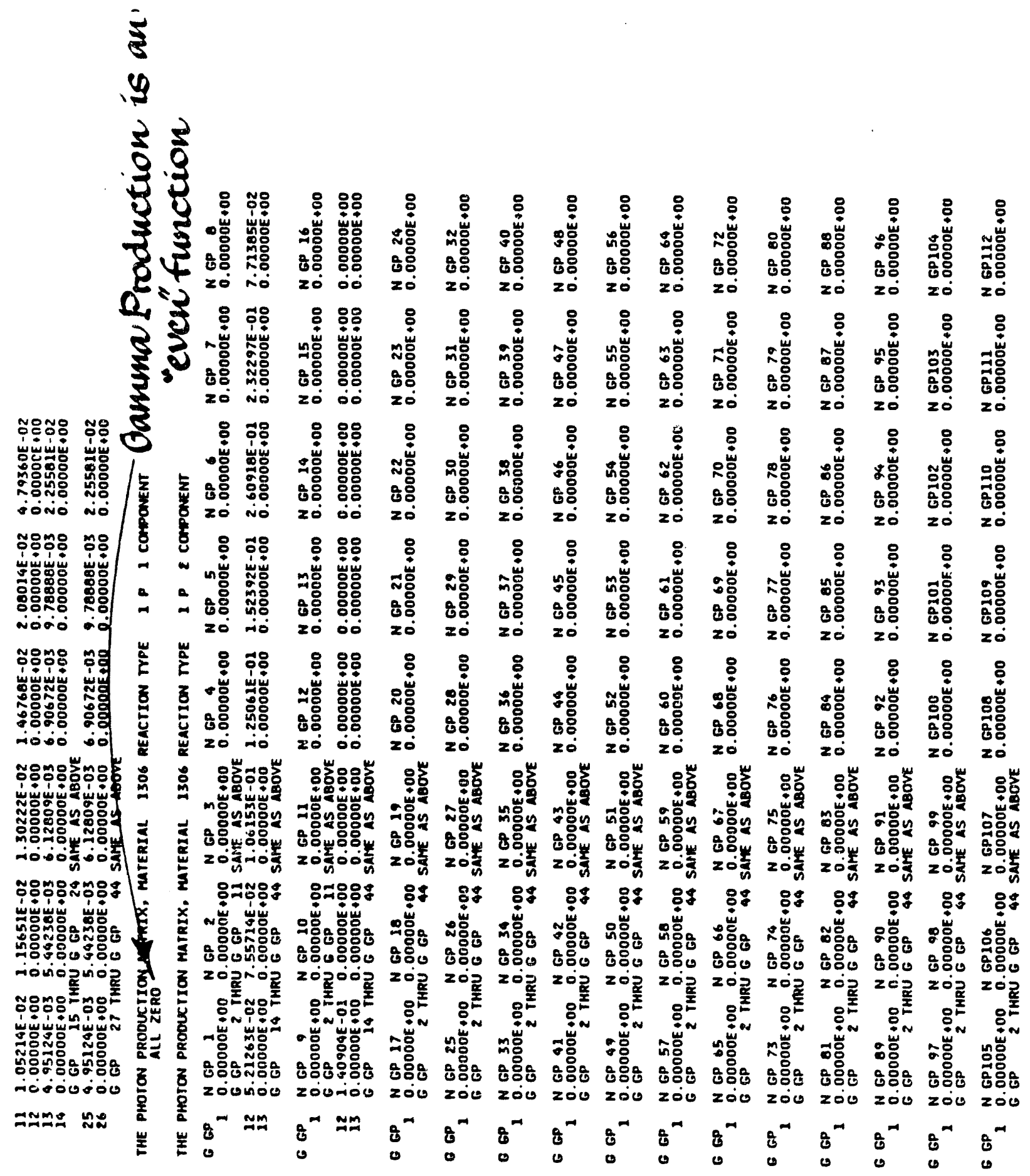




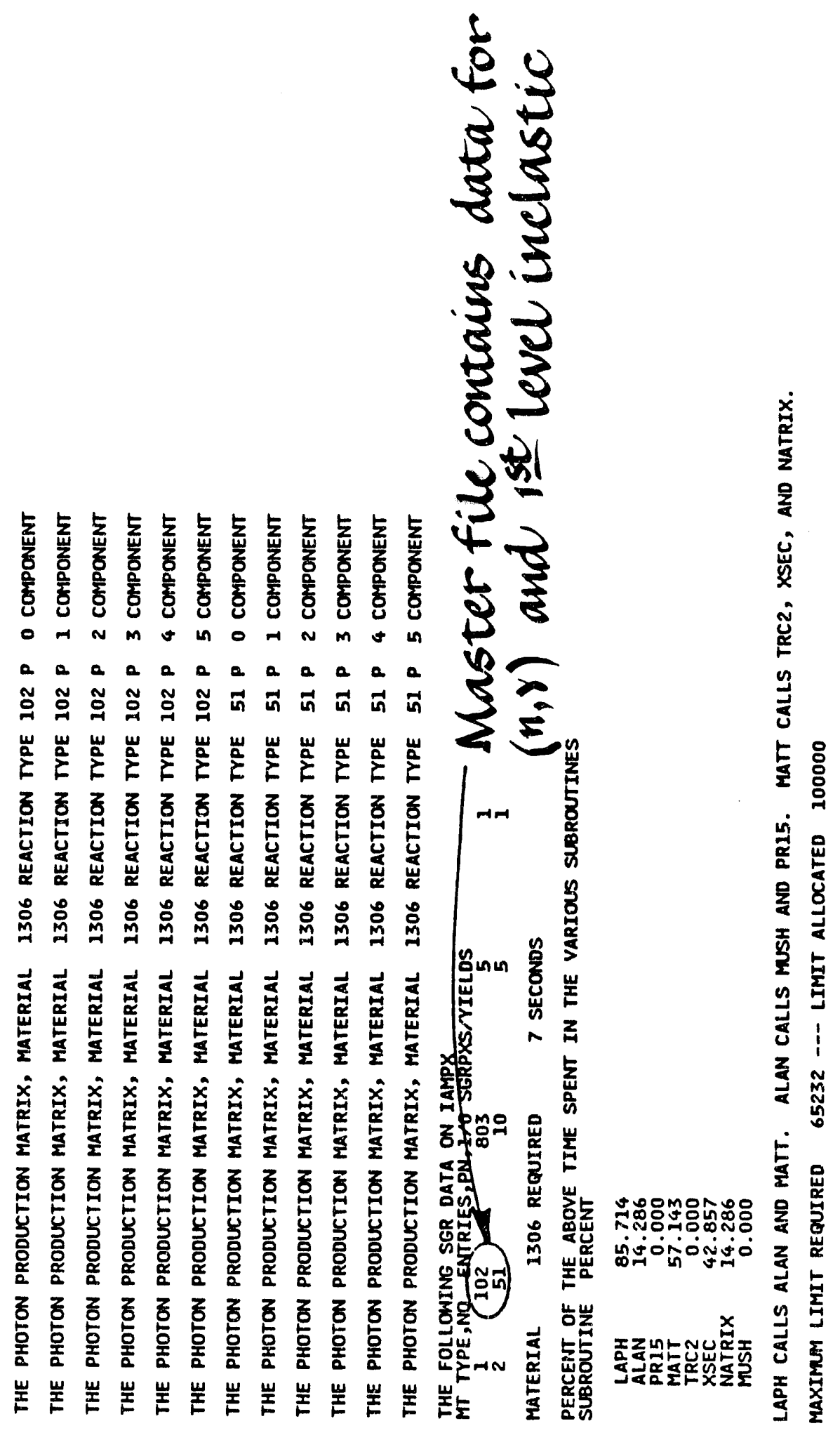




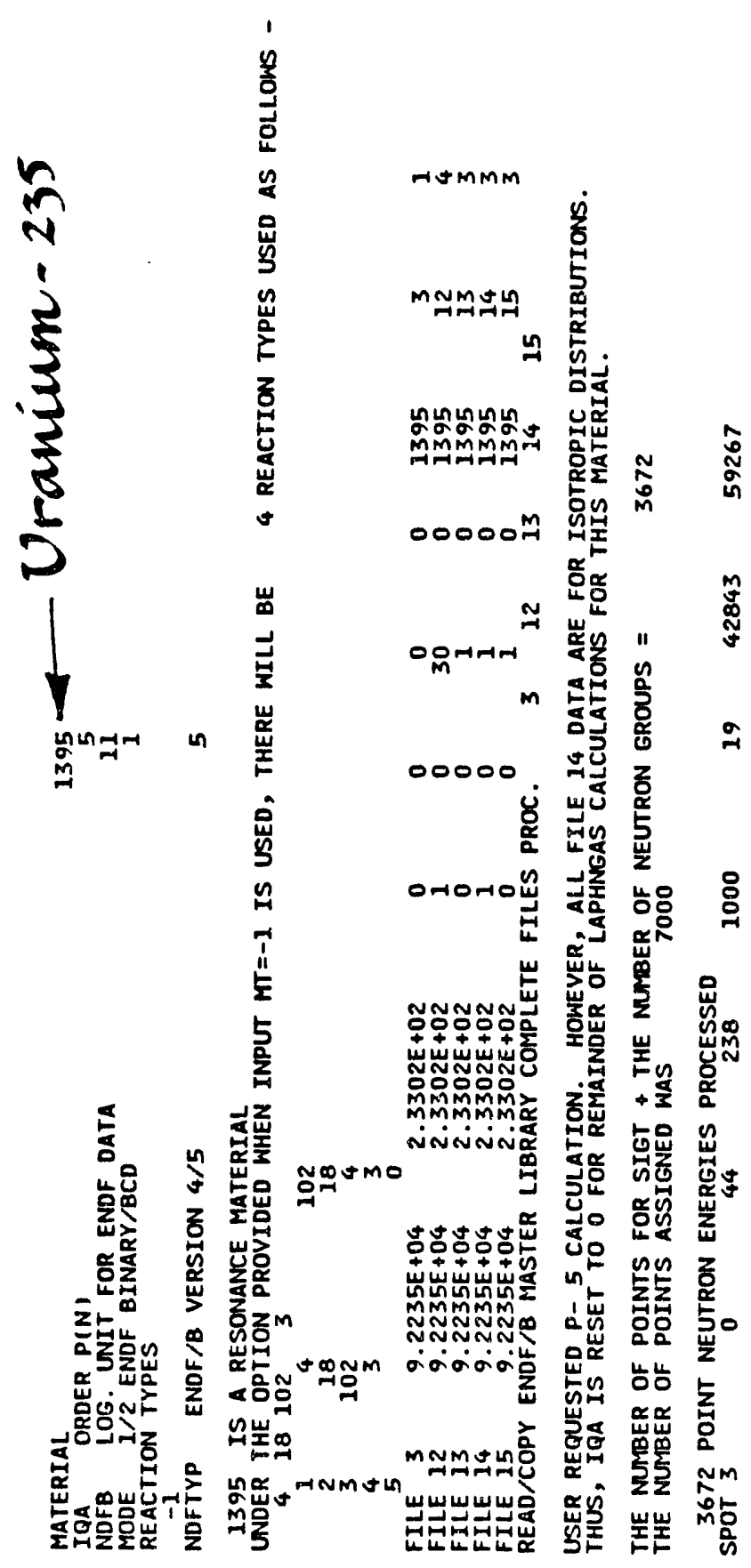




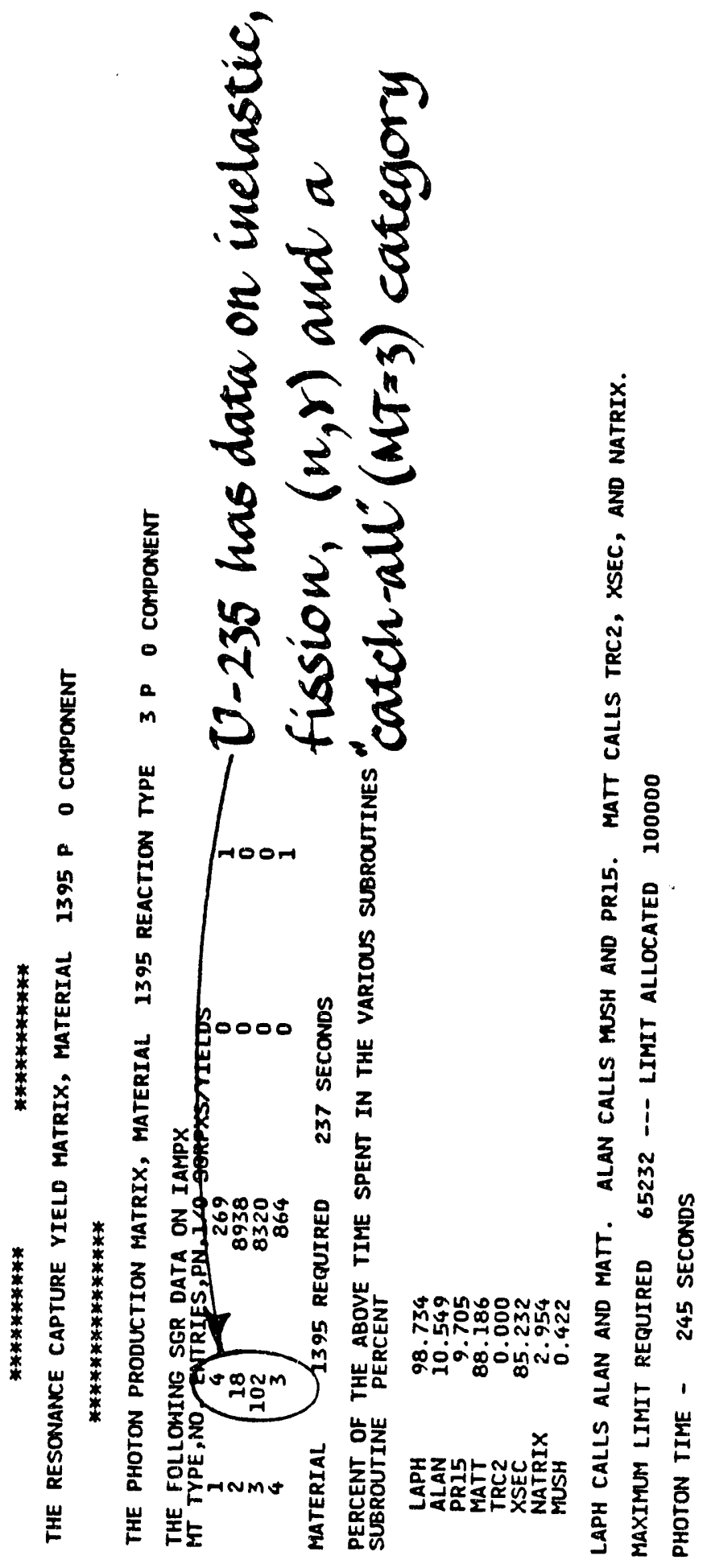


The input for Subtask 2 is shown on the following pages, followed by portions of the output. 


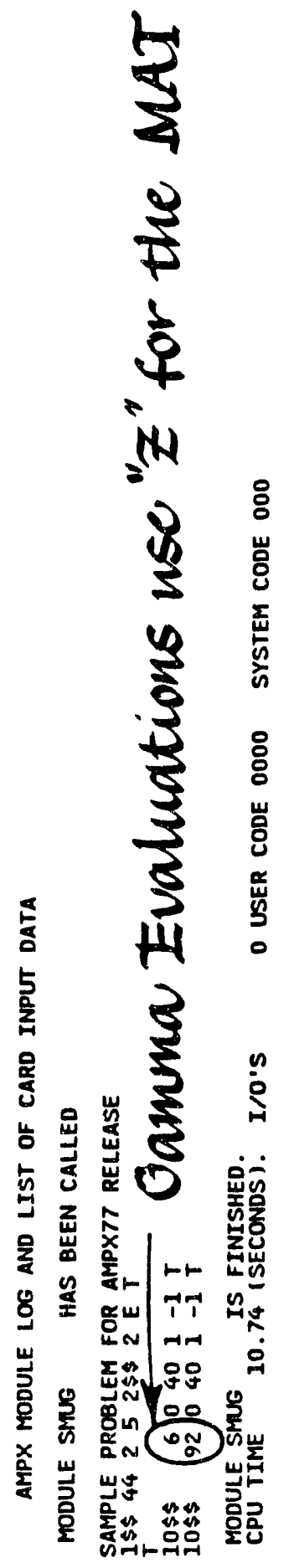

SAMPLE PROBLEMS 


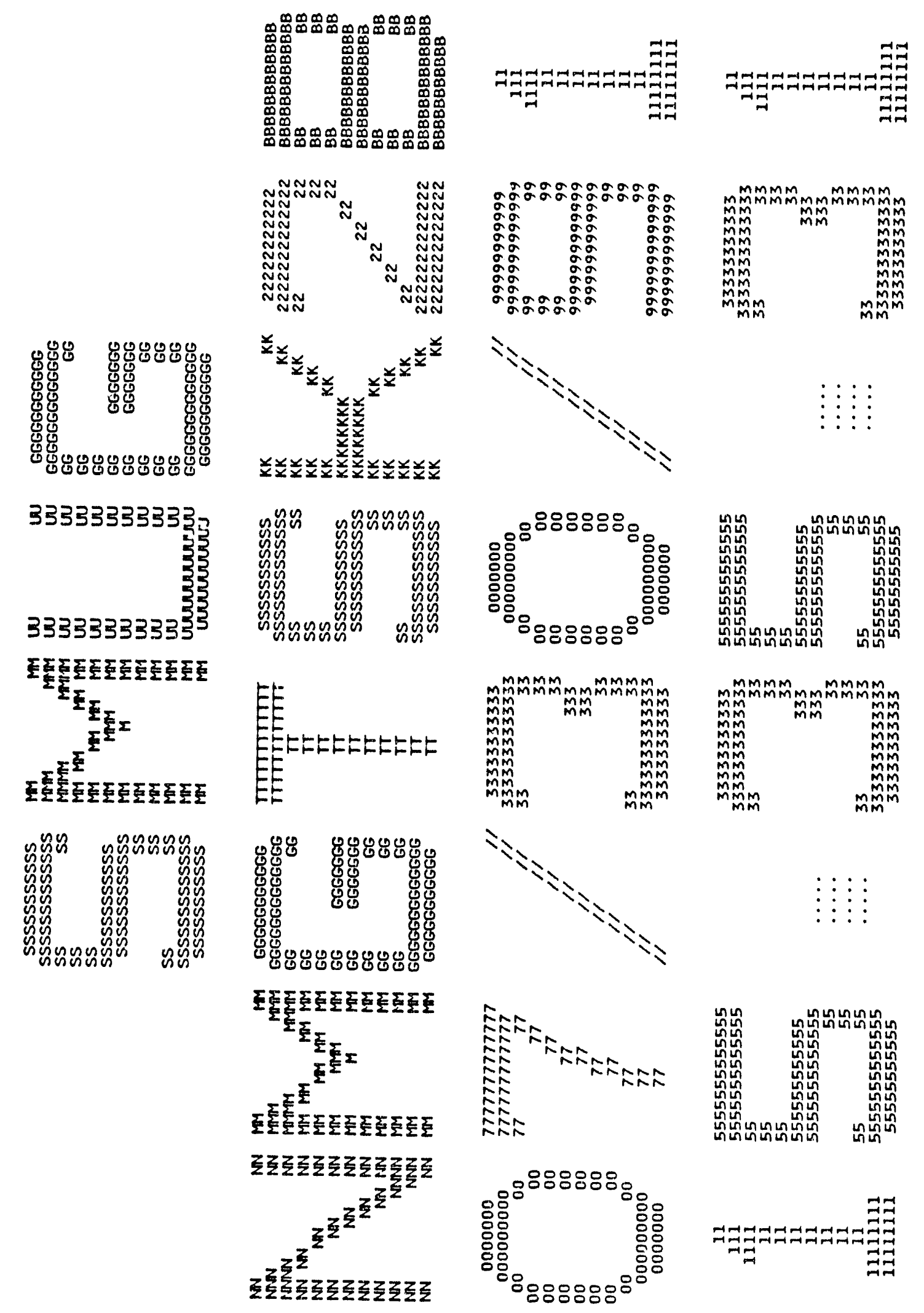




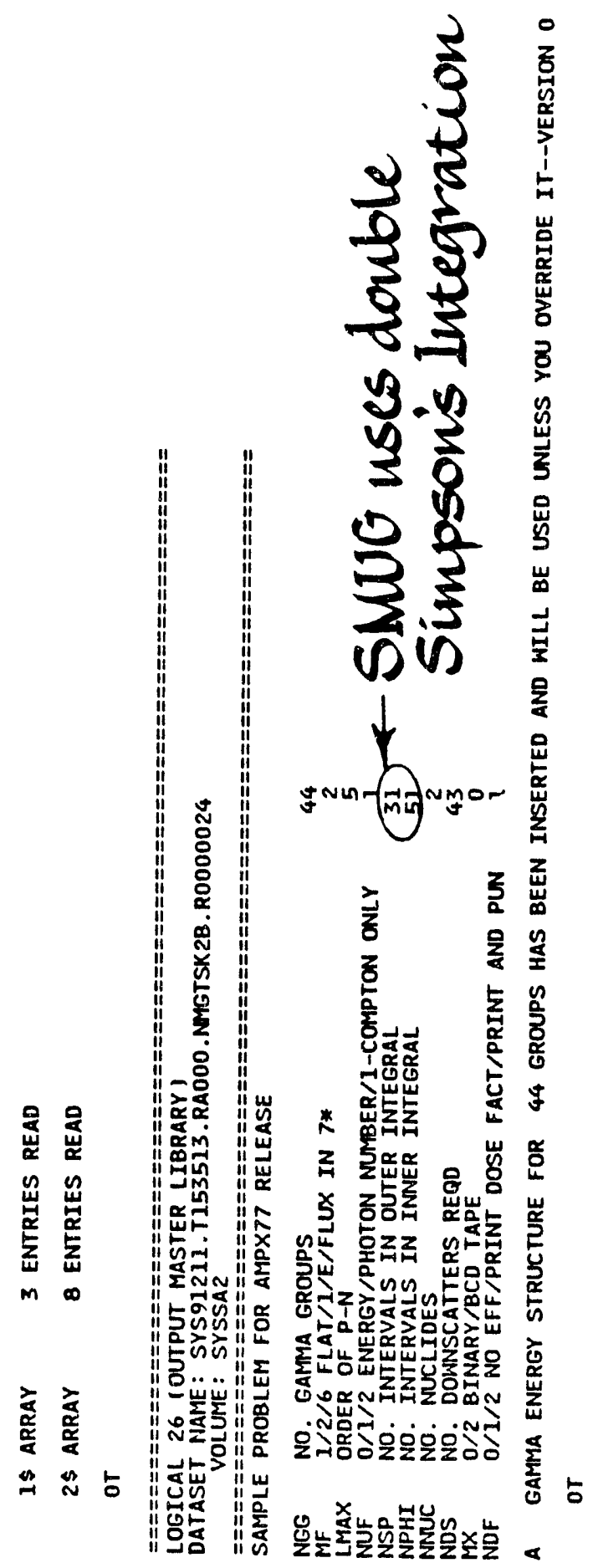

SAMPLE PROBLEMS 

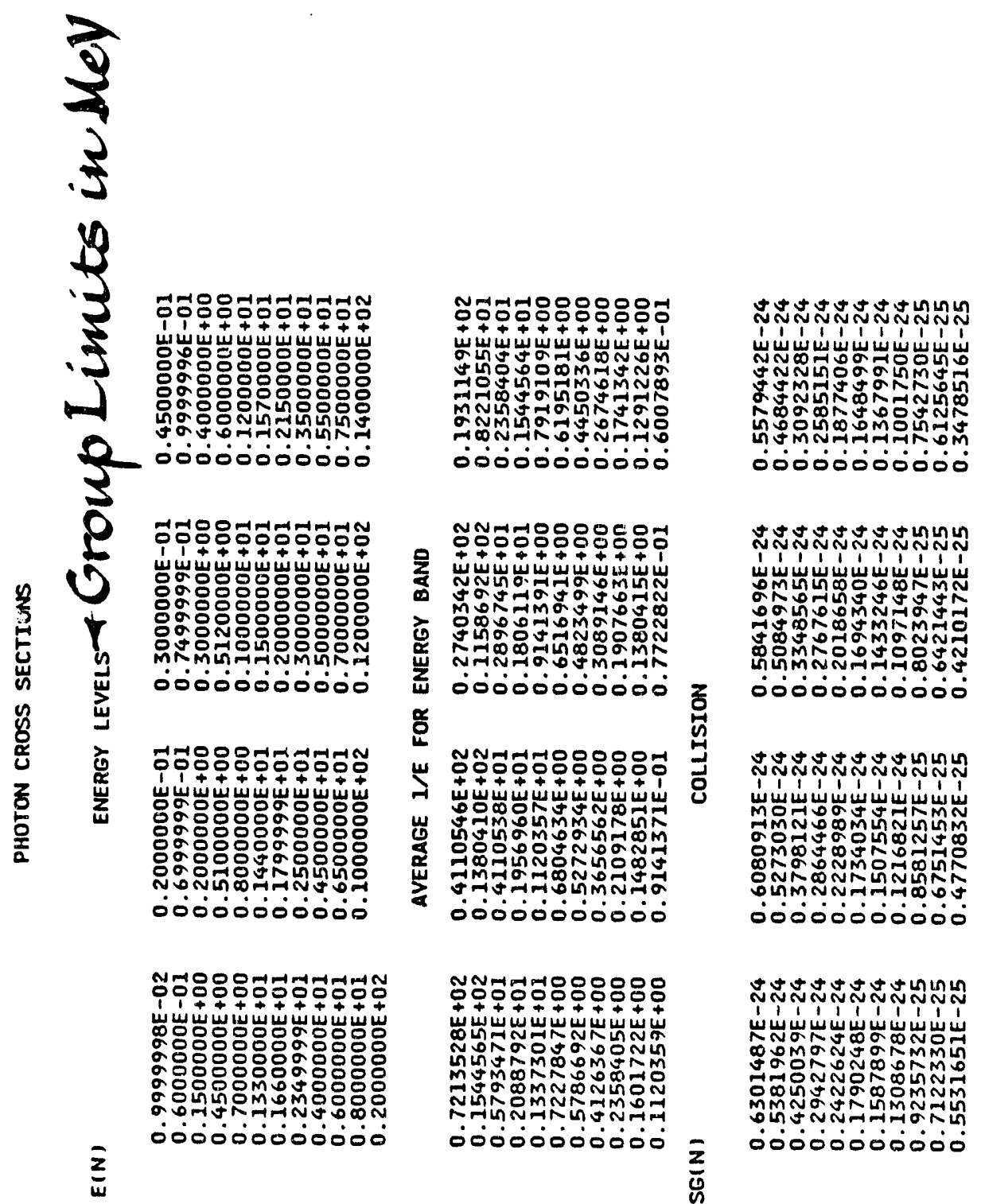

\%

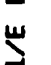
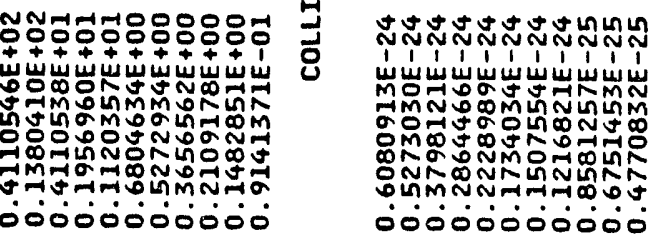

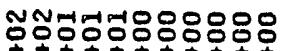

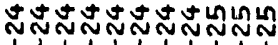

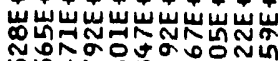

NoNo

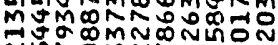

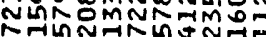

00000000000

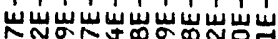

DoMaNs TNMML

TONNONDNN PMN N 00000000000 \& 

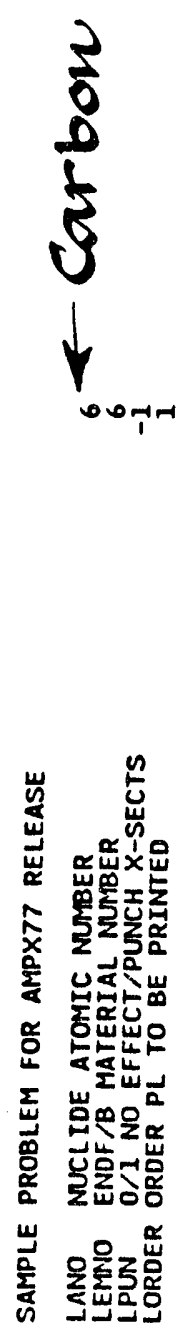

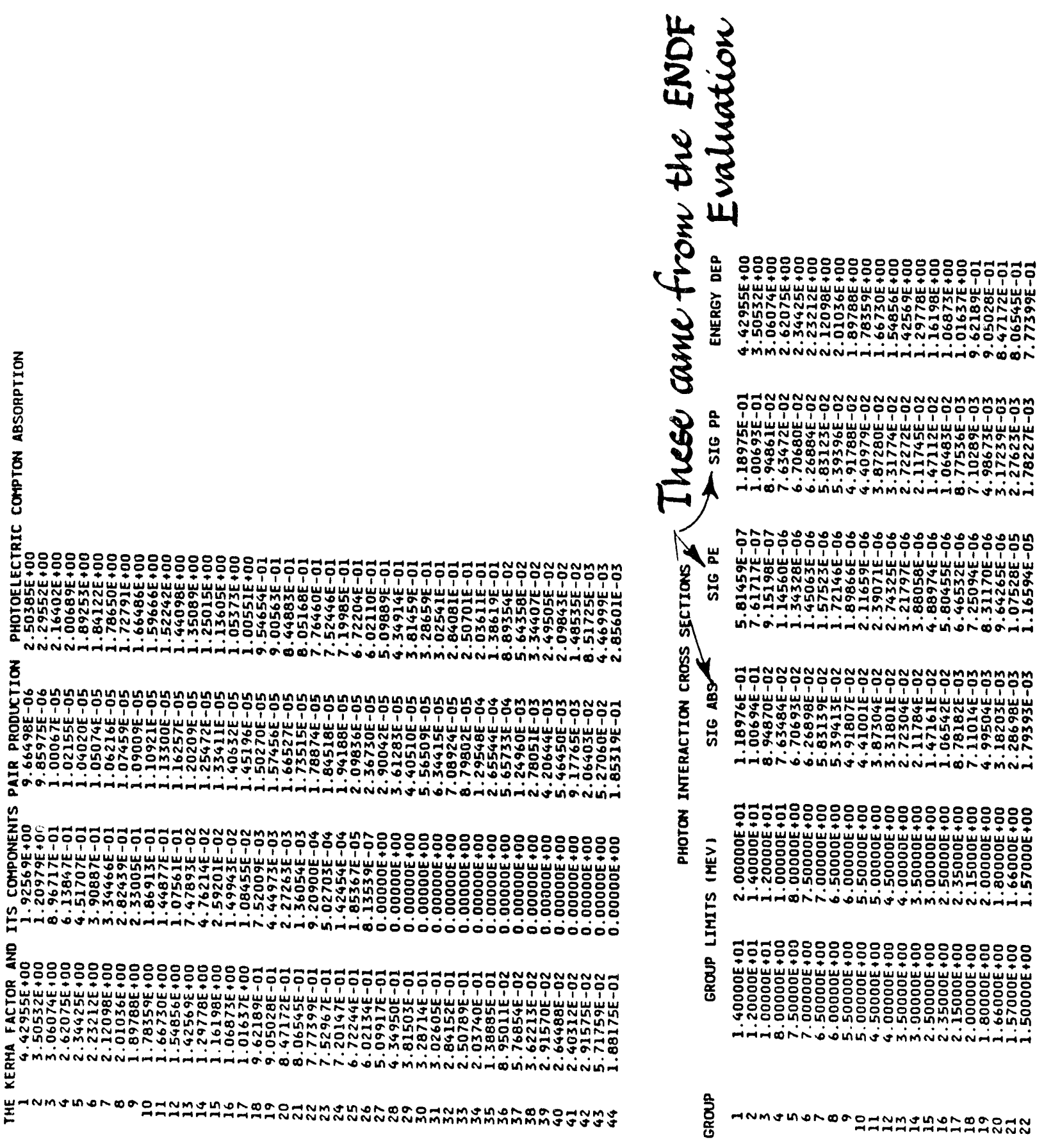

\section{SAMPLE PROBLEMS}



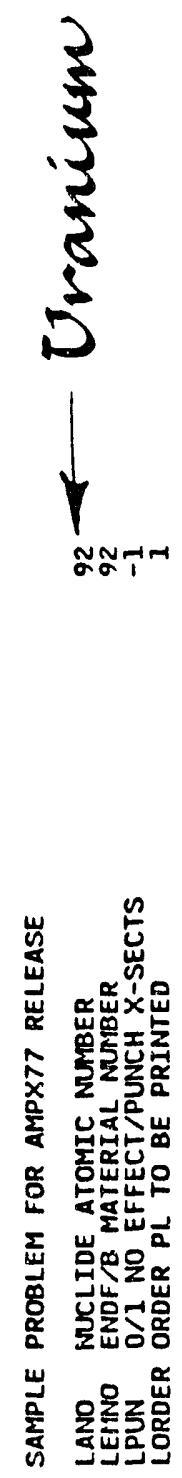

SAMPLE PROBLEMS 

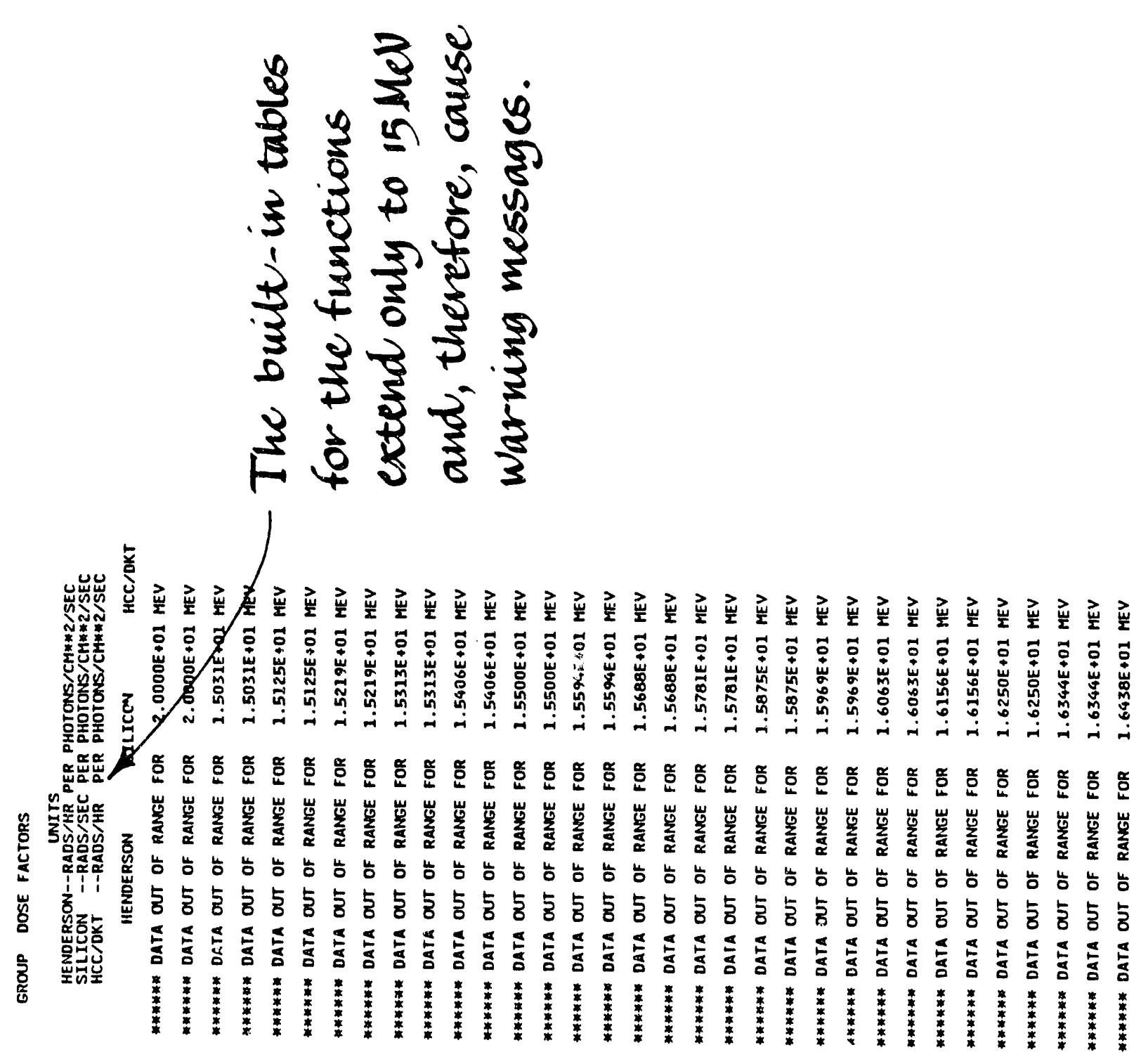

SAMPLE PROBLEMS 
The input for Subtask 3 is shown on the following pages, followed by portions of the output. 


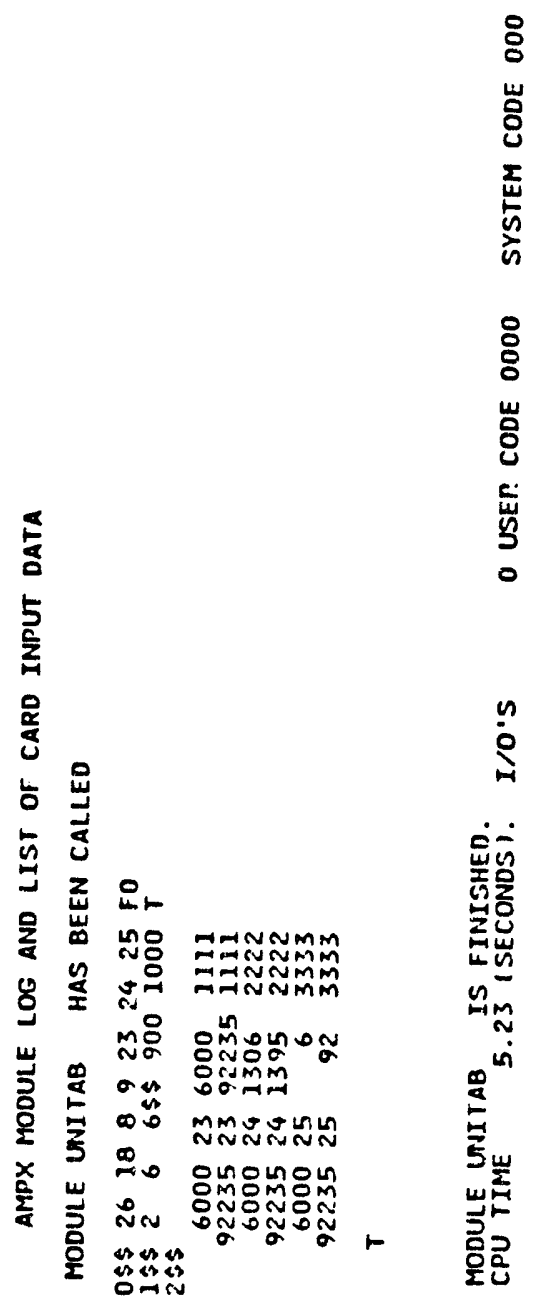



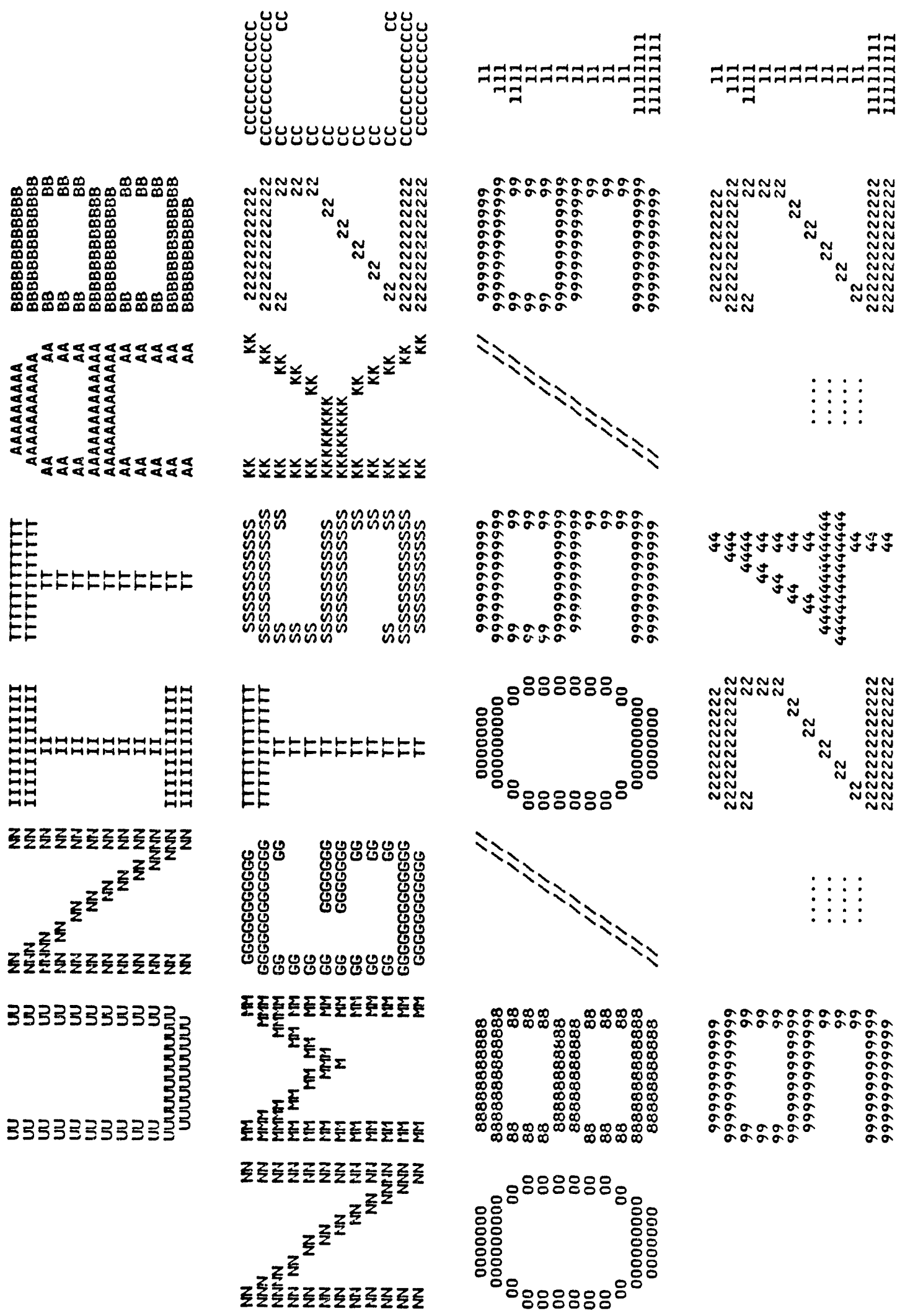


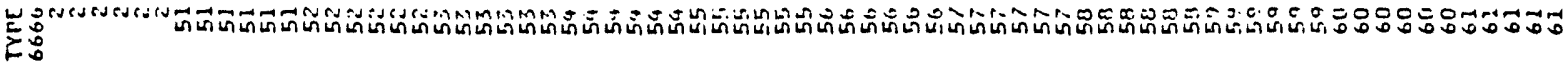
QำM \&

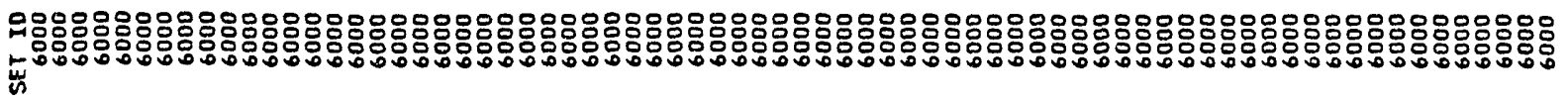

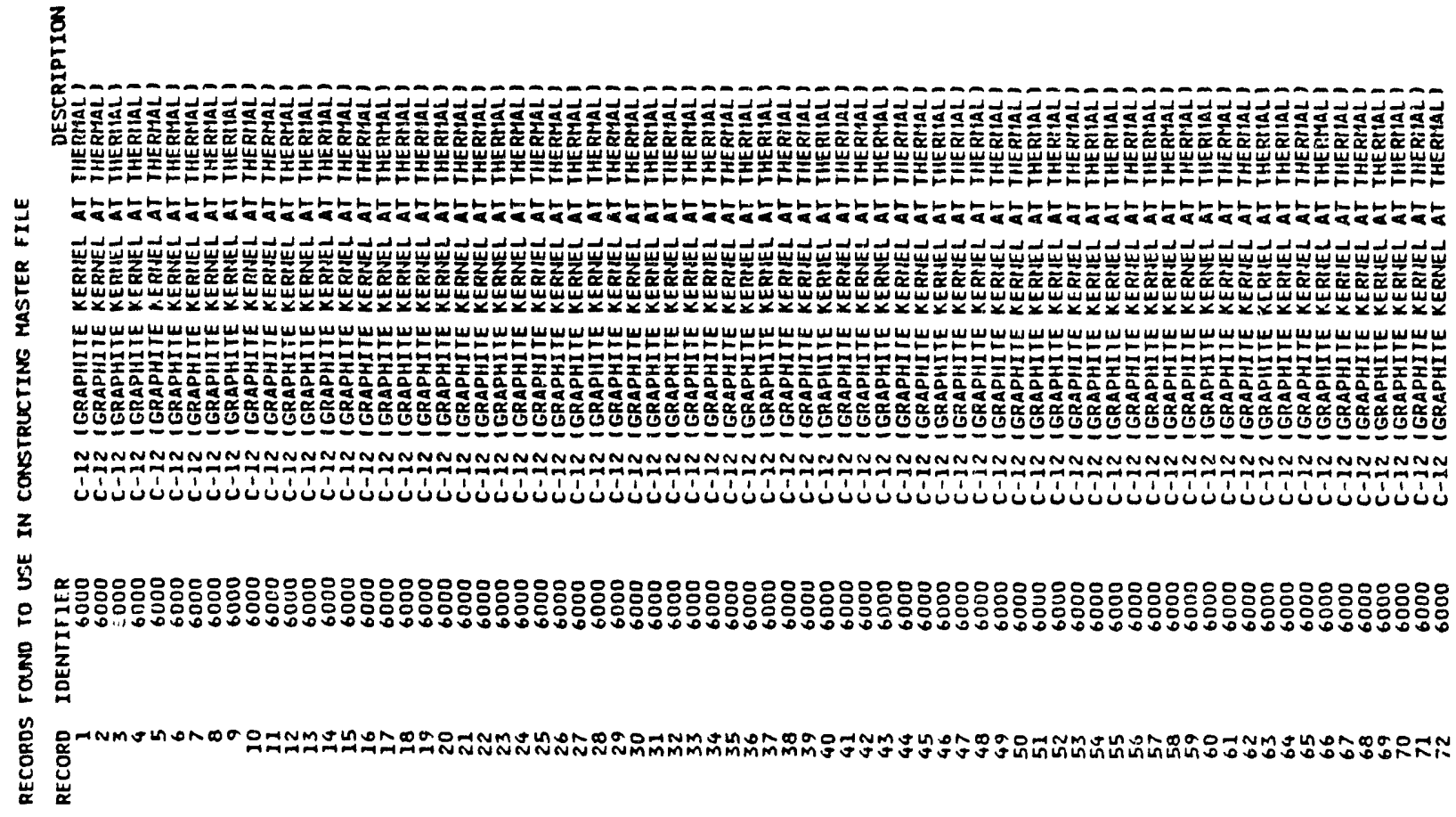




\section{SAMPLE PROBLEMS.3 PERFORM A RESONANCE SELF- SHIELDING CALCULATION}

With the limited palate of nuclides processed in Task 1 (graphite and ${ }^{235} \mathrm{U}$ ), we will assume a reactor core consisting of $1-\mathrm{cm}$-diam. ${ }^{235} \mathrm{U}$ rods inserted in a graphite moderator and arranged in a square lattice with a pitch of $2 \mathrm{~cm}$ (see Fig. 6.SAMPLE PROBLEMS.6).

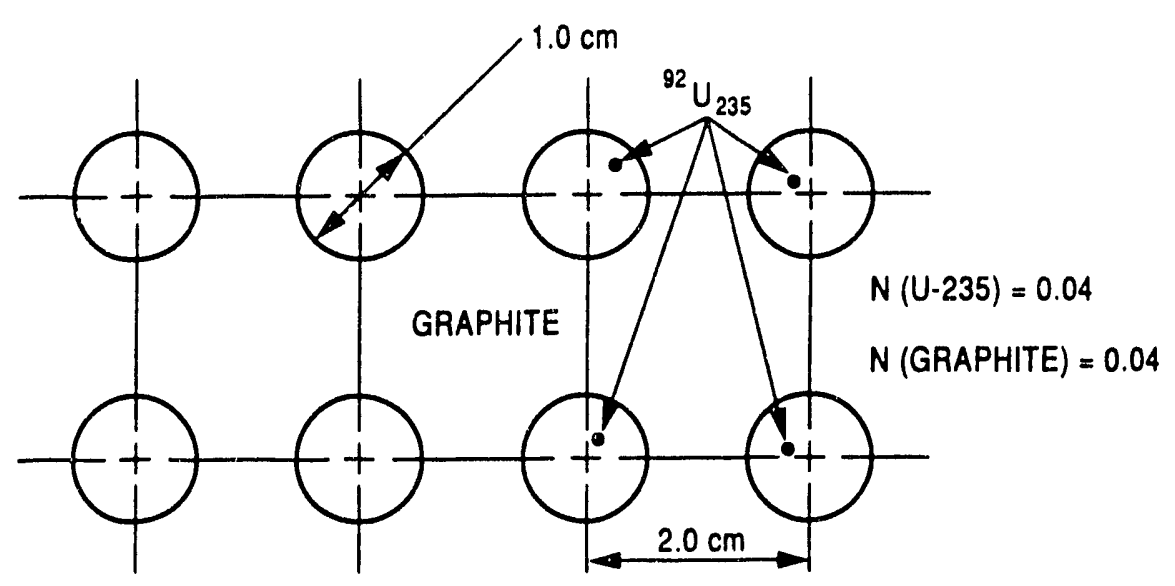

Fig. 6.SAMPLE PROBLEMS.6. Hypothetical reactor lattice.

The first step is to determine a representative "Wigner-Seitz" cell in cylindrical geometry which preserves the volume of a square cell from the lattice above, shown in Fig. 6.SAMPLE PROBLEMS.7 below.
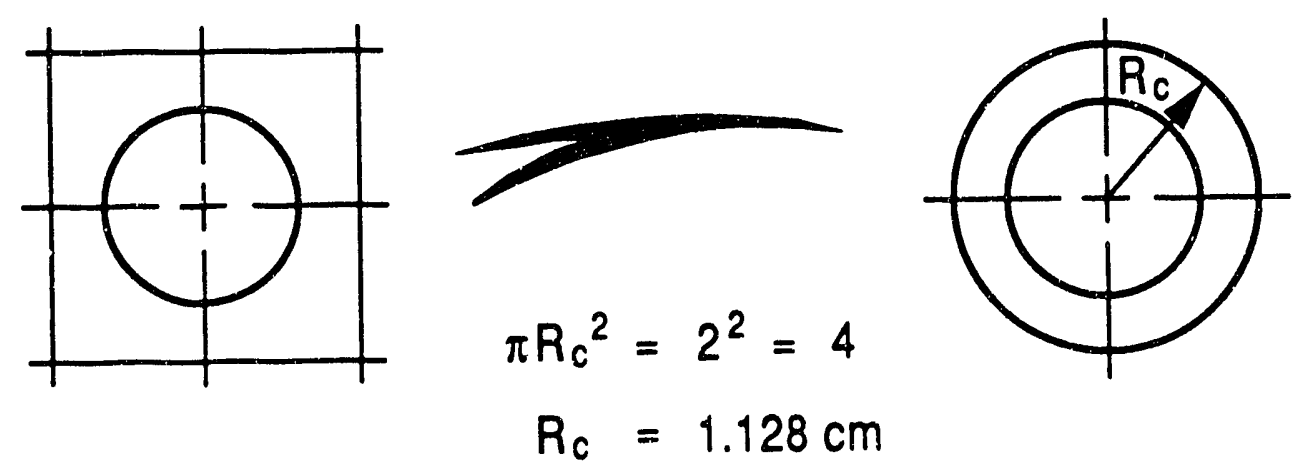

Fig. 6.SAMPLE PROBLEMS.7. Wigner-Seitz cell approximation. 
This is the geometry for our resonance calculation.

This section will show how the resonance calculation could be mocked for three situations:

a. the integral transport theory code, ROLAIDS,

b. the integral transport theory code, NITAWL, and

c. the Bondarenko method code, BONAMI.

\section{SAMPLE PROBLEMS.3.1 Sample Resonance Calculation by ROLAIDS}

To perform this calculation, we need first to obtain a file with point cross sections for carbon and ${ }^{235} \mathrm{U}$.

A possible procedure is shown in Fig. 6.SAMPLE PROBLEMS.8. Here, POLIDENT is used to generate point cross sections for ${ }^{235} \mathrm{U}$ and to pick up those for carbon. These are input to ROLAIDS, which performs the resonance calculation and group-averages the data to produce self-shielded cross sections on a file that is passed to CLAROL, which corrects the original master library.

\section{SAMPLE PROBLEMS.3.2 Sample Resonance Calculation by NITAWL}

In this case, all that is required is to prepare the input data for NITAWL. Among other things, this requires that one obtain a value for a Dancoff factor. This can be obtained by accessing tables from ANL-5800 (Fast Reactor Constants), where we find a factor for two isolated $1-\mathrm{cm}$ rods separated by $2 \mathrm{~cm}$ in graphite. This value is 0.0035 (based on $\lambda=\frac{1}{0.04(4.8)}=5.2, \rho=0.5, d=2.0$ ). Since each rod sees four rods at this spacing, we multiply the value by 4 . There are four rods each rod sees at a spacing of $2 \sqrt{2} \mathrm{~cm}$. The Dancoff value for this system is essentially zero. Therefore, we use 0.014 for our Dancoff value.

A procedure to use NITAWL is shown in Fig. 6.SAMPLE PROBLEMS.9.

\section{SAMPLE PROBLEMS.3.3 Sample Resonance Calculation by BONAMI}

In the sample runs for Task 1, Bondarenko factors were produced for ${ }^{235} \mathrm{U}$ for the unresolved resonance range. BONAMI can be used to self-shield these factors. A procedure is shown in Fig. 6.SAMPLE PROBLEMS.10. 


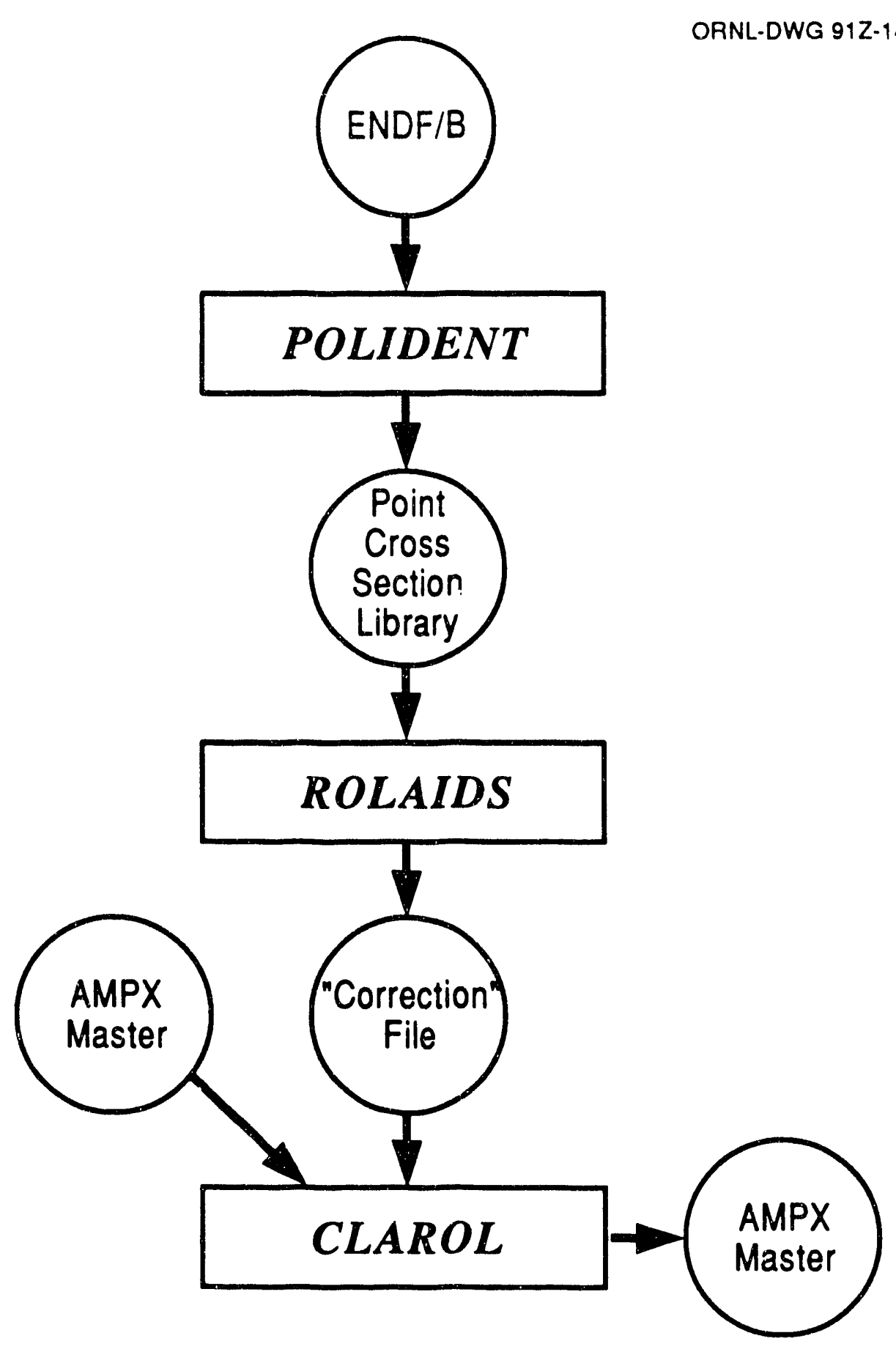

Fig. 6.SAMPLE PROBLEMS.8. Procedure to use ROLAIDS for resonance shielding. 


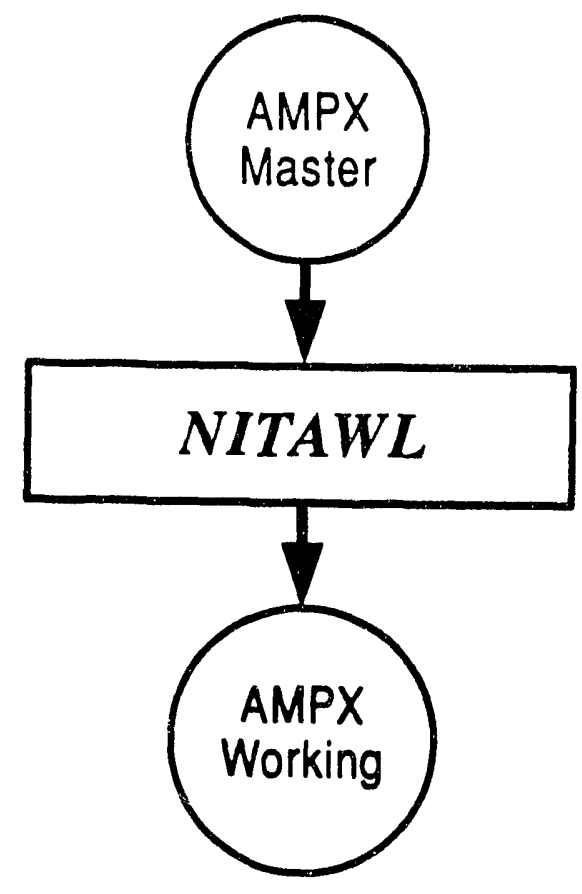

ORNL-DWG 91Z-14379

Fig. 6.SAMPLE PROBLEMS.9. Procedure to use NITAWL for resonance self-shielding.

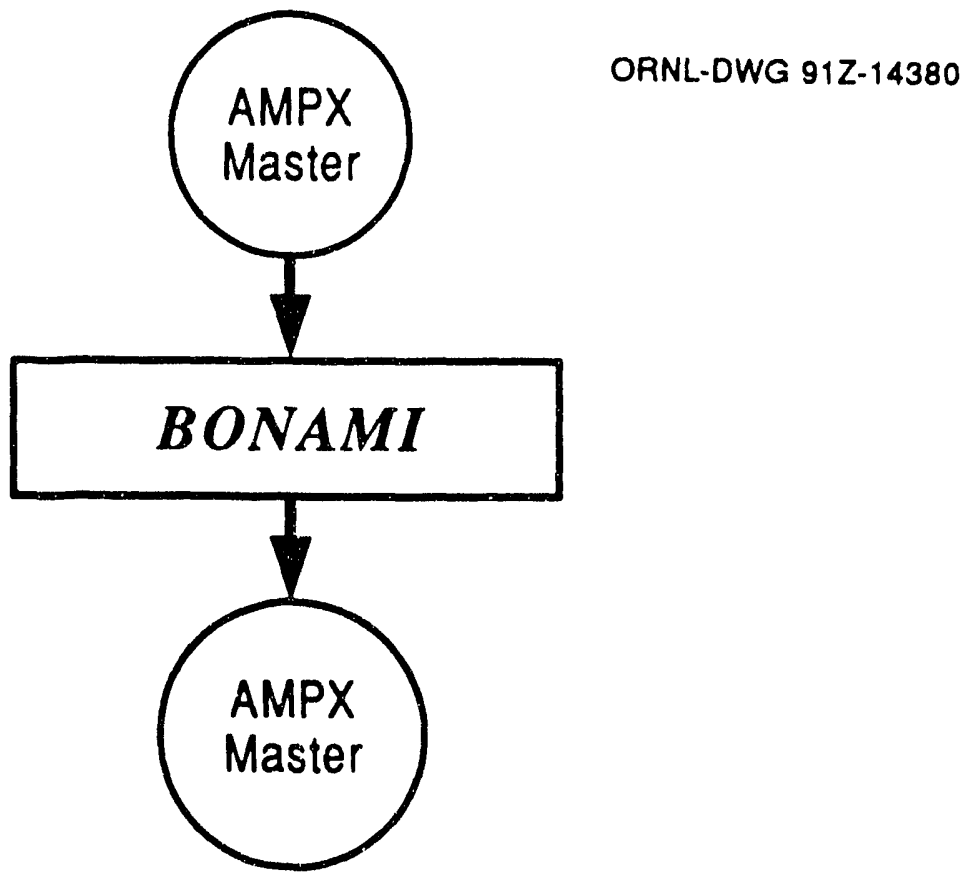

Fig. 6.SAMPLE PROBLEMS.10. Procedure to use BONAMI for resonance self-shielding. 
The input for the cases is given below, followed by portions of the output from the various modules.

AMPX MODULE LOG AND LIST OF CARD INPUT DATA

MODULE POLIOENT HAS BEEN CALLED

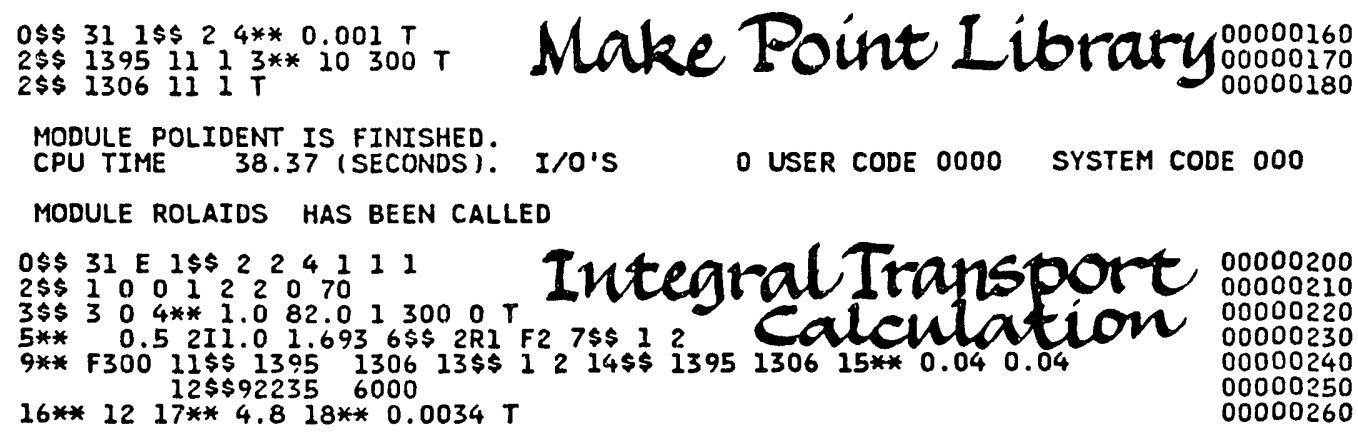

MODULE ROLAIDS IS FINISHED.
CPU TIME 60.99 (SECONDS): I/O'S

MODULE CLAROL HAS BEEN CALLED

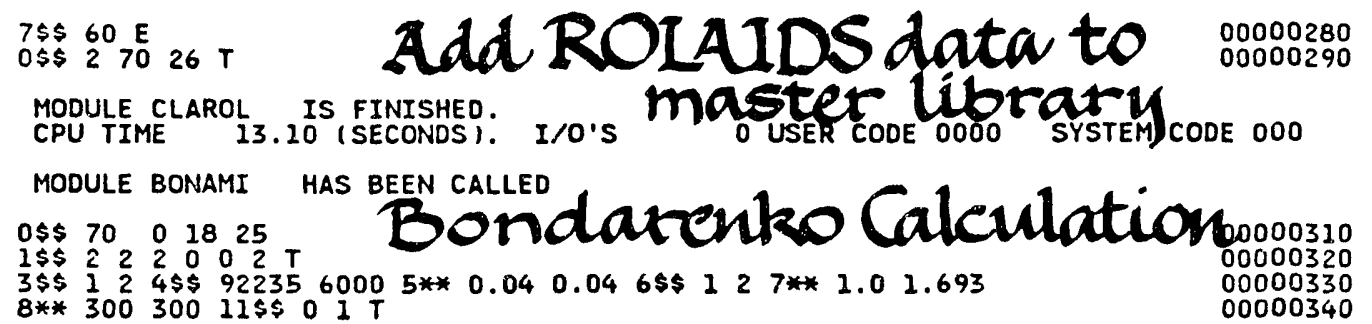

MODULE BONAMI IS FINISHED.

CPU TIME 10.03 (SECONDS). I/0'S O USER CODE 0000 SYSTEM CODE 000

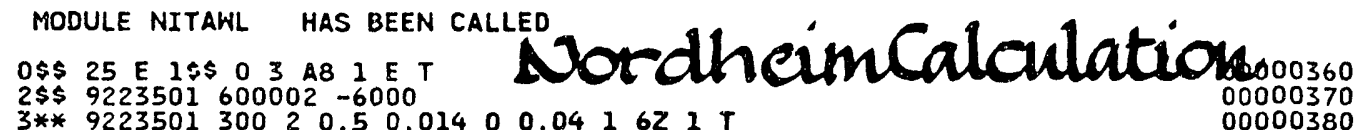

$3 * * 9223501300 \quad 20.50 .01400 .041621 \%$

MODULE NITAWL IS FINISHED.

CPU TIME 15.24 (SECONDS): I/O'S O USER CODE 0000 SYSTEM CODE OOO 

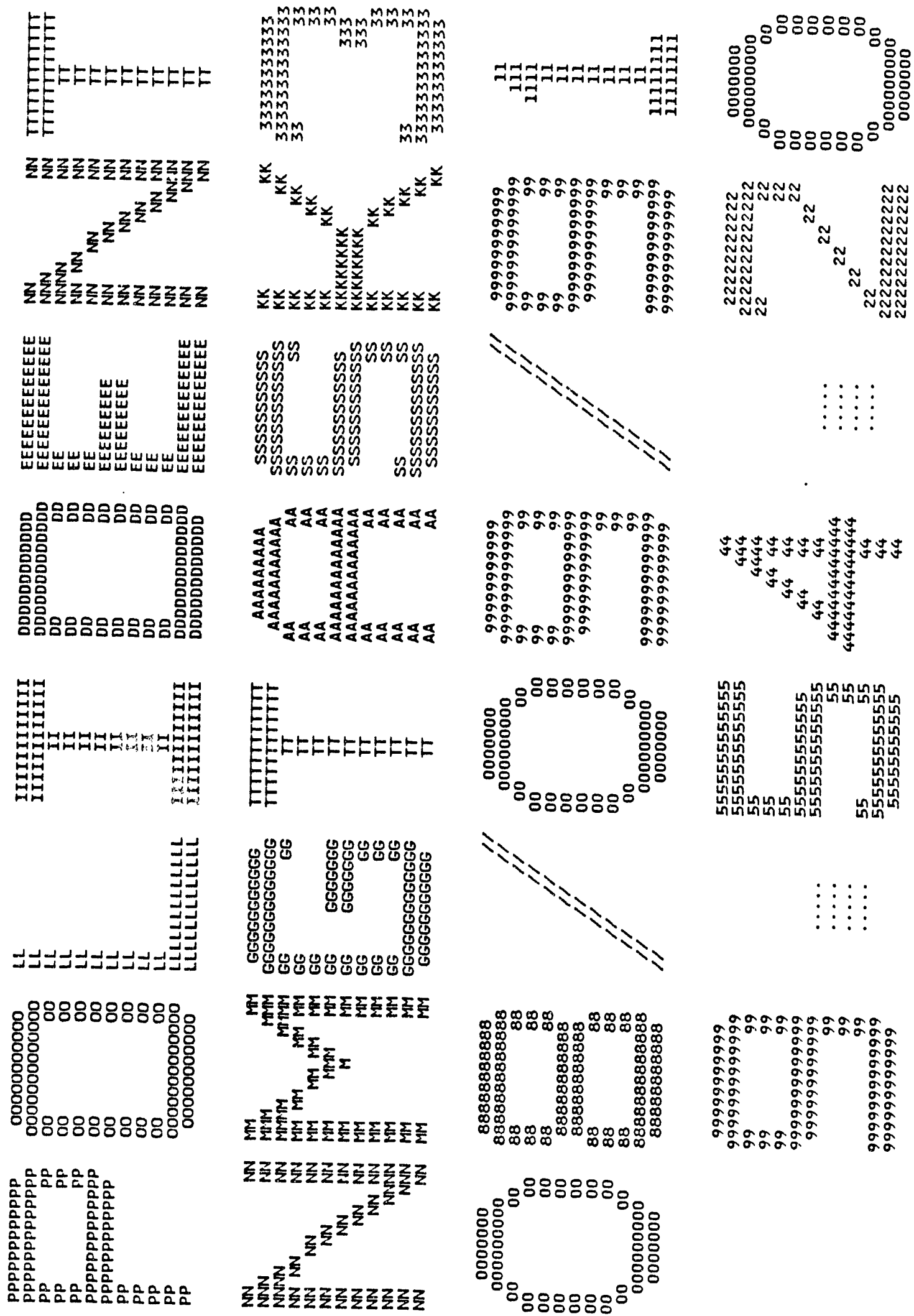

SAMPLE PROBLEMS 


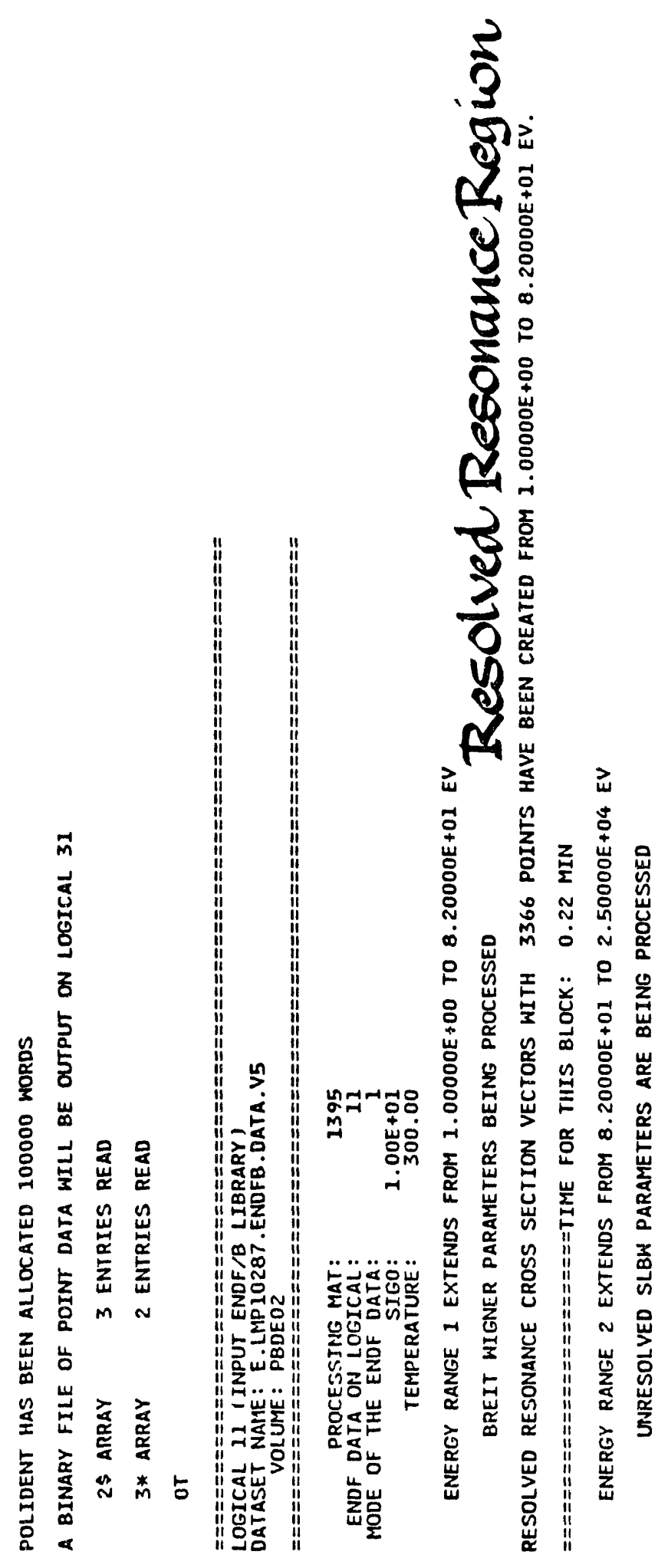



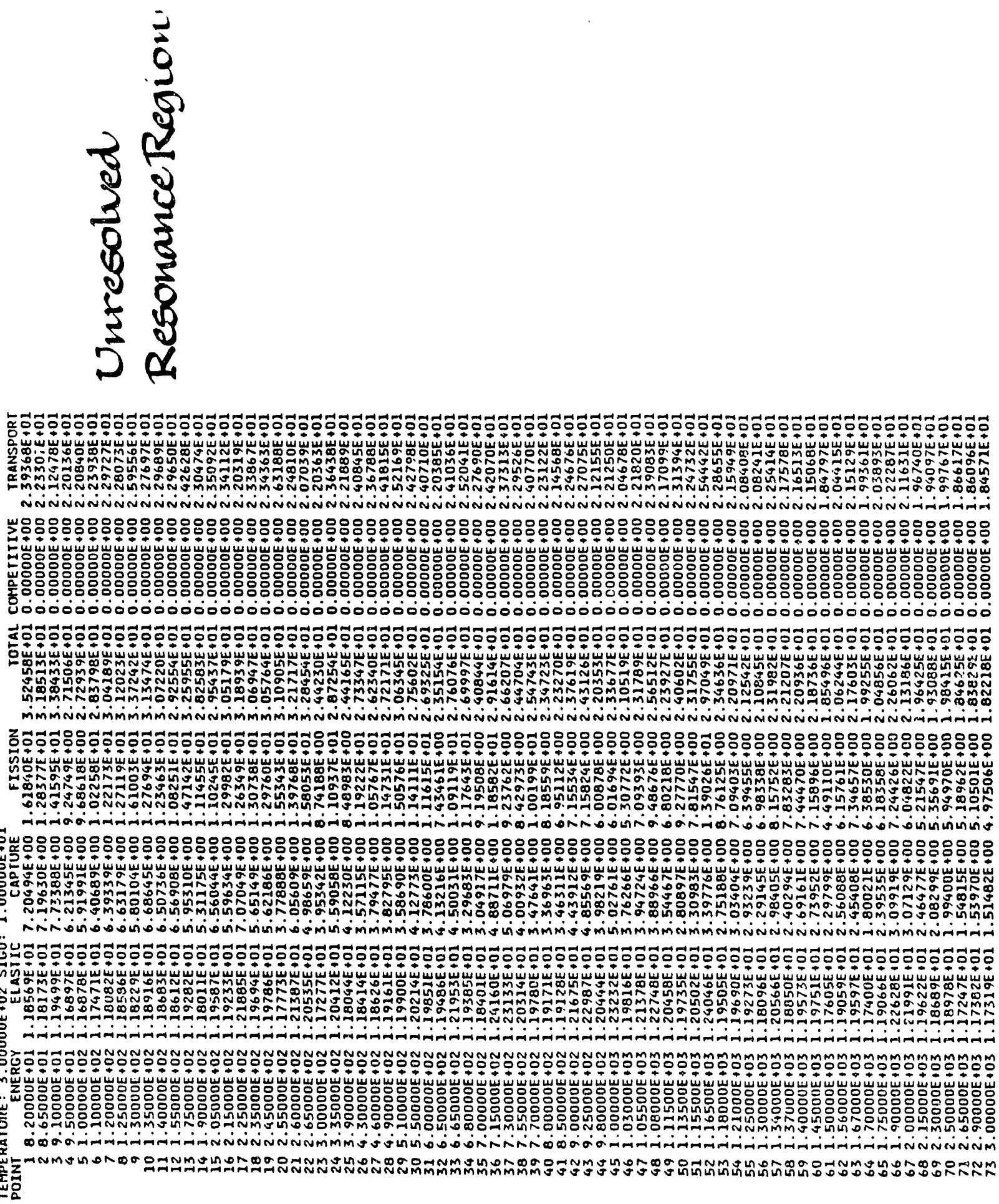

\section{SAMPLE PROBLEMS}




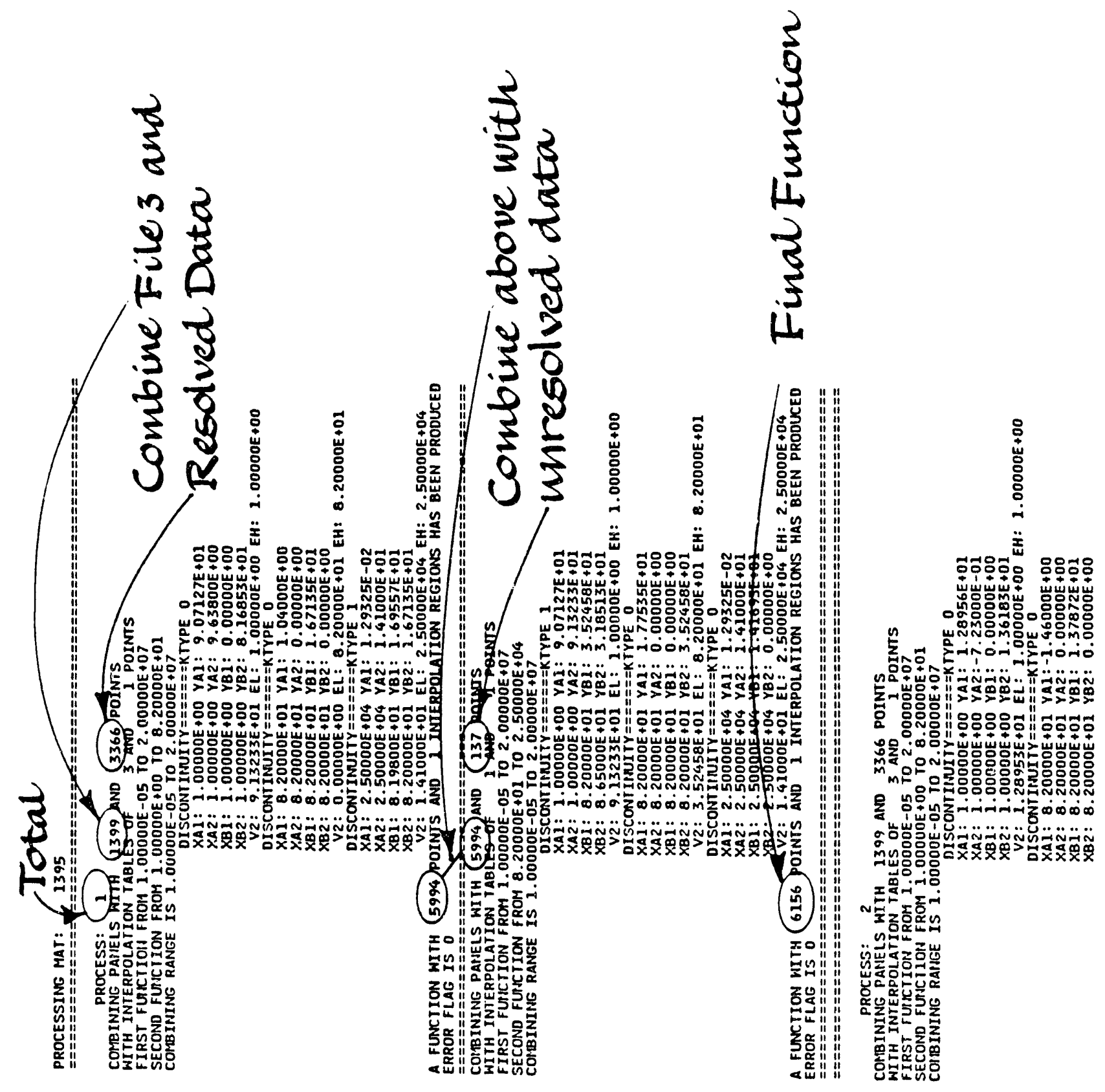




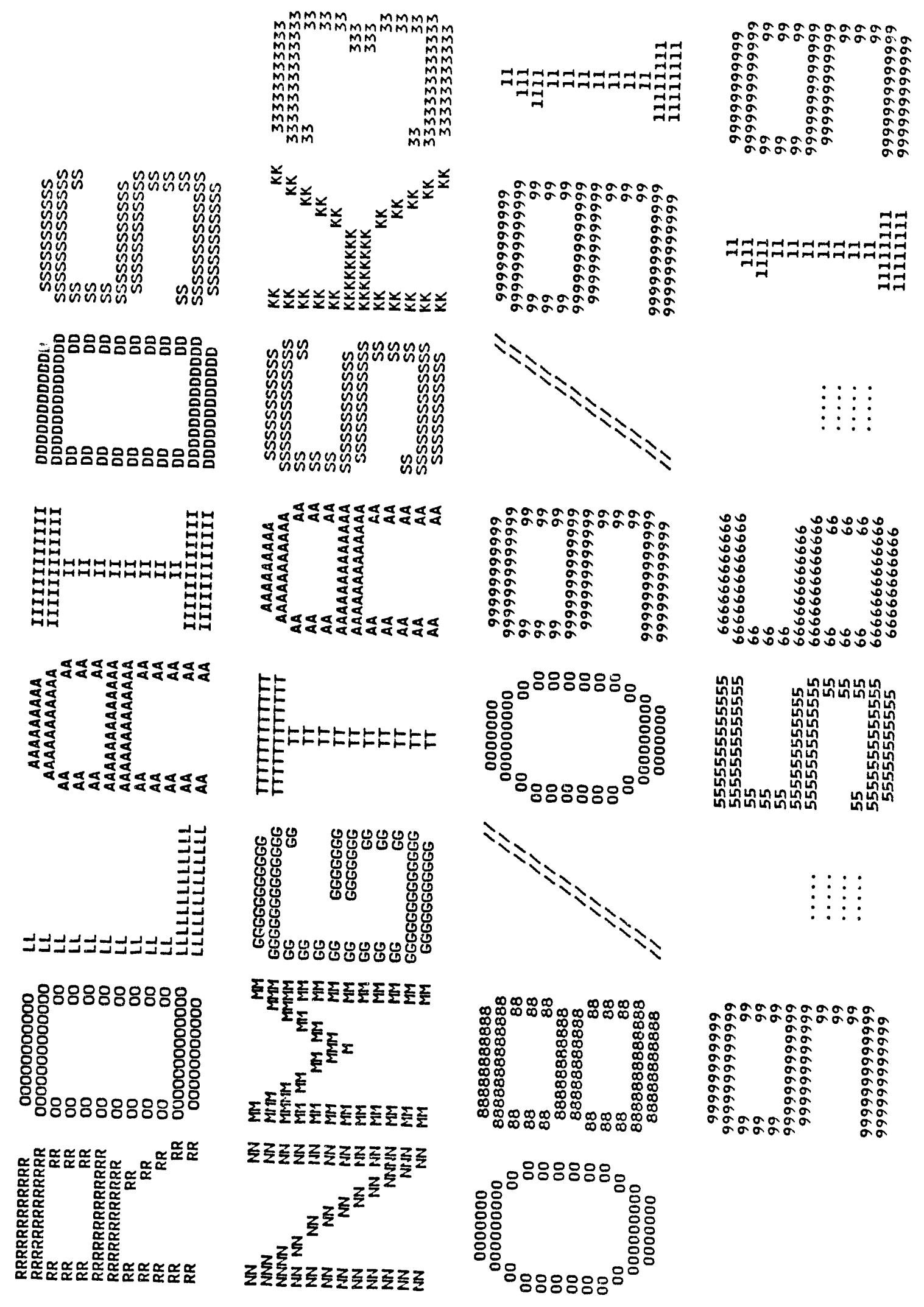



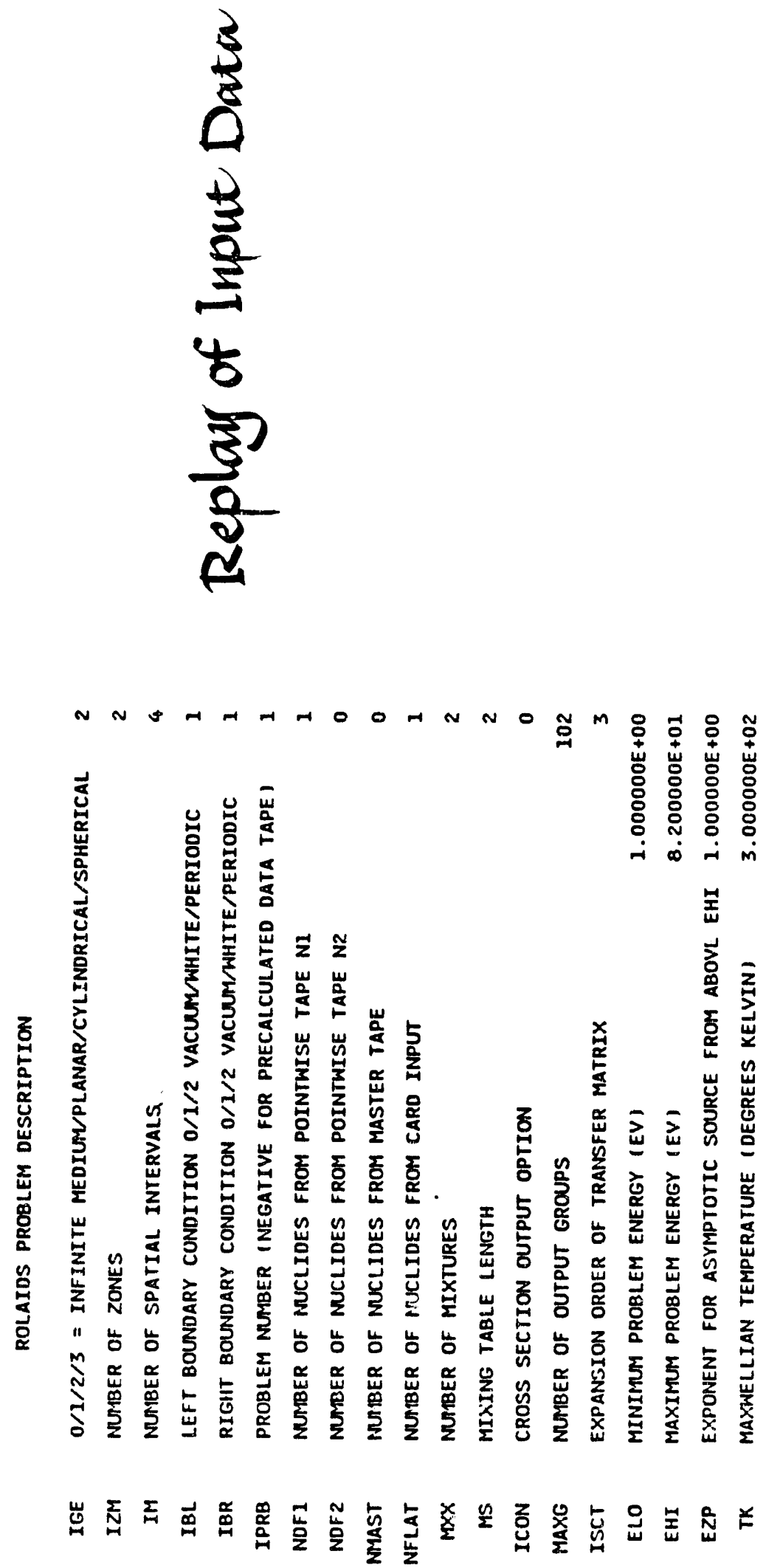

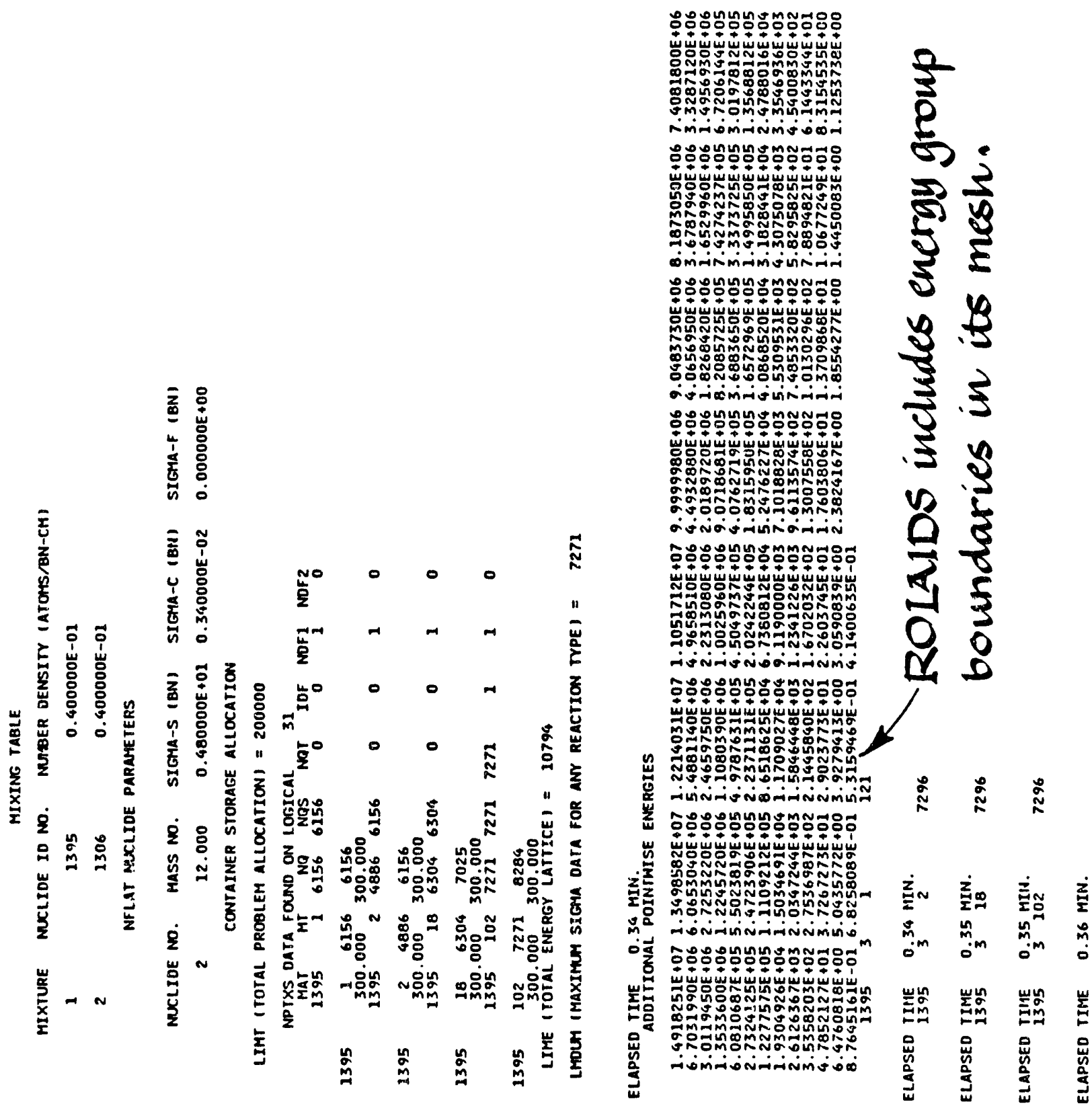


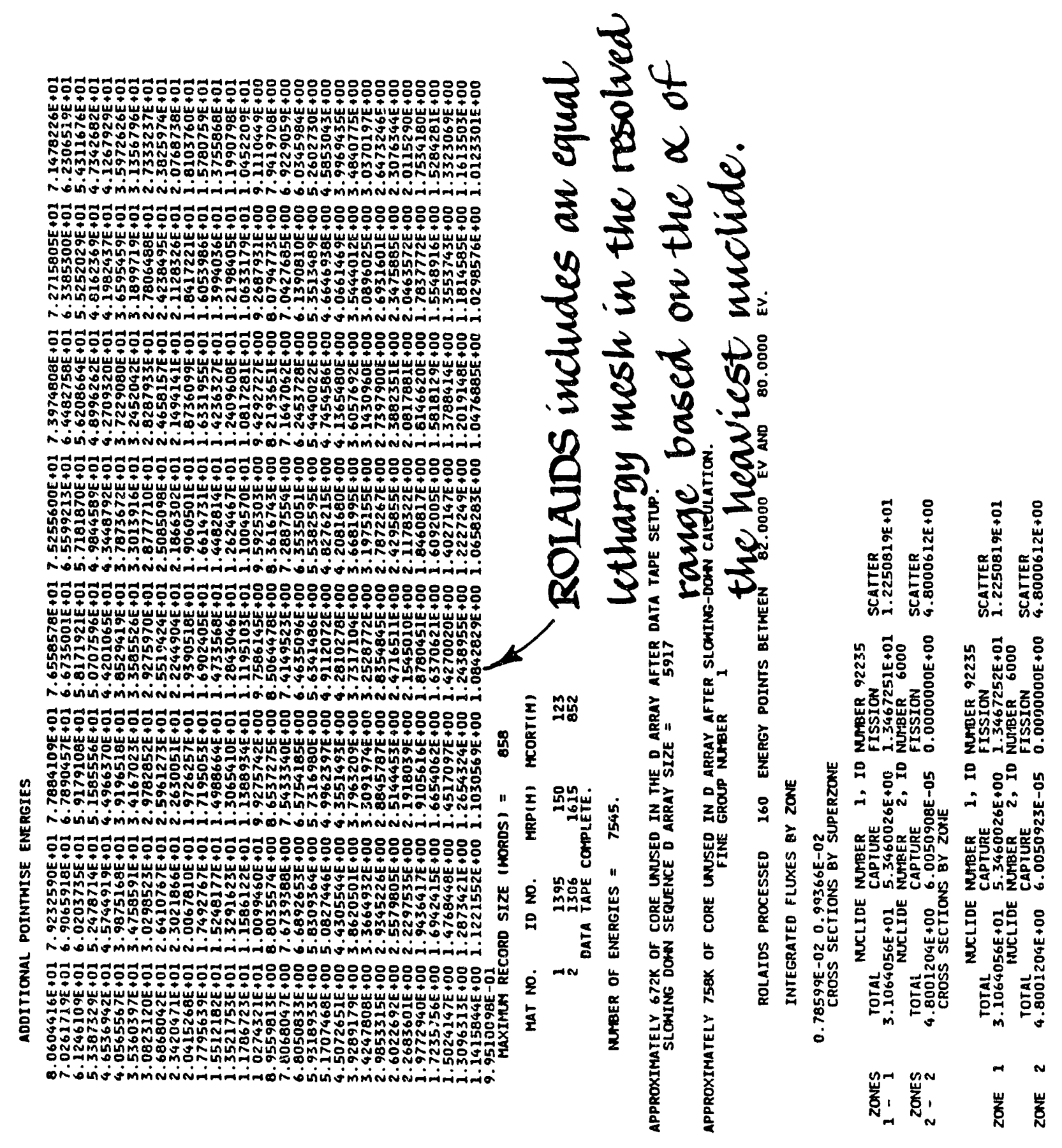




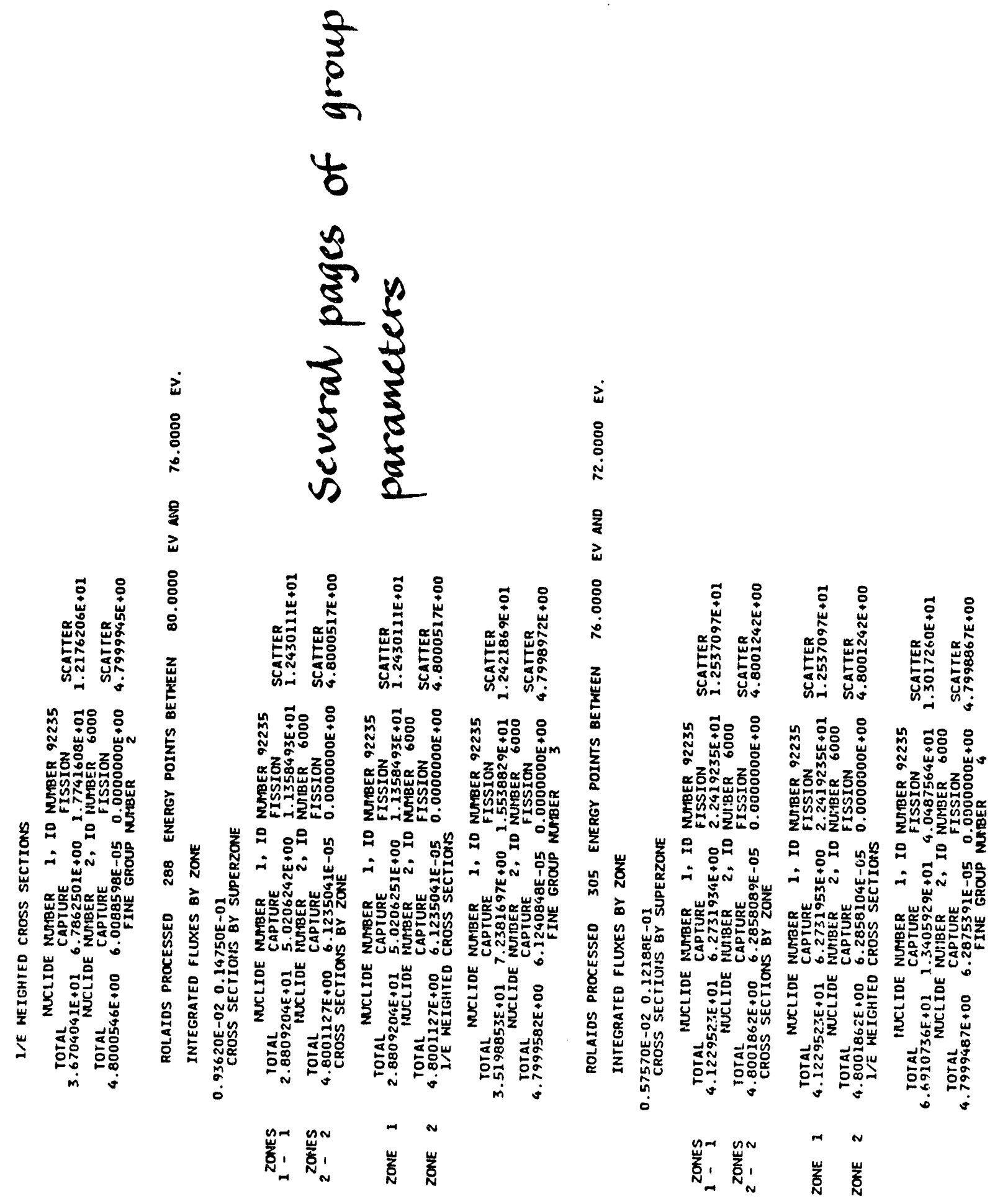




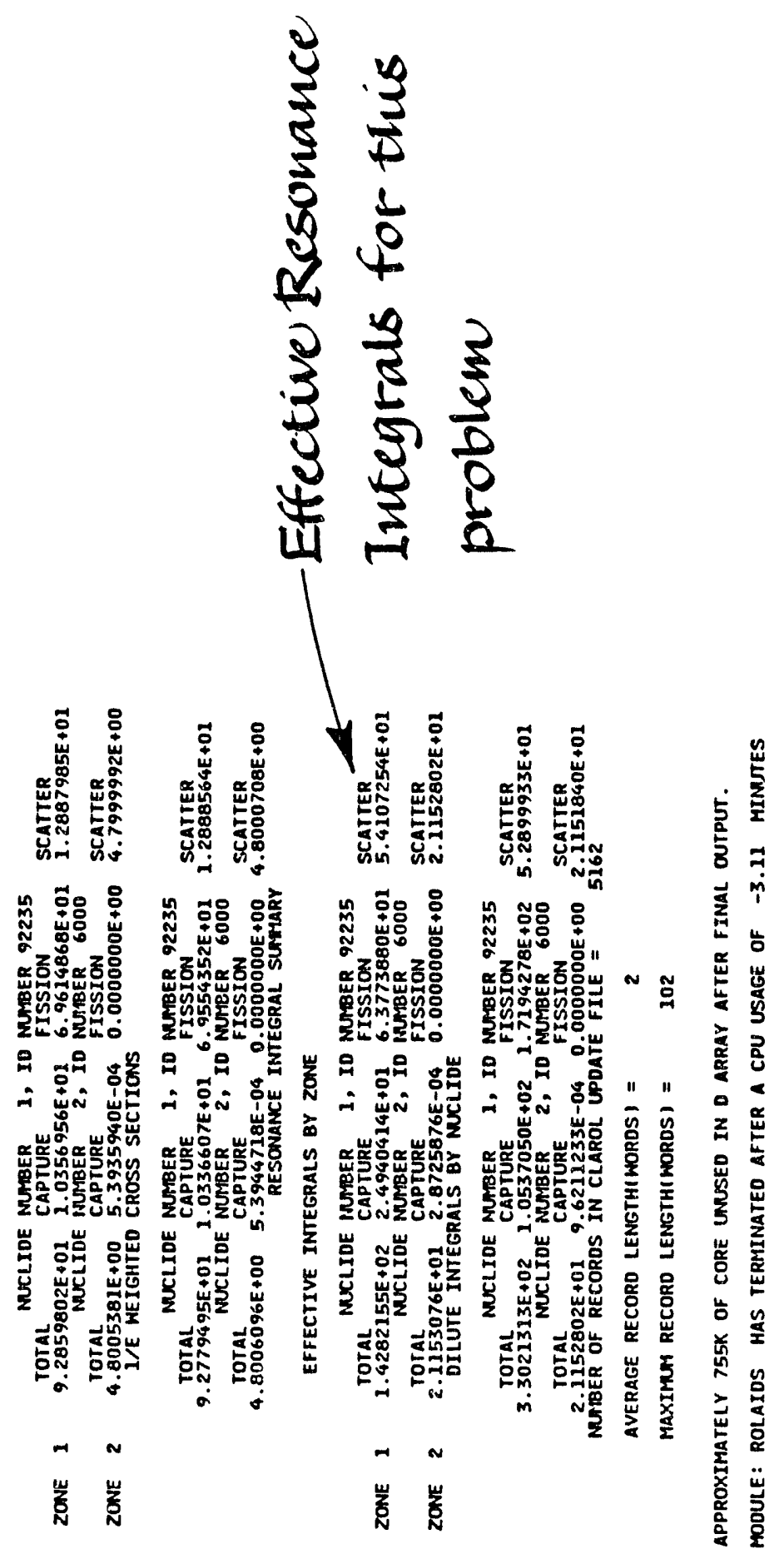




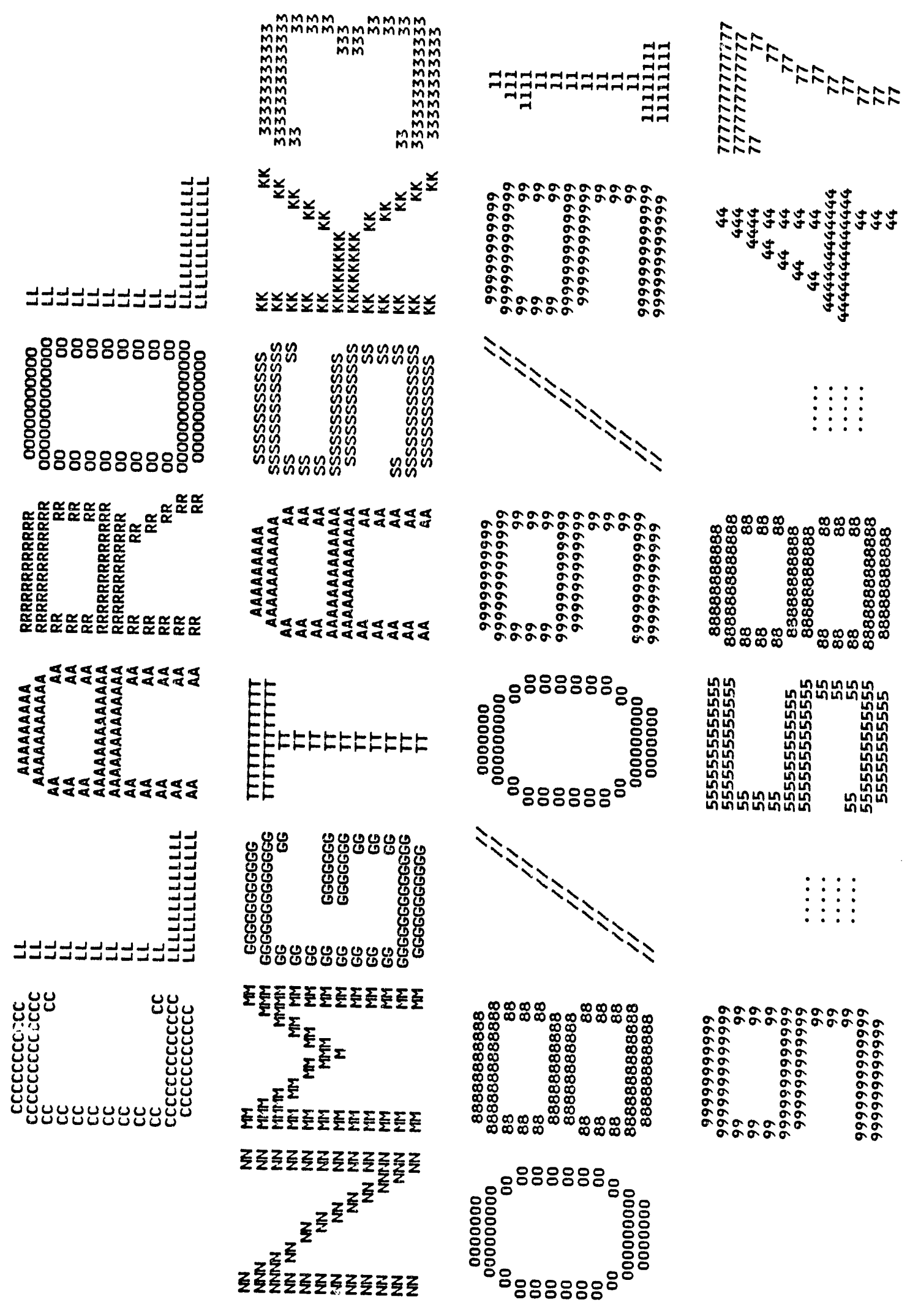




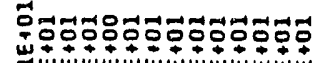


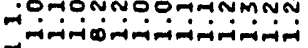

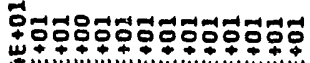

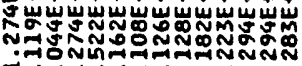

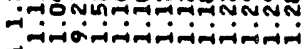

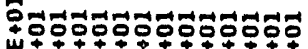

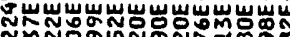

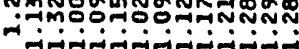

궁

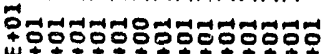

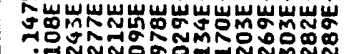
तi

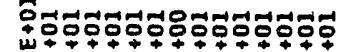

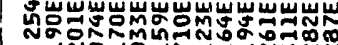
-iminiminoiniminimin

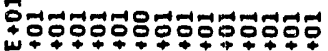
ज̂ñ

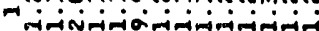

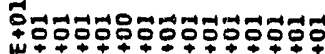

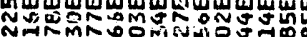

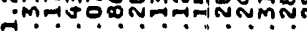

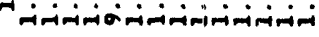
븜

엄

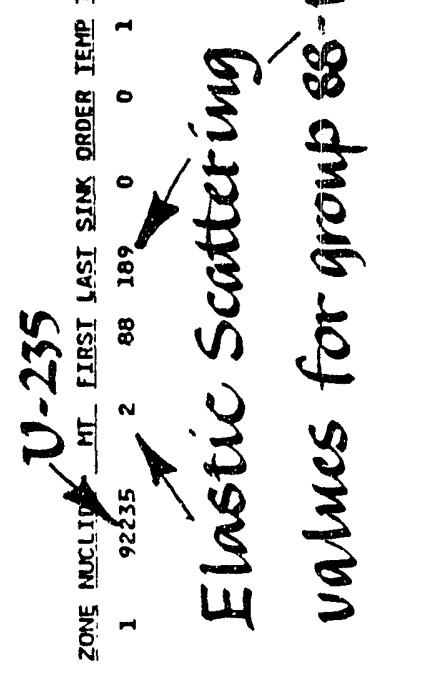

엄

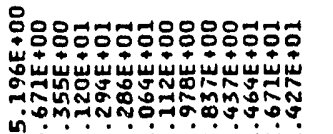
जि-iminimimininimiñ-i

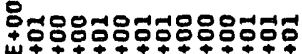

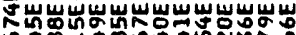

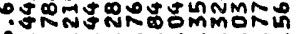

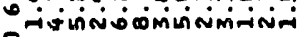
$\$ 0780880888850$

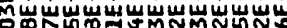

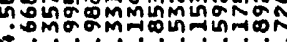
- iviónisiminimiñ

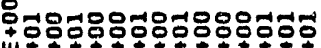

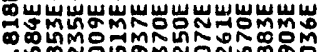
.

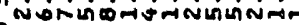

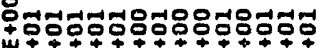

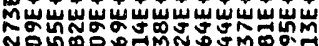
- Nimi

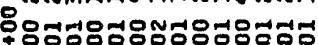

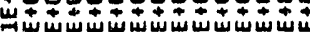

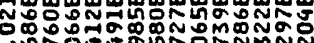
minimininsivini
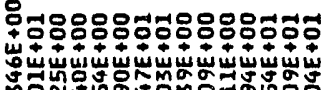
mง m

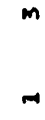

잉.

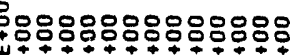

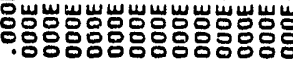

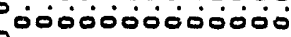
†े०8ำ

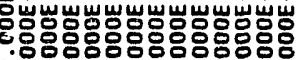
0000000000000000

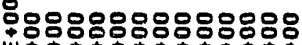

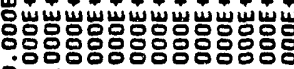
000000000000000 808080880808880

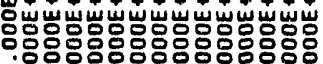
ó000000

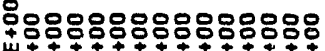

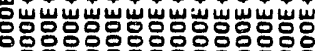
0.0. 808880800800080

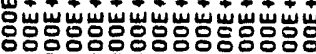
年 -00000000000000 \$80808080808880

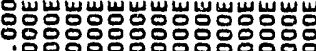
०000000000000

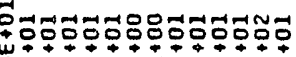

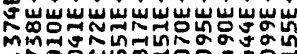
M.

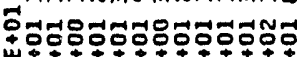

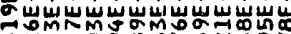

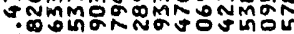
- irivivininimiñio

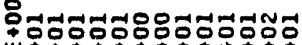
N క్రండ imininominiminivio

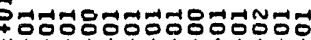

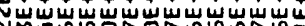
mện

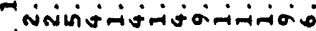

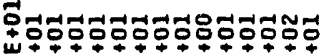

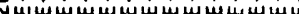

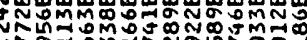
inviniminivimisatio

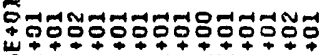

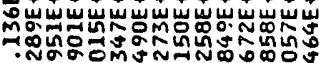

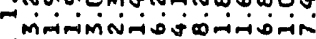

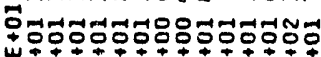

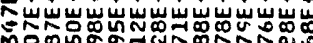

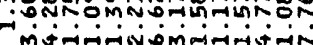
N

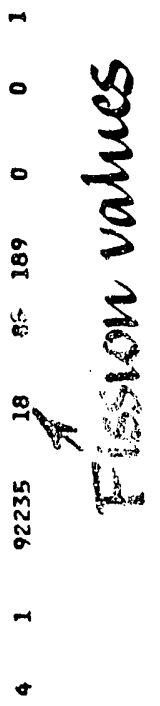

SAMPLE PROBLEMS 


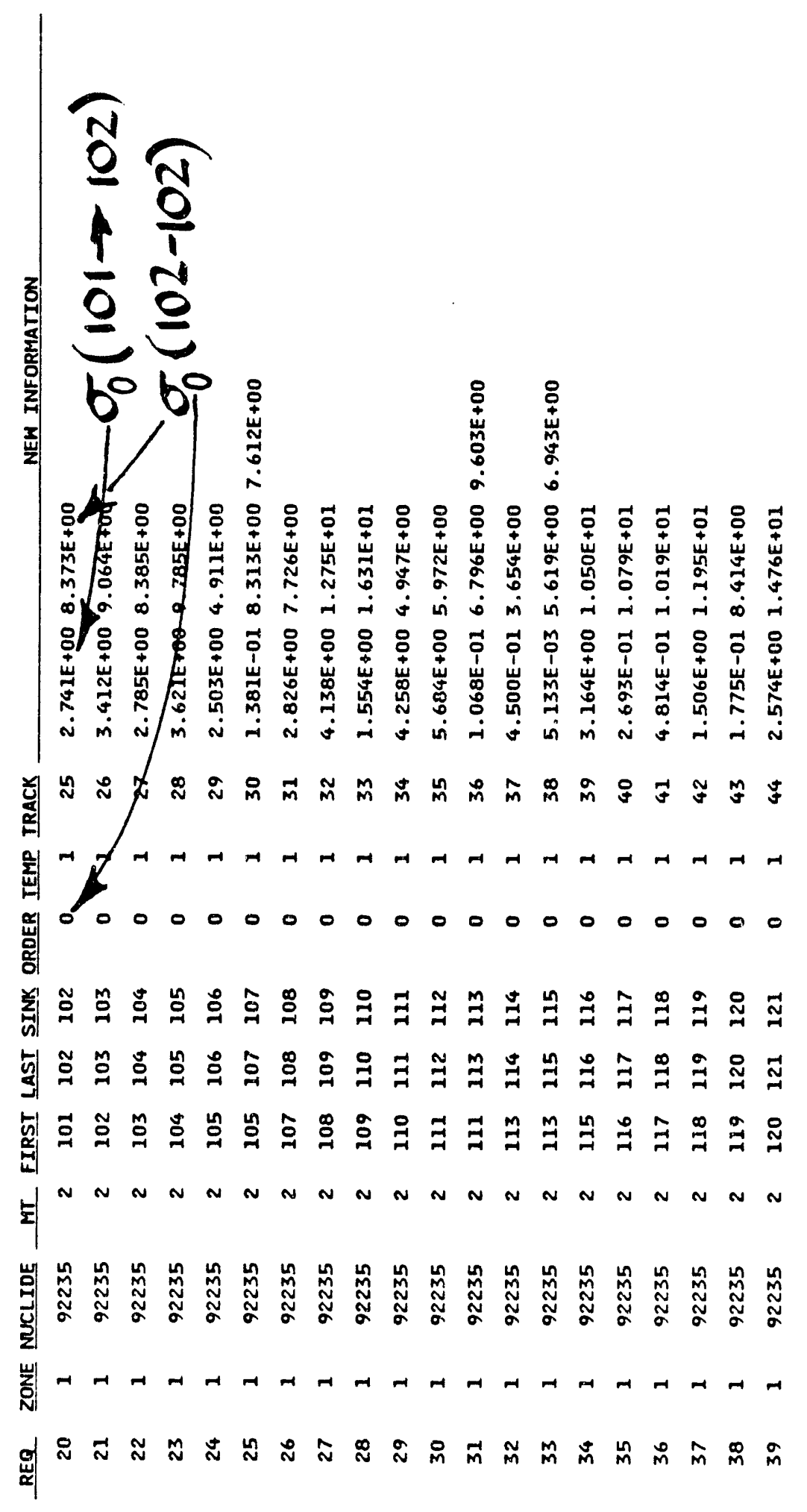



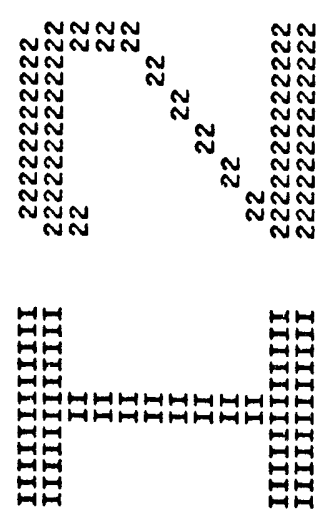

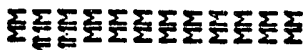
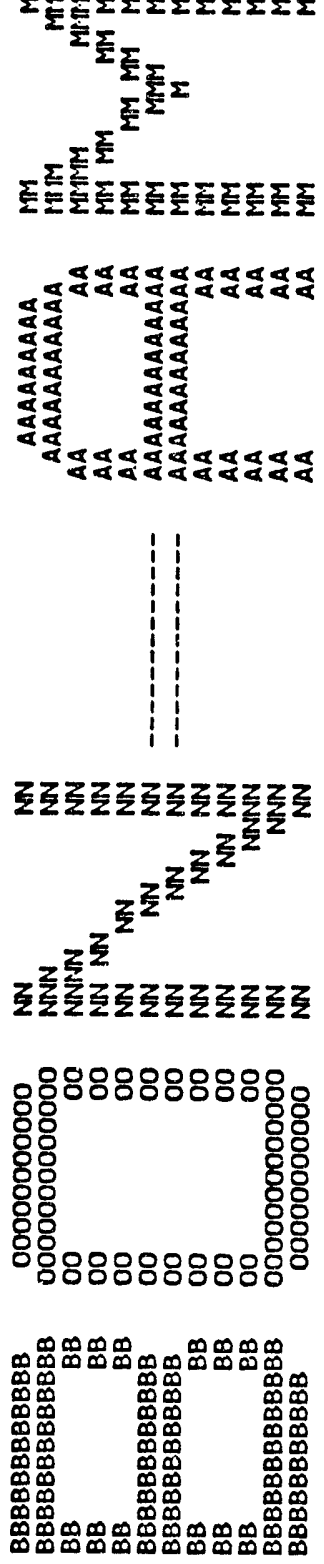
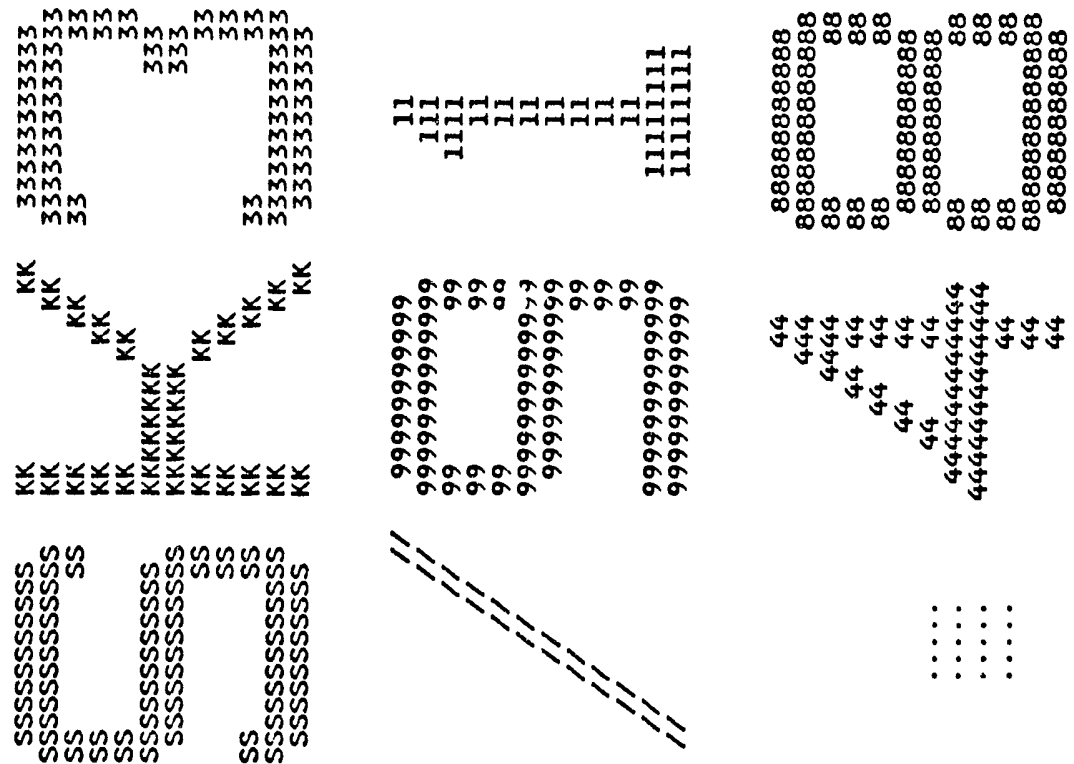

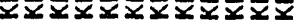
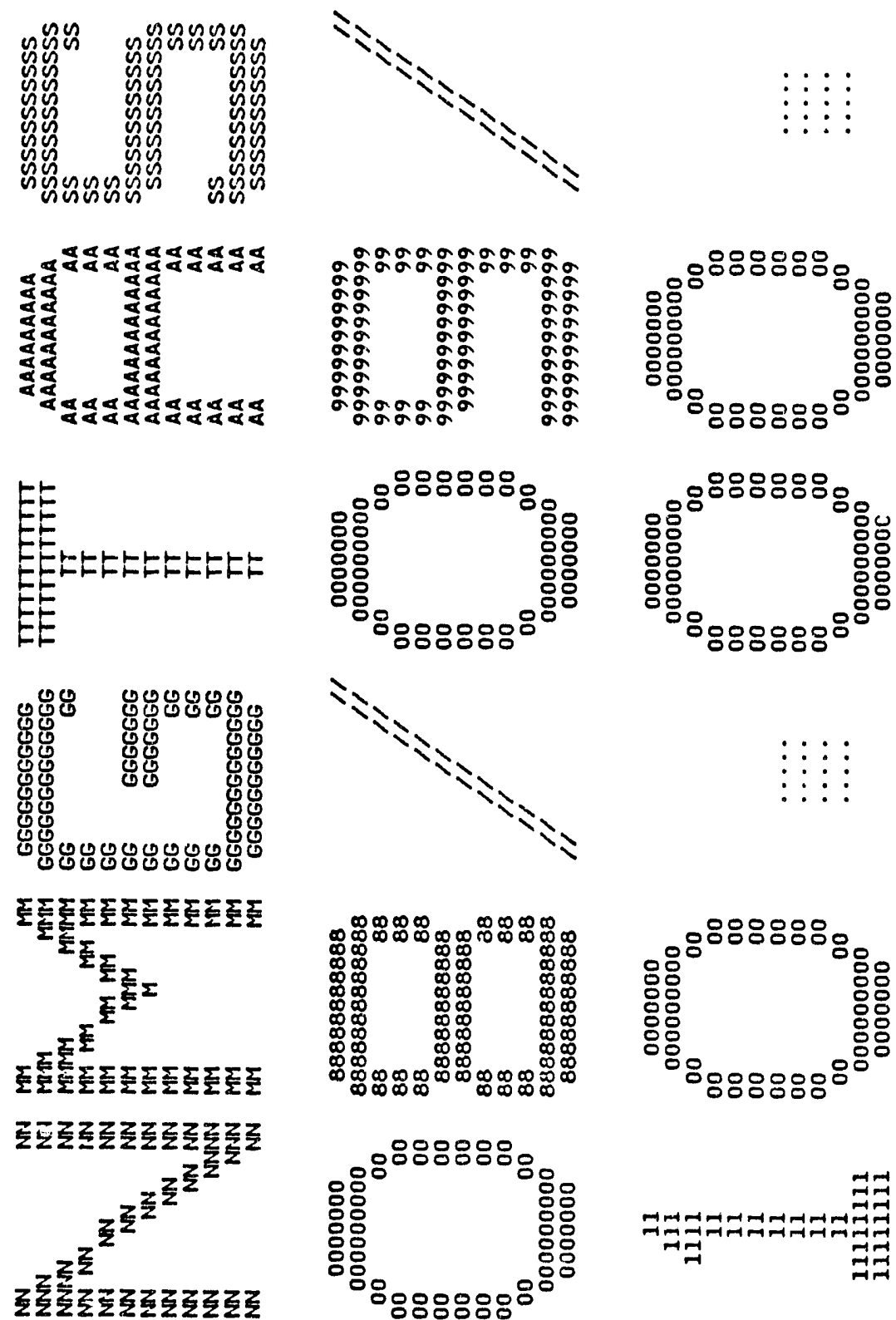
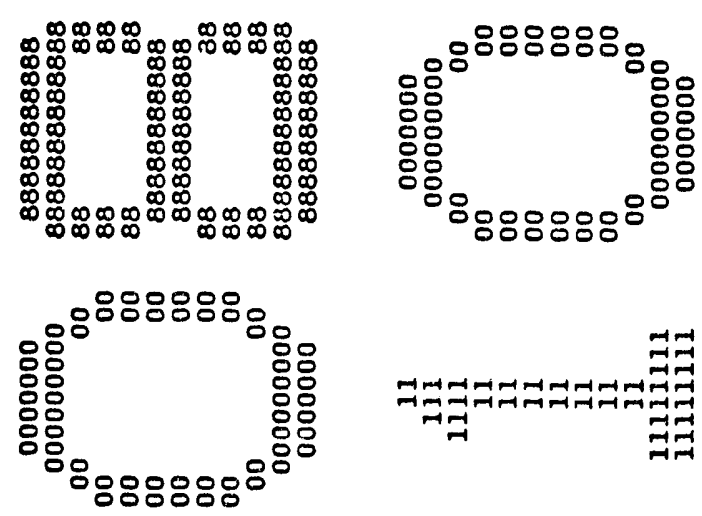


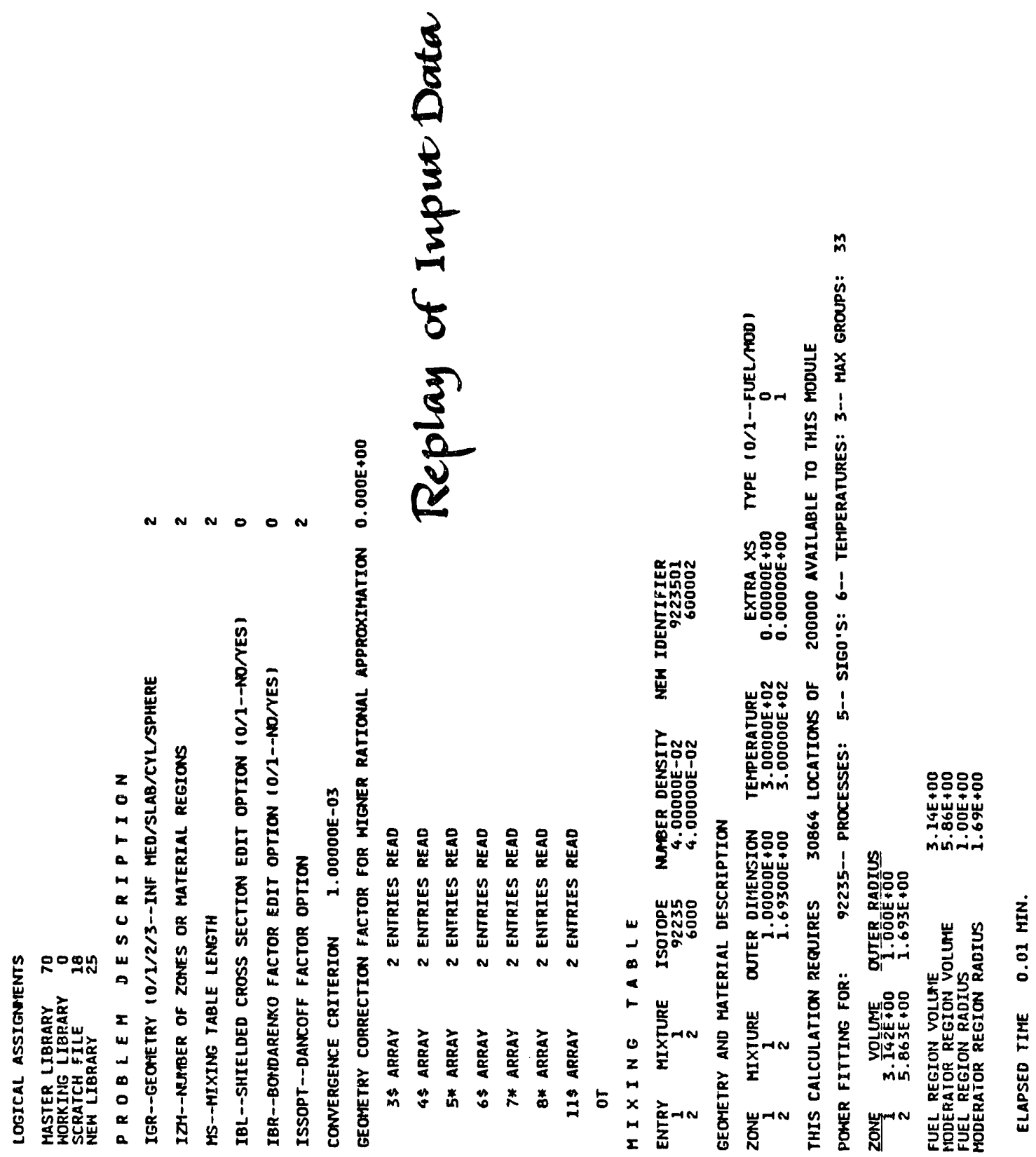




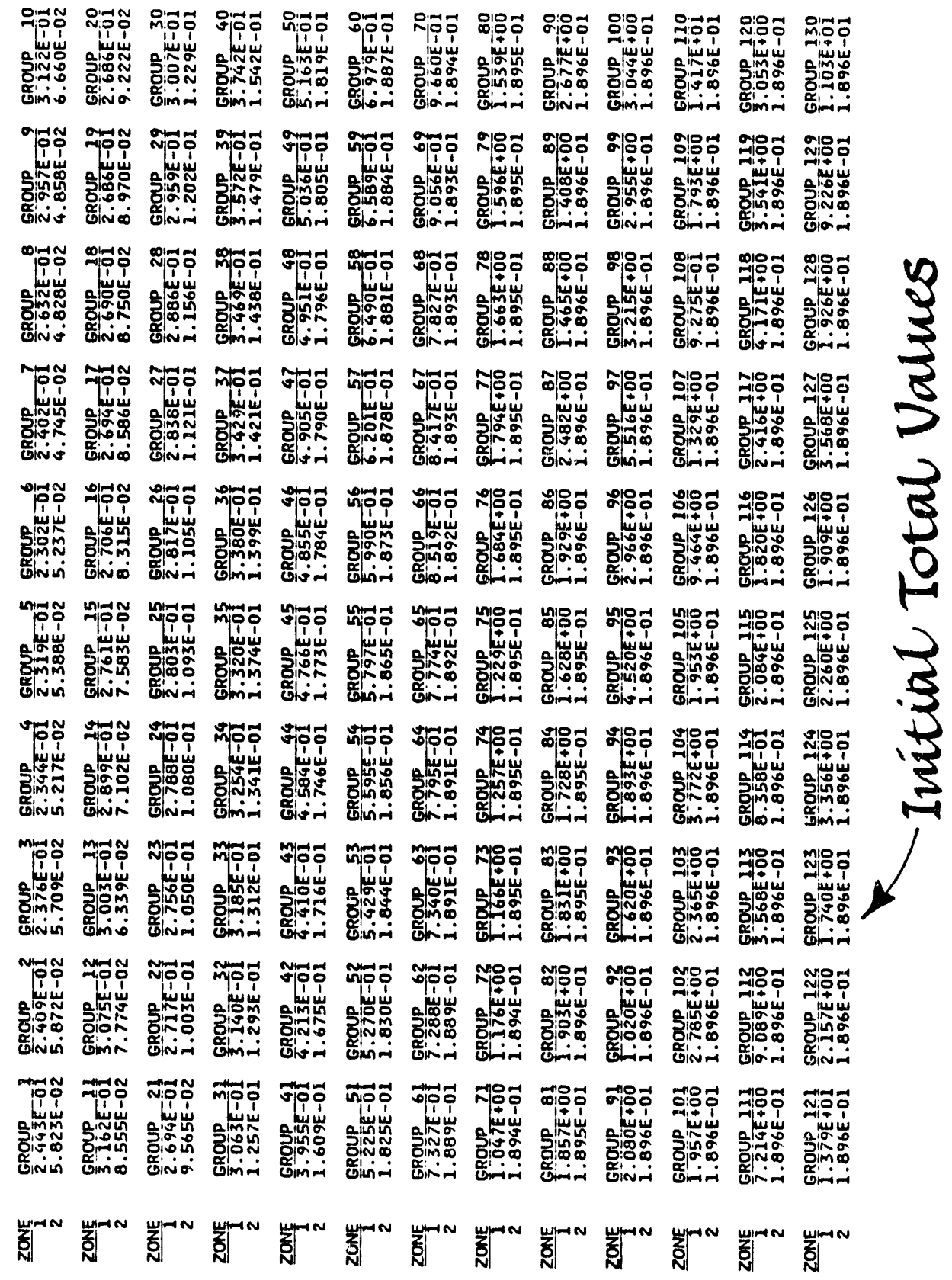




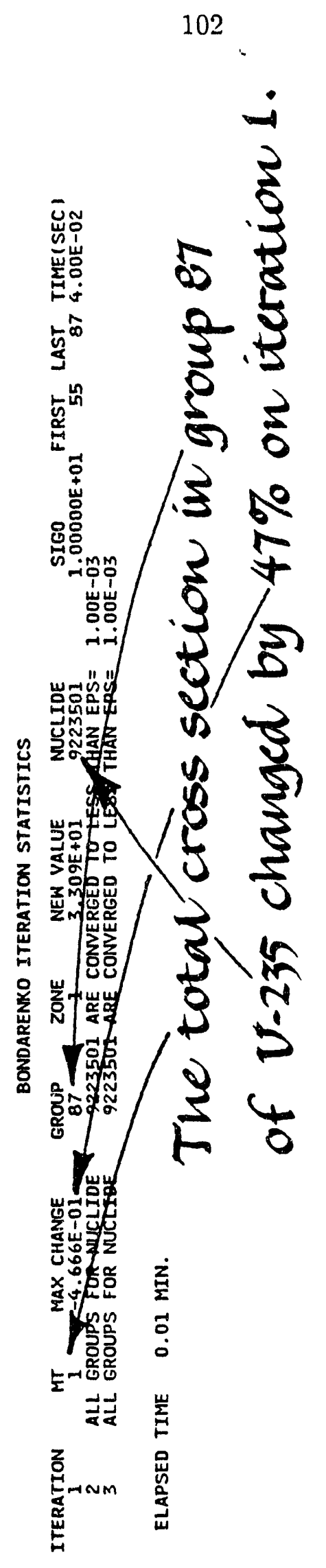

SAMPLE PROBLEMS 


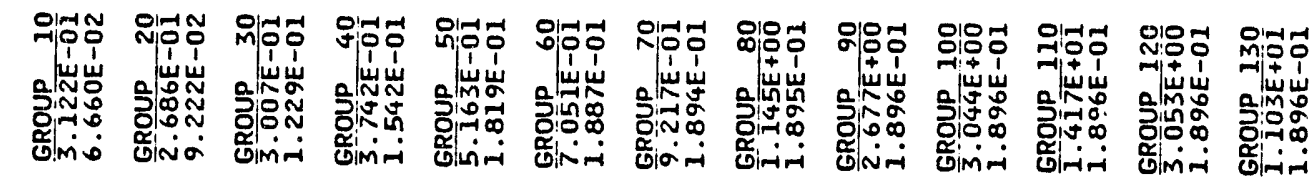

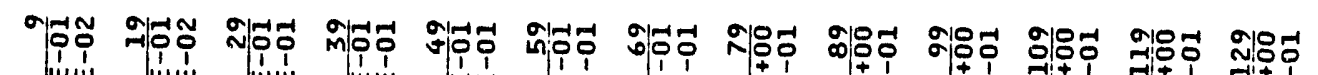

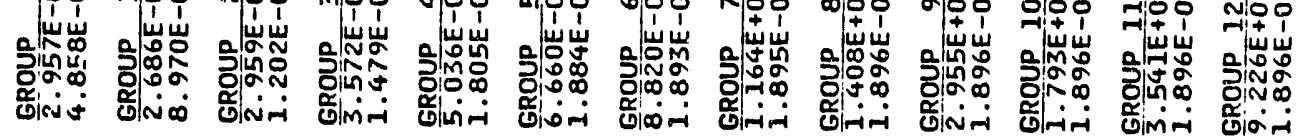

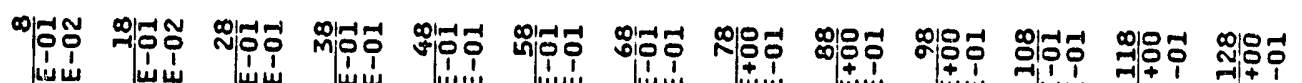

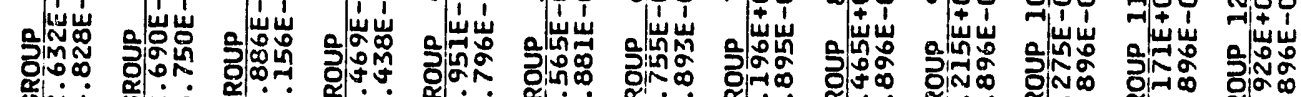

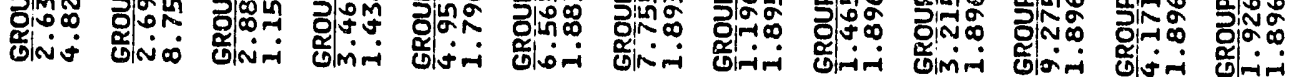

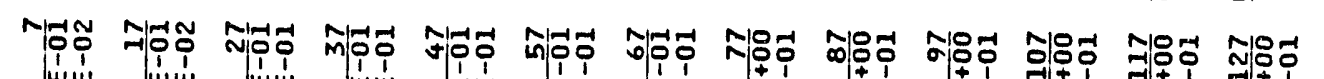

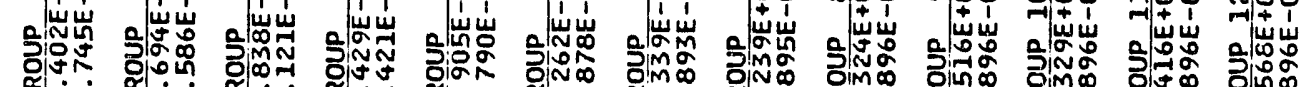

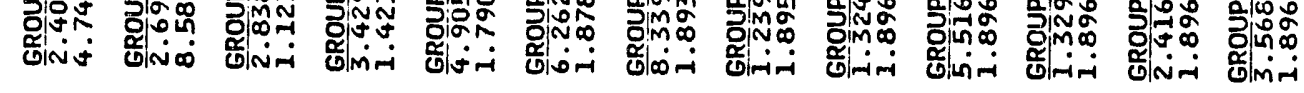

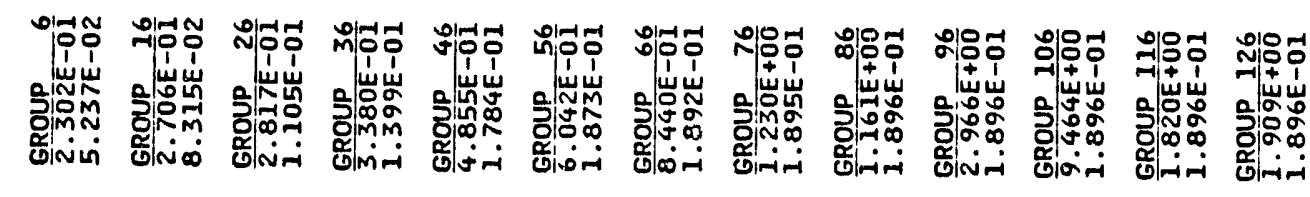

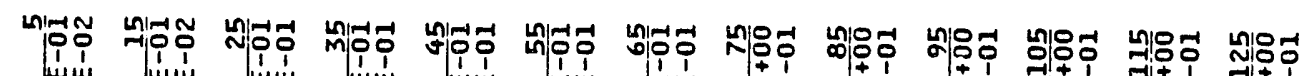
行岕

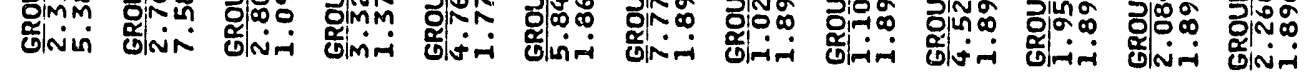

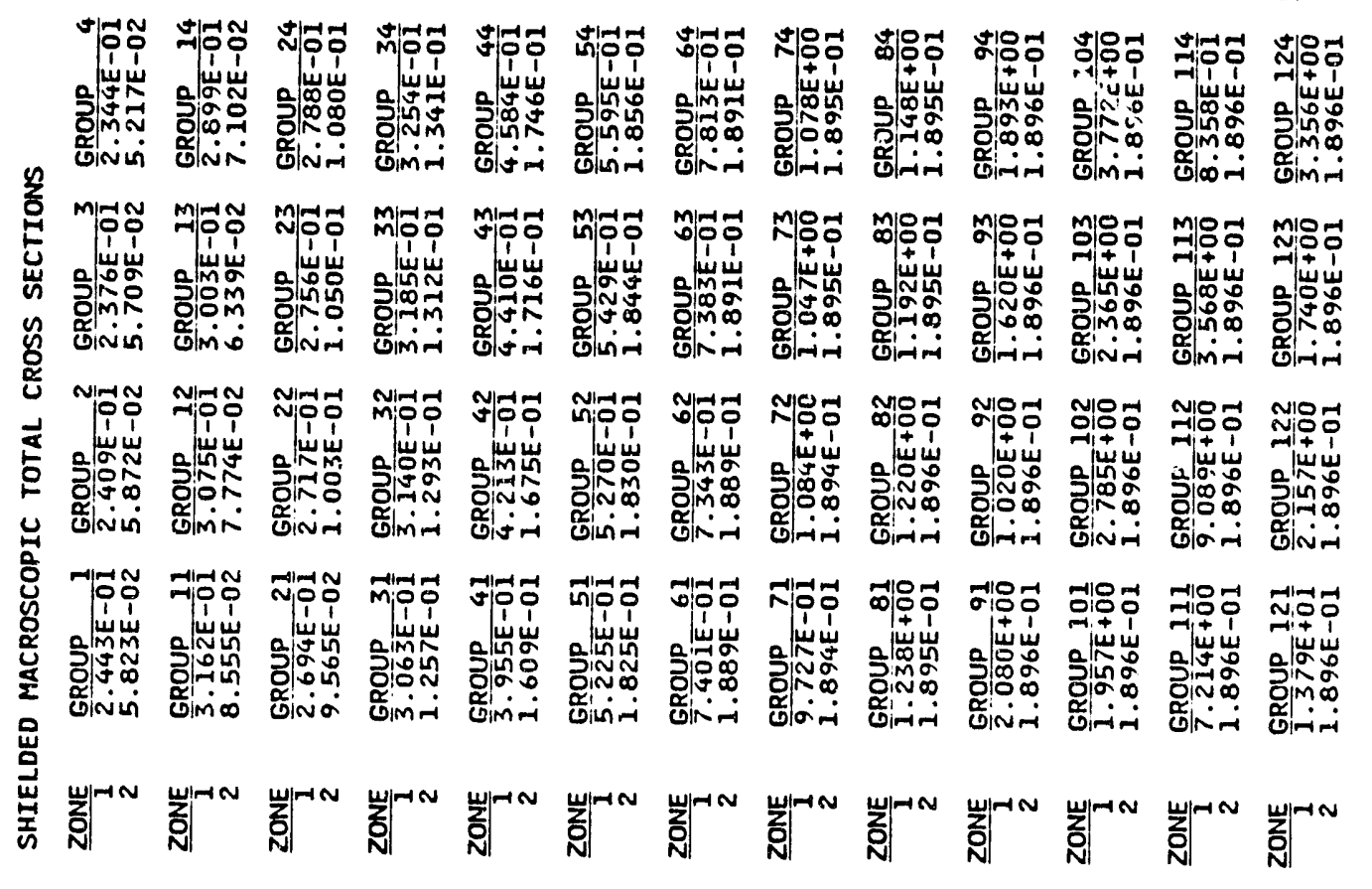

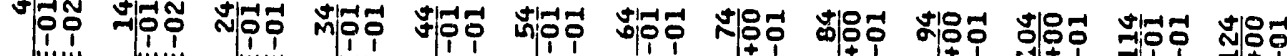

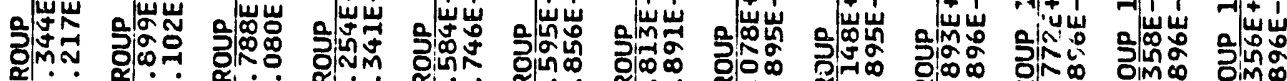

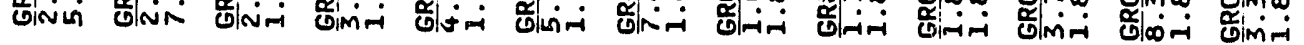
H 


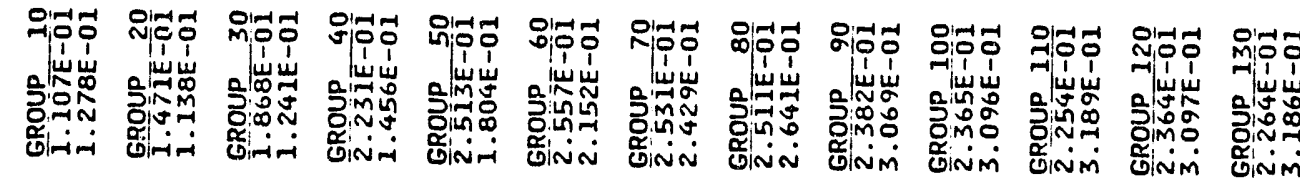

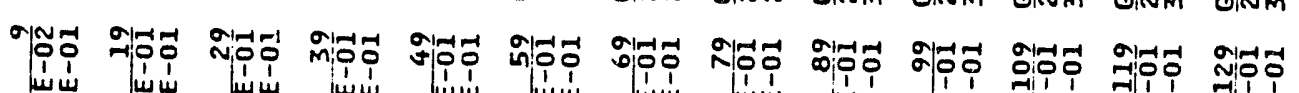
施

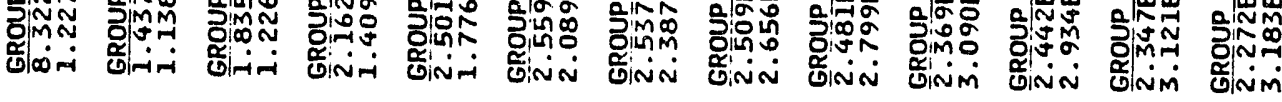

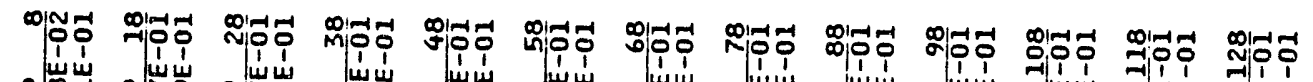
0
oñ

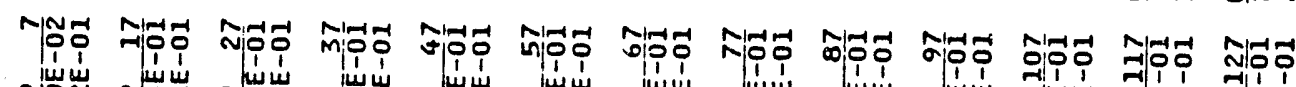

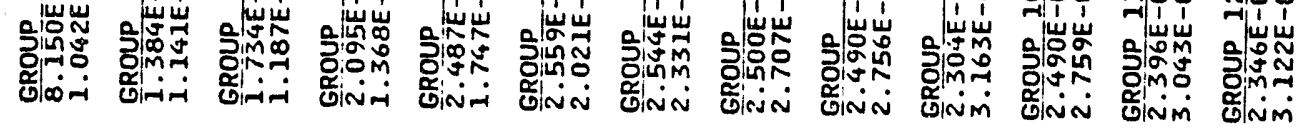

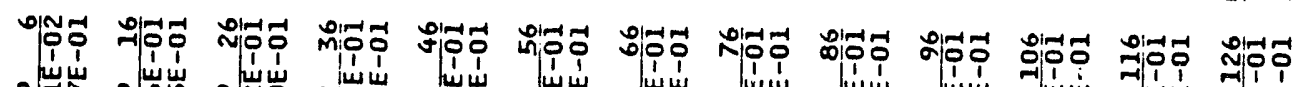

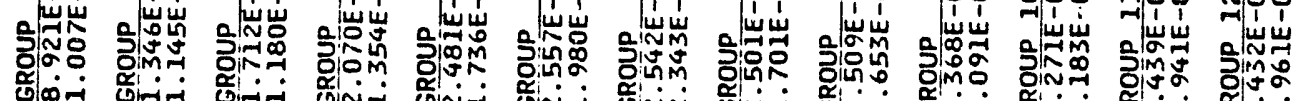

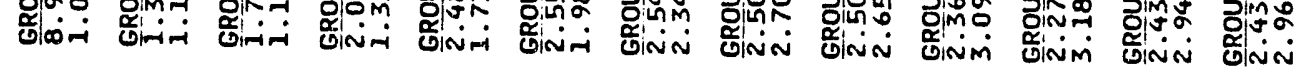

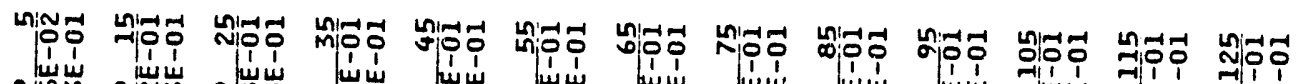

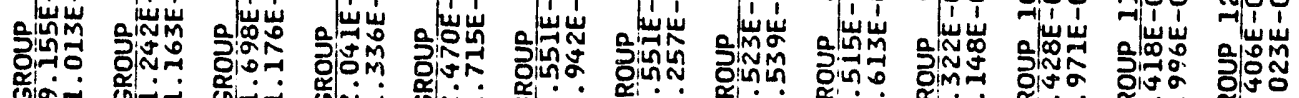

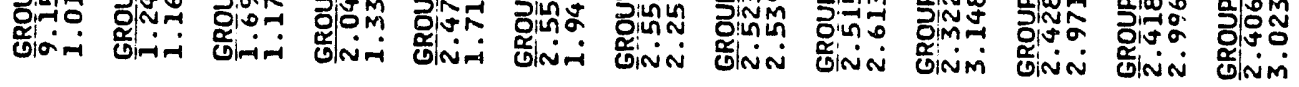

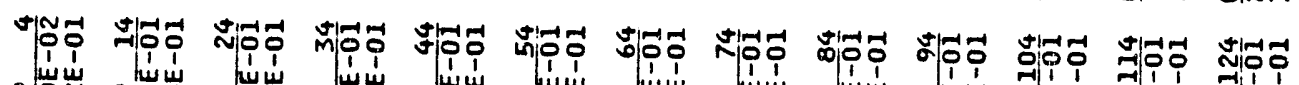

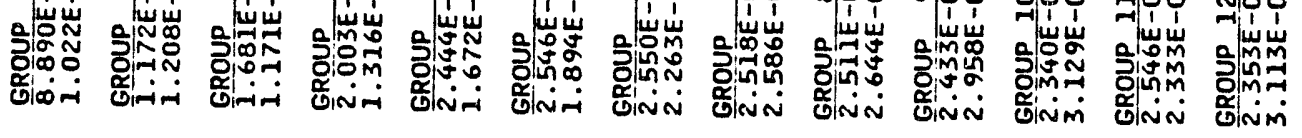

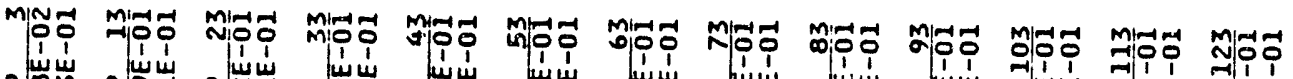

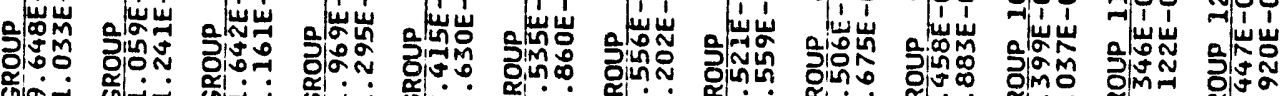

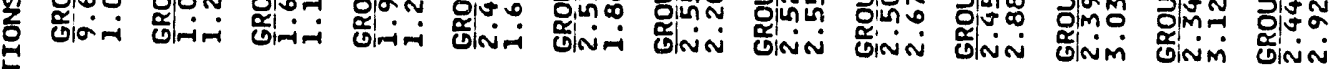

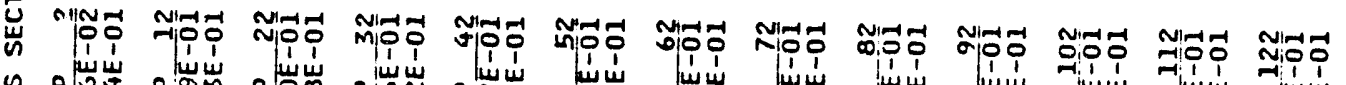

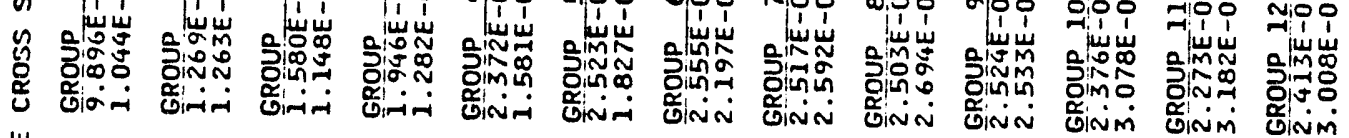

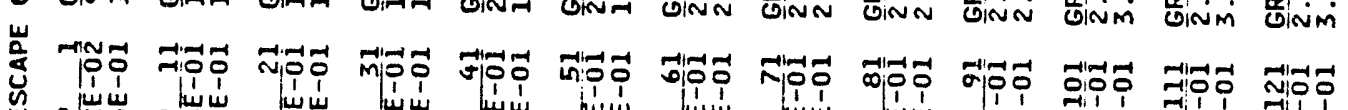

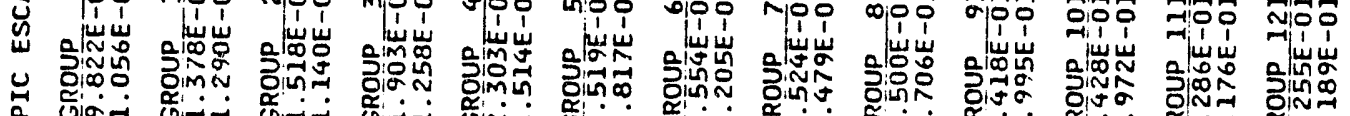

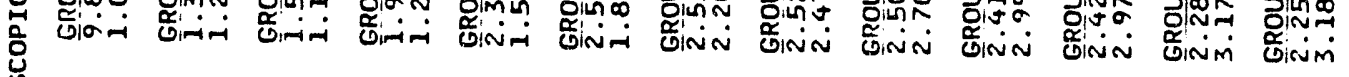
惫

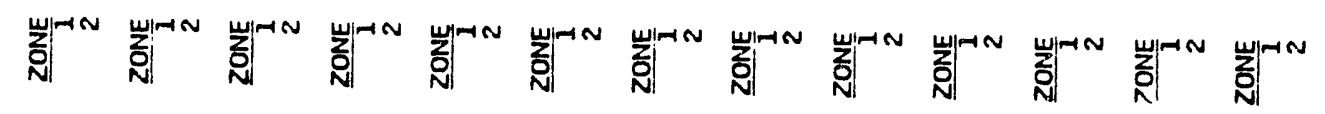

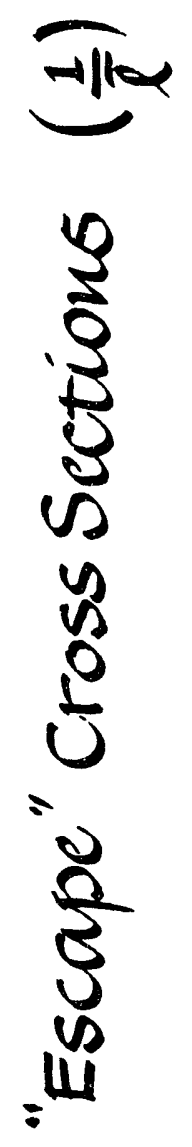

\section{SAMPLE PROBLEMS}




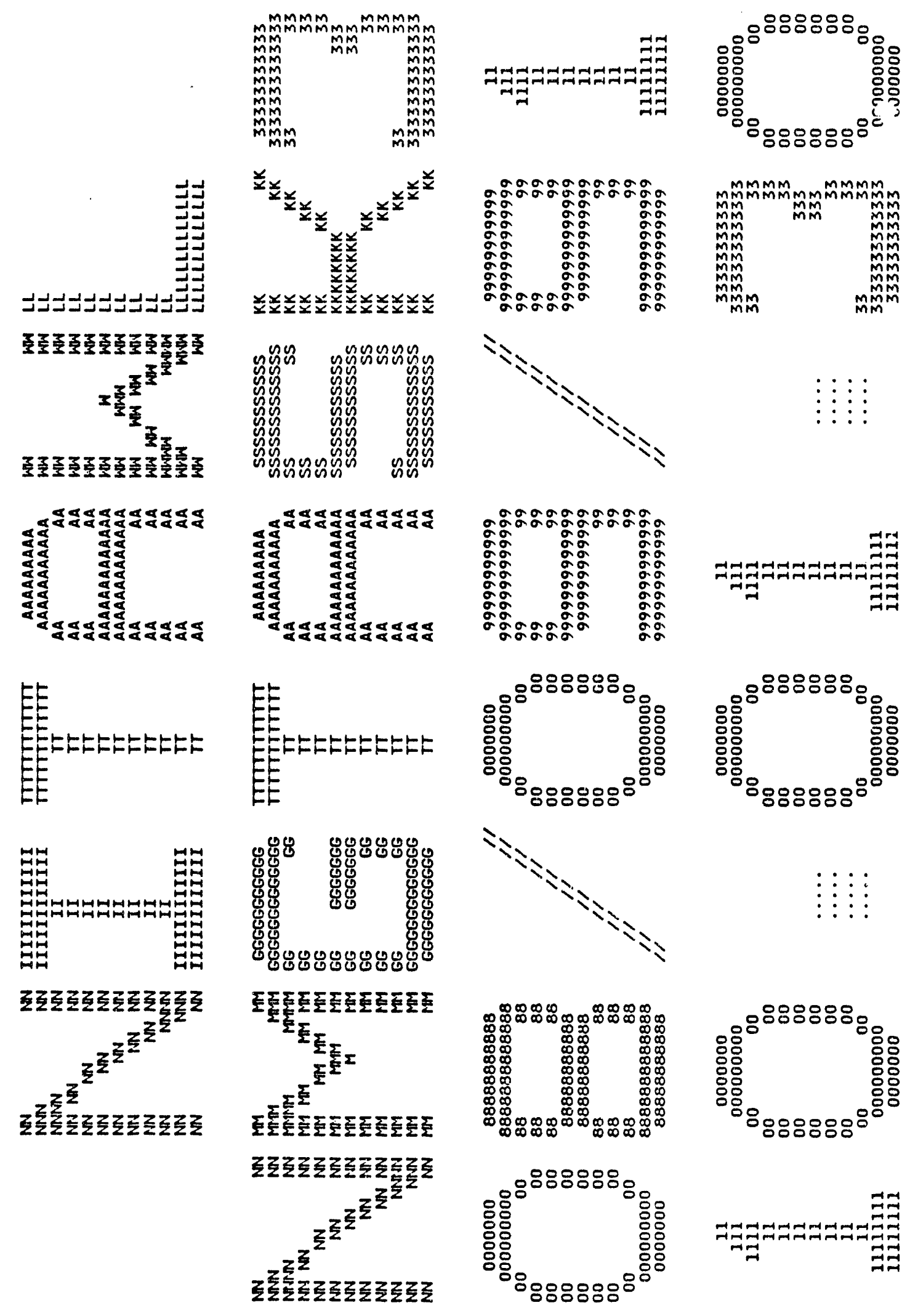


Oops!

Blank Page

SAMPLE PROBLEMS 

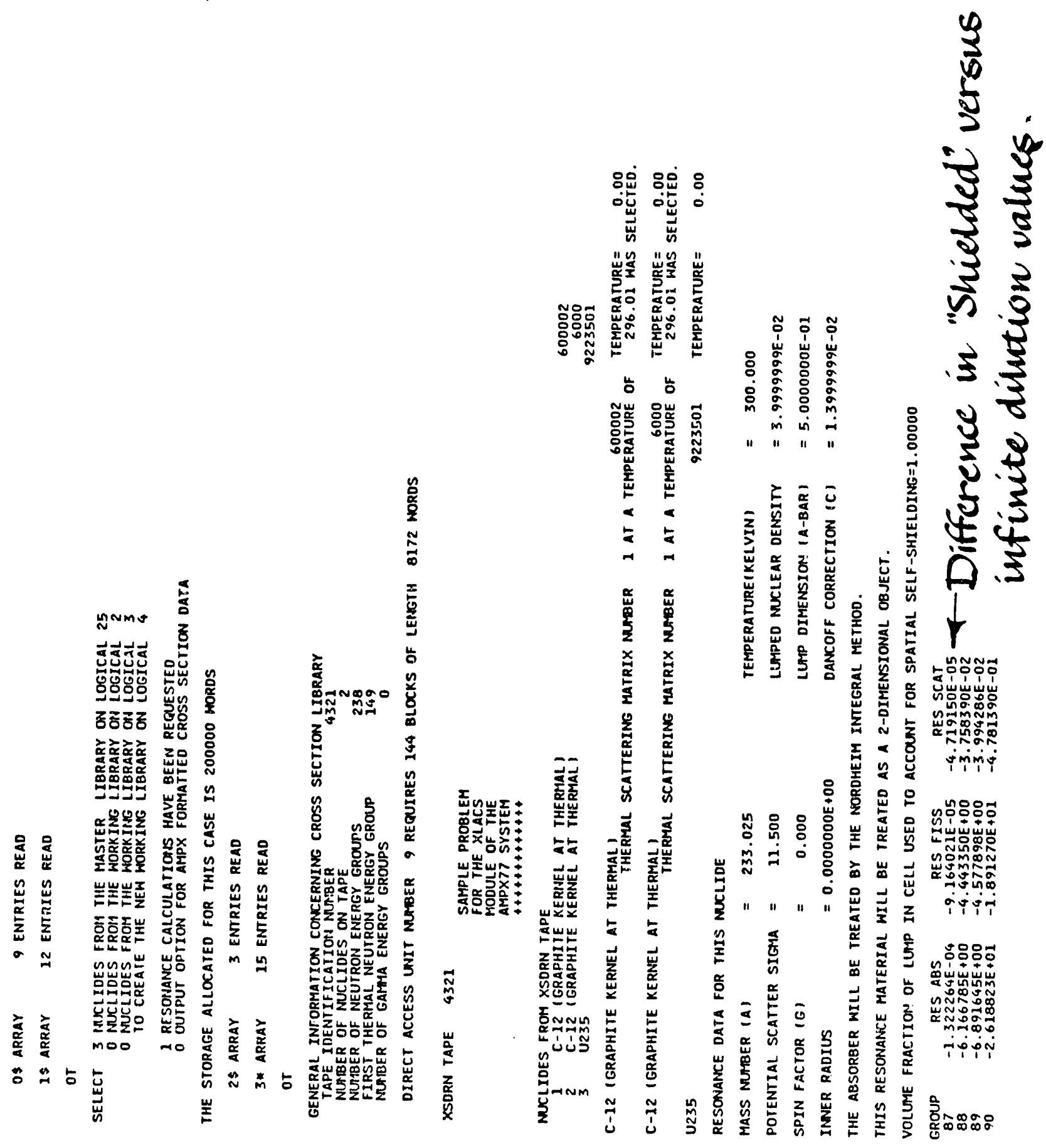


\section{SAMPLE PROBLEMS.4 HOMOGENIZE AND COLLAPSE CROSS SECTIONS}

In this task, we assume our simple reactor lattice discussed in the previous task is located in a cylindrical region that is $50 \mathrm{~cm}$ in radius and surrounded by an annular shell of 200-cm-thick graphite. See Fig. 6.SAMPLE PROBLEMS.11.

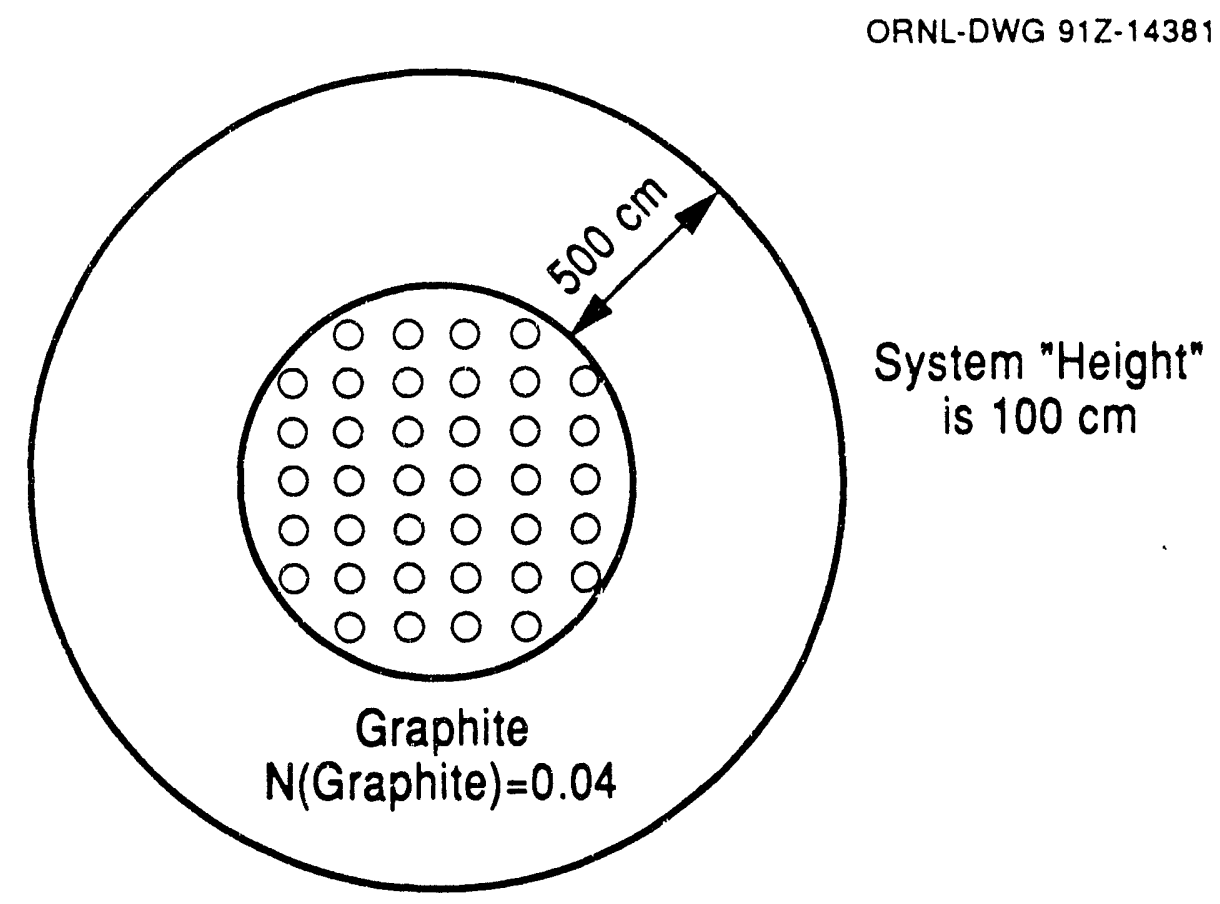

Fig. 6.SAMPLE PROBLEMS.11. Hypothetical system for demonstrating Task 4.

It was decided to produce cross sections in a 25 -group energy structure, which are collapsed consistent with using them in a homogeneous representation of the core region. This collapsing will be made in two stages:

1. The first stage will produce homogenized cross sections for the representative cell shown for Task 3.

2. The second stage will use the homogenized cross sections, along with those for graphite, in a one-dimensional cylindrical mock-up of the reactor to collapse to 25 groups.

\section{SAMPLE PROBLEMS.4.1 Homogenization of Cross Sections}

This case will use the cross sections from the resonance self-shielding calculations from the BONAMI/NITAWL segment discussed in Sect. 6.SAMPLE PROBLEMS.4.3 for ${ }^{235} \mathrm{U}$ in conjunction with those for graphite to homogenize the cell shown in Fig. 6.SAMPLE PROBLEMS.7. The procedure is shown in Fig. 6.SAMPLE PROBLEMS.12. 


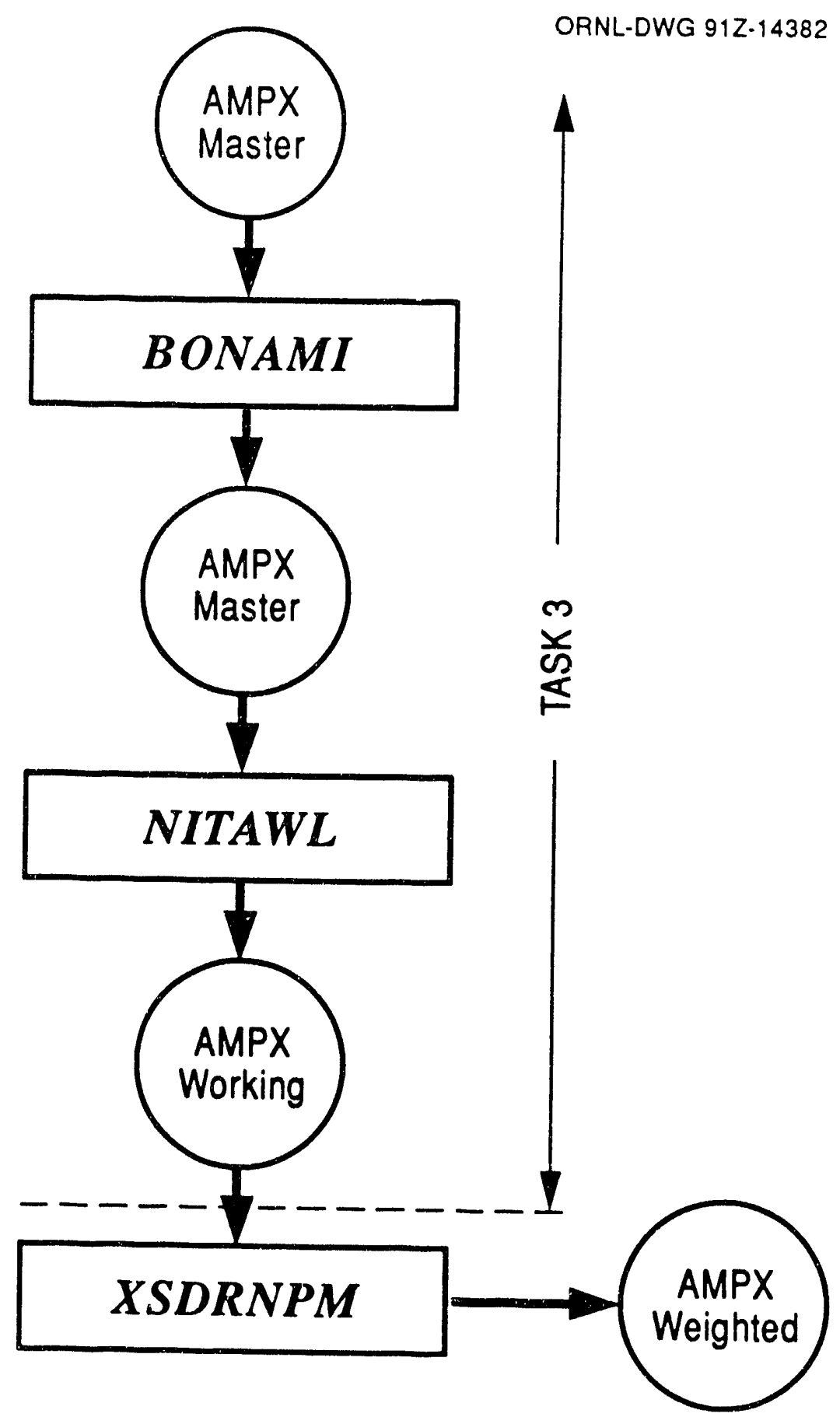

Fig. 6.SAMPLE PROBLEMS.12. Procedure for homogenizing cross sections. 


\section{SAMPLE PROBLEMS.4.2 Collapsing the 238-Group Library to 25 Groups}

The "weighted" library discussed above is combined with a set of graphite data from the master library and used in a one-dimensional mock-up of the reactor to collapse to 25 groups. The procedure is shown in Fig. 6.SAMPLE PROBLEMS.13.

The input for the combination of the two XSDRNPM cases is shown below. Note that in the NITAWL calculation discussed in Task 3 we used a special option that allows one to make one or more copies of a nuclide when it creates a working library. In this case we have made two sets for carbon because when we homogenize the lattice cell we want a set that accounts for the flux peaking and dipping in this area; however, in the large region surrounding the core region, we need a set of data without these effects. In the first "cell-weighting" XSDRNPM case, we pick up both of the carbon sets, identified by 600002 and 6000 , and mix one into the moderator region of the cell with its real number density, while we mix the other into both regions with an equal but insignificant number density. This step cancels any cell weighting on the second set and conveniently allow it to be written on the output weighted library, along with the cell-weighted set, ready for use in the second XSDRNPM case.

Portions of the output follow the input shown on the following pages.

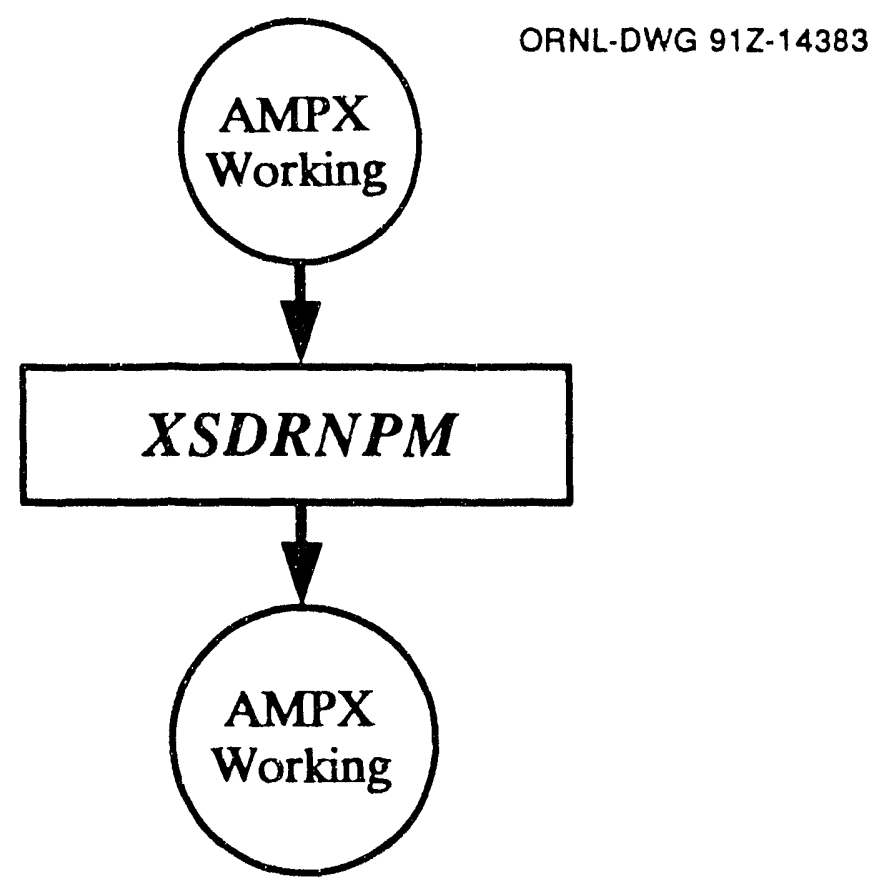

Fig. 6.SAMPLE PROBLEMS.13. Procedure to collapse cross sections. 

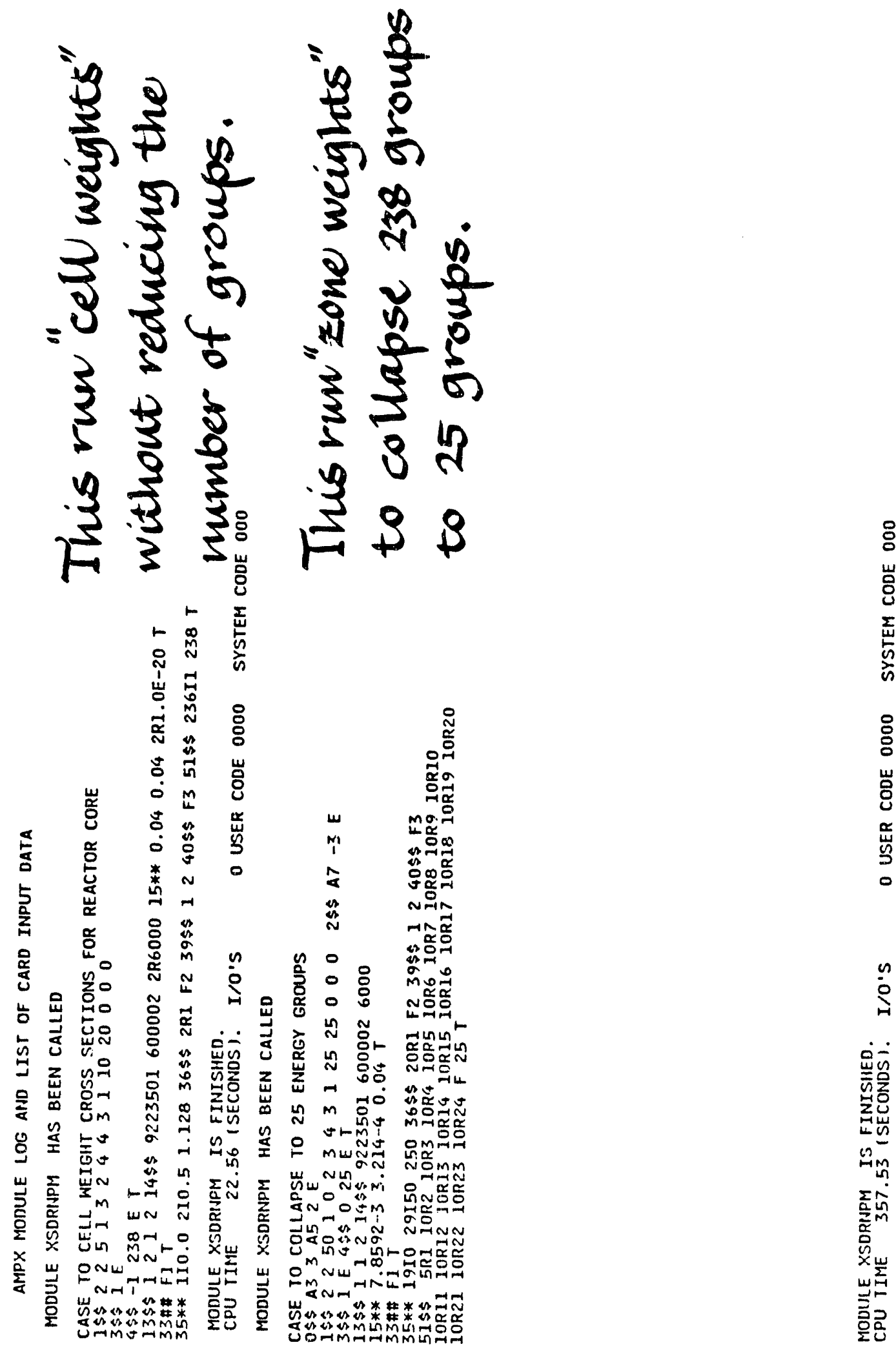

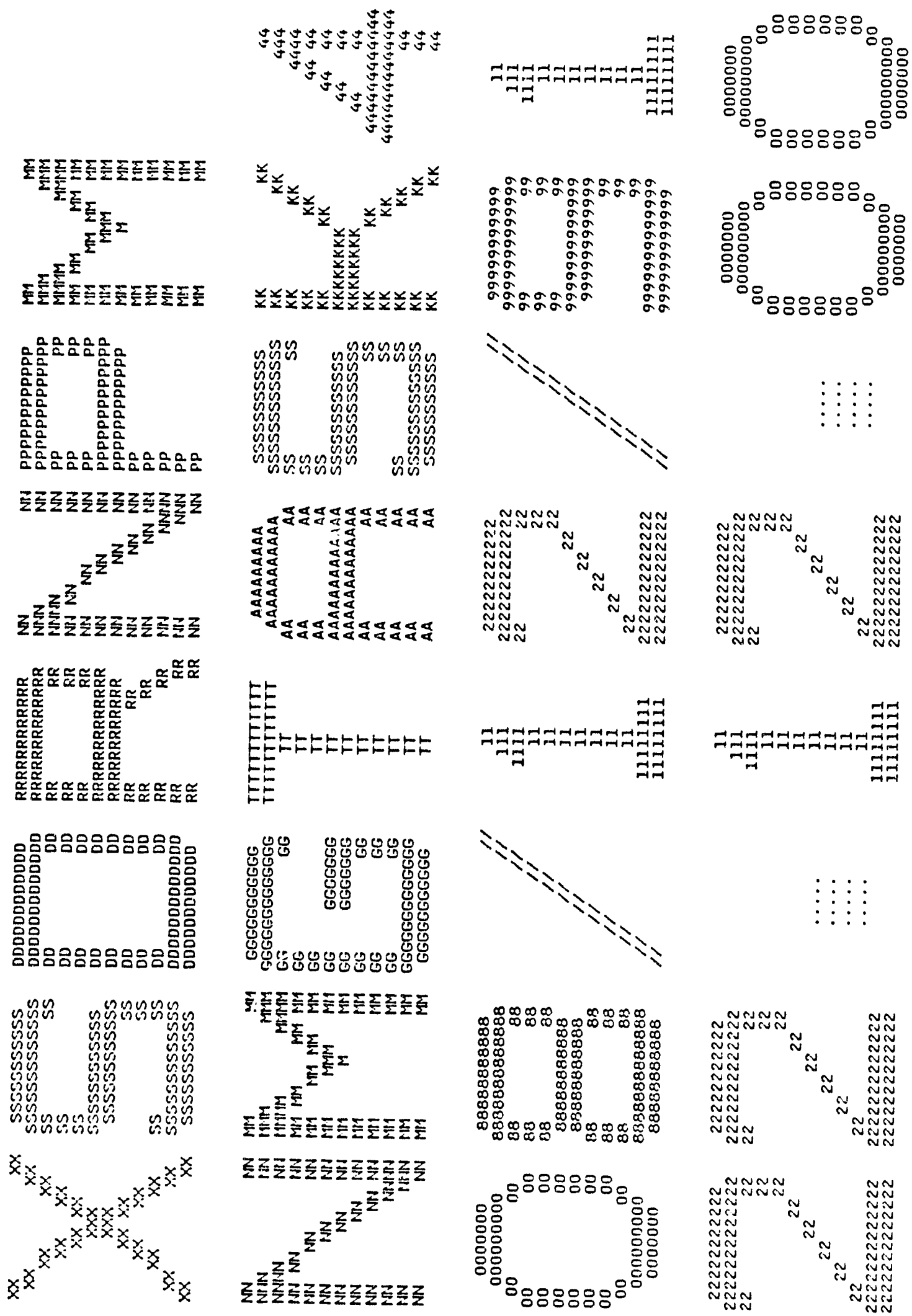


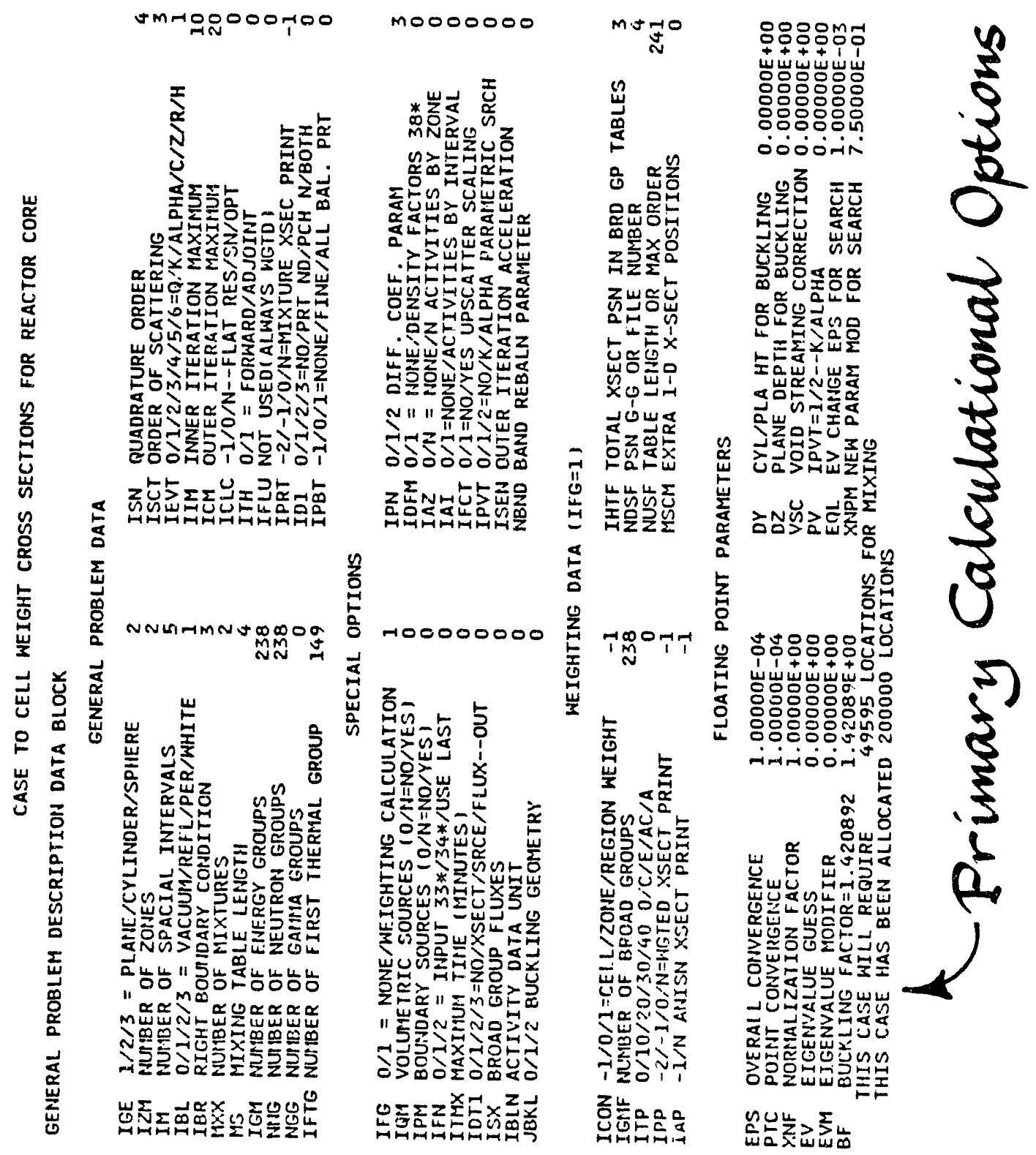




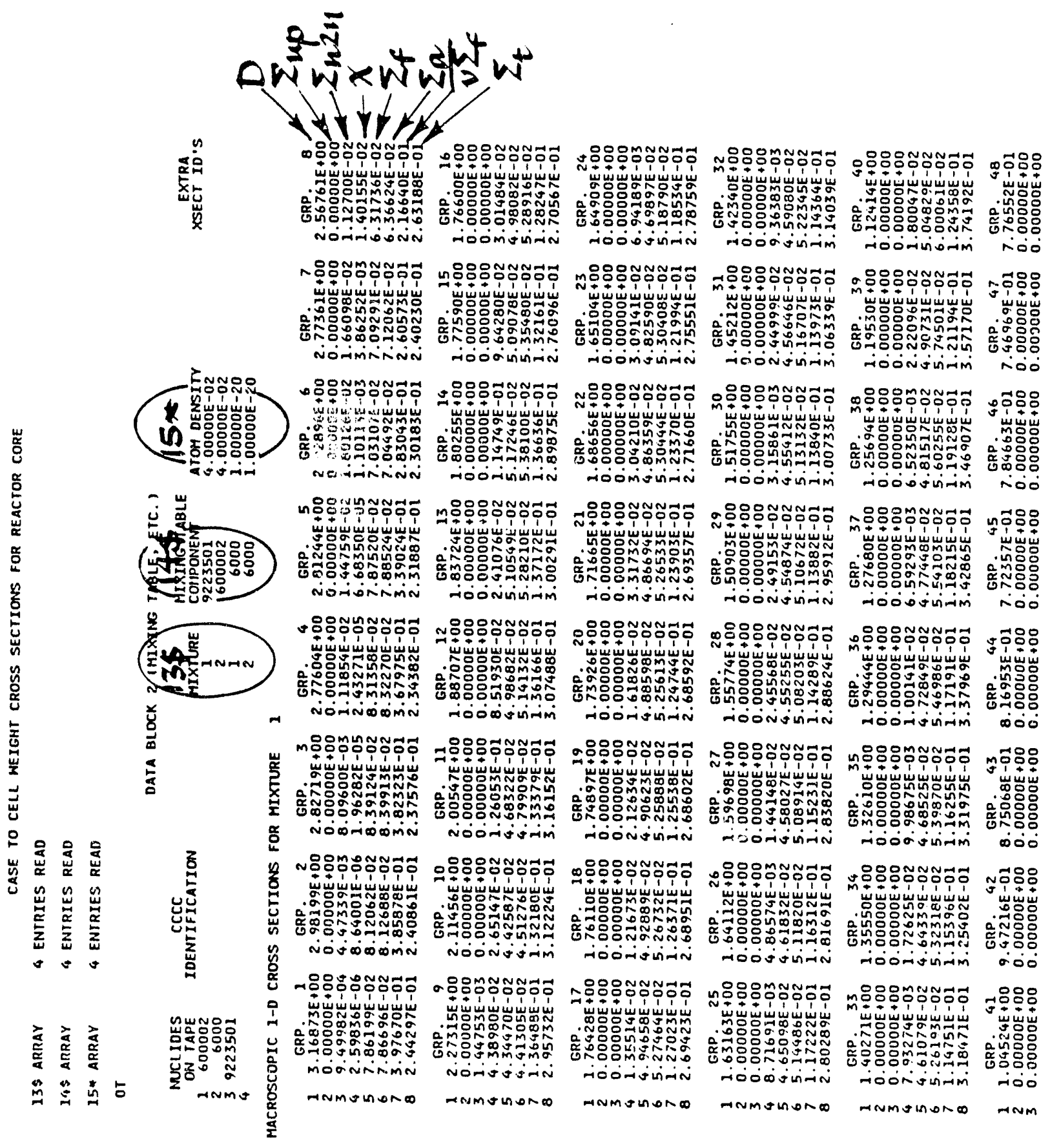




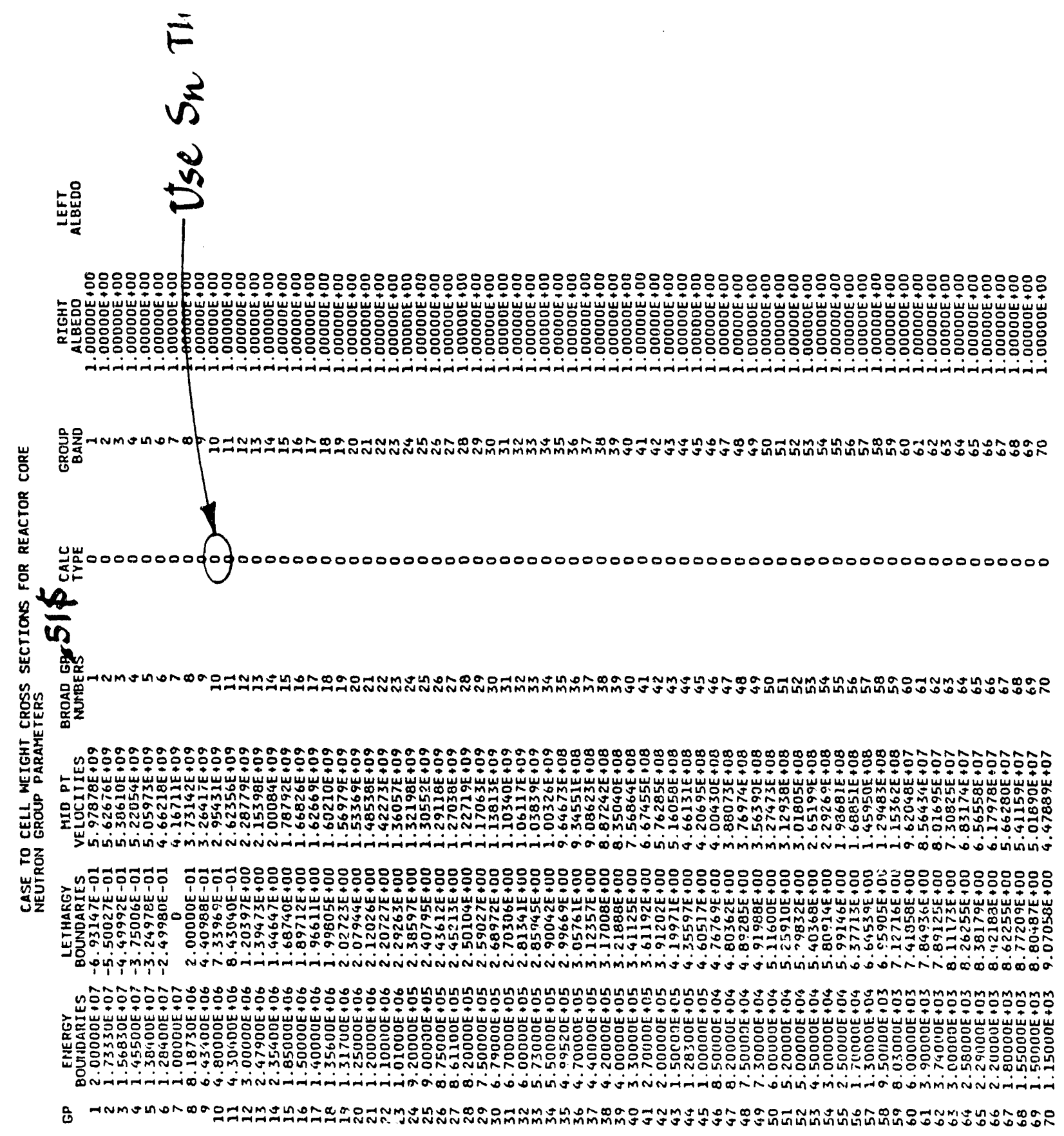




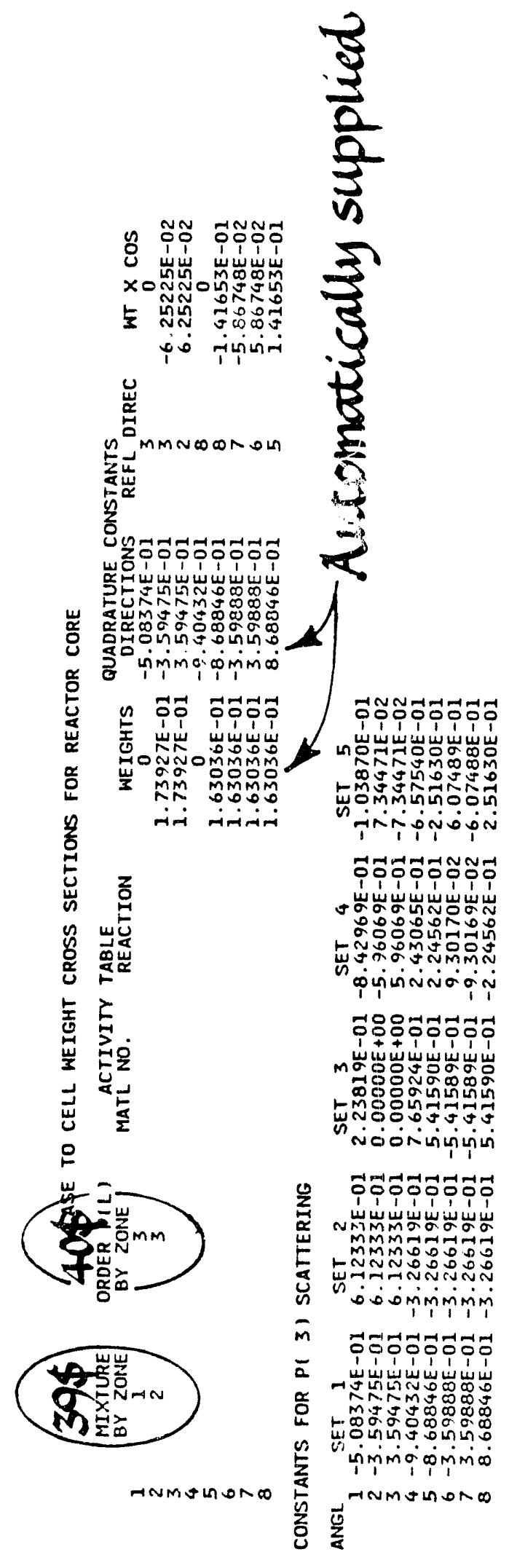


$\sum_{i=1}^{5}$
$\bar{u}$
n

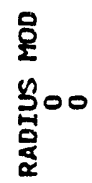

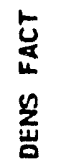

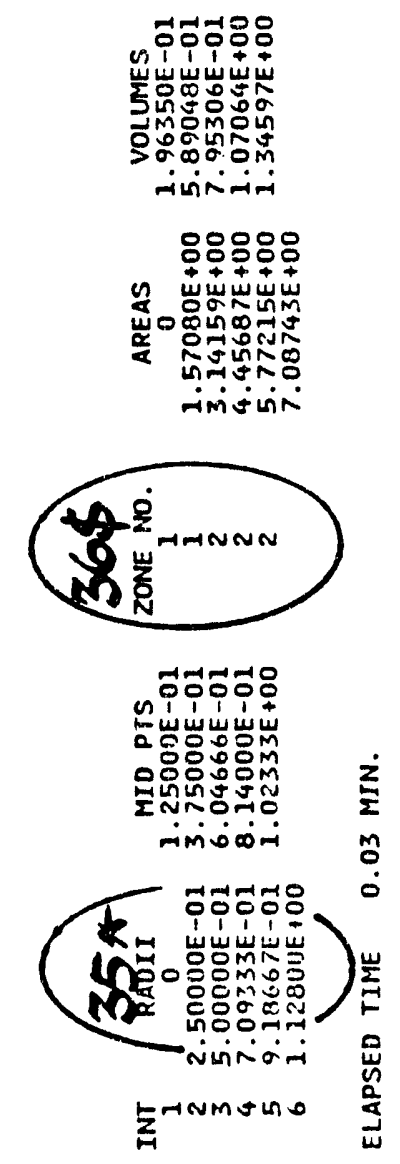

SAMPLE PROBLEMS 


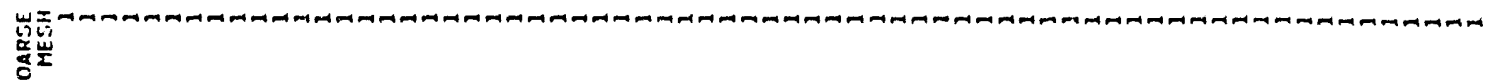

过

แ

迎它

$\dot{x}$

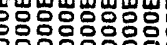

ini

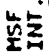

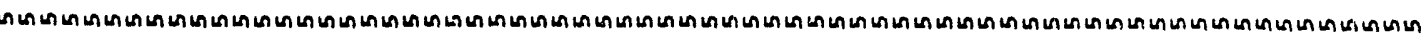

ว 三

3

$0 \dot{0} 0 \dot{0}=$

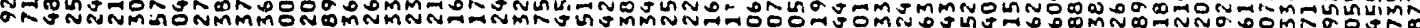

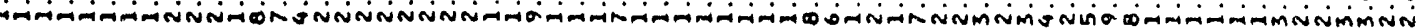

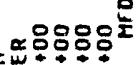

它

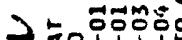

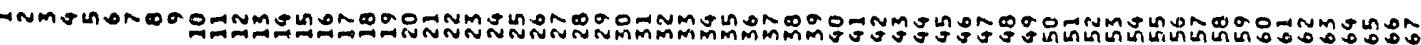

等

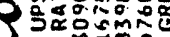

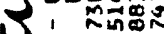

a onit

ะ 8กิ๊

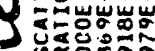

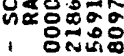

- inipi

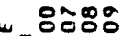

0

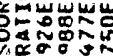

m몽

- inis

岁 유ㅇㅠㅜㅇ

บ2

- ininín

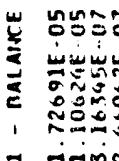

- rimín

xumen

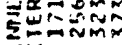

xerams

등

\section{SAMPLE PROBLEMS}




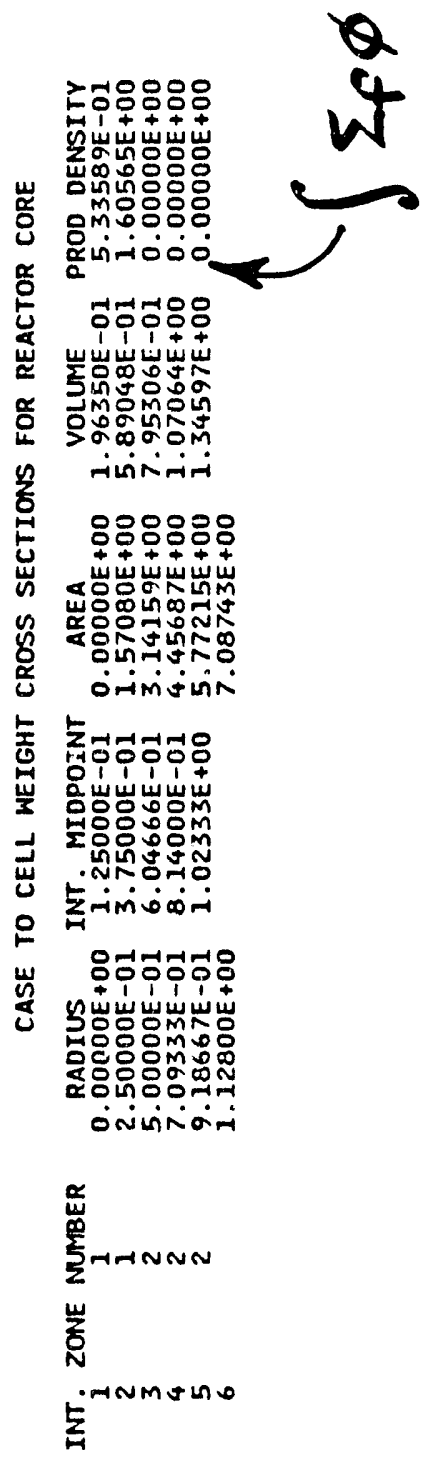



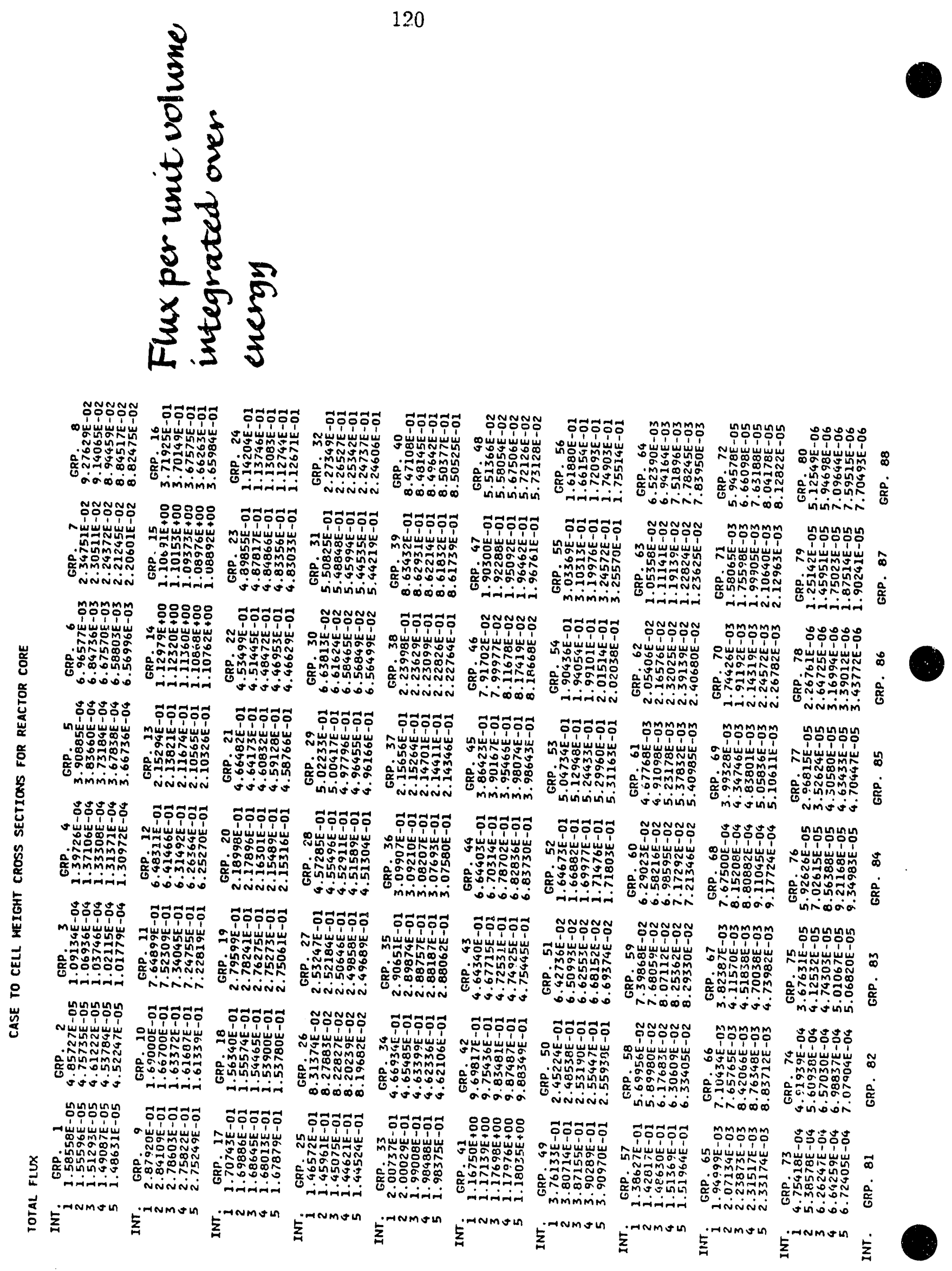

\section{SAMPLE PROBLEMS}


121

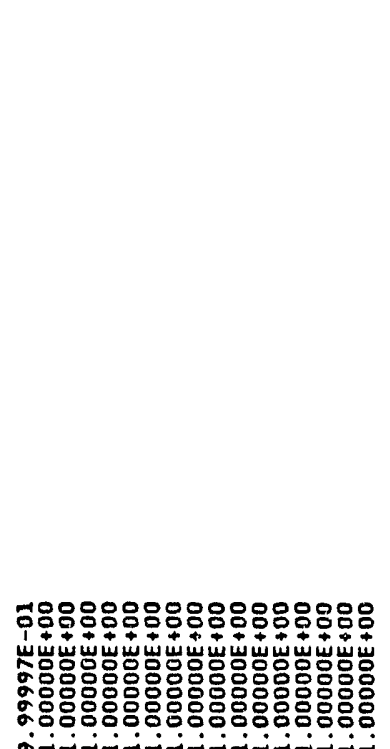

$$
\begin{aligned}
& \sum_{0}^{5} 5_{0}^{5}
\end{aligned}
$$

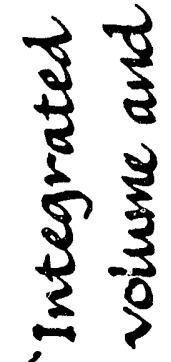

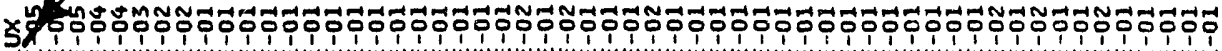

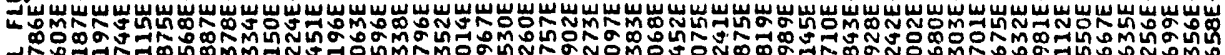
萡N

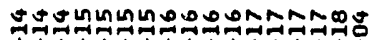

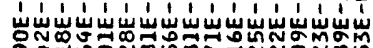

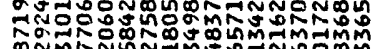

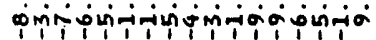

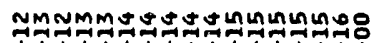

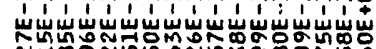
กี่ mิn

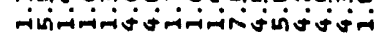

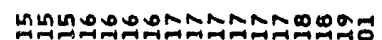

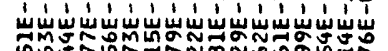
mo nôn

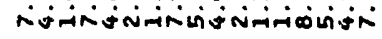

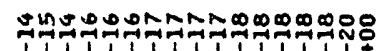

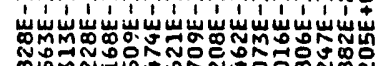

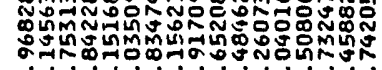

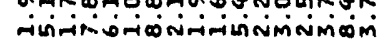

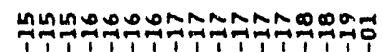

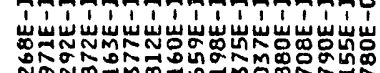

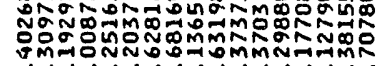

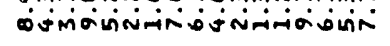

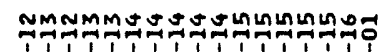

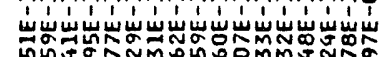

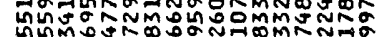

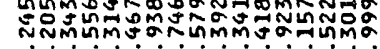

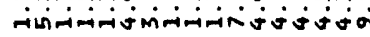

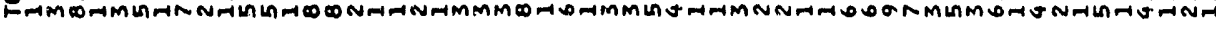

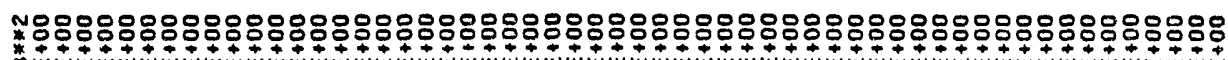

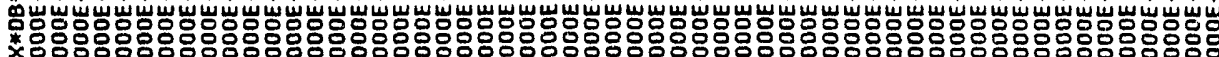

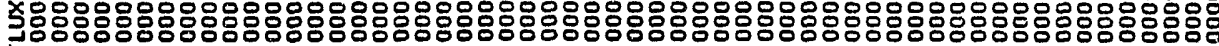
u0000000000000000000000000 00000000000000000000000000000000000

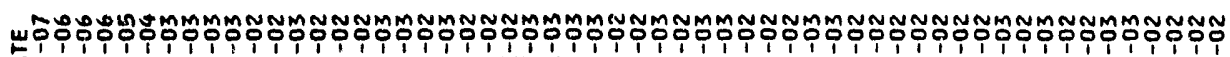

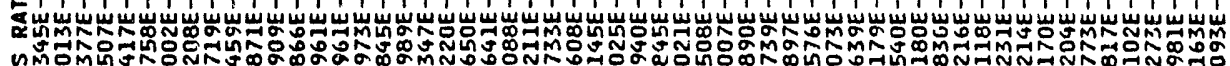
ผै

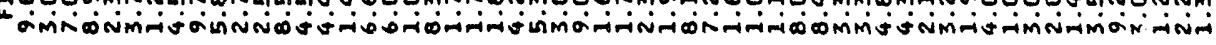

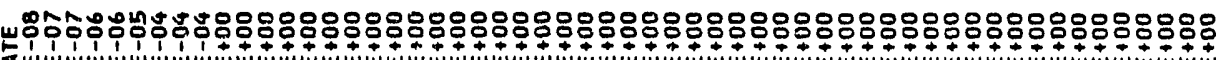

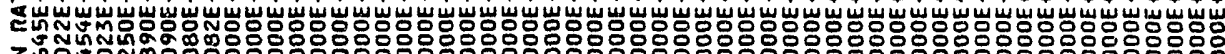
承触

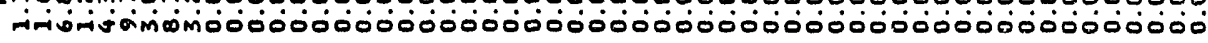

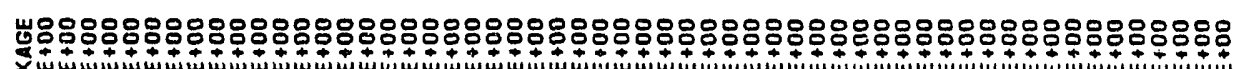

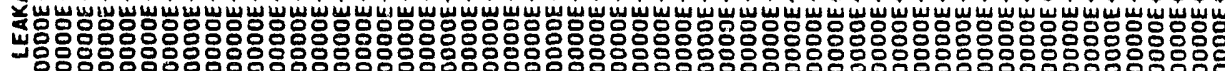

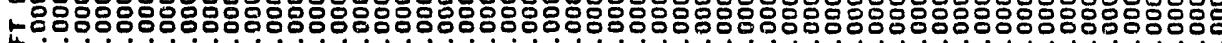
J0000000000000000000000000000000000000000000000000000000

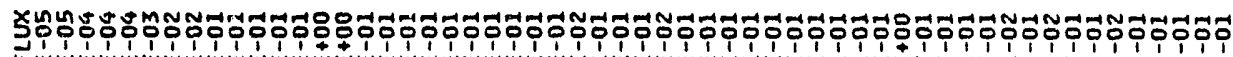
4.

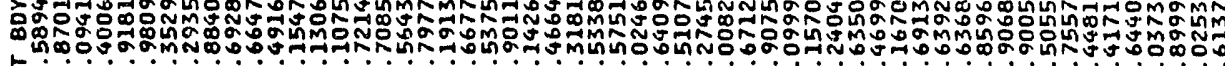

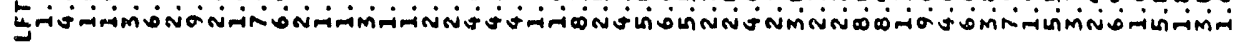

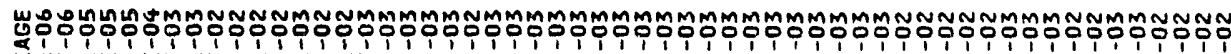
3 tô.

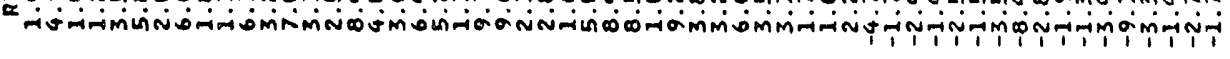

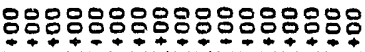

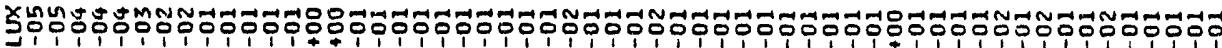

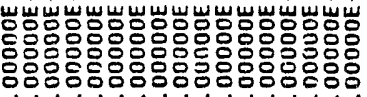

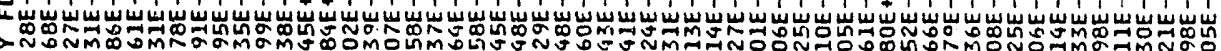

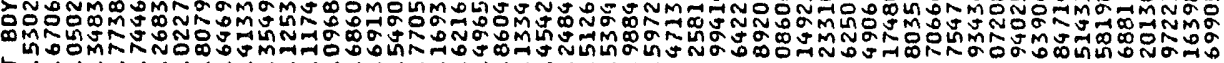
D000000000000000000

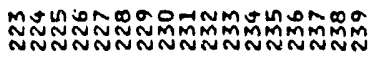

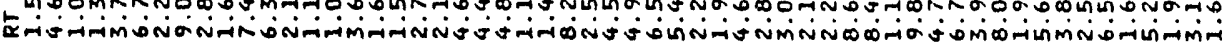
范

\author{
SAMPLE PROBLEMS
}




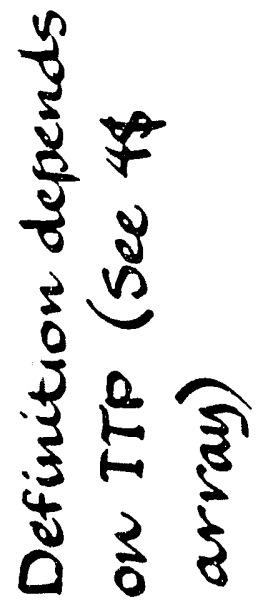

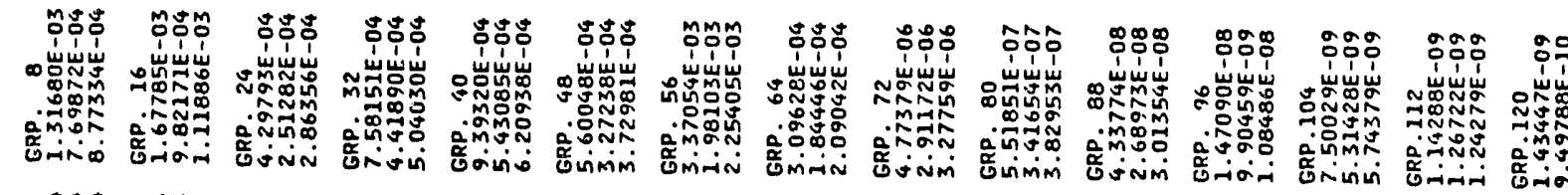

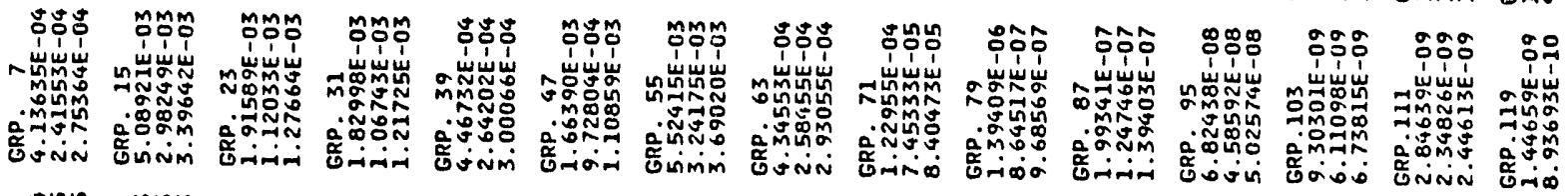

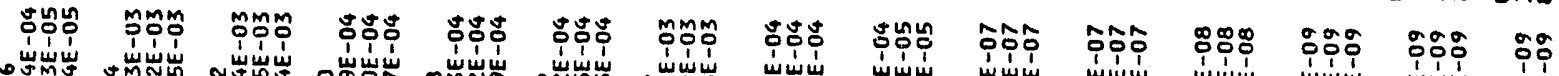

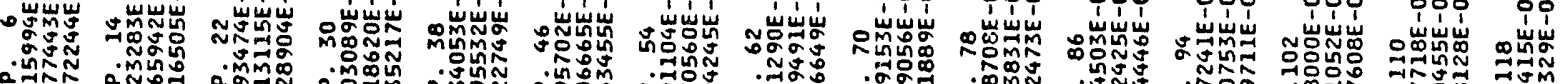

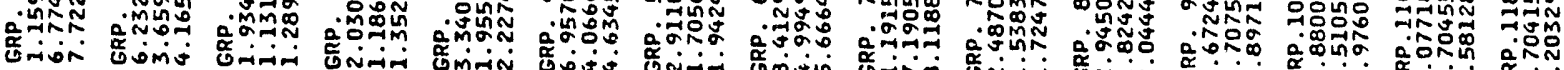

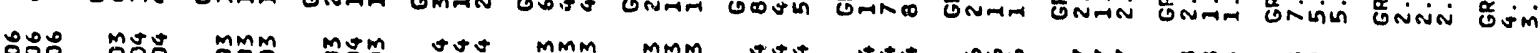

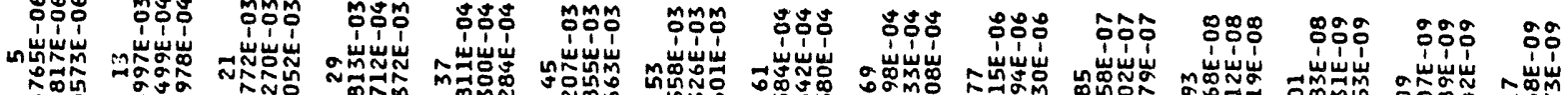

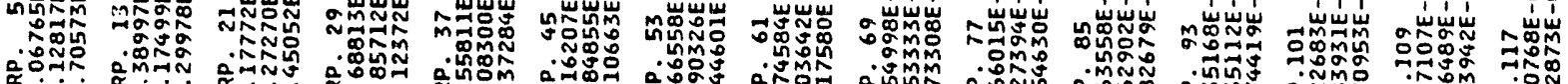

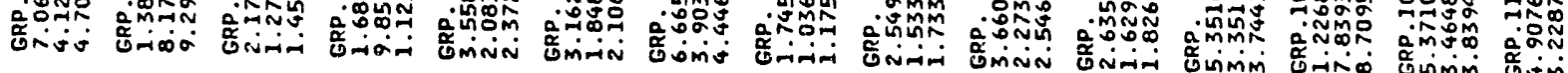
遇 mpm

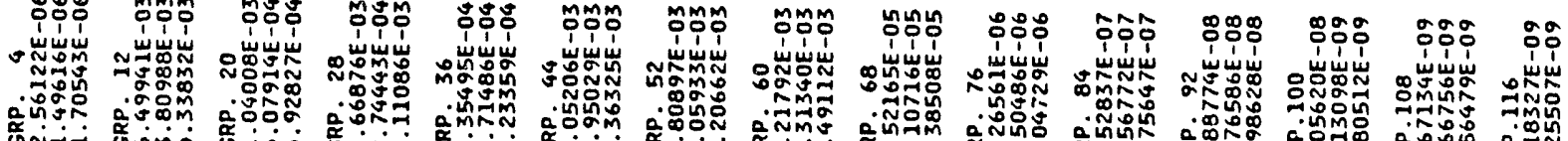

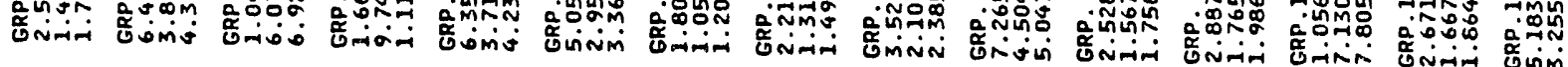

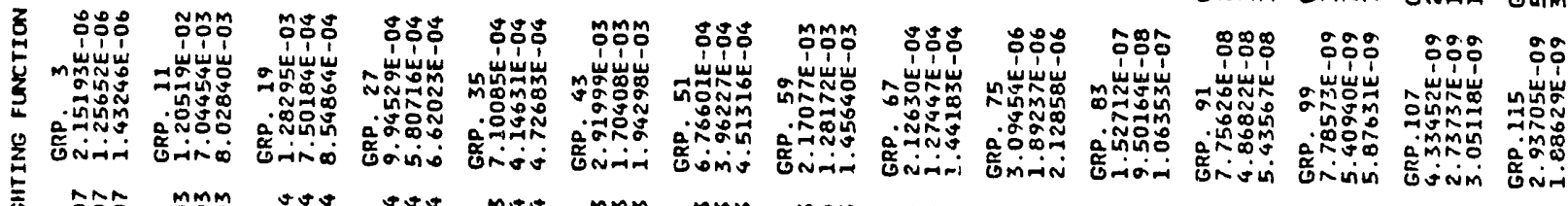

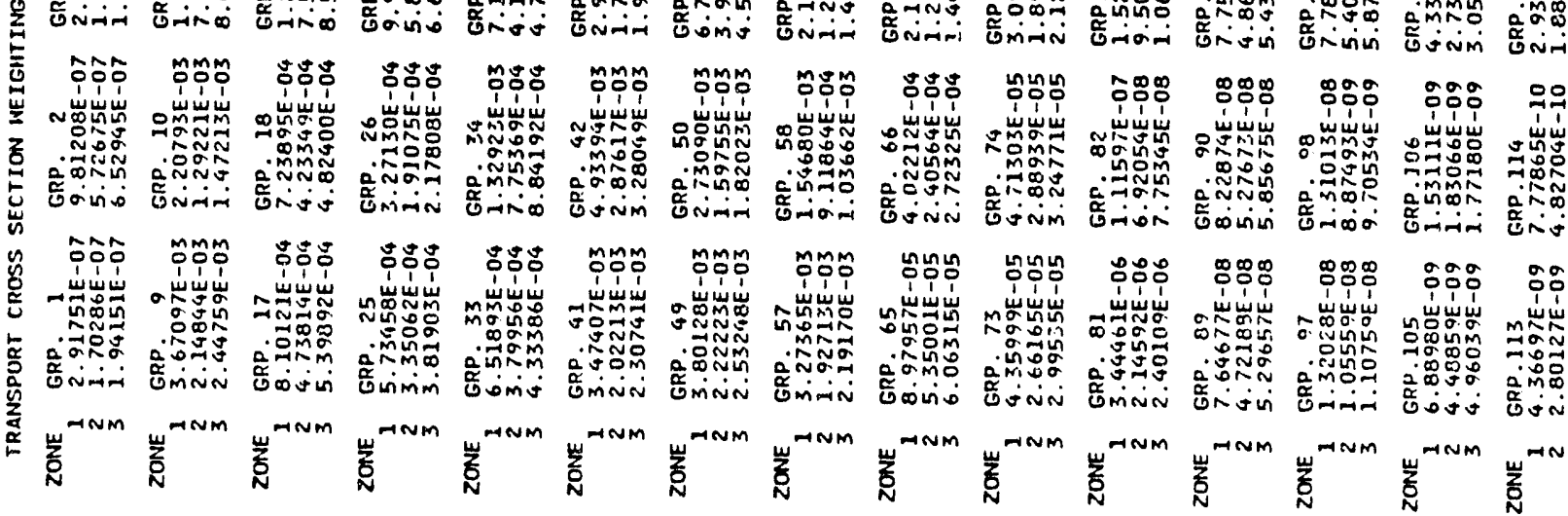




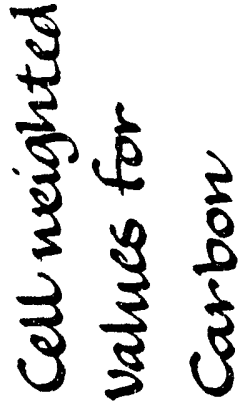

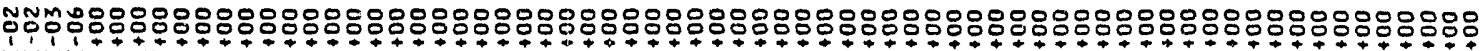

$\stackrel{m}{\stackrel{0}{\circ}}$

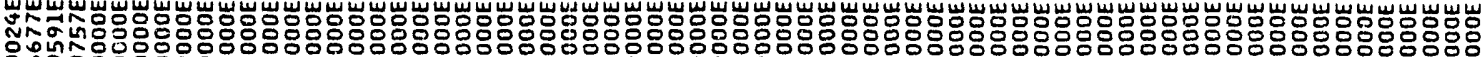

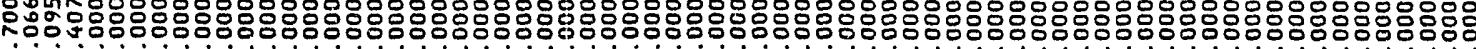
Tinmo00000000000000000000019000000000000000000000000000000000000000000

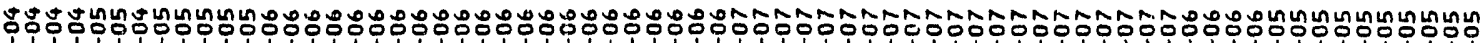

赵

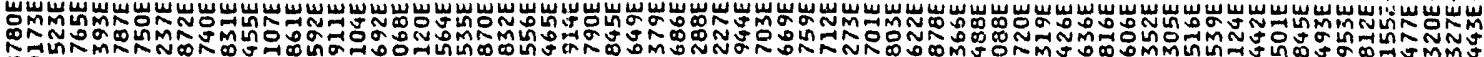

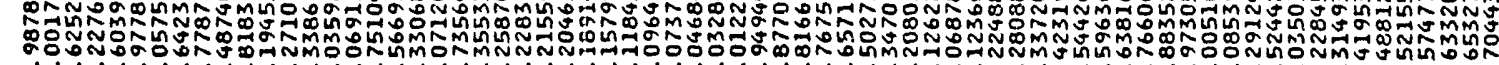

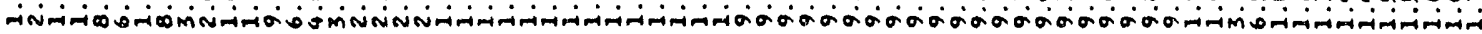

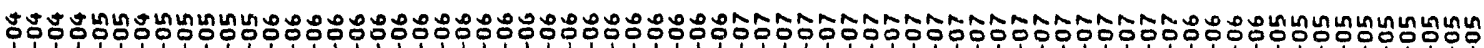

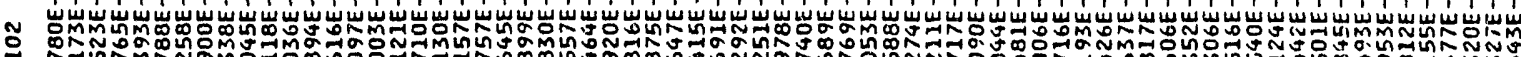

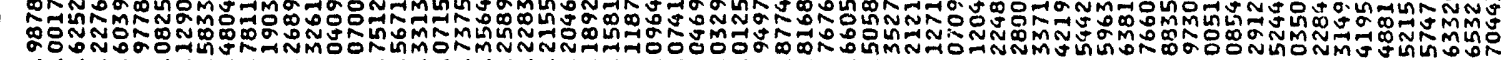

葛

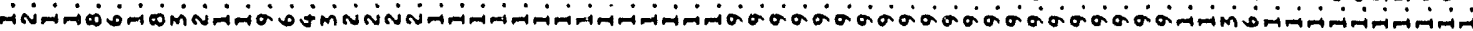

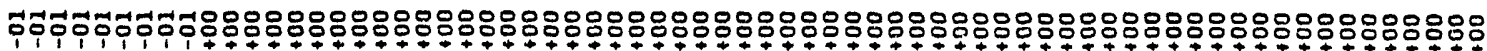

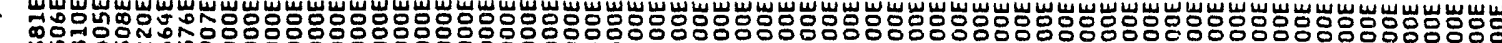
n̂n.

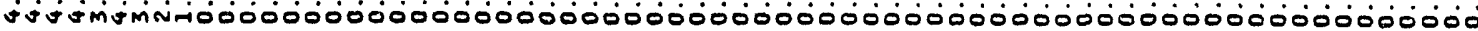

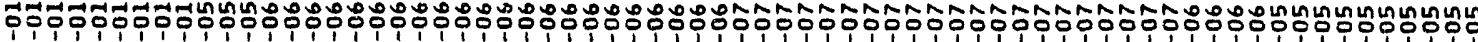

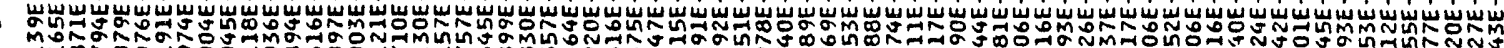
Mี

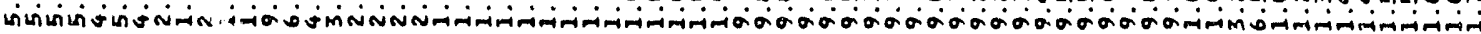

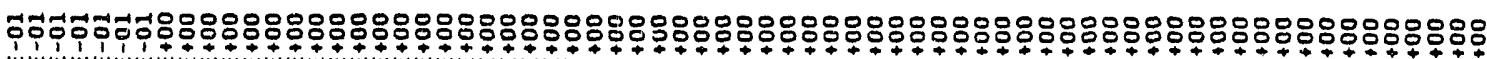

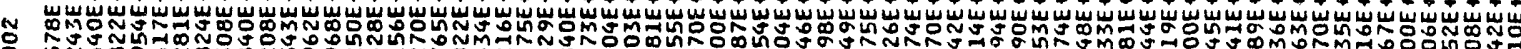

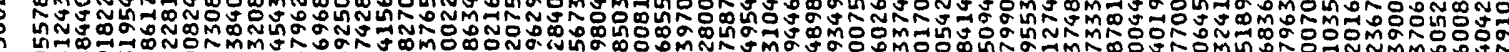

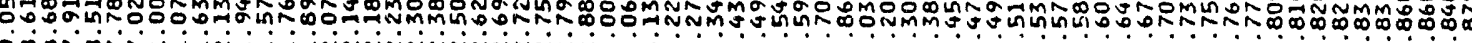

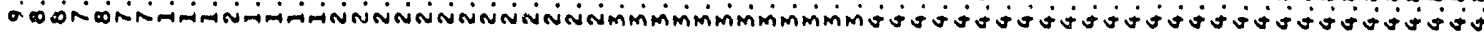

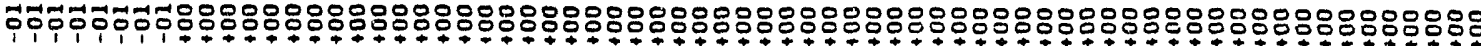

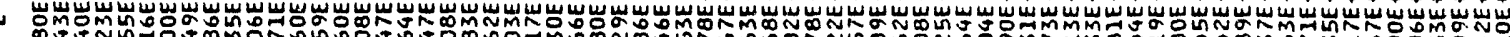

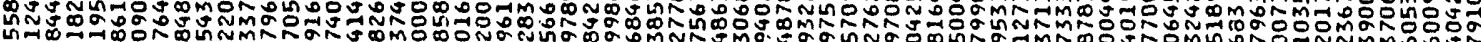
Uึ兀

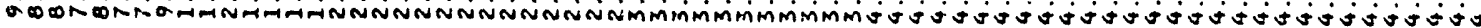

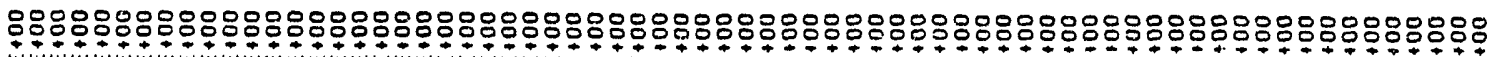

$\rightarrow$

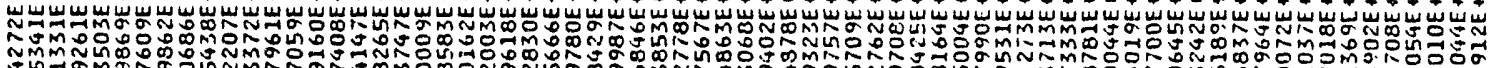

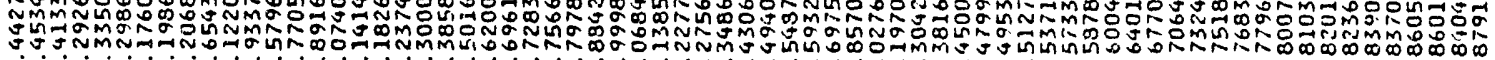
온

嵌最 


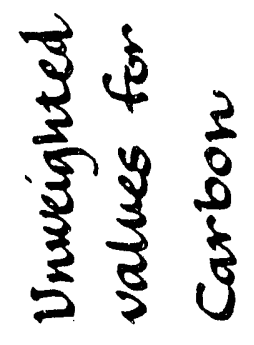

ำ

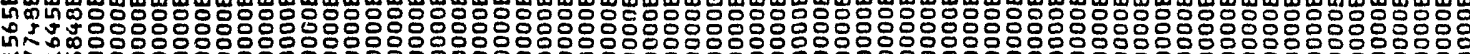

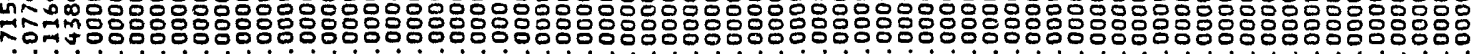
Amino0000000000000000000000000000000000000000000000000000000000000000

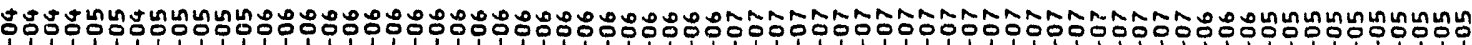

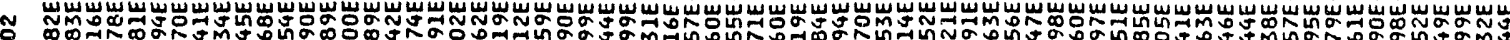

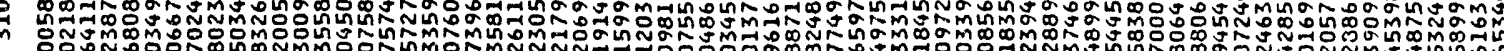

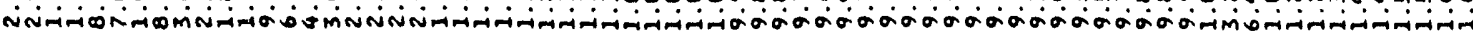

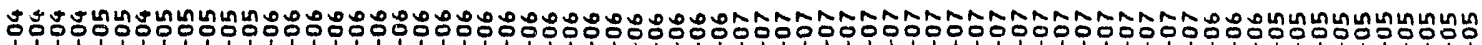

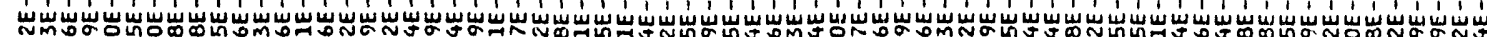
○

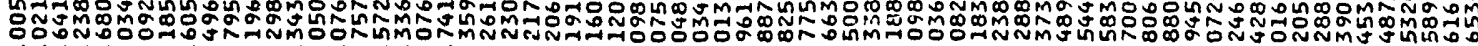
Niñ

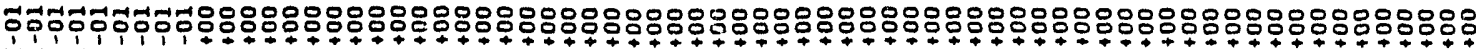

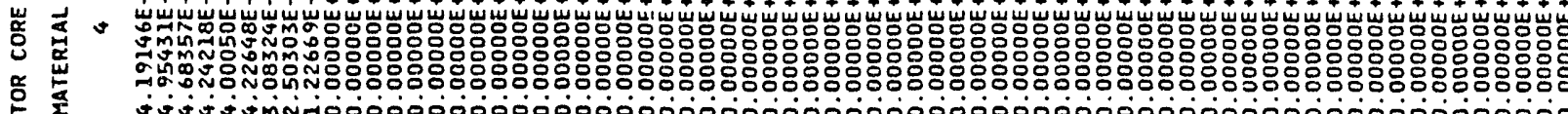

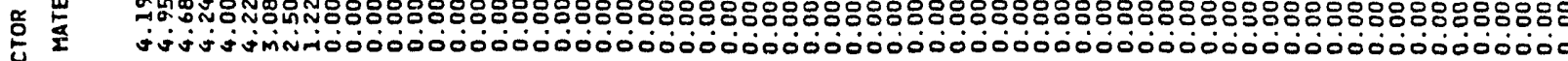

要

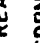

尊

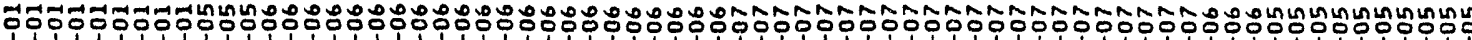

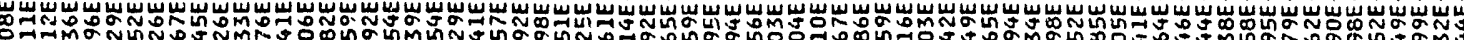

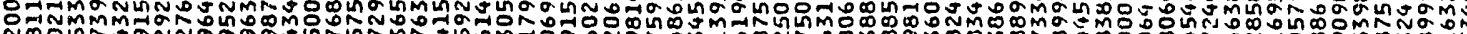

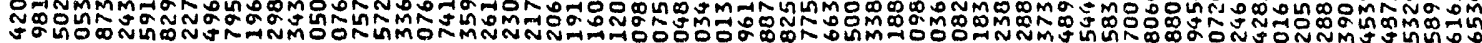

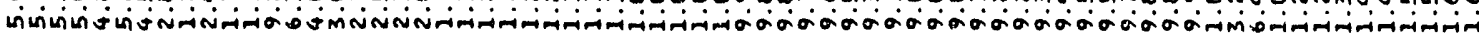

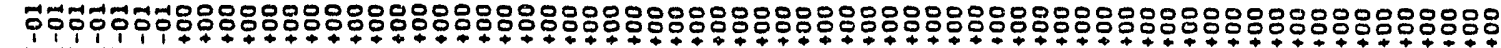

空

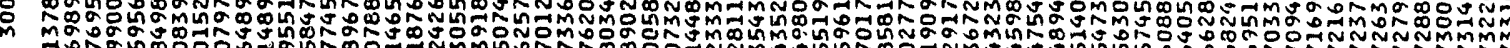

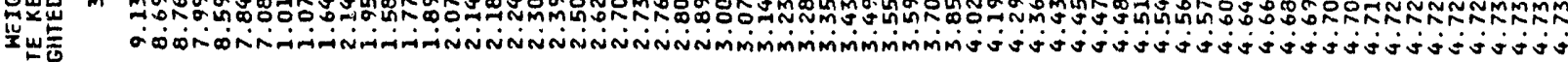

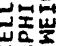

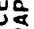

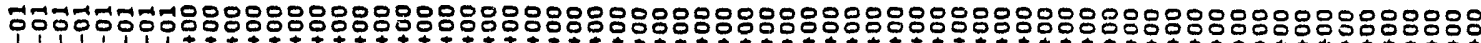

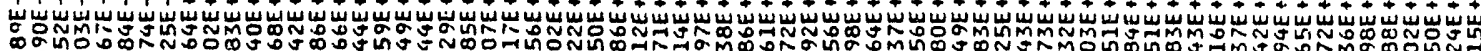
等

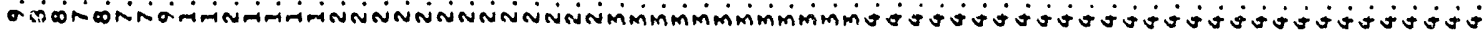

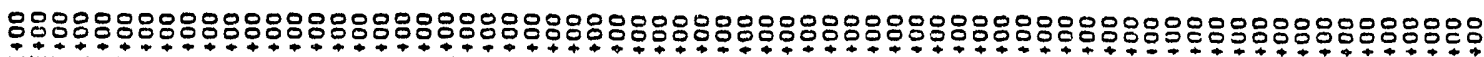

$\rightarrow$

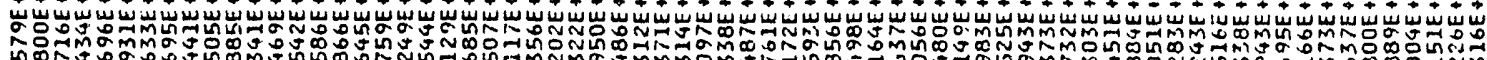

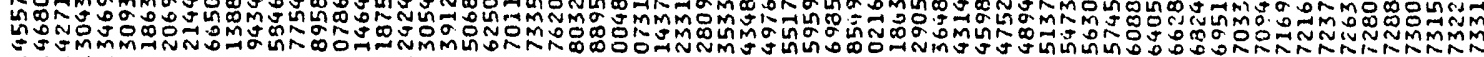

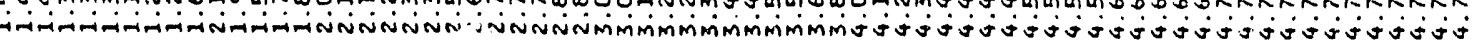
요

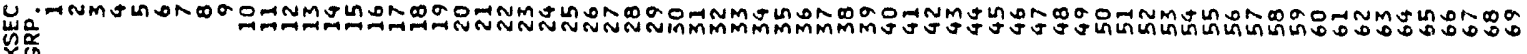

\section{SAMPLE PROBLEMS}




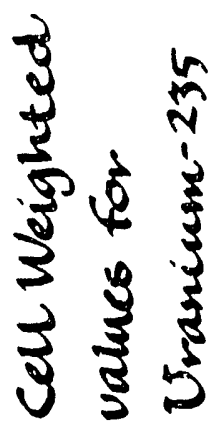

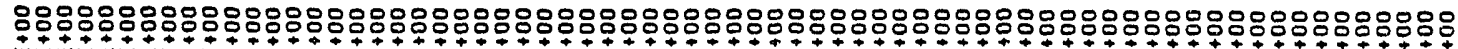

$\stackrel{\infty}{\circ}$

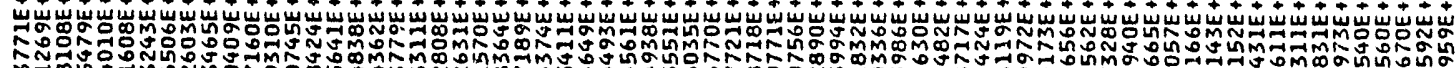
m.

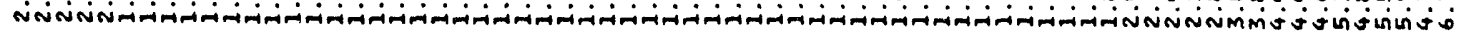

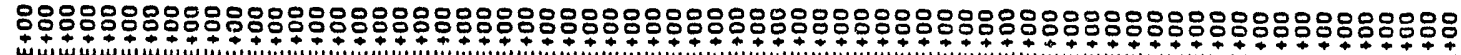

$\stackrel{\infty}{\infty}$

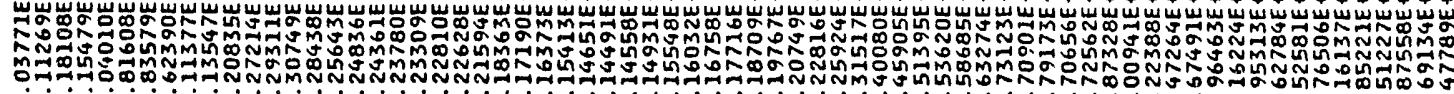

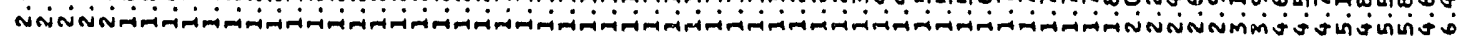

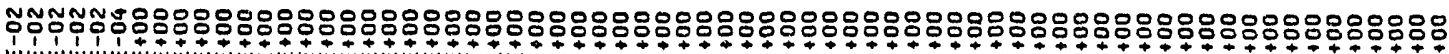

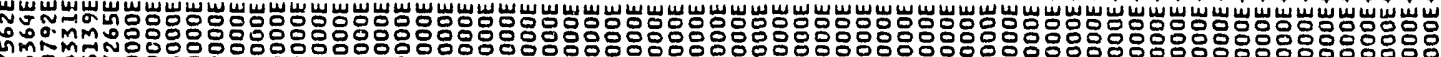

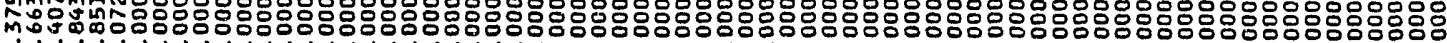

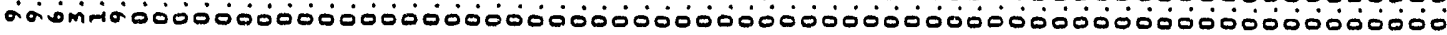

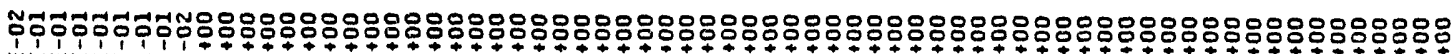

m

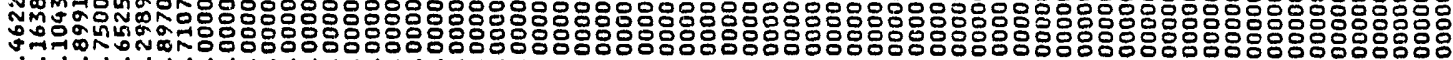

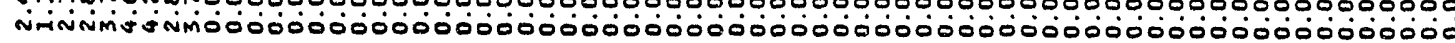

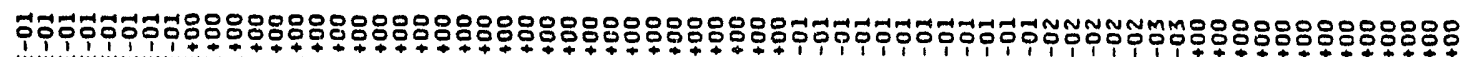

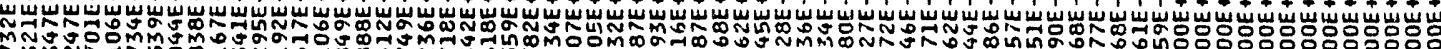

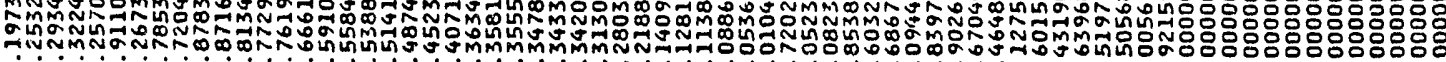

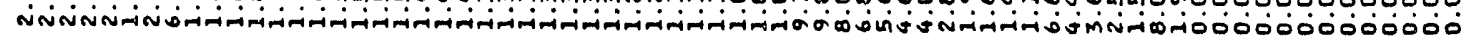

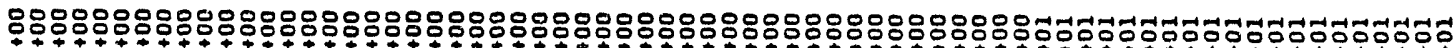

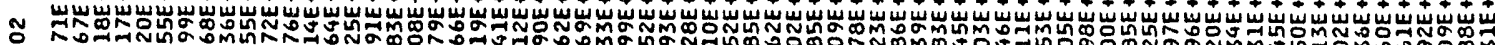

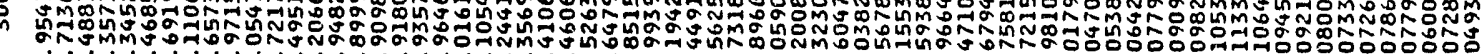

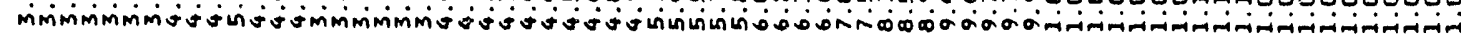

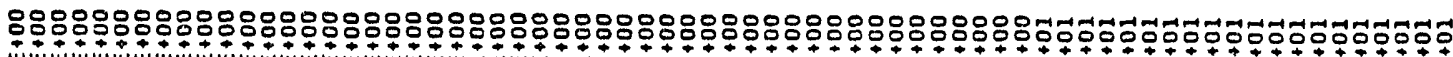

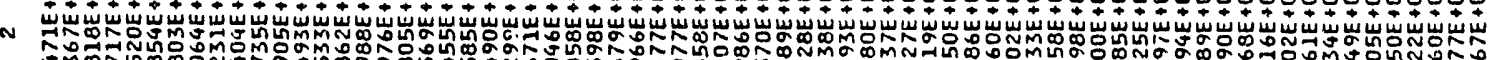

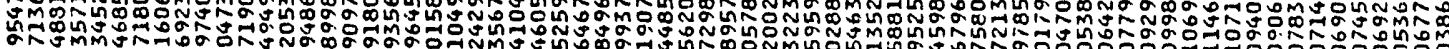

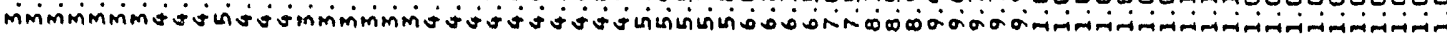

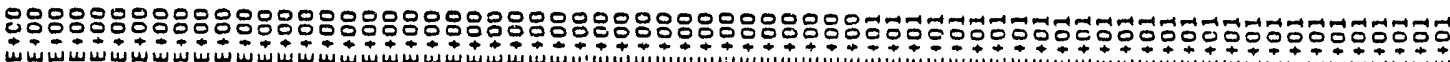

$\rightarrow$

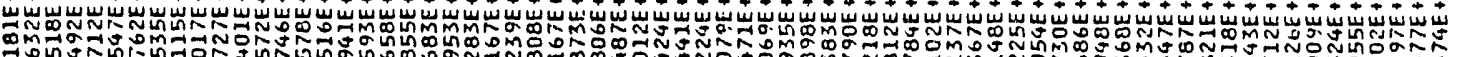

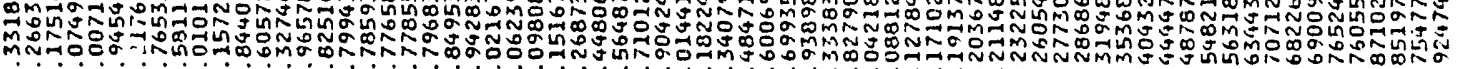

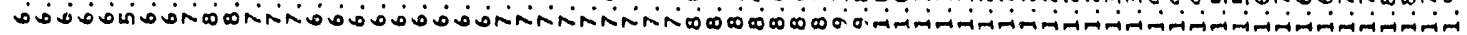
요

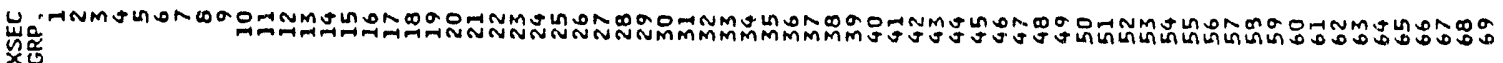



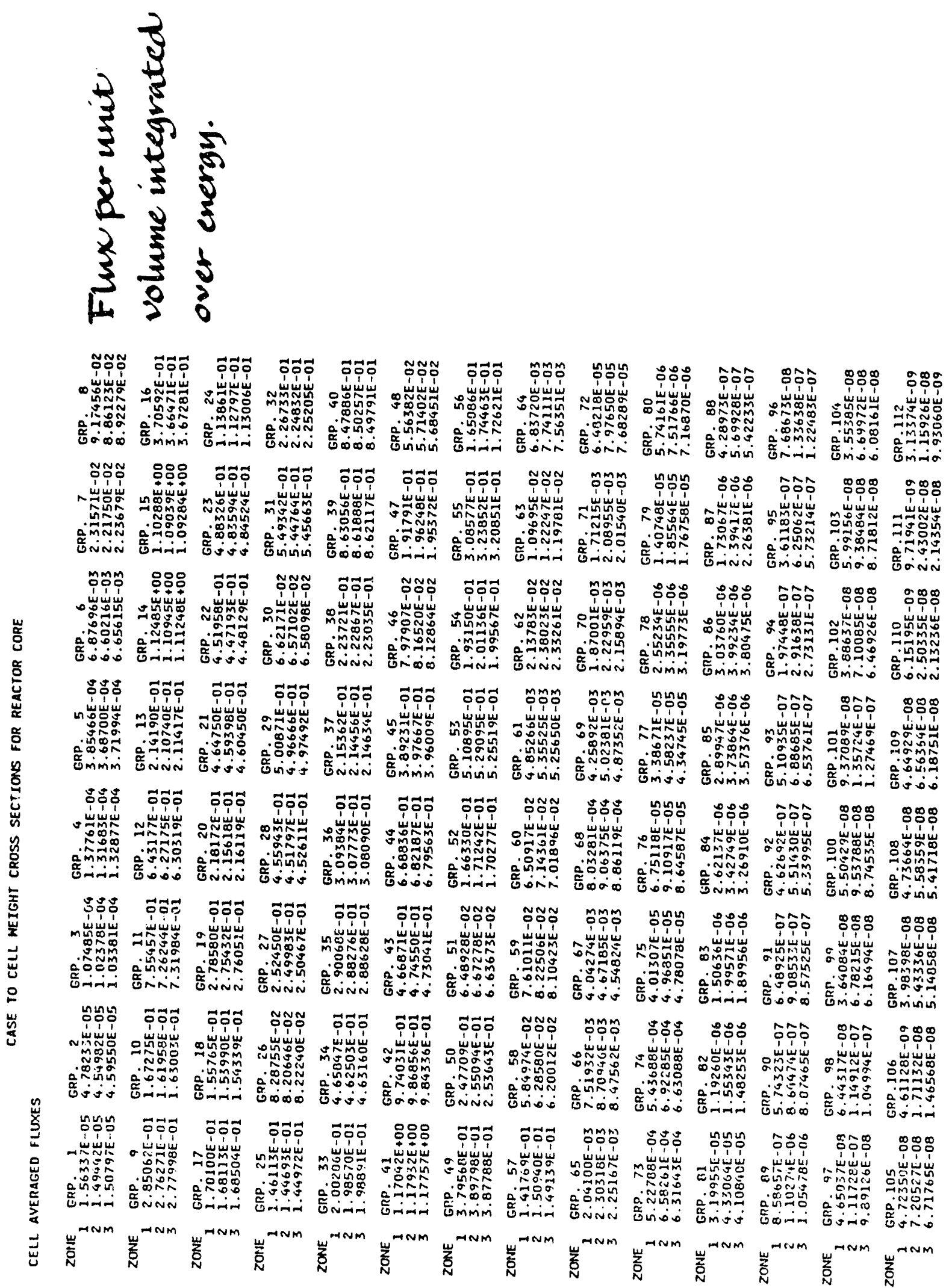

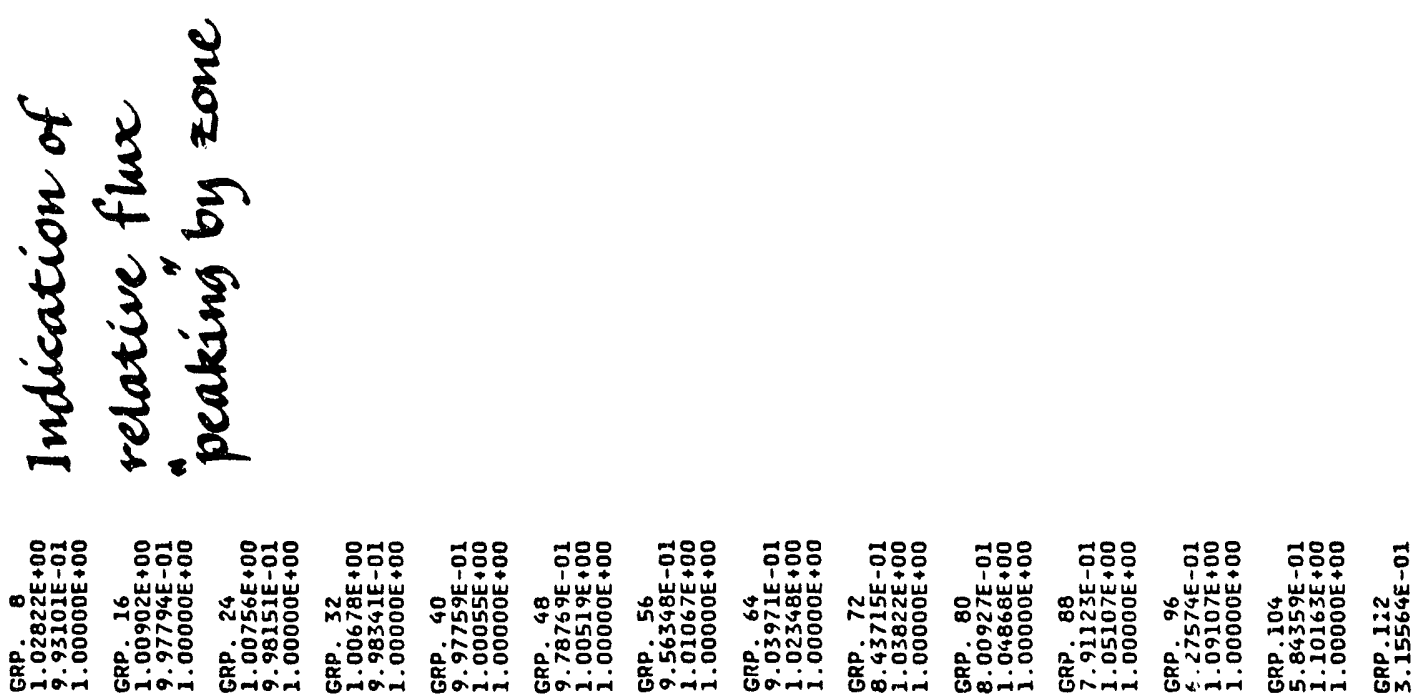

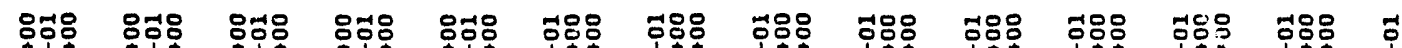

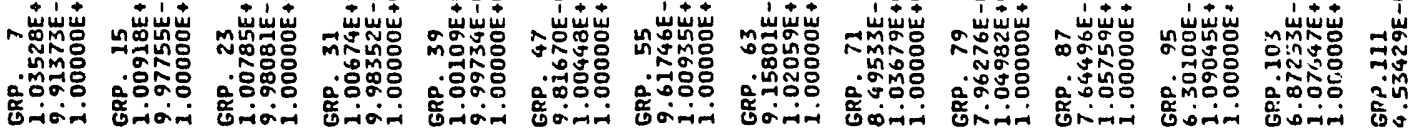

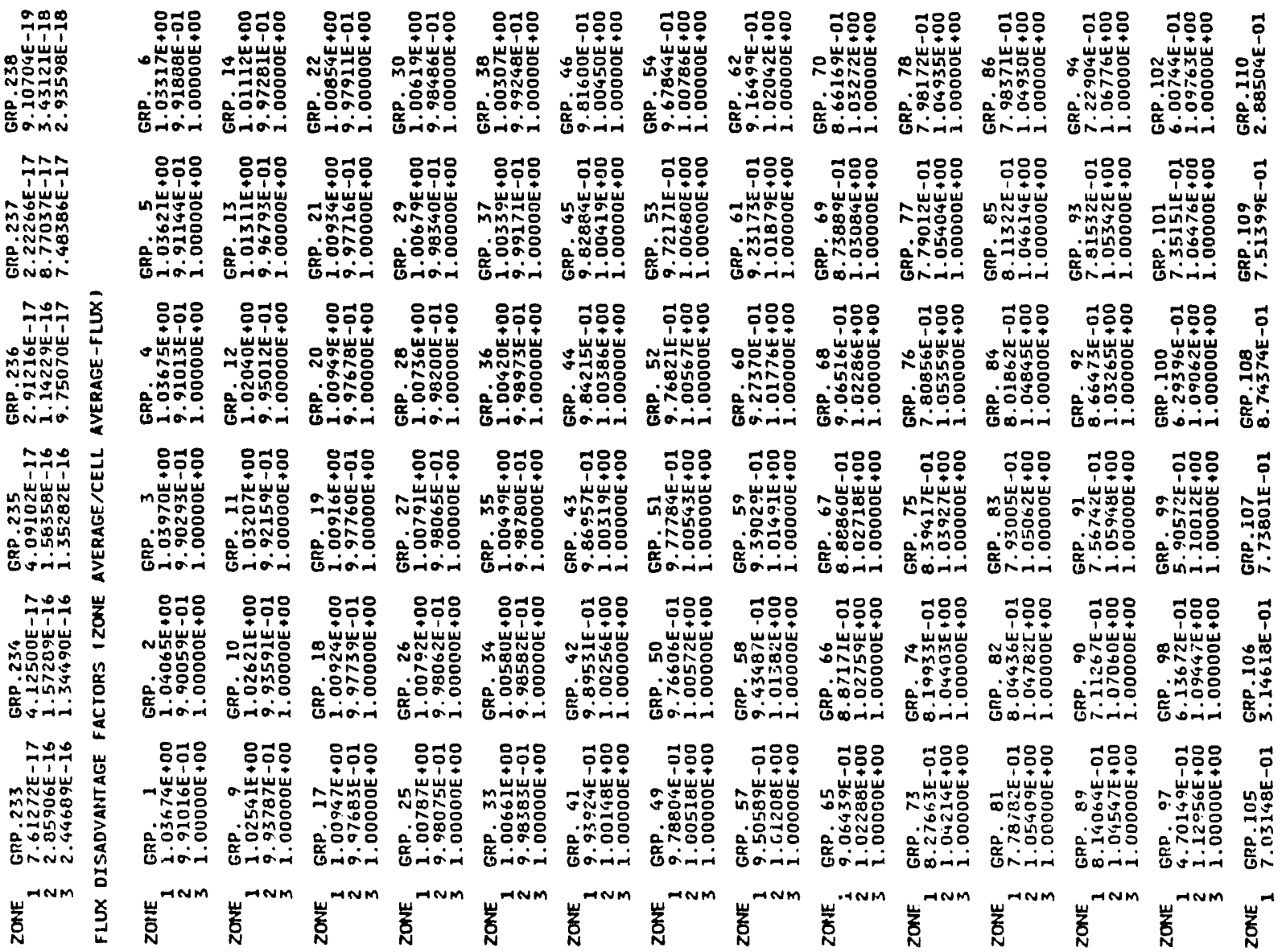



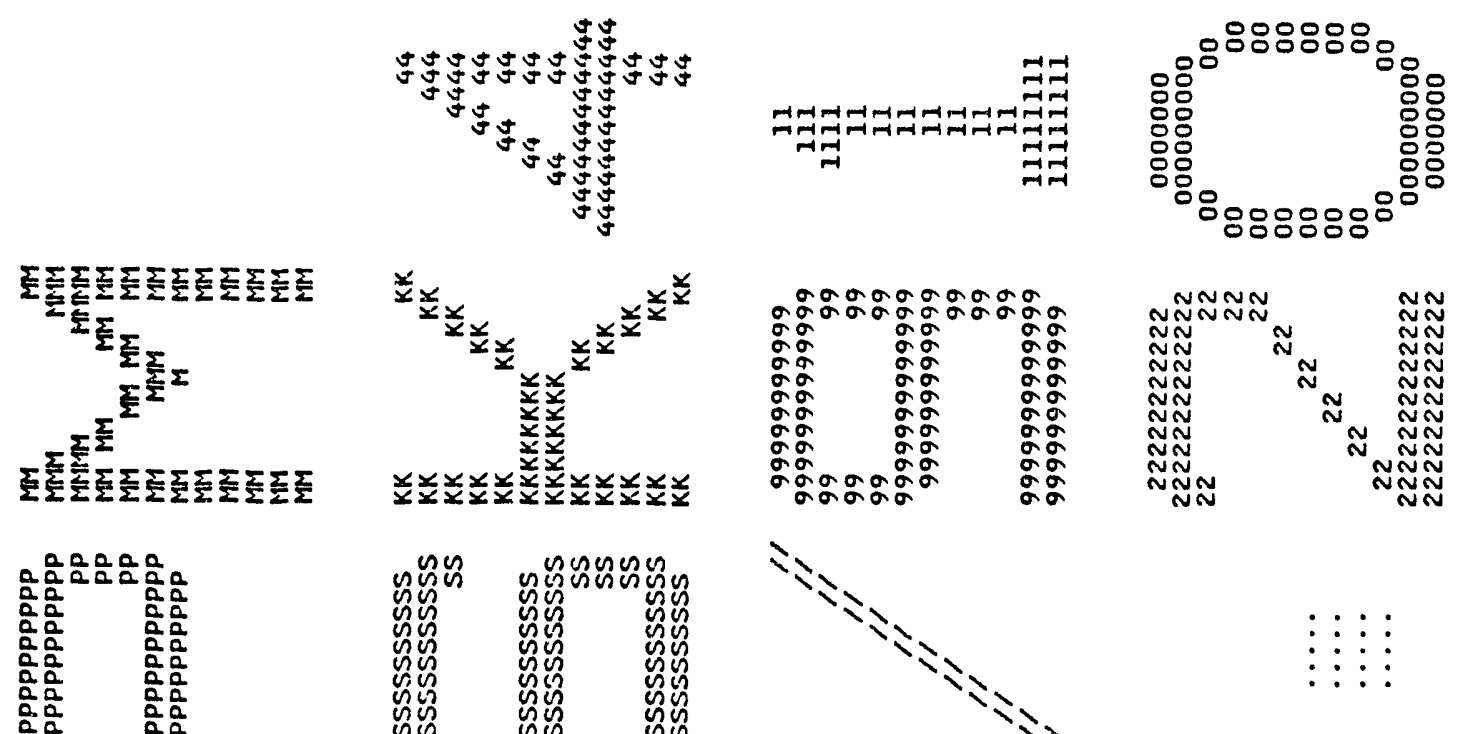

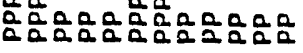
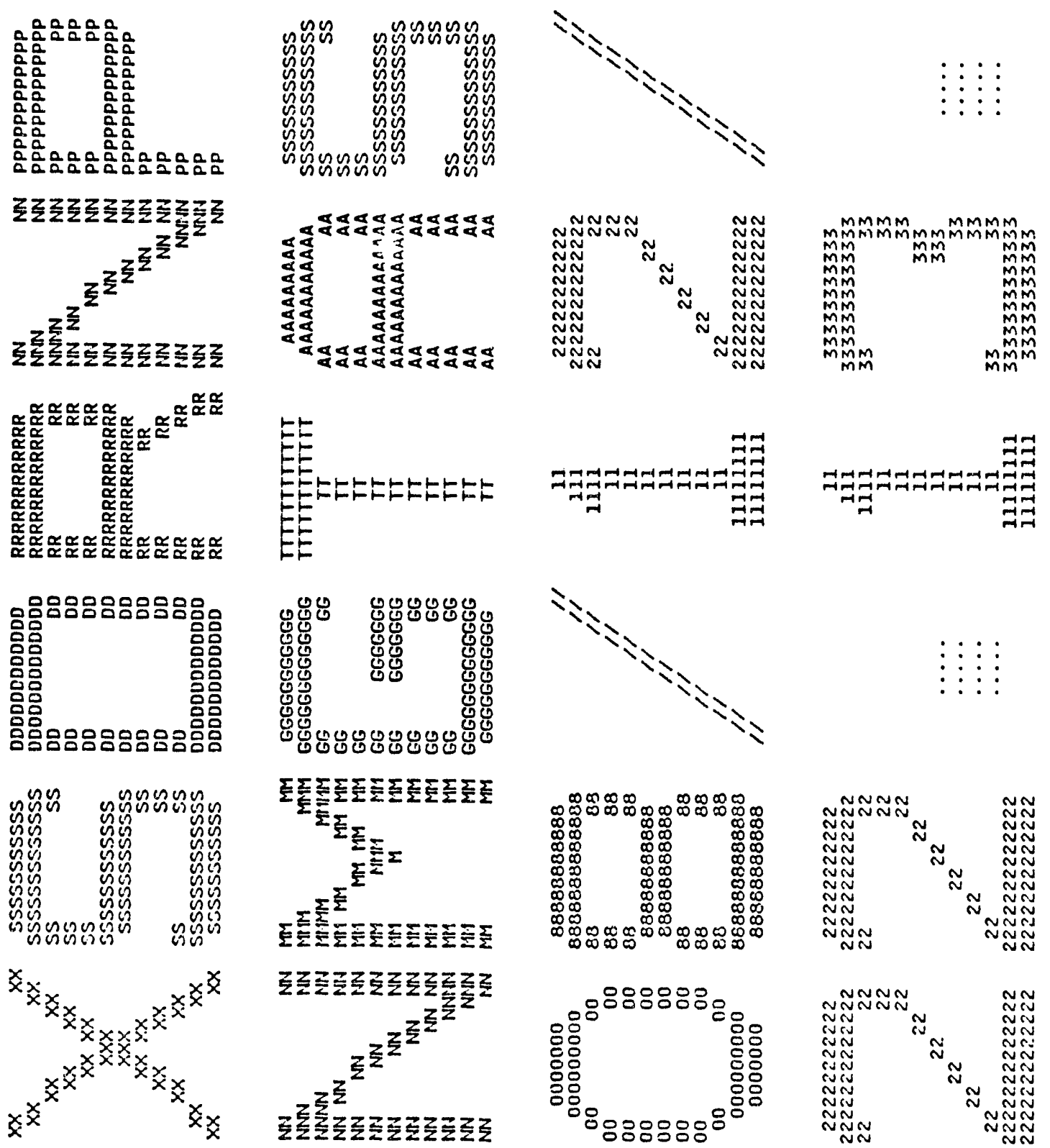


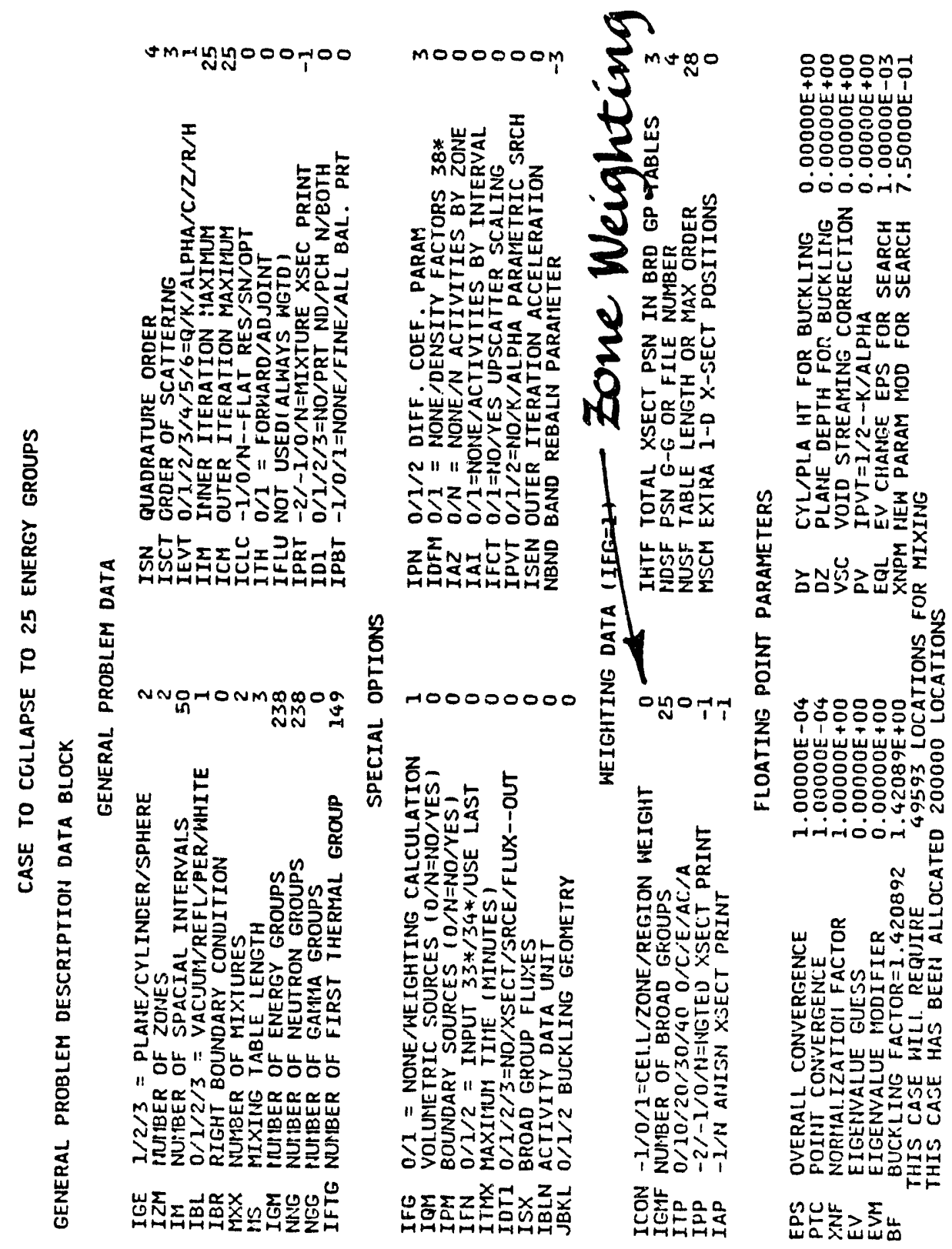




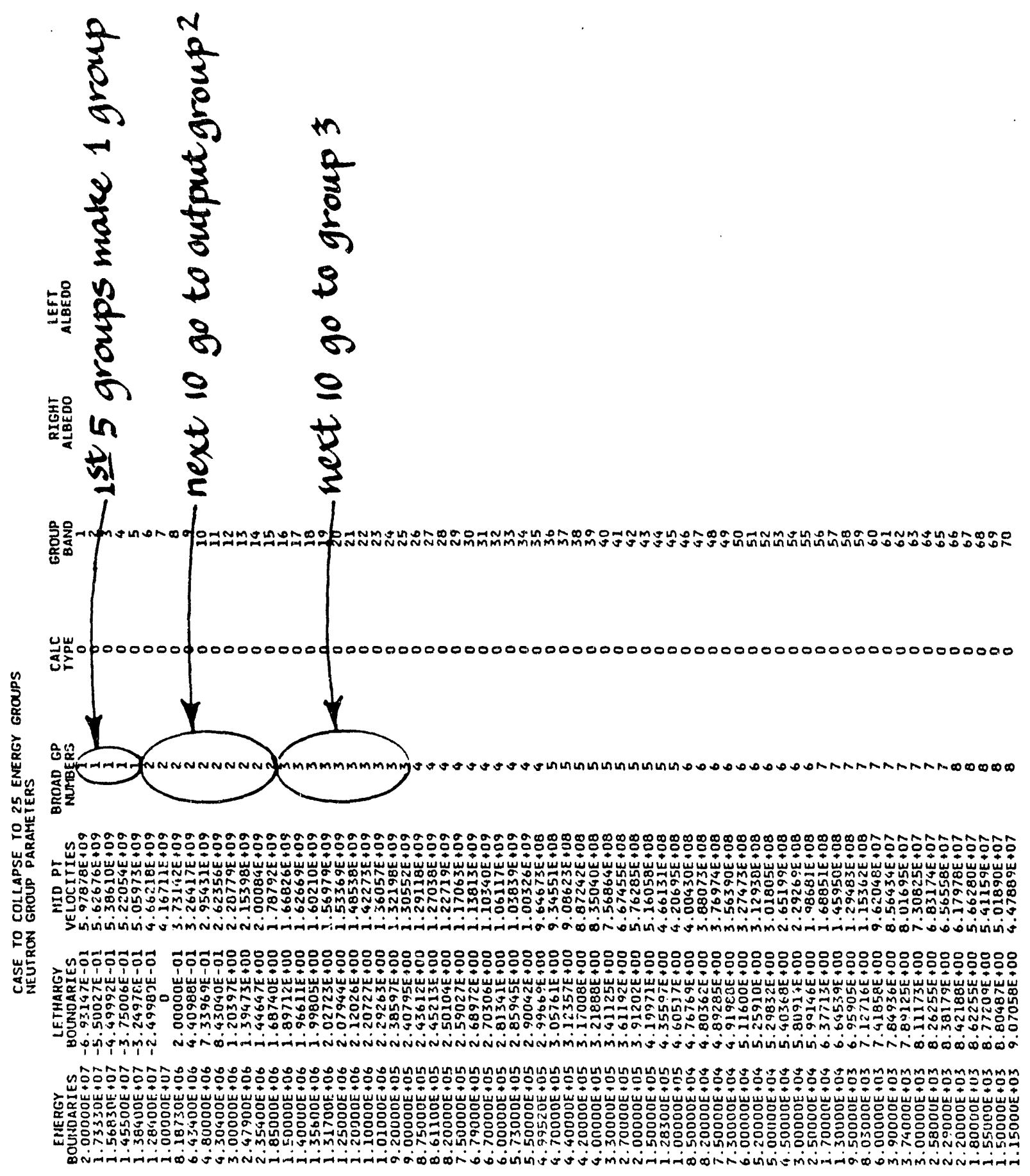

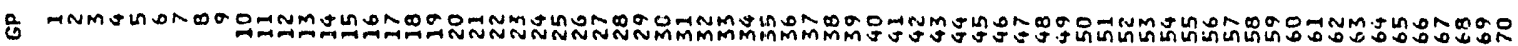

\section{SAMPLE PROBLEMS}



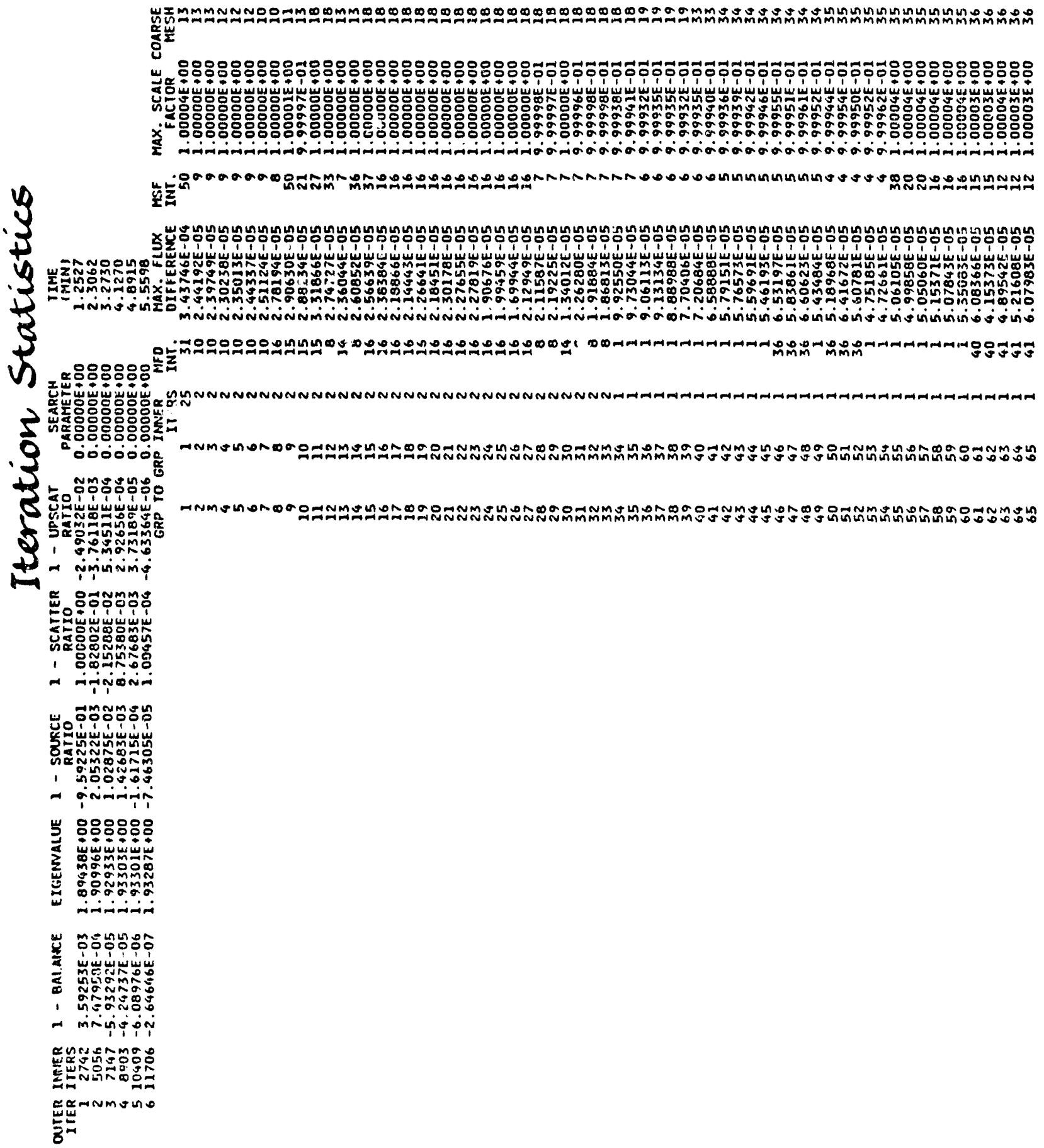


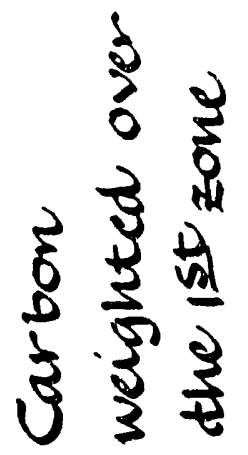

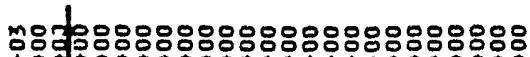

m

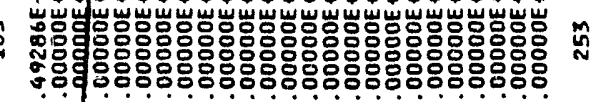
(1)

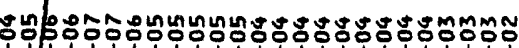

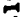

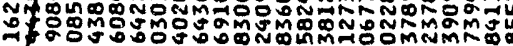
Fi (5)

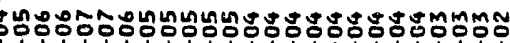

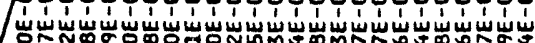

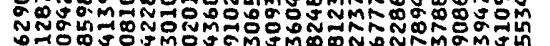

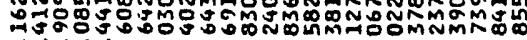

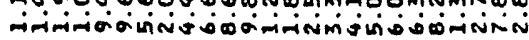

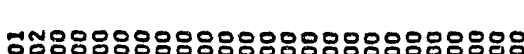

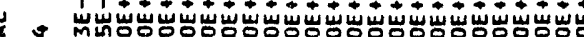

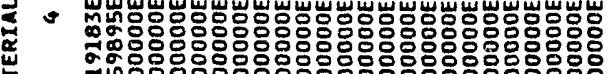

-

* 1000000000000000000000000

咅

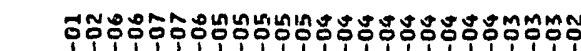

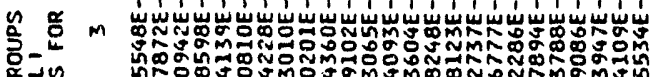

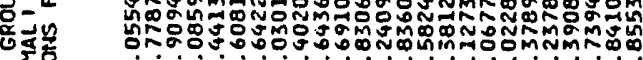

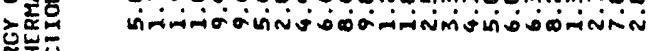

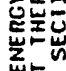

why

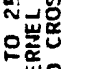

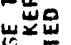

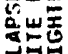

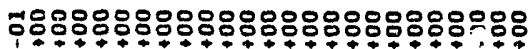

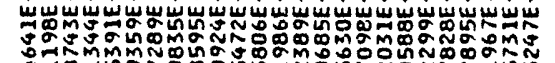

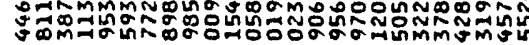

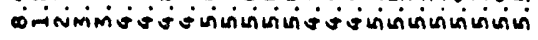

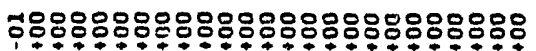

N

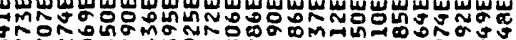
与ั0

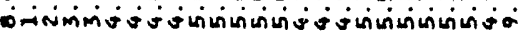

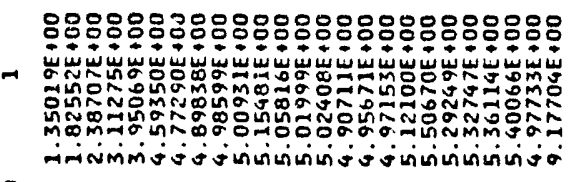

a

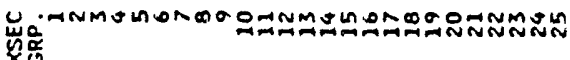

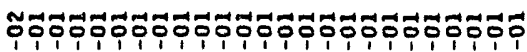

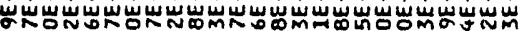

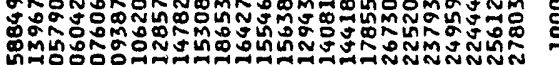

促

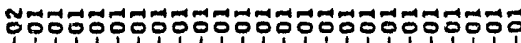

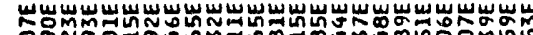

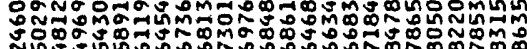

○rindina

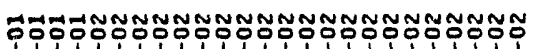

5

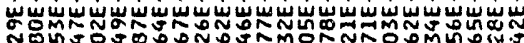

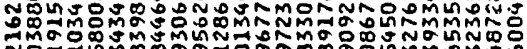
(1)

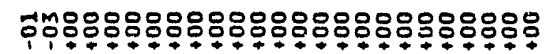

仓

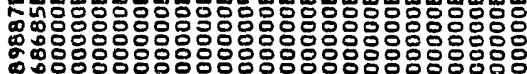

nNi00000000000000000000000

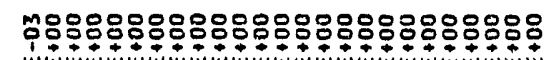

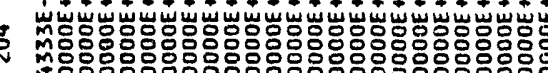

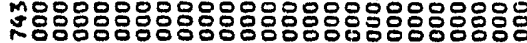

mó00000000000000000000000

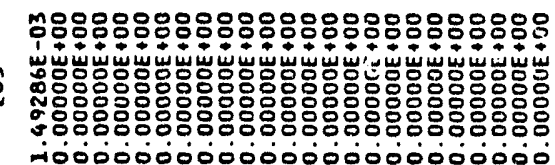

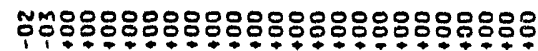

o

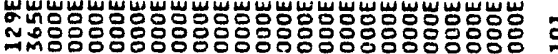

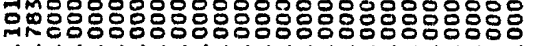

1000000000000000000000000

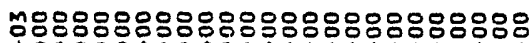

官

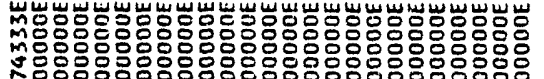

mo a

岁:

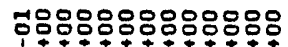

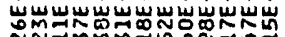

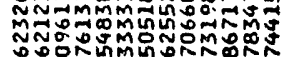

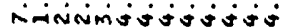

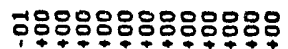

¿ w.

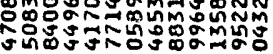

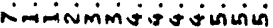

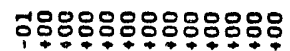

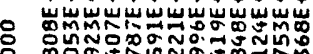

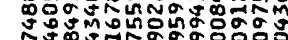
orininiminiminiso ós

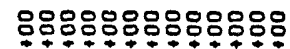

\%

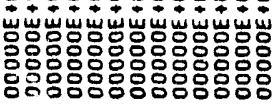

00000000000000

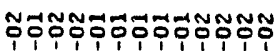

。

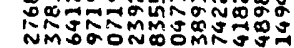
inisiniviminimivio

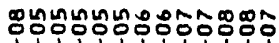

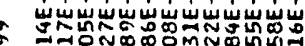

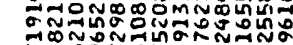

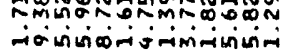

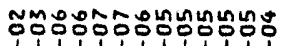

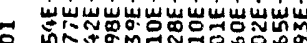

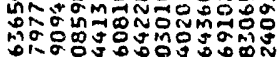
inimion incis

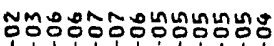

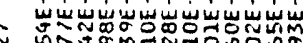
مिم

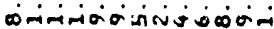
i

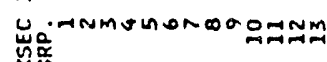

\section{SAMPLE PROBLEMS}




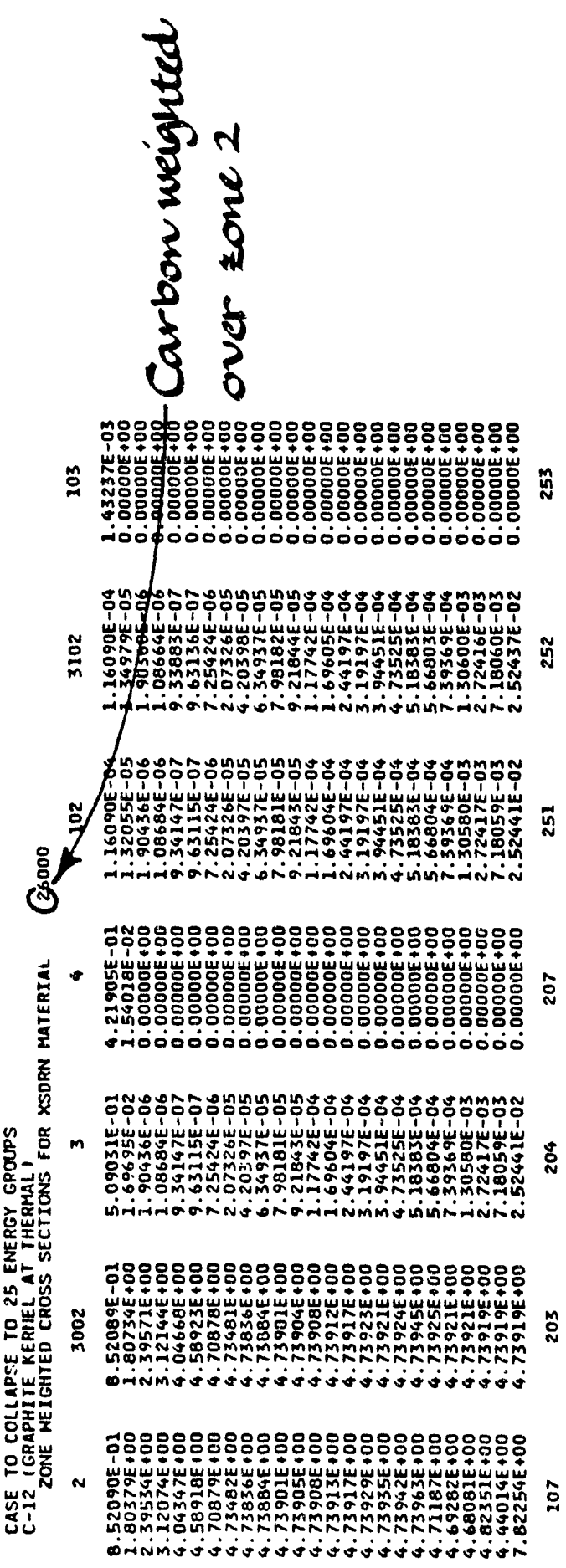

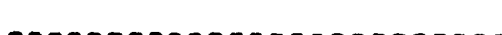

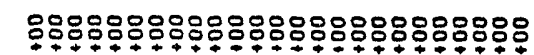

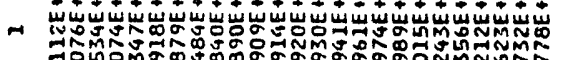

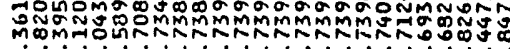

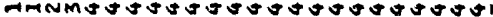

is

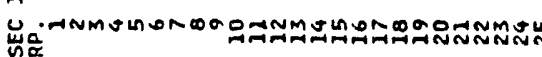

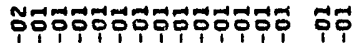

政

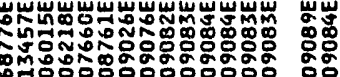
किनं-iminimini-inimi

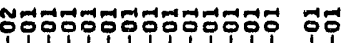

ก̃

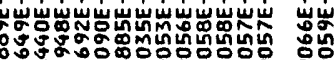

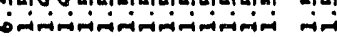

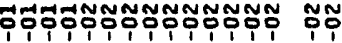

药 Sỡ

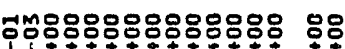
วิm. Ninjojó00000000

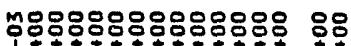

实

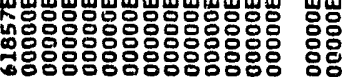

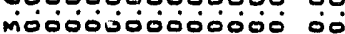

mำㅇำ

\section{西}

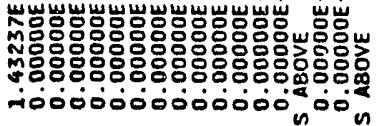

y $y$

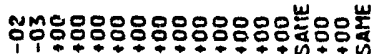

(1)

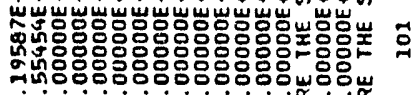

m-100000000000 N

¿

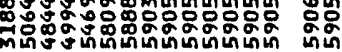

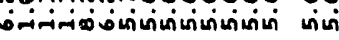

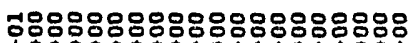

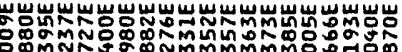

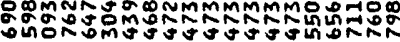

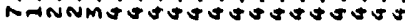

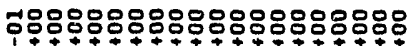

5

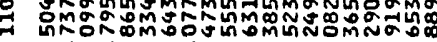

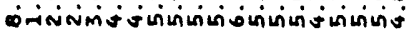

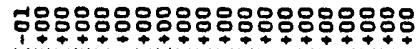

:

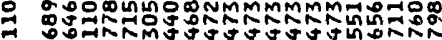

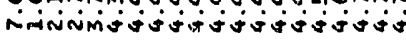

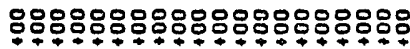

范

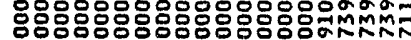
000000000000000ñن́vis

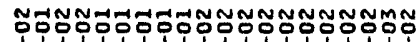

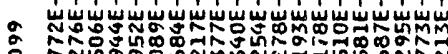
AN

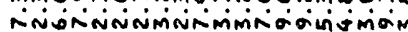

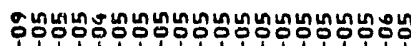
س山س ă onjo

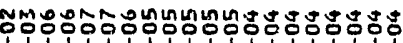

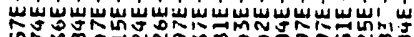

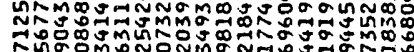
Dritaran

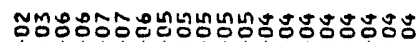

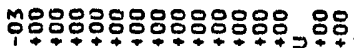

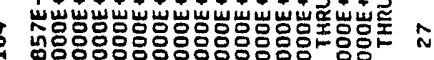

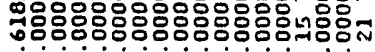
m0000000000000 00

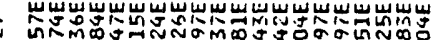

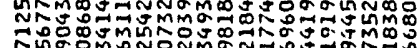

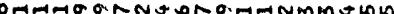
D 警. 


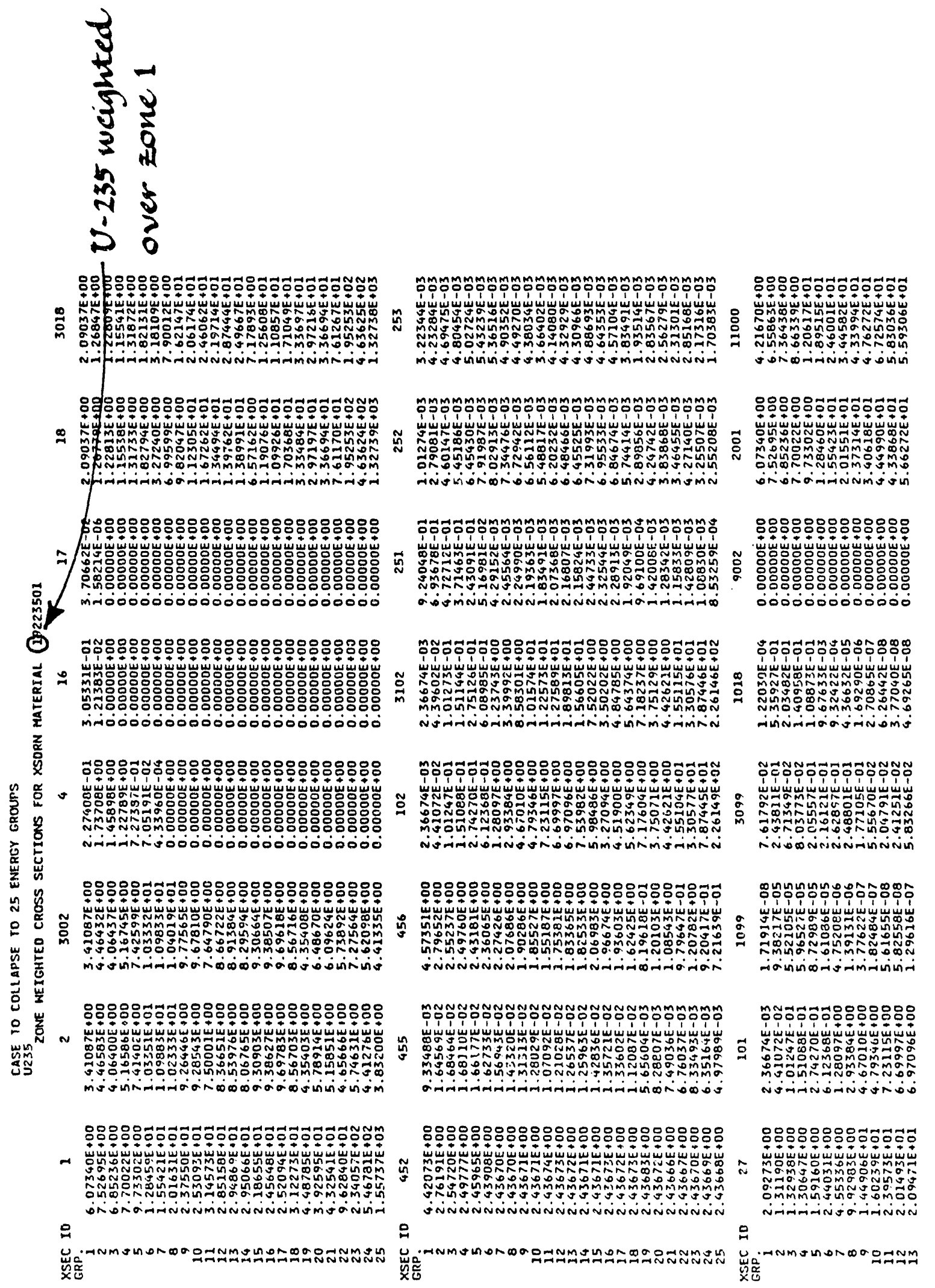

\section{SAMPLE PROBLEMS}




\section{SAMPLE PROBLEMS.5 MOVE MULTIGROUP LIBRARIES TO ANOTHER COMPUTING SYSTEM}

A very common task for a user of multigroup libraries is that of moving them from one computing system to another. Because the binary formats vary from system to system, a BCD transmittal file is typically employed.

AMPX libraries can be transmitted by using the AIM module. This module reads a master library in binary format and generates a BCD-formatted library that it can also read to recreate the master library. The procedure is shown in Fig. 6.SAMPLE PROBLEMS.14.

The input for the AIM module is trivial (unless the user elects to generate the BCD input to create a master file). It generally consists of data that identify where the files are to be located and one parameter that specifies which way the format conversion will be made.

The input for a case to convert the library produced by Task 2 to a BCD file is shown below. In this case, we start with a master library on logical unit 70 containing just the ${ }^{235} \mathrm{U}$ data, convert this to a BCD file on logical unit 71 using an AIM run, then take logical 71 and pass it back through AIM to recreate a binary master file on logical unit 2, after which we run ALE to demonstrate that the file is readable and structured properly.

Portions of the output follow the input data. 


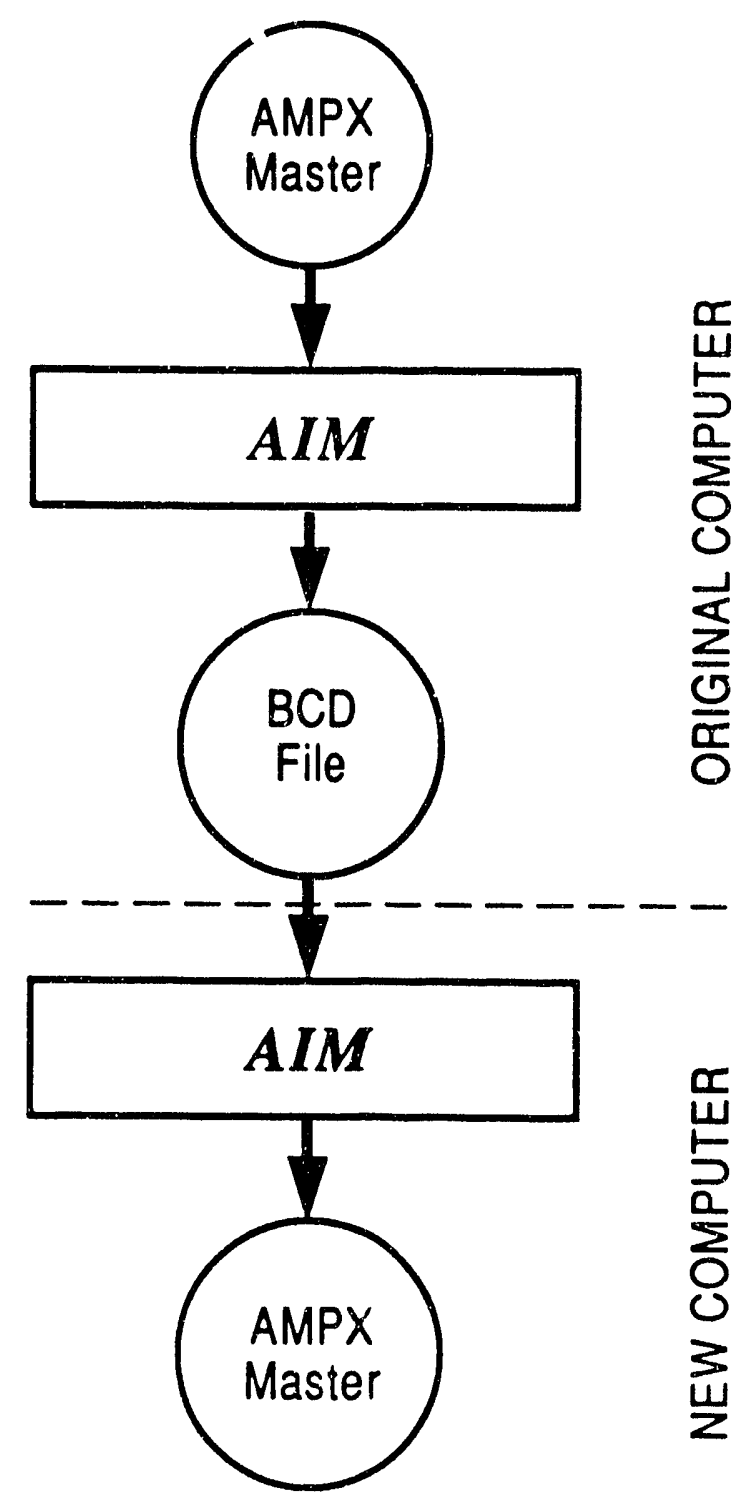

Fig. 6.SAMPLE PROBLEMS.14. Procedure for moving an AMPX master to a new computer. 


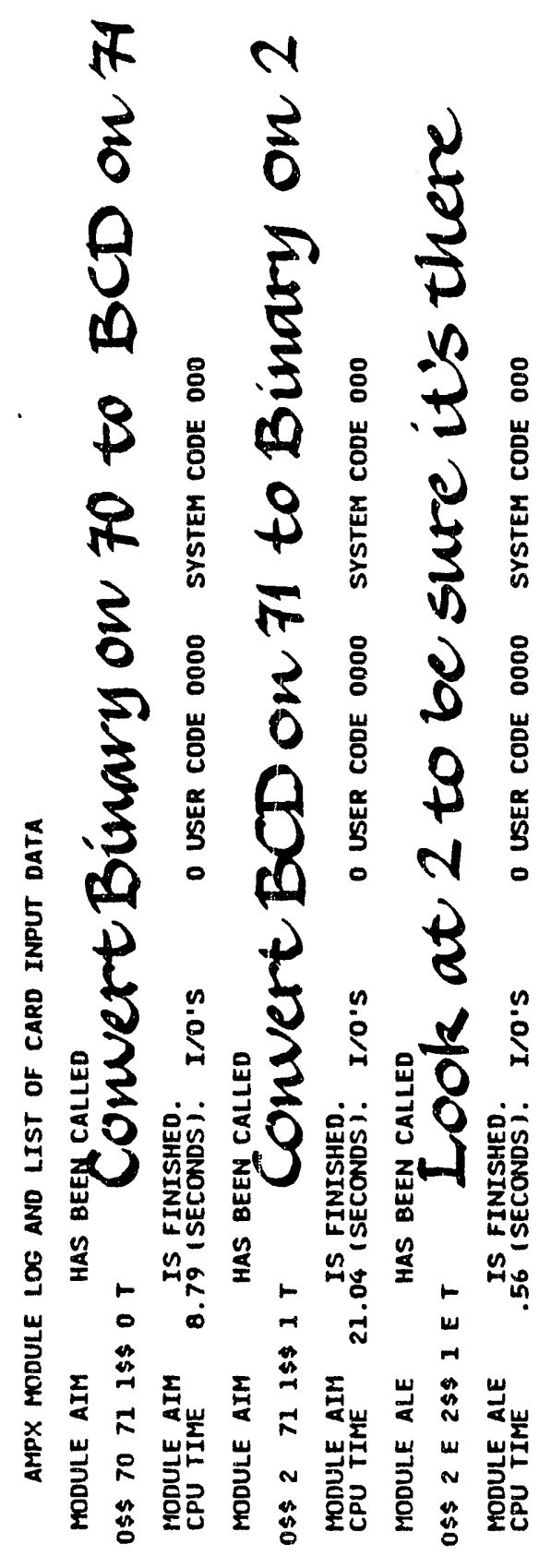



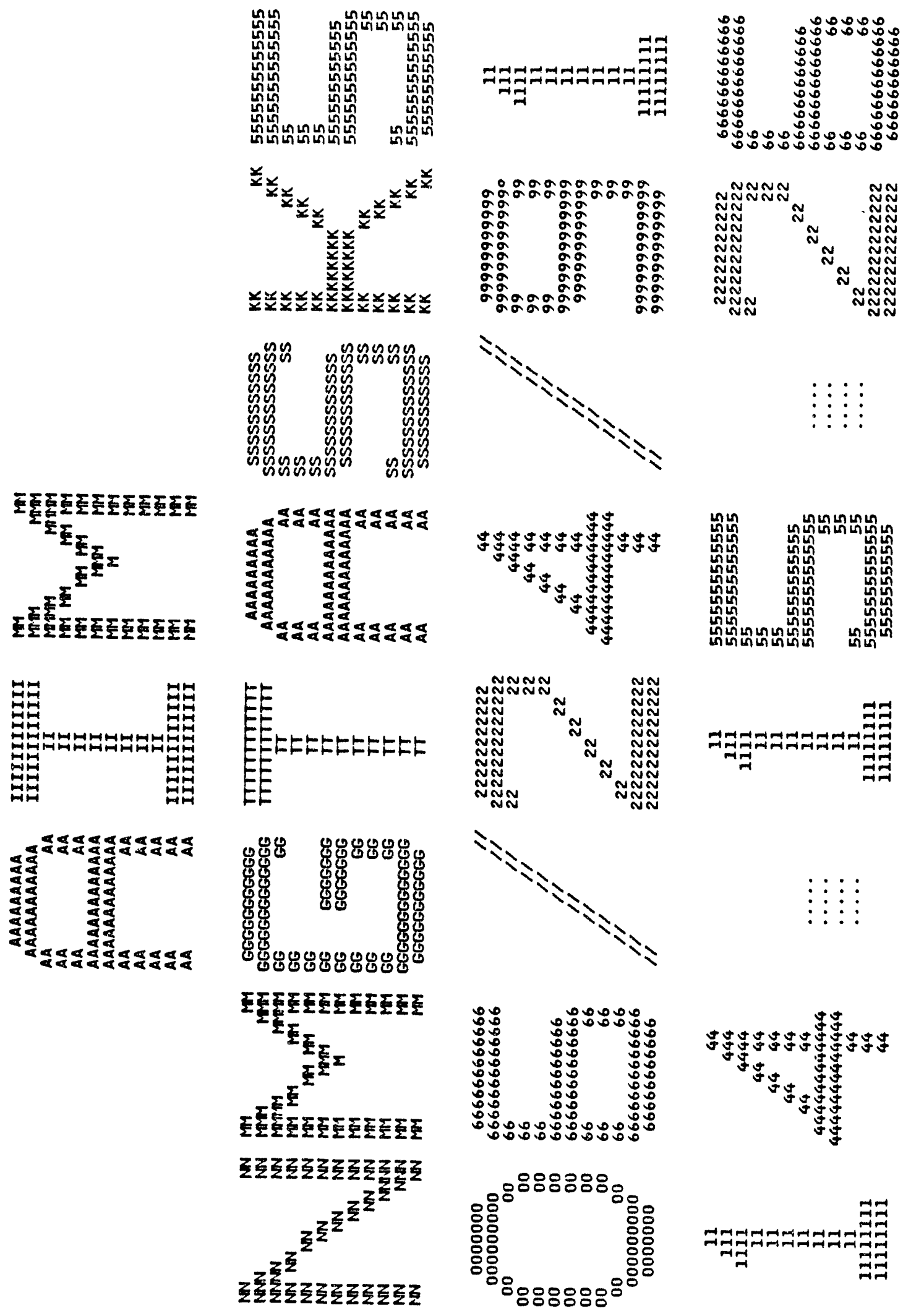


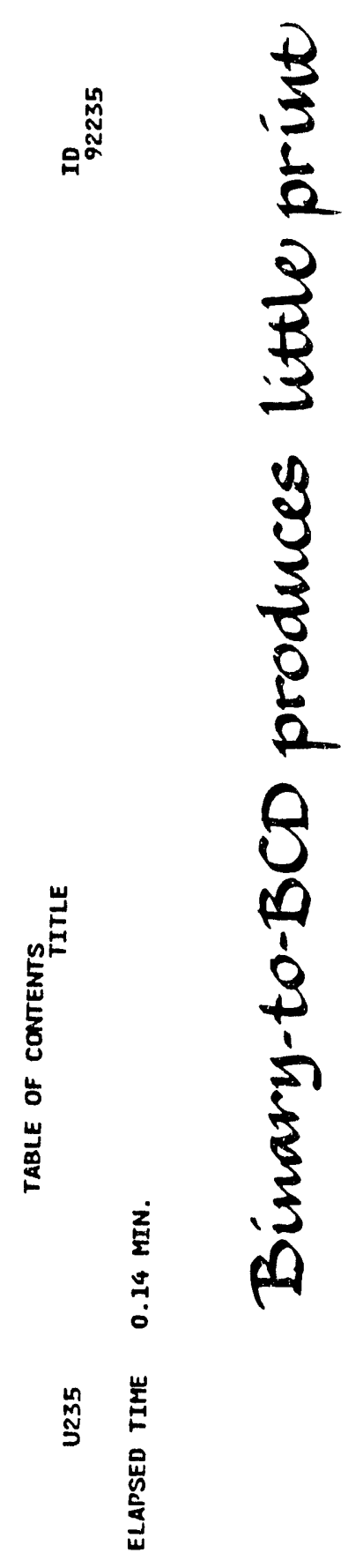



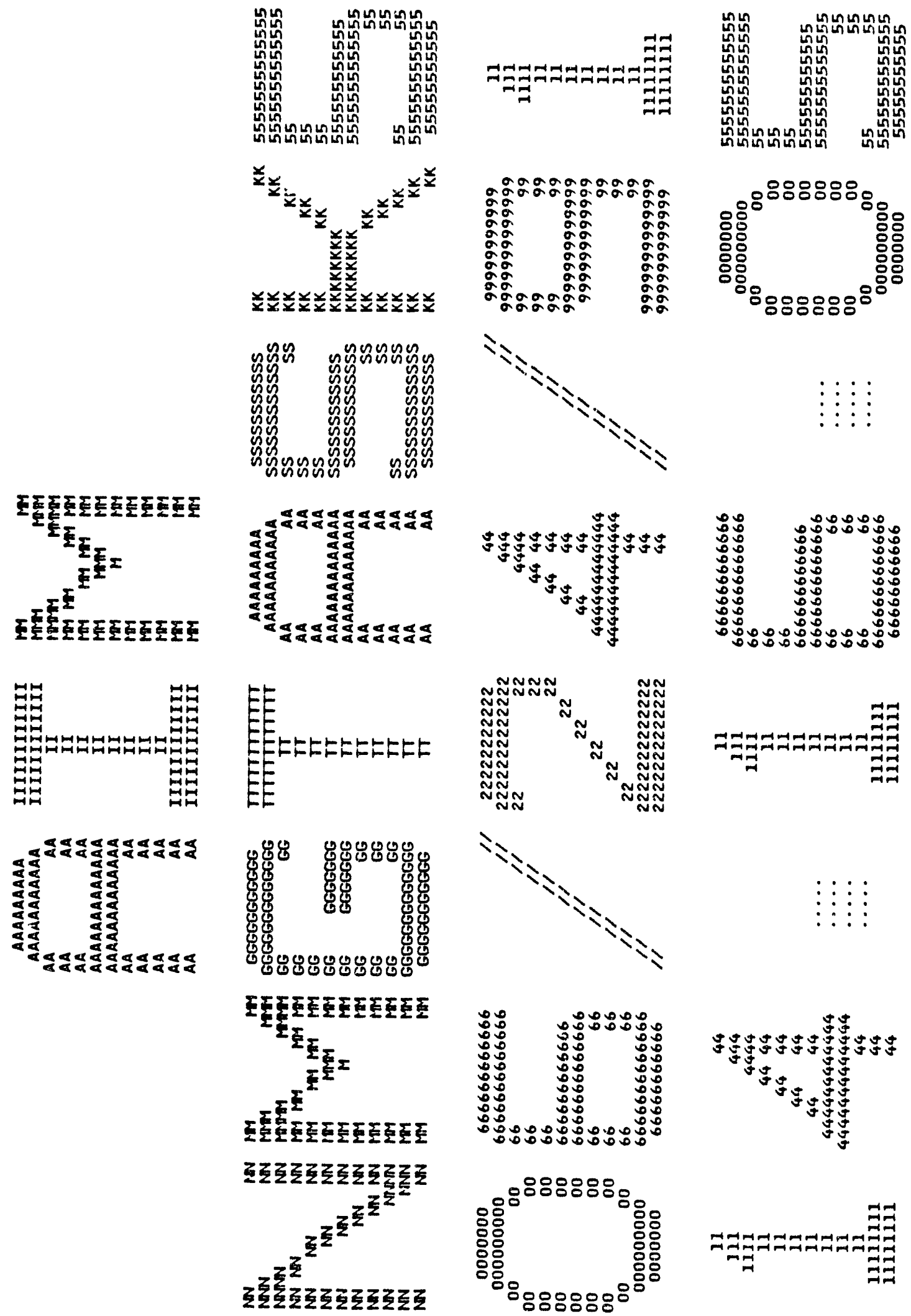


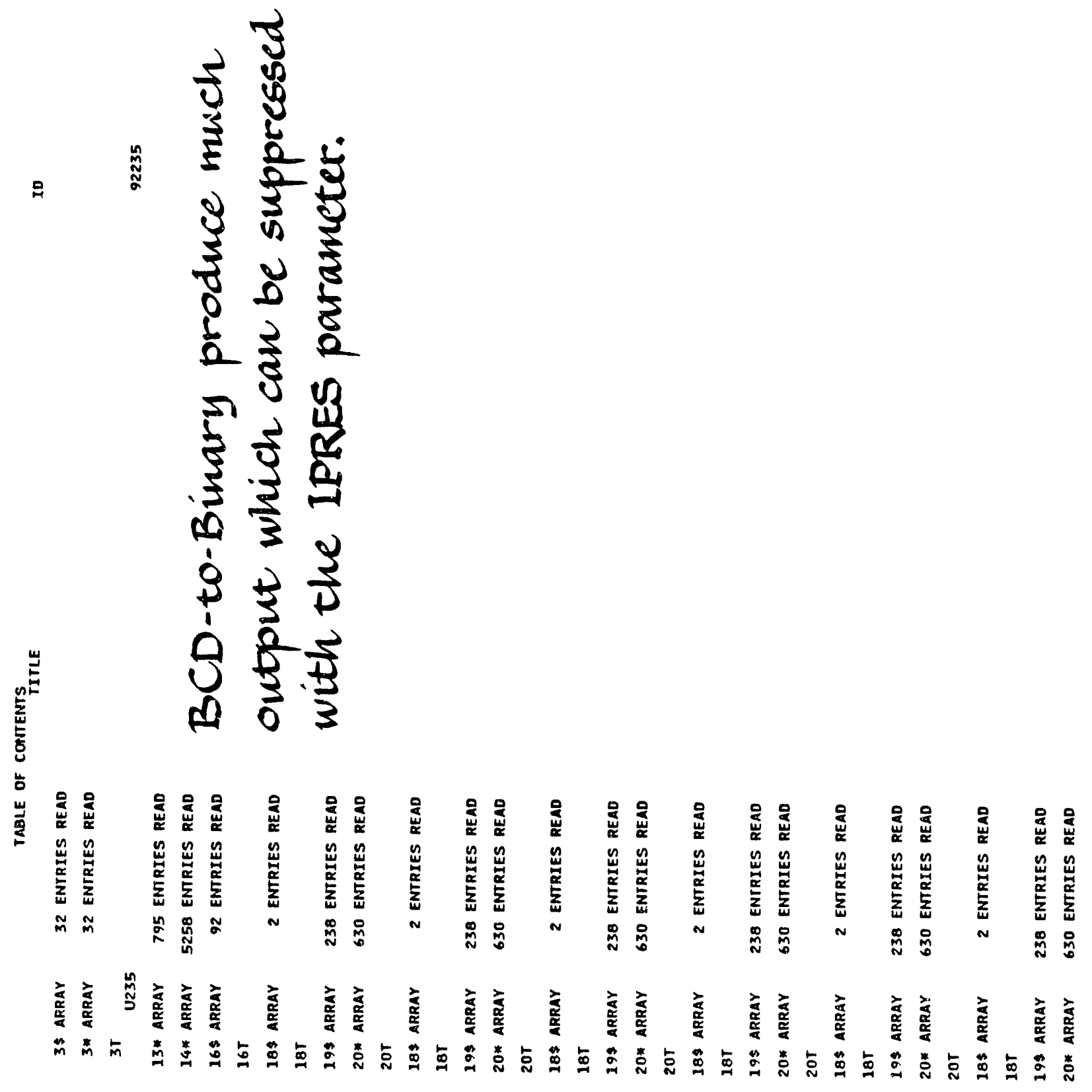




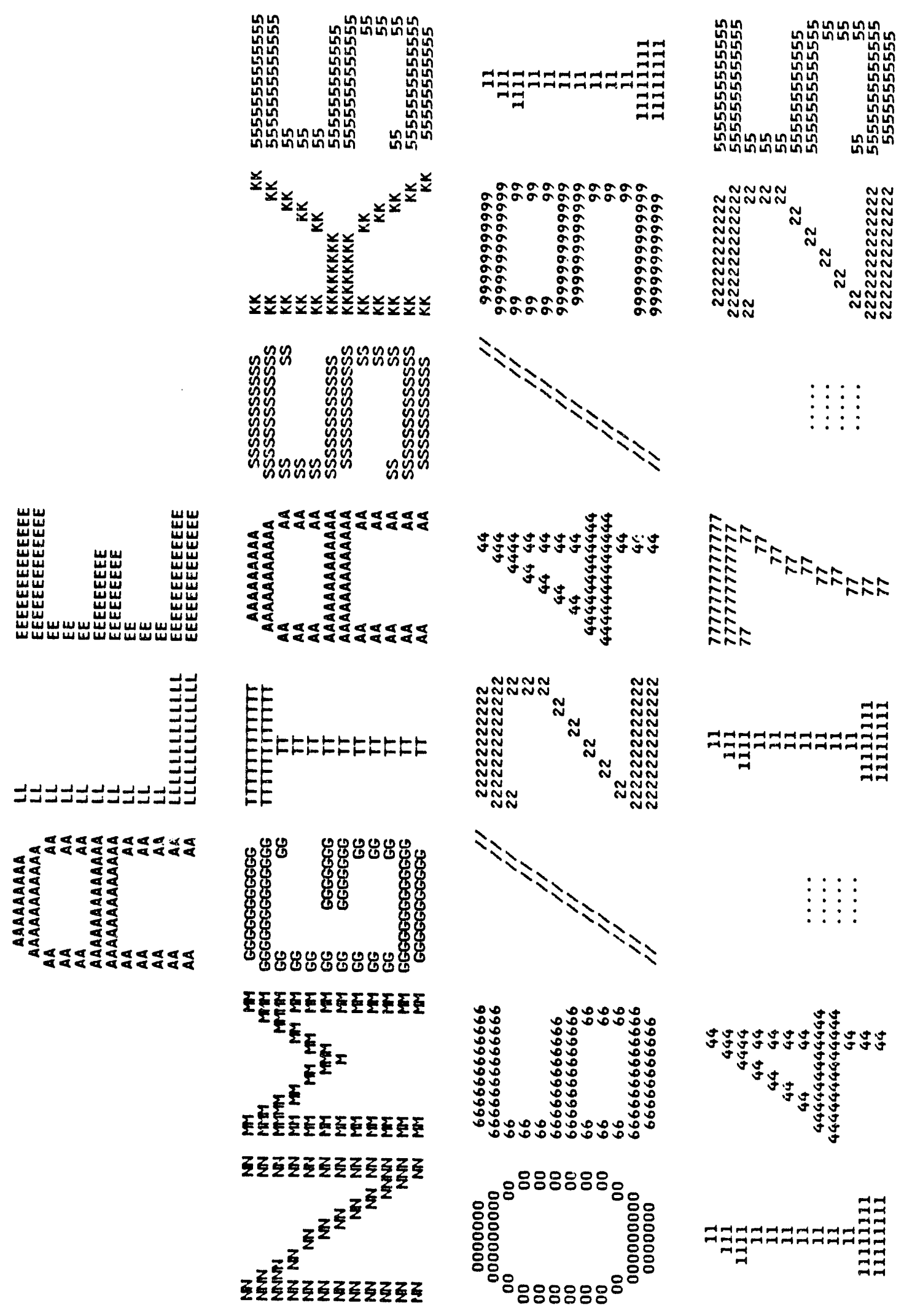




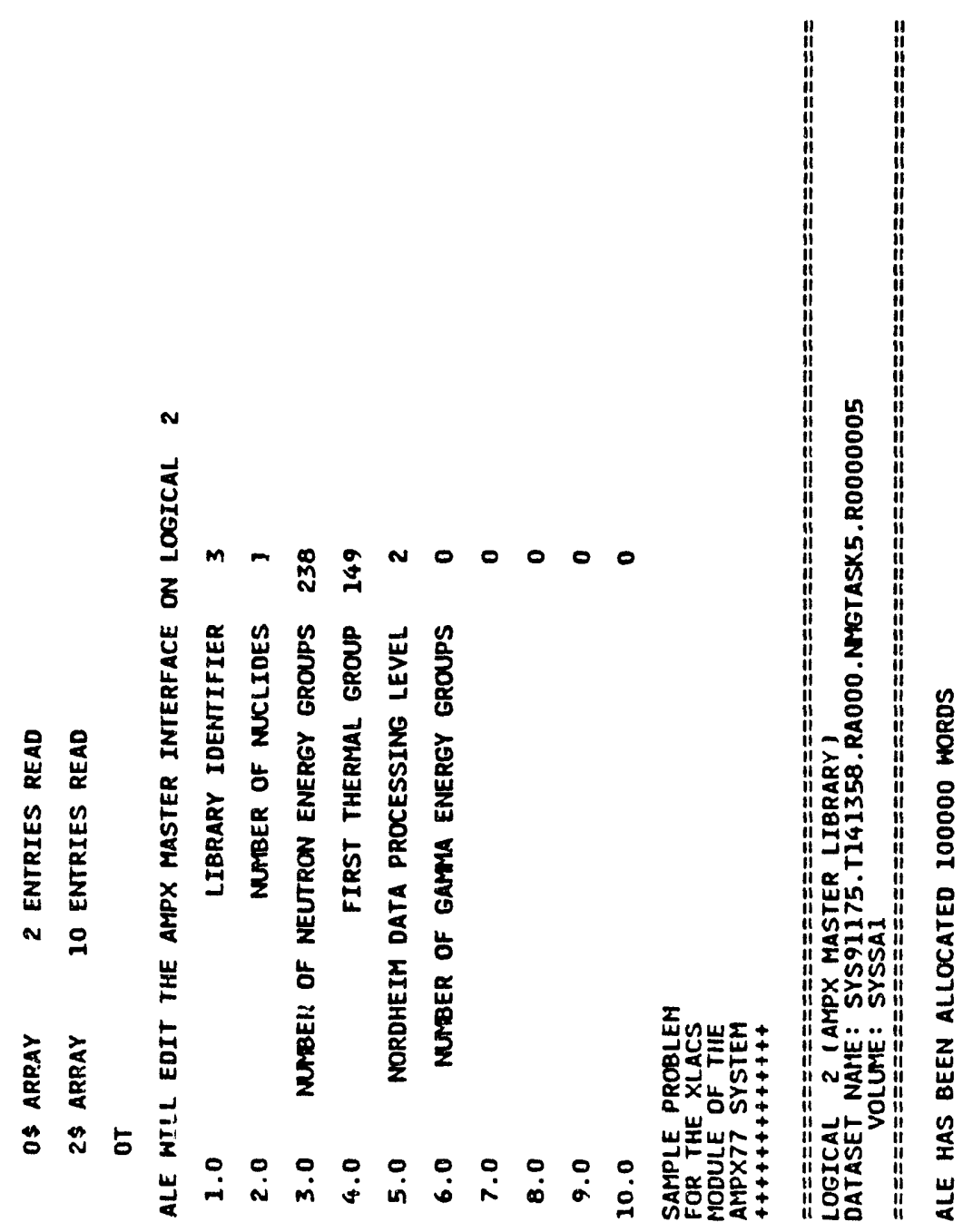

SAMPLE PROBLEMS 

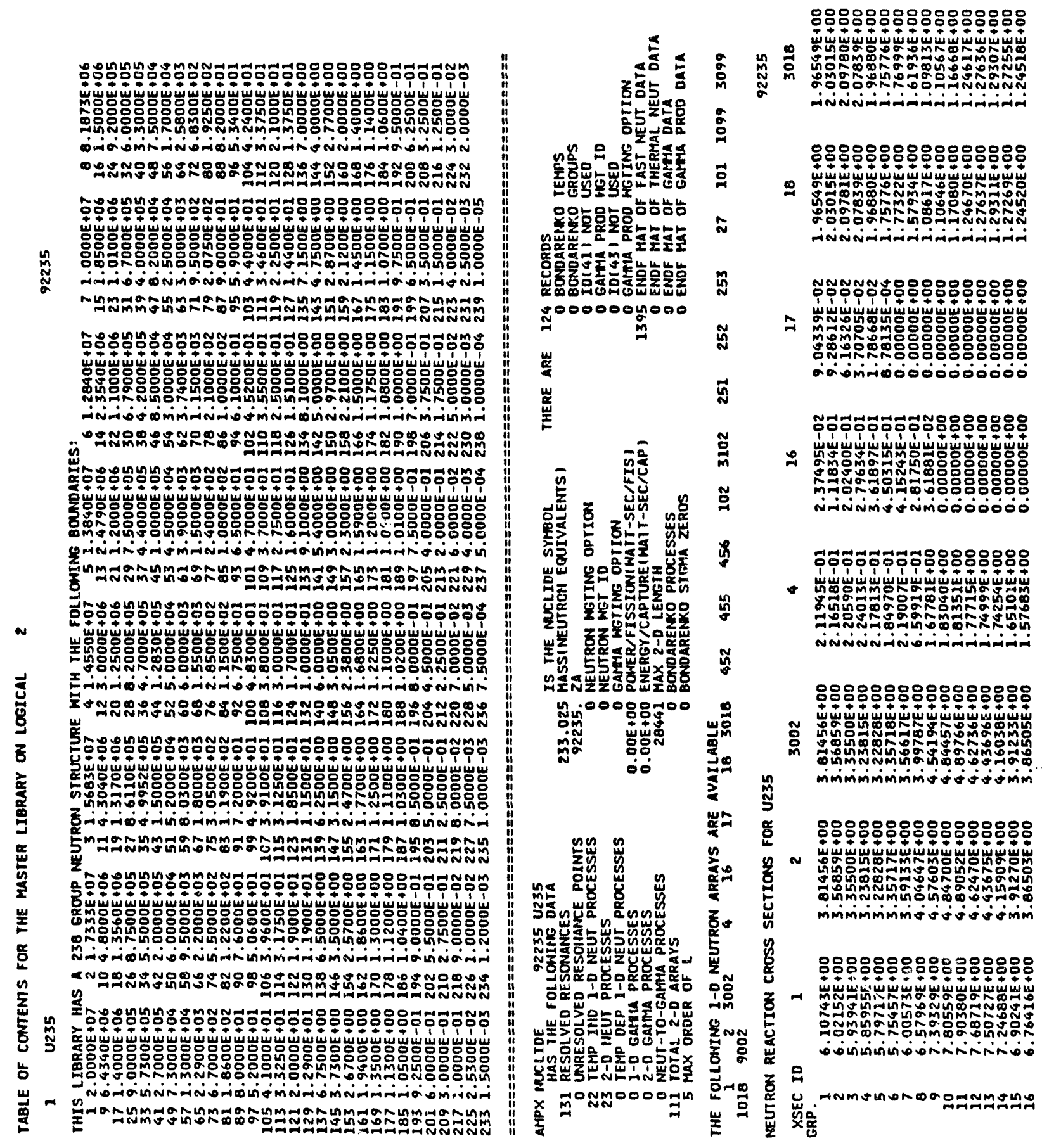


\section{SAMPLE PROBLEMS.6 AVERAGE POINT CROSS SECTIONS}

A user of cross sections is frequently faced with the task of having a function that is known as an array of energies and values which is needed in a multigroup format. Several modules in AMPX-77 relate to this task.

Suppose, for example, that one wants to average a function that varies as 1 over the velocity of the neutron over some simple weighting spectrum. (This procedure is widely employed when it has been recognized that, for thermal systems, many important data collections, such as fission products, are dominated by nuclides whose thermal cross sections vary as $1 / \mathrm{v}$.)

The procedure for this task is shown in Fig. 6.SAMPLE PROBLEMS.15.

The input for this case is shown below, followed by portions of the output. Here we run CASTROL to put the cross sections in a format that the VEL program knows how to access, followed by a JERGENS run to create a simple weighting function, also written in a format VEL understands; then VEL is run to perform the group averaging and to write them on a master library on logical unit 1, after which an ALE run is made to ensure the data are there and in proper format. 


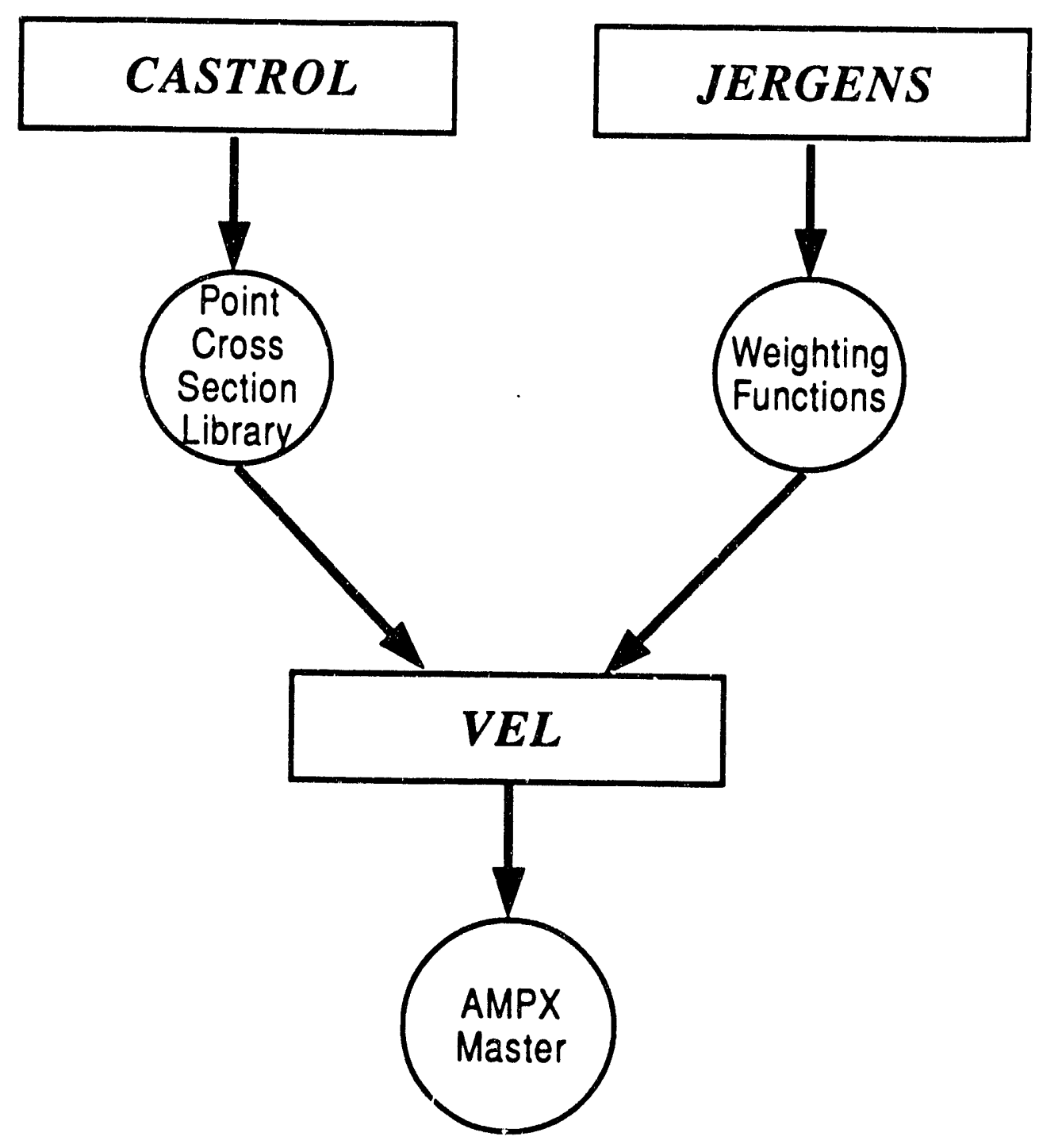

Fig. 6.SAMPLE PROBLEMS.15. Procedure to weight a point cross-section array. 


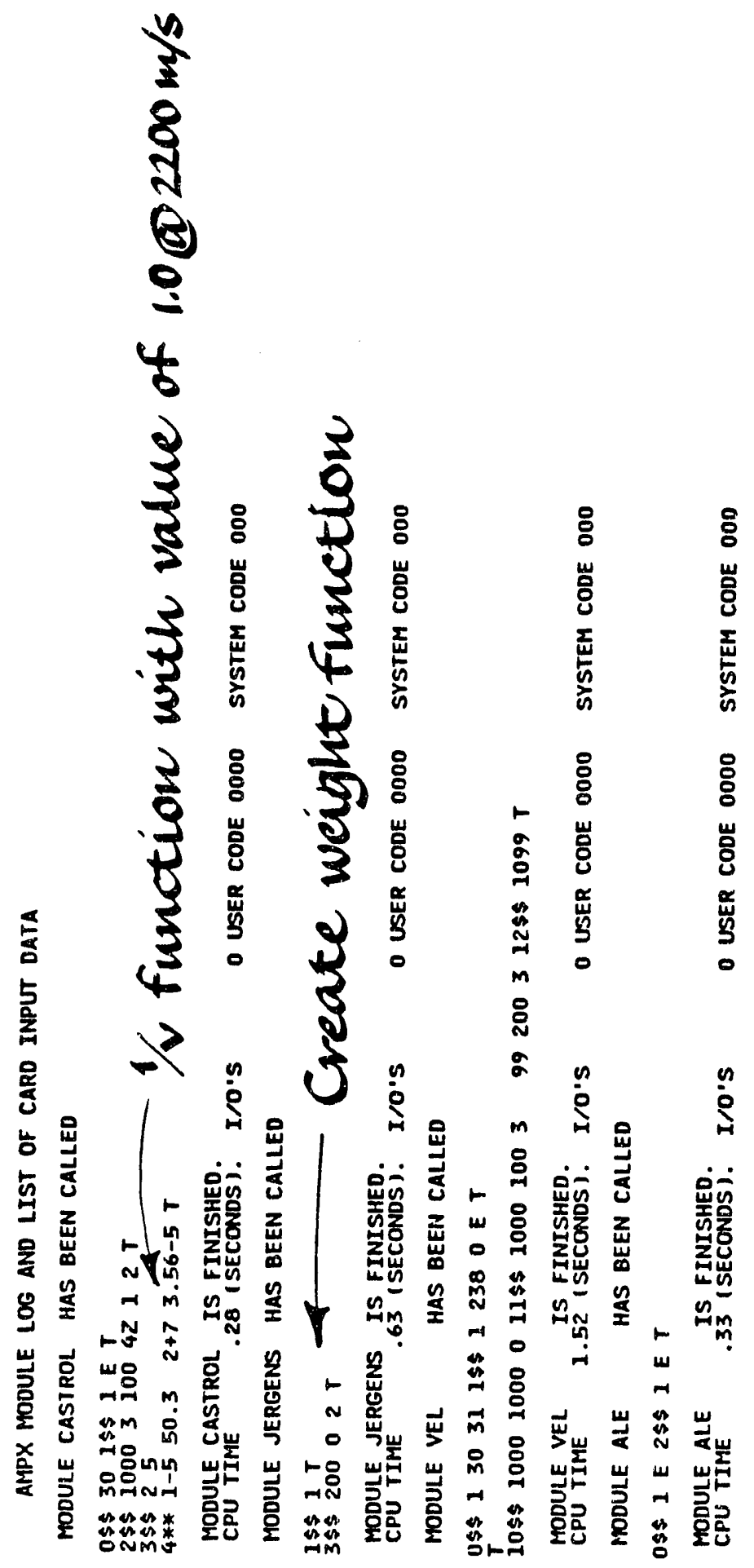



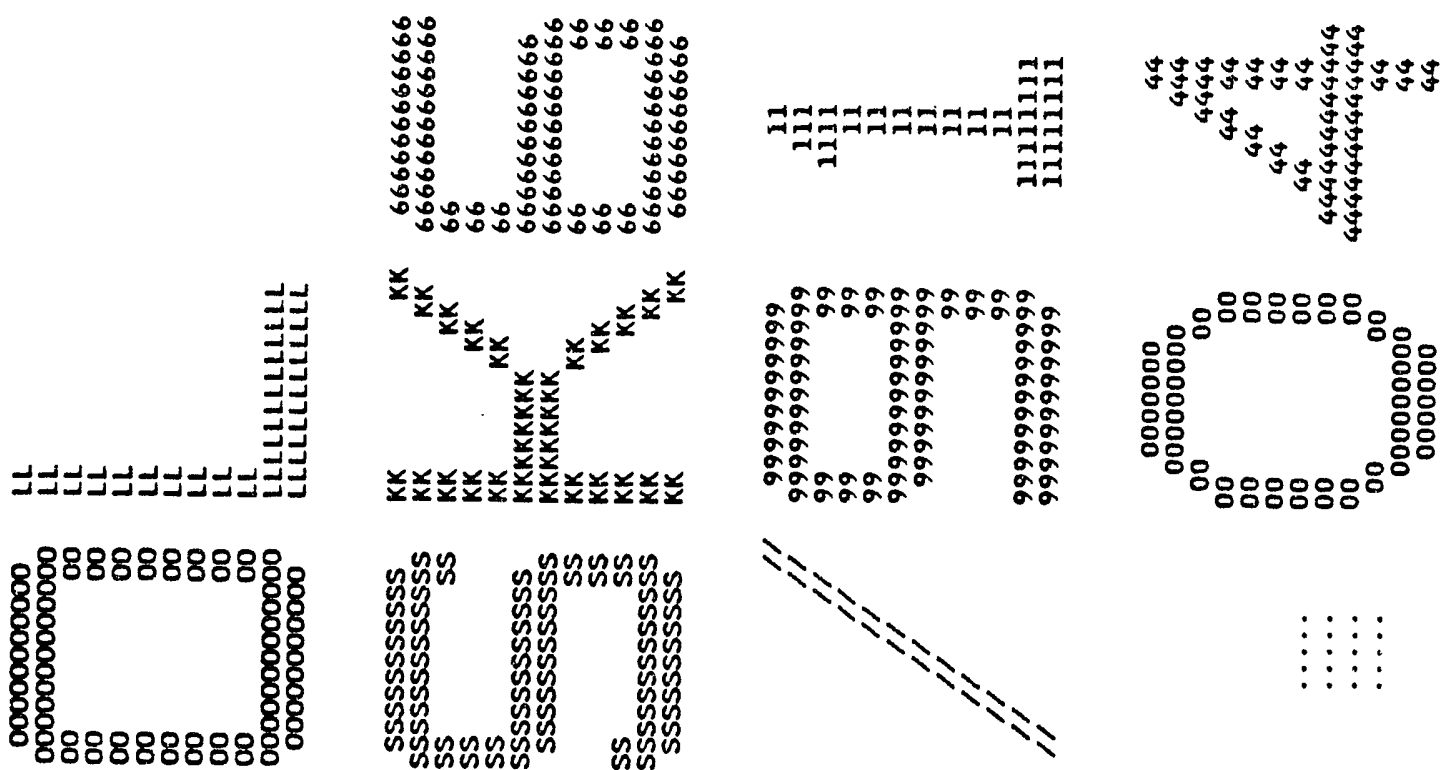

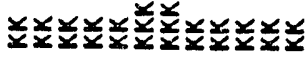
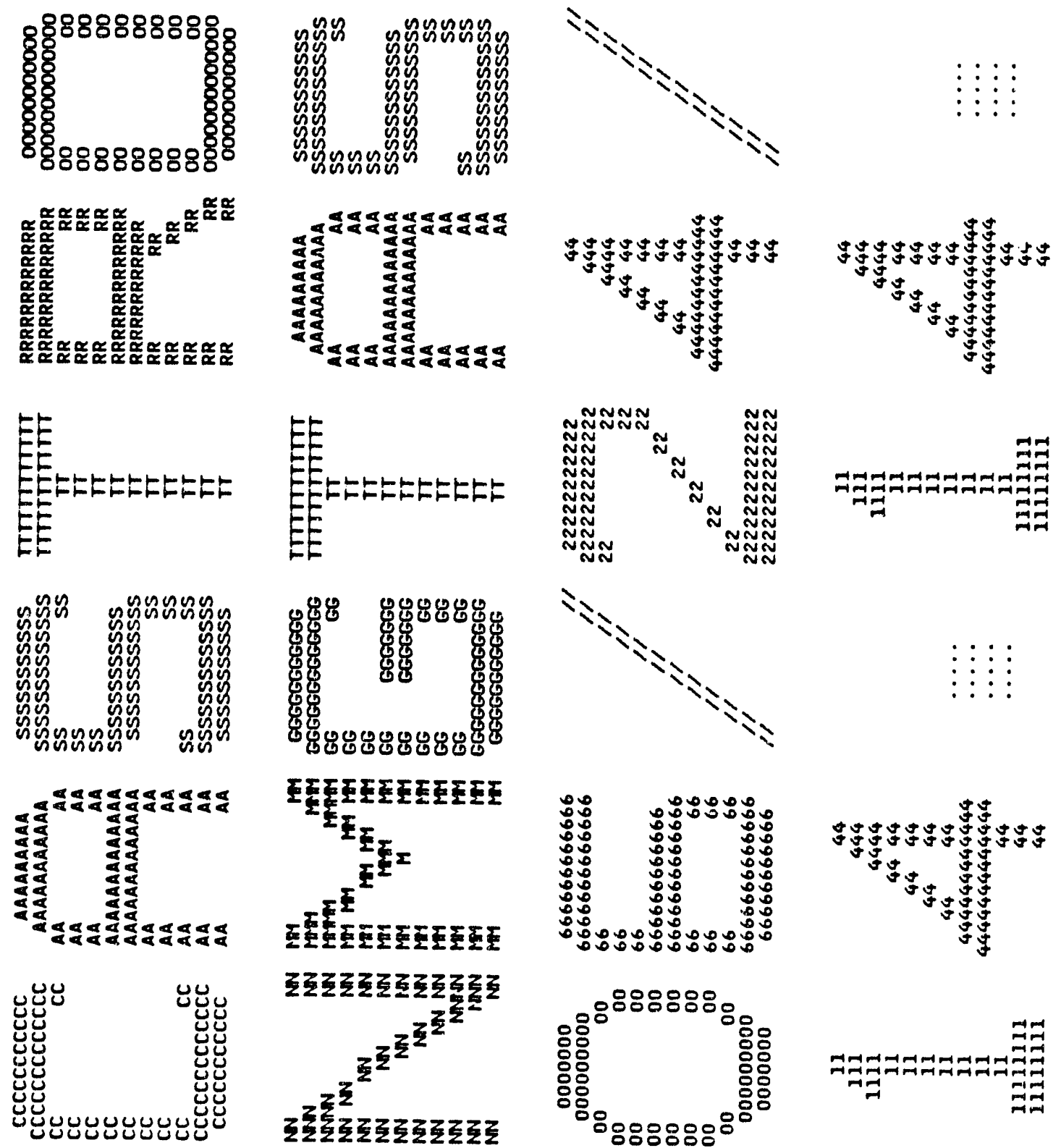

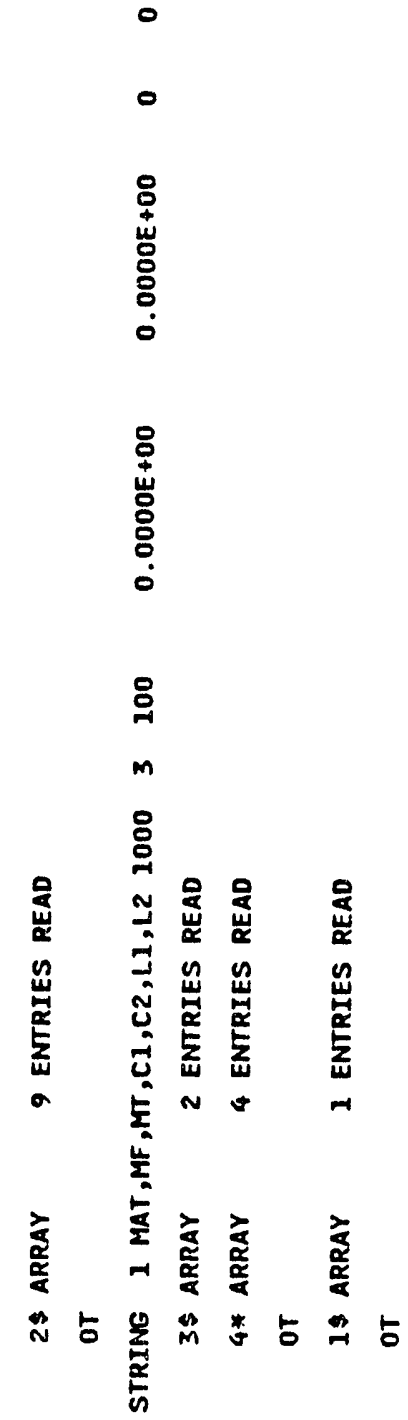


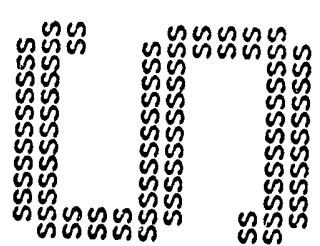

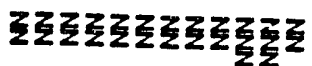

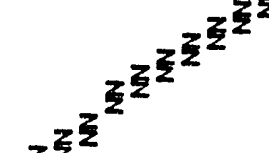

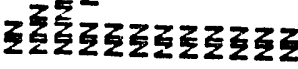
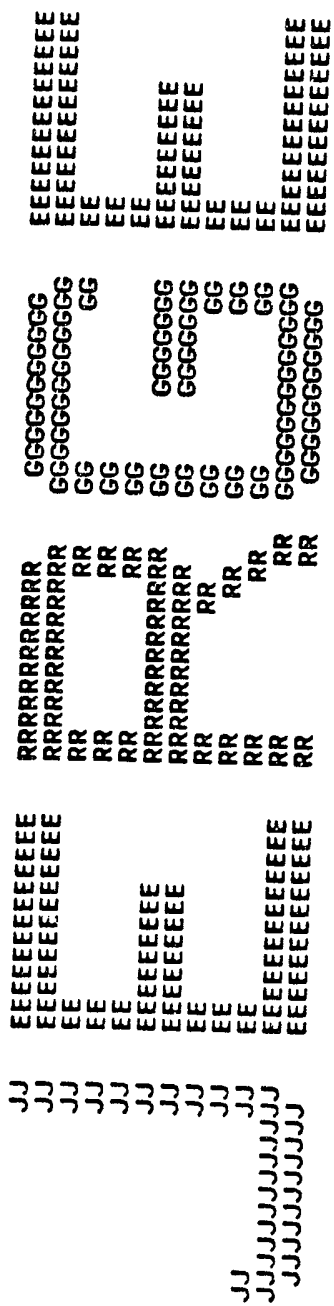
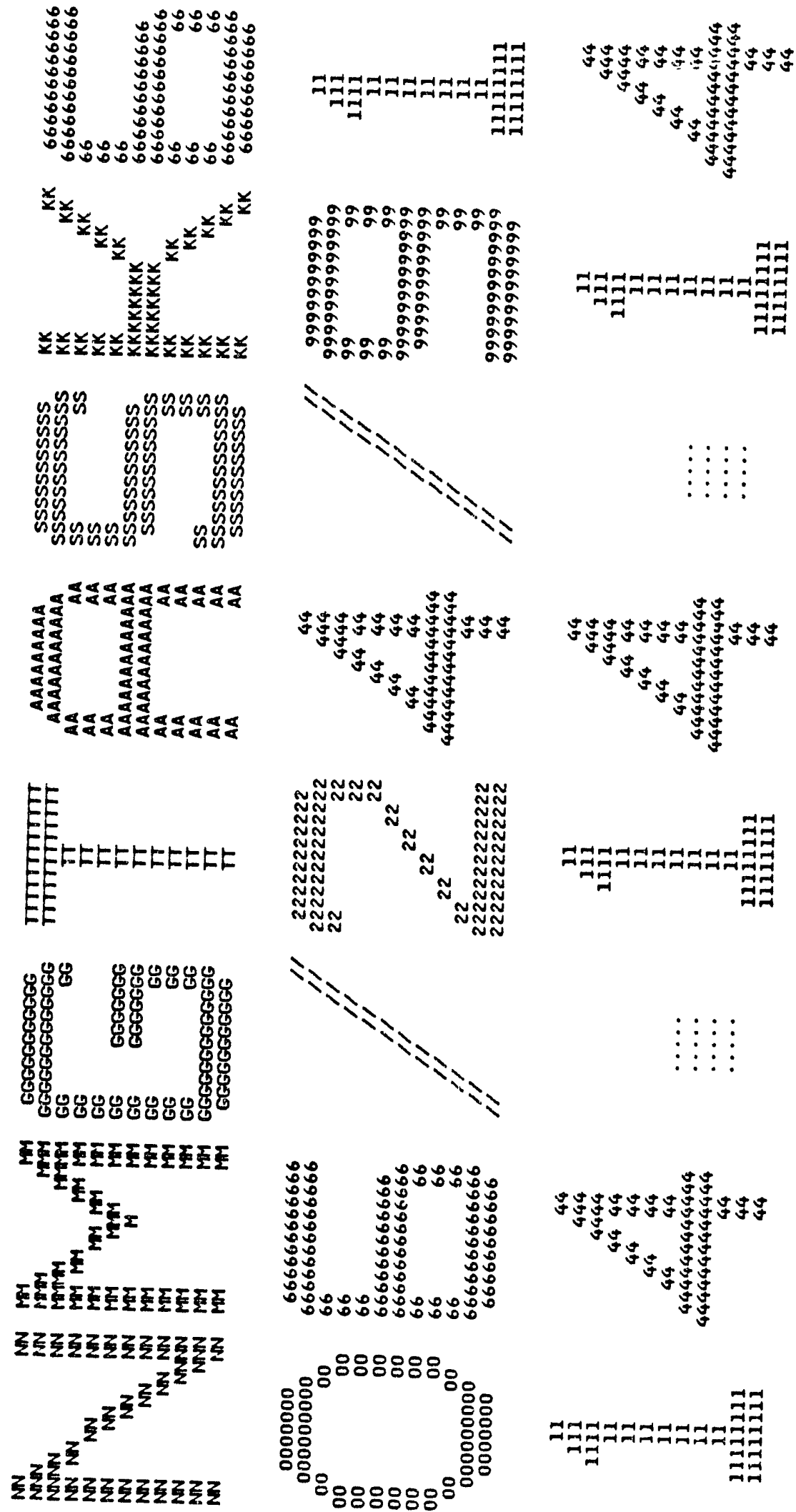


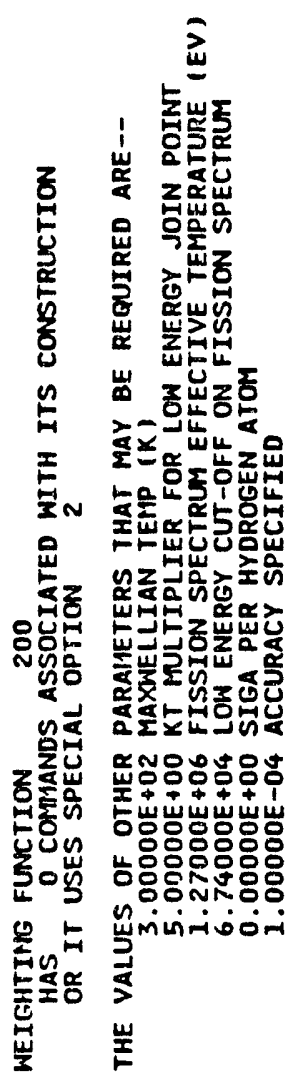


岁 mommmmmmmmmmmmmmmmmmmmmmmmmmmmmmm t

2 مूMN Oำ

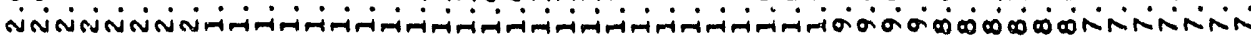

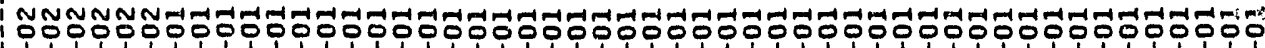

嵌 M̂Non

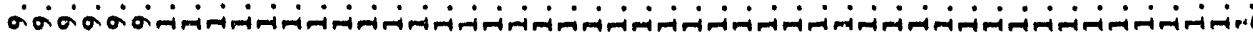

5

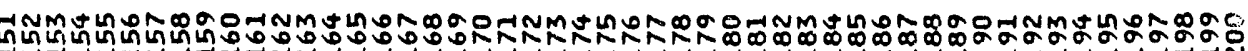

L

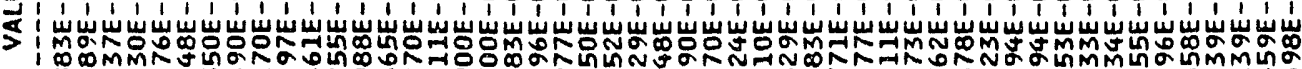

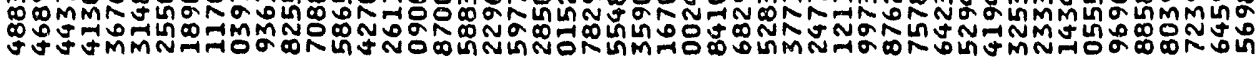

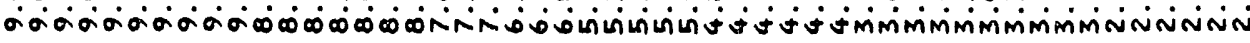

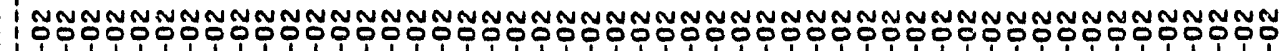

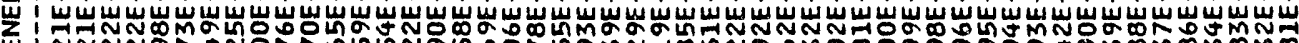
N N N No T NO م๓⿴囗大

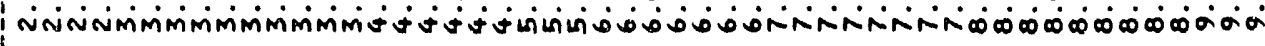

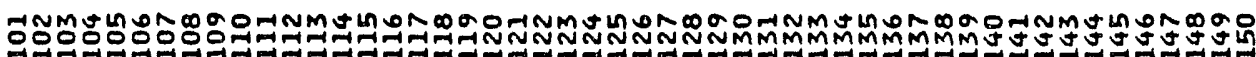

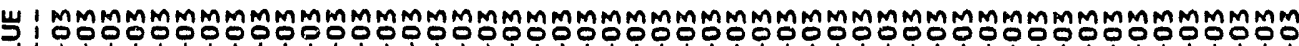
J|

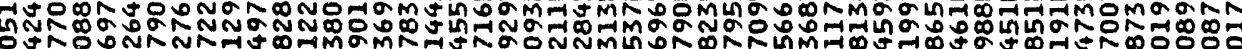

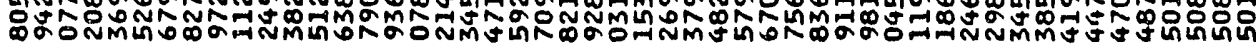

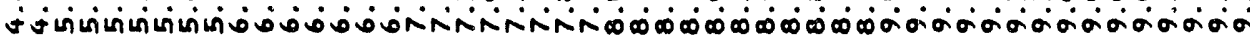
Mmmmmmmmmmmminmm

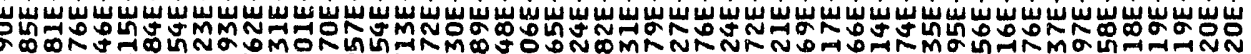
on

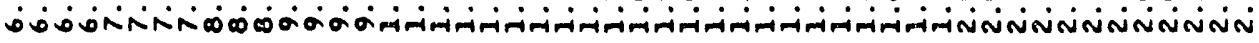
:

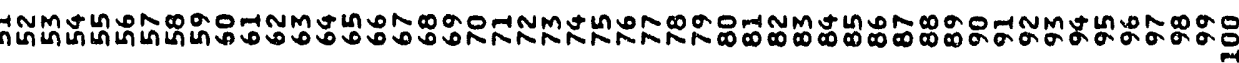

岁 |

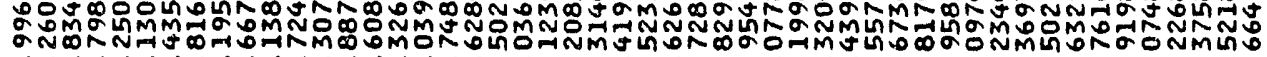
Oinmino

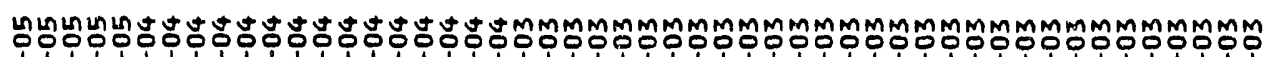
T. | oñ - inmmino 总

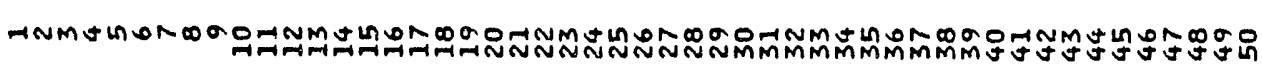



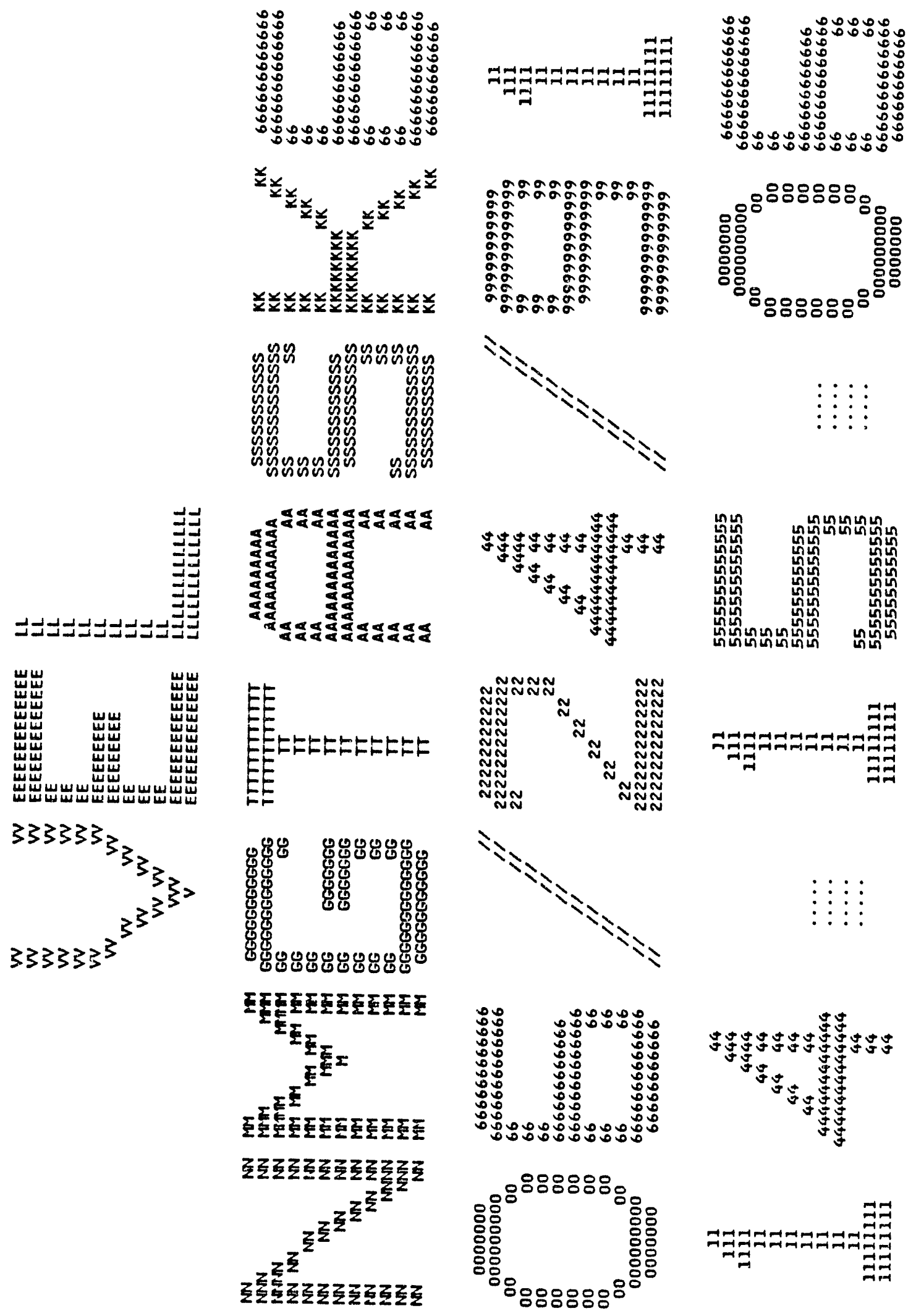

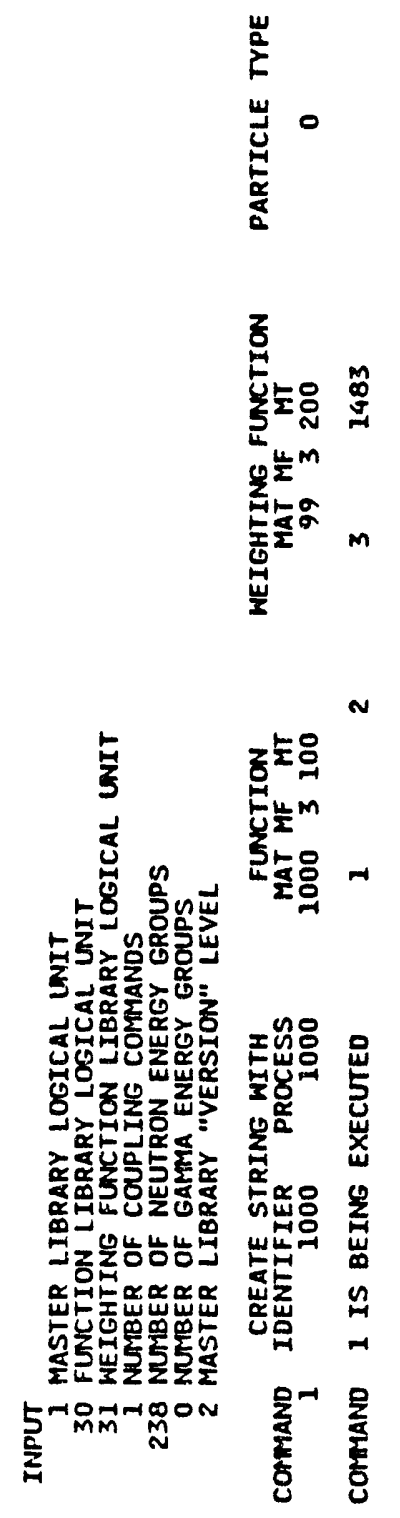

SAMPLE PROBLEMS 
总

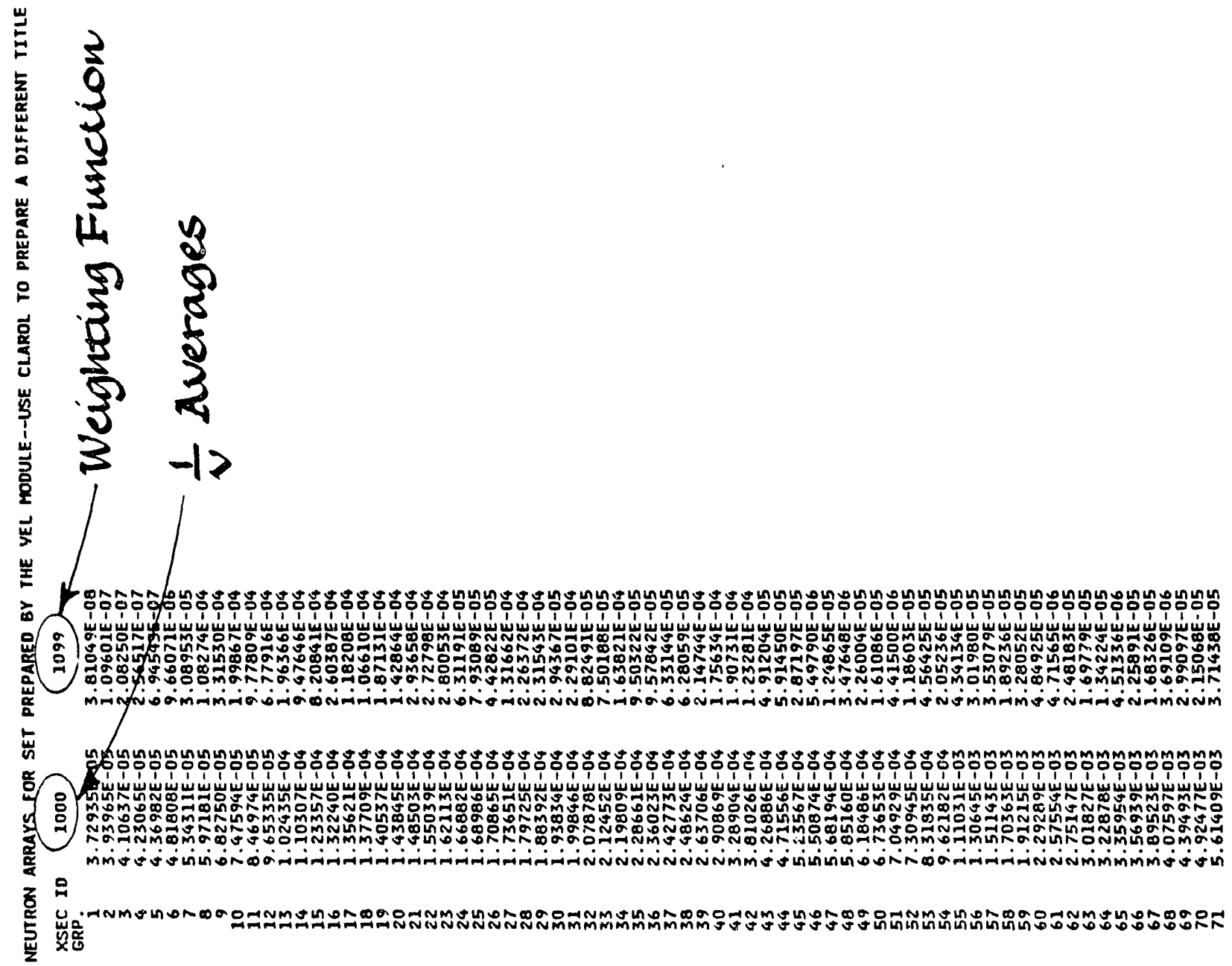



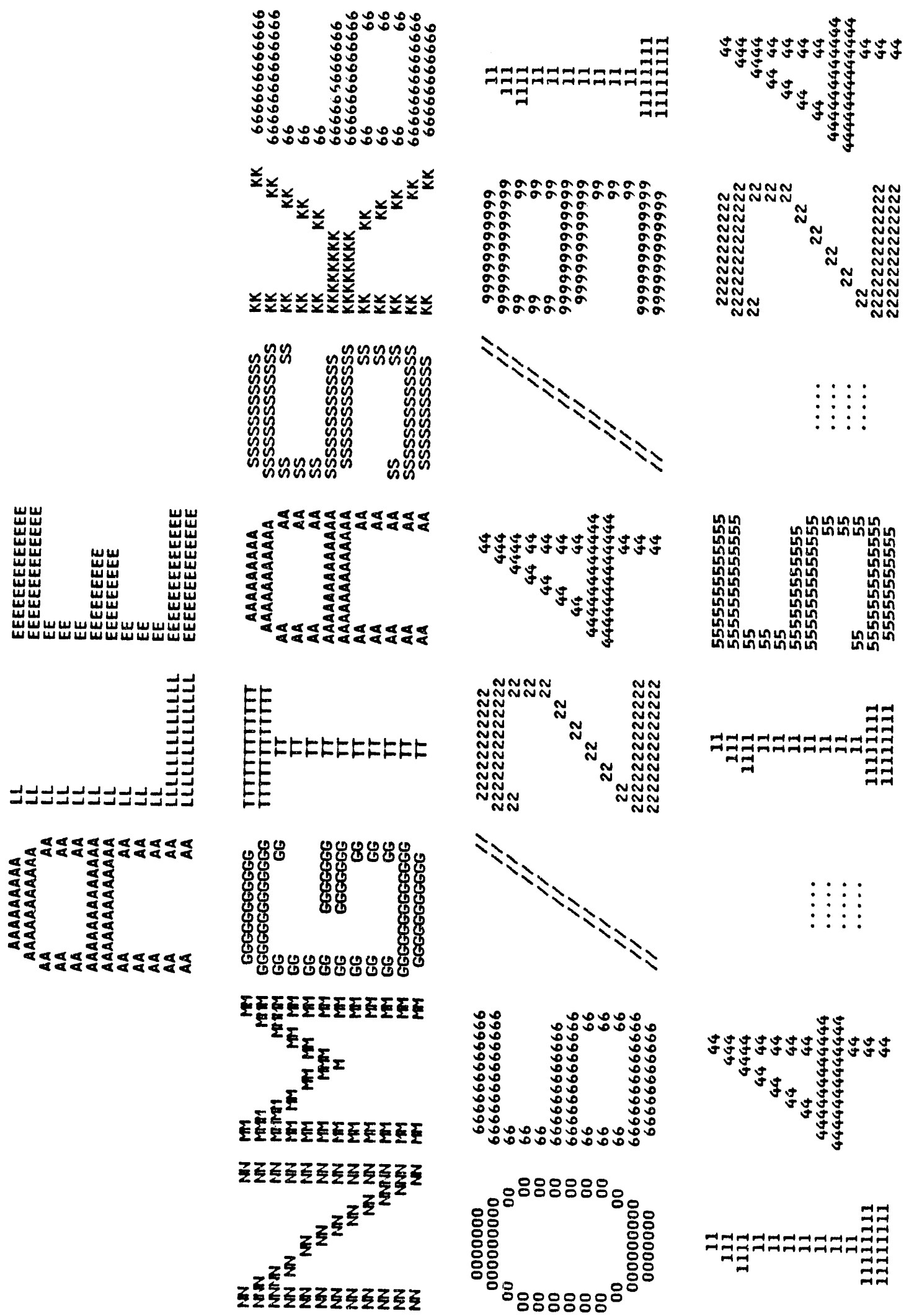

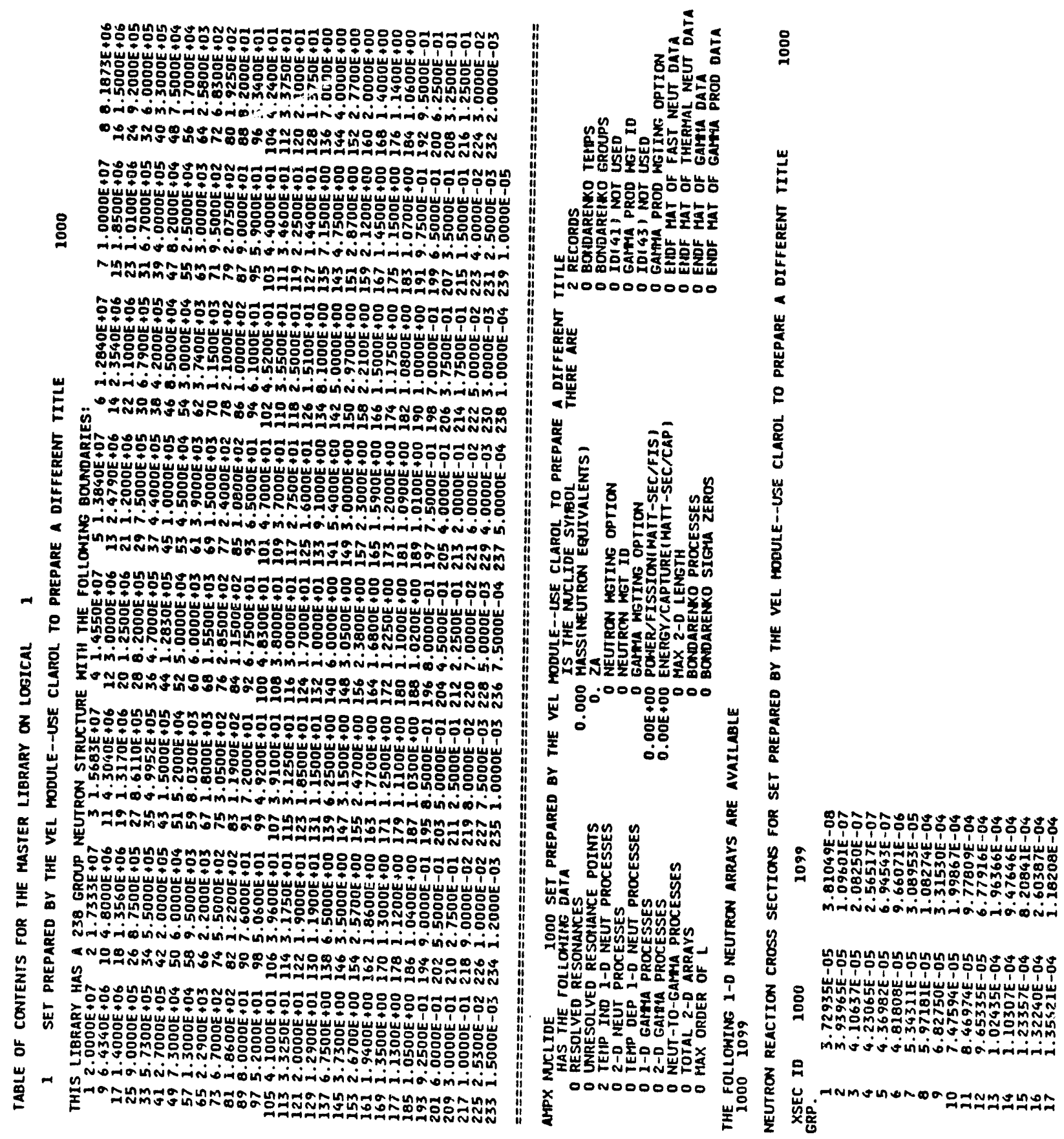

\section{SAMPLE PROBLEMS}




\section{SAMPLE PROBLEMS.7 CONVERT THE CROSS SECTIONS ON AN AMPX MASTER LIBRARY FOR USE IN THE ANISN PROGRAM}

This example assumes that we want to use the ANISN program and that we have a set of cross sections for carbon on an AMPX master library that we wish to convert. This master library was part of those produced in the runs described in Sect. 6.SAMPLE PROBLEMS.1.

As you will recall, these cross sections had 238 energy groups, with 90 of those being "thermal" groups. Let us assume that our ANISN library will be constructed such that every possible transfer from the original library will have a position in the final library and that we want to have the group-averaged values for $(n, \gamma),(n, p)$, elastic scattering, inelastic scattering, and $(n, 2 n)$ also included.

ANISN expects its cross sections to be formatted in a rectangular array with the structure discussed in Sect. INFORMATION.3 of this document.

Each group has a column of values that is IHM words long. The total cross section is in position IHT in the column, $\overline{\nu \sigma_{f}}$ is in IHT-1, $\sigma_{a}$ is in IHT-2, and positions above this are available for other processes, if necessary. The values for scatter to a group are located in the positions below IHT, with the within-group term located ir position IHS.

In our example, three of the positions down to IHT are usurped, as described above. We want to add space for $(n, \gamma),(n, p)$, elastic, inelastic, and $(n, 2 n)$; therefore, our IHT is in position 8 . With 90 thermal groups, we can have a maximum of 89 upscatter values, though many of these will certainly be zero for carbon. To include all positions, IHS will be in

$$
\begin{aligned}
\text { IHS } & =\text { IHT }+ \text { upscatters }+1 \\
& =8+89+1=98
\end{aligned}
$$

Also with 238 groups, there are 237 possible downscatters; hence, IHM is

$$
\begin{aligned}
\text { IHM } & =\text { IHS }+ \text { downscatters } \\
& =98+237=235 .
\end{aligned}
$$

The procedure we will use to generate the ANISN values is shown in Fig. 6.SAMPLE PROBLEMS.16.

The input data for this task are shown below, followed by portions of the output it generated. Here we run NITAWL to extract data for carbon from logical 60 and to write it on a working library, which ALPO can read, on logical 4. ALPO then reads this and makes an ANISN binary library on logical unit 20. 
ORNL-DWG 91Z-14386

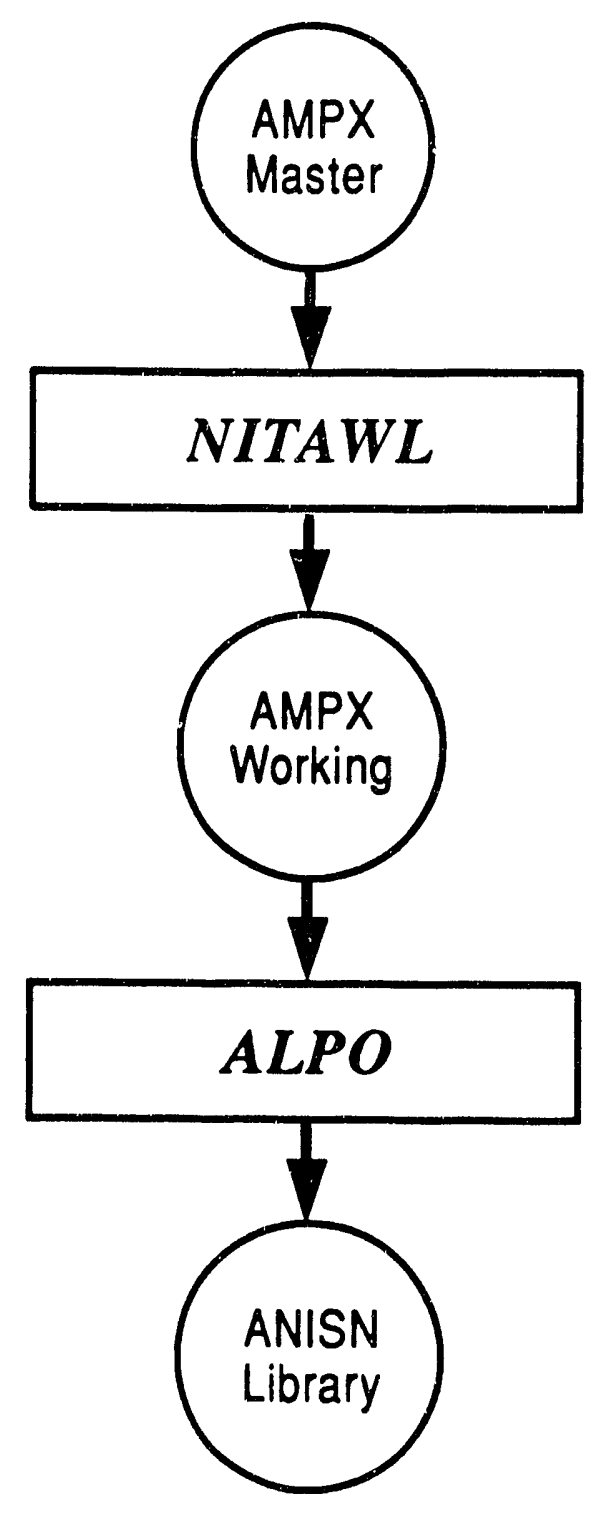

Fig. 6.SAMPLE PROBLEMS.16. Procedure to make ANISN cross sections. 


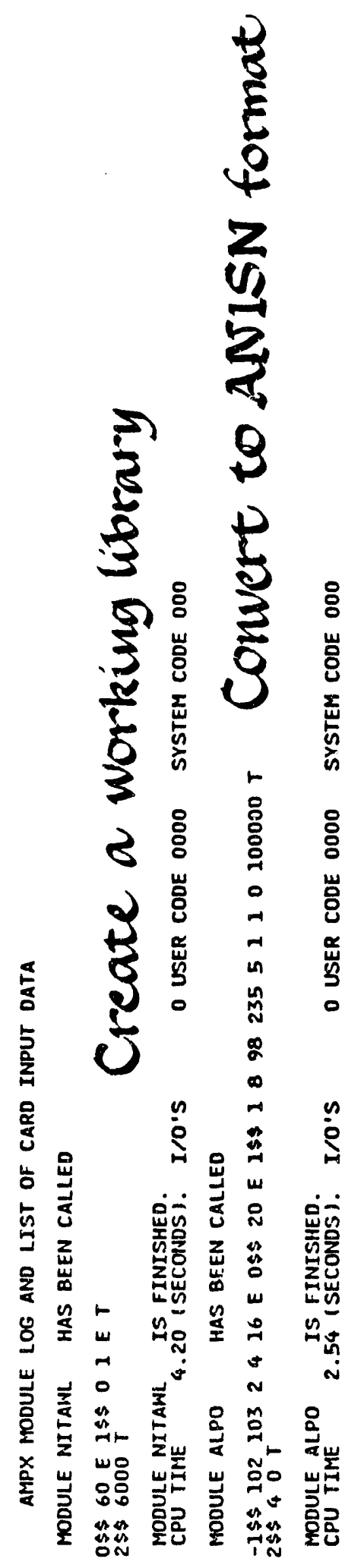




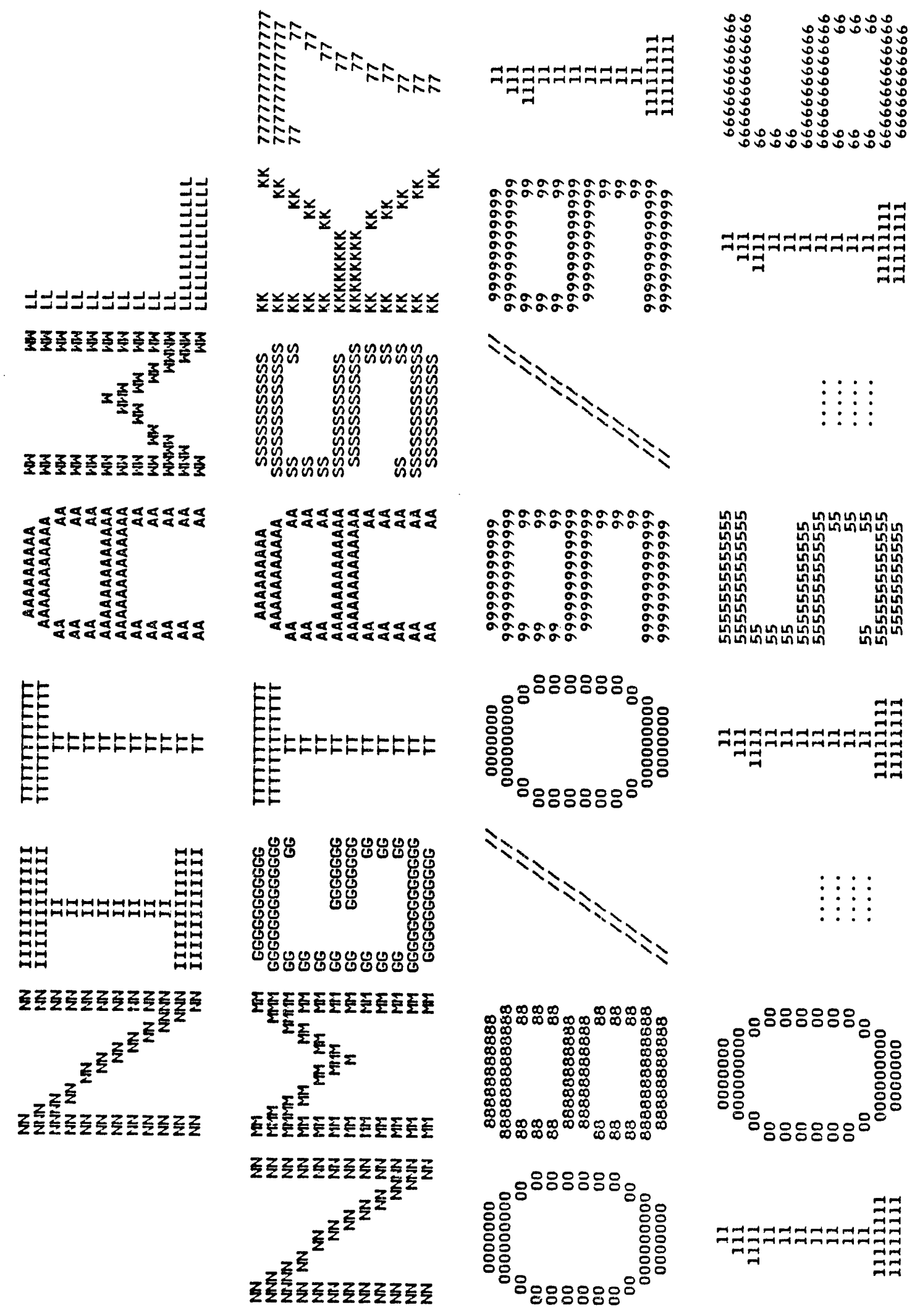




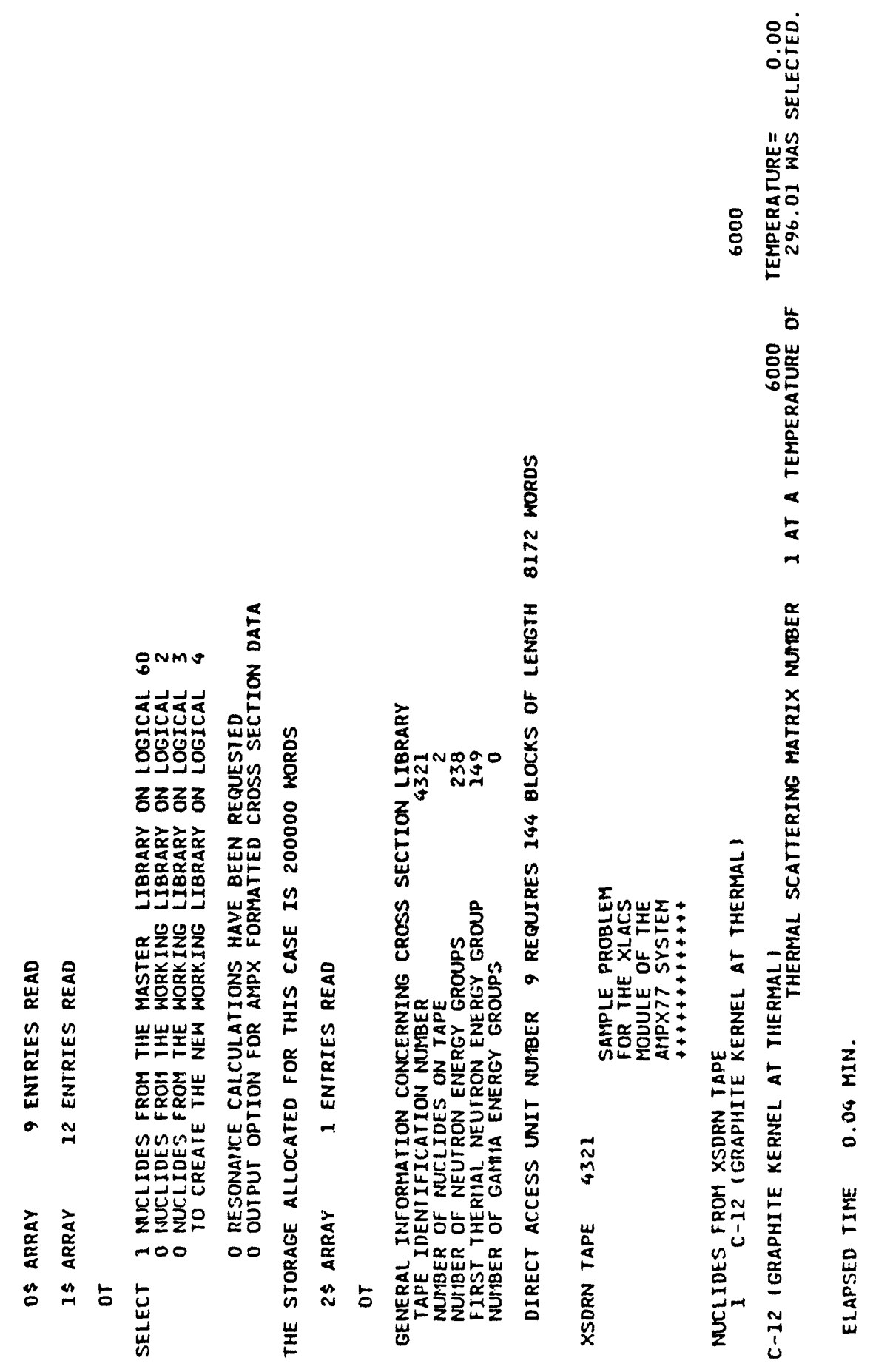


Hov $\frac{8}{8}$

우

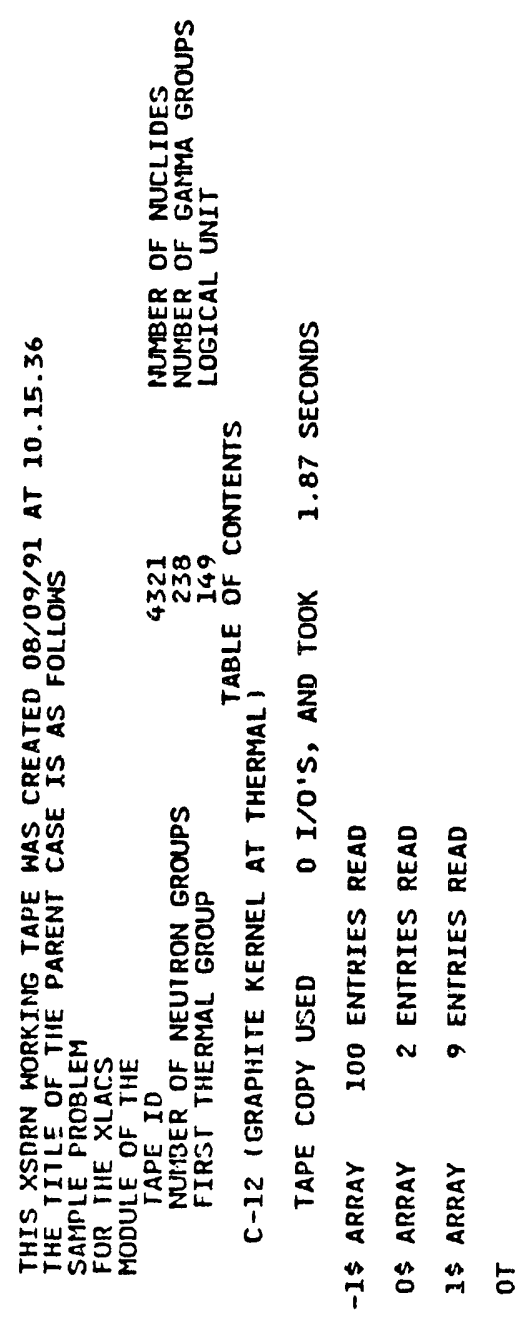




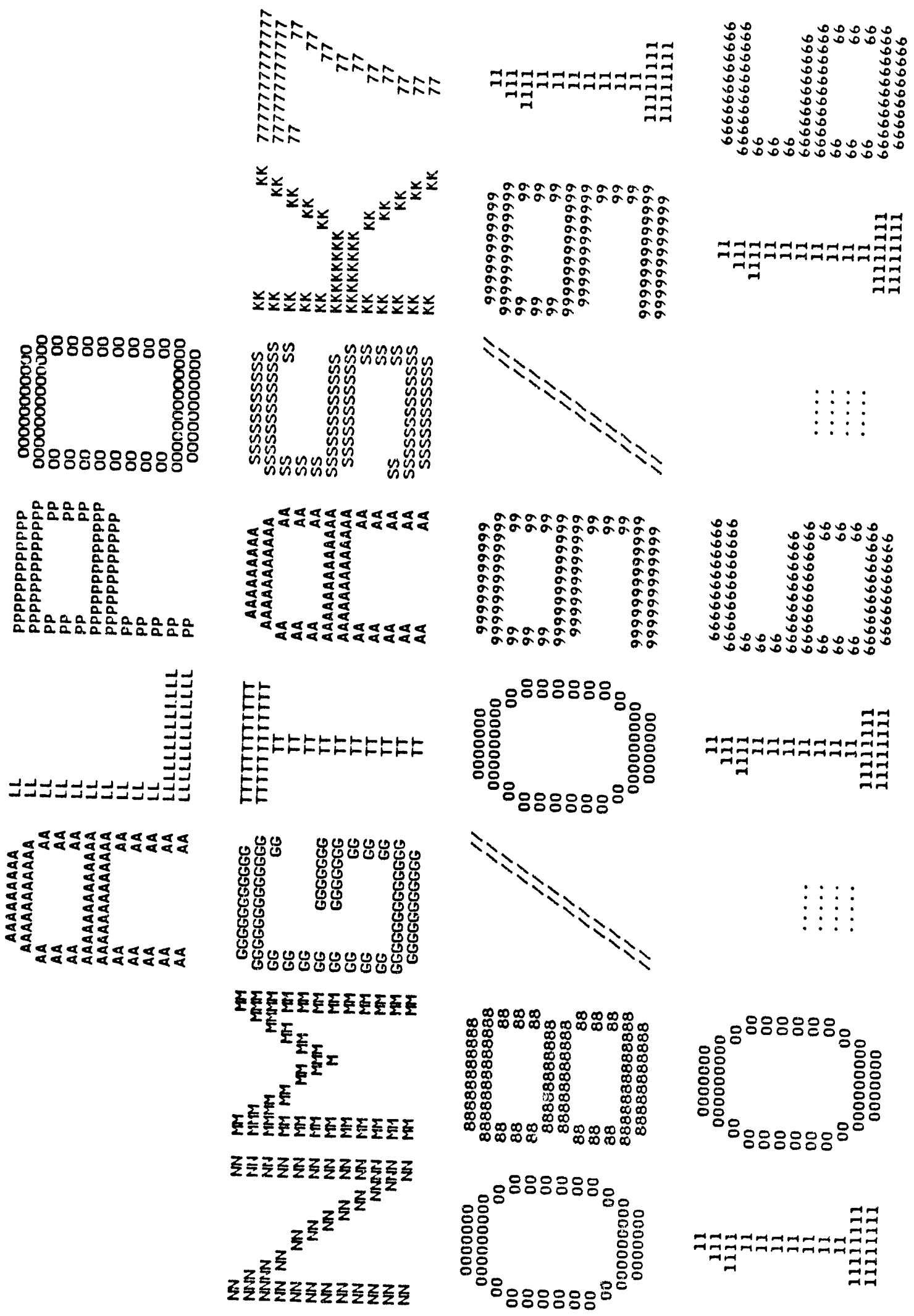




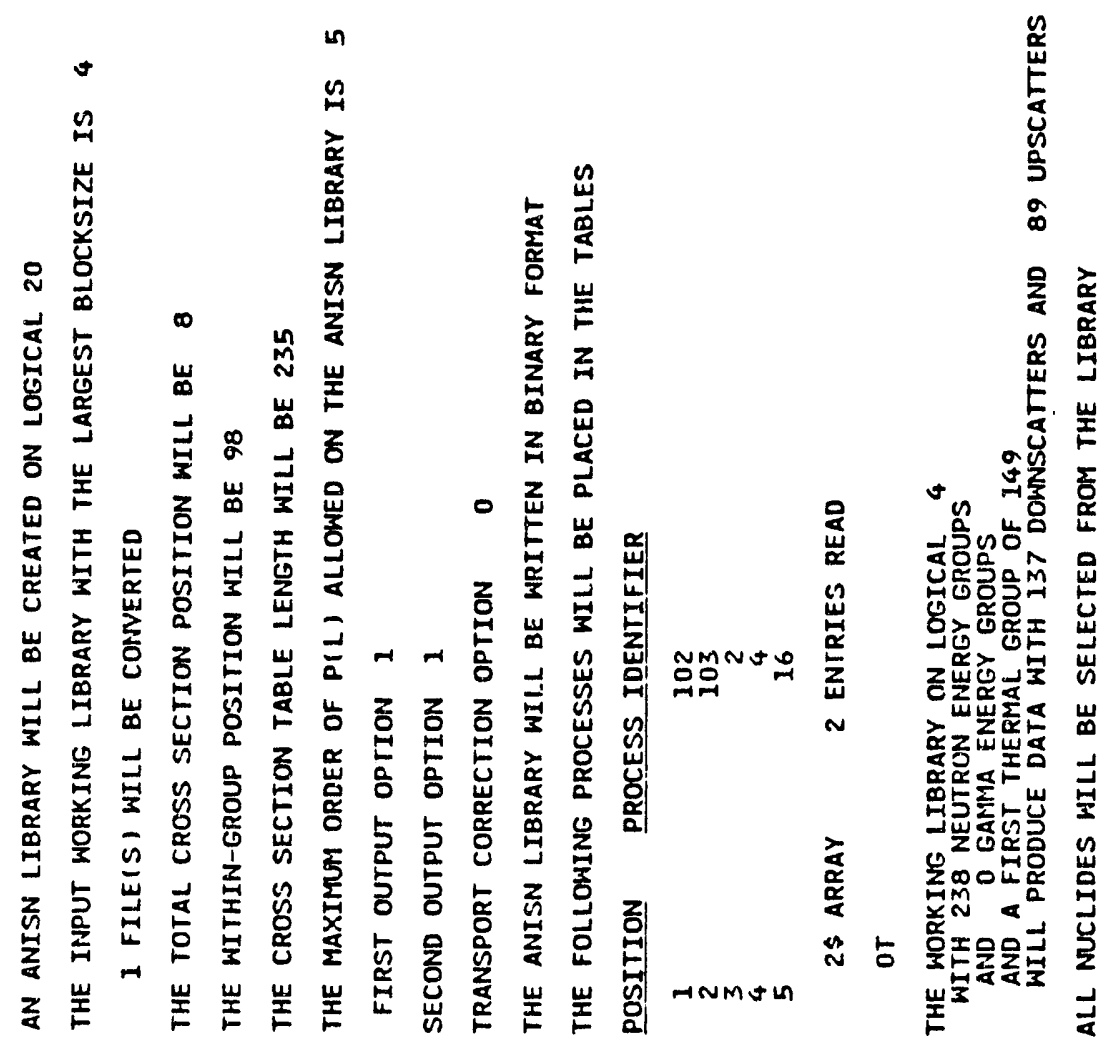




$$
\begin{aligned}
& \begin{array}{l}
\frac{3}{2} \\
\frac{4}{8} \\
0
\end{array} \\
& \text {. } \frac{13}{4}
\end{aligned}
$$

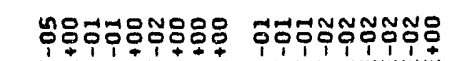

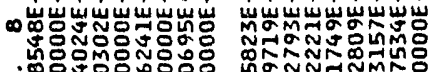

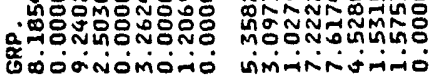

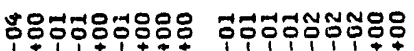

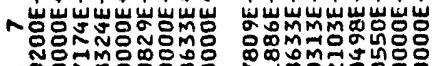

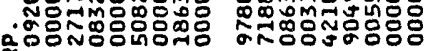

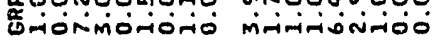

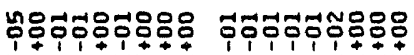

-

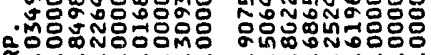

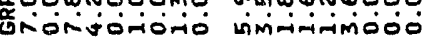

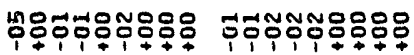

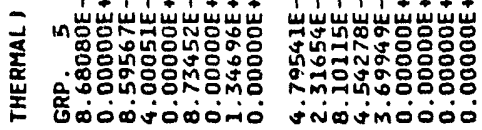

-

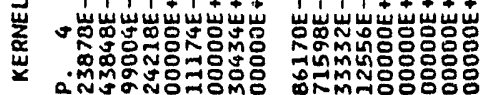

w

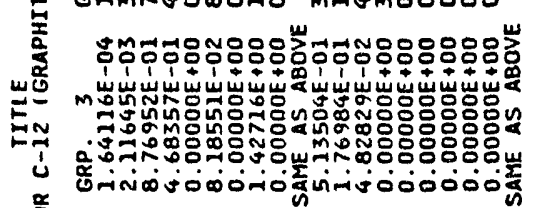

总

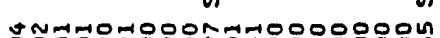

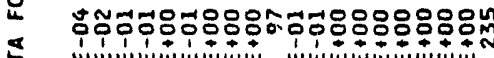

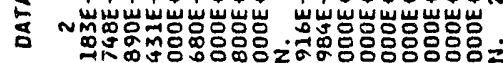

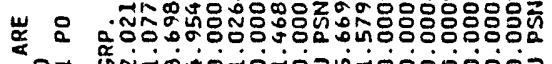

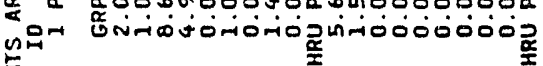

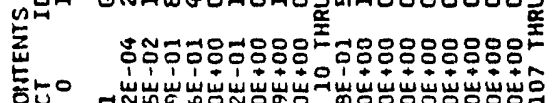

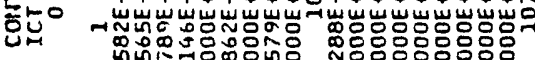

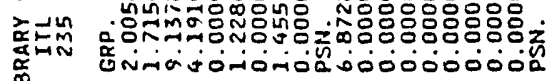

至

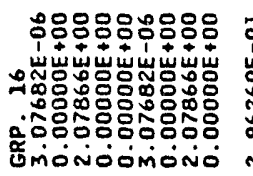

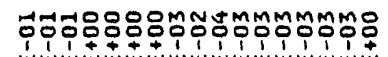

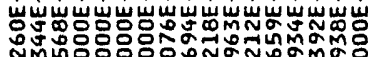

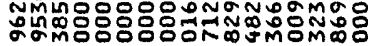

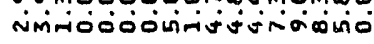

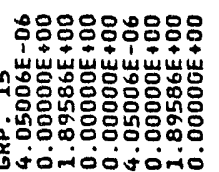

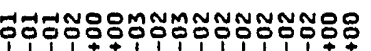

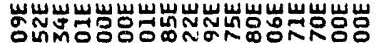

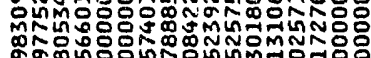

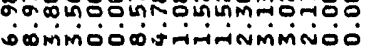

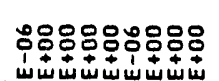

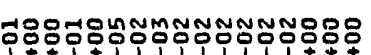

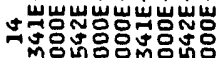

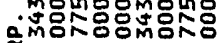

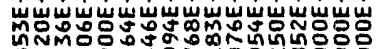

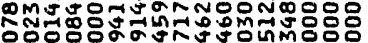

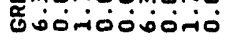

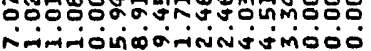

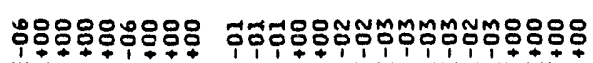

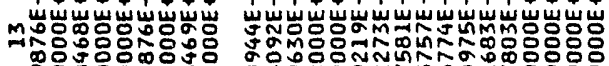
. Nơํํำ

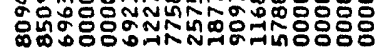

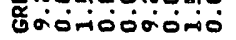

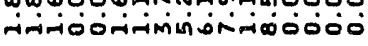

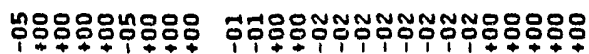

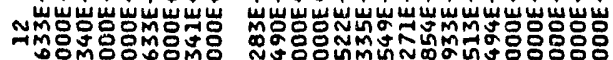

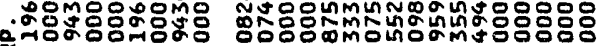

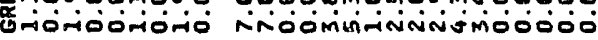

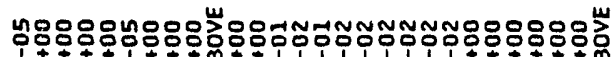

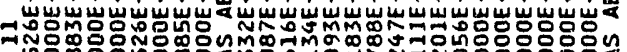
Nombon. ơ

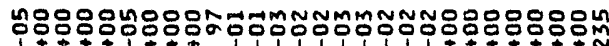

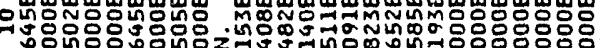

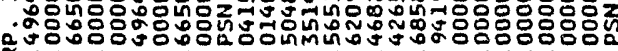

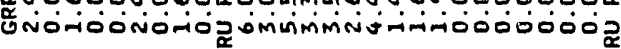

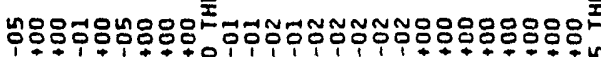

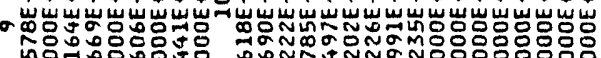
.

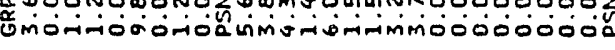

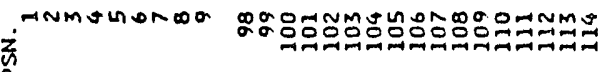

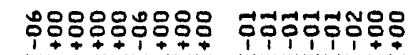

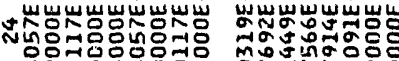
คํํำำดัด ơ

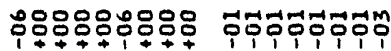

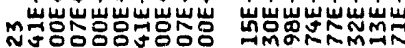

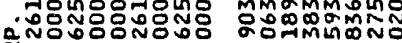

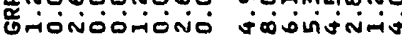

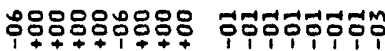

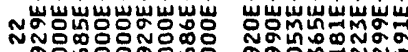

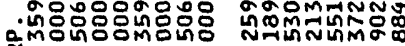

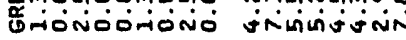

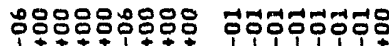

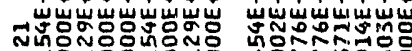

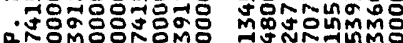
Éronioorionio frivinivio

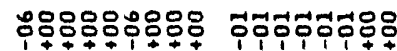

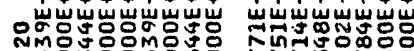

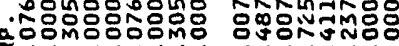

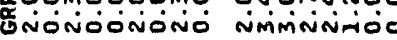

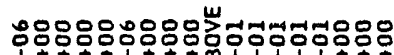

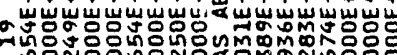
în.

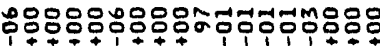

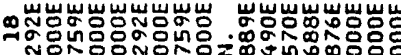

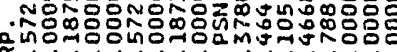

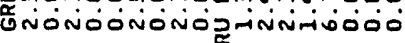

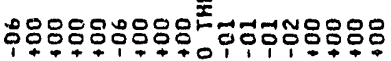

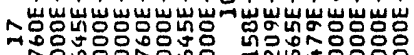

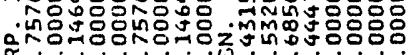

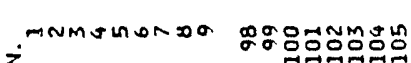
$i_{n}$

\section{SAMPLE PROBLEMS}




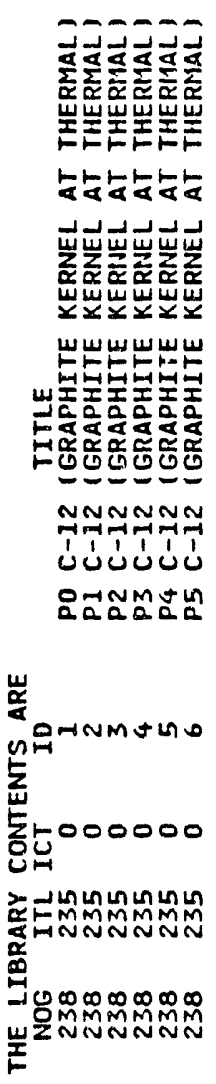




\section{FIDO (ANISN) INPUT SYSTEM}

The FIDO (Flexible Input Data Option) input method is designed to allow the entering or modifying of large data arrays with minimum effort. Special advantage is taken of patterns of repetition or symmetry wherever possible. The FIDO system was patterned after the input method used with the FLOCO coding system at Los Alamos and was first applied to the DTF-II code. Since that time, numerous features requested by users have been added, a free-field option has been developed, and the application of FIDO has spread to innumerable codes.

The data are entered in units called "arrays." An array comprises a group of contiguous storage locations which are to be filled with data at one time. In the AMPX input instructions (Sect. 3.INSTRUCTIONS), the number of entries in each array is denoted within []'s. The arrays usually correspond on a one-to-one basis with FORTRAN arrays used in the program. A group of one or more arrays read with a single call to the FIDO package forms a "block," and the special delimiter, "T," is required to signify the end of each block. Arrays within a block may be read in any order with respect to each other, but an array belonging to one block must not be shifted to another. The same array can be filled with "0" using a special option, and then a few scattered locations could be changed by reading in a new set of data for that array. If no entries to the arrays in a block are required, the delimiter alone satisfies the input requirement.

To facilitate data preparation, many "default" data values have been programmed into the AMPX modules. Where such values exist, the values are denoted in Sect. 3.INSTRUCTIONS within ( )'s. The user can always override the "default" value by entering a data value in the appropriate array.

Three major types of input are available: fixed-field input, free-field input, and user-field input.

\section{FIDO.1 FIXED-FIELD INPUT}

Each card is divided into six 12-column data fields, each of which is divided into three subfields. The following sketch illustrates a typical data field. The three subfields always comprise 2,1 , and 9 columns, respectively.

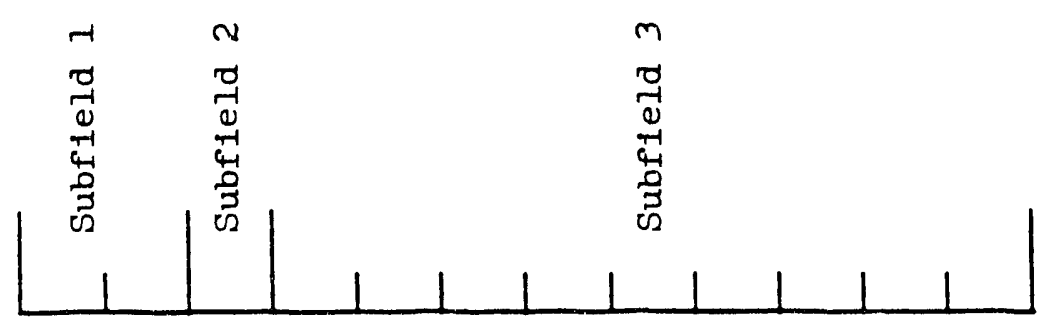


To begin the first array of a block, an array originator field is placed in any field on a card:

Subfield 1: An integer array identifier < 100 specifying the data array to be read in

Subfield 2: An array-type indicator:

"\$" if the array is integer data

"*" if the array is real data

Subfield 3: Blank

Data are then placed in successive fields until the required number of entries has been satisfied. A sample data sheet, shown on the next page, illustrates this input.

In entering data, it is convenient to think of an "index" or "pointer" that is under control of the user, and which specifies the position in the array into which the next data entry is to go. The pointer is always positioned at array location No. 1 by entering the array criginator field. The pointer subsequently moves according to the data operator chosen. Blank fields are a special case in that they do not cause any data modification and do not move the pointer.

A data field has the following form:

Subfield 1: The data numerator, an integer $<100$. We refer to this entry as $N_{1}$ in the following discussion.

Subfield 2: One of the special data operators listed below.

Subfield 3: A nine-character data entry, to be read in F9.0 format. It will be converted to an integer if the array is a "\$" array or if a special array operator such as $Q$ is being used. Note that an exponent is permissible but not required. Likewise, a decimal is permissible bitt not required. If no decimal is supplied it is assumed to be immediately to the left of the exponent, if any, and otherwise to the right of the last column. This entry is referred to as $\mathrm{N}_{3}$ in the following discussion.

A list of data operators and their effect on the array being input follows:

The data operator field is blank when one wants to make a single entry of data. The data entry in the third subfield is entered in the location indicated by the pointer, and the pointer is advanced by one. If the data portion in $\mathrm{N}_{3}$ is blank, the field is ignored.

A "+" or "-" indicates exponentiation. The data entry in the third field is multiplied by $10^{ \pm N_{1}}$, where $N_{1}$ is the data numerator in the first subfield, and the sign is in the data operator field. The pointer advances by one. In cases where an exponent is needed, this option allows the entering of more significant figures than the blank option.

Note that the use of " $\&$ " for a " + " is no longer allowed.

An " $R$ " indicates that the data entry is to be repeated $N_{1}$ times. The pointer advances by $\mathrm{N}_{1}$. 


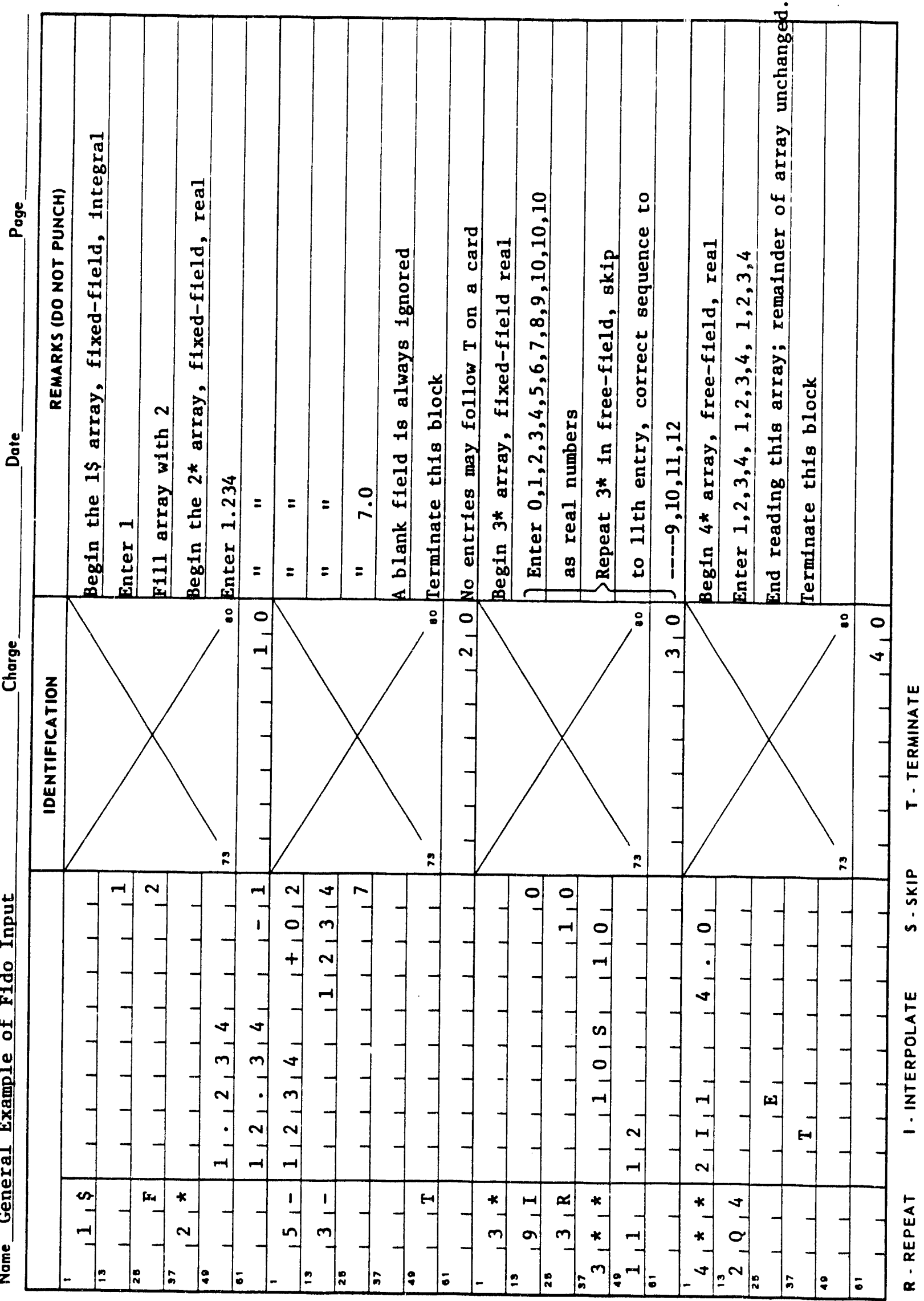

FIDO 
An " $\mathrm{I}$ " indicates linear interpolation. The data numerator, $N_{1}$, indicates the number of interpolated points to be supplied. The data entry in the third subfield is entered, followed by $\mathrm{N}_{1}$ interpolated entries equally spaced between that value and the data entry found in the third subfield of the next nonblank field. The pointer is advanced by $\mathrm{N}_{1}+1$. The field following an "I" field is then processed normally, according to its own data operator. The "I" entry is especially valuable for specifying a spatial mesh. In "\$" arrays, interpolated values will be rounded to the nearest integer.

An " $L$ " indicates logarithmic interpolation. The effect is the same as that of "I," except that the resulting data are cvenly separated in log-space. This method is especially convenient for specifying energy meshes that use equal lethargy bins.

$A$ " $Q$ " is used to repeat sequences of numbers. The length of the sequence is given by the third subfield, $N_{3}$. The sequence of $N_{3}$ entries is to be repeated $N_{1}$ times. The pointer advances by $N_{1}{ }^{*} N_{3}$. If either $N_{1}$ or $N_{3}$ is 0 , then a sequence of $N_{1}+$ $\mathrm{N}_{3}$ is repeated one time only, and the pointer advances by $\mathrm{N}_{1}+\mathrm{N}_{3}$. This feature is especially valuable for geometry specification.

The "N" option has the same effect as the " $Q$," except that the order of the sequence is reversed each time it is entered. This feature is valuable for the type of symmetry possessed by $S_{n}$ quadrature coefficients.

$A n$ " $M$ " has the same effect as " $N$," except that the sign of each entry in the sequence is reversed each time the sequence is entered. For example, the entries

$\begin{array}{llll}1 & 2 & 3 & 2 \mathrm{M} 2\end{array}$

would be equivalent to

$\begin{array}{llllllll}1 & 2 & 3 & -3 & -2 & 2 & 3 .\end{array}$

This option is also useful in entering quadrature coefficients. $\mathrm{N}_{3}$.

A " $Z$ " causes $N_{1}+N_{3}$ locations to be set to 0 . The pointer is advanced by $N_{1}+$

A " $\mathrm{C}$ " causes the position of the last array item entered to be printed. This is the position of the pointer, less 1 . The pointer is not moved.

An "O" causes the print trigger to be changed. The trigger is originally off. Successive " $O$ " fields turn it on and off alternately. When the trigger is on, each card image is listed as it is read.

An " $S$ " indicates that the pointer is to skip $N_{1}$ positions leaving those array positions unchanged. If the third subfield is blank, the pointer is advanced by $N_{1}$. If the third subfield is nonblank that data entry is entered following the skip, and the pointer is advanced by $\mathrm{N}_{1}+1$.

An "A" moves the pointer to the position, $N_{3}$, specified in the third subfield.

An " $F$ " fills the remainder of the array with the datum entered in the third subfield.

An " $E$ " skips over the remainder of the array. The array length criterion is always satisfied by an $E$, no matter how many entries have been specified. No more entries to an array may be given following an "E," except that data entry may be restarted with an "A." 
The reading of data to an array is terminated when a new array origin field is supplied or when the block is terminated. If an incorrect number of positions has been filled, an error edit is given, and a flag is set which will later abort execution of the problem. FIDO then continues with the next array if an array origin was read. Otherwise, it returns control to the calling program.

A block termination consists of a field having " $\mathrm{T}$ " in the second subfield. All entries following " $T$ " on a card are ignored, and control is returned from FIDO to the calling program.

Comment cards can be entered within a block by placing an apostrophe (') in column 1. Then columns $2-80$ will be listed, with column 2 being used for printer carriage control. Such cards have no effect on the data array or pointer.

\section{FIDO.2 FREE-FIELD INPUT}

With free-field input, data are witten without fixed restrictions as to field and subfield size and positioning on the card. The options used with fixed-field input are available, although some are slightly restricted in form. Data arrays using fixed- and free-field input can be intermingled at will within a given block.

In general, the free-field form of input is much easier to use and to read.

The concept of three subfields per field is still applicable to free-field input, but if no entry for a field is required, no reserve space is needed. Only columns 1-72 may be used, as with fixed-field input.

The array originator field can begin in any position. The array identifiers and type indicators are used as a fixed-field input. The type indicator is entered twice, to designate free-field input (i.e., "\$\$" or "**"). The blank third subfield required in fixed-field input is not required. For example:

$31^{* *}$

indicates that array 31, a real-data array, will follow in free-field format. A "\#\#" type indicator can be used to input real double-precision arrays.

Data fields may follow the array origin field immediately. The data field entries are identical to the fixed-field entries with the following restrictions:

1. Any number of blanks may separate fields, but at least one blank must follow a third subfield entry if one is used.

2. If both first- and second-subfield entries are used, no blanks may separate them (i.e., $24 \mathrm{~S}$, but not $24 \mathrm{~S}$ ).

3. Numbers written with exponents must not have imbedded blanks (i.e., $0.0 \mathrm{E}+4$, $1.0 \mathrm{E} 4$, or even $1+4$, but not $1.0 \mathrm{E} 4$ ).

4. In third-subfield data entries, only 9 digits, including the decimal but not including the exponent field, can be used (i.e., 123456.89E07, but not 123456.789E07).

5. The Z entry must be of the form $738 \mathrm{Z}$, not $\mathrm{Z} 738$, or $738 \mathrm{Z}$.

6 . The + or - data operators are not needed and are not available.

7. The $Q, N$, and $M$ entries are restricted: $3 Q 4,1 N 4$, or $M 4$, but not $4 Q, 4 N$, or $4 M$. 


\section{FIDO.3 USER-FIELD INPUT}

If the user follows the array identifier in the array originator field with the character "U" or "V," the input format is to be specified by the user. If " $U$ " is specified, the FORTRAN format to be used must be supplied in columns 1-72 of the next card. The format must be enclosed by the usual parentheses. Then the data for the entire array must follow on successive cards. The rules of ordinary FORTRAN input as to exponients, blanks, etc., apply. If the array data do not fill the last card, the remainder must be left blank.

A "V" has the same effect as a " $U$ " except that the format read in the last preceding "U," array is used. 


\section{INTERNAL DISTRIBUTION}

1. D. M. D'Aquila

2. C. W. Alexander

3. J. W. Arwood

4. J. M. Barnes

5. S. M. Bowman

6. B. I. Broadhead

7. J. A. Bucholz

8. R. L. Childs

9. S. N. Cramer

10. E. C. Crume

11. R. D. Dabbs

12. L. Deleanu

13. F. C. Difillippo

14. H. L. Dodds, Jr.

15. M. B. Emmett

16. W. W. Engle

17. G. F. Flanagan

18-19. W. E. Ford III (2)

20. V. M. Forsberg

21. P. B. Fox

22-23. N. M. Greene (2)

24. G. R. Handley

25. O. W. Hermann

26. D. M. Hetrick

27. L. M. Hively

28. C. M. Hopper

29. D. T. Ingersoll

30. J. C. Ingram

31. J. O. Johnson

32. W. C. Jordan

33. F. B. Kam

34. H. T. Kerr

35. N. F. Landers

36. N. M. Larson

37. R. P. Leinius

38. R. A. Lillie

39. S. B. Ludwig

40. R. E. Maerker
41. J. B. Manneschmidt

42. J. F. Manneschmidt

43. S. K. Martin

44. L. C. Masters

45. D. Mueller

46. L. F. Norris

47. J. V. Pace

48-52. C. V. Parks (5)

53. L. M. Petrie, Jr.

54. R. T. Primm

55. D. A. Reed

56. J.-P. Renier

57. W. A. Rhoades

58. R. C. Robinson

59. R. B. Rothrock

60. R. W. Roussin

61. J. C. Ryman

62-64. C. H. Shappert (3)

65. C. O. Slater

66. O. L. Smith

67. M. S. Smith

68. G. R. Smolen

69. R. V. Stachowiak

70. C. A. Sweet

71. J. S. Tang

72. J. C. Turner

73. M. W. Waddell

74. R. M. Westfall

75. J. E. White

76. G. E. Whitesides

77. L. R. Williams

78. B. A. Worley

79. R. Q. Wright

80-104. Radiation Shielding Information Ctr.

105. Laboratory Records Dept.

106. Laboratory Records, ORNL-RC

107. Document Reference Section

108. Central Research Library

109. ORNL Patent Section 


\section{EXTERNAL DISTRIBUTION}

110. Office of Assistant Manager for Energy Research and Development, DOE Field Office, Oak Ridge, P.O. Box 2001, Oak Ridge, Tenn. 37831

111-112. Office of Scientific and Technical Information, P.O. Box 2001, Oak Ridge, Tenn. 37831

113. Dr. E. D. Arthur, T-DO, MS B-210, Los Alamos National Laboratory, Los Alamos, N.M. 87545

114. Dr. Alan M. Baxter, Reactor Engineering, General Atomics, 3550 General Atomics Court, P.O. Box 85608, San Diego, Calif. 92138-5608

115. Dr. Martin J. Berger, Center for Radiation Research, Photon and Charged Particle Data Center, National Institute of Standards and Technology, Gaithersburg, Md. 20878

116. Mr. Sam E. Berk, Office of Energy Research, Reactors and Systems Radiation, ER-5333, U.S. Department of Energy, Washington, DC 20585

117. Dr. R. C. Block, Dept. of Nuclear Engineering, Rensselaer Polytechnic Institute, Troy, N.Y. 12180

118. Dr. M. C. Brady, Sandia National Laboratories, P.O. Box 5800, Division 6643, Albuquerque, N.M. 87185

119. Dr. Robert Breen, Bldg. 3, Electric Power Research Institute, P.O. Box 10412, Palo Alto, Calif. 94303

120. Dr. Allan D. Carlson, RADP C311, National Institute of Standards and Technology, Gaithersburg, Md. 20878

121. Dr. Edward T. Cheng, Suite 110, TSI Research Corp., 225 Stevens Ave., Solana Beach, Calif. 92075

122. Dr. Charles Cowan, MS S15, Advanced Nuclear Technology, General Electric Co., 6835 Visa Del Oro, San Jose, Calif. 95119

123. Dermit E. Cullen, Lawrence Livermore National Laboratory, Livermore, Calif. 94551

124. Dr. Ulrich Decher, GC-28, Combustion Engineering, Inc., 1000 Prospect Hill Rd., P.O. Box 500, Windsor, Conn. 06095

125. Dr. Piet de Leege, Delft University of Technology, Interfaculty Reactor Institute, Mekelweg 15, NL-2629-JB Delft, The Netherlands

126. Dr. Charles L. Dunford, Dept. of Nuclear Energy, National Nuclear Data Center, Bldg. 197-D, Brookhaven National Laboratory, Upton, N.Y. 19973

127. Mr. Colin Durston, Nuclear Fuel Division, MS MOB 3-2, Westinghouse Electric Corp., P.O. Box 3912, Pittsburgh, Pa. 15230

128. Dr. Talmadge R. England, T2, MS B-243, Los Alamos National Laboratory, Los Alamos, N.M. 87545

129. Dr. Richard B. Firestone, Isotopes Project, Bldg. 50-A, Rm. 6102, Lawrence Berkeley Laboratory, University of California, Berkeley, Calif. 94720

130. Dr. Donald G. Gardner, Div. of Nuclear Chemistry, MS L-234, Lawrence Livermore National Laboratory, Livermore, Calif. 94551

131. Dr. Hans Gruppelaar, ECN, Petten, Physics Dept., Netherlands Energy Research Foundation, ECN, P.O. Box 1, 1755 ZG, Petten NH, The Netherlands

132. Mr. Philip B. Hemmig, Advanced Reactor Technology, NE-542, GTN, U.S. Department of Energy, Washington, D.C. 20585

133. Dr. Norman E. Holden, Department of Nuclear Energy, Bldg. 750, Brookhaven National Laboratory, Upton, N.Y. 11973

134. Mr. Alf Jonsson, Dept. 5318-2403, Combustion Engineering, Inc., 1000 Prospect Hill Rd., P.O. Box 500, Windsor, Conn. 06095 
135. Dr. Albert Kahler, MS ZAP 34-F, Bettis Atomic Power Laboratory, P.O. Box 79, West Mifflin, Pa. 15122

136. Dr. Cecil R. Lubitz, Bldg. F-16, Knolls Atomic Power Laboratory, P.O. Box 1072, Schenectady, N.Y. 12301

137. Dr. Robert MacFarlane, T2, MS B-243, Los Alamos National Laboratory, Los Alamos, N.M. 87545

138. Dr. Fred M. Mann, HO-36, Hanford Engineering Development Laboratory, P.O. Box 1970, Richland, Wash. 99352

139. Dr. F. J. McCrosson, Bldg. 773-41A, Savannah River Laboratory, Aiken, SC 29808

140. Dr. R. D. McKnight, Bldg. 208, Argonne National Laboratory, 9700 S. Cass Ave., Argonne, IL 60439

141. Ms. Victoria McLane, Dept. of Nuclear Energy, National Nuclear Data Center, Bldg. 197 D, Brookhaven National Laboratory, Upton, N.Y. 19973

142. Dr. Michael S. Milgram, Dept. of Reactor Physics, Chalk River Nuclear Laboratory, Atomic Energy of Canada Ltd., Chalk River, Ontario, KOJ 1JO CANADA

143. Dr. G. C. Panini, ENEA, TIB/FICS/DATINV, via Ercolani 8, 40138 Bologna, Italy

144. Dr. Sol Pearlstein, Dept. of Nuclear Energy, National Nuclear Data Center, Bldg. 197-D, Brookhaven National Laboratory, Upton, N.Y. 19973

145. Dr. Sterrett T. Perkins, MS L-298, Lawrence Livermore National Laboratory, Livermore, Calif. 94551

146. Dr. W. P. Poenitz, Div. of Engineering Physics, Argonne National Laboratory-West, P.O. Box 2528, Idaho Falls, Id. 83403-2528

147. Dr. John M. Ryskamp, Engineering Laboratory, MS 3515, EG\&G Idaho, Inc., P.O. Box 1625, Idaho Falls, Id. 83415

148. Mr. Joseph Sapir, MS B-220, Los Alamos National Laboratory, Los Alamos, N.M. 87545

149. Dr. Enrico Sartori, NEA Data Bank, 91191 Gif-sur-Yvette Cedex, France

150. Dr. Massimo Salvatores, Commissariat a l'Energie Atomique, Centre d'Etudes Nucleaires de Cadarache, DRNR/SPCI/LEPh, 13108 St. Paul lez Durance Cedex, France

151. Dr. Robert Schenter, HO-36, Isotope Production, Hanford Engineering Development Laboratory, P.O. Box 1970, Richland, Wash. 99352

152. Dr. Robert E. Seamon, Group X-6, MS B-226, Los Alamos National Laboratory, Los Alamos, N.M. 87545

153. Dr. Alan Bowen Smith, APD-316, Argonne National Laboratory, 9700 S. Cass Ave., Argonne, Ill. 60439

154. Dr. Eugene Specht, MS HB23, Rockwell International Corp., 6633 Canoga Ave., Canoga Park, Calif. 91303

155. Dr. Stanley L. Whetstone, Div. of Nuclear Physics, ER-23, GTN, U.S. Department of Energy, Washington, D.C. 20585

156. Dr. Roger M. White, Dept. of Physics, MS L-298, Lawrence Livermore National Laboratory, Livermore, Calif. 94551

157. Prof. Mark Williams, Nuclear Science Center, Louisiana State University, Baton Rouge, La. 70803

158. Dr. W. A. Wittkopf, Div. of Fuel Engineering, Babcock \& Wilcox Co., P.O. Box 10935, 3315 Old Forest Rd., Lynchburg, Va. 24506

159. Dr. Phillip G. Young, Group T-2, MS B-243, Los Alamos National Laboratory, Los Alamos, N.M. 87545

160. Dr. Mahmoud Youssef, School of Engineering and Applied Science, 6288 Boelter Hall, University of California, Los Angeles, Calif. 90024 

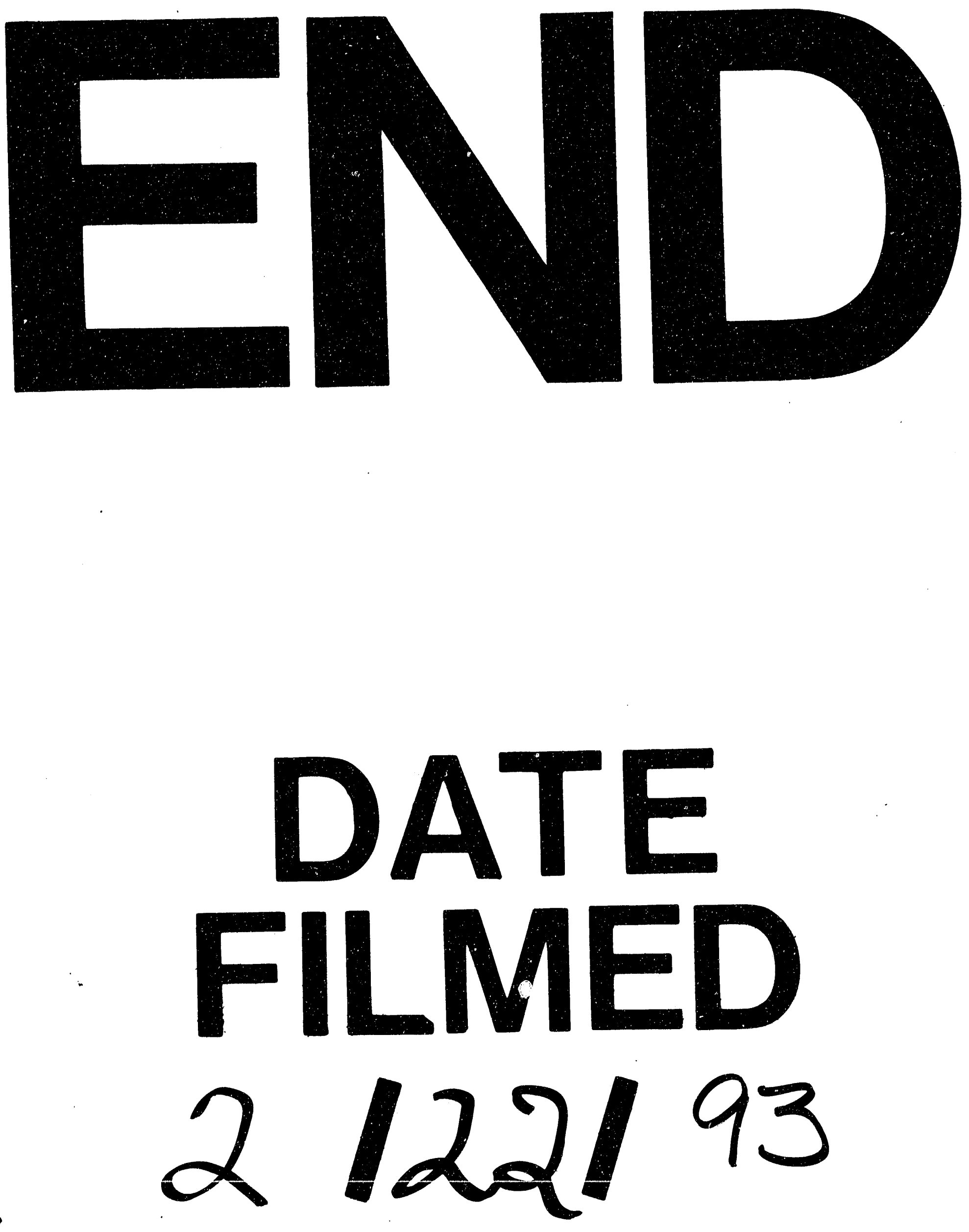
